\title{
Medication Therapy Management
}

\section{Prepared for:}

Agency for Healthcare Research and Quality

U.S. Department of Health and Human Services

540 Gaither Road

Rockville, MD 20850

www.ahrq.gov

\section{Contract No. xxx-xx-xxxx}

Prepared by:

$<$ Name $>$ Evidence-based Practice Center

$<$ City, State $>$

Investigators:

First and Last Names, X.X.

First and Last Names, X.X.

\section{AHRQ Publication No. xx-EHCxxx}

\section{$<$ Month Year $>$}


This report is based on research conducted by the XXXXX Evidence-based Practice Center (EPC) under contract to the Agency for Healthcare Research and Quality (AHRQ), Rockville, MD (Contract No. XXX-20XX-XXXXX). The findings and conclusions in this document are those of the authors, who are responsible for its contents; the findings and conclusions do not necessarily represent the views of AHRQ. Therefore, no statement in this report should be construed as an official position of AHRQ or of the U.S. Department of Health and Human Services.

The information in this report is intended to help health care decisionmakers - patients and clinicians, health system leaders, and policymakers, among others - make well informed decisions and thereby improve the quality of health care services. This report is not intended to be a substitute for the application of clinical judgment. Anyone who makes decisions concerning the provision of clinical care should consider this report in the same way as any medical reference and in conjunction with all other pertinent information, i.e., in the context of available resources and circumstances presented by individual patients.

This report may be used, in whole or in part, as the basis for development of clinical practice guidelines and other quality enhancement tools, or as a basis for reimbursement and coverage policies. AHRQ or U.S. Department of Health and Human Services endorsement of such derivative products may not be stated or implied.

This report may periodically be assessed for the urgency to update. If an assessment is done, the resulting surveillance report describing the methodology and findings will be found on the Effective Health Care Program website at: www.effectivehealthcare.ahrq.gov. Search on the title of the report.>

This document is in the public domain and may be used and reprinted without special permission. Citation of the source is appreciated.

Persons using assistive technology may not be able to fully access information in this report. For assistance contact [insert program email address].

None of the investigators has any affiliations or financial involvement that conflicts with the material presented in this report.

$<$ Add a suggested citation of your report: $>$

$<$ Standard citation:>

Suggested citation: $<$ Authors $>$. $<$ Topic in Title Caps $>$. Evidence Report/Technology

Assessment No. $<\#>$. (Prepared by the $<$ EPC Name $>$ Evidence-based Practice Center under Contract No. <\#\#>.) AHRQ Publication No. XX-EHCXXX-EF. Rockville, MD: Agency for Healthcare Research and Quality. $<$ Month Year>. www.effectivehealthcare.ahrq.gov. 


\section{Preface}

The Agency for Healthcare Research and Quality (AHRQ), through its Evidence-based Practice Centers (EPCs), sponsors the development of systematic reviews to assist public- and private-sector organizations in their efforts to improve the quality of health care in the United States. These reviews provide comprehensive, science-based information on common, costly medical conditions, and new health care technologies and strategies.

Systematic reviews are the building blocks underlying evidence-based practice; they focus attention on the strength and limits of evidence from research studies about the effectiveness and safety of a clinical intervention. In the context of developing recommendations for practice, systematic reviews can help clarify whether assertions about the value of the intervention are based on strong evidence from clinical studies. For more information about AHRQ EPC systematic reviews, see www.effectivehealthcare.ahrq.gov/reference/purpose.cfm

AHRQ expects that these systematic reviews will be helpful to health plans, providers, purchasers, government programs, and the health care system as a whole. Transparency and stakeholder input are essential to the Effective Health Care Program. Please visit the Web site (www.effectivehealthcare.ahrq.gov) to see draft research questions and reports or to join an email list to learn about new program products and opportunities for input.

We welcome comments on this systematic review. They may be sent by mail to the Task Order Officer named below at: Agency for Healthcare Research and Quality, 540 Gaither Road, Rockville, MD 20850, or by email to epc@ahrq.hhs.gov.

Richard Kronick, Ph.D.

Director, Agency for Healthcare Research and Quality

Stephanie Chang M.D., M.P.H. Director, EPC Program

Center for Outcomes and Evidence

Agency for Healthcare Research and Quality
Jean Slutsky, P.A., M.S.P.H.

Director, Center for Outcomes and Evidence

Agency for Healthcare Research and Quality

Carmen Y. Kelly, Pharm.D., M.P.H., R.Ph.

Task Order Officer

Center for Outcomes and Evidence

Agency for Healthcare Research and Quality

\section{Acknowledgments}

The authors gratefully acknowledge the following individuals for their contributions to this project: $<$ Acknowledgments $>$. 


\section{Key Informants}

In designing the study questions, the EPC consulted several Key Informants who represent the end-users of research. The EPC sought the Key Informant input on the priority areas for research and synthesis. Key Informants are not involved in the analysis of the evidence or the writing of the report. Therefore, in the end, study questions, design, methodological approaches, and/or conclusions do not necessarily represent the views of individual Key Informants.

Key Informants must disclose any financial conflicts of interest greater than $\$ 10,000$ and any other relevant business or professional conflicts of interest. Because of their role as end-users, individuals with potential conflicts may be retained. The TOO and the EPC work to balance, manage, or mitigate any conflicts of interest.

The list of Key Informants who participated in developing this report follows:

$<$ Name $>$

$<$ Place $>$

$<$ City $>,<$ ST $>$

\section{Technical Expert Panel}

In designing the study questions and methodology at the outset of this report, the EPC consulted several technical and content experts. Broad expertise and perspectives were sought. Divergent and conflicted opinions are common and perceived as healthy scientific discourse that results in a thoughtful, relevant systematic review. Therefore, in the end, study questions, design, methodologic approaches, and/or conclusions do not necessarily represent the views of individual technical and content experts.

Technical Experts must disclose any financial conflicts of interest greater than $\$ 10,000$ and any other relevant business or professional conflicts of interest. Because of their unique clinical or content expertise, individuals with potential conflicts may be retained. The TOO and the EPC work to balance, manage, or mitigate any potential conflicts of interest identified.

The list of Technical Experts who participated in developing this report follows:

$$
\begin{aligned}
& <\text { Name }> \\
& <\text { Place }> \\
& <\text { City }>,<\text { ST }> \\
& <\text { Name }> \\
& <\text { Place }> \\
& <\text { City }>,<\text { ST }>
\end{aligned}
$$

\section{Peer Reviewers}

Prior to publication of the final evidence report, EPCs sought input from independent Peer Reviewers without financial conflicts of interest. However, the conclusions and synthesis of the scientific literature presented in this report does not necessarily represent the views of individual reviewers.

Peer Reviewers must disclose any financial conflicts of interest greater than $\$ 10,000$ and any other relevant business or professional conflicts of interest. Because of their unique clinical or content 
expertise, individuals with potential non-financial conflicts may be retained. The TOO and the EPC work to balance, manage, or mitigate any potential non-financial conflicts of interest identified.

The list of Peer Reviewers follows:

$<$ Name $>$

$<$ Place $>$

$<$ City $>,<$ ST $>$

$<$ Name $>$

$<$ Place $>$

$<$ City $>,<$ ST $>$ 


\section{Medication Therapy Management}

\section{Structured Abstract}

Objectives: To describe intervention components and implementation features (Key Question 1 [KQ 1]) for medication therapy management (MTM); assess the effectiveness of MTM on intermediate, patient-centered, or resource utilization outcomes (KQ 2); identify intervention features (KQ 3) and patient characteristics (KQ 4) that moderate the effect of an intervention on outcomes; and assess harms associated with interventions (KQ 5).

Data Sources: MEDLINE, Cochrane Library, International Pharmaceutical Abstracts (IPA), grey literature, additional studies from reference lists and technical experts.

Review Methods: Two trained reviewers selected, extracted data from, and rated the risk of bias of relevant trials and systematic reviews. We used random-effects models to estimate pooled effects for outcomes with three or more similar studies with a low or medium risk of bias. For other outcomes, we synthesized the data qualitatively.

Results: We included 36 eligible studies (19 randomized controlled trials, 3 controlled clinical trials, and 14 cohort studies) reported in 42 articles. Evidence was insufficient on the effect of MTM on most outcomes. For a limited number of outcomes, we found evidence that MTM results in improvement when compared with usual care (low strength). Specifically, these outcomes include medication appropriateness, the rate of hospitalization among heart failure patients with home medicines review, and the use of generic medications for patients receiving MTM from community pharmacy when compared with educational mailings.

Similarly, we found sufficient evidence to conclude that MTM conferred no benefit for a limited number of outcomes. When MTM is implemented in settings with a broad range of patients, it does not reduce the number of hospitalizations (low strength of evidence). MTM does not improve most measures of health-related quality of life (low strength of evidence).

We found evidence on four intervention components and intervention features: one study provided information on each feature and yielded insufficient evidence for most outcomes with two exceptions. MTM programs with pharmacist access to brief clinical summaries from the medical record reduced the mean number of adverse drug events when compared with basic MTM programs without such access (low strength of evidence). Community pharmacists increase the generic dispensing ratio more than call-center-based pharmacists (low strength of evidence). Similarly, the evidence on harms associated with MTM was limited to one study each on confusion and inconvenience and was rated as insufficient.

Conclusions: The evidence base is insufficient to address the effectiveness of MTM on most outcomes. Given the widespread implementation of MTM and urgent need for actionable information, funders may wish to weigh the relative value of information on overall effectiveness, effectiveness of implementation features, and program implementation and accountability when commissioning new research. 


\section{Contents}

Executive Summary

ES-1

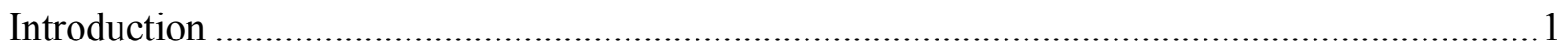

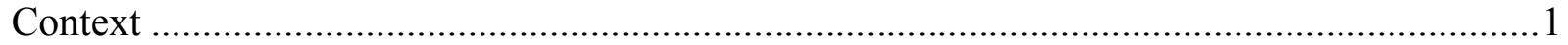

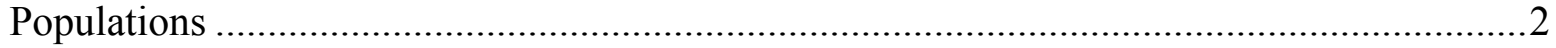

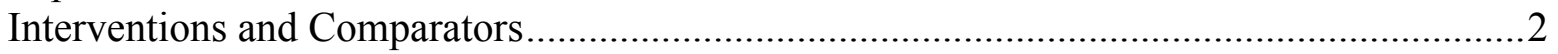

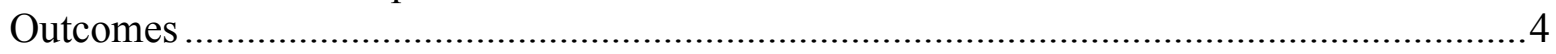

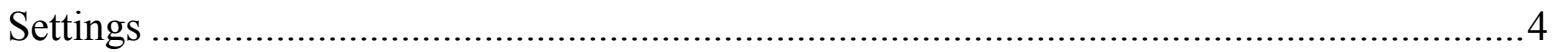

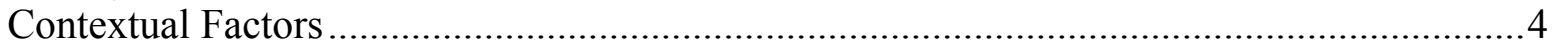

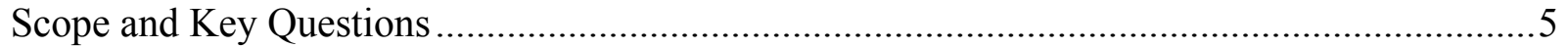

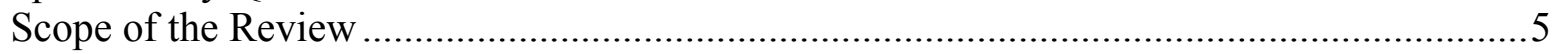

Relevance of Research Question to Clinical Decisionmaking or Policymaking......................5

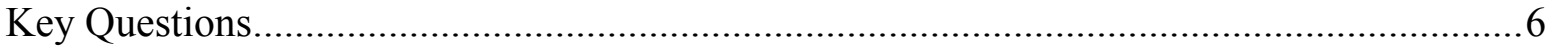

Populations, Interventions, Comparators, Outcomes, Timing, and Setting (PICOTS) ................ 7

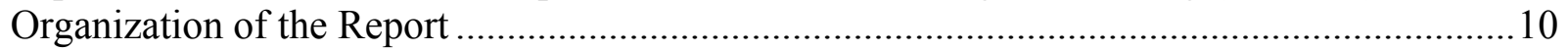

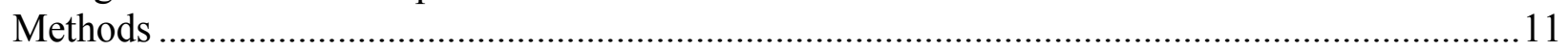

Topic Refinement and Review Protocol.............................................................................1

Literature Search and Identification Strategy …………........................................................11

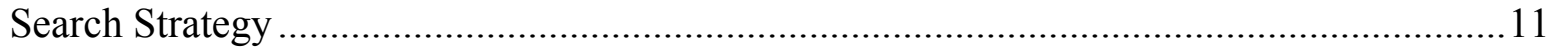

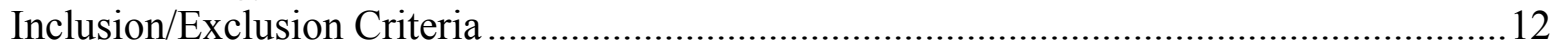

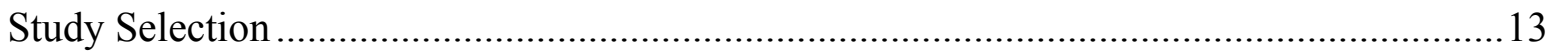

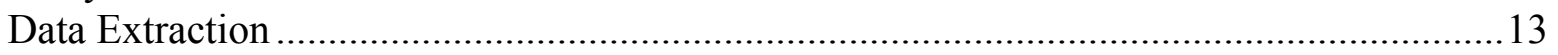

Assessment of Risk of Bias of Individual Studies...............................................................15

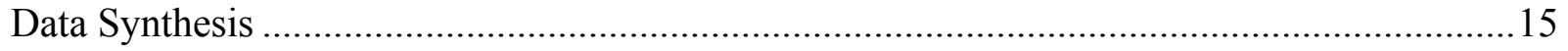

Grading Strength of Evidence for Individual Comparisons and Outcomes ..............................16

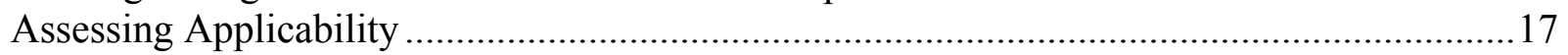

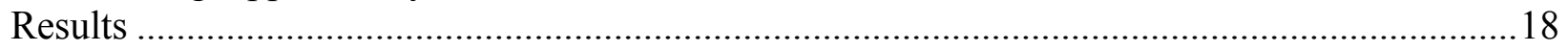

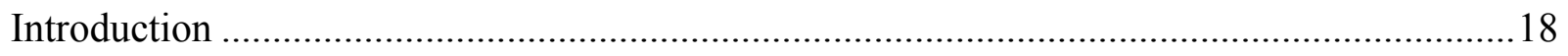

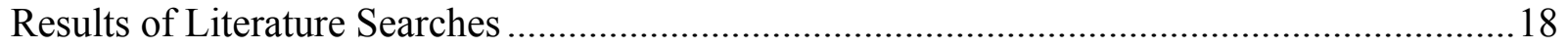

Key Question 1: Components and Implementation Features of MTM Interventions ...............20

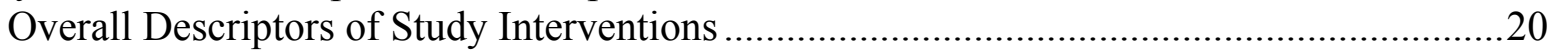

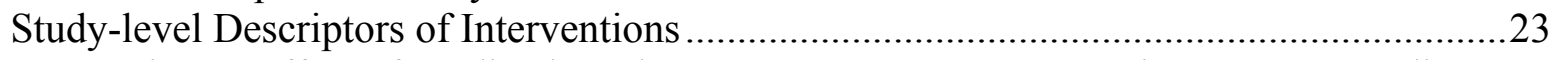

Key Question 2: Effect of Medication Therapy Management Interventions on Intermediate,

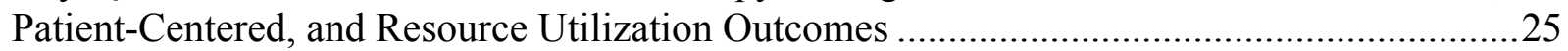

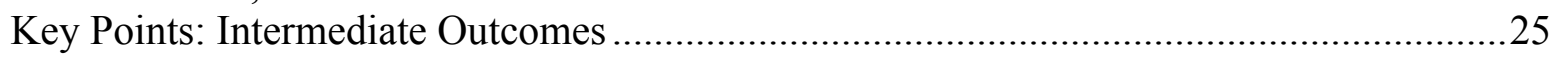

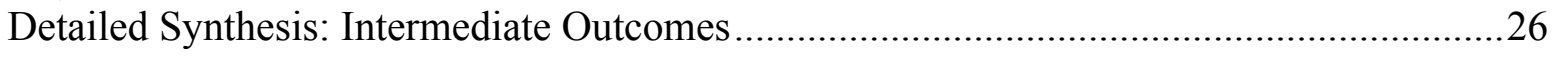

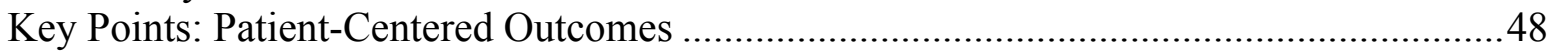

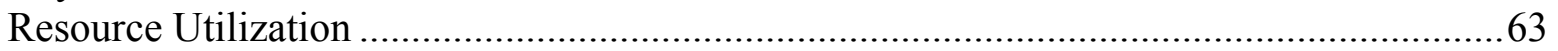

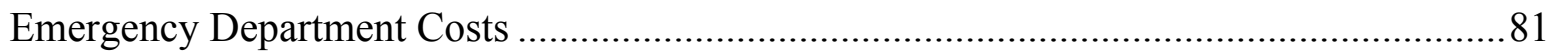

Key Question 3: Outcomes of Medication Therapy Management by Intervention Features ....86

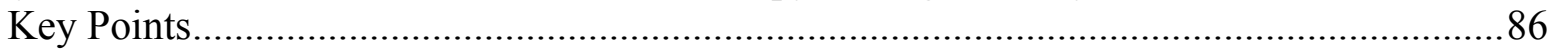

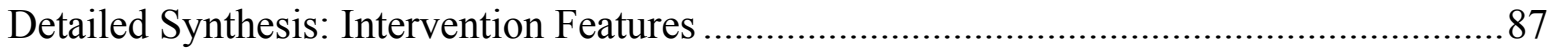

Key Question 4. Outcomes of MTM by Patient Characteristics ...............................................8

Key Question 5. Harms of Medication Therapy Management Interventions.............................8 89

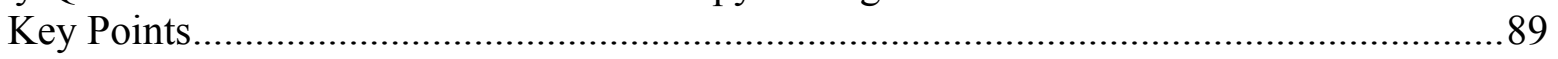




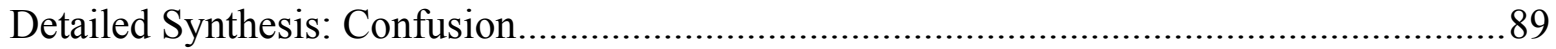

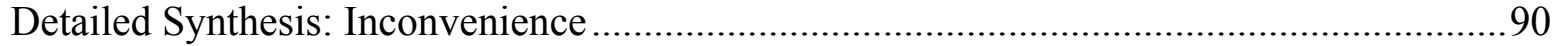

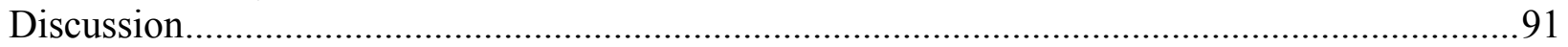

Key Findings and Strength of Evidence ……………….................................................. 91

KQ 1: Intervention Components and Implementation Features ...........................................91

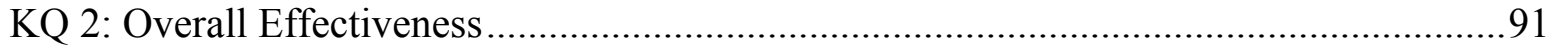

KQ 3: Effectiveness of MTM by Intervention Features .....................................................94

KQ 4: Effectiveness of MTM by Patient Characteristics ...................................................95

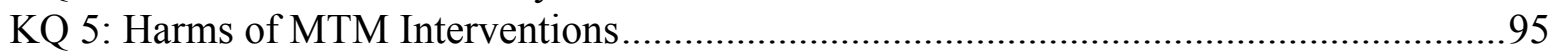

Findings in Relation to What Is Already Known ................................................................99

Applicability of the Findings..............................................................................................96

Implications for Clinical Practice and Policymakers ............................................................99

Limitations of the Comparative Effectiveness Review Process .................................................98

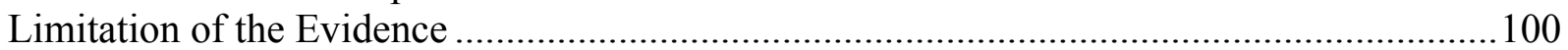

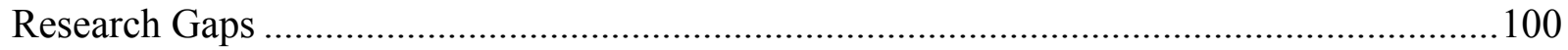

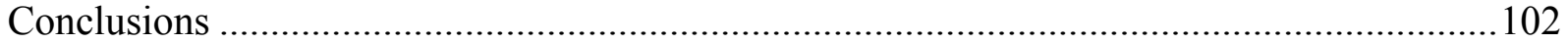

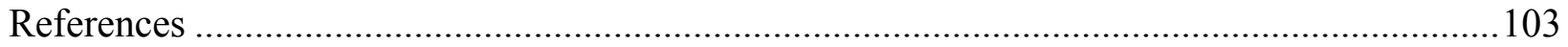

\section{Tables}

Table A. Populations, interventions, comparators, outcomes, timing, and settings ...................ES-3

Table B. Definitions of the grades of overall strength of evidence ............................................

Table 1. Populations, interventions, comparators, outcomes, timing, and settings .........................

Table 2. Literature search terms for medication therapy management studies ..............................12

Table 3. Inclusion/exclusion criteria for medication therapy management studies .......................14

Table 4. Definitions of the grades of overall strength of evidence …………................................16

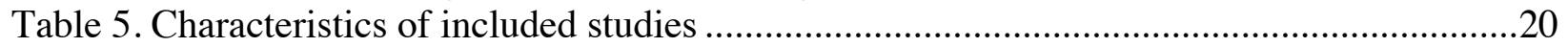

Table 6. Characteristics of medication therapy management interventions ..................................21

Table 7. Characteristics of medication therapy management studies by type of patient population (broad focus or narrow focus on conditions or diagnoses) .......................................22

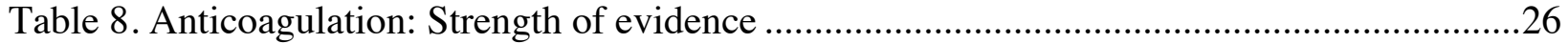

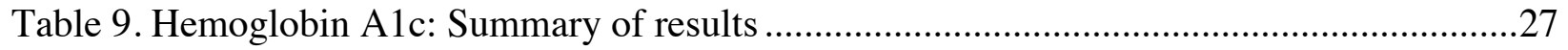

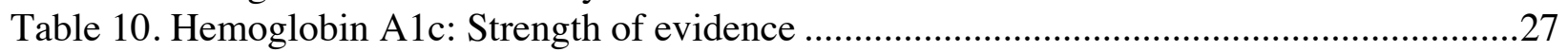

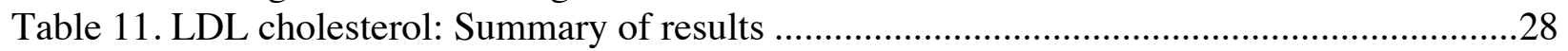

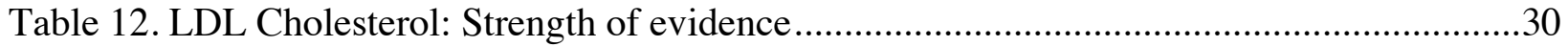

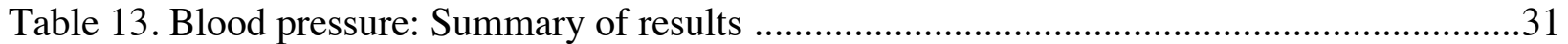

Table 14. Achieving blood pressure goal: Strength of evidence .................................................32

Table 15. Mean change in systolic and diastolic blood pressure: Strength of evidence ................33

Table 16. Drug therapy problems identified: Summary of results ................................................34

Table 17. Drug therapy problems identified: Strength of evidence ………………......................34

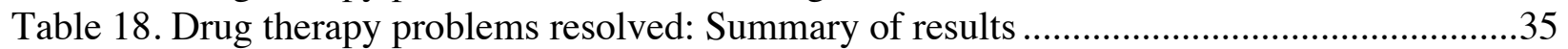

Table 19. Drug therapy problems resolved: Strength of evidence ………………….....................36

Table 20. Medication Adherence: Summary of results grouped by type of adherence outcome 
Table 21. Adherence Outcome Type 1-Proportion of Patients Adherent based on a Threshold of

Percentage of Pills Taken: Strength of Evidence . .40

Table 22. Adherence Outcome Type 2-Absolute Measure of Adherence as Percentage of Prescribed Doses Taken: Strength of Evidence .............................................................................4

Table 23. Adherence outcome type 3--self-reported scales: Strength of evidence .......................41

Table 24. Adherence outcome miscellaneous: Strength of evidence ............................................41

Table 25. Medication appropriateness scales: Summary of results ..............................................42

Table 26. Medication appropriateness for individual medications: Summary of results ...............45

Table 27. Medication appropriateness scales: Strength of evidence .............................................46

Table 28. Medication appropriateness for individual medications: strength of evidence .............47

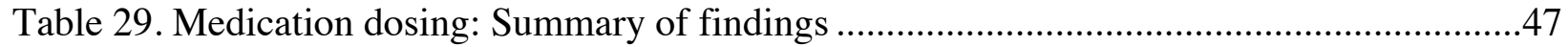

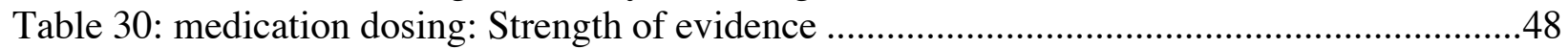

Table 31. Adverse events: Summary of results .......................................................................49

Table 32. Adverse drug events: Strength of evidence ..............................................................50

Table 33. Cognitive, affective, and physical function: Strength of evidence ...............................51

Table 34. All-cause mortality: Summary of results ..............................................................52

Table 35. All-cause mortality: Strength of evidence ......................................................................53

Table 36. Gastrointestinal bleeding events: Strength of evidence …….......................................53

Table 37. Scores on SF-36 measures: Summary of effects from meta-analyses.............................54

Table 38. Condition-specific quality-of-life: Summary of results .............................................57

Table 39. Condition-specific quality of life: Strength of evidence ...........................................57

Table 40. Patient satisfaction: Summary of results ..................................................................58

Table 41. Patient satisfaction: Strength of evidence ………..................................................63

Table 42. Number of medications: Strength of evidence …………........................................64

Table 43. Use of generic medications: Summary of results ..........................................................65

Table 44. Use of generics for telephone-based MTM versus usual care or educational mailings:

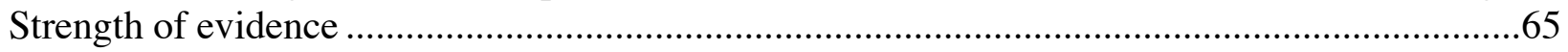

Table 45. Use of generics for community pharmacy-based MTM versus educational mailings:

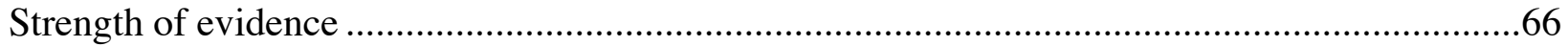

Table 46. Measures used in studies of costs of medications .......................................................66

Table 47. Patient copayments: Summary of results ……….................................................69

Table 48. Patient copayments: Strength of evidence ...................................................................69

Table 49. Total expenditures on medications by insurers: Summary of results .............................70

Table 50. Health plan expenditures: Strength of evidence ..........................................................71

Table 51. Total outlays on medications: Summary of results ......................................................

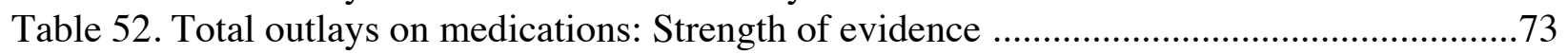

Table 53. Medication and other costs: Summary of results .......................................................74

Table 54. Medication and other costs: Strength of evidence ........................................................75

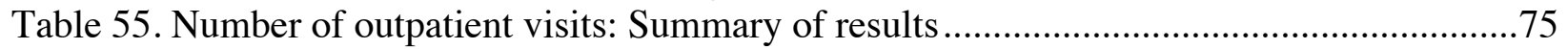

Table 56. Number of outpatient visits: Strength of evidence ………...........................................77

Table 57. Costs of outpatient visits: Summary of results ............................................................78

Table 58. Costs of outpatient resource utilization: Strength of evidence ……………..................78

Table 59. Number of laboratory tests: Summary of results ..........................................................79

Table 60. Number of laboratory tests: Strength of evidence ........................................................

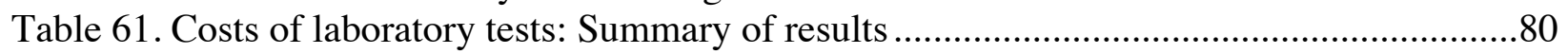

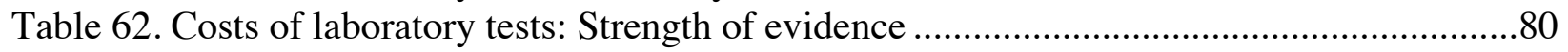


Table 63. Emergency department visits: Summary of results ................................................81

Table 64. Emergency department visits: Strength of evidence ................................................81

Table 65. Costs of emergency department visits: Summary of results .....................................82

Table 66. Cost of emergency department visits: Strength of evidence .....................................82

Table 67. Hospitalizations: Mean number, risk and rates .......................................................8.

Table 68. Mean number of hospitalizations: Strength of evidence ….....................................85

Table 69. Percentage of patients hospitalized: Strength of evidence .......................................85

Table 70. Rate of hospitalization: Strength of evidence .........................................................85

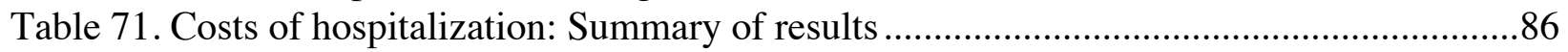

Table 72. Cost of hospitalization: Strength of evidence ............................................................86

Table 73. Length of hospital stay: Strength of evidence ......................................................86

Table 74. Access to patient records (basic MTM versus enhanced MTM): Strength of evidence87

Table 75. Pharmacy intensity of adoption: Strength of evidence ................................................8

Table 76. Community pharmacy versus call center: Strength of evidence ................................89

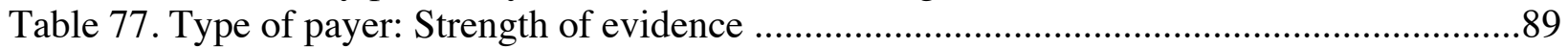

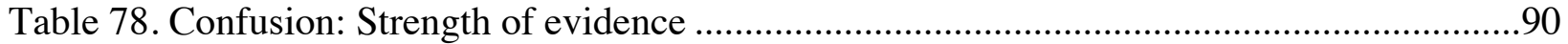

Table 79. Confusion: Strength of evidence ............................................................................90

Table 80. Summary of findings and strength of evidence for intermediate outcomes of MTM

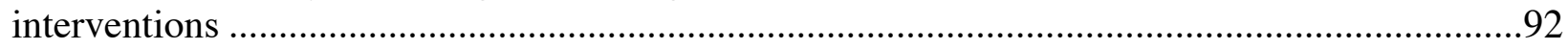

Table 81. Summary of findings and strength of evidence for patient-centered outcomes of MTM

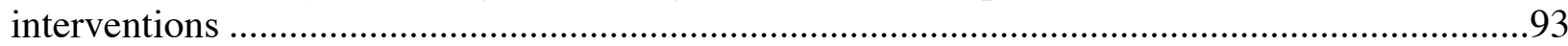

Table 82. Summary of findings and strength of evidence for resource-utilization outcomes of

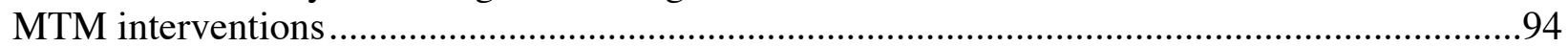

\section{Figures}

Figure A. Analytic framework for medication therapy management ..................................ES-2

Figure B. Disposition of articles on medical therapy management (PRISMA figure) .............ES-9

Figure 1. Analytic framework for medication therapy management.....................................6

Figure 2. Disposition of articles on medical therapy management (PRISMA figure) ................19

\section{Appendixes}

Appendix A. Literature Search Strategies

Appendix B. Abstract and Full-Text Review Form Templates

Appendix C. List of Studies Excluded after Full-Text Level Review

Appendix D. List of Studies on Hold

Appendix E. Evidence Tables

Appendix F. Risk of Bias Evaluations and Rationale

Appendix G. Meta-analyses 


\section{Executive Summary}

\section{Background}

Used appropriately, medications can alleviate distressing symptoms that compromise physical and psychological well-being, help prevent the onset of many acute and chronic health illnesses, and improve patient health outcomes. Too often, however, medications are not used appropriately. ${ }^{1-3}$ In the United States in 2001, an estimated 4.3 million ambulatory visits were for adverse drug events. ${ }^{4}$ In addition to problems involving adverse drug events, many patients do not receive optimal pharmaceutical prescriptions. Even when optimal therapy is prescribed, patient inability to adhere closely to medication regimens may lead to poor health outcomes. ${ }^{5}$

Medication-related problems are especially pronounced among older adults. ${ }^{6}$ Individuals 65 years or older constitute 13 percent of the U.S. population, but they consume more than 30 percent of all prescription medications. ${ }^{6,7}$ A 2006 report found that nearly 60 percent of people in this age group were taking five or more medications and that nearly 20 percent were taking 10 or more medications, ${ }^{8}$ placing them at increased risk for experiencing adverse drug events.

Medication therapy management (MTM) services are intended to address issues of polypharmacy, preventable adverse drug events, medication adherence, and medication misuse. ${ }^{9}$ MTM is the current term that represents services that have evolved out of the philosophy and processes first implemented in the early 1990s as "pharmaceutical care." In 2008, 11 national pharmacy organizations achieved a consensus framework for MTM services and established 5 core elements for MTM in practice; these included a medication therapy review, a personal medication record, a medication action plan, intervention and/or referral, and documentation and follow-up. ${ }^{9}$

In the United States, the Medicare Prescription Drug, Improvement, and Modernization Act of 2003 (Public Law 108-173) ${ }^{10}$ established the requirements that sponsors of Part D Prescription Drug Benefit Plan have to meet with respect to cost, quality, and the requirements for MTM programs. The evolution from pharmaceutical care research interventions to large-scale MTM programs in routine practice represents a journey involving multiple practice settings, patient populations, and intervention components and features. Over time, standards for these services in routine practice have evolved, as have standards for describing and conducting research studies involving these interventions. Thus, we established a broad scope for this comparative effectiveness review and did not limit our perspective to Medicare Part D-defined MTM programs.

\section{Scope and Key Questions}

MTM is a complex intervention with numerous and differing components. This review seeks to catalog MTM intervention components, assess the overall effectiveness of MTM in comparison with usual care, examine the factors under which MTM is effective and optimally delivered, determine what types of patients are likely to benefit from MTM services, and clarify what types of patients may be at risk of harms from the program.

The KQs are listed below and placed in relation to another and the PICOs in the analytic framework (Figure A). Specific details regarding patient population, intervention components, and outcomes are provided in the section that follows the analytic framework. 


\section{Analytic Framework}

Figure A. Analytic framework for medication therapy management

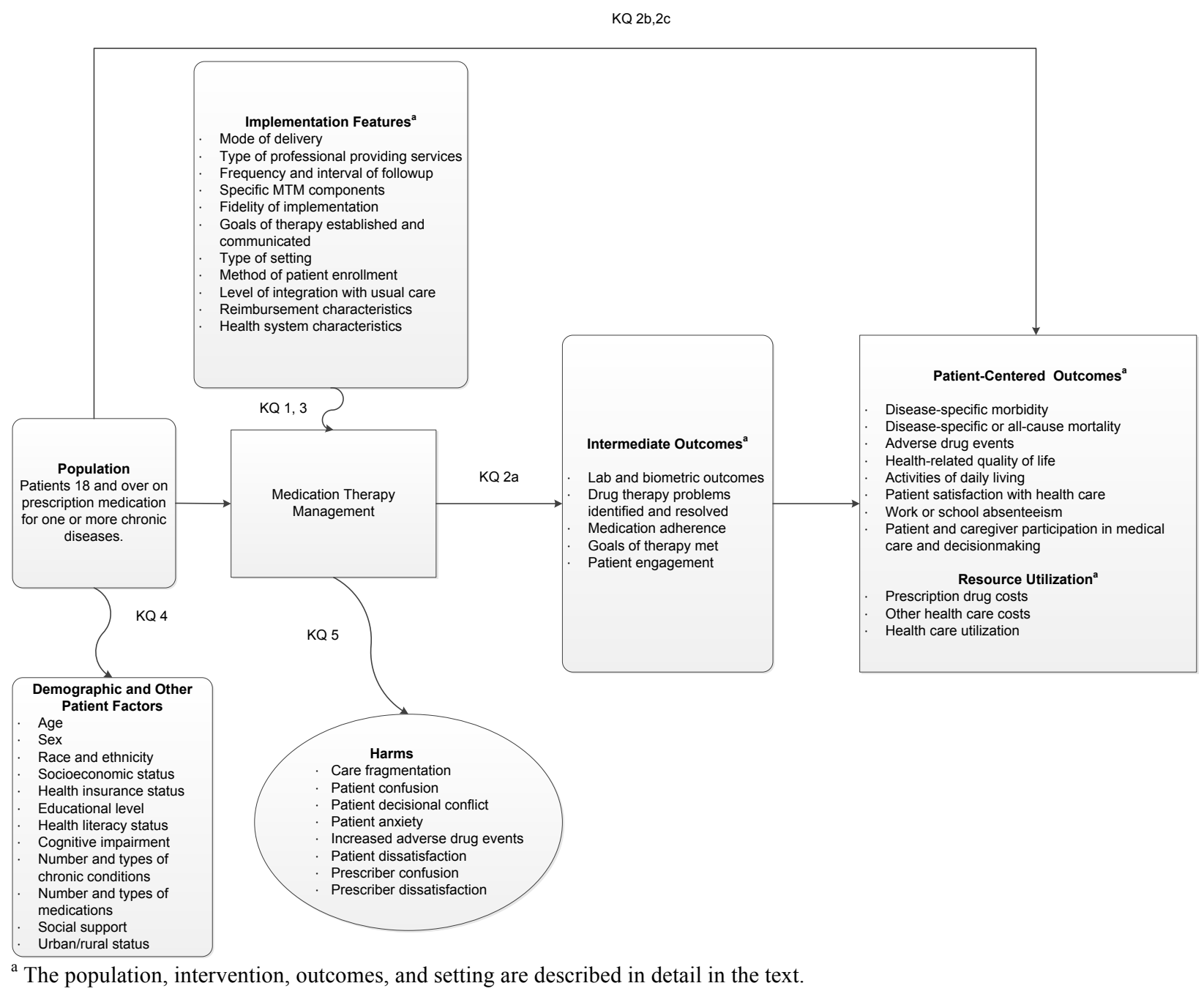

Abbreviations: $\mathrm{KQ}=$ key question; $\mathrm{MTM}=$ medication therapy management

Question 1: What are the components and implementation features of MTM interventions?

Question 2: In adults with one or more chronic diseases who are taking prescription medications, is MTM effective in improving the following:

a. Intermediate outcomes, including biometric and laboratory measures, drug therapy problems identified, drug therapy problems resolved, medication adherence, goals of therapy met, and patient engagement in medication management?

b. Patient-centered outcomes, such as disease-specific morbidity, disease-specific or allcause mortality, adverse drug events, health-related quality of life, activities of daily living, patient satisfaction with health care, work or school absenteeism, and patient and caregiver participation in medical care and decisionmaking? 
c. Resource utilization, such as prescription drug costs, other health care costs, and health care utilization?

Question 3: Does the effectiveness of MTM differ by MTM components and implementation features?

Question 4: Does the effectiveness of MTM differ by patient characteristics, including but not limited to patient demographics and numbers and types of conditions and medications?

Question 5: Are there harms of MTM, and if so, what are they?

\section{Populations, Interventions, Comparators, Outcomes, Timing, and Setting (PICOTS)}

Table A lays out the PICOTS for this review. For this review, we take a broad perspective on the population and interventions evaluated; we did not require CMS Part D MTM eligibility criteria. Specifically, we did not require multiple chronic conditions or a minimum number or level of expenditures on prescription drugs. We included randomized and controlled clinical trials, systematic reviews, and prospective and retrospective cohort studies.

Table A. Populations, interventions, comparators, outcomes, timing, and settings

\begin{tabular}{lc}
\hline PICOTS & Criteria \\
\hline Populations & $\circ$ Patients ages 18 or older with one or more chronic conditions requiring the use of prescription \\
& medication to manage symptoms or prevent progression of chronic disease \\
$\circ$ & Patient characteristics that may influence intervention effectiveness: \\
& - Age, sex, race and ethnicity, socioeconomic status, health insurance status, education level, \\
& health literacy status, cognitive impairment, number and types of chronic conditions, social \\
& support, and urban/rural status \\
\hline Interventions & Explicitly termed $M T M$ services, generally provided as a bundle of related services, that include \\
& at a minimum the following four elements: \\
- & Comprehensive medication review \\
- & Patient-directed medication management action plan with or without an equivalent prescriber- \\
& directed action plan \\
- & Patient-directed education and counseling or other resources to enhance understanding of \\
& the use of medication \\
- & Coordination of care, including prescriber-directed interventions; documentation of MTM \\
& services for use by the patient's other providers; and referral to other providers, clinicians, or \\
& resources when appropriate ${ }^{11}$
\end{tabular}


Table A. Populations, interventions, comparators, outcomes, timing, and settings (continued)

\begin{tabular}{|c|c|}
\hline PICOTS & Criteria \\
\hline $\begin{array}{l}\text { Interventions } \\
\text { (continued) }\end{array}$ & 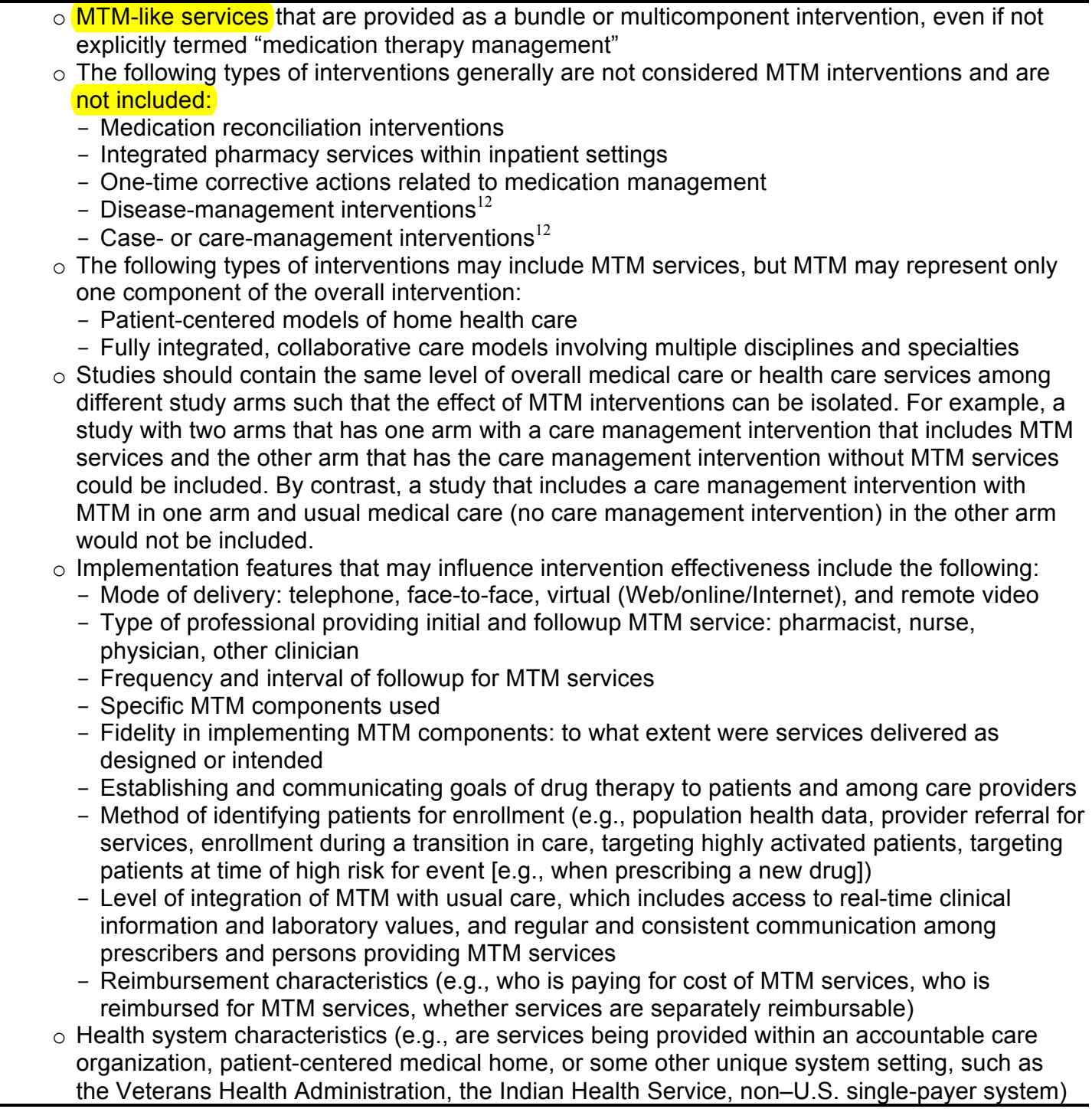 \\
\hline Comparators & $\begin{array}{l}\text { O Usual care, as defined by the studies } \\
\text { Individual components of MTM services (e.g., MTM services with four components vs. a single } \\
\text { component) } \\
\text { Different bundles of MTM services } \\
\text { Same MTM services provided by different health care professionals (e.g., pharmacist, } \\
\text { physician, nurse, other) } \\
\text { Same bundles of MTM services delivered by different modes (e.g., telephone or in person) } \\
\text { Same MTM services provided at different intensities, frequencies, or level of integration with } \\
\text { prescribers }\end{array}$ \\
\hline
\end{tabular}


Table A. Populations, interventions, comparators, outcomes, timing, and settings (continued)

\begin{tabular}{|c|c|}
\hline PICOTS & Criteria \\
\hline Outcomes & 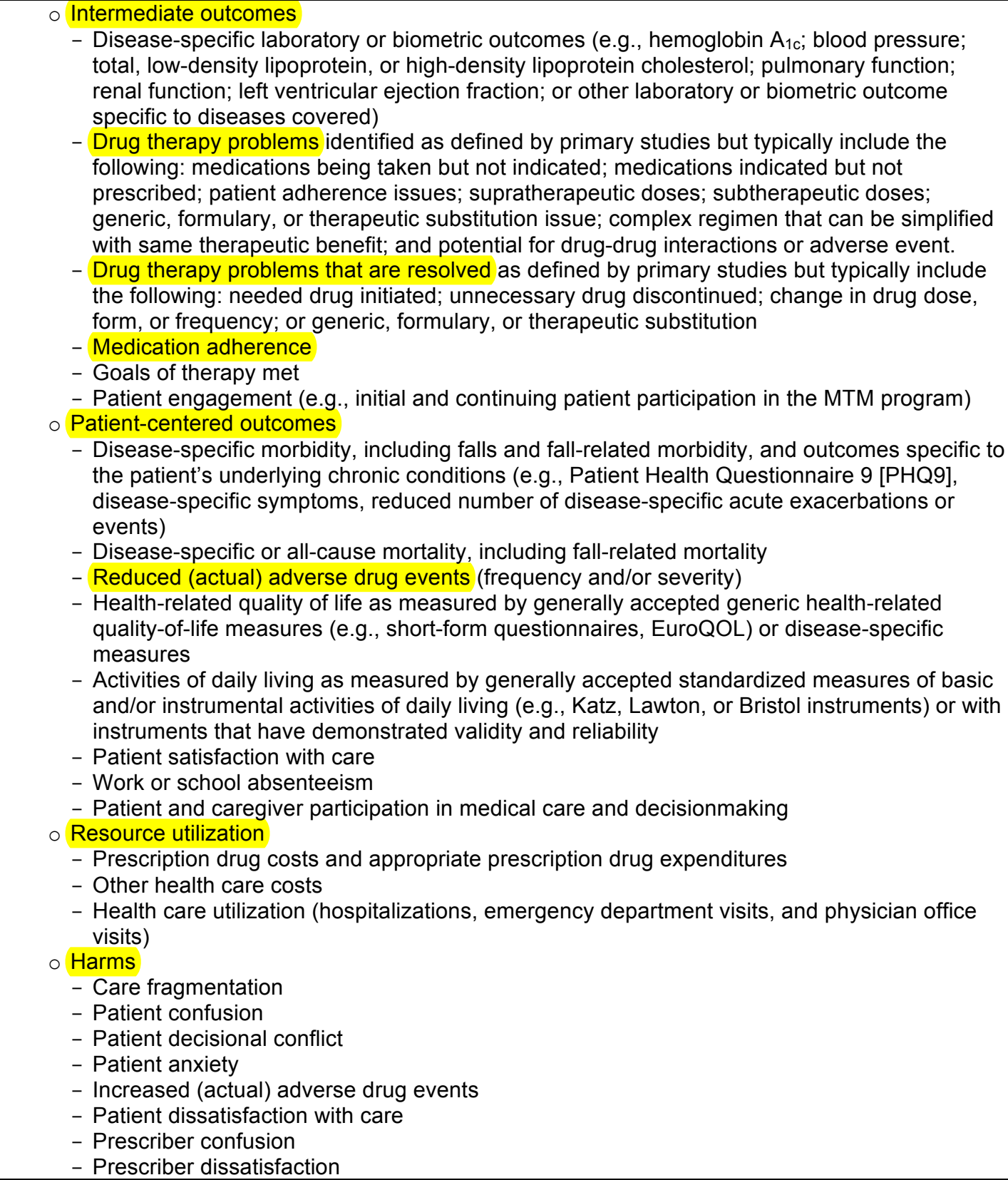 \\
\hline Timing & $\begin{array}{l}\text { Interventions should have at least two separately identifiable episodes of MTM services (either } \\
\text { patient or provider directed or both), with any interval of time in between episodes. } \\
\text { For studies that report outcomes at different points in time, we only considered outcomes } \\
\text { measured after the second episode of care. }\end{array}$ \\
\hline
\end{tabular}


Table A. Populations, interventions, comparators, outcomes, timing, and settings (continued)

\begin{tabular}{lc}
\hline PICOTS & Criteria \\
\hline Setting & Ambulatory settings (e.g., outpatient clinics or private physician offices), long-term-care setting, \\
& or retail pharmacy settings) \\
$\circ$ & However, the MTM intervention itself may be delivered by home visits, telephone, via the Web, \\
& or in other non-face-to-face modalities, such as video teleconferencing. \\
$\circ$ & MTM services that are delivered mostly in inpatient settings are not included. \\
$\circ$ & Interventions conducted in the United States and other countries and are published in English \\
& are included.
\end{tabular}

\section{Methods}

\section{Topic Refinement and Review Protocol}

The topic of this report and preliminary key questions (KQs) arose through a nomination from the Pharmacy Quality Alliance. Key Informants representing several clinical and scientific disciplines provided input on the initial KQs; we revised them as needed. An initial draft of the revised KQs was posted for public comment from March 6, 2013, through April 2, 2013, on the AHRQ Effective Health Care program Web site. We received comments from 23 professional organizations and individuals and further revised KQs as appropriate.

\section{Literature Search and Identification Strategy}

To identify articles relevant to each KQ, we began with a focused MEDLINE ${ }^{\circledR}$ search for MTM interventions using a combination of medical subject headings and title and abstract keywords and limiting the search to English-language and human-only studies. We also searched the Cochrane Library and the International Pharmaceutical Abstracts database using analogous search terms. We selected these databases based on preliminary searches and consultation with content experts. We conducted quality checks to ensure that the searches identified known studies (i.e., studies identified during topic nomination and refinement). Based on these quality checks, we revised and ran additional searches (specifically, drug therapy management, drug therapy problem, and medications management) to avoid missing articles that might prove eligible for this systematic review.

In addition, we searched the gray literature for unpublished studies relevant to this review and included studies that met all the inclusion criteria and contained enough methodological information to assess risk of bias. Specifically, sources of gray literature included ClinicalTrials.gov, the World Health Organization's International Clinical Trials Registry Platform, Health Services Research Projects in Progress (HSRProj), the National Institutes of Health Research Portfolio Online Reporting Tools, the Database of Promoting Health Effectiveness Reviews, the New York Academy of Medicine Grey Literature Report, and CMS.gov.

We reviewed our search strategy with an independent information specialist and the Technical Expert Panel and supplemented it according to their recommendations. In addition, to avoid retrieval bias, we manually searched the reference lists of landmark studies and background articles on this topic to identify any relevant citations that our electronic searches might have missed.

Two trained members of the research team independently reviewed each of the titles and abstracts against the inclusion/exclusion criteria listed in Table A. We applied the same criteria to systematic reviews and primary studies. For each article that either or both reviewers chose to 
include based on the abstract review, two reviewers reviewed their full texts for eligibility against our inclusion/exclusion criteria. During full-text review, if both reviewers agreed that a study did not meet the eligibility criteria (including designation of high risk of bias), we excluded the study. Reviewers resolved conflicts by discussion and consensus or by consulting a third member of the review team.

For studies that met our inclusion criteria, a trained reviewer abstracted information into structured evidence tables; a second senior member of the team reviewed all data abstractions for completeness and accuracy. Reviewers resolved conflicts by discussion and consensus or by consulting a third member of the review team.

\section{Assessment of Risk of Bias of Individual Studies}

To assess the risk of bias of individual studies, we used predefined criteria developed by AHRQ. ${ }^{13}$ For randomized controlled trials (RCTs), we relied on the risk-of-bias tool developed by the Cochrane Collaboration. ${ }^{14}$ We assessed the risk of bias of observational studies using an item bank developed by RTI International. ${ }^{15}$

In general terms, results of a study with low risk of bias are considered valid. A study with medium risk of bias is susceptible to some bias but probably not sufficient to invalidate its results. A study with high risk of bias has significant methodological flaws (e.g., stemming from serious errors in design or analysis) that may invalidate its results. Primary concerns for our review included selection bias, confounding, performance bias, detection bias, and attrition bias. Specifically, we evaluated studies on the adequacy of randomization, allocation concealment, similarity of groups at baseline, masking, attrition, whether intention-to-treat analysis was used, method of handling dropouts and missing data, validity and reliability of outcome measures, and treatment fidelity.

We excluded studies that we deemed at high risk of bias from our main data synthesis and main analyses. We included them for sensitivity analyses; in cases when we had no other available or credible evidence, we included in the report a brief synopsis of studies assessed as high risk of bias.

\section{Data Synthesis}

When we found three or more similar studies for a comparison of interest, we conducted meta-analysis of the data from those studies. For all analyses, we used random-effects models to estimate pooled or comparative effects. To determine whether quantitative analyses were appropriate, we assessed the clinical and methodological heterogeneity of the studies under consideration following established guidance; ${ }^{16}$ that is, we qualitatively assessed the PICOTS of the included studies, looking for similarities and differences. When we conducted quantitative syntheses (i.e., meta-analysis), we assessed statistical heterogeneity in effects between studies by calculating the chi-squared statistic and the $\mathrm{I}^{2}$ statistic (the proportion of variation in study estimates attributable to heterogeneity). The importance of the observed value of $\mathrm{I}^{2}$ depends on the magnitude and direction of effects and on the strength of evidence for heterogeneity (e.g., the $\mathrm{p}$-value from the chi-squared test or a confidence interval for $\mathrm{I}^{2}$ ). Where relevant, we examined potential sources of heterogeneity using sensitivity analysis.

When quantitative analyses were not appropriate (e.g., because of heterogeneity, insufficient numbers of similar studies, or insufficiency or variation in outcome reporting), we synthesized the data qualitatively. Whenever possible, we computed confidence intervals for individual outcomes. 
Numerous articles about both trials and observational studies often did not provide complete information about findings (e.g., 95 percent confidence intervals; statistical significance values, or between-group data). In many cases, therefore, we had to calculate odds ratios, mean differences, or standardized mean differences, the relevant 95 percent confidence intervals, and p-values.

\section{Grading Strength of Evidence for Individual Comparisons and Outcomes}

We graded the strength of evidence based on the guidance established for the AHRQ Evidence-based Practice Center program. ${ }^{17}$ Developed to grade the overall strength of a body of evidence, this approach incorporates four key domains: study limitations (includes study design and aggregate quality), consistency, directness, and precision of the evidence. It also considers other optional domains that may be relevant for some scenarios, such as a dose-response association, plausible confounding that would decrease the observed effect, strength of association (magnitude of effect), and publication bias. We evaluated optimal information size criteria to make judgments about precision based on guidance from Guyatt and colleagues ${ }^{18}$ and based our grades on low or medium risk-of-bias RCTs or observational studies unless none were available, based on guidance from the Grading of Recommendations Assessment, Development and Evaluation (GRADE) Working Group ${ }^{19}$ and the AHRQ EPC program. ${ }^{17}$

Table B describes the grades of evidence that can be assigned. ${ }^{20}$ Grades reflect the strength of the body of evidence to answer the KQs on the overall effectiveness, comparative effectiveness, and harms of the interventions examined in this review. Two reviewers assessed each domain for each major outcome resolved any differences by consensus discussion or referral to a third, senior member of the team. We graded the strength of evidence for the outcomes deemed to be of greatest importance to decisionmakers and those commonly reported in the literature; we did not grade the strength of evidence for KQ 1 (on components and features of MTM services).

Table B. Definitions of the grades of overall strength of evidence

\begin{tabular}{ll}
\hline Grade & Definition \\
\hline High & $\begin{array}{l}\text { High confidence that the evidence reflects the true effect. Further research is very unlikely } \\
\text { to change our confidence in the estimate of effect. }\end{array}$ \\
\hline Moderate & $\begin{array}{l}\text { Moderate confidence that the evidence reflects the true effect. Further research may } \\
\text { change our confidence in the estimate of the effect and may change the estimate. }\end{array}$ \\
\hline Low & $\begin{array}{l}\text { Low confidence that the evidence reflects the true effect. Further research is likely to } \\
\text { change our confidence in the estimate of the effect and is likely to change the estimate. }\end{array}$ \\
\hline Insufficient & Evidence either is unavailable or does not permit estimation of an effect. \\
\hline
\end{tabular}

\section{Assessing Applicability}

We assessed applicability of the evidence following guidance from the Methods Guide for Effectiveness and Comparative Effectiveness Reviews. ${ }^{21}$ We used the PICOTS framework to explore factors that affect applicability. Some factors identified a priori that may limit the applicability of evidence include the following: age and health status of enrolled populations, health insurance coverage and access to health care, and complexity and intensity of the MTM intervention. 


\section{Results}

We provide a summary of results by KQ below. Detailed descriptions of included studies, key points, detailed synthesis, summary tables, and expanded SOE tables that include the magnitude of effect can be found in the full report. Our summary of results below presents the SOE grades.

\section{Results of Literature Searches}

Figure B presents our literature search results through June 27, 2013; for the draft report, we identified 2,129 unduplicated citations. In addition, we identified 99 publications through grey literature searches, suggestions from technical experts or public comments received during topic refinement, or hand searches of included studies. After applying our eligibility and exclusion criteria to titles and abstracts of all 2,228 identified citations, we obtained full-text copies of 328 published articles. We reapplied our inclusion criteria and excluded 286 of these articles from further review before doing the risk-of-bias assessment. The 42 articles included after full-text review represent 36 studies.

\section{Figure B. Disposition of articles on medical therapy management (PRISMA figure)}

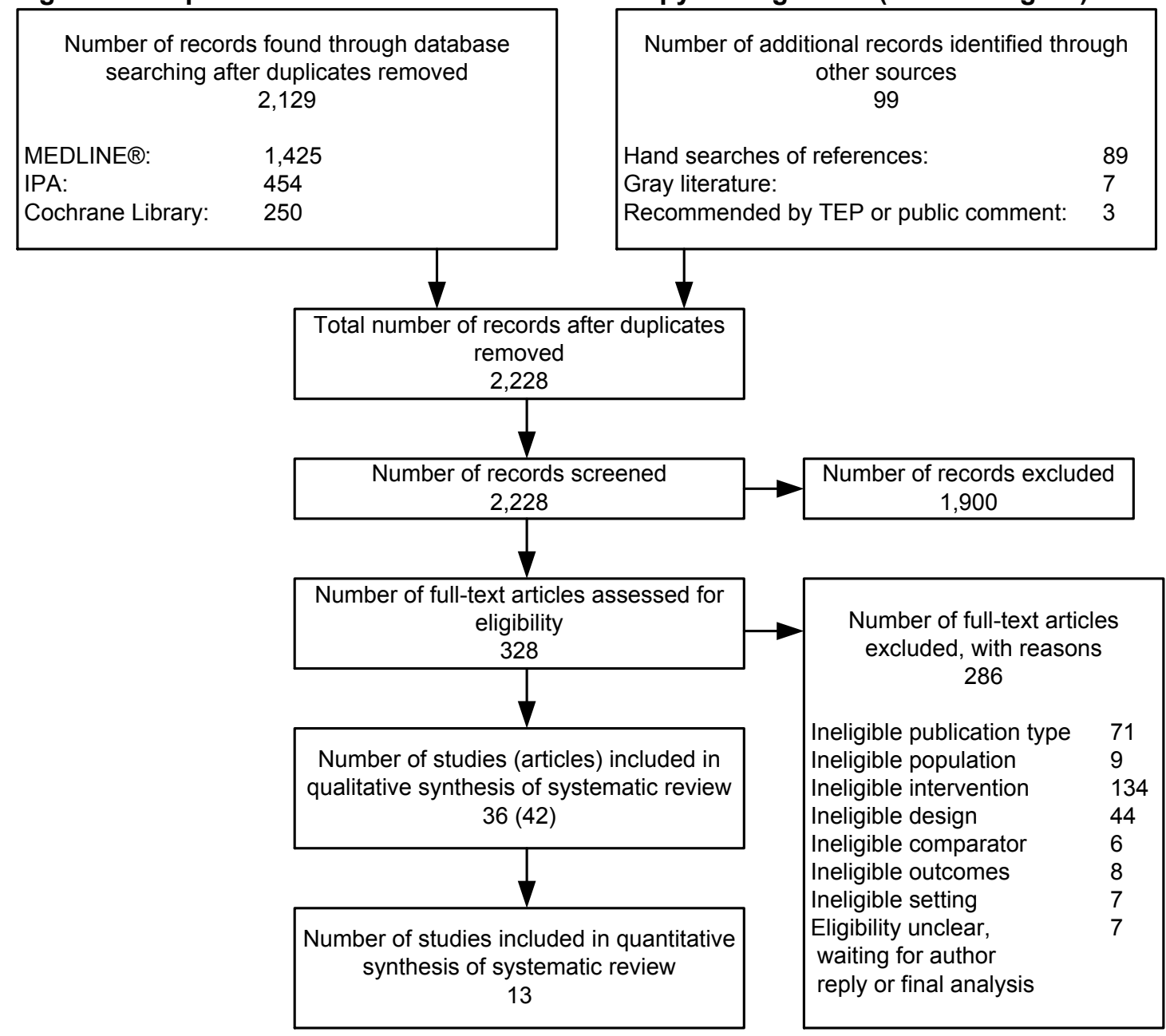

Abbreviations: IPA, International Pharmaceutical Abstracts; PICOTS, populations, interventions, comparators, outcomes, timing, settings; TEP, technical expert panel. 
This evidence base consisted of 36 studies (19 randomized controlled trials [RCTs] trials and 17 observational studies) reported in 42 articles. Most RCTs compared an MTM intervention with usual care rather than with a different active intervention; most observational studies were cohort studies. Numerous studies had methods problems that led us to rate them as having a medium or high risk of bias; only a few studies were of low risk of bias. When possible (enough studies similar in intervention, populations, and outcomes measured), we conducted metaanalyses of data from RCTs or cohort studies separately; when relevant, we did two sets, one with and one without the high risk-of-bias trials.

Because of the wide variation in types of interventions classified as MTM, we first catalogued intervention components and implementation features of MTM interventions (Key Question [KQ] 1). We then evaluated the effect of MTM on intermediate, patient-centered, and resource utilization outcomes (KQ 2). We also reviewed the evidence to identify how these effects might vary by specific intervention components and features (KQ 3) and patient characteristics (KQ 4). Finally, we reviewed the evidence on harms associated with MTM (KQ 5).

Below, we summarize the main findings and strength of evidence, where applicable. We then discuss the findings in relationship to what is already known, applicability of the findings, implications for decisionmaking, limitations, research gaps, and conclusions.

\section{Key Findings and Strength of Evidence}

\section{KQ 1: Intervention Components and Implementation Features}

Nearly two-thirds of included studies were broadly focused on patients with a wide-ranging collection of conditions; the remaining studies were narrowly focused on patients with a specific condition. All studies used a pharmacist as the interventionist. Services were provided face-toface in just over half of included studies. Included studies provided interventions in a variety of clinical settings, including community pharmacies, centralized pharmacies or pharmacy call centers, and outpatient medical clinics, and some used home visits; half of the narrowly focused interventions were delivered exclusively in an outpatient medical clinic.

Whether termed "pharmaceutical care" or "MTM," studies did not describe intervention components and features in a consistent manner or in sufficient detail. These drawbacks were especially prevalent for intervention intensity and frequency, method of patient enrollment for services, level of integration with usual care, and reimbursement characteristics for rendered MTM services. KQ 1 was descriptive in nature, so we did not grade strength of evidence.

\section{KQ 2: Overall Effectiveness}

Of the 36 studies included in this review, we rated 14 as high risk of bias overall; that is, concerns about randomization failure, confounding, or overall attrition increased the risk of bias for all outcomes. In addition, we rated some studies that were otherwise of low or medium risk of bias as high for individual outcomes, chiefly because of measurement bias. These instances are specified in the relevant results section in Chapter 3.

We rated the strength of evidence for each outcome from low- or medium risk-of-bias studies when available. MTM significantly improved medication appropriateness assessed in general (Table C). However, we did not find evidence of benefit for any other intermediate outcomes on which we had data. No studies addressed either goals of therapy or patient engagement. 
Table C. Summary of findings and strength of evidence for intermediate outcomes of MTM interventions

\begin{tabular}{|c|c|c|c|}
\hline Intermediate Outcome & $\begin{array}{l}\text { Study Design: } \\
\text { No. Studies (N } \\
\text { Analyzed) }\end{array}$ & Results & $\begin{array}{l}\text { Strength of } \\
\text { Evidence }\end{array}$ \\
\hline Anticoagulation & RCT: $1(10)$ & Imprecise & Insufficient \\
\hline Hemoglobin A1C & RCT: 2 (102) & Inconsistent, imprecise & Insufficient \\
\hline Low density lipoprotein cholesterol & RCT: $1(38)$ & Imprecise & Insufficient \\
\hline $\begin{array}{l}\text { Hypertension: achieving blood } \\
\text { pressure goals }\end{array}$ & RCT: 1 (44) & Imprecise & Insufficient \\
\hline $\begin{array}{l}\text { Hypertension: systolic blood } \\
\text { pressure }\end{array}$ & RCT: 1 (23) & Imprecise & Insufficient \\
\hline $\begin{array}{l}\text { Hypertension: diastolic blood } \\
\text { pressure }\end{array}$ & RCT: 1 (23) & Imprecise & Insufficient \\
\hline Drug therapy problems identified & $\begin{array}{l}\text { RCT: } 1 \text { (332) } \\
\text { Cohort: } 2 \text { (668) }\end{array}$ & Indirect, imprecise, high study limitations & Insufficient \\
\hline Drug therapy problems resolved & Cohort: $1(120)$ & Indirect, imprecise, high study limitations & Insufficient \\
\hline Medication adherence & $\begin{array}{l}\text { RCT: } 8(2,415) \\
\text { Cohort: } 2(1,493)\end{array}$ & $\begin{array}{l}\text { Inconsistent, imprecise (across } \\
\text { heterogeneous measures) }\end{array}$ & Insufficient \\
\hline $\begin{array}{l}\text { Medication Appropriateness General } \\
\text { Index Scores }\end{array}$ & RCT: 1 (208) & $\begin{array}{l}\text { Improvement in MTM group from score of } \\
17.7 \text { to } 13.4 \text { and to } 12.8 \text { at } 3 \text { and } 12 \\
\text { months, respectively }\end{array}$ & Low for benefi \\
\hline Medication-specific appropriateness & RCT: 2 (261) & Indirect, imprecise, inconsistent & Insufficient \\
\hline Medication dosing & RCT: 2 (90) & Inconsistent, imprecise & Insufficient \\
\hline Goals of therapy & 0 & NA & NA \\
\hline Patient engagement & 0 & NA & NA \\
\hline
\end{tabular}

Similarly, we did not have evidence of benefit for most patient-centered outcomes (Table D). MTM did not improve most measures of health-related quality of life (low strength of evidence for no benefit). We graded the "vitality" domain of the Medical Outcomes Study Short-Form (SF36) questionnaire as insufficient for this domain. For the SF-36, neither the other seven domains nor the two component scores (physical health, mental health) showed significant benefit from MTM interventions. The various patient satisfaction items also showed no impact from MTM programs (low strength of evidence for no benefit). 
Table D. Summary of findings and strength of evidence for patient-centered outcomes of MTM interventions

\begin{tabular}{|c|c|c|c|}
\hline Patient-Centered Outcome & $\begin{array}{l}\text { Study Design: } \\
\text { No. Studies (N } \\
\text { Analyzed) }\end{array}$ & Results & $\begin{array}{l}\text { Strength of } \\
\text { Evidence }\end{array}$ \\
\hline Adverse drug events & RCT: 2 (806) & Inconsistent, imprecise & Insufficient \\
\hline $\begin{array}{l}\text { Cognitive, affective, and physical } \\
\text { function }\end{array}$ & RCT: 1 (133) & Imprecise & Insufficient \\
\hline Mortality & $\begin{array}{l}\text { RCT: } 2 \text { (335) } \\
\text { Cohort: } 1 \text { (795) }\end{array}$ & Inconsistent, imprecise & Insufficient \\
\hline Gastrointestinal bleeding events & Cohort: $1(1,373)$ & High study limitations & Insufficient \\
\hline $\begin{array}{l}\text { General health-related quality of life } \\
\text { domains other than vitality }\end{array}$ & RCT: $4(1,169)$ & $\begin{array}{l}\text { Variable mean difference with confidence } \\
\text { intervals consistently spanning the null } \\
\text { effect }\end{array}$ & $\begin{array}{l}\text { Low for no } \\
\text { benefit }\end{array}$ \\
\hline $\begin{array}{l}\text { General health-related quality of life } \\
\text { vitality domain }\end{array}$ & RCT: $4(1,169)$ & Imprecise & Insufficient \\
\hline $\begin{array}{l}\text { Condition-specific health-related } \\
\text { quality of life (diabetes) }\end{array}$ & RCT: 1 (73) & Imprecise & Insufficient \\
\hline Patient satisfaction & RCT: 3. $(1,625)$ & $\begin{array}{l}\text { No differences on } 17 \text { or } 21 \text { items of patient } \\
\text { satisfaction }\end{array}$ & $\begin{array}{l}\text { Low for no } \\
\text { benefit }\end{array}$ \\
\hline Activities of daily living & 0 & NA & $\mathrm{NA}$ \\
\hline Work or school absenteeism & 0 & NA & $\mathrm{NA}$ \\
\hline $\begin{array}{l}\text { Patient and caregiver participation in } \\
\text { medical care and decisionmaking }\end{array}$ & 0 & NA & NA \\
\hline \multicolumn{4}{|c|}{$\begin{array}{l}\text { Abbreviations: } \mathrm{CI}=\text { confidence interval, } \mathrm{MTM}=\text { medication therapy management; } \mathrm{N}=\text { number; } \mathrm{NA}=\text { not applicable; } \mathrm{RCT}= \\
\text { randomized controlled trial. }\end{array}$} \\
\hline \multicolumn{4}{|c|}{$\begin{array}{l}\text { Outcomes related to using health resources were similarly not much influenced by MTM } \\
\text { interventions (Table E). Two exceptions may merit attention: (1) the use of generic medications } \\
\text { for patients receiving MTM from community pharmacy when compared with educational } \\
\text { mailings (low for benefit from the community pharmacy approach) and (2) the rate of } \\
\text { hospitalization among heart failure patients with home medicines review when compared with } \\
\text { usual care. When MTM was implemented in settings with a broad range of patients, it did not } \\
\text { reduce the number of hospitalizations (low strength of evidence). }\end{array}$} \\
\hline
\end{tabular}


Table E. Summary of findings and strength of evidence for resource-utilization outcomes of MTM interventions

\begin{tabular}{|c|c|c|c|}
\hline Use of Resources Outcomes & $\begin{array}{l}\text { Study Design: } \\
\text { No. Studies (N } \\
\text { Analyzed) }\end{array}$ & Results & $\begin{array}{l}\text { Strength of } \\
\text { Evidence }\end{array}$ \\
\hline Number of high-risk medications & Cohort: $1(2,211)$ & High study limitations & Insufficient \\
\hline $\begin{array}{l}\text { Use of generic medications for call- } \\
\text { center pharmacy-based MTM vs. } \\
\text { educational mailings }\end{array}$ & $\begin{array}{l}2 ; 97,124 \\
(75,166)\end{array}$ & High study limitations, inconsistent & Insufficient \\
\hline $\begin{array}{l}\text { Use of generic medications for } \\
\text { community pharmacy-based MTM } \\
\text { vs. educational mailings }\end{array}$ & $\begin{array}{l}\text { Cohort: } 1 \\
(73,793)\end{array}$ & $\begin{array}{l}\text { Calculated mean difference in weighted } \\
\text { generic dispensing ratio: } 1.2(95 \% \mathrm{Cl} \text { : } \\
0.724 \text { to } 1.676 \text { to; } \mathrm{p}<0.001)\end{array}$ & $\begin{array}{l}\text { Low for benefit for } \\
\text { community } \\
\text { pharmacy }\end{array}$ \\
\hline $\begin{array}{l}\text { Medication costs: patient } \\
\text { copayments }\end{array}$ & NRCT: $1(1,626)$ & High study limitations, indirect & Insufficient \\
\hline $\begin{array}{l}\text { Medication costs: health plan } \\
\text { expenditures }\end{array}$ & $\begin{array}{l}\text { RCT: } 2 \text { (835); } \\
\text { NRCT \& Cohort: } \\
2(1,746)\end{array}$ & Indirect, imprecise & Insufficient \\
\hline Medication costs: total outlays & RCT: $3(1,975)$ & Inconsistent, indirect, imprecise & Insufficient \\
\hline $\begin{array}{l}\text { Medication costs: medication costs } \\
\text { plus other expenditures }\end{array}$ & $\begin{array}{l}\text { RCT: } 2 \text { (>779); } \\
\text { NRCT: } 1 \text { (675) }\end{array}$ & Indirect, imprecise & Insufficient \\
\hline Number of outpatient visits & RCT: $2(2,038)$ & Inconsistent, imprecise & Insufficient \\
\hline Outpatient costs & RCT: $2(1,842)$ & Inconsistent, indirect, imprecise & Insufficient \\
\hline Number of laboratory tests & RCT: $2(1,842)$ & Inconsistent, indirect, imprecise & Insufficient \\
\hline Costs of laboratory tests & RCT: $2(1,842)$ & Inconsistent, indirect, imprecise & Insufficient \\
\hline $\begin{array}{l}\text { Number of emergency department } \\
\text { visits }\end{array}$ & $\begin{array}{l}\text { RCT: } 2(1,344) \\
\text { Observational: } 1 \\
(795)\end{array}$ & Inconsistent, imprecise & Insufficient \\
\hline $\begin{array}{l}\text { Costs of emergency department } \\
\text { visits }\end{array}$ & RCT: 1 (779) & Imprecise & Insufficient \\
\hline Hospitalization: number & RCT: $2(2,398)$ & $\begin{array}{l}\text { Mean difference of } 0.038(95 \% \mathrm{Cl} \\
-0.005 \text { to } 0.080)\end{array}$ & Low for no benefit \\
\hline Hospitalization: percentage & RCT: 2 (710) & Inconsistent. Imprecise & Insufficient \\
\hline $\begin{array}{l}\text { Hospitalization: rate (patients with } \\
\text { heart failure and home medicine } \\
\text { review) }\end{array}$ & Cohort: $1(5,717)$ & $\begin{array}{l}\text { Adjusted HR (95\% Cl): } 0.55 \text { (0.39 to } \\
0.77)\end{array}$ & Low for benefit \\
\hline Costs of hospitalization & RCT: $2(1,842)$ & Inconsistent, imprecise & Insufficient \\
\hline Length of hospital stay & RCT: 1 (46) & High study limitations, imprecise & Insufficient \\
\hline
\end{tabular}

Over all three categories of outcomes, each of which had a substantial number of individual measures, MTM improved outcomes in only a couple of instances. Study limitations and lack of precision of the estimates of effects limited the strength of evidence considerably. As discussed later, even the minimal findings of effectiveness are at best only narrowly applicable.

\section{KQ 3: Effectiveness of MTM by Intervention Features}

We found evidence from one study each on four intervention features: (1) access of pharmacists to patient records ${ }^{22}$ (2) community pharmacy versus call center ${ }^{23}$ (3) level of intensity of intervention, ${ }^{24}$ and (4) type of payer (private vs. Medicaid). ${ }^{25}$ With the exception of the study on pharmacists' access to patient records, we rated these studies as high risk of bias. Evidence was insufficient for most outcomes for the first two intervention features, with two exceptions. First, MTM delivered by community pharmacists increased the weighted generic dispensing ratio when compared with call-center pharmacists (low strength of evidence). Second, enhanced MTM with pharmacists' access to patient records reduced the mean number of adverse drug events; this finding suggested benefit when compared with basic MTM (low strength of 
evidence). We found insufficient evidence for all outcomes for intensity of intervention and type of payer.

\section{KQ 4: Effectiveness of MTM by Patient Characteristics}

We did not identify any studies that analyzed outcomes of MTM by patient characteristics.

\section{KQ 5: Harms of MTM Interventions}

Lack of precision and the limitations of a single high risk-of-bias study meant that evidence was insufficient to judge whether MTM resulted in greater confusion ${ }^{26}$ or inconvenience $^{27,28}$ than usual care. We found no evidence on other prespecified harms, specifically including care fragmentation, patient decisional conflict, patient anxiety, increased (actual) adverse drug events, prescriber confusion, and prescriber dissatisfaction.

\section{Discussion}

\section{Key Findings}

We identified 36 studies that offered information on a range of intermediate outcomes, patientcentered outcomes, and resource utilization. Evidence was insufficient on the effect of MTM on most outcomes. For a limited number of outcomes, we found enough evidence to show that MTM results in improvement when compared with usual care (low strength). Specifically, these outcomes include medication appropriateness, the rate of hospitalization among heart failure patients with home medicines review when compared with usual care, and the use of generic medications for patients receiving MTM from community pharmacies when compared with educational mailings. Similarly, we found sufficient evidence to conclude that MTM conferred no benefit for a limited number of outcomes. When MTM is implemented in settings with a broad range of patients, it does not reduce the number of hospitalizations (low strength of evidence). We found evidence on four intervention components and intervention features: one study provided information on each feature and yielded insufficient evidence for most outcomes with two exceptions. MTM programs with pharmacist access to brief clinical summaries from the medical records reduce the mean number of adverse drug events when compared with basic MTM programs without such access (low strength of evidence). Community pharmacists increase the generic dispensing ratio more than call-center-based pharmacists (low strength of evidence). Similarly, the evidence on harms associated with MTM was limited to one study each on confusion and inconvenience and was rated as insufficient.

\section{Findings in Relation to What Is Already Known}

Our findings contrast with conclusions that Chisholm-Burns and colleagues reached in a recent systematic review. ${ }^{29}$ In that review, the authors concluded that "Pharmacist-provided direct patient care has favorable effects across various patient outcomes, health care settings, and disease states."29, p. 923 Several differences between the Chisholm-Burns review and the current review may account for the discrepant conclusions. First, the Chisholm-Burns review included all studies that cited evidence of pharmacist involvement in direct patient care. The interventions examined included chronic disease management and prospective and retrospective drug utilization review; we excluded these types of efforts because our intended focus was on the MTM intervention itself. Notably, the Chisholm-Burns review did not use the term "medication 
therapy management" to categorize the interventions in the articles they reviewed. Second, approximately 30 percent of the papers in the Chisholm-Burns review were conducted entirely in institutional settings. In contrast, we did not identify any studies within institutional settings that met our MTM intervention definition criteria. Third, the Chisholm-Burns review included a total of 298 articles and did not omit from their analyses studies with a high risk of bias; by contrast, we based our strength-of-evidence grades in this review on only those studies with no more than medium risk of bias.

The striking differences between the conclusions reached in these two reviews emphasize two important needs for both conceptual and practical efforts to systematically review MTM programs. The first is to create a systematic system for classifying the different types of direct patient care services that pharmacists can provide. The second is to develop consensus guidelines for describing intervention features in publications reporting findings from evaluation studies. Progress on these two steps would enable systematic reviews to differentiate better between different types of services and avoid the problem of overgeneralizing review results.

The Centers for Medicare \& Medicaid Services (CMS) supported a large evaluation of MTM programs that we were unable to include in this draft because of the timing of the release of that report. ${ }^{30}$ We will include it in our final report along with our update of the published and grey literature. Although we have not yet incorporated the findings of this large evaluation into our systematic review, we note that the report finds that MTM improved patient adherence to medication regimens and the quality of prescribing. Our review did not find sufficient evidence to evaluate the effect of MTM on improved adherence, but we did find low strength of evidence that MTM improves medication appropriateness, which is conceptually similar to "quality of prescribing." The discrepancy between the CMS findings regarding adherence and findings of studies included thus far in our review may reflect the greater precision that the CMS investigators might have had in their use of pharmaceutical prescription refill records to assess adherence when compared with other studies that primarily used self-report to assess adherence. The report also found some reduction in resource use, but these results were for patients with diabetes or congestive heart failure. Our review found that for patients with heart failure, MTM was likely to reduce hospitalization rates, but we found no effect on mean number of hospitalizations for broadly defined populations.

\section{Applicability of the Findings}

This body of evidence has significant clinical and methodological heterogeneity, which limits the ability to make any universal statements about effectiveness. However, the range of study designs, which includes RCTs, nonrandomized trials, and cohort studies, enhances the applicability of findings for real-world settings. ${ }^{31}$ Included studies ranged from relatively small interventions in single clinics provided by a single interventionist to evaluations of MTM services delivered on a large scale through integrated health systems or health plans as a Medicare Part D or other drug plan benefit. This diversity of studies enhanced the applicability of findings to a wide variety of settings, including outpatient clinics, community pharmacies, and centralized pharmacy call centers. A few studies conducted outside the United States included MTM as part of a home visits program; findings from this model may not be directly applicable within the United States.

The studies in this review are broadly applicable to a range of chronically ill, adult patient populations. The majority of interventions were directed at populations with multiple and common chronic conditions, such as diabetes, chronic heart failure, and hypertension. Several 
specifically targeted adults aged 65 years or older. Few studies reported sociodemographic characteristics beyond age and sex; thus, the applicability of findings to specific populations (e.g., rural, low socioeconomic status, cognitively impaired, uninsured) is unknown. The nature of the MTM intervention, which includes involving patients as active participants in the process, limits the extent to which findings can be generalized beyond patients who agreed to participate in such interventions. Patients who agree to participate may be systematically different from those who decline to be in such a program. For that reason, the impact of such interventions at a population or health-plan level may be limited by the degree of uptake among interested patients.

The intervention used across most studies can be characterized as complex and moderately resource intensive. Components involve identifying applicable patients; initially assessing patients; providing counseling, education, and care coordination; and following patients over time. These services were provided per protocol in some studies and as needed or ad hoc in others. Most studies described intervention components in terms of "pharmaceutical care model" components or Medicare Part D MTM program criteria, but few provided detailed descriptions or measurement of implementation fidelity.

The comparator arm in all studies was usual medical care. This does not typically include distinct MTM services by health care providers other than prescribing providers (not common for the time period covered by most of the studies). Models of collaborative health care delivery are evolving, and the changing roles and training of pharmacists increase the potential applicability of MTM interventions in future models of health care.

The broad sets of outcomes evaluated across this body of evidence spanned a substantial range of both intermediate and health outcomes as well as outcomes related to resource use. Proximal and intermediate outcomes included number of drugs, identification of drug therapy problems, appropriateness of medication prescribing, and laboratory or biometric markers of disease control (e.g., hypertension, hemoglobin A1c, low-density lipoprotein cholesterol). Patient-centered outcomes focused on numerous measures of quality of life as well as adverse drug events. Many studies also reported outcomes involving health care resource use and expenditures (e.g., number and costs of hospitalizations, emergency department visits, outpatient visits).

Most studies did not, however, clearly indicate the expected, desired, or intended direction of effect on most resource use outcomes, making the applicability of using these interventions to reduce drug-related health care costs or expenditures difficult to assess. For example, whether one should expect the number of medications prescribed for heart failure to increase or decrease under the careful scrutiny of an MTM intervention is not clear.

The focus of outcome measurement in many studies was the short-term identification and characterization of drug therapy problems and their resolution; these endpoints are thought to be the outcomes most sensitive to change as a result of receiving MTM services. However, by design, because identification of drug therapy problems is a part of the MTM intervention itself, differences between the nature of the intervention and that of the control programs mean that measuring these outcomes cannot be as rigorous in a usual care comparison group as it is in the intervention group. In fact, many studies were able to measure only changes in this outcome in the intervention group. Hence, many studies failed to demonstrate a direct analytic link between the resolution of drug therapy problems as a result of MTM and impact on intermediate outcomes, patient-centered outcomes, and resource utilization. Thus, the applicability of studies that demonstrate an impact on the resolution of drug therapy problems is limited. 


\section{Implications for Clinical Practice and Policymakers}

Although we found the evidence insufficient in general to draw definitive conclusions about the comparative effectiveness of MTM for most outcomes that we evaluated, our findings do suggest some implications for practice and policy. MTM is already in widespread practice and is now shaped in the United States largely by Medicare Part D policy: this presents both challenges and opportunities. MTM programs of the future, sponsored and administered by Part D drug benefit plans, may be less integrated into routine health care for Medicare beneficiaries than many of the pharmaceutical care interventions included in our review. We were unable to answer definitively whether level of integration matters for effectiveness, but policymakers may need to consider expectations about the impact that MTM might have on patient-centered outcomes and resource use in the context of other health care delivery transformation activities or quality improvement initiatives that are also occurring. More integration of MTM services with other activities may be effective; however, the more integrated MTM becomes within routine medical care, the more difficult it becomes to isolate it as a discrete intervention for evaluation.

Policymakers could thus consider whether MTM services should be positioned as a contributor to overall improvement in processes of care, health status, and costs or positioned as an intervention to which effects can be discretely attributed. Improvements in medication appropriateness or drug therapy regimens may not always translate into improvements in health or costs, and even if they do, secular improvements in other areas of quality improvement may make measuring outcomes attributable to MTM very challenging.

Future training of MTM providers would benefit from a better understanding of which MTM components really matter. At the moment, such information is lacking. Policymakers and funders who wish to understand the comparative effectiveness of different MTM components could encourage rigorous program evaluation designs that fit within the context of the real-world implementation of these programs. For example, positive deviance analyses ${ }^{32}$ with rigorous measurement of implementation features or stepped wedge trial designs ${ }^{33}$ may be useful approaches.

A typical approach for evaluating complex interventions is to identify the "core" components for standardization, while allowing for flexibility for peripheral components or variations in implementation. In complex practice-based innovations, such flexibility may reflect desirable (or unavoidable) adaptations to local circumstances. Policy governing MTM programs may warrant modifications to permit investigators to conduct rigorous and innovative evaluative designs to identify core components or effectiveness-enhancing modifications. As future research and evaluation elucidates these components or enhancements, policy will need to evolve to keep pace with best practices.

Finally, considering both patients' and prescribers' perspectives in future design and delivery of MTM services may be needed. In our current analytic framework, MTM interventions require a significant element of engagement by both patients and prescribers if the interventions are to have a reasonable likelihood of improving outcomes. Although "opt in" strategies may increase the reach of such interventions, keeping patients (and their prescribing providers) engaged in the intervention over a reasonable amount of time may be the key to translating the potential of MTM interventions into actual improvements. 


\section{Limitations of the Comparative Effectiveness Review Process}

The constraints for populations, interventions, and settings that we imposed on this systematic review may limit its applicability as discussed above. During topic refinement and based on technical expert panel inputs and public comment, we expanded the scope by removing an exclusion criterion that would have required MTM interventions to have been directed at a patient population with two or more chronic conditions. As a result, we did include studies that focused on one chronic condition. Because of the prevalence of certain chronic conditions in the adult population, and particularly among Medicare beneficiaries, we think this decision was sensible and permitted us to examine a broader evidence base than would otherwise have been the case.

Although we tried to distinguish MTM from disease or case management interventions, making this distinction was challenging. We created a threshold for what intervention components were required to be present for this distinction. Specifically, we elected to emphasize whether the intervention entailed a comprehensive review of all medications; for that reason, we did not constrain studies of interest to those that targeted a single medication or drug regimen or that focused on a single condition such as diabetes or hypertension.

When we were unable to determine which medications the interventionist had reviewed, we wrote to the authors for additional information. We chose to pursue authors in an effort to permit us to use studies that had been designed as MTM but did not describe the comprehensive medication review component in detail.

Our approach may have been overly inclusive because it led us to include studies that addressed a single disease, as long as the pharmacist reviewed all medications. For example, 12 of the 36 studies were relatively narrowly focused; four of these addressed patients with chronic heart failure and two addressed patients with either hypertension or hypertension and diabetes. The remaining six studies focused on post-transplant patients (kidney, lung), diabetes, glucocorticoid-induced osteoporosis, and hemodialysis. The fact that we drew the line at only one intervention component criterion resulted in an approach that was inclusive of these more narrowly focused (albeit often termed "MTM") studies and may render our results less applicable to MTM interventions targeted to patients with a wide range of chronic conditions.

Also based on feedback during the process of setting out the scope of this review, we chose to include interventions that were broader than the Medicare Part D MTM-defined interventions. Put another way, we broadened our view of patient populations and intervention criteria, and we allowed studies not conducted in the United States into the evidence base. This decision led us to include interventions described as "pharmaceutical care," which were generally based on the pharmaceutical care model as described and refined by Strand and associates; ${ }^{9}$ it also permitted us to examine investigations with elements of pharmaceutical care or MTM that did not specifically label the intervention as either MTM or pharmaceutical care. These studies were often described as "clinical pharmacist interventions."

Furthermore, all the non-U.S. studies involved interventions within single-payer health systems. Hence, the interventions in this review constitute a more heterogeneous group than if we had allowed only those labeled as Medicare Part D MTM programs. This is both a limitation and a strength. Although our approach makes results more challenging to interpret, it enhances our ability not to miss interventions that include MTM components but lack the descriptor term MTM.

Studies did not often explicitly describe certain MTM components. In cases when we could not determine whether investigators had provided certain MTM components (such as patient 
education and counseling, medication action plan, or coordination with other health care providers), we again contacted the authors to gain additional information that would allow us to make an informed decision. We were fairly permissive in interpreting the presence of the MTM intervention components other than comprehensive medication review (e.g., medication action plan). The main reason is that we recognized that terms describing some components have evolved over time and may have been absent from the lexicon in earlier years or implicitly conveyed by authors by simply using the terms "MTM" or pharmaceutical care to describe their intervention.

Our approach to categorizing interventions for KQ 1 relied primarily on the short descriptions in published manuscripts and those we were able to obtain via email inquiries. Their similarities or differences substituted for any overarching taxonomy, because none that we considered seemed to fit our purpose. Thus, we have introduced intervention labels that, admittedly, do not fully describe or account for clinical heterogeneity among interventions. This approach limits our ability to make definitive statements about the effectiveness of various intervention components. We believe that the clusters and categorizations we used are useful heuristics, but some may regard them more as hypothesis generating than as reflecting settled principles of classification.

Finally, our search process was complicated by having to ensure coverage of all terms that could be used to describe MTM interventions over time. Adding to this challenge was our effort to examine the gray literature, where we thought we might find studies tilted toward effectiveness and real-world program evaluation. As it turned out, studies of these types of interventions were not indexed similarly; for that reason, we needed to rely heavily on hand searches of citation lists from key background articles to identify possibly relevant studies for inclusion. Thus, we may have missed some studies that might have qualified for inclusion. Given the considerable diversity in the evidence base we did have, however, and the general lack of data supporting effectiveness of MTM, we do not think that any potential missed studies would have changed our conclusions in any material way. No meta-analyses included more than five studies; as a result, we did not examine included studies for publication bias quantitatively.

\section{Limitations of the Evidence}

As a body of evidence, the MTM literature evaluated in this review has measured numerous outcomes. As indicated in previous sections, very few outcomes, with the exception of harms, remain completely unexamined. Of the 36 studies in this review, we rated 22 as having medium or low risk of bias. The 36 studies included 19 trials and 3 nonrandomized controlled studies. In other words, the literature on this topic is not marked by failure to consider important outcomes, universally high risk of bias, or pervasively weak designs.

Despite these advantages, we were unable to identify sufficient evidence on the majority of hypothesized outcomes of MTM. In several instances, our inability to rate evidence as higher than insufficient came from indirect, inconsistent, and imprecise evidence. The choice of outcome measures in this body of evidence limited our ability to come to conclusions in some instances. For example, some studies did not focus on changes that proponents might expect MTM services to produce. Because effective MTM can either increase or decrease expenditures or use of services based on the needs of the patient, studies that did not prespecify the expected direction of change had no way to interpret their results as an appropriate change. Studies that demonstrated inconsistent results in direction of change (i.e., some showing an increase in resource use and others showing a decrease) may well have been consistent in terms of 
appropriate change, but because they generally failed to establish a priori the direction in which they expected to find an effect, we rated such evidence as indirect and inconsistent.

Similarly, studies often used nonstandardized or idiosyncratic measures for outcomes such as adverse events, adherence, and expenditures or costs; this tendency limited our ability to metaanalyze results. When studies focused on specific outcomes, they were often significantly underpowered to detect differences between groups (that is, they did not meet optimal information size criteria). As a result, we rated several studies as imprecise.

MTM intervention studies are largely practice based and incorporate substantial heterogeneity in specific intervention elements and in patient populations targeted. Yet the evidence is sharply constrained in its ability to inform questions of the effectiveness of specific MTM components or intervention features (KQ 3 in our review) because study designs did not often capitalize on variants in MTM programs for a prospective evaluation of outcomes by those variants. Neither did they measure fidelity to intended MTM elements for post-hoc evaluation. Similarly, the relatively untargeted nature of the MTM interventions meant that, in many studies, only small numbers of patients had any one specific condition, and most studies did not measure patient characteristics beyond age and sex, thus limiting our ability to address KQ 4 in our review. For this reason, the evidence we identified for this review was most relevant for KQ 2.

\section{Research Gaps}

In many bodies of research, questions regarding the comparative effectiveness of specific intervention components or implementation features are best answered after clear evidence of the overall effectiveness of the intervention relative to usual care has been established. Our review largely indicates insufficient evidence on the primary question of effectiveness relative to usual care. By definition, this limited what we could say about comparative effectiveness.

Nonetheless, the widespread implementation of MTM coexists with the urgent need for actionable information for policy, program policies, and training. This clinical and policy environment means that new research cannot afford to address causal claims relative to usual care first, followed by comparative effectiveness of the intervention elements in a relatively controlled environment, and finally, program evaluation of real-world implementation, all in sequential order.

In choosing among various research goals, therefore, funders may wish to consider the relative value of new evidence on overall effectiveness, effectiveness of implementation features, and program implementation and accountability. Trial research in narrow clinical settings can address questions of effectiveness but may lack applicability to real-world implementation. Likewise, evaluations of real-world programs with variable fidelity to interventions can answer questions about process and implementation, but they offer limited information on effectiveness.

For new studies focusing on causal claims, a critical gap relates to the failure to specify the expected direction of effect. New research requires a strong theoretical foundation to help specify causal mechanisms and hypothesized effects. Without such an edifice, future research will continue to produce inconsistent and uninterpretable results.

Heightened attention to causal mechanisms will also help researchers convey their understanding of what outcomes these types of interventions are likely to influence. For instance, how should researchers wishing to establish direct causal links between MTM programs and outcomes evaluate distal outcomes such as patient-centered outcomes and resource utilization? This effort requires a better understanding of the relationship between proximal outcomes like "drug therapy problems identified and resolved" and distal outcomes. For instance, MTM may 
reduce outpatient visits to address side effects. MTM may also result in the need for further testing and evaluation for some patients, which could, in turn, result in more rather than fewer outpatient visits. Unless the nature of change resulting from MTM is specified in relation to goals of drug therapy, studies cannot assert benefit or harm. Further, drug therapy problems are diverse and may not all have the same causal relationship to health, quality of life, patient satisfaction, or resource use outcomes. Furthermore, a causal model of these distal outcomes may need to take into account the competing or complementary contributions of MTM, new models of health care delivery (e.g., patient-centered medical homes), and other quality improvement interventions.

Investigators embarking on new studies focusing on causal links between MTM and outcomes may wish to consider the limitations of studies based on secondary data from existing MTM programs that use opt-in/opt-out patient enrollment mechanisms. Although these studies may provide invaluable information on process measures such as patient engagement, underlying issues of confounding severely limit the validity of causal claims from such studies.

Regardless of the goal of their future research, investigators should consider issues of sample size to ensure precision of their results. This issue is particularly relevant when evaluating outcomes likely to occur in smaller subgroups of patients. Innovative designs (e.g., stepped wedge trials) can permit both rigor and adequate sample size within the context of real-world implementation. With careful attention to fidelity, such designs may also inform questions of the effectiveness of intervention components and implementation features. Such designs may also help inform our understanding of critical training elements for MTM service providers.

Regarding research gaps for specific outcomes such as patient satisfaction, measures specific to the types of services provided through MTM (e.g., patient education about medications) or to the proximal outcomes that MTM is intended to achieve (e.g., reduced medication side effects, improved disease control) may offer better insights into the effects of MTM. Similarly, a medication-related instrument may better measure patients' concerns that are directly related to medication use (e.g., experience of side-effects, intrusiveness of the medication regimen) than generic tools.

\section{Conclusions}

The evidence base is insufficient to address the effectiveness of MTM on most outcomes. Given the widespread implementation of MTM and urgent need for actionable information, funders may wish to weight the relative value of information on overall effectiveness, effectiveness of implementation features, and program implementation and accountability when commissioning new research. 


\section{References}

1. Adams K, Corrigan E, eds. Priority areas for quality improvement. Washington, DC:

Institute of Medicine of the National Academies, The National Academies Press; 2003.

2. Kohn LT, Corrigan J, Donaldson MS, eds. To Err is Human: Building A Safer Health System. Washington, DC: National Academy Press; 2000.

3. McGlynn EA, Asch SM, Adams J, et al. The quality of health care delivered to adults in the United States. N Engl J Med. 2003 Jun 26;348(26):2635-45. PMID: 12826639.

4. Zhan C, Arispe I, Kelley E, et al. Ambulatory care visits for treating adverse drug effects in the United States, 1995-2001. Jt Comm J Qual Patient Saf. 2005 Jul;31(7):372-8. PMID: 16130980.

5. Warholak TL, Nau DP. Quality and Safety in Pharmacy Practice. New York, NY: McGraw-Hill Medical; 2010.

6. Gurwitz JH, Rochon P, Food and Drug Administration. Improving the quality of medication use in elderly patients: a not-sosimple prescription. Arch Intern Med. 2002 Aug 12-26;162(15):1670-2. PMID: 12153368 .

7. Mahan D. Out-of-Bounds: Rising Prescription Drug Prices for Seniors. Families USA Foundation Publication No. 03-106. Washington, DC: July 2003.

8. Kaufman DW, Kelly JP, Rosenberg L, et al. Recent patterns of medication use in the ambulatory adult population of the United States: the Slone survey. JAMA. 2002 Jan 16;287(3):337-44. PMID: 11790213.

9. Pellegrino AN, Martin MT, Tilton JJ, et al. Medication therapy management services: definitions and outcomes. Drugs. 2009;69(4):393-406. PMID: 19323584.

10. 108th U.S. Congress. Medicare Prescription Drug, Improvement, and Modernization Act of 2003. 2003.

11. Tudor CG. Contract Year 2013 Medication Therapy Management (MTM) Program Guidance and Submission Instructions. Baltimore, MD: Centers for Medicare \& Medicaid Services; 2012. http:www.cms.gov/Medicare/Prescription-
Drug-

Coverage/PrescriptionDrugCovGenIn/Down loads/Memo-Contract-Year-2013Medication-Therapy-Management-MTMProgram-Submission-v041012.pdf.

12. Bodenheimer T, Berry-Millett R. Care management of patients with complex health care needs. Robert Wood Johnson Research Synthesis Report No. 19. San Francisco, CA: December 2009.

http://www.rwjf.org/content/dam/farm/repor ts/issue briefs/2009/rwjf49853/subassets/rw jf49853 1.

13. Viswanathan M, Ansari MT, Berkman ND, et al. Assessing the Risk of Bias of Individual Studies in Systematic Reviews of Health Care Interventions. AHRQ Publication No. 12-EHC047-EF. Rockville, MD: Agency for Healthcare Research and Quality Methods Guide for Comparative Effectiveness Reviews; March 2012. www.effectivehealthcare.ahrq.gov/.

14. Higgins JP, Altman DG, Gotzsche PC, et al. The Cochrane Collaboration's tool for assessing risk of bias in randomised trials. BMJ. 2011;343:d5928. PMID: 22008217.

15. Viswanathan M, Berkman ND. Development of the RTI item bank on risk of bias and precision of observational studies. J Clin Epidemiol. 2012 Feb;65(2):163-78. PMID: 21959223.

16. West SL, Gartlehner G, Mansfield AJ, et al. Comparative effectiveness review methods: Clinical heterogeneity. Agency for Healthcare Research and Quality. Methods Research Paper. AHRQ Publication No. 10EHC070-EF. Rockville, MD: September 2010. http://effectivehealthcare.ahrq.gov/.

17. Berkman ND, Lohr KN, Ansari M, et al. Grading the Strength of a Body of Evidence When Assessing Health Care Interventions for the Effective Health Care Program of the Agency for Healthcare Research and Quality: An Update. Methods Guide for Comparative Effectiveness Reviews (Prepared by the RTI-UNC Evidence-based Practice Center under Contract No. 2902007-10056-I) AHRQ Publication No. 13(14)-EHC130-EF. Rockville, MD: Agency for Healthcare Research and Quality; November 2013. 
www.effectivehealthcare.ahrq.gov/reports/fi nal.cfm.

18. Guyatt GH, Oxman AD, Kunz R, et al. GRADE guidelines 6. Rating the quality of evidence--imprecision. J Clin Epidemiol. 2011 Dec;64(12):1283-93. PMID: 21839614.

19. Guyatt GH, Oxman AD, Vist G, et al. GRADE guidelines: 4. Rating the quality of evidence--study limitations (risk of bias). J Clin Epidemiol. 2011 Apr;64(4):407-15. PMID: 21247734.

20. Owens DK, Lohr KN, Atkins D, et al. AHRQ series paper 5: grading the strength of a body of evidence when comparing medical interventions--Agency for Healthcare Research and Quality and the Effective Health-Care Program. J Clin Epidemiol. 2010 May;63(5):513-23. PMID: 19595577.

21. Atkins D, Chang SM, Gartlehner G, et al. Assessing applicability when comparing medical interventions: AHRQ and the Effective Health Care Program. J Clin Epidemiol. 2011 Nov;64(11):1198-207. PMID: 21463926.

22. Touchette DR, Masica AL, Dolor RJ, et al. Safety-focused medication therapy management: a randomized controlled trial. J Am Pharm Assoc (2003). 2012 SepOct;52(5):603-12. PMID: 23023840.

23. Chrischilles EA, Carter BL, Lund BC, et al. Evaluation of the Iowa Medicaid pharmaceutical case management program. J Am Pharm Assoc (2003). 2004 MayJun;44(3):337-49. PMID: 15191244.

24. Winston S, Lin YS. Impact on drug cost and use of Medicare part D of medication therapy management services delivered in 2007. J Am Pharm Assoc (2003). 2009 NovDec;49(6):813-20. PMID: 19858047.

25. Witry MJ, Doucette WR, Gainer KL. Evaluation of the pharmaceutical case management program implemented in a private sector health plan. J Am Pharm Assoc (2003). 2011 Sep-Oct;51(5):631-5. PMID: 21896463.

26. Blennerhassett JD, Cusack BM, Smith CD, et al. A novel medicines management pathway. Journal of Pharmacy Practice and Research (Australia). 2006;36(Mar):175-9. PMID: 44-00783.

27. Carter BL, Barnette DJ, Chrischilles E, et al. Evaluation of hypertensive patients after care provided by community pharmacists in a rural setting. Pharmacotherapy. 1997 NovDec;17(6):1274-85. PMID: 9399611.

28. Barnette DJ, Murphy CM, Carter BL. Clinical skill development for community pharmacists. J Am Pharm Assoc (Wash). 1996 Sep;NS36(9):573-80. PMID: 8824077.

29. Chisholm-Burns MA, Kim Lee J, Spivey CA, et al. US pharmacists' effect as team members on patient care: systematic review and meta-analyses. Med Care. 2010 Oct;48(10):923-33. PMID: 20720510.

30. Marrufo G, Dixit A, Perlroth D, et al. Medication Therapy Management in a Chronically Ill Population: Interim Report. (Prepared by Acumen LLC under Contract \#HHSM-500-2011-00012I/TOT\#0001.) Baltimore, MD: Services CfMM; 2013.

31. Atkins D, Chang S, Gartlehner G, et al. Assessing the applicability of studies when comparing medical interventions. Methods Guide for Comparative Effectiveness Reviews. AHRQ Publication No. 10(12)EHC063-EF. Rockville, MD: Agency for Healthcare Research and Quality.; 2011.

32. Bradley EH, Curry LA, Ramanadhan S, et al. Research in action: using positive deviance to improve quality of health care. Implement Sci. 2009;4:25. PMID: 19426507.

33. Brown CA, Lilford RJ. The stepped wedge trial design: A systematic review. BMC Med Res Methodol. 2006;6:54. PMID: 17092344. 


\section{Introduction}

\section{Context}

Used appropriately, medications can alleviate distressing symptoms that compromise physical and psychological well-being, help prevent the onset of many acute and chronic health illnesses, and improve patient health outcomes. Too often, however, medications are not used appropriately. The Institute of Medicine and other prominent organizations have recognized that medication-related problems plague our health care system. ${ }^{1-3}$ In the United States in 2001, an estimated 4.3 million ambulatory visits were for adverse drug events. ${ }^{4}$ A cohort study of Medicare enrollees estimated the overall rate of adverse drug events at 50.1 per 1,000 personyears. ${ }^{5}$ The study rated more than one-third of the adverse drug events as serious, lifethreatening, or fatal; more than 40 percent of these more severe adverse drug events were classified as preventable. Another study found that more than 12 percent of hospitalized patients experienced an adverse drug event within 3 weeks following hospital discharge. ${ }^{6}$

In addition to problems involving adverse drug events, many patients are not prescribed optimal treatment for conditions such as high blood pressure and hyperlipidemia that increase their risk of developing cardiovascular disease. Moreover, even when optimal therapy is prescribed, patient inability to adhere closely to medication regimens may lead to poor health outcomes. $^{7}$

Medication-related problems are especially pronounced among older adults. ${ }^{5}$ Individuals 65 years or older constitute 13 percent of the U.S. population, but they consume more than 30 percent of all prescription medications. ${ }^{5,8}$ A 2006 report found that nearly 60 percent of people in this age group were taking five or more medications and that nearly 20 percent were taking 10 or more medications, ${ }^{9}$ placing them at increased risk for experiencing adverse drug events. Moreover, these figures reflect a substantial increase in the prevalence of polypharmacy since 1998. ${ }^{9}$

Medication therapy management (MTM) services are intended to address issues of polypharmacy, preventable adverse drug events, medication adherence, and medication misuse. ${ }^{10}$ MTM services are designed to be distinct from medication-dispensing services; in particular, they employ a patient-centric and comprehensive approach, rather than an individual product or episodic perspective. ${ }^{11}$ MTM is the current term that represents services that have evolved out of the philosophy and processes first implemented in the early 1990s as "pharmaceutical care." ${ }^{\text {" In }}$ 2008, 11 national pharmacy organizations achieved a consensus framework for MTM services and established 5 core elements for MTM in practice, including: a medication therapy review, a personal medication record, a medication action plan, intervention and/or referral, and documentation and follow-up. ${ }^{10}$

The Medicare Prescription Drug, Improvement, and Modernization Act of 2003 (Public Law 108-173) ${ }^{12}$ established the requirements that Part D Prescription Drug Benefit Plan sponsors have to meet with respect to cost, quality, and the requirements for MTM programs. The law established oversight by the Centers for Medicare \& Medicaid Services (CMS) and provided a general framework for MTM programs but allowed Part D Plan sponsors flexibility in design, including the patient eligibility criteria for services. The CMS requirements for Part D MTM programs have evolved since their implementation in 2006.

The evolution from pharmaceutical care research interventions to large-scale MTM programs in routine practice represents a journey along a continuum of practice settings, patient populations, and intervention components and features. Over time, standards for these services in 
routine practice have evolved, as have standards for describing and conducting research studies involving these interventions. Thus, we established a broad scope for this comparative effectiveness review and did not limit our perspective to Medicare Part D-defined MTM programs. Throughout this review, we will use the term MTM to describe the general class of intervention. However, when describing individual studies included in this review, we will defer to the terms used by the study author to describe the intervention they were evaluating (e.g., pharmaceutical care, clinical pharmacy services).

\section{Populations}

Adult patients with multiple chronic conditions who take many different prescription or nonprescription medications, herbal products, or diet supplements (and combinations of these) are the target population for MTM services. ${ }^{11}$ Because older adults are more likely to take multiple medications, MTM services generally target them.

CMS required that MTM programs target Medicare Part D enrollees, who have multiple chronic diseases, are taking multiple Part D drugs, and are likely to incur annual costs for covered Part D drugs that exceed a predetermined level ("annual cost threshold"). Beginning in 2010, CMS established both a ceiling and floor for the minimum number of diseases and medications that plans may require for eligibility into their MTM program.

To be eligible for CMS reimbursement, MTM programs originally had to offer services for at least four of seven core chronic diseases: hypertension, chronic heart failure, diabetes, dyslipidemia, respiratory disease (e.g., asthma, chronic obstructive pulmonary disease), bone disease (e.g., osteoporosis, osteoarthritis, rheumatoid arthritis), and mental health diseases. As of January 2013, this criterion specifies at least five of nine core chronic conditions-Alzheimer's disease and end-stage renal disease were the added conditions. Programs may require no more than eight Part D drugs, although they may set the maximum at any number between two and eight. CMS set the annual cost threshold at $\$ 4,000$ in 2006 , lowered it to $\$ 3,000$ in 2010, and increased it by an annual percentage each year beginning in 2012. The cost threshold for 2013 is $\$ 3,144$.

CMS-reimbursable MTM services are required for both community-dwelling beneficiaries and beneficiaries in long-term care settings. Although initial MTM programs were designed as "opt-in," more recently, MTM programs must enroll eligible Medicare Part D beneficiaries using only an opt-out approach. Furthermore, MTM enrollees can refuse individual MTM services without having to disenroll from the MTM program.

CMS eligibility criteria requirements are designed to meet a minimum threshold. The MTM program can also offer MTM services to beneficiaries who do not meet the CMS Part D criteria. The Veterans Health Administration (VHA) includes MTM as one of several clinical activities provided to VHA health beneficiaries by VHA pharmacy services. ${ }^{13}$ The VHA does not specify patient eligibility criteria for MTM services. Non-Part D MTM programs and single research studies of MTM or pharmaceutical care interventions may define their own patient eligibility criteria, which may or may not be similar to current CMS criteria, for example, requiring only one chronic condition to be eligible for services.

\section{Interventions and Comparators}

Several pharmacy organizations have proposed operational features to describe MTM services and best practices for delivering MTM. ${ }^{10,11}$ These features can be summarized as follows: 
- A comprehensive medication review (CMR) to identify and resolve medication-related problems that may include the generation of a personal medication report, which is a written list of the patient's prescription and nonprescription drugs, herbal products, and dietary supplements.

- A medication action or treatment plan developed in collaboration with the patient.

- Education, counseling, and resources to enhance patients' understanding about using the medication and to improve adherence.

- Coordination of care, including documenting MTM services and providing that documentation to the patient's other providers and referring patients to other providers as needed.

CMS requires that each beneficiary enrolled in the MTM program be offered a minimum level of MTM services. These include:

- interventions for both beneficiaries and prescribers;

- an annual CMR with written summaries in CMS's standardized format:

$\circ$ The beneficiary's CMR must include an interactive, person-to-person, or telehealth consultation that is performed by a pharmacist or other qualified provider (e.g., a nurse or a physician) and may result in a recommended medication action plan.

$\circ$ If a beneficiary is offered the annual CMR and is unable to accept the offer to participate, the pharmacist or other qualified provider may perform the CMR with the beneficiary's prescriber, caregiver, or other authorized individual; and

- quarterly targeted medication reviews with follow-up interventions when necessary.

CMS expects the CMR to meet the following professional service definition: "a systematic process of collecting patient-specific information, assessing medication therapies to identify medication-related problems, and developing a prioritized list of medication-related problems, and creating a plan to resolve them with the patient, caregiver, and/or prescriber." ${ }^{\text {, p. p. } 6}$ In addition, CMS expects the CMR to be "an interactive person-to-person or telehealth medication review and consultation conducted in real time between the patient and/or other authorized individual, such as [a] prescriber or caregiver, and the pharmacist or other qualified provider. It is designed to improve patients' knowledge of their prescriptions, over-the-counter medications, herbal therapies, and dietary supplements; identify and address problems or concerns that patients may have; and empower patients to self-manage their medications and their health conditions." 14 , p.6 Written summaries of the CMR are to be provided in CMS's standardized written format that includes a beneficiary cover letter, medication action plan, and personal medication list. ${ }^{15}$ The service-level expectations of a CMR align closely with the definition of MTMs contained in the official health-reporting nomenclature of Current Procedural Terminology. ${ }^{\circledR 16,17}$

Disease-management, case-management, and self-management interventions have components that overlap with MTM components - for example, provision of education and counseling to increase medication adherence. Our preliminary literature search yielded many interventions that can be classified as one of these three types of interventions. To increase the usefulness of this review to stakeholders, we need to exclude disease-management, casemanagement, and self-management intervention studies by applying stringent intervention- 
definition criteria. This will enhance our ability to draw conclusions about the effectiveness of MTM services.

\section{Outcomes}

MTM is thought to influence a wide variety of outcomes. Some MTM services relate to health care-delivery issues, such as medication costs, use of other health care services, and the costs of those services (e.g., emergency department visits or hospitalizations). Other MTM services relate to intermediate health outcomes measured typically by laboratory or other biometric tests for the main chronic conditions of interest to CMS; these may include hemoglobin $\mathrm{A}_{1 \mathrm{c}}$, blood pressure, cholesterol (e.g., total, low-density lipoprotein, and high-density lipoprotein cholesterol), and cardiac function (e.g., left ventricular ejection fraction). Finally, still other MTM services relate to patient-centered outcomes (e.g., morbidity, mortality, reduced adverse drug events, missed days of work or school, patient satisfaction with care, health-related quality of life). ${ }^{18}$ Thus, the impact of MTM on health care utilization, intermediate outcomes, and patient-centered outcomes may derive from improved medication adherence, fewer drugrelated adverse events, and better or more efficient coordination of care.

\section{Settings}

MTM services can be delivered in a variety of settings. These include ambulatory care settings (e.g., outpatient clinics, physician practices), retail pharmacies in the community, and long-term care settings such as assisted living or skilled nursing facilities. In addition, telephonebased MTM services may be provided to community-dwelling adults by professional staff (often pharmacists) employed by pharmacy benefits management companies or other commercial health care companies that have centralized call centers. The setting in which MTM is delivered may depend on the type of provider delivering the service.

One or two specific components of MTM may be delivered within an inpatient setting; medication reconciliation at discharge is an example. However, MTM is designed as a longitudinal intervention. For that reason, it is not an intervention delivered exclusively within inpatient settings.

\section{Contextual Factors}

CMS guidelines require that MTM be delivered by a pharmacist or other qualified health care provider. CMS requires MTM plan sponsors to submit information about the MTM program each year, and plan sponsors must indicate which types of providers deliver MTM services within their plan by selecting one or more of the following provider types:

- local pharmacist

- long-term care consultant pharmacist

- plan sponsor pharmacist

- plan benefit manager pharmacist

- MTM vendor local pharmacist

- MTM vendor in-house pharmacist

- physician

- physician assistant

- registered nurse 
- licensed practical nurse

- nurse practitioner

Professional pharmacy organizations have been actively involved in proposing delivery models, standards, and recommendations for MTM services. Pharmacist training varies considerably. Before the 1990s, individuals could become registered pharmacists with a bachelor of science (B.S.) degree that required a minimum of 5 years of study. Current regulations require that individuals have a doctor of pharmacy (Pharm.D.) degree, which requires a minimum of 6 years of study and provides more clinical training than B.S. programs. In addition, many Pharm.D. graduates pursue advanced training through residency, fellowship, and certificate programs. Some of these programs focus on areas such as MTM. The influence that provider type, education, and MTM-specific training have on MTM effectiveness is unknown.

Numerous factors other than clinical specialty may affect the quality of MTM services. Mode, frequency, and interval of delivery may influence MTM success, as may specific MTM components and the fidelity of their implementation. One key factor is how well an MTM provider understands the patient-specific goals of medication therapy. Integrating MTM services with usual care may help ensure that the goals of MTM are achieved. Integration of services and usual care refers to the ability of the MTM provider to communicate well with patients and multiple prescribers and ease of access to patients' medical records.

Health care reimbursement systems may also influence the delivery of MTM services. Not all private insurers cover MTM services. The degree to which MTM component services differ for Medicare beneficiaries when compared with non-Medicare beneficiaries is not known.

Finally, certain patient populations may have considerable difficulty accessing or participating in MTM services. Examples include individuals who are homebound, individuals who have physical or cognitive disabilities, patients without health insurance, and patients living in rural areas.

\section{Scope and Key Questions}

\section{Scope of the Review}

MTM is a complex intervention with numerous and differing components. This review seeks to catalog MTM intervention components, assess the overall effectiveness of MTM in comparison with usual care, examine the factors under which MTM is effective and optimally delivered, assess what types of patients are likely to benefit from MTM services, and assess what types of patients may be at risk of harms from the program.

\section{Relevance of Research Question to Clinical Decisionmaking or Policymaking}

The Key Questions (KQs) we address are highly relevant to both clinical decisionmaking and policies regarding MTM services. Identifying demonstrably effective models and components of MTM services will help patients and their health care providers achieve important intermediate and long-term health-related outcomes. Our findings will help providers of MTM services, particularly pharmacists and pharmacy benefit managers, understand what works well in which settings and with which patients; the findings will have the potential to improve the efficiency of delivery and thus improve the value of MTM services. Lastly, a better understanding of the 
comparative effectiveness of MTM services will assist CMS with future revisions or enhancements to the policies governing coverage for MTM services.

\section{Key Questions}

The KQs are listed below and placed in relation to another and the PICOs in the analytic framework (Figure 1). Specific details regarding patient population, intervention components, and outcomes are provided in the section that follows the analytic framework.

\section{Analytic Framework}

Figure 1. Analytic framework for medication therapy management

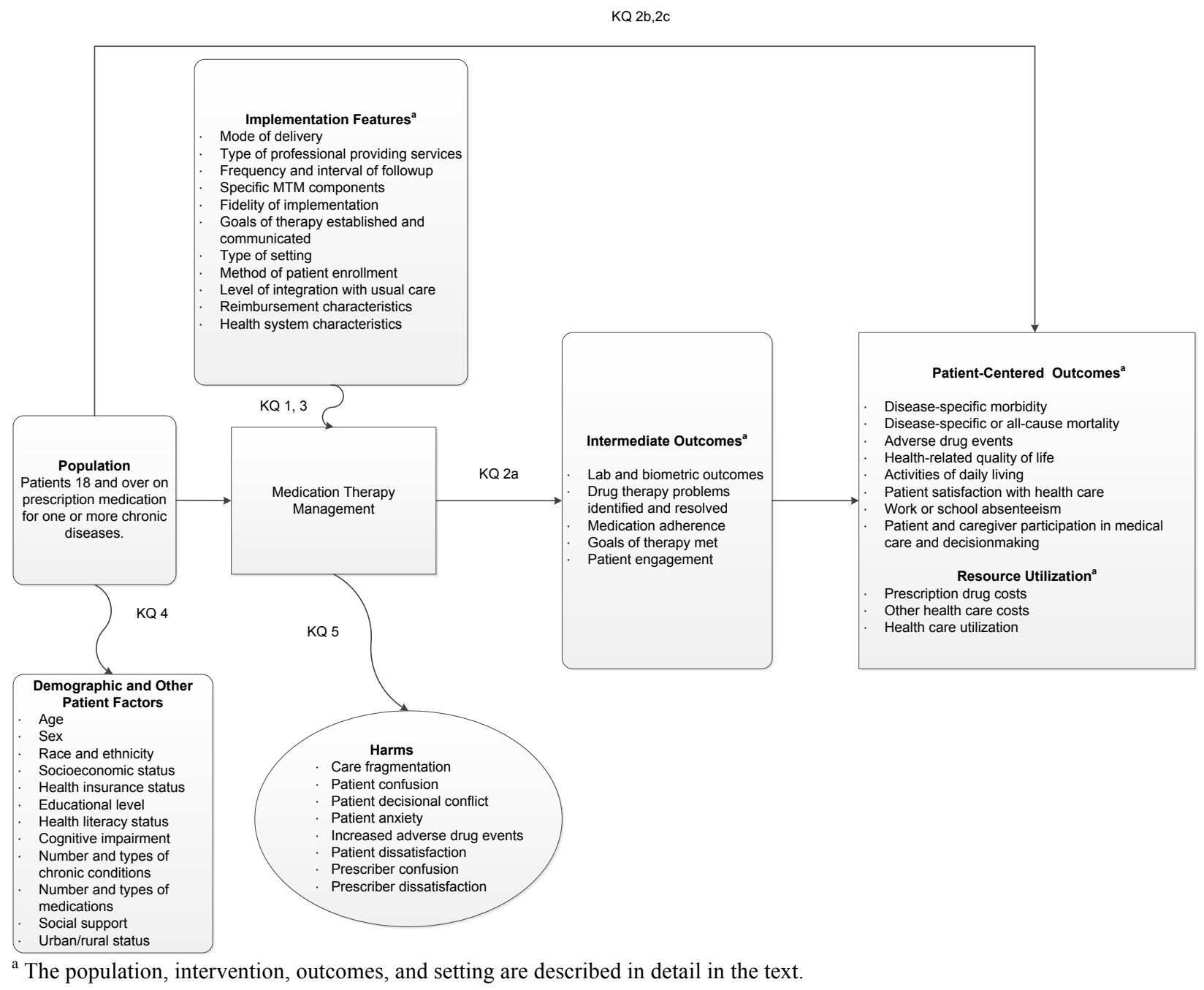

Abbreviations: $\mathrm{KQ}=$ key question; $\mathrm{MTM}=$ medication therapy management

Question 1: What are the components and implementation features of MTM interventions?

Question 2: In adults with one or more chronic diseases who are taking prescription medications, is MTM effective in improving the following: 
a. Intermediate outcomes, including biometric and laboratory measures, drug therapy problems identified, drug therapy problems resolved, medication adherence, goals of therapy met, and patient engagement in medication management?

b. Patient-centered outcomes, such as disease-specific morbidity, disease-specific or allcause mortality, adverse drug events, health-related quality of life, activities of daily living, patient satisfaction with health care, work or school absenteeism, and patient and caregiver participation in medical care and decisionmaking?

c. Resource utilization, such as prescription drug costs, other health care costs, and health care utilization?

Question 3: Does the effectiveness of MTM differ by MTM components and implementation features?

Question 4: Does the effectiveness of MTM differ by patient characteristics, including but not limited to patient demographics and numbers and types of conditions and medications?

Question 5: Are there harms of MTM, and if so, what are they?

\section{Populations, Interventions, Comparators, Outcomes, Timing, and Setting (PICOTS)}

Table 1 lays out the PICOTS for this review. For this review, we take a broad perspective on the population and interventions evaluated; we do not limit the review to interventions and populations meeting CMS Part D MTM eligibility criteria. Specifically, we did not require multiple chronic conditions or a minimum number or level of expenditures on prescription drugs.

Table 1. Populations, interventions, comparators, outcomes, timing, and settings

\begin{tabular}{lc}
\hline PICOTS & Criteria \\
\hline Populations & $\circ$ Patients aged 18 or older with one or more chronic conditions requiring the use of prescription \\
& medication to manage symptoms or prevent progression of chronic disease \\
$\circ$ & Patient characteristics that may influence intervention effectiveness: \\
& - Age, sex, race and ethnicity, socioeconomic status, health insurance status, education level, \\
& health literacy status, cognitive impairment, number and types of chronic conditions, social \\
& support, and urban/rural status \\
\hline Interventions & Explicitly termed $M T M$ services, generally provided as a bundle of related services, that include \\
& at a minimum the following four elements: \\
& - Comprehensive medication review \\
& - Patient-directed medication management action plan with or without an equivalent prescriber- \\
& directed action plan \\
& - Patient-directed education and counseling or other resources to enhance understanding of \\
& the use of medication \\
& - Coordination of care, including prescriber-directed interventions; documentation of MTM \\
& services for use by the patient's other providers; and referral to other providers, clinicians, or \\
& resources when appropriate ${ }^{14}$
\end{tabular}


Table 1. Populations, interventions, comparators, outcomes, timing, and settings (continued)

\begin{tabular}{|c|c|}
\hline PICOTS & Criteria \\
\hline $\begin{array}{l}\text { Interventions } \\
\text { (continued) }\end{array}$ & 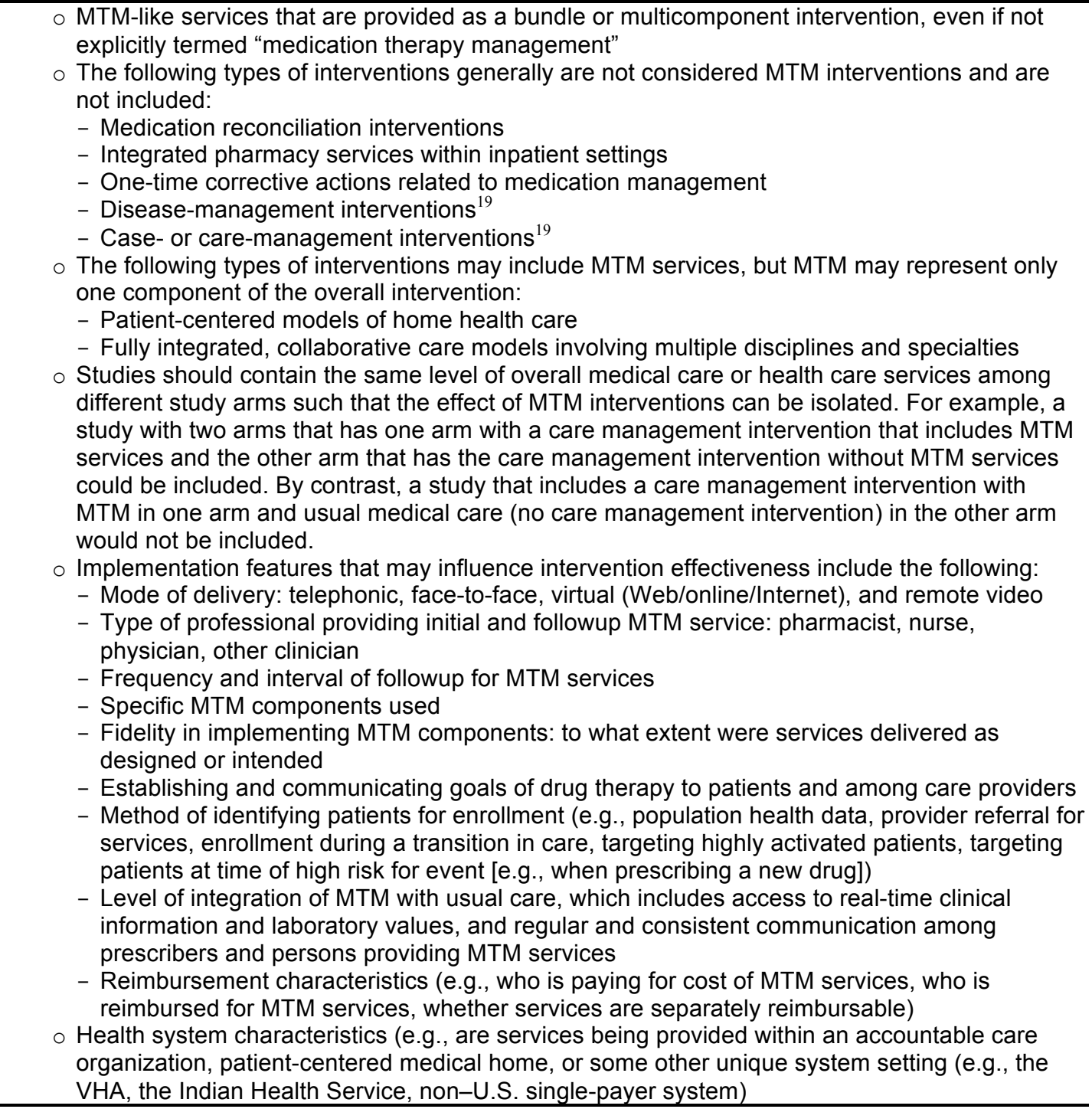 \\
\hline Comparators & $\begin{array}{l}\text { - Usual care, as defined by the studies } \\
\text { Individual components of MTM services (e.g., MTM services with four components vs. a single } \\
\text { component) } \\
\text { Different bundles of MTM services } \\
\text { - Same MTM services provided by different health care professionals (e.g., pharmacist, } \\
\text { physician, nurse, other) } \\
\text { Same bundles of MTM services delivered by different modes (e.g., telephone or in person) } \\
\text { Same MTM services provided at different intensities, frequencies, or level of integration with } \\
\text { prescribers }\end{array}$ \\
\hline
\end{tabular}


Table 1. Populations, interventions, comparators, outcomes, timing, and settings (continued)

\begin{tabular}{|c|c|}
\hline PICOTS & Criteria \\
\hline Outcomes & 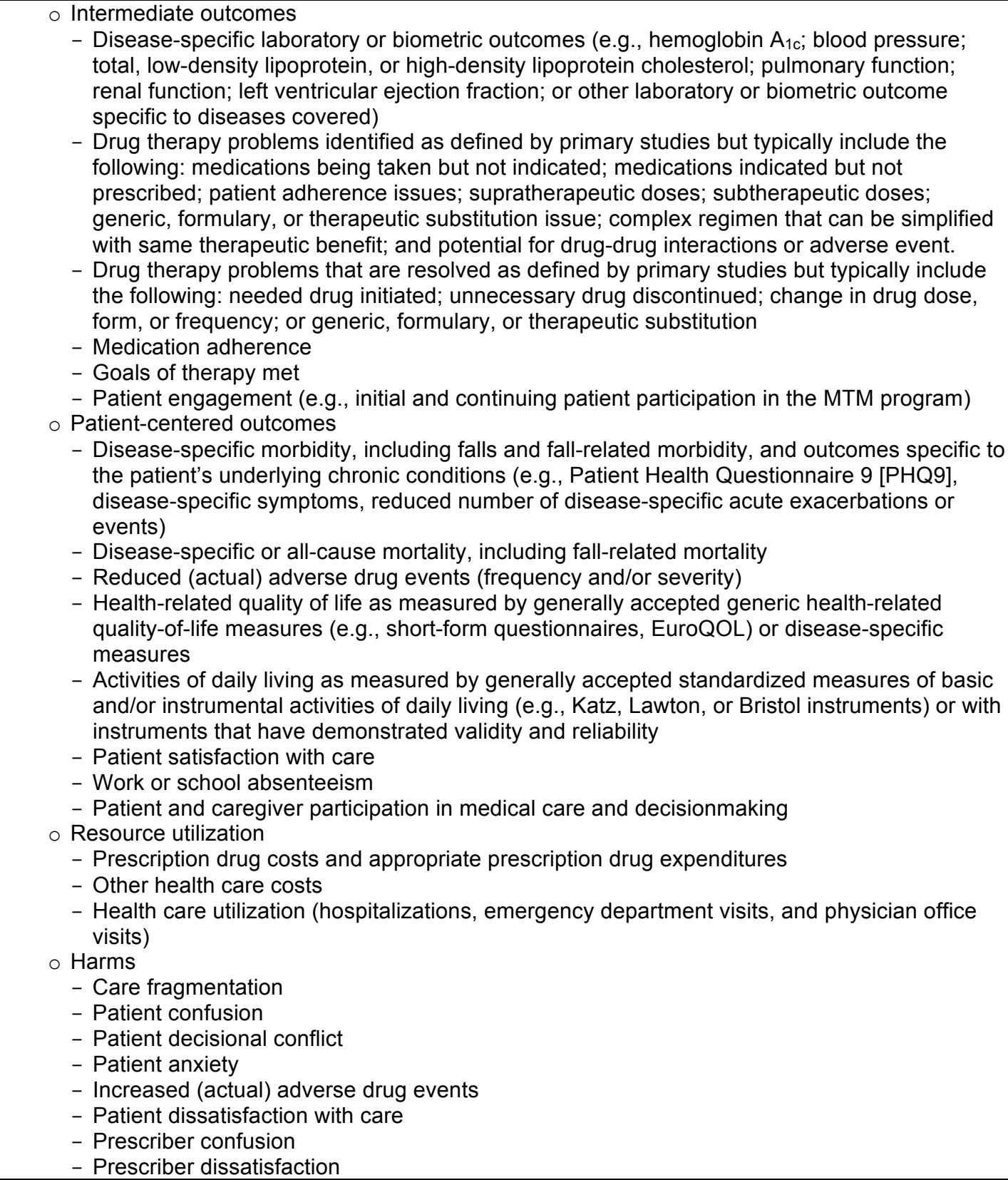 \\
\hline Timing & $\begin{array}{l}\text { Interventions should have at least two separately identifiable episodes of MTM services (either } \\
\text { patient or provider directed or both), with any interval of time in between episodes. } \\
\text { For studies that report outcomes at different points in time, we only considered outcomes } \\
\text { measured after the second episode of care. }\end{array}$ \\
\hline
\end{tabular}


Table 1. Populations, interventions, comparators, outcomes, timing, and settings (continued)

\begin{tabular}{lc}
\hline PICOTS $\quad$ Criteria \\
\hline Setting & Ambulatory settings (e.g., outpatient clinics or private physician offices), long-term care setting, \\
& or retail pharmacy settings) \\
$\circ$ & However, the MTM intervention itself may be delivered by home visits, telephone, via the Web, \\
& or in other non-face-to-face modalities, such as video teleconferencing. \\
$\circ$ & MTM services that are delivered mostly in inpatient settings are not included. \\
$\circ$ & Interventions conducted in the United States and other countries and are published in English \\
are included.
\end{tabular}

\section{Organization of the Report}

The remainder of this report describes our methods, presents the results of our synthesis of the literature, discusses our conclusions, and provides other information relevant to the interpretation of this work. The Methods section describes our scientific approach for this systematic review in detail. The Results section presents our findings for all five of the KQs and includes summary and strength-of-evidence tables. In the Discussion section, we summarize the findings and discuss the implications for clinical practice and further research. A complete list of references, acronyms, and abbreviations follows the Discussion section.

This report contains the following appendixes: Appendix A contains the exact search strings we used in our literature searches. Appendix B documents the title and abstract and full-text review forms. Studies excluded at the stage of reviewing full-text articles with reasons for exclusion are presented in Appendix C. Studies that are awaiting further information from authors are presented in Appendix D. Evidence tables appear in Appendix E. Appendix F lists studies rated high risk of bias and reasons for excluding them from relevant KQ analyses. Quantitative analyses are presented in Appendix G. 


\section{Methods}

The methods for this comparative effectiveness review (CER) on medication therapy management (MTM) follow the methods suggested in the Agency for Healthcare Research and Quality (AHRQ) Methods Guide for Effectiveness and Comparative Effectiveness Reviews (available at http://www.effectivehealthcare.ahrq.gov/methodsguide.cfm). We specified methods and analyses a priori in a protocol posted on the AHRQ website, ${ }^{20}$ following a standard framework for specifying population, interventions, comparators, outcomes, and settings (PICOTS). The main sections in this chapter reflect the elements of the protocol established for the CER; certain methods map to the PRISMA (Preferred Reporting Items for Systematic Reviews and Meta-Analyses) checklist. ${ }^{21} \mathrm{We}$ describe below instances in which our a priori methods required further specification during the project.

\section{Topic Refinement and Review Protocol}

The topic of this report and preliminary key questions (KQs) arose through a nomination from the Pharmacy Quality Alliance. Key Informants representing several clinical and scientific disciplines provided input on the initial KQs; we revised them as needed. An initial draft of the revised KQs was posted for public comment from March 6, 2013, through April 2, 2013, on the AHRQ Effective Health Care program Web site. We received comments from 23 professional organizations and individuals and further revised KQs as appropriate. Specifically, we

1. added a new KQ (KQ 1) to describe the components and implementation features of MTM interventions,

2. included additional intermediate outcomes in KQ 2,

3. reworded KQ 3 to include MTM components,

4. specified MTM components and implementation features for KQ 3 in the PICOTS,

5. specified additional patient characteristics for KQ 4 in the PICOTS, and

6. rephrased KQ 5 to make the response conditional on identifying whether any harms of MTM exist.

\section{Literature Search and Identification Strategy}

\section{Search Strategy}

To identify articles relevant to each KQ, we began with a focused MEDLINE ${ }^{\circledR}$ search for MTM interventions using a combination of medical subject headings $\left(\mathrm{MeSH}^{\circledR}\right)$ and title and abstract keywords and limiting the search to English-language and human-only studies (Table 2). We also searched the Cochrane Library and the International Pharmaceutical Abstracts database using analogous search terms (Appendix A). We selected these databases based on preliminary searches and consultation with content experts. We conducted quality checks to ensure that the searches identified known studies (i.e., studies identified during topic nomination and refinement). Based on these quality checks, we revised and ran additional searches (specifically, drug therapy management, drug therapy problem, and medications management) to avoid missing articles that might prove eligible for this CER. 
In addition, we searched the gray literature for unpublished studies relevant to this review and included studies that met all the inclusion criteria and contained enough methodological information to assess risk of bias. Specifically, sources of gray literature included ClinicalTrials.gov, the World Health Organization's International Clinical Trials Registry Platform, Health Services Research Projects in Progress (HSRProj), the National Institutes of Health Research Portfolio Online Reporting Tools, the Database of Promoting Health Effectiveness Reviews, the New York Academy of Medicine Grey Literature Report, and CMS.gov. AHRQ's Scientific Resource Center managed the process of submitting requests for scientific information packets, which contain information about MTM programs and services of interest from relevant providers.

We reviewed our search strategy with an independent information specialist and the Technical Expert Panel and supplemented it according to their recommendations. In addition, to avoid retrieval bias, we manually searched the reference lists of landmark studies and background articles on this topic to identify any relevant citations that our electronic searches might have missed.

We will conduct an updated literature search (of the same databases searched initially) concurrent with the peer review process. We will also investigate any literature the peer reviewers or the public suggest and, if appropriate, incorporate additional studies into the final review. The appropriateness of those studies will be determined using the methods and criteria described above.

We will include pooled estimates of effect or other relevant results from systematic reviews in the update search that meet our inclusion/exclusion criteria. We will evaluate the quality of included systematic reviews using the AMSTAR tool. ${ }^{22}$ If appropriate and feasible, we may update the results of these reviews quantitatively or qualitatively. Should identified systematic reviews use inclusion or exclusion criteria that differ from ours, we will review their reference lists to ensure that we include all relevant studies.

\section{Inclusion/Exclusion Criteria}

We specified our inclusion and exclusion criteria based on the population, intervention, outcome, timing, and settings identified through the topic refinement exercise. We excluded studies published in languages other than English. We excluded study designs without control groups to ensure that our pool of included studies can inform the causal link between the intervention and outcomes. 
In conducting the review, we found that we needed to define the intervention with greater specificity than originally thought so that we could include MTM interventions but exclude disease management interventions. Specifically, we required that included studies had conducted a comprehensive, rather than condition-specific, medication review, as required in our PICOTS criteria. Although we had not planned to contact study authors routinely for additional information, the lack of clarity regarding intervention elements in numerous published studies necessitated our contacting authors. For these studies, we based our decisions on inclusion or exclusion based on email communication. (Appendix D specifies the studies or publications for which we sought such information but received no response from authors as of the time the draft report was submitted for peer review.)

\section{Study Selection}

Pairs of trained members of the research team reviewed each title and abstract independently against our inclusion/exclusion criteria. Studies marked for possible inclusion by either reviewer underwent a full-text review. For studies that lack adequate information to determine inclusion or exclusion, we retrieved the full text and then made the determination.

We retrieved and reviewed the full text of all included titles during the title/abstract review phase. Two trained members of the team independently reviewed each full-text article for inclusion or exclusion based on the eligibility criteria specified in Table 3. If both reviewers agreed that a study did not meet the eligibility criteria, they excluded the study. If the reviewers disagreed, they discussed differences to achieve a consensus. If they could not reach consensus, a third senior member of the review team resolved the conflict. We tracked all results in an EndNote $^{\circledR}$ (Thomson Reuters, New York, NY) database, and we will deposit them in the Systematic Review Data Repository at the end of the study. We recorded the reason that each excluded full-text publication did not satisfy the eligibility criteria. Appendix $\mathrm{C}$ lists all studies excluded at this stage together with the reason(s) for exclusion.

\section{Data Extraction}

For studies that met our inclusion criteria, we abstracted relevant information into evidence tables (Appendix E). We piloted our approach with a sample of studies and revised the form thereafter. We designed data abstraction forms to gather pertinent information from each article, including the characteristics of the study populations, interventions, comparators, outcomes, timing, settings, study designs, methods, and results. A second member of the team reviewed all data abstractions for completeness and accuracy. (Relevant forms can be found in Appendix B.) 
Table 3. Inclusion/exclusion criteria for medication therapy management studies

\begin{tabular}{|c|c|c|}
\hline Category & Inclusion & Exclusion \\
\hline Population & $\begin{array}{l}\text { Patients aged } 18 \text { or older with one or more } \\
\text { conditions requiring the regular use of prescription } \\
\text { medication to manage symptoms or prevent } \\
\text { progression of chronic disease }\end{array}$ & $\begin{array}{l}\text { - Children under age } 18 \\
\text { - Adults with acute conditions }\end{array}$ \\
\hline Interventions & $\begin{array}{l}\text { - Those specified in the PICOTS criteria listed in } \\
\text { Table } 1 \text { (Introduction) } \\
\text { - More complex interventions with an MTM } \\
\text { component that are compared with identical } \\
\text { interventions without an MTM component } \\
\text { (including care management and disease } \\
\text { management) }\end{array}$ & $\begin{array}{l}\text { - Drug therapy services for a single drug } \\
\text { (e.g., warfarin clinics, statin clinics) } \\
\text { - Interventions in which the effect of the } \\
\text { MTM component cannot be isolated (e.g., } \\
\text { case management or disease } \\
\text { management with an MTM component) } \\
\text { - Self-management programs } \\
\text { - Isolated medication reconciliation } \\
\text { interventions } \\
\text { - Integrated pharmacy services within } \\
\text { inpatient settings } \\
\text { - One-time corrective interventions related } \\
\text { to medication management }\end{array}$ \\
\hline $\begin{array}{l}\text { Control } \\
\text { interventions }\end{array}$ & $\begin{array}{l}\text { - Those specified in the PICOTS criteria listed in } \\
\text { Table } 1 \text { (Introduction) }\end{array}$ & \\
\hline Outcomes & $\begin{array}{l}\text { - Those specified in the PICOTS criteria listed in } \\
\text { Table } 1 \text { (Introduction) }\end{array}$ & $\begin{array}{l}\text { - Studies that do not include at least one of } \\
\text { the outcomes listed under the inclusion } \\
\text { criteria }\end{array}$ \\
\hline $\begin{array}{l}\text { Timing of } \\
\text { intervention and } \\
\text { followup }\end{array}$ & $\begin{array}{l}\text { - Interventions should have at least two } \\
\text { separately identifiable episodes of MTM } \\
\text { services (either patient directed or provider } \\
\text { directed or both) with or without specifying any } \\
\text { certain amount of time between those episodes } \\
\text { - For studies that report outcomes at different } \\
\text { points in time, we considered only outcomes } \\
\text { measured after the second episode of care. }\end{array}$ & $\begin{array}{l}\text { - Studies that measure outcomes only after } \\
\text { one episode of MTM care }\end{array}$ \\
\hline Settings & $\begin{array}{l}\text { - Ambulatory (e.g., outpatient clinics, private } \\
\text { physician offices, or retail pharmacy settings) } \\
\text { and long-term care settings } \\
\text { - May be delivered by telephone, via the Web, or } \\
\text { in other non-face-to-face modalities, such as } \\
\text { video teleconferencing } \\
\text { - Interventions conducted in the United States } \\
\text { and other countries will be included }\end{array}$ & $\begin{array}{l}\text { - Inpatient settings, if delivery of MTM } \\
\text { services occurs almost exclusively in the } \\
\text { inpatient setting }\end{array}$ \\
\hline Geography & - No limits & - Not applicable \\
\hline Dates of search & $\begin{array}{l}\text { - No limits; searches will be updated while the } \\
\text { draft report is out for peer review }\end{array}$ & - Not applicable \\
\hline Study designs & $\begin{aligned} \text { - Original research } \\
\text { - Eligible study designs include: } \\
-\quad \text { Randomized controlled trials } \\
-\quad \text { Nonrandomized controlled trials } \\
-\quad \text { Prospective controlled cohort studies } \\
-\quad \text { Retrospective controlled cohort studies } \\
-\quad \text { Case-control studies } \\
-\quad \text { Systematic reviews and meta-analyses }\end{aligned}$ & $\begin{array}{l}\text { - Case series } \\
\text { - Case reports } \\
\text { - Nonsystematic reviews } \\
\text { - Studies without a control group }\end{array}$ \\
\hline Study duration & No limits & Not applicable \\
\hline $\begin{array}{l}\text { Publication } \\
\text { language }\end{array}$ & English & All other languages \\
\hline Publication type & Any publication reporting primary data & Publications not reporting primary data \\
\hline
\end{tabular}




\section{Assessment of Risk of Bias of Individual Studies}

To assess the risk of bias of individual studies, we used predefined criteria developed by AHRQ. ${ }^{23}$ For randomized controlled trials, we relied on the risk-of-bias tool developed by the Cochrane Collaboration. ${ }^{24} \mathrm{We}$ assessed the risk of bias of observational studies using an item bank developed by RTI International. ${ }^{25}$

In general terms, results of a study with low risk of bias are considered valid. A study with medium risk of bias is susceptible to some bias but probably not sufficient to invalidate its results. A study with high risk of bias has significant methodological flaws (e.g., stemming from serious errors in design or analysis) that may invalidate its results. Primary concerns for our review included selection bias, confounding, performance bias, detection bias, and attrition bias. Specifically, we evaluated studies on the adequacy of randomization, allocation concealment, similarity of groups at baseline, masking, attrition, whether intention-to-treat analysis was used, method of handling dropouts and missing data, validity and reliability of outcome measures, and treatment fidelity.

We excluded studies that we deemed at high risk of bias from our main data synthesis and main analyses. We included them for sensitivity analyses; in cases when we had no other available or credible evidence, we included in the report a brief synopsis of studies assessed as high risk of bias.

\section{Data Synthesis}

When we found three or more similar studies for a comparison of interest, we conducted meta-analysis of the data from those studies. For all analyses, we used random-effects models to estimate pooled or comparative effects. To determine whether quantitative analyses were appropriate, we assessed the clinical and methodological heterogeneity of the studies under consideration following established guidance; ${ }^{26}$ that is, we qualitatively assessed the PICOTS of the included studies, looking for similarities and differences. When we conducted quantitative syntheses (i.e., meta-analysis), we assessed statistical heterogeneity in effects between studies by calculating the chi-squared statistic and the $\mathrm{I}^{2}$ statistic (the proportion of variation in study estimates attributable to heterogeneity). The importance of the observed value of $\mathrm{I}^{2}$ depends on the magnitude and direction of effects and on the strength of evidence for heterogeneity (e.g., the $\mathrm{p}$-value from the chi-squared test or a confidence interval for $\mathrm{I}^{2}$ ). Where relevant, we examined potential sources of heterogeneity using sensitivity analysis.

When quantitative analyses were not appropriate (e.g., because of heterogeneity, insufficient numbers of similar studies, or insufficiency or variation in outcome reporting), we synthesized the data qualitatively. Whenever possible, we computed confidence intervals for individual outcomes.

Because numerous articles about both trials and observational studies often did not provide complete information about findings (e.g., 95 percent confidence intervals; statistical significance values, or between-group data), in many cases we had to calculate odds ratios, mean differences, or standardized mean differences; the relevant 95 percent confidence intervals; and p-values. In all such cases in which we calculated data, we specify this in the Results chapter; information not specifically called out as "calculated" is taken from the original articles. 


\section{Grading Strength of Evidence for Individual Comparisons and Outcomes}

We graded the strength of evidence based on the guidance established for the AHRQ Evidence-based Practice Center (EPC) program. ${ }^{27}$ Developed to grade the overall strength of a body of evidence, this approach incorporates four key domains: study limitations (includes study design and aggregate quality), consistency, directness, and precision of the evidence.

Consistency refers to similarity in direction or magnitude of effect. Study limitations refer to the risk of bias from study design or study conduct. Directness refers to whether evidence links the intervention directly to the health outcome. Precision refers to the certainty around the estimate of effect, after accounting for sample size and number of events. It also considers other optional domains that may be relevant for some scenarios, such as a dose-response association, plausible confounding that would decrease the observed effect, strength of association (magnitude of effect), and publication bias. Dose-response refers to a pattern of a larger effect with greater exposure. Plausible confounding refers to situations where, had these confounders not been present, the observed effect would have been even larger than the one observed. Strength of association refers to instances where the observed effect is large enough that it cannot have occurred solely as a result of bias from potential confounding factors. We evaluated optimal information size criteria to make judgments about precision and based our grades on low or medium risk-of-bias studies unless none were available.

Our approach is consistent with current strength of evidence guidance developed by GRADE and AHRQ EPCs. The GRADE guidance explicitly discourages the inclusion and averaging of risk of bias across studies with different underlying risk of bias criteria. Rather, it suggests considering including only studies with a lower risk of bias. ${ }^{28}$ Likewise, the AHRQ EPC guidance notes that reviewers may focus "strength of evidence on the subset of studies that provide the least limited, most direct, and most reliable evidence for an outcome or comparison, after analysis of all the evidence."27, p. 20

Table 4 describes the grades of evidence that can be assigned. ${ }^{29}$ Grades reflect the strength of the body of evidence to answer the KQs on the comparative effectiveness, efficacy, and harms of the interventions examined in this review. Two reviewers assessed each domain for each key outcome resolved any differences by consensus discussion or referral to a third, senior member of the team. We graded the strength of evidence for the outcomes deemed to be of greatest importance to decisionmakers and those commonly reported in the literature; we did not grade the strength of evidence for KQ 1 (on components and features of MTM services).

Table 4. Definitions of the grades of overall strength of evidence

\begin{tabular}{ll}
\hline Grade & Definition \\
\hline High & $\begin{array}{l}\text { High confidence that the evidence reflects the true effect. Further research is very unlikely } \\
\text { to change our confidence in the estimate of effect. }\end{array}$ \\
\hline Moderate & $\begin{array}{l}\text { Moderate confidence that the evidence reflects the true effect. Further research may } \\
\text { change our confidence in the estimate of the effect and may change the estimate. }\end{array}$ \\
\hline Low & $\begin{array}{l}\text { Low confidence that the evidence reflects the true effect. Further research is likely to } \\
\text { change our confidence in the estimate of the effect and is likely to change the estimate. }\end{array}$ \\
\hline Insufficient & Evidence either is unavailable or does not permit estimation of an effect. \\
\hline
\end{tabular}




\section{Assessing Applicability}

We assessed applicability of the evidence following guidance from the Methods Guide for Effectiveness and Comparative Effectiveness Reviews. ${ }^{30}$ We used the PICOTS framework to explore factors that affect applicability. Some factors identified a priori that may limit the applicability of evidence include the following: age and health status of enrolled populations, health insurance coverage and access to health care, and complexity and intensity of the MTM intervention.

\section{Peer Review and Public Commentary}

[To be completed after peer review] 


\section{Results}

\section{Introduction}

This section of this comparative effectiveness review (CER) on medication therapy management (MTM) first presents the results of the literature searches. We then document the results for each Key Question (KQ). KQ 1 describes MTM intervention characteristics. KQ 2 presents evidence on the effectiveness of MTM interventions, focusing on intermediate outcomes, then patient-centered (health) outcomes, and then use of health care resources or costs. The presentation of KQ 3 summarizes the evidence by intervention components and implementation features; KQ 4 summarizes evidence by patient characteristics. KQ 5 examines the evidence on harms of MTM programs. Appendix E has two parts pertaining to these KQs: the first part has the lengthy descriptions of the design of all included studies (for KQ 1); the second presents the evidence tables, organized by outcome, for the remaining KQs.

Generally, for KQs 2 through 5, the text gives key points and the related strength of evidence grades, followed by a detailed synthesis of the relevant studies. We also present pairs of tables for each outcome. One gives basic summary information about the results of included studies, indicating whether the quantitative data had been what the investigators reported or were calculated by us. The other table in these sets documents the strength of evidence grades for major outcomes (showing the ratings for required domains and, in a small number of cases, any ratings for optional domains). Appendix F contains the tables documenting how we arrived at risk of bias assessments for individual studies.

Most data can be found in tables and are not repeated in text. As noted in Methods, we focus on studies of low or medium risk of bias; when we need to summarize information for studies of high risk of bias, we note the principal problems leading to that rating.

Finally, our inclusion criteria for study designs were expansive and included randomized controlled trials (RCTs) and a variety of observational studies (nonrandomized controlled trials, cohort studies and the like). We use "studies" to refer to all types of investigations; we specify RCTs (or non-RCTs) as appropriate.

\section{Results of Literature Searches}

Figure 2 presents our literature search results. Literature searches through June 27, 2013, for the draft report, identified 2,129 unduplicated citations. Appendix A provides a list of all search terms used and the results of each literature search. In addition, we identified 99 publications through grey literature searches, suggestions from technical experts or public comments received during topic refinement, or hand searches of included studies. After applying our eligibility and exclusion criteria to titles and abstracts of all 2,228 identified citations, we obtained full-text copies of 328 published articles. We reapplied our inclusion criteria and excluded 286 of these articles from further review before doing the risk-of-bias assessment. Appendix C provides a list of excluded studies and reasons for exclusion at the full-text stage. Appendix D lists the studies with too little information for us to be able to make a decision on inclusion or exclusion; these are the studies from which we sought further information directly from authors but did not receive a response before sending this draft out for peer review.

The 42 articles included after full-text review represent 36 studies. Evidence tables for these 36 studies are provided in Appendix E. 
Figure 2. Disposition of articles on medical therapy management (PRISMA figure)

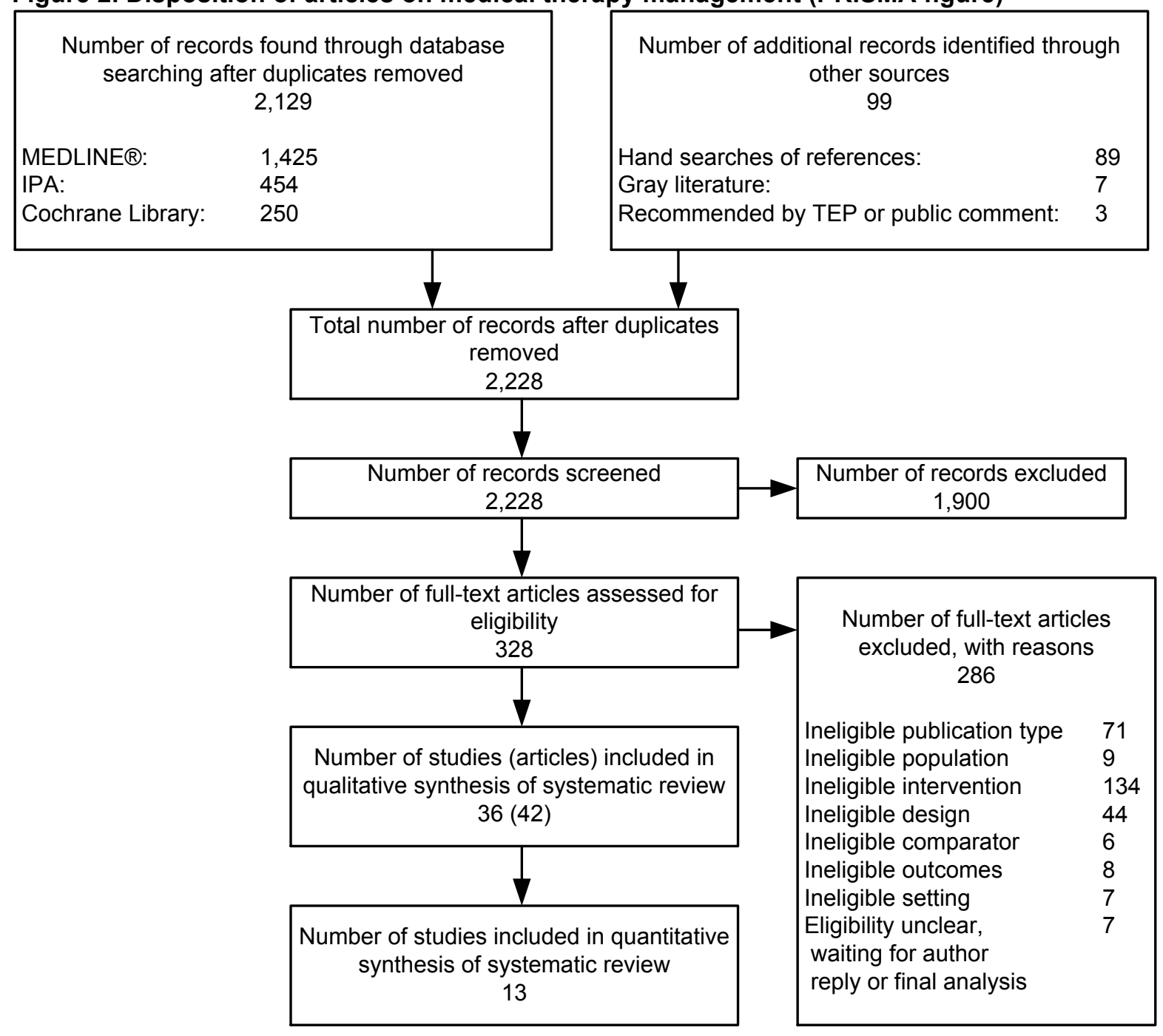

Abbreviations: IPA, International Pharmaceutical Abstracts; PICOTS, populations, interventions, comparators, outcomes, timing, settings; TEP, technical expert panel.

The Agency for Healthcare Research and Quality (AHRQ) Effective Health Care Program Scientific Resource Center placed the request for scientific information packets (SIPs) in the Federal Register on September 16, 2013; it posted them for 30 days. The final version of this CER will include any relevant data from responses to this request.

Table 5 summarizes study characteristics of included studies. Overall, 28 (77.8 percent) of included studies were conducted in the US, and 16 (44.4 percent) were conducted prior to the 2003 Medicare Modernization Act, which established the framework for Medicare Part D MTM programs. Just over half of included studies used an RCT design (either parallel or cluster group), three ( 8.3 percent) used a non-randomized controlled trial design, and the remaining studies (38.9 percent) used a cohort study design. Only three studies used an active treatment comparison group. Intermediate outcomes were the most commonly reported outcomes. Of the 36 studies, two were considered low risk of bias (5.6 percent), 21 were considered medium risk of bias (55.6 percent) and 14 (38.9 percent) were considered high risk of bias. 
Table 5. Characteristics of included studies

\begin{tabular}{ll}
\hline Study Characteristic & $\mathbf{N ~ ( \% )}$ \\
\hline Country & $28(77.8)$ \\
\hline US & $8(22.2)$ \\
\hline Non-US & $1(2.8)$ \\
\hline Multiple & $16(44.4)$ \\
\hline Conducted prior to 2003 Medicare Modernization Act & $14(38.9)$ \\
\hline Study Design & $5(13.9)$ \\
\hline RCT-parallel group & $3(8.3)$ \\
\hline RCT-cluster group & $14(38.9)$ \\
\hline Non-randomized Controlled Trial & $3(8.3)$ \\
\hline Cohort Study & $29(80.6)$ \\
\hline Used an active treatment comparison arm & $17(47.2)$ \\
\hline Outcomes Measured & $25(69.4)$ \\
\hline \begin{tabular}{l} 
Intermediate outcomes (e.g., disease specific lab values, medication adherence, \\
\hline Prug therapy problems)
\end{tabular} \\
\hline Utilization and Economic Outcomes \\
\hline Risk of bias & $2(5.6)$ \\
\hline Low & $20(55.6)$ \\
\hline Medium & $14(38.9)$ \\
\hline High & \\
\hline Abreviatentered outcomes (e.g., health outcomes, quality of life, patient satisfaction) & \\
\hline
\end{tabular}

Abbreviations: US = United States

\section{Key Question 1: Components and Implementation Features of MTM Interventions}

KQ 1 was designed to synthesize descriptive findings regarding MTM intervention components and implementation features, which have been identified as important factors related to effectiveness of these interventions. Because this report is a CER, our study inclusion criteria included a requirement for a control or comparison arm. For that reason, our synthesis of descriptive findings related to MTM components and implementation features is limited to investigations that comparatively evaluated MTM; that is, it does not include all studies of MTM interventions, many of which we had excluded because of the lack of a comparison arm. Thus, our findings represent a somewhat circumscribed lens for the descriptive part of this review.

Synthesizing intervention components and implementation features across this body of evidence was challenging. Mainly, studies did not consistently describe the intervention characteristics or implementation features in sufficient detail to allow us to determine the extent to which certain components were used, at which intervals, and at what intensity. Even studies published after the 2003 Medicare Modernization Act, which formalized aspects of pharmaceutical care, lacked sufficient reporting detail in many cases.

\section{Overall Descriptors of Study Interventions}

Table 6 specifies the components and implementation features from our analytic framework (Figure 1 in Introduction). It also gives our assessment of the suitability or feasibility of synthesis, based on information available in the included studies across the entire evidence base. 
Table 6. Characteristics of medication therapy management interventions

\begin{tabular}{llll}
\hline $\begin{array}{l}\text { Characteristic of the MTM Intervention } \\
\text { (Specified in Analytic Framework in } \\
\text { Introduction) }\end{array}$ & $\begin{array}{l}\text { Summarize in Tables } \\
\text { and Synthesize With } \\
\text { Counts }\end{array}$ & $\begin{array}{l}\text { Summarize in Table } \\
\text { but Not Synthesize } \\
\text { With Counts }\end{array}$ & $\begin{array}{l}\text { Neither Summarize in } \\
\text { Tables Nor Synthesize } \\
\text { With Counts }\end{array}$ \\
\hline Mode of delivery & $\mathrm{X}$ & $\mathrm{X}$ \\
\hline Type of professional providing services & $\mathrm{X}$ & $\mathrm{X}$ \\
\hline Frequency and interval of followup & $\mathrm{X}$ & $\mathrm{X}$ \\
\hline Specific MTM components & & $\mathrm{X}$ \\
\hline Fidelity of implementation & & $\mathrm{X}$ \\
\hline $\begin{array}{l}\text { Goals of therapy established and } \\
\text { communicated }\end{array}$ & $\mathrm{X}$ & \\
\hline Type of setting & $\mathrm{X}$ & \\
\hline Method of patient enrollment & $\mathrm{X}$ & \\
\hline Level of integration with usual care & & \\
\hline Reimbursement characteristics & & \\
\hline Health system characteristics & & \\
\hline
\end{tabular}

Abbreviations: MTM =medication therapy management.

In the best case, we can summarize data in tables and synthesize the information with actual counts across the body of evidence. This is true for mode of delivery, type of professional giving the services, details about followup, settings, modes of reimbursement, and characteristics of health systems. Somewhat less can be done with methods for enrolling patients and level of integrating MTM with usual care, so information is just included in study-level summary tables (but not synthesized with actual counts across the body of evidence). Finally, information on specific MTM components, fidelity of implementation, and MTM goals was so inconsistent or sparse that we could not either synthesize or include information in summary tables.

Table 7 summarizes the intervention characteristics and features that were reported consistently enough to be synthesized with counts and frequencies - namely, those in Table 6 with an X in the first column. It also notes whether the investigators used the phrase "pharmaceutical care" or the phrase "medication therapy management" to refer to the program tested. For details about intervention followup, the information in Table 7 is "as designed" (i.e., however the investigators described their initial intentions).

During our abstraction process, we identified two distinct categories of interventions. One category, of 24 studies, used a broad pharmaceutical care approach or MTM intervention in serving their patient populations; that is, they did not focus specifically on any one disease or clinical condition. We refer to these studies in the review and Table 7 as "broadly focused." The other category, with 12 studies, involved interventions evaluated in the context of a single chronic condition (e.g., chronic heart failure, diabetes) or provided in a highly specialized clinic setting (e.g., transplant clinics). In these studies, the investigators implemented a pharmaceutical care approach or MTM intervention that was comprehensive and attended to the patient's complete drug therapy regimen, but the focus of component interventions (e.g., education, counseling, care coordination) and outcomes measured may have been specific to diseases or conditions. We refer to these studies as "narrowly focused." 
Table 7. Characteristics of medication therapy management studies by type of patient population (broad focus or narrow focus on conditions or diagnoses)

\begin{tabular}{|c|c|c|c|c|}
\hline $\begin{array}{l}\text { MTM } \\
\text { Intervention }\end{array}$ & Characteristic of the Intervention & $\begin{array}{l}\text { Overall } \\
(\mathbf{N}=36) \\
\mathbf{N}(\%)\end{array}$ & $\begin{array}{l}\text { Broad } \\
\text { Focus } \\
(\mathrm{N}=24) \\
\mathrm{N}(\%)\end{array}$ & $\begin{array}{l}\text { Narrow } \\
\text { Focus } \\
(\mathrm{N}=12) \\
\mathrm{N}(\%)\end{array}$ \\
\hline \multirow{3}{*}{$\begin{array}{l}\text { Phrase used to } \\
\text { describe } \\
\text { intervention }\end{array}$} & Pharmaceutical care & $14(39)$ & $8(33)$ & $6(50)$ \\
\hline & Medication therapy management & $12(33)$ & $11(46)$ & $1(8)$ \\
\hline & Other & $10(28)$ & $5(21)$ & $5(42)$ \\
\hline \multirow[t]{4}{*}{ Mode of delivery } & Face-to-face only & $19(53)$ & $10(42)$ & $9(75)$ \\
\hline & Telephone only & $6(17)$ & $6(25)$ & 0 \\
\hline & Mixture of face-to-face and telephone & $9(25)$ & $7(29)$ & $2(17)$ \\
\hline & Not reported & $2(6)$ & $1(4)$ & $1(8)$ \\
\hline Professional & Pharmacist as interventionist & $36(100)$ & $24(100)$ & $12(100)$ \\
\hline \multirow{6}{*}{$\begin{array}{l}\text { Frequency of } \\
\text { followup as } \\
\text { designed }\end{array}$} & One time with followup as needed & $6(17)$ & $5(21)$ & $1(8)$ \\
\hline & Two times & $6(17)$ & $6(25)$ & 0 \\
\hline & Three times & $4(11)$ & $2(8)$ & $2(17)$ \\
\hline & Every 4 to 8 weeks for between 4 and 24 months & $6(17)$ & 0 & $6(50)$ \\
\hline & $\begin{array}{l}\text { Varied based on trigger (e.g., refill, physician visit, continuous } \\
\text { enrollment for certain duration) }\end{array}$ & $3(8)$ & $3(13)$ & 0 \\
\hline & Not reported & $11(31)$ & $8(33)$ & $3(25)$ \\
\hline \multirow[t]{6}{*}{ Clinical settings } & Community pharmacy & $6(17)$ & $3(13)$ & $3(25)$ \\
\hline & Centralized pharmacy & $4(11)$ & $4(17)$ & 0 \\
\hline & Outpatient medical clinic & $14(39)$ & $8(33)$ & $6(50)$ \\
\hline & Home visits & $4(11)$ & $2(8)$ & $2(17)$ \\
\hline & Integrated health system & $4(11)$ & $4(17)$ & 0 \\
\hline & Multiple settings & $4(11)$ & $3(13)$ & $1(8)$ \\
\hline \multirow{4}{*}{$\begin{array}{l}\text { Reimbursement } \\
\text { characteristics }\end{array}$} & Services provided through Medicare Part D benefit & $7(19)$ & $7(29)$ & 0 \\
\hline & Services provided through some other health plan benefit & $4(11)$ & $2(8)$ & $2(17)$ \\
\hline & Services provided through study-related funding & $3(8)$ & $3(13)$ & 0 \\
\hline & Reimbursement details not reported & $22(61)$ & $12(50)$ & $10(83)$ \\
\hline \multirow{6}{*}{$\begin{array}{l}\text { Health system } \\
\text { characteristics }\end{array}$} & Single payer system (outside US) & $7(19)$ & $4(17)$ & $3(25)$ \\
\hline & Academic medical center & $8(22)$ & $4(17)$ & $4(33)$ \\
\hline & Integrated health system & $9(25)$ & $8(33)$ & $1(8)$ \\
\hline & Health plan & $8(22)$ & $7(29)$ & $1(8)$ \\
\hline & $\begin{array}{l}\text { Pharmacies independent of medical care system or health } \\
\text { plans }\end{array}$ & $2(6)$ & 0 & $2(17)$ \\
\hline & Other & $2(6)$ & $1(4)$ & $1(8)$ \\
\hline
\end{tabular}

Abbreviations: $\mathrm{N}=$ number; $\mathrm{US}=$ United States.

In many cases, to distinguish narrowly focused MTM studies from case- or diseasemanagement interventions, we had to contact study authors to clarify that their intervention included a comprehensive drug therapy assessment and drug therapy intervention beyond the single target condition of interest. The distinction between these broad-focus and narrow-focus categories may be important for interpretation of the effectiveness of these types of interventions. Studies included in this review used "medication therapy management" to describe the intervention (Table 7) in only 12 of the 36 studies. With respect to mode of delivery (Table 7), six broadly focused studies used only telephone contact; ${ }^{31-37}$ by contrast, no narrowly focused studies used only telephone contact. Nine studies (seven broad; two narrow) used a mixture of face-to-face and telephone contact. ${ }^{38-49}$ The studies using a mixture of modes often used face-toface delivery for the initial consultation and did follow-up contacts by telephone. Except for the two studies that did not report mode of delivery, ${ }^{50-52}$ the remaining studies used only face-to-face delivery in pharmacies, clinics, or homes. 
All included studies used a pharmacist as the interventionist (Table 7). In some studies, however, the interventionist was described as a community pharmacy resident or ambulatory care pharmacy resident.

Table 7 also summarizes the intervention frequency and interval of follow-up as designed, not as may have actually occurred, and these features also differed across studies. Of the 36 included studies, however, 11 did not report the designed frequency of contact and interval of followup. Only six studies reported on the actual frequency and interval of follow-up. ${ }^{38,40-43,47,53-}$ ${ }^{55}$ Studies evaluating real-world experience with these types of interventions often included a minimum contact threshold for inclusion of patients in the data analysis, but the intervention duration and interval of followup was open-ended and determined by clinical need, as is typical in real-world practice.

Included studies provided interventions in a variety of clinical settings including community pharmacies, centralized pharmacies or pharmacy call centers, outpatient medical clinics, and some used home visits (Table 7). Half of the narrowly focused interventions were delivered exclusively in an outpatient medical clinic. ${ }^{48,49,53,56-60}$

Concerning reimbursement, of the 36 studies in the evidence base, 22 did not report on reimbursement at all. Of the remaining 14 studies, 11 reported that pharmaceutical care or MTM was a covered benefit to patients; pharmacist services were reimbursed through an existing mechanism (e.g., Medicare Part D or other health care benefit). ${ }^{31-37,40-43,46,61-63}$ Three studies clearly indicated that pharmacist services were reimbursed through pilot, grant, or study-related funding. ${ }^{38,47,64}$ Among the 12 broad studies with such information, 10 used either Medicare Part $\mathrm{D}$ or internal study funding. The two narrow studies with reimbursement information used some type of health plan funding.

Finally, the context of the MTM services also varied in terms of features of the health system or organization in which they were provided. Academic medical centers, integrated health care delivery systems, health plans, and single payer health care systems outside the United States were all represented in this evidence base.

\section{Study-level Descriptors of Interventions}

In Appendix E (Part 1), we have provided study-level summaries to describe the included interventions. Those tables (Table E.1 and E.2) document: interventions and the amount of integration with usual practice; method of identifying patients for receipt of MTM services; setting, mode of delivery, frequency and interval of followup; and health care system and reimbursement context. Table E. 1 describes the 24 broad-focus studies; Table E. 2 describes the remaining 12 narrow-focus studies (and additionally specifies the particular focus). We summarize the main elements in text below.

Of the dozen narrow-focus studies, four addressed chronic heart failure and two addressed hypertension or hypertension and diabetes. The remaining six studies focused on post-transplant patients (kidney; lung), diabetes alone, glucocorticoid-induced osteoporosis, and hemodialysis.

Interventions described as pharmaceutical care were generally based on the pharmaceutical care model as described and refined by Strand and associates ${ }^{40-43,50-54,56-60,63,65-70}$ Interventions termed medication therapy management (i.e., MTM) were generally based on criteria defined for the Medicare Part D program, which includes elements of the pharmaceutical care model. ${ }^{31-}$ $38,46,47,55,64,71$ The remaining interventions included elements of pharmaceutical care or MTM but did not specifically label the intervention as either one or the other. ${ }^{39,44,45,48,49,61,62,72-74}$ These studies were often described as "clinical pharmacist interventions." 
The level with which pharmaceutical care or MTM services were integrated with usual care has two main element: (1) the degree of access that the interventionist had to clinical information in the patient's medical record, such as laboratory results, diagnoses, and progress notes; (2) the method and process of communication between the interventionist and prescribers. Providing these programs within an outpatient medical clinic, presumably where the patient is also receiving medical care, is one such marker of integration, particularly when the study indicated that the pharmacist was part of a multidisciplinary care team. Some studies, however, described the pharmacy or pharmacist simply as co-located in a clinic. In these instances, we do not know whether the level of integration with medical care would be any higher than if the pharmacist had been located in a community pharmacy. Thus, we could not rely solely on clinical setting as a marker of integration with usual care.

Because many studies did not provide sufficient details regarding specific components of the intervention, whether termed pharmaceutical care, MTM, or clinical pharmacist intervention, we were unable to synthesize the use of specific intervention components beyond the components we required for study inclusion.

Only three studies used an active treatment comparator group. ${ }^{46,47,55}$ All other studies (regardless of focus) compared pharmaceutical care or MTM with usual care. This factor also impeded our assessing the effectiveness of individual intervention components. Furthermore, almost no study reported on the fidelity with which intervention components were delivered (relative to the original design or intention), including whether goals of drug therapy were established and communicated.

The methods by which patients were identified and offered pharmaceutical care or MTM services has been proposed as a moderator of effectiveness; the aim is to target patients most likely to benefit. These factors may include, for example, patients using drugs with narrow therapeutic windows, complex drug regimens, or patient characteristics such as age, cognitive status, or social situation. With respect to data sources that studies used to identify and then enroll patients for services, pharmacy prescription records (at a community pharmacy, clinic, or health plan) were the most common source. Except for the studies evaluating Medicare Part D MTM programs, few studies used the same criteria for identifying patients for enrollment. Most required either some degree of regimen complexity, such as the number of drugs taken or use of one or more drugs considered high risk for adverse events. Most studies using pharmacy data or claims mailed or telephoned eligible patients to provide information about enrollment in an MTM program. For Medicare Part D MTM programs, "opt out" is another variation of enrollment for these services. Patients meeting eligibility criteria are enrolled for services unless they specifically "opt-out." Some studies relied solely on provider referral, patient self-referral, or routine medical record screening at time of a provider visit to identify patients for services. Only one study enrolled patients in services during a transition in care from an inpatient to a home setting. ${ }^{54}$

Tables E1 and E2 also provide study-level detail on intervention setting, mode of delivery, frequency and interval of followup and health care system and reimbursement characteristics, which were summarized overall in Table 7 and in the preceding section. 


\section{Key Question 2: Effect of Medication Therapy Management Interventions on Intermediate, Patient-Centered, and Resource Utilization Outcomes}

We present below key findings and a detailed synthesis of intermediate, patient-centered, and resource utilization outcomes separately. (These outcomes were specified in Table 1 of the Introduction.) When possible (a minimum of three reasonably similar studies for a given intervention or outcome), we pooled study results and document those findings below. When studies were too heterogeneous to pool, we present effect sizes for individual studies whenever possible in summary tables for each outcome that was reported in two or more studies. We also provide strength of evidence tables to support our findings.

Because in many cases the investigators did not report a full set of findings that compared changes over time between intervention and comparisons groups or other details that would permit full analysis, we calculated various statistics ourselves. In these cases, we present in the tables below only these calculated findings and related statistical levels, and we note this explicitly in the tables or text (as "calculated"). The underlying data from the study article(s) can be found in the evidence tables in Appendix E.

\section{Key Points: Intermediate Outcomes}

- Evidence was insufficient to evaluate the effect of MTM on anticoagulation after 12 months due to an imprecise, single RCT body of evidence with medium limitations.

- Evidence was insufficient to evaluate the effect of MTM on hemoglobin A1C after 6 to 12 months due to and inconsistent and imprecise body of evidence from two RCTs with medium limitations.

- Evidence was insufficient to evaluate the effect of MTM for decreasing low-density lipoprotein (LDL) cholesterol after 6 to 24 months due to an imprecise, single RCT body of evidence with medium limitations.

- Evidence was insufficient to evaluate the effect of MTM for reducing blood pressure (BP) after 4 to 12 months due to an imprecise, single RCT body of evidence with medium limitations.

- Several studies did not report outcomes such as drug therapy problems identified and resolved for both intervention and control groups. As a result, limited evidence addresses the effectiveness of MTM compared with usual care in improving these important intermediate outcomes. Study limitations, inconsistency, and lack of precision led us to conclude that the evidence is insufficient to judge the effectiveness of MTM in improving these outcomes when compared with usual care.

- Evidence was insufficient to evaluate the effect of MTM on medication adherence (as defined in several ways) as a result of inconsistent and imprecise evidence. The number of trials, consistency, and study limitations varied by specific adherence measure.

- MTM increases the appropriate use of medications as measured by overall scores on appropriateness indices (low strength of evidence).

- Evidence was insufficient for effect of MTM on medication dosing as a result of inconsistent, indirect, and imprecise evidence from two trials with medium study limitations. 


\section{Detailed Synthesis: Intermediate Outcomes}

\section{Anticoagulation}

One RCT (medium risk of bias) reported on the effects of a pharmaceutical care intervention on anticoagulation among patients in family medicine clinics in a rural community after 12 months of followup. ${ }^{68}$ This intervention was conducted with 81 patients at high risk for medication-related problems; however, this outcome was reported only for the four patients in the intervention arm and the six patients in the control arm who were taking anticoagulants. The percentage of subjects who achieved a therapeutic international normalized ratio (INR) differed significantly between the intervention and control arms (100 percent versus 16.7 percent $(\mathrm{p}=0.048)$; calculated odds ratio [OR], 32.94; 95\% confidence interval [CI], 1.06 to 1,021.35). Because of imprecision (wide confidence intervals) and unknown consistency, we graded the evidence as insufficient to evaluate the effectiveness of MTM on improving therapeutic anticoagulation (Table 8).

Table 8. Anticoagulation: Strength of evidence

\begin{tabular}{|c|c|c|c|c|c|c|c|}
\hline $\begin{array}{l}\text { Study } \\
\text { Design }\end{array}$ & $\begin{array}{l}\text { Number of } \\
\text { Studies; } \\
\text { Subjects } \\
\text { (Analyzed) }\end{array}$ & $\begin{array}{l}\text { Study } \\
\text { Limitations }\end{array}$ & Consistency & Directness & Precision & $\begin{array}{l}\text { Findings and } \\
\text { Direction } \\
\text { [Magnitude] of } \\
\text { Effect }\end{array}$ & $\begin{array}{l}\text { Strength of } \\
\text { Evidence }\end{array}$ \\
\hline RCT & $\begin{array}{l}1 ; 81 \\
(10)\end{array}$ & Medium & $\begin{array}{l}\text { Consistency } \\
\text { unknown-single } \\
\text { study }\end{array}$ & Direct & Imprecise & $\begin{array}{l}\text { Therapeutic } \\
\text { INR achieved: } \\
100 \% \text { vs. } \\
16.7 \% \\
p=0.048\end{array}$ & Insufficient \\
\hline
\end{tabular}

Abbreviations: INR, international normalized ratio; RCT, randomized controlled trial; vs. = versus

\section{Hemoglobin A1C}

Two RCTs and two cohort studies reported on outcomes related to hemoglobin A1c (HbA1c) among patients with diabetes (Table 9). One RCT (medium risk of bias) reported no significant difference in mean HbA1c between intervention (pharmaceutical care) and control patients in an Australian outpatient hospital diabetes clinic at 6 months. ${ }^{58}$ The other RCT (medium risk of bias) reported on changes in the percentage of patients with diabetes who achieved a HbAlc of $\leq 7.5$ percent at 12 months among patients at high risk for medication-related problems seen in family medicine practices in a rural community. ${ }^{68}$ The percentage of patients at goal did not differ significantly between intervention and control arms at baseline (23.1 versus 56.3 , calculated $\mathrm{p}=0.08$ ) but was significantly different at followup (100 versus 26.7 , calculated OR, 56.455; $95 \%$ CI, 2.811 to 1,133.912. $\mathrm{p}=0.008$,). The two cohort studies (high risk of bias because of selfselection of participants into the intervention arm) were conducted primarily by telephone within large, integrated US health care systems. Of these studies, one study did not report any significant difference in the percentage of patients achieving a HbAlc of less than 7 percent at 6 months $;{ }^{35}$ the other reported no significant change in mean HbAlc at 6 months. ${ }^{32}$ 
Table 9. Hemoglobin A1c: Summary of results

\begin{tabular}{|c|c|c|c|c|}
\hline $\begin{array}{l}\text { Study } \\
\text { Design/Risk of } \\
\text { Bias }\end{array}$ & Study Arms & N Analyzed ${ }^{a}$ & $\begin{array}{l}\text { Outcome Reported } \\
\text { by Study and Time } \\
\text { Period }\end{array}$ & Results \\
\hline $\begin{array}{l}\text { Clifford et al. } \\
2002^{58} \\
\text { RCT/Medium }\end{array}$ & $\begin{array}{l}\text { G1: Pharmaceutical care } \\
\text { G2: Standard care }\end{array}$ & $\begin{array}{l}\text { G1:48 } \\
\text { G2:25 }\end{array}$ & $\begin{array}{l}\text { Mean } \mathrm{HbA} 1 \mathrm{c} \text { at } 6 \\
\text { months. }\end{array}$ & $\begin{array}{l}\text { Calculated mean difference: } \\
-0.20 \\
95 \% \mathrm{Cl}:-0.927 \text { to } 0.527 \\
p=0.590\end{array}$ \\
\hline $\begin{array}{l}\text { Taylor et al., } \\
2003^{68} \\
\text { RCT/Medium }\end{array}$ & $\begin{array}{l}\text { G1: Pharmaceutical care } \\
\text { G2: Standard care }\end{array}$ & $\begin{array}{l}\text { G1: } 13^{a} \\
\text { G2: } 16^{a}\end{array}$ & $\begin{array}{l}\text { Percentage with } \\
\mathrm{HbA} 1 \mathrm{c} \text { at goal } \\
\text { (defined as less } \\
\text { than or equal to } \\
7.5 \% \text { ) at baseline } \\
\text { and at } 12 \text { months. }\end{array}$ & $\begin{array}{l}\text { Calculated OR: } 56.45595 \% \mathrm{Cl} \text { : } \\
2.811 \text { to } 1,133.912, p=0.008\end{array}$ \\
\hline $\begin{array}{l}\text { Pindolia et al., } \\
2009^{35} \\
\text { Cohort } \\
\text { study/High }\end{array}$ & $\begin{array}{l}\text { G1: Opted in to a telephone- } \\
\text { based MTM Program } \\
\text { G2: Usual medical care } \\
\text { (opted out of MTM program) }\end{array}$ & $\begin{array}{l}\text { G1: } N^{a} \\
\text { G2: } N^{a}\end{array}$ & $\begin{array}{l}\text { Change in } \\
\text { percentage of } \\
\text { patients with } \mathrm{HbA} 1 \mathrm{c} \\
\text { less than } 7 \text { at } 6 \\
\text { months }\end{array}$ & $\begin{array}{l}\text { G1: }+3 \\
\text { G2: }+7 \\
\text { Between-group p: inferred to } \\
\text { be NS, exact } p \text { NR } \\
\text { Within-group p: NR }\end{array}$ \\
\hline $\begin{array}{l}\text { Jeong et al., } \\
2007^{32} \\
\text { Cohort } \\
\text { study/High }\end{array}$ & $\begin{array}{l}\text { G1: Participants in Part D } \\
\text { Medicare MTM program } \\
\text { G2: Control subjects eligible } \\
\text { for Part D MTM program but } \\
\text { declined enrollment } \\
\text { G3: Control subjects without } \\
\text { Part D Medicare as their } \\
\text { primary drug benefit }\end{array}$ & $\begin{array}{l}\text { G1: } 1,211^{a} \\
\text { G2: } 1,000^{a} \\
\text { G3: } 743^{a}\end{array}$ & $\begin{array}{l}\text { Mean change in } \\
\text { HbA1c at } 6 \text { months }\end{array}$ & $\begin{array}{l}\text { Calculated mean difference: } \\
\text { G1 vs. G3: } 0.004 \text {, } \\
95 \% \text { Cl: }-0.087 \text { to } 0.095 \\
p=0.931 \\
\text { Calculated mean difference of } \\
\text { G1 vs. G2: } 0.041 \\
95 \% \mathrm{Cl}:-0.043 \text { to } 0.125 \\
p=0.337\end{array}$ \\
\hline
\end{tabular}

${ }^{\mathrm{a}}$ The study included more subjects than the number analyzed and reported in this column, but the investigators assessed this outcome only among patients with diabetes within each study arm.

Abbreviations: $\mathrm{CI}=$ confidence interval; $\mathrm{DM}=$ diabetes mellitus; $\mathrm{G}=$ group; $\mathrm{HbA} 1 \mathrm{C}=$ hemoglobin $\mathrm{A} 1 \mathrm{C}$ or glycosolated hemoglobin, $\mathrm{MTM}=$ medication therapy management; $\mathrm{NR}=$ not reported; $\mathrm{NS}=$ not sufficient; $\mathrm{OR}=$ odds ratio; $\mathrm{RCT}=$ randomized controlled trial.

Based on direct, but inconsistent and imprecise, evidence from the two RCTs, both with medium limitations (Table 10) we concluded that the strength of evidence is insufficient to evaluate the effectiveness of MTM interventions to improve mean HbA1c levels or increase the percent of patients achieving a goal HbAlc level.

Table 10. Hemoglobin A1c: Strength of evidence

\begin{tabular}{|c|c|c|c|c|c|c|}
\hline $\begin{array}{l}\text { Study } \\
\text { Design }\end{array}$ & $\begin{array}{l}\text { Number of } \\
\text { Studies; } \\
\text { Subjects } \\
\text { (Analyzed) }\end{array}$ & $\begin{array}{l}\text { Study } \\
\text { Limitations }\end{array}$ & Consistency & Directness & Precision & $\begin{array}{ll}\text { Findings and } & \text { Strength of } \\
\text { Direction } & \text { Evidence } \\
\text { [Magnitude] of Effect } & \end{array}$ \\
\hline RCT & $\begin{array}{l}2 ; 154 \\
(102)\end{array}$ & Medium & Inconsistent & Direct & Imprecise & $\begin{array}{l}\text { One trial: no change in Insufficient } \\
\text { mean } \mathrm{HbA} 1 \mathrm{c} \text { at } 6 \\
\text { months. One trial: } \\
\text { significantly greater } \\
\text { percentage of patients } \\
\text { with } \mathrm{HbA} 1 \mathrm{c}>7.5 \text { at } 12 \\
\text { months. }\end{array}$ \\
\hline
\end{tabular}

Abbreviations: HbA1c= hemoglobin A1c; RCT $=$ randomized controlled trial 


\section{LDL Cholesterol}

One RCT and four cohort studies reported on outcomes related to LDL cholesterol (Table 11). The RCT (medium risk of bias), reported the percentage of patients with dyslipidemia who achieved an LDL cholesterol goal based on Adult Treatment Panel III (ATPIII) criteria for lipid management among patients at high risk for medication-related problems in a rural community. ${ }^{68}$ The intervention and control groups did not differ significantly in percentage at goal at baseline ( 10.5 percent versus 15.8 percent, $\mathrm{p}=0.631)$ but differed significantly at 12 months $(77.8$ percent versus 5.9 percent, $p=0.001$; calculated OR, 56.00; 95\% CI, 5.583 to 561.753).

Table 11. LDL cholesterol: Summary of results

\begin{tabular}{|c|c|c|c|c|}
\hline $\begin{array}{l}\text { Study } \\
\text { Design/Risk of } \\
\text { Bias }\end{array}$ & Study Arms & $\begin{array}{l}\mathrm{N} \text { of Subjects } \\
\text { Analyzed }\end{array}$ & $\begin{array}{l}\text { Outcome Reported } \\
\text { by Study and Time } \\
\text { Period }\end{array}$ & Results \\
\hline $\begin{array}{l}\text { Taylor et al., } \\
2003^{68} \\
\text { RCT/Medium }\end{array}$ & $\begin{array}{l}\text { G1: Pharmaceutical care } \\
\text { G2: Standard care }\end{array}$ & $\begin{array}{l}\text { Followup (N } \\
\text { inferred from } \\
\text { percentage in } \\
\text { results) } \\
\mathrm{G} 1: 18^{\mathrm{a}} \\
\mathrm{G} 2: 17^{\mathrm{a}}\end{array}$ & $\begin{array}{l}\text { Percentage of } \\
\text { patients at LDL-C goal } \\
\text { based on ATPIII } \\
\text { criteria at } 12 \text { months. }\end{array}$ & $\begin{array}{l}\text { Calculated OR: } 56.00 \\
95 \% \mathrm{Cl}: 5.583 \text { to } 561.753 \\
p=0.001\end{array}$ \\
\hline $\begin{array}{l}\text { Isetts et al., } \\
2008^{64} \\
\text { Cohort study/High }\end{array}$ & $\begin{array}{l}\text { G1: MTM services provided } \\
\text { by health plan in existing } \\
\text { medical care clinics in } \\
\text { collaboration with primary } \\
\text { care providers. } \\
\text { G2: Usual medical care } \\
\text { without MTM }\end{array}$ & $\begin{array}{l}\text { G1: } 128 \\
\text { G2: } 126\end{array}$ & $\begin{array}{l}\text { Percentage of } \\
\text { patients meeting } \\
\text { HEDIS measures } \\
\text { related to cholesterol } \\
\text { control after } \\
\text { cardiovascular event } \\
\text { at } 12 \text { months. }\end{array}$ & $\begin{array}{l}\text { Calculated OR: } 2.544, \\
95 \% \mathrm{Cl}: 1.52 \text { to } 4.256 \\
p=0.001\end{array}$ \\
\hline $\begin{array}{l}\text { Pindolia et al., } \\
2009^{35} \\
\text { Cohort study/High }\end{array}$ & $\begin{array}{l}\text { G1: Opted in to a } \\
\text { telephone-based MTM } \\
\text { program } \\
\text { G2: Usual medical care } \\
\text { (opted out of MTM } \\
\text { program) }\end{array}$ & $\begin{array}{l}\text { G1: } N^{a} \\
G 2: N^{a} \\
\text { (outcome } \\
\text { assessed only } \\
\text { among patients } \\
\text { with coronary } \\
\text { artery disease) } \\
\end{array}$ & $\begin{array}{l}\text { Change in percentage } \\
\text { of patients with LDL-C } \\
>100 \mathrm{mg} / \mathrm{dl} \text { at } \\
6 \text { months. }\end{array}$ & $\begin{array}{l}\text { G1: }-5 \\
\text { G2: }+7 \\
\text { p: NR and could not be } \\
\text { calculated. }\end{array}$ \\
\hline \multirow[t]{2}{*}{$\begin{array}{l}\text { Fox et al. } 2009^{31} \\
\text { Cohort study/High }\end{array}$} & $\begin{array}{l}\text { G1: MTM program provided } \\
\text { through a health plan } \\
\text { G2: Usual medical care } \\
\text { (eligible but opt-out from } \\
\text { MTM program) }\end{array}$ & $\begin{array}{l}\text { G1: } 255 \\
\text { G2: } 56 \\
\text { G1: } 215 \\
\text { G2: } 46\end{array}$ & $\begin{array}{l}\text { Percentage of } \\
\text { patients with diabetes } \\
\text { with LDL-C }>100 \\
\text { mg/dl at } 12 \text { to } 24 \\
\text { months. }\end{array}$ & $\begin{array}{l}\text { Calculated OR: } 2.228 \\
95 \% \mathrm{Cl}: 1.238 \text { to } 4.008 \\
\text { calculated } p=0.008\end{array}$ \\
\hline & & & $\begin{array}{l}\text { Mean (SD) LDL-C at } \\
12 \text { to } 24 \text { months. }\end{array}$ & $\begin{array}{l}\text { Calculated mean } \\
\text { difference: }-7.4 \\
95 \% \mathrm{Cl}:-17.297 \text { to } 2.497 \\
\text { p: } 0.33 \text { as reported by } \\
\text { study authors, } p=0.143 \text { as } \\
\text { calculated }\end{array}$ \\
\hline
\end{tabular}


Table 11. LDL cholesterol: Summary of results (continued)

\begin{tabular}{|c|c|c|c|c|}
\hline $\begin{array}{l}\text { Study } \\
\text { Design/Risk of } \\
\text { Bias }\end{array}$ & Study Arms & $\begin{array}{l}\mathrm{N} \text { of Subjects } \\
\text { Analyzed }\end{array}$ & $\begin{array}{l}\text { Outcome Reported } \\
\text { by Study and Time } \\
\text { Period }\end{array}$ & Results \\
\hline $\begin{array}{l}\text { Jeong et al., } \\
2007^{32} \\
\text { Cohort study/High }\end{array}$ & $\begin{array}{l}\text { G1: Participants in Part D } \\
\text { Medicare MTM program } \\
\text { G2: Control subjects eligible } \\
\text { for Part D MTM program } \\
\text { but declined enrollment } \\
\text { G3: Control subjects } \\
\text { without Part D Medicare as } \\
\text { their primary drug benefit }\end{array}$ & $\begin{array}{l}\text { G1: NR } \\
\text { G2: NR } \\
\text { G3: NR }\end{array}$ & $\begin{array}{l}\text { Mean LDL-C at } 6 \\
\text { months }\end{array}$ & $\begin{array}{l}\text { Baseline: } \\
\text { G1: } 94.2 \\
\text { G2: } 95.6 \\
\text { G3: } 91.9 \\
\text { Followup: } \\
\text { G1: } 87.4 \\
\text { G2: } 92.5 \\
\text { G3: } 90.2 \\
\text { p value unable to be } \\
\text { reported }\end{array}$ \\
\hline & & & $\begin{array}{l}\text { Percentage of } \\
\text { patients at goal } \\
\text { (defined as less than } \\
100 \mathrm{mg} / \mathrm{dl} \text { ) at } 6 \text { months }\end{array}$ & $\begin{array}{l}\text { Baseline: } \\
\text { G1:62 } \\
\text { G2:62 } \\
\text { G3:67 }\end{array}$ \\
\hline & & & & $\begin{array}{l}\text { Followup: } \\
\text { G1:73 } \\
\text { G2:67 } \\
\text { G2:69 } \\
\text { p value unable to be } \\
\text { reported }\end{array}$ \\
\hline
\end{tabular}

${ }^{\mathrm{a}}$ The investigators assessed this outcome only among patients with hyperlipidemia, diabetes, or coronary artery disease within each study arm but did not report the specific number analyzed.

${ }^{\mathrm{b}} \mathrm{p}$ values reported as $<0.001$ for G1 versus G2 and G1 versus G3, but unclear whether these refer to between-group differences at followup in LDL-C, between group differences in LDL-C change, or to between-group differences in change in percent at LDL$\mathrm{C}$ goal. Calculated mean differences and OR were unable to be calculated due to absence of SD and number analyzed.

Abbreviations: ATPIII = Adult Treatment Panel III (Expert Panel on Detection, Evaluation, and Treatment of High Blood Cholesterol); HEDIS = Healthcare Effectiveness Data and Information Set; LDL-C = low density lipoprotein cholesterol; MTM $=$ medication therapy management; $\mathrm{NR}=$ not reported; $\mathrm{OR}=$ odds ratio; $\mathrm{RCT}=$ randomized controlled trial.

To assess whether other studies might replicate the unexpectedly high odds of improvement from this trial, we also evaluated the findings from the four cohort studies. All studies (high risk of bias because of selection bias and baseline characteristics of groups not reported or not adjusted for) evaluated MTM programs within large, integrated US health care systems. ${ }^{31,32,35,64}$ With the exception of one study, ${ }^{35}$ they reported a direction of effect similar to that reported in the RCT but at a much smaller magnitude. The one study reporting an opposite direction of effect did not provide the data necessary to calculate whether the difference between groups was significant. A random-effect meta-analysis (Appendix G-1) of the three remaining cohort studies $^{31,32,64}$ included the Jeong et al. study ${ }^{32}$ with the assumption that reported Ns for other outcomes applied to this outcome as well. Our analysis yielded an OR of 1.848 (95\% CI, 1.146 to $\left.2.980, \mathrm{p}=0.012 ; \mathrm{I}^{2}=76.55\right)$. One explanation for the high level of heterogeneity is the variation in sample sizes across the cohort studies. Removing the large cohort study ${ }^{32}$ reduced the $\mathrm{I}^{2}$ estimate to 0 ; the pooled estimate of effect continued to indicate benefit from MTM (OR, 2.401; $95 \% \mathrm{CI}, 1.630$ to $3.536 ; \mathrm{p}<0.001)$.

Overall, we concluded that the strength of evidence is insufficient for the effectiveness of MTM interventions on lowering mean LDL-cholesterol levels or increasing the percentage of patients achieving a LDL-cholesterol goal, based on direct but imprecise evidence from single study body of evidence with medium limitations (Table 12). 
Table 12. LDL Cholesterol: Strength of evidence

\begin{tabular}{|c|c|c|c|c|c|c|c|}
\hline $\begin{array}{l}\text { Study } \\
\text { Design }\end{array}$ & $\begin{array}{l}\text { Number of } \\
\text { Studies; } \\
\text { Subjects } \\
\text { (Analyzed) }\end{array}$ & $\begin{array}{l}\text { Study } \\
\text { Limitations }\end{array}$ & Consistency & Directness & Precision & $\begin{array}{l}\text { Findings and } \\
\text { Direction } \\
\text { [Magnitude] of } \\
\text { Effect }\end{array}$ & $\begin{array}{l}\text { Strength of } \\
\text { Evidence }\end{array}$ \\
\hline RCT & $\begin{array}{l}1 ; 81 \\
(38)\end{array}$ & Medium & $\begin{array}{l}\text { Consistency } \\
\text { Unknown - } \\
\text { single study- }\end{array}$ & Direct & Imprecise & $\begin{array}{l}\text { Significantly } \\
\text { greater } \\
\text { percentage of } \\
\text { patients at LDL- } \\
\text { C goal in MTM } \\
\text { group at } 12 \\
\text { months }(77.8 \% \\
\text { vs. } 5.9 \% \text {, } \\
\text { p<0.001, } \\
\text { Calculated OR: } \\
56.00,95 \% \mathrm{Cl} \text { : } \\
5.583 \text { to } \\
561.753) \text {. }\end{array}$ & Insufficient \\
\hline
\end{tabular}

Abbreviations: LDL-C = low density lipoprotein cholesterol; MTM = medication therapy management; $\mathrm{OR}=$ odds ratio; $\mathrm{RCT}=$ randomized controlled trial

\section{Blood Pressure}

In all, we identified six, mostly small, studies that measured blood pressure outcomes using various followup periods (Table 13). This evidence base consisted of four RCTs and two cohort studies; the outcomes involved achieving blood pressure goals or becoming normotensive, and changes in systolic or diastolic blood pressure levels (SBP; DBP) or both. Of these studies, two RCTs were rated medium risk of bias (listed first in Table 13). The remaining four studies were all high risk of bias but do offer some additional or contextual information useful for interpreting the results from the two medium risk of bias RCTs.

\section{Normotensive or BP Goal Attainment}

One RCT (medium risk of bias), conducted among of a small number of patients at high risk of medication-related problems receiving pharmaceutical care through family medicine clinics in a rural community, reported a significant difference in the number of patients at blood pressure goal $(\mathrm{SBP} \leq 140 \mathrm{~mm} \mathrm{Hg}$ and $\mathrm{DBP} \leq 90 \mathrm{~mm} \mathrm{Hg})$ at 12 months $(91.7$ percent versus 27.6 percent, calculated OR $28.875,95 \%$ CI 5.486 to $151.993, \mathrm{p}<0.001) .{ }^{68}$ Two other trials (both high risk of bias) also demonstrated benefit from MTM for a similar outcome but with a lower magnitude of effect. $^{71,72}$

We conducted a random-effects meta-analysis that combined data from these three trials (Appendix G-2). It produced an odds ratio (OR) of 8.683 with wide confidence intervals and a high $\mathrm{I}^{2}$ (which indicates that much of the observed heterogeneity is real; $95 \% \mathrm{CI}, 1.665$ to $45.276, p=0.01 ; \mathrm{Q}, 6.151 ; \mathrm{I}^{2}, 67.48(\mathrm{p}=0.046)$. The two cohort studies (also both high risk of bias) reported findings that were consistent in direction of effect with the trials. $56,57,64$ 
Table 13. Blood pressure: Summary of results

\begin{tabular}{|c|c|c|c|c|}
\hline $\begin{array}{l}\text { Study } \\
\text { Design/Risk of } \\
\text { Bias }\end{array}$ & Study Arms & N Analyzed ${ }^{a}$ & $\begin{array}{l}\text { Outcome Reported by } \\
\text { Study and Time } \\
\text { Period }\end{array}$ & Results \\
\hline \multirow[t]{2}{*}{$\begin{array}{l}\text { Chisholm et al. } \\
2002^{48} \\
\text { RCT/Medium }\end{array}$} & \multirow{2}{*}{$\begin{array}{l}\text { G1: Clinical pharmacy } \\
\text { services within a kidney } \\
\text { transplant clinic. } \\
\text { G2: Usual medical care } \\
\text { in the kidney transplant } \\
\text { clinic. }\end{array}$} & $\begin{array}{l}\text { G1: } 13 \\
\text { G2: } 10\end{array}$ & $\begin{array}{l}\text { Mean SBP }(\mathrm{mm} \mathrm{Hg}) \text { at } \\
\text { quarterly points in time } \\
\text { for } 12 \text { months. }\end{array}$ & $\begin{array}{l}\text { Calculated mean difference: } \\
-22.1 \text {, } \\
95 \% \mathrm{Cl}:-43.896 \text { to }-0.304, \\
p=0.047\end{array}$ \\
\hline & & & $\begin{array}{l}\text { Mean DBP }(\mathrm{mm} \mathrm{Hg}) \text { at } \\
\text { quarterly points in time } \\
\text { for } 12 \text { months. }\end{array}$ & $\begin{array}{l}\text { Calculated mean difference: } \\
-18.5 \\
95 \% \mathrm{Cl}:-29.039 \text { to }-7.961, \\
p=0.001\end{array}$ \\
\hline $\begin{array}{l}\text { Taylor et al., } \\
2003^{68} \\
\text { RCT/Medium }\end{array}$ & $\begin{array}{l}\text { G1: Pharmaceutical care } \\
\text { G2: Standard care }\end{array}$ & $\begin{array}{l}\text { G1: } 24^{a} \\
\text { G2: } 20^{a}\end{array}$ & $\begin{array}{l}\text { Percentage of patients } \\
\text { with SBP and DBP at } \\
\text { goal at } 12 \text { months. }\end{array}$ & $\begin{array}{l}\text { Calculated OR: } 28.875 \\
95 \% \mathrm{Cl}: 5.486 \text { to } 151.993 \\
p<0.001\end{array}$ \\
\hline \multirow[t]{3}{*}{$\begin{array}{l}\text { Park et al. } \\
1996{ }^{72} \\
\text { RCT/High }\end{array}$} & \multirow{3}{*}{$\begin{array}{l}\text { G1: Community- } \\
\text { pharmacy } \\
\text { pharmaceutical care } \\
\text { program } \\
\text { G2: Usual care }\end{array}$} & \multirow[t]{3}{*}{$\begin{array}{l}\mathrm{G} 1: 23 \\
\mathrm{G} 2: 26\end{array}$} & $\begin{array}{l}\text { Percentage of patients } \\
\text { who were normotensive } \\
(\text { SBP }<140 \text { and DBP } \\
<90)\end{array}$ & $\begin{array}{l}\text { Calculated OR: } 2.455 \\
95 \% \text { Cl: } 0.764 \text { to } 7.888 \\
p=0.132\end{array}$ \\
\hline & & & $\begin{array}{l}\text { Mean SBP }(\mathrm{mm} \mathrm{Hg}) \text { at } \\
4 \text { months. }\end{array}$ & $\begin{array}{l}\text { Calculated mean difference: } \\
-13.0 \\
95 \% \mathrm{Cl}:-23.739 \text { to }-2.261 \text {, } \\
p=0.018\end{array}$ \\
\hline & & & $\begin{array}{l}\text { Mean (SD) DBP }(\mathrm{mm} \\
\mathrm{Hg}) \text { at } 4 \text { months }\end{array}$ & $\begin{array}{l}\text { Calculated mean difference: } \\
-4.90 \\
95 \% \mathrm{Cl}:-10.3 \text { to } 0.50 \\
p=0.075\end{array}$ \\
\hline \multirow[t]{2}{*}{$\begin{array}{l}\text { Planas et al. } \\
2009^{71} \\
\text { RCT/High }\end{array}$} & \multirow{2}{*}{$\begin{array}{l}\text { G1: Community } \\
\text { pharmacy hypertension } \\
\text { MTM program for } \\
\text { patients with diabetes } \\
\text { G2: Control group (BP } \\
\text { recorded, informed of BP } \\
\text { goals at } 3 \text { times during } \\
\text { study) }\end{array}$} & \multirow{2}{*}{$\begin{array}{l}\text { G1: } 25 \\
\text { G2: } 15\end{array}$} & $\begin{array}{l}\text { OR }(95 \% \mathrm{CI}) \text { for } \\
\text { intervention group } \\
\text { participant achieving BP } \\
\text { goal relative to control } \\
\text { group. }\end{array}$ & $\begin{array}{l}\text { OR: } 12.9(1.5 \text { to } 113.8) \\
p=0.021\end{array}$ \\
\hline & & & $\begin{array}{l}\text { Mean change in SBP } \\
(\mathrm{mm} \mathrm{Hg}) \text { at } 9 \text { months }\end{array}$ & $\begin{array}{l}\text { Between-group difference: } \\
-20.0(95 \% \mathrm{Cl}-32.7 \text { to }-7.4) \mathrm{p} \text { : } \\
0.003\end{array}$ \\
\hline \multirow{3}{*}{$\begin{array}{l}\text { Carter et al., } \\
997^{56} \\
\text { Barnette et al. } \\
1996^{57} \\
\text { Cohort study/High }\end{array}$} & \multirow{3}{*}{$\begin{array}{l}\text { G1: Pharmacy-based } \\
\text { pharmaceutical care } \\
\text { G2: Usual medical care }\end{array}$} & \multirow[t]{3}{*}{$\begin{array}{l}\mathrm{G} 1: 25 \\
\mathrm{G} 2: 26\end{array}$} & $\begin{array}{l}\text { Percentage with blood } \\
\text { pressure control }\end{array}$ & $\begin{array}{l}\text { Calculated OR: } 1.558 \\
95 \% \mathrm{Cl}: 0.496 \text { to } 4.898 \\
p=0.448\end{array}$ \\
\hline & & & $\begin{array}{l}\text { Mean SBP }(\mathrm{mm} \mathrm{Hg}) \text { at } \\
6 \text { months }\end{array}$ & $\begin{array}{l}\text { Calculated mean difference: } \\
-9.00 \\
95 \% \mathrm{Cl}:-19.451 \text { to } 1.451 \\
p=0.0914\end{array}$ \\
\hline & & & $\begin{array}{l}\text { Mean DBP }(\mathrm{mm} \mathrm{Hg}) \text { at } \\
6 \text { months. }\end{array}$ & $\begin{array}{l}\text { Calculated mean difference: } \\
-1.00 ; 95 \% \mathrm{Cl}:-5.977 \text { to } 3.977 \text {, } \\
p=0.694\end{array}$ \\
\hline $\begin{array}{l}\text { Isetts et al., } \\
2008^{64} \\
\text { Cohort study/High }\end{array}$ & $\begin{array}{l}\text { G1: MTM services } \\
\text { provided by health plan } \\
\text { in existing medical care } \\
\text { clinics in collaboration } \\
\text { with primary care } \\
\text { providers. } \\
\text { G2: Usual medical care } \\
\text { without MTM }\end{array}$ & $\begin{array}{l}\text { G1: } 128 \\
\text { G2: } 126\end{array}$ & $\begin{array}{l}\text { Percentage of patients } \\
\text { meeting HEDIS } \\
\text { measures related to } \\
\text { hypertension } \\
\text { management at } 12 \\
\text { months. }\end{array}$ & $\begin{array}{l}\text { Calculated OR: } 1.728 \\
95 \% \mathrm{Cl}: 1.026 \text { to } 2.911 \\
p=0.04\end{array}$ \\
\hline
\end{tabular}

Abbreviations: ATPIII=Adult Treatment Panel III (Expert Panel on Detection, Evaluation, and Treatment of High Blood Cholesterol); BP, blood pressure; $\mathrm{CI}=$ confidence interval; $\mathrm{DBP}=$ diastolic blood pressure; $\mathrm{G}=$ group; HEDIS= Healthcare Effectiveness Data and Information Set; $\mathrm{mm} \mathrm{Hg}=$ millimeters of mercury (a unit of pressure); MTM, medication therapy management; $\mathrm{NR}=$ not reported; $\mathrm{OR}$, odds ratio; $\mathrm{RCT}=$ randomized controlled trial; $\mathrm{SBP}=$ systolic blood pressure

\footnotetext{
${ }^{a}$ The study had more participants but this outcomes was measured in only the number of patients specified.
} 
Overall, we concluded that the strength of evidence is insufficient for the effectiveness of MTM interventions to increase the percentage of patients achieving a blood pressure goal or becoming normotensive based on a single study body of evidence with medium limitations and an imprecise estimate (Table 14).

Table 14. Achieving blood pressure goal: Strength of evidence

\begin{tabular}{|c|c|c|c|c|c|c|c|}
\hline $\begin{array}{l}\text { Study } \\
\text { Design }\end{array}$ & $\begin{array}{l}\text { Number of } \\
\text { Studies; } \\
\text { Subjects } \\
\text { (Analyzed) }\end{array}$ & $\begin{array}{l}\text { Study } \\
\text { Limitations }\end{array}$ & Consistency & Directness & Precision & $\begin{array}{l}\text { Findings and } \\
\text { Direction } \\
\text { [Magnitude] of } \\
\text { Effect }\end{array}$ & $\begin{array}{l}\text { Strength of } \\
\text { Evidence }\end{array}$ \\
\hline RCT & $\begin{array}{l}1 ; 81 \\
(44)\end{array}$ & Medium & $\begin{array}{l}\text { Consistency } \\
\text { unknown-single } \\
\text { study }\end{array}$ & Direct & Imprecise & $\begin{array}{l}\text { OR: } 28.875 \\
(95 \% \mathrm{Cl}, 5.486 \\
\text { to } 151.993) \\
\text { favoring MTM } \\
\text { over usual care }\end{array}$ & Insufficient \\
\hline
\end{tabular}

Abbreviations: $\mathrm{CI}=$ confidence interval; MTM, medication therapy management; OR, odds ratio; RCT= randomized controlled trial,

\section{Systolic and Diastolic Blood Pressure Levels}

Four studies reported on SBP outcomes. One RCT (medium risk of bias), which was conducted among patients receiving medical care in a post-kidney transplant clinic, reported significantly a lower mean difference in SBP of $-22.1 \mathrm{~mm} \mathrm{Hg}$ at 12 months for the intervention group compared with the usual care group $(95 \% \mathrm{CI},-43.896$ to $-0.304 ; \mathrm{p}=0.047) .{ }^{48} \mathrm{We}$ conducted a random-effects meta-analysis of this and the two high-risk-of-bias RCTs measuring SBP outcomes. ${ }^{71,72}$ The estimated mean difference was -16.774 between intervention and control groups (95\% CI, -24.346 to -9.202; $<<0.001 ; \mathrm{Q}, 0.970 ; \mathrm{I}^{2}, 0$ (p=0.616)) (Appendix G-3). The single high risk-of-bias cohort study also reported improved SBP levels in the intervention arm, but wide confidence intervals spanned the null effect.

We found similar results for DBP levels from the three studies that reported this outcome. The medium risk-of-bias trial reported a mean difference of $-18.50 \mathrm{~mm} \mathrm{Hg} \mathrm{(95 \%} \mathrm{CI,} \mathrm{-29.039} \mathrm{to} \mathrm{-}$ $7.961, p=0.001) .^{48}$ The other trial ${ }^{72}$ and the cohort study ${ }^{56,57}$ (both high risk of bias) both reported benefits from MTM interventions, but both had wide confidence intervals that spanned the null effect.

We concluded that the strength of evidence is insufficient for the effectiveness of MTM interventions to reduce SBP and DBP based on a single study body of evidence with medium limitations and an imprecise estimate (Table 15). 
Table 15. Mean change in systolic and diastolic blood pressure: Strength of evidence

\begin{tabular}{|c|c|c|c|c|c|c|c|}
\hline $\begin{array}{l}\text { Study } \\
\text { Design }\end{array}$ & $\begin{array}{l}\text { Number of } \\
\text { Studies; } \\
\text { Subjects } \\
\text { (Analyzed) }\end{array}$ & $\begin{array}{l}\text { Study } \\
\text { Limitations }\end{array}$ & Consistency & Directness & Precision & $\begin{array}{l}\text { Findings and } \\
\text { Direction } \\
\text { [Magnitude] of } \\
\text { Effect }\end{array}$ & $\begin{array}{l}\text { Strength of } \\
\text { Evidence }\end{array}$ \\
\hline \multirow[t]{2}{*}{ RCT } & $\begin{array}{l}1 ; 26 \\
(23)\end{array}$ & Medium & $\begin{array}{l}\text { Consistency } \\
\text { unknown- } \\
\text { single study }\end{array}$ & Direct & Imprecise & $\begin{array}{l}\text { Calculated mean } \\
\text { difference SBP: } \\
-22.1 \mathrm{~mm} \mathrm{Hg} \text {, } \\
95 \% \mathrm{Cl}:-43.896 \\
\text { to }-0.304, \\
p=0.047\end{array}$ & Insufficient \\
\hline & & & & & & $\begin{array}{l}\text { Calculated mean } \\
\text { difference DBP: } \\
-18.5 \mathrm{~mm} \mathrm{Hg} \\
95 \% \mathrm{Cl}:-29.039 \\
\text { to }-7.961 \\
p=0.001\end{array}$ & \\
\hline
\end{tabular}

Abbreviations: $\mathrm{CI}=$ confidence interval; $\mathrm{DBP}=$ diastolic blood pressure; $\mathrm{mm} \mathrm{Hg}=$ millimeters of mercury (a unit of pressure); $\mathrm{RCT}=$ randomized controlled trial, $\mathrm{SBP}=$ systolic blood pressure

\section{Drug Therapy Problems Identified}

In all, 11 studies addressed the question of the effectiveness of MTM for identifying drug therapy problems. Of these, eight provided information on drug therapy problems only from the intervention arm. ${ }^{36,38,44,50,58-60,63,72}$ Thus, these studies cannot inform the question of the comparative effectiveness of MTM.

The three remaining comparative studies (one trial, two cohort studies) reported findings about the effectiveness of MTM when compared with usual care (Table 16). We rated all three high risk of bias for various reasons: uncontrolled selection bias from the comparison of patients who refused services to patients who accepted services $;{ }^{37}$ bias associated with the specific measure and failure to control for patient-level clustering in a comparison of all drug related problems $;{ }^{73}$ and failure to control for differences at baseline. ${ }^{53}$

These three studies also did not specify their expected direction of effect. We inferred that the studies expected to find fewer drug therapy problems after the completion of the intervention because the interventions were (apparently) specifically designed to identify and then resolve drug therapy problems. However, studies measuring outcomes during an MTM intervention might, instead, expect to find more drug therapy problems in the intervention arm because the intervention led to greater discovery of various problems. Consequently, we treated the evidence as inconsistent. Given high study limitations, inconsistency (single trial), indirectness, and lack of precision, evidence was insufficient to draw any conclusions about the effect on MTM interventions on drug therapy problems identified (Table 17). 
Table 16. Drug therapy problems identified: Summary of results

\begin{tabular}{|c|c|c|c|c|}
\hline $\begin{array}{l}\text { Study } \\
\text { Design/Risk of } \\
\text { Bias } \\
\end{array}$ & Study Arms & N Analyzed & $\begin{array}{l}\text { Outcome and Time } \\
\text { Period }\end{array}$ & Results \\
\hline $\begin{array}{l}\text { Krska et al., } \\
2001^{73} \\
\text { RCT/High }\end{array}$ & $\begin{array}{l}\text { G1: Pharmacist-led } \\
\text { medication review } \\
\text { G2: Usual care including } \\
\text { identification of } \\
\text { pharmaceutical care } \\
\text { issues, but no plan }\end{array}$ & $\begin{array}{l}\text { G1: } 168 \\
\text { G2: } 164\end{array}$ & $\begin{array}{l}\text { Number of drug therapy } \\
\text { problems identified for } \\
\text { each study arm at } 3 \\
\text { months }\end{array}$ & $\begin{array}{l}\text { G1: } 1,206 \\
\text { G2: } 1,380\end{array}$ \\
\hline $\begin{array}{l}\text { Harrison et al., } \\
2012^{53} \\
\text { Cohort/High }\end{array}$ & $\begin{array}{l}\text { G1: Pharmaceutical care } \\
\text { G2: Standard care }\end{array}$ & $\begin{array}{l}\text { G1: } 43 \\
\text { G2: } 43\end{array}$ & $\begin{array}{l}\text { Drug therapy problems } \\
\text { identified per visit, } \\
\text { followup } 2 \text { weeks after } \\
\text { intervention (SD) }\end{array}$ & $\begin{array}{l}\text { G1 baseline: } 0.51 \pm 0.64 \\
\text { G1 followup: } 1.05 \pm 1.34 \\
\text { G2: } 0.74 \pm 0.81 \\
\text { Reported } p=0.19 \text { for } \\
\text { pharmaceutical care vs. standard } \\
\text { care, not controlling for differences } \\
\text { between } \mathrm{G} 1 \text { at baseline and } \\
\mathrm{G} 2 ; \text { Cls for change not calculated } \\
\text { because study does not report } \\
\text { baseline } \mathrm{G} 2 \text { values }\end{array}$ \\
\hline $\begin{array}{l}\text { Welch et al., } \\
2009^{37} \\
\text { Cohort/High }\end{array}$ & $\begin{array}{l}\text { G1: MTM program } \\
\text { provided to home-based } \\
\text { beneficiaries } \\
\text { G2: No-MTM control } \\
\text { group (voluntary opt-out) }\end{array}$ & $\begin{array}{l}\text { G1: } 459 \\
\text { G2: } 123\end{array}$ & $\begin{array}{l}\text { Percentage with at least } \\
1 \text { potential drug therapy } \\
\text { problem during MTM } \\
\text { process (timing unclear) }\end{array}$ & $\begin{array}{l}\text { G1: } 89.8 \% \\
\text { G2: } 83.7 \% \\
\text { Risk difference=6.1\%, calculated } \\
p=0.062\end{array}$ \\
\hline
\end{tabular}

Table 17. Drug therapy problems identified: Strength of evidence

\begin{tabular}{|c|c|c|c|c|c|c|c|}
\hline $\begin{array}{l}\text { Study } \\
\text { Design }\end{array}$ & $\begin{array}{l}\text { Number of } \\
\text { Studies; } \\
\text { Subjects } \\
\text { (Analyzed) }\end{array}$ & $\begin{array}{l}\text { Study } \\
\text { Limitations }\end{array}$ & Consistency & Directness & Precision & $\begin{array}{l}\text { Findings and } \\
\text { Direction } \\
\text { [Magnitude] of } \\
\text { Effect }\end{array}$ & $\begin{array}{l}\text { Strength of } \\
\text { Evidence }\end{array}$ \\
\hline$\overline{R C T}$ & $1 ; 381(332)$ & High & $\begin{array}{l}\text { Consistency } \\
\text { unknown-single } \\
\text { study }\end{array}$ & Indirect & Imprecise & $\begin{array}{l}\text { Cannot be } \\
\text { determined } \\
\text { because of unit of } \\
\text { analysis issues }\end{array}$ & Insufficient \\
\hline Cohort & $2 ; 990(668)$ & High & Inconsistent & Indirect & Imprecise & $\begin{array}{l}\text { Direction and } \\
\text { magnitude of } \\
\text { effect varied by } \\
\text { design and } \\
\text { measure }\end{array}$ & Insufficient \\
\hline
\end{tabular}

Abbreviation: $\mathrm{RCT}=$ randomized controlled trial

\section{Drug Therapy Problems Resolved}

In all, we identified nine studies that attempted to report on whether MTM programs resolved drug therapy problems that were identified. Of these, six studies provided information only from the intervention arm. ${ }^{35,38,40,43,59,60,64,72}$ Thus, as with drug therapy problems identified, they cannot inform the question of the comparative effectiveness of MTM interventions. Three other studies (two RCTs, one cohort study) provided information on the effectiveness of MTM for resolving drug therapy problems when compared with usual care (Table 18). The cohort study (medium risk of bias) found a significant effect of MTM on the difference in drug therapy problems identified between baseline and a 6-month followup; the investigators interpreted the change in number of drug therapy problems identified over time as drug therapy problems resolved between baseline and followup. ${ }^{33,34}$ The two RCTs both had a high risk of bias for several reasons: failure to control for patient-level clustering ${ }^{73}$ or country-level clustering ${ }^{51,52}$ in a 
comparison of all drug related problems; attrition; ${ }^{51,52}$ or failure to control for differences at baseline. $^{51,52}$

Table 18. Drug therapy problems resolved: Summary of results

\begin{tabular}{|c|c|c|c|c|}
\hline $\begin{array}{l}\text { Study } \\
\text { Design/Risk of Bias }\end{array}$ & Study Arms & N Analyzed & $\begin{array}{l}\text { Outcome and Time } \\
\text { Period }\end{array}$ & Results \\
\hline $\begin{array}{l}\text { Krska et al., } 2001^{73} \\
\text { RCT/High }\end{array}$ & $\begin{array}{l}\text { G1: Pharmacist-led } \\
\text { medication review } \\
\text { G2: Usual care } \\
\text { including identification } \\
\text { of pharmaceutical care } \\
\text { issues, but no plan }\end{array}$ & $\begin{array}{l}\text { G1: } 168 \\
\text { G2: } 164\end{array}$ & $\begin{array}{l}\text { Drug therapy problems } \\
\text { wholly or partially } \\
\text { resolved at } 3 \text { months }\end{array}$ & $\begin{array}{l}\text { G1: } 998 \\
\text { G2: } 569\end{array}$ \\
\hline \multirow[t]{4}{*}{$\begin{array}{l}\text { Bernsten et al., } \\
2001^{51,52} \\
\text { RCT/High }\end{array}$} & \multirow[t]{4}{*}{$\begin{array}{l}\text { G1: Structured } \\
\text { pharmaceutical care } \\
\text { program in community } \\
\text { pharmacy } \\
\text { G2: Usual community } \\
\text { pharmacy services }\end{array}$} & $\begin{array}{l}\text { Baseline } \\
\text { G1: } 1290 \\
\text { G2: } 1164\end{array}$ & $\begin{array}{l}\text { Number of changes in } \\
\text { therapy at baseline }\end{array}$ & $\begin{array}{l}\text { Baseline } \\
\text { Calculated mean difference: } \\
0.2 \text {, } \\
95 \% \mathrm{Cl}: 0.101 \text { to } 0.299, \\
\mathrm{p}<0.001\end{array}$ \\
\hline & & $\begin{array}{l}6 \text { months } \\
\text { G1: } 1024 \\
\text { G2: } 953\end{array}$ & $\begin{array}{l}\text { Number of changes in } \\
\text { therapy at } 6 \text { months }\end{array}$ & $\begin{array}{l}6 \text { months } \\
\text { Calculated mean difference: } \\
0.4 \text {, } \\
95 \% \mathrm{Cl}: 0.257 \text { to } 0.543 \text {, } \\
p<0.001\end{array}$ \\
\hline & & $\begin{array}{l}12 \text { months } \\
\text { G1: } 863 \\
\text { G2: } 764\end{array}$ & $\begin{array}{l}\text { Number of changes in } \\
\text { therapy at } 12 \text { months }\end{array}$ & $\begin{array}{l}12 \text { months } \\
\text { Calculated mean difference: } \\
0.1 \text {, } \\
95 \% \mathrm{Cl}:-0.051 \text { to } 0.251, \\
p=0.195\end{array}$ \\
\hline & & $\begin{array}{l}18 \text { months } \\
\text { G1: } 704 \\
\text { G2: } 636\end{array}$ & $\begin{array}{l}\text { Number of changes in } \\
\text { therapy at } 18 \text { months }\end{array}$ & $\begin{array}{l}18 \text { months } \\
\text { Calculated mean difference: } \\
0, \\
95 \% \mathrm{Cl}:-0.156 \text { to } 0.156, \\
p=1.0\end{array}$ \\
\hline $\begin{array}{l}\text { Moczygemba et al., } \\
2011^{33} \\
\text { Moczygemba et al., } \\
2008^{34}\end{array}$ & $\begin{array}{l}\text { G1: Opt-in telephone } \\
\text { MTM program } \\
\text { G2: No-MTM control } \\
\text { group }\end{array}$ & $\begin{array}{l}\text { G1: } 60 \\
\text { G2: } 60\end{array}$ & $\begin{array}{l}\text { Medication and health- } \\
\text { related problems } \\
\text { identified at baseline } \\
\text { and } 6 \text { months }\end{array}$ & $\begin{array}{l}\text { Calculated mean } \\
\text { difference: }-1.00(95 \% \mathrm{Cl} \text { : } \\
-1.967 \text { to }-0.033), p=0.04\end{array}$ \\
\hline Cohort/Medium & & & & \\
\hline
\end{tabular}

Abbreviations: $\mathrm{CI}=$ confidence interval; $\mathrm{MTM}=$ medication therapy management; NS = not significant; RCT = randomized controlled trial; SMD = standardized mean difference

Together (or taking the medium risk of bias cohort study alone), these studies offer insufficient evidence, based on study limitations, inconsistency, and imprecision, to judge the effectiveness of MTM on resolving drug therapy problems (Table 19). 
Table 19. Drug therapy problems resolved: Strength of evidence

\begin{tabular}{|c|c|c|c|c|c|c|c|}
\hline $\begin{array}{l}\text { Study } \\
\text { design }\end{array}$ & $\begin{array}{l}\text { Number of } \\
\text { Studies; } \\
\text { Subjects } \\
\text { (Analyzed) }\end{array}$ & $\begin{array}{l}\text { Study } \\
\text { Limitations }\end{array}$ & Consistency & Directness & Precision & $\begin{array}{l}\text { Findings and } \\
\text { Direction } \\
\text { [Magnitude] of } \\
\text { Effect }\end{array}$ & $\begin{array}{l}\text { Strength of } \\
\text { Evidence }\end{array}$ \\
\hline Cohort & $1 ; 132(120)$ & High & $\begin{array}{l}\text { Consistency } \\
\text { unknown-single } \\
\text { study }\end{array}$ & Indirect & Imprecise & $\begin{array}{l}\text { Calculated } \\
\text { mean } \\
\text { difference:-1.00 } \\
(95 \% \mathrm{Cl} \text { : } \\
-1.967 \text { to }- \\
0.033), p=0.04\end{array}$ & Insufficient \\
\hline
\end{tabular}

Abbreviations: $\mathrm{CI}=$ confidence intervals;

\section{Medication Adherence}

Eleven studies reported on the effects of MTM interventions on adherence outcomes. $^{33,35,37,39,45,51,67-69,71,72}$ One prospective cohort study reported nonadherence determined during MTM (during a mock MTM chart review for the control group) ${ }^{37}$ any adherence differences noted between the two groups were unlikely to be attributable to MTM effects. Moreover, the description of nonadherence used in that study (percentage of patients "nonadherent" per chart review) cannot be interpreted because of a lack of a clear definition. For these reasons, we excluded this study from further analysis.

The 10 remaining studies in the analysis are described in Table 20. Of these 10 studies, eight were RCTs ${ }^{37,39,45,51,67-69,71,72}$; one was a prospective cohort study ${ }^{35}$ and one was a retrospective cohort study ${ }^{33}$. Most studies assessed one of three different adherence outcomes: (1) the proportion of patients who, based on a threshold of between 75 percent and 80 percent of prescribed doses taken, were deemed to be adherent ${ }^{35,68}$; (2) the percentage of prescribed doses taken $^{33,71,72}$; and (3) the scores from an adherence scale score (such as the Morisky Scale). ${ }^{39,51,69}$ Two studies assessed miscellaneous aspects of medication-taking behavior ${ }^{45,67}$ these included "remembering to take medication," a medication-taking behavior subscore, and or determining the number of medications (not pills) for which the participant's reported manner of taking (number of pills and frequency per day) exactly matched the prescribed directions. When studies did not report statistical significance, we calculated the standard difference in means, standard errors, and $95 \%$ confidence intervals based on raw data. 
Table 20. Medication Adherence: Summary of results grouped by type of adherence outcome

\begin{tabular}{|c|c|c|c|c|c|}
\hline Outcome Type & $\begin{array}{l}\text { Study } \\
\text { Design/Risk } \\
\text { of Bias }\end{array}$ & Study Arms & $\begin{array}{l}\mathbf{N} \\
\text { Analyzed }\end{array}$ & $\begin{array}{l}\text { Outcome and Time } \\
\text { Period }\end{array}$ & Results \\
\hline \multirow{3}{*}{$\begin{array}{l}\text { Outcome Type } \\
\text { 1: Proportion of } \\
\text { patients } \\
\text { adherent based } \\
\text { on a threshold of } \\
\text { percentage of } \\
\text { pills taken }\end{array}$} & $\begin{array}{l}\text { Taylor et al., } \\
2003^{68} \\
\text { RCT/Medium } \\
\end{array}$ & $\begin{array}{l}\text { G1: } \\
\text { Pharmaceutical } \\
\text { care } \\
\text { G2: Standard } \\
\text { care }\end{array}$ & $\begin{array}{l}\text { G1:33 } \\
\text { G2:36 }\end{array}$ & $\begin{array}{l}\text { Percentage of patients } \\
\text { adherent defined as self- } \\
\text { reported taking } 80 \% \text { or } \\
\text { more of medications } 12 \\
\text { months after baseline }\end{array}$ & $\begin{array}{l}\text { Calculated OR: } 9.277 \\
95 \% \text { CI: } 0.480 \text { to } \\
179.263 ; p=0.140\end{array}$ \\
\hline & $\begin{array}{l}\text { Pindolia et al., } \\
2009^{35} \\
\text { Cohort } \\
\text { study/High }\end{array}$ & $\begin{array}{l}\text { G1: Telephone- } \\
\text { based MTM } \\
\text { program } \\
\text { G2: Patients } \\
\text { eligible for MTM } \\
\text { program who } \\
\text { declined }\end{array}$ & $\begin{array}{l}\text { G1: } 292 \\
\text { G2: } 1,081 \\
\text { (study year } \\
\text { 1) }\end{array}$ & $\begin{array}{l}\text { Percentage of CHF } \\
\text { patients who were adherent } \\
\text { to at least } 75 \% \text { of } \\
\text { ACE/ARB medications } \\
\text { based on } 2006 \text { claims data: } \\
\text { Measured during } 6 \text { months } \\
\text { post-MTM enrollment }\end{array}$ & $\begin{array}{l}\text { Calculated OR: } 1.088 \\
95 \% \mathrm{Cl}: 0.834 \text { to } 1.417 \\
\mathrm{P}=0.533\end{array}$ \\
\hline & & enrollment & & $\begin{array}{l}\text { Percentage of CHF } \\
\text { patients who were adherent } \\
\text { to at least } 75 \% \text { of beta } \\
\text { blocker medications based } \\
\text { on } 2006 \text { claims data: } \\
\text { Measured during } 6 \text { months } \\
\text { post-MTM enrollment c }\end{array}$ & $\begin{array}{l}\text { Calculated OR: } 1.174 \\
95 \% \mathrm{Cl} 0.89 \text { to } 1.54 \\
\mathrm{P}=0.252\end{array}$ \\
\hline \multirow[t]{2}{*}{$\begin{array}{l}\text { Outcome Type } \\
\text { 2: Absolute } \\
\text { measure of } \\
\text { adherence as } \\
\text { percentage of } \\
\text { prescribed } \\
\text { doses taken }\end{array}$} & $\begin{array}{l}\text { Moczygemba, } \\
2011^{33} \\
\text { Moczygemba, } \\
2008^{34} \\
\text { Retrospective } \\
\text { cohort/ } \\
\text { Medium }\end{array}$ & $\begin{array}{l}\text { G1: Opt-in } \\
\text { telephone Scott } \\
\text { \& White Health } \\
\text { Plan MTM } \\
\text { program } \\
\text { G2: No-MTM } \\
\text { control group }\end{array}$ & $\begin{array}{l}\text { G1: } 60 \\
\text { G2: } 60\end{array}$ & $\begin{array}{l}\text { Percentage prescribed } \\
\text { doses taken: Overall } \\
\text { average MPR across all } \\
\text { medications measured at } 6 \\
\text { months before MTM } \\
\text { participation (i.e., baseline) } \\
\text { and } 6 \text { months after MTM } \\
\text { (i.e., followup) using } \\
\text { pharmacy data }\end{array}$ & $\begin{array}{l}\text { Calculated mean } \\
\text { difference: }-0.020 \\
\text { 95\% Cl: }-0.78 \text { to } 0.038 \\
\mathrm{P}=0.502\end{array}$ \\
\hline & $\begin{array}{l}\text { Planas et al } \\
2009^{71}\end{array}$ & $\begin{array}{l}\text { G1: } \\
\text { Collaborative } \\
\text { home-based } \\
\text { medication } \\
\text { review } \\
\text { G2: No } \\
\text { medication } \\
\text { review received }\end{array}$ & $\begin{array}{l}\text { G1: } 25 \\
\text { G2: } 15\end{array}$ & $\begin{array}{l}\text { Percent mean adherence } \\
\text { (percentage of prescribed } \\
\text { doses taken) to } \\
\text { antihypertensive } \\
\text { medication } \\
\text { Measured twice (9 months } \\
\text { before and } 9 \text { months after } \\
\text { baseline visit) and } \\
\text { continuously using } \\
\text { medication acquisition } \\
\text { method, in which days' } \\
\text { supply of medication is } \\
\text { compared with dates } \\
\text { medication was filled using } \\
\text { pharmacy refill data. }\end{array}$ & $\begin{array}{l}\text { Calculated mean } \\
\text { difference from baseline } \\
\text { to } 9 \text { months: } 0.077 \\
95 \% \mathrm{Cl}:-0.127 \text { to } 0.281 \\
\mathrm{P}=0.46\end{array}$ \\
\hline
\end{tabular}


Table 20. Medication Adherence: Summary of results by type of adherence outcome (continued)

\begin{tabular}{|c|c|c|c|c|c|}
\hline Outcome Type & $\begin{array}{l}\text { Study } \\
\text { Design/Risk } \\
\text { of Bias }\end{array}$ & Study Arms & $\begin{array}{l}\mathrm{N} \\
\text { Analyzed }\end{array}$ & $\begin{array}{l}\text { Outcome and Time } \\
\text { Period }\end{array}$ & Results \\
\hline $\begin{array}{l}\text { Outcome Type } \\
\text { 2: Absolute } \\
\text { measure of } \\
\text { adherence as } \\
\text { percentage of } \\
\text { prescribed } \\
\text { doses taken } \\
\text { (continued) }\end{array}$ & $\begin{array}{l}\text { Park, } 1996^{72} \\
\text { RCT/high }\end{array}$ & $\begin{array}{l}\text { G1: } \\
\text { Comprehensive } \\
\text { pharmaceutical } \\
\text { care } \\
\text { G2: Usual care }\end{array}$ & $\begin{array}{l}\text { Visit } 1 \\
\text { G1: } 7 \\
\text { G2: } 5 \\
\text { Visit } 2 \\
\text { G1: } 21 \\
\text { G2: } 23 \\
\text { Visit } 3 \\
\text { G1: } 23 \\
\text { G2: } 20 \\
\text { Visit } 4 \\
\text { G1: } 21 \\
\text { G2: } 22\end{array}$ & $\begin{array}{l}\text { Mean percent compliance } \\
\text { (percentage of prescribed } \\
\text { pills taken) from pharmacist } \\
\text { report of pill counts } \\
4 \text { month timeframe }\end{array}$ & $\begin{array}{l}\text { Calculated mean } \\
\text { difference for change } \\
\text { from baseline to Visit } 4 \text { : } \\
-0.023 \\
95 \% \mathrm{Cl}:-0.175 \text { to } \\
0.129 p=0.767\end{array}$ \\
\hline \multirow[t]{3}{*}{$\begin{array}{l}\text { Outcome Type } \\
\text { 3: Self-reported } \\
\text { Adherence using } \\
\text { Morisky Scale }\end{array}$} & $\begin{array}{l}\text { Bernsten, } \\
2001^{51} ; \\
\text { Sturgess, } \\
2003^{52} \\
\text { RCT/ High } \\
\text { (pooled data) }\end{array}$ & $\begin{array}{l}\text { G1: Structured } \\
\text { community } \\
\text { pharmacy- } \\
\text { based } \\
\text { pharmaceutical } \\
\text { care program } \\
\text { G2: Usual } \\
\text { community } \\
\text { pharmacy } \\
\text { services }\end{array}$ & $\begin{array}{l}\text { Pooled } \\
\text { sample } \\
\text { (excluding } \\
\text { The } \\
\text { Netherlands } \\
\text { because no } \\
\text { baseline } \\
\text { adherence } \\
\text { data } \\
\text { collected) } \\
\text { Baseline } \\
\text { G1: } 867 \\
\text { G2: } 748 \\
18 \text { months } \\
\text { G1: } 792 \\
\text { G2: } 758\end{array}$ & $\begin{array}{l}\text { Medication adherence: self- } \\
\text { reported as assessed by } \\
\text { Morisky Scale } \\
\text { (Note: Percentage of } \\
\text { participants who were } \\
\text { adherent defined as } \\
\text { patients responded that } \\
\text { they "never" experienced } \\
\text { any aspects of } \\
\text { noncompliance on the 4- } \\
\text { item scale with a 4-point } \\
\text { response option per item) }\end{array}$ & $\begin{array}{l}\text { Pooled sample } \\
\text { (percentage adherent) } \\
\text { OR at baseline: } 0.82, \\
\text { calculated } 95 \% \mathrm{Cl} \text { : } \\
0.666 \text { to } 1.0, \mathrm{p}=0.050 \\
\text { Calculated OR at } 18 \\
\text { months: } 1.084,95 \% \mathrm{Cl} \text { : } \\
0.883 \text { to } 1.332, \mathrm{p}= \\
0.440\end{array}$ \\
\hline & $\begin{array}{l}\text { Volume et al. } \\
2001^{69} \text { and } \\
\text { Kassam }^{70} \\
\text { RCT/Medium }\end{array}$ & $\begin{array}{l}\text { G1: } \\
\text { Comprehensive } \\
\text { pharmaceutical } \\
\text { care services } \\
\text { G2: Traditional } \\
\text { pharmacy care }\end{array}$ & $\begin{array}{l}\text { T1: } N=363 \\
\text { G1: } 159 \\
\text { G2: } 204 \text { T2: } \\
N=317 \mathrm{~T} 3: \\
\mathrm{N}=292 \\
\text { Estimated } \\
\text { by group } \\
\text { based on } \\
\text { overall } \\
\text { retention } \\
\text { G1: } 127 \\
\text { G2: } 163\end{array}$ & $\begin{array}{l}\text { Self-reported adherence } \\
\text { using the Morisky Scale } \\
\text { made up of four } \\
\text { dichotomous items where } \\
\text { summary score is } 0-4 \text { with } \\
\text { lower scores being better } \\
\text { adherence } \\
12 \text { to } 13 \text { months after } \\
\text { intervention }\end{array}$ & $\begin{array}{l}\text { Calculated mean } \\
\text { difference } \\
0.090 \\
95 \% \mathrm{Cl}:-0.076 \text { to } \\
0.256 \\
\mathrm{P}=0.289\end{array}$ \\
\hline & & & $\begin{array}{l}\text { Note: } \\
\text { T=time }\end{array}$ & & \\
\hline
\end{tabular}


Table 20. Medication Adherence: Summary of results by type of adherence outcome (continued)

\begin{tabular}{|c|c|c|c|c|c|}
\hline Outcome Type & $\begin{array}{l}\text { Study } \\
\text { Design/Risk } \\
\text { of Bias } \\
\end{array}$ & Study Arms & $\begin{array}{l}\mathbf{N} \\
\text { Analyzed }\end{array}$ & $\begin{array}{l}\text { Outcome and Time } \\
\text { Period }\end{array}$ & Results \\
\hline $\begin{array}{l}\text { Outcome Type } \\
\text { 3: Self-reported } \\
\text { Adherence using } \\
\text { Morisky Scale } \\
\text { (continued) }\end{array}$ & $\begin{array}{l}\text { Jameson et al., } \\
1995^{39} \\
\text { RCT/High } \\
\text { (Medium for } \\
\text { study overall } \\
\text { but high for } \\
\text { adherence } \\
\text { because of } \\
\text { poor outcome } \\
\text { measure)) }\end{array}$ & $\begin{array}{l}\text { G1: } \\
\text { Consultation } \\
\text { with a clinical } \\
\text { pharmacist } \\
\text { within a primary } \\
\text { care office. } \\
\text { G2: Standard } \\
\text { medical care at } \\
\text { the primary care } \\
\text { office. }\end{array}$ & $\begin{array}{l}\text { G1: } 27 \\
\text { G2: } 29\end{array}$ & $\begin{array}{l}\text { Self-reported composite } \\
\text { "understanding and } \\
\text { compliance" } 0-12 \text { score at } \\
\text { baseline and at } 6 \text { months } \\
\text { (no further information on } \\
\text { measure used) } \\
\text { Change in self-reported } \\
\text { composite score over } 6 \\
\text { months with negative score } \\
\text { representing improvement }\end{array}$ & $\begin{array}{l}\text { Baseline Means Scale } \\
\text { Score (SD not reported) } \\
\text { G1: } 2.3 \\
\text { G2: } 2.3 \\
\text { 95\% Cl: NR } \\
\text { p: NS } \\
6 \text { months } \\
\text { G1: } 0.6 \\
\text { G2: } 2.1 \\
\text { 95\% Cl: NR } \\
\text { p: NS } \\
\text { G1: }-1.6 \\
\text { G2: }-0.2 \\
\text { 95\% Cl: NR } \\
\text { p: NS }\end{array}$ \\
\hline \multirow[t]{2}{*}{$\begin{array}{l}\text { Miscellaneous } \\
\text { Adherence } \\
\text { Outcomes }\end{array}$} & $\begin{array}{l}\text { Hanlon et al., } \\
1996^{67} \\
\text { RCT/Medium } \\
\text { (Low for study } \\
\text { overall but } \\
\text { medium for } \\
\text { adherence } \\
\text { because of } \\
\text { lack of } \\
\text { information } \\
\text { about and } \\
\text { precision of } \\
\text { adherence } \\
\text { measure) }\end{array}$ & $\begin{array}{l}\text { G1: Usual care, } \\
\text { plus clinical } \\
\text { pharmacist care. } \\
\text { G2: Usual care } \\
\text { in the General } \\
\text { Medicine Clinic }\end{array}$ & $\begin{array}{l}\text { G1: } 86 \\
\text { G2: } 83\end{array}$ & $\begin{array}{l}\text { Self-reported medication } \\
\text { compliance with } 12-\text { month } \\
\text { time frame, assessed by } \\
\text { determining whether the } \\
\text { way patients said they took } \\
\text { their medicine (in terms of } \\
\text { number of pills and daily } \\
\text { frequency) matched how } \\
\text { the medication was } \\
\text { prescribed. Compliance } \\
\text { was defined as the } \\
\text { proportion of medications } \\
\text { for which the patients' } \\
\text { response agreed with the } \\
\text { directions. }\end{array}$ & $\begin{array}{l}\text { Calculated OR: } 1.076, \\
95 \% \mathrm{Cl}: 0.527 \text { to } 2.197 \text {, } \\
\text { p: } 0.84\end{array}$ \\
\hline & $\begin{array}{l}\text { Sidel, } 1990^{45} \\
\text { RCT/Medium }\end{array}$ & $\begin{array}{l}\text { G1: Received at } \\
\text { least } 2 \\
\text { pharmacist visits } \\
\text { involving } \\
\text { medication } \\
\text { review, patient- } \\
\text { specific } \\
\text { education and } \\
\text { counseling; } \\
\text { follow- up patient } \\
\text { telephone calls } \\
\text { and contacting of } \\
\text { physicians by } \\
\text { pharmacists as } \\
\text { needed } \\
\text { G2: Contacted } \\
\text { only to complete } \\
\text { the survey. }\end{array}$ & $\begin{array}{l}\text { G1: } 92 \\
\text { G2: } 104 \\
\text { t } \\
\text { f }\end{array}$ & $\begin{array}{l}\text { Change from baseline to 6- } \\
\text { month followup in } \\
\text { Medication-taking Behavior } \\
\text { Subscore (negative scores } \\
\text { indicate improvement, } \\
\text { which means decreased } \\
\text { risk) } \\
\text { Change at } \\
6 \text { months in normative } \\
\text { score for Remembering to } \\
\text { take Medicine }\end{array}$ & $\begin{array}{l}\text { G1: }-3.47 \\
\text { G2: }-4.38 \\
95 \% \text { Cl: NR } \\
P<0.001 \text { for within- } \\
\text { group differences } \\
p=0.52 \text { for between- } \\
\text { group differences } \\
\text { G1: } 0.09 \\
\text { G2: }-0.19 \\
95 \% \text { Cl: NR } \\
P=0.52\end{array}$ \\
\hline
\end{tabular}

Abbreviations: $\mathrm{ACE}=$ angiotension converting enzyme inhibitor; $\mathrm{ARB}=$ angiotension receptor blocker; $\mathrm{CHF}=$ congestive heart failure; $\mathrm{HbA} 1 \mathrm{C}=$ hemoglobin $\mathrm{A} 1 \mathrm{C}$ or glycosolated hemoglobin; $\mathrm{MPR}=$ medication possession ratio; $\mathrm{MTM}=$ medication therapy management; $\mathrm{NR}=$ not reported; $\mathrm{RCT}=$ randomized controlled trial. 
Of the two studies assessing the proportion of patients who achieved threshold adherence levels, one was a small RCT (medium risk of bias); ${ }^{68}$ the other was a relatively large cohort study (high risk of bias). ${ }^{35}$ Neither study found statistically significant effects of MTM on adherence. Of the three studies that assessed MTM effects on percentage of prescribed doses taken, two were small RCTs (both high risk of bias) ${ }^{71,72}$ the other was a small retrospective cohort study (medium risk of bias). ${ }^{33}$ None of these studies found a statistically significant effect of MTM on adherence. All three studies that assessed adherence using self-reported adherence scales were small RCTs (one medium risk of bias ${ }^{69}$; two high risk of bias ${ }^{39,51}$ ). None found a statistically significant effect of MTM on adherence, although one high risk of bias study, ${ }^{6}$ did not account for the marked baseline differences, hence may have missed a statistically signficant difference in change in adherence. This same study (high risk of bias) reported that a statistically significant increase in the percentage of individuals who changed from nonadherent to adherent over 18 months (15.25 percent in the intervention group and 12.2 percent in the control group; $\mathrm{p}=0.028) ;{ }^{51}$ however, this assessment did not take into account the percentage in each group that changed from adherent to nonadherent. Finally, the two RCTs (both medium risk of bias) that assessed miscellaneous aspects of adherence found no statistically significant differences between groups in adherence outcomes assessed. ${ }^{45,67}$ Hence, none of the 11 studies that assessed effects of MTM on adherence found a statistically significant effect.

Overall, we concluded that evidence is insufficient to draw conclusions about the effectiveness of MTM for improving the proportion of patients who, based on a threshold of 80 percent of prescribed doses taken, were adherent at 6 to 12 months based on direct, imprecise evidence from one small RCT (Table 21). The findings are consistent with the direct but imprecise evidence from one large prospective cohort study with high study limitations. Strength of evidence is also insufficient for improving the absolute percentage of prescribed doses taken at 6 months (mean adherence), based on direct, imprecise evidence from one small retrospective cohort study (Table 22). This conclusion is consistent with findings from two small high risk of bias RCTs that provided direct, imprecise evidence of these effects at 4 to 9 months. Of note, however, is that these two trials had a high level of study limitations and reported opposite directions of effect on absolute percentage of prescribed doses taken, both with nonsignificant differences between groups.

Table 21. Adherence Outcome Type 1-Proportion of Patients Adherent based on a Threshold of Percentage of Pills Taken: Strength of Evidence

\begin{tabular}{|c|c|c|c|c|c|c|c|}
\hline $\begin{array}{l}\text { Study } \\
\text { Design }\end{array}$ & $\begin{array}{l}\text { Number of } \\
\text { Studies; } \\
\text { Subjects } \\
\text { (Analyzed) }\end{array}$ & $\begin{array}{l}\text { Study } \\
\text { Limitations }\end{array}$ & Consistency & Directness & Precision & $\begin{array}{l}\text { Findings and } \\
\text { Direction } \\
\text { [Magnitude] of } \\
\text { Effect }\end{array}$ & $\begin{array}{l}\text { Strength } \\
\text { of } \\
\text { Evidence }\end{array}$ \\
\hline$\overline{R C T}$ & $1 ; 81(69)$ & Medium & $\begin{array}{l}\text { Unknown } \\
\text { (single study) }\end{array}$ & Direct & Imprecise & $\begin{array}{l}100 \% \text { of intervention } \\
\text { patients and } 88.9 \% \text { of } \\
\text { controls were } \\
\text { adherent; } \\
P=0.115\end{array}$ & Insufficient \\
\hline
\end{tabular}

Abbreviations: $\mathrm{RCT}=$ randomized controlled trial. 
Table 22. Adherence Outcome Type 2-Absolute Measure of Adherence as Percentage of Prescribed Doses Taken: Strength of Evidence

\begin{tabular}{|c|c|c|c|c|c|c|c|}
\hline $\begin{array}{l}\text { Study } \\
\text { Design }\end{array}$ & $\begin{array}{l}\text { Number of } \\
\text { Studies; } \\
\text { Subjects } \\
\text { (Analyzed) }\end{array}$ & $\begin{array}{l}\text { Study } \\
\text { Limitations }\end{array}$ & Consistency & Directness & Precision & $\begin{array}{l}\text { Findings and } \\
\text { Direction } \\
\text { [Magnitude] of } \\
\text { Effect }\end{array}$ & $\begin{array}{l}\text { Strength } \\
\text { of } \\
\text { Evidence }\end{array}$ \\
\hline $\begin{array}{l}\text { Obser- } \\
\text { vational }\end{array}$ & $1 ; 132(120)$ & High & $\begin{array}{l}\text { Unknown } \\
\text { (single study) }\end{array}$ & Direct & Imprecise & $\begin{array}{l}\text { SMD: }-0.1226 \\
95 \% \mathrm{Cl}:-0.4808 \text { to } \\
0.2355 \mathrm{p}=0.50\end{array}$ & Insufficient \\
\hline
\end{tabular}

Abbreviations: $\mathrm{CI}=$ confidence interval; $\mathrm{SMD}=$ standardized mean difference

Evidence is also insufficient about improving medication adherence as measured by selfreported scales at 6 to 18 months, based on inconsistent, direct, imprecise evidence from three RCTs (one small, one medium, one large) (Table 23). Finally (Table 24), regarding miscellaneous medications taking behaviors, such as remembering to take medication, a medication-taking behavior subscore, and the proportion of medications matched with instructions, we concluded that evidence was insufficient for the effect of MTM on these outcomes, based on evidence from two RCTs that was direct but imprecise and inconsistent. Although the significant degree of heterogeneity across adherence measures precluded our ability to assess strength of evidence across all adherence studies, we note that considering the body of evidence for the effect of MTM on adherence, taken together, results from all studies were nonsignificant with small magnitudes of effect. Across studies, the direction of effect was inconsistent. Hence, considering the adherence studies as a whole, there appears to be insufficient evidence regarding an effect of MTM on adherence.

Table 23. Adherence outcome type 3--self-reported scales: Strength of evidence

\begin{tabular}{|c|c|c|c|c|c|c|c|}
\hline $\begin{array}{l}\text { Study } \\
\text { Design }\end{array}$ & $\begin{array}{l}\text { Number of } \\
\text { Studies; } \\
\text { Subjects } \\
\text { (Analyzed) }\end{array}$ & $\begin{array}{l}\text { Study } \\
\text { Limitations }\end{array}$ & Consistency & Directness & Precision & $\begin{array}{l}\text { Findings and } \\
\text { Direction } \\
\text { [Magnitude] of } \\
\text { Effect }\end{array}$ & $\begin{array}{l}\text { Strength of } \\
\text { Evidence }\end{array}$ \\
\hline RCT & $\begin{array}{l}3 ; 2,881 \\
(1,898)\end{array}$ & High & Inconsistent & Direct & Imprecise & $\begin{array}{l}\text { Three studies; one } \\
\text { larger with small and } \\
\text { not statistically } \\
\text { significant } \\
\text { improvement in MTM } \\
\text { group and high risk } \\
\text { of bias; two with } \\
\text { opposite direction of } \\
\text { effect, both with non- } \\
\text { significant differences } \\
\text { between groups }\end{array}$ & Insufficient \\
\hline
\end{tabular}

Abbreviations: $\mathrm{RCT}=$ randomized controlled trial.

Table 24. Adherence outcome miscellaneous: Strength of evidence

\begin{tabular}{|c|c|c|c|c|c|c|c|}
\hline $\begin{array}{l}\text { Study } \\
\text { Design }\end{array}$ & $\begin{array}{l}\text { Number of } \\
\text { Studies; } \\
\text { Subjects } \\
\text { (Analyzed) }\end{array}$ & $\begin{array}{l}\text { Study } \\
\text { Limitations }\end{array}$ & Consistency & Directness & Precision & $\begin{array}{l}\text { Findings and } \\
\text { Direction } \\
\text { [Magnitude] of } \\
\text { Effect }\end{array}$ & $\begin{array}{l}\text { Strength of } \\
\text { Evidence }\end{array}$ \\
\hline $\mathrm{RCT}$ & $\begin{array}{l}2 ; 492 \\
(404)\end{array}$ & Medium & Inconsistent & Direct & Imprecise & $\begin{array}{l}\text { Two studies with } \\
\text { opposite direction of } \\
\text { effect, both with non- } \\
\text { significant differences } \\
\text { between groups. }\end{array}$ & Insufficient \\
\hline
\end{tabular}

Abbreviations: $\mathrm{RCT}=$ randomized controlled trial. 


\section{Medication Appropriateness}

Five studies (four RCTs, ${ }^{49,50,67,68}$ one cohort study ${ }^{56}$ ) reported on the effects of MTM interventions on medication appropriateness (Table 25 and Table 26). Of these studies, three assessed medication appropriateness across a broad spectrum of regimens; ${ }^{56,67,68}$ the other two trials assessed appropriateness for specific medications. ${ }^{4,50}$

For the three broader studies, two trials used the Medication Appropriateness Index (MAI). ${ }^{67,68}$ One of these reported results for the full scale and for each item of the index (each item asks about a different aspect of medication appropriateness) individually; ${ }^{67}$ the other trial reported results only for each of the individual items. ${ }^{68}$ The cohort study of broad regimens used a panel of three pharmacists to rate the appropriateness of the various antihypertensive regimens on a visual analogue scale. ${ }^{56}$

As shown in Table 25 , one RCT (low risk of bias) ${ }^{67}$ found a statistically significant improvement in the MAI Scale at 3 and 12 months' followup. The small cohort study (high risk of bias) reported no statistically significant improvement in the three appropriateness scores assessed for blood pressure regimens (appropriateness of regimens, of dosing intervals, and of dosages) although it was very underpowered. ${ }^{56}$

Table 25. Medication appropriateness scales: Summary of results

\begin{tabular}{|c|c|c|c|c|}
\hline $\begin{array}{l}\text { Study } \\
\text { Design/Risk of Bias }\end{array}$ & Study Arms & N Analyzed & $\begin{array}{l}\text { Outcome and Time } \\
\text { Period }\end{array}$ & Results \\
\hline $\begin{array}{l}\text { Hanlon et al., } 1196^{67} \\
\text { RCT/Low }\end{array}$ & $\begin{array}{l}\text { G1: Usual care, plus } \\
\text { clinical pharmacist } \\
\text { care. } \\
\text { G2: Usual care in the } \\
\text { General Medicine } \\
\text { Clinic }\end{array}$ & $\begin{array}{l}\text { G1: } 105 \\
\text { G2: } 103\end{array}$ & $\begin{array}{l}\text { Covariate-adjusted } \\
\text { Medication } \\
\text { Appropriateness Index } \\
\text { assessed at baseline, } 3 \text {, } \\
12 \text { months by blinded } \\
\text { research pharmacist }\end{array}$ & $\begin{array}{l}\text { Baseline } \\
\text { G1: } 17.7(0.6) \\
\text { G2: } 17.6(0.6) \\
3 \text { months } \\
\text { G1: } 13.4(0.6) \\
\text { G2: } 16.5(0.6) \\
95 \% \mathrm{Cl} \text { : NR } \\
\text { p<0.0006 for between- group } \\
\text { differences, controlling for } \\
\text { baseline and other covariates } \\
\text { 12 months } \\
\text { G1: } 12.8(0.7) \\
\text { G2: } 16.7(0.7) \\
95 \% C l: \text { NR } \\
\text { p<0.0006 for between- group } \\
\text { differences, controlling for } \\
\text { baseline and other covariates }\end{array}$ \\
\hline
\end{tabular}


Table 25. Medication appropriateness scales: Summary of results (continued)

\begin{tabular}{|c|c|c|c|c|}
\hline $\begin{array}{l}\text { Study } \\
\text { Design/Risk of Bias }\end{array}$ & Study Arms & N Analyzed & $\begin{array}{l}\text { Outcome and Time } \\
\text { Period }\end{array}$ & Results \\
\hline $\begin{array}{l}\text { Hanlon et al., } 1196^{67} \\
\text { RCT/Low } \\
\text { (continued) }\end{array}$ & & $\begin{array}{l}\text { G1: } 105 \\
\text { G2: } 103\end{array}$ & $\begin{array}{l}\text { Change in covariate- } \\
\text { adjusted Medication } \\
\text { Appropriateness Index } \\
\text { assessed at baseline, } 3 \text {, } \\
12 \text { months by blinded } \\
\text { research pharmacist }\end{array}$ & $\begin{array}{l}3 \text { months change in outcome } \\
\text { G1: }-4.3 \\
\text { G2: }-1.1 \\
95 \% \mathrm{Cl} \text { : NR } \\
24 \% \text { improvement in } \\
\text { intervention group and } 6 \% \\
\text { improvement in control group } \\
\text { p= } 0.0006 \\
12 \text { months change in outcome } \\
\text { G1: }-4.9 \\
\text { G2: }-0.9 \\
95 \% \text { Cl: NR } \\
28 \% \text { improvement in } \\
\text { intervention group and } 5 \% \\
\text { improvement in control group } \\
\text { p=0.0002 }\end{array}$ \\
\hline \multirow[t]{2}{*}{$\begin{array}{l}\text { Carter et al., } \\
1997^{56} \\
\text { Barnette et al. } 1996^{57} \\
\text { Cohort study/High }\end{array}$} & $\begin{array}{l}\text { G1: Pharmacy-based } \\
\text { pharmaceutical care } \\
\text { G2: Usual medical } \\
\text { care }\end{array}$ & $\begin{array}{l}\text { G1: } 25 \\
\text { G2: } 26\end{array}$ & $\begin{array}{l}\text { Appropriateness of BP } \\
\text { regimen } \\
\text { A blinded review panel } \\
\text { of three pharmacists } \\
\text { evaluated cases in } \\
\text { random order on a } \\
\text { visual analog scale, } \\
\text { using medical records. } \\
\text { The investigators } \\
\text { averaged and } \\
\text { converted scores to a } \\
\text { numerical value by } \\
\text { measuring the distance } \\
\text { from the best option. } \\
\text { Score arranged from } 0 \\
\text { to } 16.2 \text {. Higher scores } \\
\text { are better. } \\
\text { Appropriateness of daily } \\
\text { dosage }\end{array}$ & $\begin{array}{l}\text { BP regimen } \\
\text { Baseline } \\
\text { G1: } 8.7(4.7) \\
\text { G2: } 10.3(4.8) \\
\text { Follow-up } \\
\text { G1: } 10.9(4.5) \\
\text { G2: } 10.1(5.2) \\
\text { p for change scores NR }\end{array}$ \\
\hline & & & $\begin{array}{l}\text { Appropriateness of } \\
\text { dosing interval }\end{array}$ & $\begin{array}{l}\text { Daily dosage } \\
\text { Baseline } \\
\text { G1: } 11.6(4.5) \\
\text { G2: } 12.6(4.5) \\
\text { Followup } \\
\text { G1: } 13.4(3.7) \\
\text { G2: } 13.2(4.1) \\
\text { p for change scores NR } \\
\text { Dosing interval } \\
\text { Baseline } \\
\text { G1: } 13.8(4.3) \\
\text { G2: } 13.4(4.6) \\
\text { Follow-up } \\
\text { G1: } 15.1(2.3) \\
\text { G2: } 13.8(4.1) \\
\text { p for change scores NR }\end{array}$ \\
\hline
\end{tabular}

Abbreviations: $\mathrm{BP}=$ blood pressure; $\mathrm{NR}=$ not reported; $\mathrm{RCT}=$ randomized controlled trial. 
Of note, one ${ }^{67}$ (low risk of bias) of the two trials reporting the effect of MTM on general medication appropriateness scales, also provided descriptive data, by intervention group, regarding the proportion of inappropriate prescriptions for each of 10 items on the MAI (which address different aspects of appropriateness). These findings are reported in Appendix E. While one is unable to draw conclusions regarding the findings because they report percentages with prescriptions (rather than "per patient") as the unit of analysis, they do suggest that some items are likely driving the improvements in MAI in the MTM group more than others. Specifically, six aspects of medication prescription appropriateness: drug indication; dosage; practicality of directions; drug-drug interactions; duplication; duration of therapy seem to show greater improvement in inappropriate prescriptions than do those for four other aspects: effective medication; correctness of directions; drug-disease interactions; expense of medication. Similarly, another study ${ }^{68}$ which did not report on the full MAI scale, also reported data regarding the effect of MTM on individual MAI items (Appendix E). The ability to interpret these descriptive findings is not only, like the other study, ${ }^{67}$ hampered by the use of prescriptions rather than patients as the unit of analysis, but also is limited by the marked baseline differences that existed between intervention groups.

Two RCTs (both medium risk of bias) assess the appropriateness of regimens for specific medications for specific conditions (Table 26). One assessed, among patients at risk for glucocorticoid-induced osteoporosis, the percentages of patients receiving each of three indicated regimens; ${ }^{50}$ the investigators found, at 9-month followup, a statistically significant improvement in the percentage appropriately prescribed calcium supplements among MTM recipients compared with controls but not for bisphosphonate or estrogen drug therapy. The other trial assessed the use of angiotensin-conversion enzyme (ACE) inhibitors among heart failure patients ${ }^{49}$. The pharmaceutical care program had a significant effect on the mean percentage of target dose achieved and on the proportion receiving an appropriate alternative medicine among the subsample; such services did not produce a significant effect on the percentage of patients who received an ACE inhibitor. 
Table 26. Medication appropriateness for individual medications: Summary of results

\begin{tabular}{|c|c|c|c|c|}
\hline $\begin{array}{l}\text { Study } \\
\text { Design/Risk of Bias }\end{array}$ & Study Arms & $\mathrm{N}$ analyzed & $\begin{array}{l}\text { Outcome and Time } \\
\text { Period }\end{array}$ & Results \\
\hline $\begin{array}{l}\text { McDonough, } 2005^{50} \\
\text { cluster-randomized } \\
\text { RCT/Medium }\end{array}$ & $\begin{array}{l}\text { G1: pharmaceutical } \\
\text { care provided by } \\
\text { pharmacist in a } \\
\text { community pharmacy } \\
\text { G2: usual care } \\
\text { Patients at risk for } \\
\text { glucocorticoid-induced } \\
\text { osteoporosis) }\end{array}$ & $\begin{array}{l}\text { Baseline } \\
\text { G1: } 70 \\
\text { G2: } 26 \\
\text { Follow Up } \\
\text { G1:61 } \\
\text { G2:19 }\end{array}$ & $\begin{array}{l}\text { 9-month followup } \\
\text { Percentage of patients } \\
\text { taking calcium } \\
\text { supplements }\end{array}$ & $\begin{array}{l}\text { Baseline } \\
\text { G1: } 38.6 \\
\text { G2: } 38.5 \\
\text { p for between-- group } \\
\text { differences at baseline } \\
\text { presumed not significant }{ }^{\text {a }} \\
\text { Followup } \\
\text { G1:55.7 ( } p<0.05 \text { for within- } \\
\text { group difference from } \\
\text { baseline) } \\
\text { G2: } 31.6 \\
\text { p<0.05 for change in outcome } \\
\text { between groups from baseline } \\
\text { to followup } \\
\text { Baseline } \\
\text { G1: } 17.1 \\
\text { G2: } 0 \\
\text { p<0.05 for between-group } \\
\text { difference at baseline } \\
\text { Followup } \\
\text { G1: } 26.2 \text { ( } p<0.05 \text { for within- } \\
\text { group difference from } \\
\text { baseline) } \\
\text { G2: } 10.5 \\
\text { p: NS for between-group } \\
\text { difference at followup; change } \\
\text { in outcome between baseline } \\
\text { and follow-up was NS between } \\
\text { groups }\end{array}$ \\
\hline & & & $\begin{array}{l}\text { Percentage of patients } \\
\text { on estrogen drug } \\
\text { therapy }\end{array}$ & $\begin{array}{l}\text { Baseline } \\
\text { G1: } 12.9 \\
\text { G2: } 0 \\
\text { P NS for between-group } \\
\text { difference at baseline } \\
\text { Followup } \\
\text { G1: } 16.4 \text { ( } p<0.05 \text { for within- } \\
\text { group difference baseline) } \\
\text { G2: } 0 \\
\text { p: NS for between-group } \\
\text { difference at followup; change } \\
\text { in outcome between baseline } \\
\text { and follow-up was NS between } \\
\text { groups. }\end{array}$ \\
\hline
\end{tabular}


Table 26. Medication appropriateness for individual medications: Summary of results (continued)

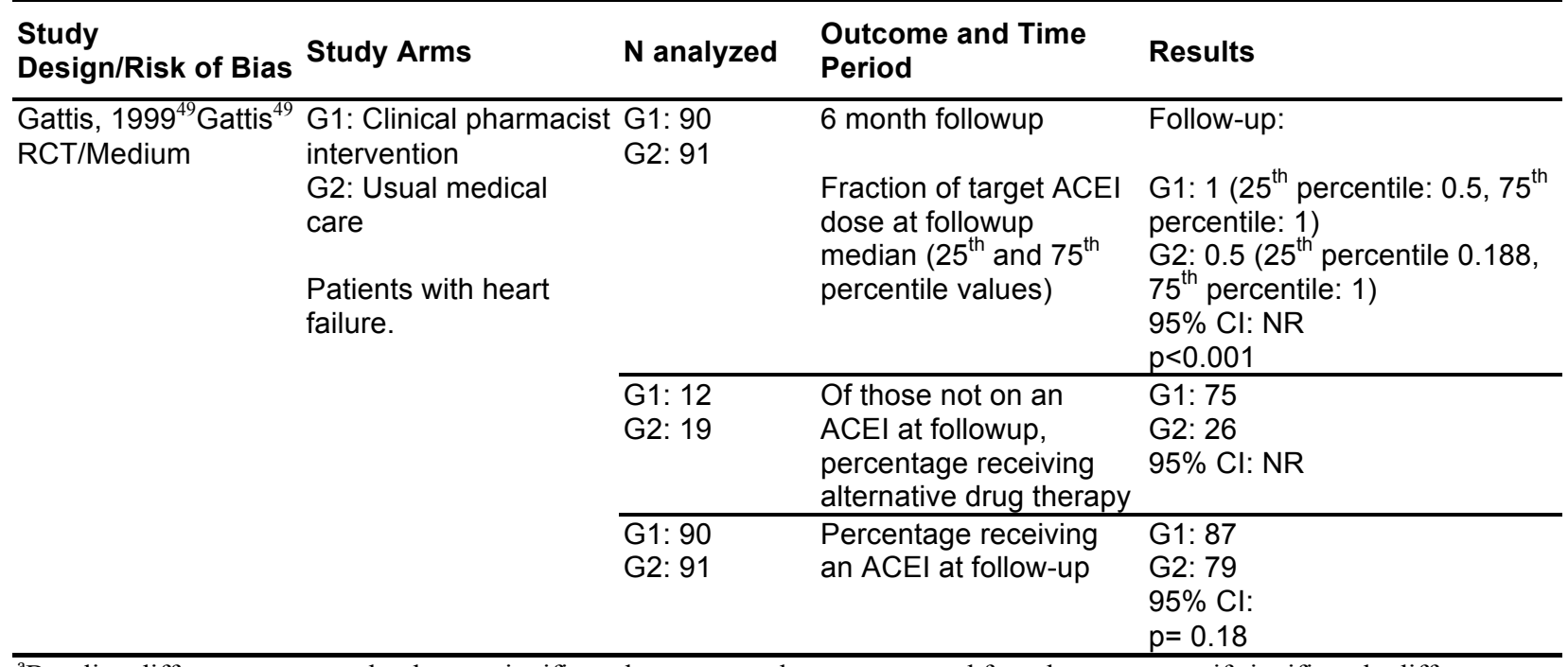

${ }^{\mathrm{a}}$ Baseline differences assumed to be nonsignificant because p-value was reported for other outcomes if significantly different between groups.

Abbreviations: ACEI, angiotensin-converting enzyme inhibitor; NR, not reported; NS. nonsignificant; RCT, randomized controlled trial

Overall, we concluded that the strength of evidence is low for the effect of MTM on medication appropriateness (measured by continuous scores on index) at 3 and 12 months based on indirect, precise evidence from one small RCT (Table 27). The findings are consistent with the direction of effect (indirect, imprecise evidence) from a small prospective cohort study with high study limitations.

Table 27. Medication appropriateness scales: Strength of evidence

\begin{tabular}{|c|c|c|c|c|c|c|c|}
\hline $\begin{array}{l}\text { Study } \\
\text { Design }\end{array}$ & $\begin{array}{l}\text { Number of } \\
\text { Studies; } \\
\text { Subjects } \\
\text { (Analyzed) }\end{array}$ & $\begin{array}{l}\text { Study } \\
\text { Limitations }\end{array}$ & $\begin{array}{l}\text { Consistenc } \\
\text { y }\end{array}$ & Directness & Precision & $\begin{array}{l}\text { Findings and } \\
\text { Direction } \\
\text { [Magnitude] } \\
\text { of Effect }\end{array}$ & $\begin{array}{l}\text { Strength } \\
\text { of } \\
\text { Evidence }\end{array}$ \\
\hline $\mathrm{RCT}$ & $\begin{array}{l}1 ; 208 \\
(208)\end{array}$ & Low & $\begin{array}{l}\text { Unknown } \\
\text { (single } \\
\text { study) }\end{array}$ & Direct & Precise & $\begin{array}{l}\text { Improvement } \\
\text { in MTM group } \\
\text { from score of } \\
17.7 \text { to } 13.4 \\
\text { and to } 12.8 \text { in } \\
3,12 \text { months } \\
\text { respectively } \\
p<0.0006 \text { for } \\
\text { between- } \\
\text { group } \\
\text { differences } \\
\text { controlling for } \\
\text { baseline and } \\
\text { other } \\
\text { covariates }\end{array}$ & Low \\
\hline
\end{tabular}

Abbreviations: $\mathrm{RCT}=$ randomized controlled trial.

Strength of evidence is insufficient for the efficacy of MTM for improving the appropriateness of medication prescriptions for specific medications (Table 28) based on findings from two small RCTs that provided indirect, imprecise evidence of these effects at 6 or 
9 months. This evidence based had medium study limitations, but the trials reported opposite directions of effect based on medication type.

Table 28. Medication appropriateness for individual medications: strength of evidence

\begin{tabular}{|c|c|c|c|c|c|c|c|}
\hline $\begin{array}{l}\text { Study } \\
\text { design }\end{array}$ & $\begin{array}{l}\text { Number of } \\
\text { Studies; } \\
\text { Subjects } \\
\text { (Analyzed) }\end{array}$ & $\begin{array}{l}\text { Study } \\
\text { Limitations }\end{array}$ & Consistency & Directness & Precision & $\begin{array}{l}\text { Findings and } \\
\text { Direction } \\
\text { [Magnitude] of } \\
\text { Effect }\end{array}$ & $\begin{array}{l}\text { Strength of } \\
\text { Evidence }\end{array}$ \\
\hline RCT & $2 ; 277(261)$ & Medium & Inconsistent & Direct & Imprecise & $\begin{array}{l}\text { Significant } \\
\text { improvement in } \\
\text { appropriateness in } \\
\text { the MTM group for } \\
\text { some medications } \\
\text { but not others. }\end{array}$ & Insufficient \\
\hline
\end{tabular}

Abbreviations: $\mathrm{RCT}=$ randomized controlled trial.

\section{Intermediate Outcome: Medication Dosing}

Two RCTs (medium risk of bias) assessed the effect of MTM on medication dosing (Table 29). ${ }^{39,48}$ A third study assessed dose adjustment we excluded it from this analysis because dosing assessed only at baseline. ${ }^{37}$

One trial of renal transplant patients compared the daily doses of three medications (cyclosporine, tacrolimus, and prednisone) for MTM and control groups; it found no statistically significant differences for any of the three medications. ${ }^{48}$ The other trial assessed changes in the number of doses that primary care patients received per day at the end of 6 months; patients in the MTM arm received 1.6 fewer doses than at baseline, whereas control patients received 2.2 more doses per day than at baseline $(p=0.007)$.

Table 29. Medication dosing: Summary of findings

\begin{tabular}{|c|c|c|c|c|}
\hline $\begin{array}{l}\text { Study } \\
\text { Design/Risk of Bias }\end{array}$ & Study Arms & N Analyzed & $\begin{array}{l}\text { Outcome and Time } \\
\text { Period }\end{array}$ & Results \\
\hline \multirow[t]{3}{*}{$\begin{array}{l}\text { Chisholm, } 2002^{48} \\
\text { RCT/Medium }\end{array}$} & \multirow{3}{*}{$\begin{array}{l}\text { G1: Clinical MTM } \\
\text { pharmacy services } \\
\text { G2: routine clinic } \\
\text { services interaction } \\
\text { with renal transplant } \\
\text { clinic team, but no } \\
\text { clinical pharmacist }\end{array}$} & $\begin{array}{l}\text { G1: } 13 \\
\text { G2: } 10\end{array}$ & $\begin{array}{l}\text { Timeframe unspecified } \\
\text { Mean daily } \\
\text { cyclosporine dose } \\
(\mathrm{mg} / \mathrm{kg})\end{array}$ & $\begin{array}{l}\text { G1: } 6.8(1.3) \\
\text { G2: } 7.1(1.2) \\
95 \% \mathrm{Cl}: \mathrm{NR} \\
\mathrm{P}=0.703\end{array}$ \\
\hline & & & $\begin{array}{l}\text { Mean daily tacrolimus } \\
\text { dose }(\mathrm{mg} / \mathrm{kg})\end{array}$ & $\begin{array}{l}\text { G1: } 0.23(0.05) \\
\text { G2: } 0.22(0.04) \\
95 \% \mathrm{Cl}: \mathrm{NR} \\
p=0.823\end{array}$ \\
\hline & & & $\begin{array}{l}\text { Mean daily prednisone } \\
\text { dose }(\mathrm{mg})\end{array}$ & $\begin{array}{l}\text { G1: } 12.3(2.8) \\
\text { G2: } 13.2(3.2) \\
95 \% \mathrm{Cl}: \mathrm{NR} \\
\mathrm{p}=0.705\end{array}$ \\
\hline $\begin{array}{l}\text { Jameson et al., } 1995^{39} \\
\text { RCT/Medium }\end{array}$ & $\begin{array}{l}\text { G1: Consultation with } \\
\text { a clinical pharmacist } \\
\text { in a primary care } \\
\text { office. } \\
\text { G2: Standard medical } \\
\text { care in a primary care } \\
\text { office. }\end{array}$ & $\begin{array}{l}\text { G1: } 27 \\
\text { G2: } 29\end{array}$ & $\begin{array}{l}\text { Change in number of } \\
\text { doses per day at } 6 \\
\text { months' followup. }\end{array}$ & $\begin{array}{l}\text { G1: }-1.6 \\
\text { G2: }+2.2 \\
95 \% \mathrm{Cl}: \mathrm{NR} \\
==0.007\end{array}$ \\
\hline
\end{tabular}


Overall, evidence was insufficient for effect of MTM on medication dosing (Table 30) based on findings from two small RCTs with medium study limitations, but inconsistent, indirect, and imprecise results.

Table 30. Medication dosing: Strength of evidence

\begin{tabular}{|c|c|c|c|c|c|c|c|}
\hline $\begin{array}{l}\text { Study } \\
\text { Design }\end{array}$ & $\begin{array}{l}\text { Number of } \\
\text { Studies; } \\
\text { Subjects } \\
\text { (Analyzed) }\end{array}$ & $\begin{array}{l}\text { Study } \\
\text { Limitations }\end{array}$ & Consistency & Directness & Precision & $\begin{array}{l}\text { Findings and } \\
\text { Direction } \\
\text { [Magnitude] } \\
\text { of Effect }\end{array}$ & $\begin{array}{l}\text { Strength of } \\
\text { Evidence }\end{array}$ \\
\hline RCT & $2 ; 79(90)$ & Medium & Inconsistent & Indirect & Imprecise & $\begin{array}{l}\text { Two RCTs } \\
\text { with opposite } \\
\text { findings: one } \\
\text { showing } \\
\text { significant } \\
\text { increase in } \\
\text { daily doses } \\
\text { and the other } \\
\text { showing no } \\
\text { difference in } \\
\text { daily doses of } \\
\text { medications }\end{array}$ & Insufficient \\
\hline
\end{tabular}

\section{Key Points: Patient-Centered Outcomes}

- Evidence was insufficient to draw conclusions about the effect of MTM on adverse drug events (two trials: low study limitations, inconsistent, imprecise); cognitive, affective, and physical function (one trial, medium study limitations, imprecise); mortality (two trials with medium study limitations, one observational study with high study limitations, inconsistent, imprecise); and gastrointestinal bleeding events (one observational study, high study limitations, imprecise).

- With one exception, MTM interventions had no benefit on SF-36 measures (low strength of evidence of no benefit); evidence was insufficient for the SF-36 domain of vitality because of imprecision.

- Evidence was insufficient to determine whether MTM interventions improved patientreported measures for patients with diabetes (one imprecise medium risk of bias trial).

- MTM interventions did not improve measures of patient satisfaction (low strength of evidence of no benefit).

\section{Adverse Drug Events}

Four RCTs ${ }^{39,55,67,68}$ and one nonrandomized trial ${ }^{66}$ reported on prevalence of adverse drug events (ADEs) following MTM or pharmaceutical care interventions (Table 31). The methods for measuring adverse events differed substantially among included studies. Further, although we assumed that the beneficial direction of effect would be for MTM to decrease ADEs, the nonrandomized trial suggested that MTM services may heighten awareness of potential adverse outcomes by patients and, thus, increase reporting of ADEs by those receiving the intervention. ${ }^{66}$ For this outcome, we rated the risk of bias for some studies ${ }^{39,66,68}$ as higher than the overall risk of bias because of measurement and detection bias with respect to the measures and methods used to ascertain this outcome. 
Table 31. Adverse events: Summary of results

\begin{tabular}{|c|c|c|c|c|}
\hline $\begin{array}{l}\text { Study } \\
\text { Design/Risk of } \\
\text { Bias }\end{array}$ & Study Arms & N Analyzed & $\begin{array}{l}\text { Outcomes Reported } \\
\text { by Study and Time } \\
\text { Period }\end{array}$ & Results \\
\hline $\begin{array}{l}\text { Hanlon et al., } \\
1996^{67} \\
\text { RCT/Low }\end{array}$ & $\begin{array}{l}\text { G1: Clinical pharmacist } \\
\text { care within a general } \\
\text { medicine clinic. } \\
\text { G2: Usual care }\end{array}$ & $\begin{array}{l}\text { G1:86 } \\
\text { G2:83 }\end{array}$ & $\begin{array}{l}\text { Percentage with an ADE } \\
\text { at } 12 \text { months }\end{array}$ & $\begin{array}{l}\text { Calculated OR: } 0.649, \\
95 \% \mathrm{Cl}: 0.366 \text { to } 1.152, \\
p=0.014\end{array}$ \\
\hline \multirow[t]{4}{*}{$\begin{array}{l}\text { Touchette et al., } \\
2012^{55} \\
\text { RCT/Low }\end{array}$} & $\begin{array}{l}\text { G1: Basic MTM } \\
\text { services (with } \\
\text { medication information } \\
\text { from patient interview) }\end{array}$ & $\begin{array}{l}\text { G1: } 211 \\
\text { G2: } 218 \\
\text { G3: } 208\end{array}$ & $\begin{array}{l}\text { Percentage of patients } \\
\text { with an ADE between } 0 \\
\text { and } 3 \text { months and OR }\end{array}$ & $\begin{array}{l}\text { G1 vs. G3: OR: } 1.629 \\
(p=0.078) \\
\text { G2 vs. G3: OR: } 0.726 \\
(p=0.278)\end{array}$ \\
\hline & $\begin{array}{l}\text { G2: Enhanced MTM } \\
\text { services (pharmacist } \\
\text { provided with 2-page }\end{array}$ & & $\begin{array}{l}\text { Percentage of patients } \\
\text { with an ADE between } 3 \\
\text { and } 6 \text { months and OR }\end{array}$ & $\begin{array}{l}\text { G1 vs. G3: OR: } 1.107 \\
(p=0.717) \\
\text { G2 vs. G3: OR: } 0.889 \\
(p=0.672)\end{array}$ \\
\hline & $\begin{array}{l}\text { clinical summary from } \\
\text { patient medical record). } \\
\text { G3: Usual pharmacy } \\
\text { care }\end{array}$ & & $\begin{array}{l}\text { Mean number (SD) of } \\
\text { ADEs per patient } \\
\text { between } 0 \text { and } 3 \\
\text { months }\end{array}$ & $\begin{array}{l}\text { G1 vs. G3: } \\
\text { Calculated SMD: } 0.165 \text {, } \\
95 \% \text { Cl: }-0.027 \text { to } 0.357 \\
p=0.110\end{array}$ \\
\hline & & & & $\begin{array}{l}\text { G2 vs. G3: } \\
\text { Calculated SMD: }-0.010 \text {, } \\
95 \% \text { Cl: }-0.200 \text { to } 0.180 \\
p=0.916\end{array}$ \\
\hline $\begin{array}{l}\text { Touchette et al., } \\
2012^{55} \\
\text { RCT/Low } \\
\text { (continued) }\end{array}$ & & & $\begin{array}{l}\text { Mean number (SD) of } \\
\text { ADEs per patient } \\
\text { between } 3 \text { and } 6 \\
\text { months }\end{array}$ & $\begin{array}{l}\text { G1 vs. G3: } \\
\text { Calculated SMD: } 0.239 \text {, } \\
95 \% \text { Cl: } 0.047 \text { to } 0.431 \\
p=0.041 \\
\text { G2 vs. G3: } \\
\text { Calculated SMD: }-0.072 \text {, } \\
95 \% \text { Cl: }-0.262 \text { to } 0.118 \\
p=0.479\end{array}$ \\
\hline $\begin{array}{l}\text { Fischer et al., } \\
2000^{66} \\
\text { NRCT/High }\end{array}$ & $\begin{array}{l}\text { G1: Comprehensive } \\
\text { drug therapy } \\
\text { management program } \\
\text { G2: Standard } \\
\text { community pharmacy } \\
\text { practice }\end{array}$ & $\begin{array}{l}\text { G1: } 201 \\
\text { G2: } 368\end{array}$ & $\begin{array}{l}\text { OR for likelihood of } \\
\text { reporting side effects or } \\
\text { problems from } \\
\text { prescription medication } \\
(95 \% \mathrm{Cl})\end{array}$ & $1.81(1.16$ to 2.83$)$ \\
\hline $\begin{array}{l}\text { Taylor et al., } \\
2003^{68} \\
\text { RCT/High }\end{array}$ & $\begin{array}{l}\text { G1: Pharmaceutical } \\
\text { care } \\
\text { G2: Standard care }\end{array}$ & $\begin{array}{l}\text { G1: } 33 \\
\text { G2: } 36\end{array}$ & $\begin{array}{l}\text { Percentage of patients } \\
\text { with at least one } \\
\text { medication } \\
\text { misadventure at } 12 \\
\text { months }\end{array}$ & $\begin{array}{l}\mathrm{G} 1: 2.8^{\mathrm{a}}(\mathrm{N}=4) \\
\mathrm{G} 2: 3.0^{\mathrm{a}}(\mathrm{N}=3) \\
\text { Calculated OR based on } \\
\text { reported percent: } 0.93,95 \% \mathrm{Cl} \text {, } \\
0.056 \text { to } 15.603, \mathrm{p}=0.0961 \\
\text { Calculated OR based on } \\
\text { reported } \mathrm{N}: 1.515(95 \% \mathrm{Cl}, \\
0.312 \text { to } 7.344), \mathrm{p}=0.606\end{array}$ \\
\hline $\begin{array}{l}\text { Jameson et al., } \\
1995^{39} \\
\text { RCT/High }\end{array}$ & $\begin{array}{l}\text { G1: Consultation with a } \\
\text { clinical pharmacist in a } \\
\text { primary care office. } \\
\text { G2: Standard medical } \\
\text { care in a primary care } \\
\text { office. }\end{array}$ & $\begin{array}{l}\text { G1: } 27 \\
\text { G2: } 29\end{array}$ & $\begin{array}{l}\text { Change in mean } \\
\text { medication side effect } \\
\text { score at } 6 \text { months. }\end{array}$ & $\begin{array}{l}\text { G1: }-3.7 \\
\text { G2: }-1.9 \\
\text { p: NS and unable to calculate. }\end{array}$ \\
\hline
\end{tabular}

${ }^{\mathrm{a}}$ The percent reported by authors cannot be generated based on the reported $\mathrm{N}$ and the reported number of events.

Abbreviations: $\mathrm{ADE}=$ adverse drug event; $\mathrm{CI}=$ confidence interval; $\mathrm{DRP}=$ drug-related problems; $\mathrm{NRCT}=$ nonrandomized controlled trial; $\mathrm{NS}=$ not significant; $\mathrm{OR}=$ odds ratio; $\mathrm{RCT}=$ randomized controlled trial; $\mathrm{SMD}=$ standardized mean difference; vs. $=$ versus 
One RCT (low risk of bias) compared clinical pharmacy care within a VA general medicine clinic to usual care $;{ }^{67}$ it found no significant difference in the number of subjects reporting an $\mathrm{ADE}$ at 12 months. Another RCT (low risk of bias) compared usual care with the provision of basic MTM services designed to mimic conditions similar to a community pharmacy with another study arm that included an enhanced intervention that provided clinical information about the patient to the pharmacist. ${ }^{55}$ It reported on outcomes for the period between 0 and 3 months and for the period between 3 and 6 months. The enhanced MTM intervention was superior to the basic MTM intervention at 3 months in the percentage of subjects reporting an ADE; however, the enhanced intervention and usual care at 3 months and three study arms at 6 months did not differ significantly. In addition, the mean number of ADEs per patient was not statistically different between 0 and 3 months across study arms, but both the enhanced MTM and usual care study arms had significantly fewer ADEs per patient than the basic MTM study arm between 3 and 6 months. This RCT found no statistical difference in mean ADEs per patient between the enhanced MTM study arm and usual care between 3 and 6 months.

The other two RCTs were considered high risk of bias for the ADE outcome. One RCT provided pharmaceutical care to patients at high risk for medication-related problems seen in family medicine practices in a rural community; ${ }^{68}$ the intervention and control arms did not differ significantly. We rated this trial as high risk of bias because it used a nonstandard measure (medication misadventure) and because the control event rates differed by a factor of 10 relative to those in RCTs with low risk of bias. The other RCT that we rated high risk of bias compared MTM intervention with usual medical care and reported no significant difference between change in medication side effect scores using a scale that the study authors had developed. ${ }^{39}$ The nonrandomized trial (high risk of bias for this outcome)compared participants who agreed to participate in a pharmaceutical care program at one of six participating community pharmacies with a group of control patients who received medications at pharmacies that did not provide pharmaceutical care services; ${ }^{66}$ study participants were significantly more likely (OR, $1.81 ; 95 \%$ CI, 1.16 to 2.83 ) to report experiencing symptoms or problems related to prescription medication than control participants, an effect the authors attributed to increased awareness of medication side effects in the intervention group. Without a clear understanding of the hypothesized mechanism of action in each study for influencing ADEs, we cannot interpret the conflicting result presented by the nonrandomized trial relative to the findings from the RCTs.

Overall, we concluded that evidence is insufficient to draw conclusions about the efficacy of MTM for reducing adverse drug events based on direct, but inconsistent and imprecise, evidence from two low-risk-of-bias RCTs (Table 32).

Table 32. Adverse drug events: Strength of evidence

\begin{tabular}{|c|c|c|c|c|c|c|c|}
\hline Study Design & $\begin{array}{l}\text { Number of } \\
\text { Studies; } \\
\text { Subjects } \\
\text { (Analyzed) }\end{array}$ & $\begin{array}{l}\text { Study } \\
\text { Limitations }\end{array}$ & Consistency & Directness & Precision & $\begin{array}{l}\text { Findings } \\
\text { and } \\
\text { Direction } \\
\text { [Magnitude] } \\
\text { of Effect }\end{array}$ & $\begin{array}{l}\text { Strength } \\
\text { of } \\
\text { Evidence }\end{array}$ \\
\hline RCT & $\begin{array}{l}2 ; 845 \\
(806)\end{array}$ & Low & Inconsistent & Direct & Imprecise & $\begin{array}{l}\text { Direction and } \\
\text { magnitude of } \\
\text { effect differs } \\
\text { between the } \\
\text { two trials. }\end{array}$ & Insufficient \\
\hline
\end{tabular}

Abbreviations: $\mathrm{RCT}=$ randomized controlled trial. 


\section{Cognitive, Affective, and Physical Function}

One RCT (medium risk of bias)reported on changes in cognitive, affective, and physical function at 6 weeks; ${ }^{74}$ the intervention provided in a general medicine outpatient clinic was designed to simplify medication regimens among cognitively intact patients ages 65 or older at high risk for medication-related adverse events. The investigators measured cognitive function using three different tests (two subtests from the Wechsler Adult Intelligence Scale [digit-symbol and digit span] and modified Randt Memory Test). They measured affective function using the Center for Epidemiological Studies Depression Scale and the Self-Rating Anxiety Scale and physical functioning using the Timed Manual Performance Test, Physical Performance Test, and Functional Reach Assessment. Patients in the intervention arm experienced no significant changes in any of these measures when compared with patients in the control arm. This finding may be explained partly by the fact that although recommendations for medication discontinuation were made in the intervention arm (on average 4.5 drug discontinuation recommendations per participant), the authors stated that intervention participants stopped taking only 1.5 drugs per participant on from their regimen. Evidence was insufficient to draw conclusions about the impact of MTM on these functional outcomes (Table 33).

Table 33. Cognitive, affective, and physical function: Strength of evidence

\begin{tabular}{|c|c|c|c|c|c|c|c|}
\hline Study design & $\begin{array}{l}\text { Number of } \\
\text { Studies; } \\
\text { Subjects } \\
\text { (Analyzed) }\end{array}$ & $\begin{array}{l}\text { Study } \\
\text { Limitations }\end{array}$ & Consistency & Directness & Precision & $\begin{array}{l}\text { Findings and } \\
\text { Direction } \\
\text { [Magnitude] of } \\
\text { Effect }\end{array}$ & $\begin{array}{l}\text { Strength of } \\
\text { Evidence }\end{array}$ \\
\hline$\overline{\mathrm{RCT}}$ & $\begin{array}{l}1 ; 140 \\
(133)\end{array}$ & Medium & $\begin{array}{l}\text { Consistency } \\
\text { unknown- } \\
\text { single study }\end{array}$ & Direct & Imprecise & $\begin{array}{l}\text { One study with no } \\
\text { significant } \\
\text { differences } \\
\text { between arms. }\end{array}$ & Insufficient \\
\hline
\end{tabular}

Abbreviations: RCT= randomized controlled trial.

\section{Mortality}

Two RCTs ${ }^{49,54}$ and one cohort study ${ }^{37}$ reported all-cause mortality outcomes following MTM interventions at 6 months (Table 34). One RCT (medium risk of bias) of patients after discharge from the hospital for heart failure compared a study arm that added a pharmacist intervention to visiting home nurse services with just the visiting home nurse services. ${ }^{54}$ The other RCT (medium risk of bias) in a university general cardiology clinic compared a study arm that included a clinical pharmacist intervention for heart failure patients with usual medical care. ${ }^{49}$ These two RCTs reported effect estimates in opposite directions, but neither was statistically significant. 
Table 34. All-cause mortality: Summary of results

\begin{tabular}{|c|c|c|c|c|}
\hline $\begin{array}{l}\text { Study } \\
\text { Design/Risk of } \\
\text { Bias }\end{array}$ & Study Arms & $\begin{array}{l}\mathrm{N} \\
\text { Analyzed }\end{array}$ & $\begin{array}{l}\text { Outcome Reported by } \\
\text { Study and Time Period }\end{array}$ & Results \\
\hline $\begin{array}{l}\text { Triller et al., } 2007^{54} \\
\text { RCT/Medium }\end{array}$ & $\begin{array}{l}\text { G1: Visiting nurse } \\
\text { association home visit } \\
\text { services plus } \\
\text { comprehensive } \\
\text { pharmaceutical care } \\
\text { services } \\
\text { G2: Visiting nurse } \\
\text { association home visit } \\
\text { services }\end{array}$ & $\begin{array}{l}\text { G1: } 77 \\
\text { G2: } 77\end{array}$ & $\begin{array}{l}\text { RR for all-cause } \\
\text { mortality within } 180 \text { days }\end{array}$ & $\begin{array}{l}\text { RR: } 1.21 \\
\text { Calculated } 95 \% \mathrm{Cl}: 0.645 \text { to } \\
2.29 \\
p=0.67\end{array}$ \\
\hline $\begin{array}{l}\text { Gattis et al., } 1992^{49} \\
\text { RCT/Medium }\end{array}$ & $\begin{array}{l}\text { G1: Clinical pharmacist } \\
\text { intervention in addition to } \\
\text { usual medical care } \\
\text { G2: Usual medical care }\end{array}$ & $\begin{array}{l}\text { G1: } 90 \\
\text { G2: } 91\end{array}$ & $\begin{array}{l}\text { OR for all-cause } \\
\text { mortality within } 6 \\
\text { months }\end{array}$ & $\begin{array}{l}\text { OR: } 0.59 \\
95 \% \mathrm{Cl}: 0.12 \text { to } 2.49 \\
p=0.48\end{array}$ \\
\hline $\begin{array}{l}\text { Welch et al., } \\
2009^{37} \\
\text { Cohort } \\
\text { study/Medium }\end{array}$ & $\begin{array}{l}\text { G1: MTM program provided } \\
\text { to home-based beneficiaries } \\
\text { G2: No-MTM control group } \\
\text { (voluntary opt-out) }\end{array}$ & $\begin{array}{l}\text { G1: } 459 \\
\text { G2: } 336\end{array}$ & $\begin{array}{l}\text { Adjusted OR for all- } \\
\text { cause mortality, within } 6 \\
\text { months (adjusted for } \\
\text { age, sex, chronic } \\
\text { disease score, specific } \\
\text { baseline utilization) }\end{array}$ & $\begin{array}{l}\text { Adjusted OR: } 0.5 \\
95 \% \mathrm{Cl}, 0.3 \text { to } 0.9 \\
p=0.044\end{array}$ \\
\hline
\end{tabular}

Abbreviations: $\mathrm{CI}=$ confidence interval; $\mathrm{G}=$ group; $\mathrm{MTM}=$ medication therapy management; OR = odds ratio; $\mathrm{RCT}=$ randomized controlled trial; $\mathrm{RR}=$ relative risk

The cohort study (Table 34) (medium risk of bias) measured mortality outcomes for beneficiaries who met MTM program eligibility and opted in to a telephone-based MTM program provided through an integrated health care system and for eligible beneficiaries who opted out of the MTM program. ${ }^{37}$ This study reported a statistically significant reduction in allcause mortality at 6 months in the intervention arm, when adjusted for age, sex, and baseline disease and health care utilization levels.

Both RCTs reporting mortality outcomes also reported a composite measure that combined all-cause mortality with another outcome as the primary study endpoint. One RCT reported a composite outcome of all-cause mortality and all-cause hospitalization at 6 months; intervention an control arms did not differ ( 62 percent versus 61 percent; RR, $0.98 ; 95 \% \mathrm{CI}, \mathrm{NR} ; \mathrm{p}=1.0$ ) ${ }^{54}$ The other RCT reported a composite outcome of all-cause mortality and nonfatal heart failure events at 6 months; patients in the intervention arm experienced a significant benefit from the program (OR, $0.221 ; 95 \% \mathrm{CI}, 0.07$ to $0.65 ; \mathrm{p}=0.005){ }^{49}$

Overall, we concluded that evidence is insufficient for the efficacy of MTM for reducing allcause mortality at 6 months based on direct, but inconsistent and imprecise, evidence from two RCTs and one observational study, all with medium study limitations (Table 35). 
Table 35. All-cause mortality: Strength of evidence

\begin{tabular}{|c|c|c|c|c|c|c|c|}
\hline $\begin{array}{l}\text { Study } \\
\text { Design }\end{array}$ & $\begin{array}{l}\text { Number of } \\
\text { Studies; } \\
\text { Subjects } \\
\text { (Analyzed) }\end{array}$ & $\begin{array}{l}\text { Study } \\
\text { Limitations }\end{array}$ & Consistency & Directness & Precision & $\begin{array}{l}\text { Findings and } \\
\text { Direction } \\
\text { [Magnitude] of } \\
\text { Effect }\end{array}$ & $\begin{array}{l}\text { Strength of } \\
\text { Evidence }\end{array}$ \\
\hline RCT & $2 ; 335(335)$ & Medium & Inconsistent & Direct & Imprecise & $\begin{array}{l}\text { Two studies with } \\
\text { opposite direction of } \\
\text { effect, both with } \\
\text { nonsignificant } \\
\text { differences between } \\
\text { groups. }\end{array}$ & Insufficient \\
\hline Observational & $1 ; 904(795)$ & High & $\begin{array}{l}\text { Consistency } \\
\text { unknown- } \\
\text { single study }\end{array}$ & Direct & Imprecise & $\begin{array}{l}\text { OR } 0.5 \\
95 \% \mathrm{Cl}, 0.3 \text { to } 0.9\end{array}$ & Insufficient \\
\hline
\end{tabular}

Abbreviations: $\mathrm{CI}=$ confidence interval; $\mathrm{NA}=$ not applicable; $\mathrm{OR}=$ odds ratio; $\mathrm{RCT}=$ randomized controlled trial.

\section{Gastrointestinal Bleeding Events}

One cohort study (high risk of bias because of selection bias) reported the relative risk reduction in gastrointestinal bleeding events among patients with a diagnosis of arthritis enrolled in a telephone-based MTM program within a large US integrated health care system. ${ }^{35}$ The investigators compared the number of gastrointestinal bleeds after 6 months between patients who did and did not enroll in the MTM program. Enrolled patients had a 60 percent relative reduction in gastrointestinal bleeds; the nonenrolled patients had no change in gastrointestinal bleeds ( $p=0.001$ for between-group difference in change in gastrointestinal bleeds).

Overall, we concluded that evidence is insufficient for the efficacy of MTM for reducing gastrointestinal bleeding events based on direct but imprecise evidence from one cohort study with high study limitations (Table 36).

Table 36. Gastrointestinal bleeding events: Strength of evidence

\begin{tabular}{|c|c|c|c|c|c|c|c|}
\hline Study Design & $\begin{array}{l}\text { Number of } \\
\text { Studies; } \\
\text { Subjects } \\
\text { (Analyzed) }\end{array}$ & $\begin{array}{l}\text { Study } \\
\text { Limitations }\end{array}$ & Consistency & Directness & Precision & $\begin{array}{l}\text { Findings and } \\
\text { Direction } \\
\text { [Magnitude] of } \\
\text { Effect }\end{array}$ & $\begin{array}{l}\text { Strength of } \\
\text { Evidence }\end{array}$ \\
\hline Observational & $\begin{array}{l}1 ; 1,388 \\
(1.373)\end{array}$ & High & $\begin{array}{l}\text { Consistency } \\
\text { unknown- } \\
\text { single study }\end{array}$ & Direct & Imprecise & $\begin{array}{l}\text { RRR } 60 \% \\
(p=0.001)\end{array}$ & Insufficient \\
\hline
\end{tabular}

Abbreviations: $\mathrm{RCT}=$ randomized controlled trial; $\mathrm{RRR}=$ relative risk reduction

\section{Self-Reported Health Status: SF-36 Measures}

\section{SF-36 Measures: Overview}

Eight RCTs ${ }^{43,44,51,67-69,72,73}$ and one cohort study ${ }^{56}$ reported health status outcomes using the Medical Outcomes Study Short-Form questionnaire (SF-36) (Table 37). The eight SF-36 domains, which combine into two components, are as follows-physical health: physical functioning, physical role functioning, bodily pain, and general health perceptions; and mental health: vitality, emotional role functioning, social role functioning, and mental health. Seven trials ${ }^{43,44,51,67,68,72,73}$ and the cohort study ${ }^{56}$ reported scores for all eight domains. One trial reported only its two component scores (i.e., physical health; mental health). ${ }^{69}$ Finally, one trial reported both component and domain scores. ${ }^{44}$ The trials differed by overall risk of bias (one, low; four, medium, and three, high); the cohort study was high risk of bias. 
Table 37. Scores on SF-36 measures: Summary of effects from meta-analyses

\begin{tabular}{|c|c|c|c|c|c|c|}
\hline $\begin{array}{l}\text { SF-36 } \\
\text { Components } \\
\text { and Domains }\end{array}$ & $\begin{array}{l}\text { Time Periods and } \\
\text { Risk of Bias for } \\
\text { Included Trials }\end{array}$ & $\begin{array}{l}\text { Number of } \\
\text { Studies }\end{array}$ & $\begin{array}{l}\text { Total } \\
\text { Number } \\
\text { With/ } \\
\text { Without } \\
\text { MTM }\end{array}$ & $\begin{array}{l}\text { Mean Difference } \\
95 \% \mathrm{Cl} \\
\text { Lower Limit to } \\
\text { Upper Limit } \\
\text { p-value }\end{array}$ & $\begin{array}{l}\text { Q-value } \\
\text { (df for Q) } \\
\text { p-value }\end{array}$ & I-squared \\
\hline \multirow[t]{2}{*}{$\begin{array}{l}\text { Physical } \\
\text { functioning } \\
\text { domain }\end{array}$} & $\begin{array}{l}\text { All time periods, low } \\
\text { or medium risk of } \\
\text { bias }\end{array}$ & $3^{43,67,68}$ & $566 / 603$ & $\begin{array}{l}1.171 \\
\text { Cl: }-3.871 \text { to } 6.214 \\
p=0.649\end{array}$ & $\begin{array}{l}3.873 \\
(2) \\
p=0.144\end{array}$ & 48.363 \\
\hline & $\begin{array}{l}\text { All time periods, all } \\
\text { risk of bias }\end{array}$ & $5^{43,44,67,68,72}$ & $968 / 1,038$ & $\begin{array}{l}-0.438 \\
\mathrm{Cl}:-2.641 \text { to } 1.765 \\
p=0.697\end{array}$ & $\begin{array}{l}4.478 \\
(4) \\
p=0.345\end{array}$ & 10.669 \\
\hline \multirow[t]{2}{*}{$\begin{array}{l}\text { Physical role } \\
\text { functioning } \\
\text { domain }\end{array}$} & $\begin{array}{l}\text { All time periods, low } \\
\text { or medium risk of } \\
\text { bias }\end{array}$ & $3^{43,67,68}$ & $566 / 603$ & $\begin{array}{l}3.392 \\
C I:-1.223 \text { to } 8.007 \\
p=0.150\end{array}$ & $\begin{array}{l}0.988 \\
(2) \\
p=0.610\end{array}$ & 0 \\
\hline & $\begin{array}{l}\text { All time periods, all } \\
\text { risk of bias }\end{array}$ & $5^{43,44,67,68,72}$ & $968 / 1,038$ & $\begin{array}{l}0.733 \\
\mathrm{Cl}:-3.429 \text { to } 4.895 \\
p=0.730\end{array}$ & $\begin{array}{l}7.238 \\
(4) \\
p=0.124\end{array}$ & 44.733 \\
\hline \multirow[t]{2}{*}{$\begin{array}{l}\text { Bodily pain } \\
\text { domain }\end{array}$} & $\begin{array}{l}\text { All time periods, low } \\
\text { or medium risk of } \\
\text { bias }\end{array}$ & $3^{43,67,68}$ & $566 / 603$ & $\begin{array}{l}3.320 \\
C I:-0.792 \text { to } 7.433 \\
p=0.114\end{array}$ & $\begin{array}{l}2.765 \\
(2) \\
p=0.251\end{array}$ & 27.658 \\
\hline & $\begin{array}{l}\text { All time periods, all } \\
\text { risk of bias }\end{array}$ & $5^{43,44,67,68,72}$ & $968 / 1,038$ & $\begin{array}{l}1.459 \\
\mathrm{Cl}:-2.793 \text { to } 5.711 \\
p=0.501\end{array}$ & $\begin{array}{l}21.061 \\
(4) \\
p<0.001\end{array}$ & 81.007 \\
\hline \multirow[t]{2}{*}{$\begin{array}{l}\text { General health } \\
\text { perceptions } \\
\text { domain }\end{array}$} & $\begin{array}{l}\text { All time periods, low } \\
\text { or medium risk of } \\
\text { bias }\end{array}$ & $3^{43,67,68}$ & $566 / 603$ & $\begin{array}{l}1.916 \\
\mathrm{Cl}:-0.007 \text { to } 3.839 \\
p=0.051\end{array}$ & $\begin{array}{l}0.856 \\
(2) \\
p=0.652\end{array}$ & 0 \\
\hline & $\begin{array}{l}\text { All time periods, all } \\
\text { risk of bias }\end{array}$ & $5^{43,44,67,68,72}$ & $968 / 1,308$ & $\begin{array}{l}2.476 \\
C I: 2.123 \text { to } 2.829 \\
p<0.001\end{array}$ & $\begin{array}{l}1.624 \\
(4) \\
p=0.804\end{array}$ & 0 \\
\hline \multirow[t]{2}{*}{ Vitality domain } & $\begin{array}{l}\text { All time periods, low } \\
\text { or medium risk of } \\
\text { bias }\end{array}$ & $3^{43,67,68}$ & $566 / 603$ & $\begin{array}{l}2.797 \\
C I: 0.655 \text { to } 4.939 \\
p=0.010\end{array}$ & $\begin{array}{l}0.965 \\
(2) \\
p=0.617\end{array}$ & 0 \\
\hline & $\begin{array}{l}\text { All time periods, all } \\
\text { risk of bias }\end{array}$ & $5^{43,44,67,68,72}$ & 9681,038 & $\begin{array}{l}1.299 \\
\mathrm{Cl}:-0.305 \text { to } 2.904 \\
p=0.112\end{array}$ & $\begin{array}{l}4.750 \\
(4) \\
p=0.314\end{array}$ & 15.793 \\
\hline \multirow[t]{2}{*}{$\begin{array}{l}\text { Social } \\
\text { functioning } \\
\text { domain }\end{array}$} & $\begin{array}{l}\text { All time periods, low } \\
\text { or medium risk of } \\
\text { bias }\end{array}$ & $3^{43,67,68}$ & $566 / 603$ & $\begin{array}{l}2.932 \\
\text { Cl: }-0.085 \text { to } 5.949 \\
p=0.057\end{array}$ & $\begin{array}{l}1.078 \\
(2) \\
p=0.583\end{array}$ & 0.000 \\
\hline & $\begin{array}{l}\text { All time periods, all } \\
\text { risk of bias }\end{array}$ & $5^{43,44,67,68,72}$ & $968 / 1,038$ & $\begin{array}{l}0.631 \\
\mathrm{Cl}: 0.290 \text { to } 0.973 \\
\mathrm{p}<0.001\end{array}$ & $\begin{array}{l}3.407 \\
(4) \\
p=0.492 \\
\end{array}$ & 0 \\
\hline \multirow[t]{2}{*}{$\begin{array}{l}\text { Emotional role } \\
\text { functioning } \\
\text { domain }\end{array}$} & $\begin{array}{l}\text { All time periods, low } \\
\text { or medium risk of } \\
\text { bias }\end{array}$ & $3^{43,67,68}$ & $566 / 603$ & $\begin{array}{l}5.386 \\
C I:-7.244 \text { to } 18.016 \\
p=0.403\end{array}$ & $\begin{array}{l}7.794 \\
(2) \\
p=0.20 \\
\end{array}$ & 74.341 \\
\hline & $\begin{array}{l}\text { All time periods, all } \\
\text { risk of bias }\end{array}$ & $5^{43,44,67,68,72}$ & $968 / 1,038$ & $\begin{array}{l}3.441 \\
C l:-4.000 \text { to } 10.882 \\
p=0.365\end{array}$ & $\begin{array}{l}18.742 \\
(4) \\
p=0.001\end{array}$ & 78.657 \\
\hline
\end{tabular}


Table 37. Scores on SF-36 measures: Summary of effects from meta-analyses (continued)

\begin{tabular}{|c|c|c|c|c|c|c|}
\hline $\begin{array}{l}\text { SF-36 } \\
\text { Components } \\
\text { and Domains }\end{array}$ & $\begin{array}{l}\text { Time Periods and } \\
\text { Risk of Bias for } \\
\text { Included Trials }\end{array}$ & $\begin{array}{l}\text { Number of } \\
\text { Studies }\end{array}$ & $\begin{array}{l}\text { Total } \\
\text { Number } \\
\text { With/ } \\
\text { Without } \\
\text { MTM }\end{array}$ & $\begin{array}{l}\text { Mean Difference } \\
95 \% \mathrm{CI} \\
\text { Lower Limit to } \\
\text { Upper Limit } \\
\text { p-value }\end{array}$ & $\begin{array}{l}\text { Q-value } \\
\text { (df for Q) } \\
\text { p-value }\end{array}$ & I-squared \\
\hline \multirow[t]{2}{*}{$\begin{array}{l}\text { Mental health } \\
\text { domain }\end{array}$} & $\begin{array}{l}\text { All time periods, low } \\
\text { or medium risk of } \\
\text { bias }\end{array}$ & $3^{43,67,68}$ & $566 / 603$ & $\begin{array}{l}1.615 \\
C I:-0.362 \text { to } 3.593 \\
p=0.109\end{array}$ & $\begin{array}{l}0.968 \\
(2) \\
p=0.616\end{array}$ & 0 \\
\hline & $\begin{array}{l}\text { All time periods, all } \\
\text { risk of bias }\end{array}$ & $5^{43,44,67,68,72}$ & $968 / 1,038$ & $\begin{array}{l}1.109 \\
\mathrm{Cl}: 0.280 \text { to } 1.928 \\
p=0.009\end{array}$ & $\begin{array}{l}1.274 \\
(4) \\
p=0.866\end{array}$ & 0 \\
\hline
\end{tabular}

Abbreviations: CI = confidence interval; MTM = medication therapy management; SF-36 = 36-Item Short Form Health Survey

One trial (medium risk of bias) focused on patients at high risk of experiencing a drug-related problem. ${ }^{43}$ This trial compared an intervention arm that included a clinical pharmacist intervention delivered in an ambulatory care clinic with usual medical care. It reported betweengroup differences with p-values less than 0.05 for four of the eight SF-36 domains (namely, bodily pain, general health perceptions, vitality, and mental health) and for a question that assessed change in health status. All these differences favored the intervention group. However, to control for multiple comparisons, the investigators set alpha at 0.01 when evaluating statistical significance. Using this more conservative alpha level, they investigators reported that only the bodily pain domain and the item assessing change in health status were statistically significant.

Of the eight remaining studies reporting results for SF-36 domains), four trials (one low risk of bias; ${ }^{67}$ three medium risk of bias ${ }^{44,68,73}$ ) reported no statistically significant between-group differences on any SF-36 score. Two trials ${ }^{51,72}$ and the cohort study ${ }^{56}$ (all high risk of bias) reported one statistically significant $(\mathrm{p}<0.05)$ between-group difference, favoring the intervention group — specifically for vitality ${ }^{72}$ - among the total of 24 comparisons examined across the three studies. Finally, for one trial (medium risk of bias overall), we rated risk of bias for the SF-36 outcomes as high because of numerous errors in the table reporting these findings (e.g., group mean not contained within 95\% CI, group mean not centered within $95 \% \mathrm{CI}$ ); ${ }^{44}$ it reported no statistically significant between-group differences on any SF-36 elements.

\section{SF-36 Measures: Meta-analyses}

Our analysis focuses on the three trials rated either low or medium risk of bias that provided sufficient data to calculate mean differences for the eight SF-36 domain scores. ${ }^{1-3} \mathrm{We}$ also conducted sensitivity analyses that included the two high risk-of-bias trials in addition. ${ }^{44,72} \mathrm{We}$ omitted one trial from the meta-analyses altogether because it reported only that none of the SF36 domains differed significantly but did not give any precise values. ${ }^{73}$ Similarly, we excluded one trial ${ }^{51}$ and the cohort study ${ }^{56}$ in the meta-analyses because they did not report standard deviations, standard errors, or exact p-values for any of the between-group comparisons; both studies reported that MTM did not produce any significant differences in anySF-36 domain. Finally, we omitted one trial from the domain-specific meta-analyses because it reported only component scores. ${ }^{69}$ To correct for the potential inflation of Type I error attributable to multiple comparisons, we used a threshold of $\alpha /$ number of tests (i.e., domains; $0.05 / 8=0.006$ ) when evaluating statistical significance. Below, we describe our findings for each SF-36 domain, focusing on the meta-analyses of just the low to medium risk of bias trials (i.e., the smaller metaanalysis). We did not conduct a meta-analysis for the SF-36 component scores because only one trial was rated as low to medium risk of bias for these outcomes. 


\section{SF-36 Domain Scores}

Physical Functioning. Results from the low and medium risk-of-bias analysis showed no benefit for the MTM interventions (mean difference: 1.17 ; $95 \% \mathrm{CI},-3.87$ to $6.21 ; \mathrm{p}=0.65$; $\mathrm{I}^{2}=48.36$ ). Adding the two high risk-of-bias studies did not alter this conclusion (mean difference:-0.44; 95\% CI, -2.64 to $1.77 ; \mathrm{p}=0.70 ; \mathrm{I}^{2}=10.67$ ) (Appendix G-4).

Physical role functioning. Results from the low and medium risk-of-bias analysis showed no benefit for the MTM interventions (mean difference: $3.39 ; 95 \% \mathrm{CI},-0.79$ to $7.43 ; \mathrm{p}=0.11$; $\mathrm{I}^{2}=27.66$ ). Adding the two other studies did not alter this conclusion (mean difference: $0.73 ; 95 \%$ CI, -3.43 to $4.90 ; \mathrm{p}=0.73 ; \mathrm{I}^{2}=44.73$ ) (Appendix G-5).

Bodily pain. Results from the low and medium risk-of-bias analysis showed no benefit for the MTM interventions (mean difference: $3.32 ; 95 \% \mathrm{CI},-1.22$ to $8.01, \mathrm{p}=0.15 ; \mathrm{I}^{2}=0$ ). Adding the two other studies did not alter this conclusion (mean difference: $1.46 ; 95 \% \mathrm{CI},-2.79$ to 5.71 ; $\mathrm{p}=0.50 ; \mathrm{I}^{2}=81.01$ ) (Appendix G-6).

General health perceptions. Results from the low and medium risk-of-bias analysis showed no benefit for the MTM interventions (mean difference: $1.92 ; 95 \% \mathrm{CI},-0.02$ to $3.84, \mathrm{p}=0.051$; $\mathrm{I}^{2}=0$ ). With the additional studies, however, results suggested a beneficial effect of MTM interventions on general health perceptions (mean difference: 2.48 ; 95\% CI, 2.12 to 2.83 , $\mathrm{p}<0.001 ; \mathrm{I}^{2}=0$ ) (Appendix G-7).

Vitality. Results of the smaller meta-analysis showed no benefit for the MTM interventions, after correcting for multiple comparisons (mean difference: $2.80 ; 95 \% \mathrm{CI}, 0.65$ to $4.94 ; \mathrm{p}=0.01$; $\mathrm{I}^{2}=0$ ). If we had set alpha at the conventional 0.05 level, our findings would demonstrate a beneficial effect of MTM interventions. Adding the two other studies to the analysis did not change the no-benefit results for the MTM interventions, even at the more conventional alpha level (mean difference: $1.30 ; 95 \% \mathrm{CI},-0.31$ to $2.90 ; \mathrm{p}=0.11 ; \mathrm{I}^{2}=15.79$ ) (Appendix G-8).

Emotional role functioning. Results from the smaller meta-analysis showed no benefit for the MTM interventions (mean difference: $5.39 ; 95 \% \mathrm{CI},-7.24$ to $18.02 ; \mathrm{p}=0.40 ; \mathrm{I}^{2}=74.34$ ). Adding the other two studies did not alter this conclusion (mean difference: $3.44 ; 95 \% \mathrm{CI},-4.00$ to $10.88 ; \mathrm{p}=0.37 ; \mathrm{I}^{2}=78.66$ ). However, the high $\mathrm{I}^{2}$ statistic for both these meta-analyses suggested considerable heterogeneity among the studies for this particular domain (Appendix G-9).

Social role functioning. Results from the low and medium risk-of-bias analysis showed no benefits from MTM interventions (mean difference: $2.93 ; 95 \% \mathrm{CI},-0.09$ to $5.95 ; \mathrm{p}=0.057 ; \mathrm{I}^{2}=0$ ). With the additional studies, however, results suggested a beneficial effect of MTM interventions (mean difference: $0.63 ; 95 \% \mathrm{CI}, 0.29$ to $0.97 ; \mathrm{p}<0.001 ; \mathrm{I}^{2}=0$ ) (Appendix G-10).

Mental health. Results from the smaller meta-analysis showed no benefit for the MTM interventions (mean difference: $1.62 ; 95 \% \mathrm{CI},-0.36$ to $3.59 ; \mathrm{p}=0.11 ; \mathrm{I}^{2}=0$ ). Adding the two other studies did not alter this conclusion, after correcting for multiple comparisons (mean difference: 1.11 ; $95 \% \mathrm{CI}, 0.28$ to $1.94, \mathrm{p}=0.009 ; \mathrm{I}^{2}=0$ ) (Appendix G-11).

Two RCTs provided data for the SF-36 physical and mental component scores. ${ }^{44,69}$ Although we rated both trials as medium risk of bias overall, we rated one of them ${ }^{44}$ as high risk of bias for the SF-36 outcomes because of errors in the table presenting these findings. None of the between-group differences examined in either study were statistically significant with alpha set at 0.05 .

\section{SF-36 Strength of Evidence Grades}

Based on the evidence from low- and medium risk of bias trials ( 4 trials; 1,343 randomized, 1,169 analyzed) with medium study limitations, consistent results, precise, and direct evidence, 
we graded the strength of evidence for the effect of MTM interventions on seven of the eight SF36 domains and the overall physical and mental component scores as low for no benefit. For the remaining domain-vitality, we judge the evidence as imprecise and rated the evidence as insufficient.

\section{Condition-Specific Quality of Life}

Two small RCTs ${ }^{58,60}$ reported condition-specific quality-of-life outcomes (Table 38). One RCT (medium risk of bias) of just patients with diabetes compared patients in a study arm that included a clinical pharmacist intervention delivered in an ambulatory care clinic with those receiving usual medical care. ${ }^{58}$ The investigators reported no significant difference in diabetesspecific quality-of-life between the intervention and control arms at the end of 6 months. The other $\mathrm{RCT}^{59,60}$ (high risk of bias) of patients with renal disease reported a significant difference at 1 year favoring the pharmaceutical care program We graded the strength of evidence, using only the medium risk of bias trial, as insufficient (single study, direct, but imprecise) (Table 39).

Table 38. Condition-specific quality-of-life: Summary of results

\begin{tabular}{|c|c|c|c|c|}
\hline $\begin{array}{l}\text { Study } \\
\text { Design/Risk of bias }\end{array}$ & Study Arms & $\mathrm{N}$ analyzed & $\begin{array}{l}\text { Outcome and Time } \\
\text { Period }\end{array}$ & Results \\
\hline $\begin{array}{l}\text { Clifford et al., } 2002^{58} \\
\text { RCT/ Medium }\end{array}$ & $\begin{array}{l}\text { G1: Collaborative } \\
\text { pharmaceutical care } \\
\text { program } \\
\text { G2: Standard } \\
\text { outpatient care for } \\
\text { diabetes }\end{array}$ & $\begin{array}{l}\text { G1: } 48 \\
\text { G2: } 25\end{array}$ & $\begin{array}{l}\text { Diabetes Quality of Life } \\
\text { instrument } \\
\text { Scale of } 1 \text { to } 5 \\
\text { Higher scores indicate } \\
\text { greater dissatisfaction, } \\
\text { worry, or impact of } \\
\text { diabetes }\end{array}$ & $\begin{array}{l}\text { Baseline } \\
\text { G1: } 2.0(0.6) \\
\text { G2: } 1.9(0.5) \\
\text { p: NS } \\
\text { 6-month followup } \\
\text { G1: } 1.9(0.5) \\
\text { G2: } 1.9(0.4) \\
p>0.15\end{array}$ \\
\hline $\begin{array}{l}\text { Pai et al., } 2009^{59} \text {; } \\
\text { Pai et al., } 2009^{60} \\
\text { RCT/High }\end{array}$ & $\begin{array}{l}\text { G1: Pharmaceutical } \\
\text { care, consisting of } \\
\text { one-on-one care, with } \\
\text { in-depth drug therapy } \\
\text { reviews conducted by } \\
\text { a clinical pharmacist } \\
\text { G2: Standard of care, } \\
\text { consisting of brief } \\
\text { therapy reviews } \\
\text { conducted by a nurse }\end{array}$ & $\begin{array}{l}\text { Baseline } \\
\text { G1: } 61 \\
\text { G2: } 44 \\
\text { Year 1: } \\
\text { G1: } 44 \\
\text { G2: } 36 \\
\text { Year 2: } \\
\text { G1: } 24 \\
\text { G2: } 32\end{array}$ & $\begin{array}{l}\text { Renal Quality of Life } \\
\text { Profile } \\
\text { Maximum score = } 172 \\
\text { Higher scores indicate } \\
\text { worsening of HRQOL }\end{array}$ & $\begin{array}{l}\text { Total Score } \\
\text { Baseline } \\
\text { G1: } 71.9(40) \\
\text { G2: } 74.5(33.5) \\
\text { Year } 1 \\
\text { G1: } 71.4(33.6) \\
\text { G2: } 87.5(30.4) \\
\text { p<0.05 for G1 vs. G2 for Y1 } \\
\text { Year } 2 \\
\text { G1: } 56.5(32.6) \\
\text { G2: } 68.8(35.8)\end{array}$ \\
\hline
\end{tabular}

Abbreviations: HRQOL = health-related quality of life; NS = not significant; RCT= randomized controlled trial.

Table 39. Condition-specific quality of life: Strength of evidence

\begin{tabular}{|c|c|c|c|c|c|c|c|}
\hline $\begin{array}{l}\text { Study } \\
\text { Design }\end{array}$ & $\begin{array}{l}\text { Number of } \\
\text { Studies; } \\
\text { Subjects } \\
\text { (Analyzed) }\end{array}$ & $\begin{array}{l}\text { Study } \\
\text { Limitations }\end{array}$ & Consistency & Directness & Precision & $\begin{array}{l}\text { Findings and } \\
\text { Direction } \\
\text { [Magnitude] of } \\
\text { Effect }\end{array}$ & $\begin{array}{l}\text { Strength } \\
\text { of } \\
\text { Evidence }\end{array}$ \\
\hline RCT & $1 ; 73(73)$ & Medium & $\begin{array}{l}\text { Consistency } \\
\text { unknown- } \\
\text { single study }\end{array}$ & Direct & Imprecise & $\begin{array}{l}\text { Nonsignificant } \\
\text { improvement of } 0.1 \\
\text { point on a } 5 \text {-point } \\
\text { scale in the } \\
\text { intervention group } \\
\text { compared to no } \\
\text { change in the control } \\
\text { group }\end{array}$ & Insufficient \\
\hline
\end{tabular}




\section{Patient Satisfaction}

Five studies reported on various patient satisfaction measures and outcomes; four were trials (including two cluster randomized trials) ${ }^{40,51,67,69}$ and one was a cohort study. ${ }^{56}$ All compared patient satisfaction outcomes for patients receiving some form of MTM intervention and patients receiving some type of usual care (Table 40). Of these studies, we rated two RCTs low or medium risk of bias, two cluster randomized trials as medium or high risk of bias; and the cohort study as high risk of bias.

One RCT (low risk of bias) focused on patients age 65 and older who were taking five or more regularly scheduled medications. ${ }^{67}$ This study compared patients who receiving clinical pharmacist intervention delivered in an ambulatory care clinic with those receiving usual outpatient care. The study reported non-significant between-group differences for two satisfaction measures (i.e., satisfaction with general health care and satisfaction with pharmacyrelated care).

Table 40. Patient satisfaction: Summary of results

\begin{tabular}{|c|c|c|c|c|}
\hline $\begin{array}{l}\text { Study } \\
\text { Design/Risk of Bias }\end{array}$ & Study Arms & N Analyzed & Outcome and Time Period & Results \\
\hline \multirow[t]{2}{*}{$\begin{array}{l}\text { Hanlon et al., } 1996^{67} \\
\text { RCT/Low }\end{array}$} & \multirow[t]{2}{*}{$\begin{array}{l}\text { G1: Usual care at } \\
\text { outpatient clinic, plus } \\
\text { clinical pharmacist } \\
\text { care. } \\
\text { G2: Usual care at } \\
\text { outpatient clinic }\end{array}$} & $\begin{array}{l}\text { G1: } 86 \\
\text { G2: } 83\end{array}$ & $\begin{array}{l}\text { General health care } \\
\text { satisfaction at 12-month } \\
\text { followup } \\
\text { (Higher scores indicate greater } \\
\text { dissatisfaction) }\end{array}$ & $\begin{array}{l}\text { G1: } 1.5(0.7) \\
G 2: 1.6(0.8) \\
p=0.70\end{array}$ \\
\hline & & & $\begin{array}{l}\text { Pharmacy-related health care } \\
\text { satisfaction at 12-month } \\
\text { followup } \\
\text { (Higher scores indicate greater } \\
\text { dissatisfaction) }\end{array}$ & $\begin{array}{l}G 1: 5.2(1.5) \\
G 2: 5.4(1.7) \\
p=0.52\end{array}$ \\
\hline $\begin{array}{l}\text { Malone et al., } 2000^{40} ; \\
\text { Ellis et al., } 2000^{41} ; \\
\text { Malone et al, } 2001^{43} ; \\
\text { Ellis et al., } 2000^{42} \\
\text { RCT/Medium }\end{array}$ & $\begin{array}{l}\text { G1: Pharmaceutical } \\
\text { care provided by } \\
\text { clinical pharmacists } \\
\text { within ambulatory VA } \\
\text { clinics } \\
\text { G2: Usual care (i.e. } \\
\text { no pharmaceutical } \\
\text { care) }\end{array}$ & $\begin{array}{l}\text { G1: } 447 \\
\text { G2: } 484\end{array}$ & $\begin{array}{l}\text { Patient satisfaction with } \\
\text { primary health care provider } \\
\text { (Higher scores indicate greater } \\
\text { satisfaction) }\end{array}$ & $\begin{array}{l}\text { Time } 1 \\
\text { G1: } 51.9(7.5) \\
\text { G2:51.9(7.5) } \\
\text { Time } 2 \\
\text { G1: } 51.7(7.3) \\
\text { G2: } 51.9(7.5) \\
p=N S\end{array}$ \\
\hline $\begin{array}{l}\text { Bernsten et al., } \\
2001^{51} \text {; } \\
\text { Sturgess et al., } 2003^{52} \\
\text { RCT, Cluster- } \\
\text { Randomized/High }\end{array}$ & $\begin{array}{l}\text { G1: Structured } \\
\text { community } \\
\text { pharmacy-based } \\
\text { pharmaceutical care } \\
\text { program } \\
\text { G2: Usual community } \\
\text { pharmacy services }\end{array}$ & $\begin{array}{l}\text { Baseline } \\
\text { G1: } 1,290 \\
\text { G2: } 1,164 \\
6 \text { months } \\
\text { G1: } 1,024 \\
\text { G2: } 953 \\
\text { 12 months } \\
\text { G1: } 863 \\
\text { G2: } 764 \\
\text { 18 months } \\
\text { G1: } 704 \\
\text { G2: } 636\end{array}$ & $\begin{array}{l}\text { Percentage rating pharmacy } \\
\text { services provided as } \\
\text { "excellent" }\end{array}$ & $\begin{array}{l}\text { Baseline } \\
\text { G1: } 66.2 \\
\text { G2: } 68.2 \\
\text { p NR } \\
\text { 6 months } \\
\text { G1: } 72.8 \\
\text { G2: } 63.7 \\
\text { p <0.05 } \\
12 \text { months } \\
\text { G1: } 73.4 \\
\text { G2: } 71.2 \\
\text { p NR } \\
\text { 18 months } \\
\text { G1: } 73.8 \\
\text { G2: } 64.6 \\
\text { p<0.05 }\end{array}$ \\
\hline
\end{tabular}


Table 40. Patient satisfaction: Summary of results (continued)

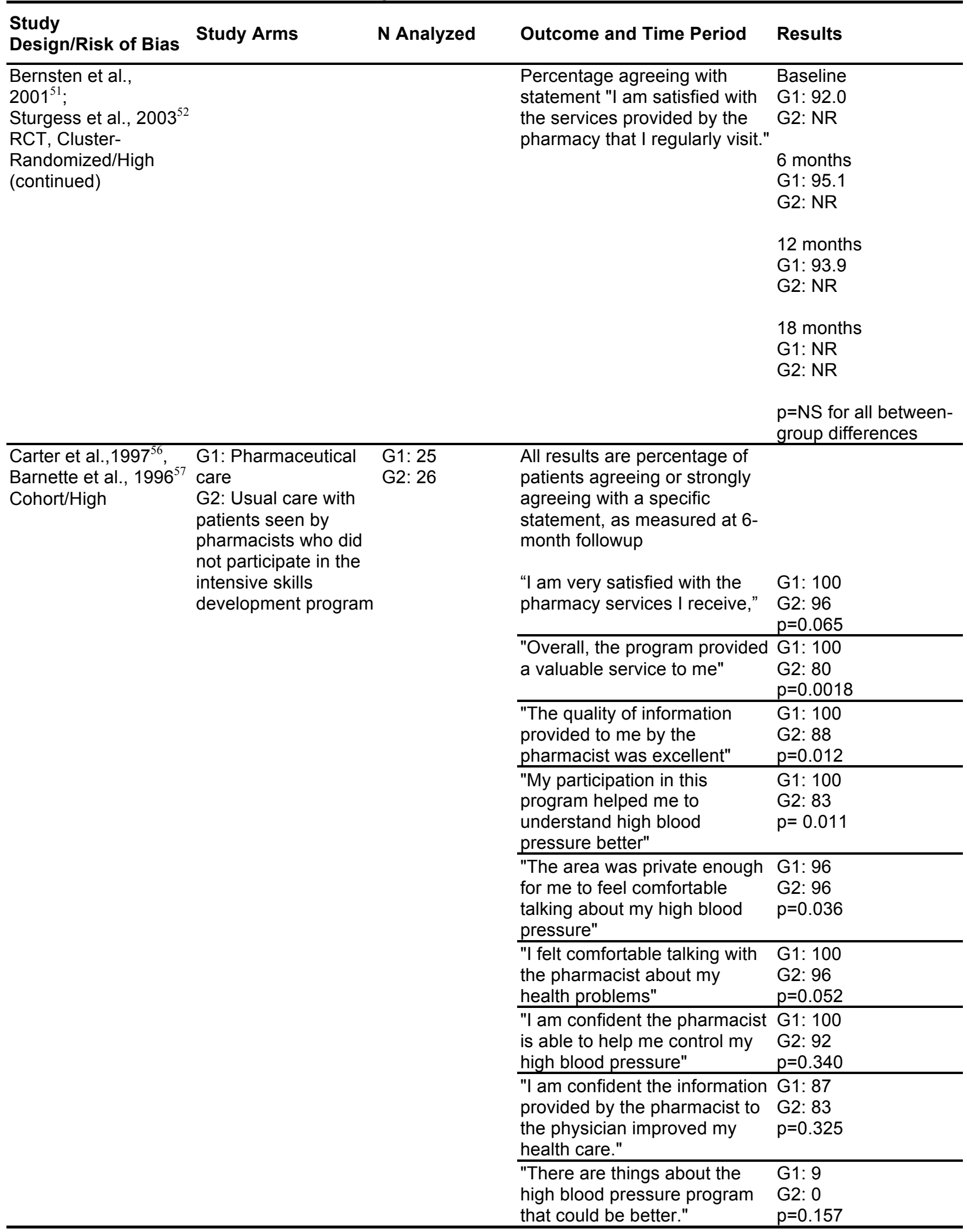


Table 40. Patient satisfaction: Summary of results (continued)

\begin{tabular}{|c|c|c|c|c|}
\hline $\begin{array}{l}\text { Study } \\
\text { Design/Risk of Bias }\end{array}$ & Study Arms & N Analyzed & Outcome and Time Period & Results \\
\hline \multirow{3}{*}{$\begin{array}{l}\text { Carter et al.,1997 } \\
\text { Barnette et al., } \\
1996^{57} \\
\text { Cohort/High } \\
\text { (continued) }\end{array}$} & & & $\begin{array}{l}\text { "I am very willing to continue } \\
\text { to see the pharmacist for help } \\
\text { with my high blood pressure } \\
\text { control." }\end{array}$ & $\begin{array}{l}\text { G1: } 95 \\
\text { G2: } 88 \\
p=0.459\end{array}$ \\
\hline & & & $\begin{array}{l}\text { "I think the pharmacist should } \\
\text { provide this type of service for } \\
\text { everyone." }\end{array}$ & $\begin{array}{l}\text { G1: } 77 \\
G 2: 75 \\
p=0.890\end{array}$ \\
\hline & & & $\begin{array}{l}\text { "I think the pharmacist should } \\
\text { be paid for this type of } \\
\text { service." }\end{array}$ & $\begin{array}{l}G 1: 91 \\
G 2: 82 \\
p=0.379\end{array}$ \\
\hline \multirow[t]{9}{*}{$\begin{array}{l}\text { Volume et al., } 2001^{69} \text {; } \\
\text { Kassam et al., } 2001^{70} \\
\text { RCT-Cluster } \\
\text { Randomized/Medium }\end{array}$} & $\begin{array}{l}\text { G1: Comprehensive } \\
\text { pharmaceutical } \\
\text { care services } \\
\text { G2: Traditional } \\
\text { pharmacy care }\end{array}$ & $\begin{array}{l}\text { Time 1: } \\
\text { N=363 } \\
\text { G1: } 159 \\
\text { G2: } 204 \\
\text { Time 2: } \\
\text { N=317 } \\
\text { G1: NR } \\
\text { G2: NR } \\
\text { Time 3: } \\
\text { N=292 } \\
\text { G1: NR } \\
\text { G2: NR }\end{array}$ & $\begin{array}{l}\text { General satisfaction } \\
\text { (Higher numbers reflect } \\
\text { greater dissatisfaction) }\end{array}$ & $\begin{array}{l}\text { Time 1: } \\
\text { G1: } 1.59(0.77) \\
\text { G2: } 1.56(0.73 \\
\text { Time 2: } \\
\text { G1: } 1.51(0.84) \\
\text { G2: } 1.57(0.72) \\
\text { Time 3: } \\
\text { G1: } 1.53(0.77) \\
\text { G2: } 1.62(0.88) \\
\text { p=NS for all between- } \\
\text { group differences }\end{array}$ \\
\hline & & & $\begin{array}{l}\text { Interpersonal skills } \\
\text { (Higher numbers reflect } \\
\text { greater dissatisfaction) }\end{array}$ & $\begin{array}{l}\text { Time 1: } \\
\text { G1: } 1.36(0.48) \\
\text { G2: } 1.37(0.53)\end{array}$ \\
\hline & & & & $\begin{array}{l}\text { Time 2: } \\
\text { G1: } 1.37(0.59) \\
\text { G2: } 1.35(0.57)\end{array}$ \\
\hline & & & & $\begin{array}{l}\text { Time } 3: \\
\text { G1: } 1.31(0.50) \\
\text { G2: } 1.45(0.72)\end{array}$ \\
\hline & & & & $\begin{array}{l}\mathrm{p}=\mathrm{NS} \text { for all between- } \\
\text { group differences }\end{array}$ \\
\hline & & & $\begin{array}{l}\text { Evaluation and goal setting } \\
\text { (Higher numbers reflect } \\
\text { greater dissatisfaction) }\end{array}$ & $\begin{array}{l}\text { Time 1: } \\
\text { G1: } 2.58(1.12) \\
\text { G2: } 2.74(1.09)\end{array}$ \\
\hline & & & & $\begin{array}{l}\text { Time } 2: \\
\text { G1: } 2.46(0.98) \\
\text { G2: } 2.98(1.24)\end{array}$ \\
\hline & & & & $\begin{array}{l}\text { Time 3: } \\
\text { G1: } 2.49(1.10) \\
\text { G2: } 2.90(1.08)\end{array}$ \\
\hline & & & & $\begin{array}{l}\mathrm{p}<0.05 \text { for between- } \\
\text { group differences in } \\
\text { score changes from } \\
\text { Time } 1 \text { to Time } 2 \text { and } \\
\text { Time } 1 \text { to Time } 3\end{array}$ \\
\hline
\end{tabular}


Table 40. Patient satisfaction: Summary of results (continued)

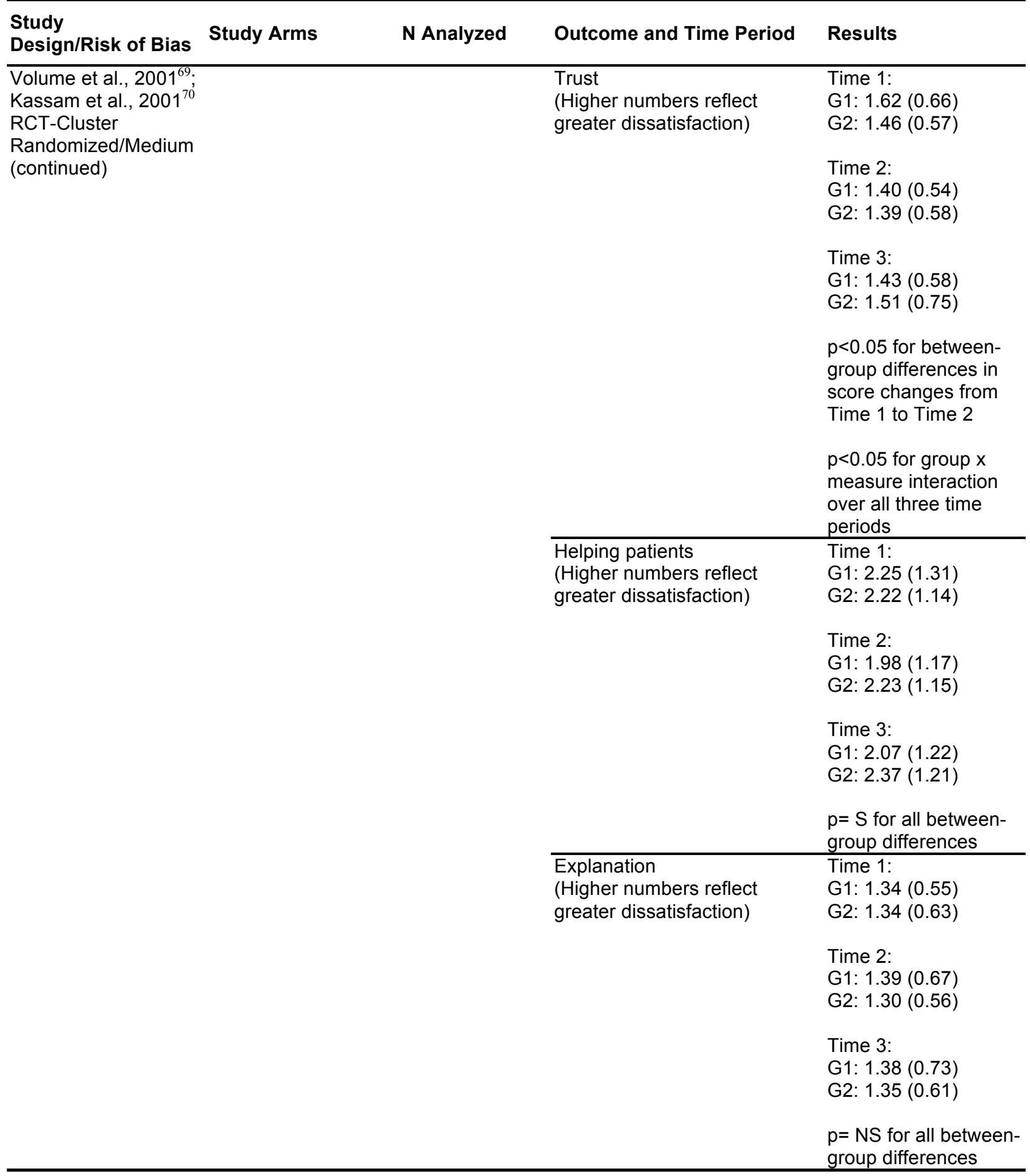


Table 40. Patient satisfaction: Summary of results (continued)

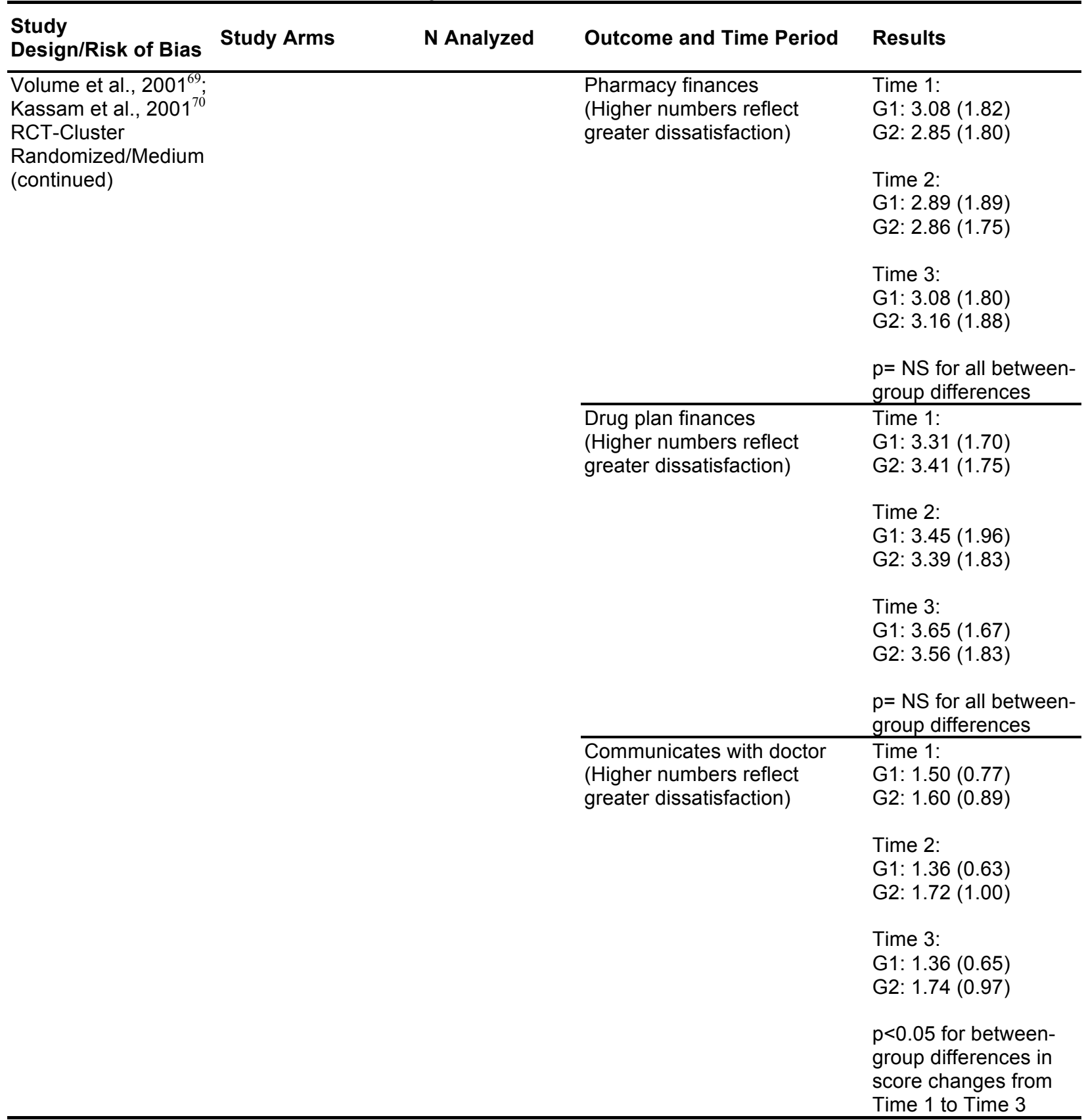

Abbreviations: $\mathrm{NR}=$ not reported; $\mathrm{NS}=$ not significant; $\mathrm{RCT}=$ randomized controlled trial

The other RCT (medium risk of bias) focused on patients at high risk of experiencing a drugrelated problem. ${ }^{43}$ This study compared patients receiving a clinical pharmacist intervention delivered in an ambulatory care clinic with those in usual medical care. The study reported a nonsignificant between-group difference on a measure assessing patient satisfaction with the primary care provider.

One cluster trial (medium risk of bias) focused on patients ages 65 and older who were taking three or more medications concurrently. ${ }^{69}$ This study evaluated a community pharmacy-based intervention and assessed nine different measures of satisfaction at baseline, at 6-7 months 
following baseline, and at 12-13 months following baseline. This study reported statistically significant between-group change in a measure labeled, Evaluation and Goal Setting. This measure included six items assessing the extent to which the pharmacist involved the patient in setting therapeutic goals. However, none of the items asked directly about patient satisfaction with the goal setting process. This study also reported a statistically significant between-group change from baseline to the 12-13 month follow-up on a measure labeled, Communicates with Doctor. This measure included two items asking about whether the patient's pharmacist and doctor work together to determine the most appropriate therapy for the patient. Neither item asked directly about patient satisfaction with the level of pharmacist-doctor communication. Finally, this study reported a statistically significant between-group change in a measure labeled, Trust. At baseline, patients in the intervention group reported lower trust in their pharmacist. Over the course of the study, their level of trust improved to the level reported by patients in the control group at baseline, accounting for the between group differences reported. The study reported no statistically significant between-group changes on the remaining six satisfaction measures, including a measure that directly assessed overall satisfaction with pharmacy services.

When grading strength of evidence, we did not consider the results from the remaining cluster trial $\mathrm{RCT}^{51}$ and the cohort study ${ }^{56}$ because they were rated as high risk of bias. We also did not consider findings from three other studies (one $\mathrm{RCT},{ }^{58}$ one nonrandomized clinical trial $^{38}$ and one cohort study ${ }^{35}$ ) because they assessed only changes in satisfaction over time in the intervention arm and did not make any between-group comparisons. Overall, we concluded that the strength of evidence for MTM interventions with respect to patient satisfaction was low for no benefit (Table 41).

Table 41. Patient satisfaction: Strength of evidence

\begin{tabular}{|c|c|c|c|c|c|c|c|}
\hline $\begin{array}{l}\text { Study } \\
\text { Design }\end{array}$ & $\begin{array}{l}\text { Number of } \\
\text { Studies; } \\
\text { Subjects } \\
\text { (Analyzed) }\end{array}$ & $\begin{array}{l}\text { Study } \\
\text { Limitations }\end{array}$ & Consistency & Directness & Precision & $\begin{array}{l}\text { Findings and Direction } \\
\text { [Magnitude] of Effect }\end{array}$ & $\begin{array}{l}\text { Strength of } \\
\text { Evidence }\end{array}$ \\
\hline RCT & $\begin{array}{l}3 ; 1,625 \\
(1,543)\end{array}$ & Medium & Consistent & Direct & Precise & $\begin{array}{l}17 \text { of } 21 \text { between- group } \\
\text { differences small and not } \\
\text { statistically significant; } 4 \\
\text { statistically significant } \\
\text { differences ranged in } \\
\text { magnitude from }-0.15 \text { to - } \\
0.36 \text {, favoring MTM }\end{array}$ & $\begin{array}{l}\text { Low for no } \\
\text { benefit }\end{array}$ \\
\hline
\end{tabular}

Abbreviations: MTM = medication therapy management; RCT = randomized controlled trial; SD = standard deviation

\section{Resource Utilization}

\section{Key Points: Resource Utilization}

- Effective MTM interventions might plausibly lead to either an increase or a decrease in resource utilization, depending on the baseline status of the patient and intended goals of the intervention. When studies did not present a clear hypothesis or expected direction of effect, we were unable to interpret changes in resource utilization outcomes as either a benefit or a harm of MTM interventions.

- Evidence was insufficient to assess the effectiveness of MTM in changing numerous measures of use of health care resources. These included the number of medications, use of generic medications for telephone-based MTM, and several different measures of medication costs; outpatient visits and costs; laboratory tests and costs; emergency 
department visits and costs; and risk of hospitalization, hospital costs, and length of hospital stay.

- Community pharmacy-based MTM interventions increase the weighted generic substitution ratio when compared with educational mailings, but the effect size is low (one cohort study, high study limitations, low strength of evidence of benefit).

- MTM interventions among patients with a variety of clinical conditions do not change in the number of hospitalizations when compared with usual care (three trials, medium study limitations, consistent, direct, precise, low strength of evidence of no benefit).

- MTM interventions in the home reduce the rate of hospitalizations for patients with heart failure (one cohort, high study limitations, direct, precise, low strength of evidence of benefit).

\section{Detailed Synthesis: Resource Utilization}

\section{Number of Medications}

Understanding whether a change in the number of medications taken following an MTM intervention is a measure of appropriate resource utilization requires knowledge of the goal of drug therapy. A decrease in the number of medications can represent regimen simplification and resolution of therapeutic duplication; thus, it can be interpreted as a measure of appropriate resource utilization. The converse - that is, an increase in number of medications - cannot, however, be interpreted as a measure of inappropriate resource use. An increase in number of medications can in fact represent appropriate use of resources when it resulted from identifying and resolving an inadequate drug regimen.

Numerous studies provided information on the number of medications at followup in intervention and control arms or on the change in number of medications between baseline and followup. . $^{3,38-40,44,46,48,51,59,60,65,67-69}$ Only one study, however, offered any context to interpret the results in the context of benefits and harms. ${ }^{63}$ In this cohort study (high risk of bias), the investigators found that those eligible for the intervention who received MTM services had a significant decrease in the prevalence of high-risk medication use that was not seen in the control group of patients eligible for the intervention but who did not receive it (-10.8 percentage points $[p<0.05$ ] versus -1.4 percentage points [no significant change]). The investigators noted, however, that the intervention arm had a higher prevalence of use of high- risk drugs at baseline and that pharmacists may have targeted these patients selectively for the intervention, suggesting confounding. Based on study limitations and unknown consistency, we graded the body of evidence as insufficient to evaluate the effect of MTM interventions on the number of medications taken (Table 42).

Table 42. Number of medications: Strength of evidence

\begin{tabular}{|c|c|c|c|c|c|c|c|}
\hline $\begin{array}{l}\text { Study } \\
\text { Design }\end{array}$ & $\begin{array}{l}\text { Number of } \\
\text { Studies; } \\
\text { Subjects } \\
\text { (Analyzed) }\end{array}$ & $\begin{array}{l}\text { Study } \\
\text { Limitations }\end{array}$ & Consistency & Directness & Precision & $\begin{array}{l}\text { Findings and } \\
\text { Direction } \\
\text { [Magnitude] of } \\
\text { Effect }\end{array}$ & $\begin{array}{l}\text { Strength } \\
\text { of } \\
\text { Evidence }\end{array}$ \\
\hline Cohort & $\begin{array}{l}1 ; 2,211 \\
(2211)\end{array}$ & High & $\begin{array}{l}\text { Consistency } \\
\text { unknown- } \\
\text { single study }\end{array}$ & Direct & Precise & $\begin{array}{l}-10.8 \\
\text { percentage } \\
\text { points }(\mathrm{p} 0<.05) \\
\text { vs. }-1.4 \\
\text { percentage } \\
\text { points (not } \\
\text { significant) }\end{array}$ & Insufficient \\
\hline
\end{tabular}




\section{Use of Generic Medications}

Two cohort studies examined the use of generic medications (Table 43); both studies evaluated telephone-based $\mathrm{MTM}^{35,46}$ and one also compared community pharmacy-based MTM with educational mailings. ${ }^{46}$ We assessed both studies as high risk of bias owing to lack of adjustment for potential confounding from study design (intervention refusers versus acceptors) ${ }^{35}$ or lack of capacity of pharmacists or inability to reach patients. ${ }^{46}$

Table 43. Use of generic medications: Summary of results

\begin{tabular}{|c|c|c|c|c|}
\hline $\begin{array}{l}\text { Study } \\
\text { Design/Risk of Bias }\end{array}$ & Study Arms & $\mathbf{N}$ analyzed & $\begin{array}{l}\text { Outcome and Time } \\
\text { Period }\end{array}$ & Results \\
\hline $\begin{array}{l}\text { Pindolia et al., } 2009^{35} \\
\text { Cohort/High }\end{array}$ & $\begin{array}{l}\text { G1: Telephone based } \\
\text { MTM program } \\
\text { (acceptors) } \\
\text { G2: Usual medical } \\
\text { care (refusers) }\end{array}$ & $\begin{array}{l}\text { G1: } 292 \\
\text { G2: } 1081\end{array}$ & $\begin{array}{l}\text { Increase in the overall } \\
\text { use of generic drugs }\end{array}$ & $\begin{array}{l}\mathrm{G} 1: 6 \% \\
\mathrm{G} 2: 3 \% \\
\text { p not calculated because } \\
\text { baseline percentages not } \\
\text { provided }\end{array}$ \\
\hline $\begin{array}{l}\text { Winston et al., } \\
2009^{46} \\
\text { Cohort/High }\end{array}$ & $\begin{array}{l}\text { G1: Community } \\
\text { pharmacy MTM } \\
\text { G2: Pharmacist-staffed } \\
\text { call center-based MTM } \\
\text { G3: Educational } \\
\text { mailings }\end{array}$ & $\begin{array}{l}\text { G1: } 21,336 \\
\text { G2: } 3,436 \\
\text { G3: } 49,021\end{array}$ & $\begin{array}{l}\text { Weighted generic } \\
\text { substitution ratio: } 30- \\
\text { day equivalent claims } \\
\text { divided by total number } \\
\text { of claims }\end{array}$ & $\begin{array}{l}\text { Calculated mean differences } \\
\text { for } \mathrm{G} 1 \text { vs. } \mathrm{G} 3: 1.2(95 \% \mathrm{Cl} \text { : } \\
0.724 \text { to } 1.676 ; p<0.001) \\
\text { Calculated mean difference for } \\
\text { G2 vs. G3: } 0.80(95 \% \mathrm{Cl} \text { - } \\
0.246 \text { to } 1.846 ; p=0.134)\end{array}$ \\
\hline
\end{tabular}

Abbreviations: $\mathrm{CI}=$ confidence interval; $\mathrm{G}=$ group; $\mathrm{MTM}=$ medication therapy management; $\mathrm{RCT}=$ randomized controlled trial.

With respect to telephone-based MTM services, we graded the body of evidence as insufficient (high study limitations, inconsistent, and imprecise) to evaluate their effect on number of generic medications (Table 44). Regarding the effect of pharmacy-based MTM intervention on the generic substitution ratio (Table 45), we graded the strength of evidence as low; the evidence was direct and precise, but a small standardized mean difference (0.04), study limitations and uncontrolled confounding limited our confidence that the estimate of effect is close to the true effect for this outcome.

Table 44. Use of generics for telephone-based MTM versus usual care or educational mailings: Strength of evidence

\begin{tabular}{|c|c|c|c|c|c|c|c|}
\hline $\begin{array}{l}\text { Study } \\
\text { Design }\end{array}$ & $\begin{array}{l}\text { Number of } \\
\text { Studies; } \\
\text { Subjects } \\
\text { (Analyzed) }\end{array}$ & $\begin{array}{l}\text { Study } \\
\text { Limitations }\end{array}$ & Consistency & Directness & Precision & $\begin{array}{l}\text { Findings and } \\
\text { Direction } \\
\text { [Magnitude] of } \\
\text { Effect }\end{array}$ & $\begin{array}{l}\text { Strength of } \\
\text { Evidence }\end{array}$ \\
\hline Cohort & $\begin{array}{l}2 ; 97,124 \\
(75,166)\end{array}$ & High & Inconsistent & Direct & Imprecise & $\begin{array}{l}\text { Calculated mean } \\
\text { difference from one } \\
\text { study: } 0.80(95 \% \\
\mathrm{Cl}:-0.246 \text { to } 1.846 ; \\
\mathrm{p}=0.134)\end{array}$ & Insufficient \\
\hline
\end{tabular}

Abbreviations: $\mathrm{CI}=$ confidence interval; $\mathrm{NA}=$ not applicable; $\mathrm{SMD}=$ standardized mean difference. 
Table 45. Use of generics for community pharmacy-based MTM versus educational mailings: Strength of evidence

\begin{tabular}{|c|c|c|c|c|c|c|c|}
\hline $\begin{array}{l}\text { Study } \\
\text { design }\end{array}$ & $\begin{array}{l}\text { Number of } \\
\text { Studies; } \\
\text { Subjects } \\
\text { (Analyzed) }\end{array}$ & $\begin{array}{l}\text { Study } \\
\text { Limitations }\end{array}$ & Consistency & Directness & Precision & $\begin{array}{l}\text { Findings and } \\
\text { Direction } \\
\text { [Magnitude] of } \\
\text { Effect }\end{array}$ & $\begin{array}{l}\text { Strength of } \\
\text { Evidence }\end{array}$ \\
\hline Cohort & $\begin{array}{l}1 ; 95,736 \\
(73,793)\end{array}$ & High & $\begin{array}{l}\text { Consistency } \\
\text { unknown- } \\
\text { single study }\end{array}$ & Direct & Precise & $\begin{array}{l}\text { Calculated mean } \\
\text { difference: } 1.2 \\
\text { (95\% Cl: } 0.724 \\
\text { to } 1.676 \text { to; } \\
\text { p<0.001) } \\
\text { (Standardized } \\
\text { mean difference: } \\
0.04 \text { ) }\end{array}$ & $\begin{array}{l}\text { Low for } \\
\text { benefit for the } \\
\text { community } \\
\text { pharmacy } \\
\text { arm }\end{array}$ \\
\hline
\end{tabular}

Abbreviations: $\mathrm{CI}=$ confidence interval; $\mathrm{SMD}=$ standardized mean difference.

\section{Medication Costs: Overview}

Eighteen studies reported data on costs of prescription medications (Table 46). We use the same language as the authors in describing their measures; they varied in study design and risk of bias and used a wide range of measures that cannot all be meaningfully combined. We categorized these outcomes in four groups; patient out-of-pocket costs (copayments), health plan costs, combined expenditures by patients and insurers, and combinations of medications and other costs. Table 46 lists studies in order by outcome category (or outcomes in some cases) and then alphabetically by author name. Later sections offer a detailed synthesis by these four categories of costs and describe the relevant studies in more detail. We were unable to categorize one high-risk-of-bias cohort study ${ }^{56,57}$ because it did not offer sufficient information on how "charges" were calculated. We note that several studies use the term "costs" although the specific measure used may not reflect true costs if they do not account for profits or subsidies. We use the same language as the authors in describing their measures.

Table 46. Measures used in studies of costs of medications

\begin{tabular}{|c|c|c|c|c|}
\hline Study & $\begin{array}{l}\text { Prescription Costs } \\
\text { to Patients }\end{array}$ & $\begin{array}{l}\text { Total Expenditures } \\
\text { on Medications by } \\
\text { Health Plan }\end{array}$ & $\begin{array}{l}\text { Total Outlays on } \\
\text { Medication }\end{array}$ & $\begin{array}{l}\text { Medication and Other } \\
\text { Costs Combined }\end{array}$ \\
\hline $\begin{array}{l}\text { Christensen et } \\
\text { al., } 2007^{38}\end{array}$ & $\begin{array}{l}\text { Difference in patient } \\
\text { copayment for } \\
\text { prescriptions over } 6 \\
\text { months }\end{array}$ & $\begin{array}{l}\text { Difference in insurer } \\
\text { payment for } \\
\text { prescriptions over } 6 \\
\text { months }\end{array}$ & & \\
\hline $\begin{array}{l}\text { Fox et al., } \\
2009^{31}\end{array}$ & $\begin{array}{l}\text { Mean Medicare Part } \\
\text { D copayment costs } \\
\text { per patient per month } \\
\text { Mean Medicare Part } \\
\text { D and non- Part D } \\
\text { copayments }\end{array}$ & $\mathrm{NA}$ & $\begin{array}{l}\text { Mean Medicare Part D } \\
\text { drug costs (total Medicare } \\
\text { Part D drug costs (patient } \\
\text { copays + insurance plan } \\
\text { medication costs + } \\
\text { dispensing fees) }\end{array}$ & NA \\
\hline $\begin{array}{l}\text { Pindolia et al., } \\
2009^{35}\end{array}$ & $\begin{array}{l}\text { Out-of-pocket } \\
\text { prescription costs per } \\
\text { health plan member }\end{array}$ & NA & $\begin{array}{l}\text { Total prescription drug } \\
\text { costs per health plan } \\
\text { member }(2006)\end{array}$ & NA \\
\hline $\begin{array}{l}\text { Chrischilles et } \\
\text { al., } 2004^{63}\end{array}$ & NA & $\begin{array}{l}\text { Mean amount billed } \\
\text { per patient for active } \\
\text { drugs (based on } \\
\text { Medicaid claims) }\end{array}$ & NA & NA \\
\hline
\end{tabular}


Table 46. Measures used in studies of costs of medications (continued)

\begin{tabular}{|c|c|c|c|c|}
\hline Study & $\begin{array}{l}\text { Prescription Costs } \\
\text { to Patients }\end{array}$ & $\begin{array}{l}\text { Total Expenditures } \\
\text { on Medications by } \\
\text { Health Plan }\end{array}$ & $\begin{array}{l}\text { Total Outlays on } \\
\text { Medication }\end{array}$ & $\begin{array}{l}\text { Medication and Other } \\
\text { Costs Combined }\end{array}$ \\
\hline $\begin{array}{l}\text { Jameson et al., } \\
1995^{39}\end{array}$ & NA & $\begin{array}{l}\text { Cost of prescription } \\
\text { drugs over } 6 \text { months, } \\
\text { based on maximum } \\
\text { allowable cost for } \\
\text { Medicaid } \\
\text { reimbursement }\end{array}$ & NA & NA \\
\hline $\begin{array}{l}\text { Moczygemba et } \\
\text { al., } 2011^{33} \\
\text { Moczygemba et } \\
\text { al., } 2008^{34}\end{array}$ & NA & $\begin{array}{l}\text { Total Part D drug } \\
\text { costs (based on } \\
\text { prescription claim } \\
\text { records, excludes } \\
\text { non-Part D drug costs }\end{array}$ & NA & \\
\hline $\begin{array}{l}\text { Sellors et al., } \\
2003^{44}\end{array}$ & NA & $\begin{array}{l}\text { Mean daily } \\
\text { medication costs to } \\
\text { the Ontario Drug } \\
\text { Benefit Program }\end{array}$ & $\begin{array}{l}\text { Mean daily medication } \\
\text { costs }\end{array}$ & $\begin{array}{l}\text { Mean cost of health care } \\
\text { resources per senior (total } \\
\text { costs, including all hospital } \\
\text { stays) }\end{array}$ \\
\hline $\begin{array}{l}\text { Krska et al., } \\
2001^{73}\end{array}$ & NA & NA & $\begin{array}{l}\text { Average monthly costs of } \\
\text { prescribed medication per } \\
\text { patient (excluding costs of } \\
\text { prescribed medicines not } \\
\text { taken) }\end{array}$ & NA \\
\hline $\begin{array}{l}\text { Malone et al., } \\
2000^{40} \text {; } \\
\text { Ellis et al., } \\
2000^{41} \text {; } \\
\text { Malone et al., } \\
2001^{43} \text {; } \\
\text { Ellis et al., } \\
2000^{42}\end{array}$ & NA & NA & $\begin{array}{l}\text { Mean drug costs } \\
\text { (calculated from Denver } \\
\text { VAMC pharmacy } \\
\text { department, individual } \\
\text { sites, or the VA Pharmacy } \\
\text { Benefits Management } \\
\text { group) }\end{array}$ & NA \\
\hline $\begin{array}{l}\text { Pai, } 2009^{59} \\
\text { Pai, } 2009^{60}\end{array}$ & NA & NA & $\begin{array}{l}\text { Mean drug costs } \\
\text { (calculated from average } \\
\text { wholesale price) }\end{array}$ & NA \\
\hline $\begin{array}{l}\text { Staresinic et al., } \\
2007^{36}\end{array}$ & NA & NA & $\begin{array}{l}\text { Total prescription cost per } \\
\text { MTM program beneficiary } \\
\text { per month ([gross drug } \\
\text { cost=ingredient cost paid } \\
+ \text { dispensing fee + sales } \\
\text { tax]/member months in } \\
\text { Part D contract) }\end{array}$ & NA \\
\hline $\begin{array}{l}\text { Welch et al., } \\
2009^{37}\end{array}$ & NA & NA & $\begin{array}{l}\text { Mean medication costs } \\
\text { per day (from data on } \\
\text { study beneficiaries' } \\
\text { purchases of ambulatory } \\
\text { prescription medications) }\end{array}$ & NA \\
\hline Williams, $2004^{74}$ & NA & NA & $\begin{array}{l}\text { Average monthly } \\
\text { wholesale price of } \\
\text { prescription and non- } \\
\text { prescription drugs }\end{array}$ & NA \\
\hline $\begin{array}{l}\text { Winston et al., } \\
2009^{46}\end{array}$ & NA & NA & $\begin{array}{l}\text { Mean drug cost per } \\
\text { patient per month (based } \\
\text { drug claims processing } \\
\text { data, total allowed } \\
\text { charges, including } \\
\text { ingredient cost paid, } \\
\text { dispensing fee, and sales } \\
\text { tax, before subtracting } \\
\text { any patient cost-sharing } \\
\text { amounts) }\end{array}$ & NA \\
\hline
\end{tabular}


Table 46. Measures used in studies of costs of medications (continued)

\begin{tabular}{|c|c|c|c|c|}
\hline Study & $\begin{array}{l}\text { Prescription Costs } \\
\text { to Patients }\end{array}$ & $\begin{array}{l}\text { Total Expenditures } \\
\text { on Medications by } \\
\text { Health Plan }\end{array}$ & $\begin{array}{l}\text { Total Outlays on } \\
\text { Medication }\end{array}$ & $\begin{array}{l}\text { Medication and Other } \\
\text { Costs Combined }\end{array}$ \\
\hline $\begin{array}{l}\text { Bernsten et al., } \\
2001^{51} \text {; } \\
\text { Sturgess et al., } \\
2003^{52}\end{array}$ & NA & NA & NA & $\begin{array}{l}\text { Mean total cost per patient } \\
\text { including (1) cost } \\
\text { associated } \\
\text { with additional time spent } \\
\text { by pharmacists; (2) cost } \\
\text { associated with contacts } \\
\text { with GPs, specialists and } \\
\text { nurses; and (3) cost of } \\
\text { hospitalizations and drugs }\end{array}$ \\
\hline $\begin{array}{l}\text { Fischer et al., } \\
2002^{65}\end{array}$ & NA & NA & NA & $\begin{array}{l}\text { Change in total charges for } \\
\text { inpatient care, outpatient } \\
\text { care, and pharmacy } \\
\text { charges }\end{array}$ \\
\hline $\begin{array}{l}\text { Triller et al., } \\
2007^{54}\end{array}$ & NA & NA & NA & $\begin{array}{l}\text { Aggregate health system } \\
\text { costs }\end{array}$ \\
\hline
\end{tabular}

Home care agency costs

Abbreviations: GPs = general practitioners; MTM = medication therapy management; NA = not applicable; VA = Veterans' Administration; VAMC $=$ Veterans' Administration Medical Center

\section{Medication Costs: Patient Copayments}

Three nonrandomized studies (one nonrandomized controlled trial [NRCT] of medium risk of bias ${ }^{38}$ and two cohort studies of high risk of bias ${ }^{31,35}$ ) studies compared the copayments for patients who refused MTM with patients who accepted MTM enrollment. These studies provided inconsistent evidence that patient medication co-payments increased following MTM. Table 47 documents the main findings; results are denominated in US dollars (\$) unless specifically identified as Canadian dollars or as another currency. We calculated mean differences between groups when the original authors did not provide those data; all currencies are rounded to two decimals (i.e., for US currency, cents).

The NRCT compared patients in the MTM arm with controls within and outside the intervention county; the control arms had declines in copayments and the MTM had increases in copayments. The two cohort studies had inconsistent and imprecise estimates of effect; one study showed an increase in copayments for the MTM arm and a decline for the control arm, ${ }^{35}$ and the other reported a smaller increase in the MTM arm than in the control arm. ${ }^{31}$ None of these studies explained whether the increase in copayment was a result of an appropriate change in medication therapy or the desired effect of the intervention. Although the results were precise in the NRCT and suggested an increase in medication copayments following MTM, the lack of directness in interpreting this outcome as a measure of appropriate resource utilization and the absence of other low and medium risk-of-bias studies to assess consistency of findings suggests insufficient evidence to judge the effect of MTM interventions on patient medication co-payment (Table 48). 
Table 47. Patient copayments: Summary of results

\begin{tabular}{|c|c|c|c|c|}
\hline $\begin{array}{l}\text { Study } \\
\text { Design/Risk } \\
\text { of Bias }\end{array}$ & Study Arms & N Analyzed & $\begin{array}{l}\text { Prescription Costs to } \\
\text { Patients }\end{array}$ & Results \\
\hline $\begin{array}{l}\text { Christensen et } \\
\text { al., } 2007^{38} \\
\text { NRCT/Medium }\end{array}$ & $\begin{array}{l}\text { G1: Patients receiving } \\
\text { pharmacist-provided-MTM } \\
\text { services } \\
\text { G2: Patients from same } \\
\text { counties as G1 who did not } \\
\text { receive intervention (control } \\
\text { group 1) } \\
\text { G3: Patients from a different } \\
\text { county than G1 who did not } \\
\text { receive intervention (control } \\
\text { group 2) }\end{array}$ & $\begin{array}{l}\text { G1: } 67 \\
\text { G2: } 669 \\
\text { G3: } 870\end{array}$ & $\begin{array}{l}\text { Mean difference in patient } \\
\text { copayment for prescriptions } \\
\text { over } 6 \text { months in } \$(S D)\end{array}$ & $\begin{array}{l}\text { Calculated mean difference } \\
\text { for } \mathrm{G} 1 \text { vs. G2: } \$ 80.40 ; 95 \% \\
\mathrm{Cl}, \$ 10.43 \text { to } \$ 150.37 \\
p=0.024 \\
\text { Calculated mean difference } \\
\text { for } \mathrm{G} 1 \text { vs. } \mathrm{G} 3=\$ 88.60 ; \\
95 \% \mathrm{Cl}, \$ 24.61 \text { to } \$ 152.59 \\
p=0.007\end{array}$ \\
\hline \multirow[t]{2}{*}{$\begin{array}{l}\text { Fox et al., } \\
2009^{31} \\
\text { Cohort/High }\end{array}$} & $\begin{array}{l}\text { G1: MTM program } \\
\text { (acceptors) } \\
\text { G2: Opt-out from MTM } \\
\text { program (refusers) }\end{array}$ & $\begin{array}{l}\text { G1: } 247 \\
\text { G2: } 50\end{array}$ & $\begin{array}{l}\text { Mean difference in Medicare } \\
\text { Part D medication copayment } \\
\text { costs per patient per month }\end{array}$ & $\begin{array}{l}\text { Calculated mean difference: } \\
-\$ 3.92 \text {, } \\
95 \% \mathrm{CI},-\$ 25.71 \text { to } \$ 17.87 \\
\mathrm{p}=0.724\end{array}$ \\
\hline & & & $\begin{array}{l}\text { Mean difference in all } \\
\text { medication copayments } \\
\text { (Medicare Part D and not } \\
\text { Part D) per patient per month }\end{array}$ & $\begin{array}{l}\text { Calculated mean difference: } \\
-\$ 1.71 \\
95 \% \mathrm{CI},-\$ 24.53 \text { to } \$ 21.11 \\
\mathrm{p}=0.883\end{array}$ \\
\hline $\begin{array}{l}\text { Pindolia et al., } \\
2009^{35} \\
\text { Cohort/High }\end{array}$ & $\begin{array}{l}\text { G1: Telephone-based MTM } \\
\text { program (acceptors) } \\
\text { G2: Usual medical care } \\
\text { (refusers) }\end{array}$ & $\begin{array}{l}\text { G1: } 292 \\
\text { G2: } 1,081\end{array}$ & $\begin{array}{l}\text { Mean out-of-pocket } \\
\text { prescription costs per health } \\
\text { plan member in \$ (assumed } \\
\text { per year, as NR in study) } \\
\text { (SD) }\end{array}$ & $\begin{array}{l}\text { Calculated mean } \\
\text { difference: } \$ 77.00 \text {; } \\
95 \% \mathrm{Cl},-\# 71.82 \text { to } \$ 225.82 \\
p=0.311\end{array}$ \\
\hline
\end{tabular}

Abbreviations: $\mathrm{CI}=$ confidence interval; $\mathrm{G}=$ group; $\mathrm{MTM}=$ medication therapy management; $\mathrm{NR}=$ not reported; $\mathrm{NRCT}=$ nonrandomized controlled trial; $\mathrm{RCT}=$ randomized controlled trial; $\mathrm{SD}=$ standard deviation; $\mathrm{SMD}$ : standardized mean difference.

Table 48. Patient copayments: Strength of evidence

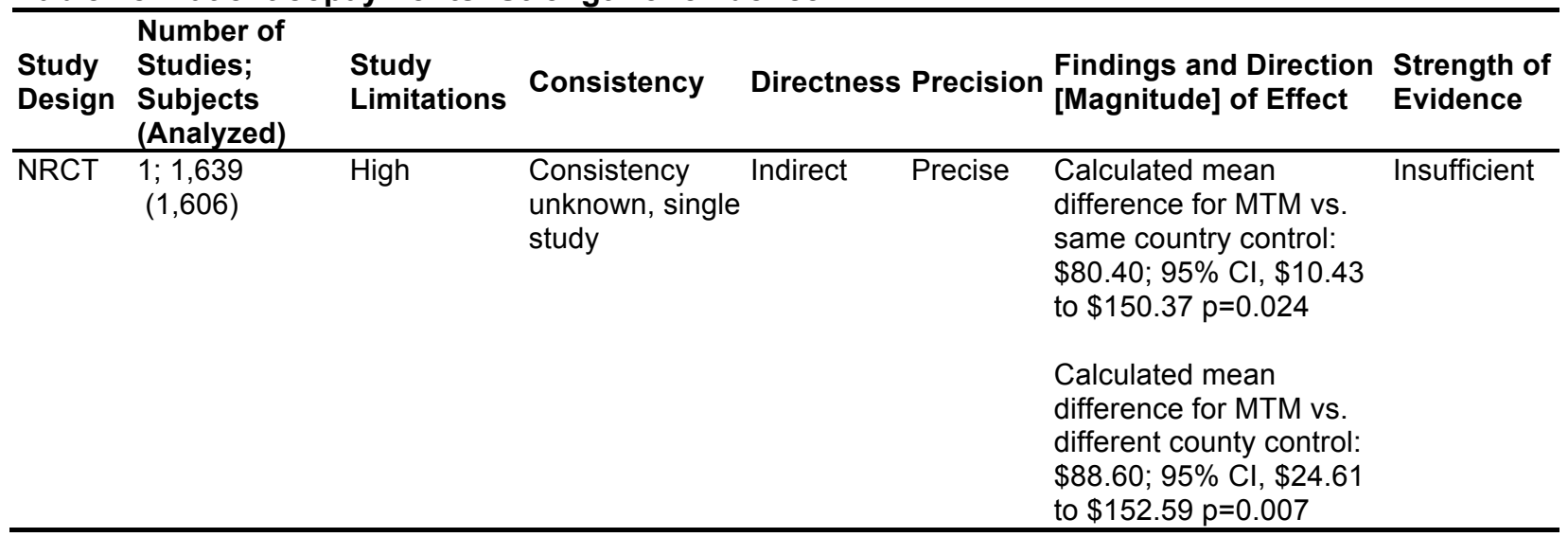

Abbreviations: NRCT = nonrandomized controlled trial.

\section{Medication Costs: Expenditures by Insurers}

Two RCTs (both medium risk of bias), ${ }^{39,44}$ the NRCT reported on above, ${ }^{38}$ and two cohort studies (one medium ${ }^{33,34}$ and one high risk of bias ${ }^{63}$ ) measured the net effect of MTM on expenditures incurred by insurers on medications (Table 49). Changes in health plan drug expenditures attributable to MTM depend on the net effect of MTM activities, which can entail adding clinically needed drugs, increasing doses or frequency, substituting therapeutically equivalent lower cost drugs, and simplifying regimens (singly or in combination). For individual 
patients, a net increase in expenditures may be the outcome of a more appropriate drug regimen. Included studies provided only the net effect on expenditures at the study arm level. All demonstrated that MTM either reduced health plan expenditures or limited the increase in expenditures over time for patients receiving the MTM intervention when compared with patients in the control or comparison arm. These results were not precise, however; confidence intervals included the null effect for all but one trial

Table 49. Total expenditures on medications by insurers: Summary of results

\begin{tabular}{|c|c|c|c|c|}
\hline $\begin{array}{l}\text { Study } \\
\text { Design/Risk of Bias }\end{array}$ & Study Arms & N Analyzed & $\begin{array}{l}\text { Prescription Costs to } \\
\text { Insurers }\end{array}$ & Results \\
\hline $\begin{array}{l}\text { Jameson et al., } \\
1995^{39} \\
\text { RCT/Medium }\end{array}$ & $\begin{array}{l}\text { G1: Pharmacotherapy } \\
\text { consultation } \\
\text { G2: Usual care }\end{array}$ & $\begin{array}{l}\text { G1: } 27 \\
\text { G2: } 29\end{array}$ & $\begin{array}{l}\text { Change in cost of } \\
\text { prescription drugs over } \\
6 \text { months, based on } \\
\text { maximum allowable } \\
\text { cost for Medicaid } \\
\text { reimbursement }\end{array}$ & $\begin{array}{l}\text { Calculated mean difference: } \\
-\$ 293.00 \\
95 \% \mathrm{Cl}:-\$ 501.50 \text { to }-\$ 84.50 \\
p<0.01\end{array}$ \\
\hline $\begin{array}{l}\text { Sellors et al., } 2003^{44} \\
\text { RCT/Medium }\end{array}$ & $\begin{array}{l}\text { G1: Pharmacist } \\
\text { consultation program } \\
\text { G2: Usual care }\end{array}$ & $\begin{array}{l}\text { G1: } 379 \\
\text { G2: } 409\end{array}$ & $\begin{array}{l}\text { Mean daily medication } \\
\text { costs per patient to the } \\
\text { Ontario Drug Benefit } \\
\text { Program (assumed } \\
\text { CAD) at } 5 \text { months }\end{array}$ & $\begin{array}{l}\text { Calculated mean difference: } \\
\$ 0.19 \\
95 \% \mathrm{Cl}:-\$ 1.52 \text { to } \$ 1.14 \\
\mathrm{p}=0.78 \\
\text { Calculated mean difference } \\
\text { over } 6 \text { months }=0.19 * 30 * 6=34\end{array}$ \\
\hline $\begin{array}{l}\text { Christensen et al., } \\
2007^{38} \\
\text { NRCT/Medium }\end{array}$ & $\begin{array}{l}\text { G1: Patients receiving } \\
\text { pharmacist-provided } \\
\text { MTM services } \\
\text { G2: Patients from } \\
\text { same counties as G1 } \\
\text { who did not receive } \\
\text { intervention (control } \\
\text { group 1) } \\
\text { G3: Patients from a } \\
\text { different county than } \\
\text { G1 who did not receive } \\
\text { intervention (control } \\
\text { group 2) }\end{array}$ & $\begin{array}{l}\text { G1: } 67 \\
\text { G2: } 669 \\
\text { G3: } 870\end{array}$ & $\begin{array}{l}\text { Mean difference in } \\
\text { amount insurer paid for } \\
\text { prescriptions over } 6 \\
\text { months }\end{array}$ & $\begin{array}{l}\text { Calculated mean difference for } \\
\text { G1 vs. G2: }-\$ 54.70 \\
95 \% \mathrm{Cl}:-\$ 287.59 \text { to } \$ 178.19 \\
p=0.645 \\
\text { Calculated mean difference for } \\
\text { G1 vs. G3: }-\$ 7.20 \text {; } \\
95 \% \mathrm{Cl}:-\$ 230.80 \text { to } \$ 216.40 \\
p=0.950\end{array}$ \\
\hline $\begin{array}{l}\text { Moczygemba et al., } \\
2011^{33} \\
\text { Moczygemba et al., } \\
2008^{34} \\
\text { Cohort/Medium }\end{array}$ & $\begin{array}{l}\text { G1: MTM-eligible } \\
\text { patients who opted into } \\
\text { a telephone MTM } \\
\text { program } \\
\text { G2: MTM-eligible } \\
\text { patients who did not } \\
\text { opt-in to the MTM } \\
\text { program. }\end{array}$ & $\begin{array}{l}\mathrm{G} 1: 60 \\
\mathrm{G} 2: 60\end{array}$ & $\begin{array}{l}\text { Mean Part D drug costs } \\
\text { (based on prescription } \\
\text { claim records, excludes } \\
\text { non-Part D drug costs) } \\
\text { (SD) at baseline and } 6 \\
\text { months }\end{array}$ & $\begin{array}{l}\text { Calculated mean difference: } \\
-\$ 276.00 \\
95 \% \mathrm{Cl}:-\$ 751.25 \text { to } \$ 199.25 \\
p=0.26\end{array}$ \\
\hline $\begin{array}{l}\text { Chrischilles et al., } \\
2004{ }^{63} \\
\text { Cohort/High }\end{array}$ & $\begin{array}{l}\text { G1: PCM-eligible } \\
\text { patients who received } \\
\text { PCM services } \\
\text { G2: PCM-eligible } \\
\text { patients who did not } \\
\text { receive PCM services }\end{array}$ & $\begin{array}{l}\text { G1: } 524 \\
\text { G2: } 1,687\end{array}$ & $\begin{array}{l}\text { Mean amount billed per } \\
\text { patient for active drugs } \\
\text { (based on Medicaid } \\
\text { claims) (SD) at baseline } \\
\text { and at } 9 \text { months }\end{array}$ & $\begin{array}{l}\text { Calculated mean difference: - } \\
\$ 0.95 \\
95 \% \text { Cl: }-\$ 58.67 \text { to } \$ 56.77 \\
P=0.974\end{array}$ \\
\hline
\end{tabular}

Abbreviations: $\mathrm{CAD}=$ Canadian dollar; $\mathrm{CI}=$ confidence interval; $\mathrm{MTM}=$ medication therapy management; $\mathrm{PCM}=$ pharmaceutical case management; $\mathrm{RCT}=$ randomized controlled trial; $\mathrm{SMD}=$ standardized mean difference.

We did not pool estimates of effect for the two trials because of heterogeneity in outcomes and setting; one trial was conducted in Canada and presented average daily costs, whereas the US-based study presented change over time. The nonrandomized studies were less heterogeneous but the pooled estimates had wide confidence intervals; the mean difference, 
without the high risk-of-bias study was $-\$ 97.55$ over 6 months $(95 \% \mathrm{CI},-\$ 306.68$ to 111.58 ; $\mathrm{p}=0.361 ; \mathrm{I}^{2}: 0$ ) (Appendix G-12). These results continued to be imprecise when we included a comparison of the intervention arm from one county versus a control arm from another county in the Christensen et al. study. ${ }^{38}$

Based on the lack of precision and directness, we rated the evidence from medium risk-ofbias studies as insufficient to evaluate the effect of MTM on expenditures by insurers (Table 50).

Table 50. Health plan expenditures: Strength of evidence

\begin{tabular}{|c|c|c|c|c|c|}
\hline $\begin{array}{l}\text { Study } \\
\text { Design }\end{array}$ & $\begin{array}{l}\text { Number of } \\
\text { Studies; } \\
\text { Subjects } \\
\text { (Analyzed) }\end{array}$ & $\begin{array}{l}\text { Study } \\
\text { Limitations }\end{array}$ & Consistency Directness Precision & $\begin{array}{l}\text { Findings and Direction } \\
\text { [Magnitude] of Effect }\end{array}$ & $\begin{array}{l}\text { Strength of } \\
\text { Evidence }\end{array}$ \\
\hline$\overline{\mathrm{RCT}}$ & $2 ; 953(835)$ & Medium & Consistent & $\begin{array}{l}\text { Mean difference varies } \\
\text { from }-\$ 34 \text { CAD to }-\$ 293 \\
\text { USD over } 6 \text { months }\end{array}$ & Insufficient \\
\hline $\begin{array}{l}\text { NRCT } \\
\text { and } \\
\text { Cohort }\end{array}$ & $2 ; 1,771(1,746)$ & High & Consistent & $\begin{array}{l}-\$ 97.55 ; 95 \% \mathrm{Cl},- \\
\$ 306.68 \text { to } 111.58 \\
p=0.361 ; I^{2}, 0\end{array}$ & Insufficient \\
\hline
\end{tabular}

Abbreviations: $\mathrm{CAD}=$ Canadian dollar; $\mathrm{CI}=$ confidence interval; $\mathrm{NRCT}=$ nonrandomized controlled trial; $\mathrm{RCT}=$ randomized controlled trial; $\mathrm{SD}=$ standard deviation, $\mathrm{USD}=\mathrm{US}$ dollar

\section{Medication Costs: Total Outlays on Medications}

Three RCTs (medium risk of bias), ${ }^{40-44,74}$ two RCTs (high risk of bias), ${ }^{59,60,73}$ and five cohort studies (high risk of bias) ${ }^{31,35-37,46}$ measured the effect of MTM on total outlays on medications. As with other data on resource use, the failure to specify the expected mechanism of action on the outcome and the predicted direction makes interpreting inconsistent results challenging. An additional challenge relates to the wide variation in data sources and degree of clarity on how investigators calculated outlays. In some studies, the specific measure used includes the combination of expenditures incurred by insurers and patients for prescription medications (Table 51). In other studies, the measure is based on wholesale costs, but whether and how the cost is split between the insurer and the patient is unclear. Results in Table 51 are denominated in US dollars unless otherwise specified, and calculated differences are rounded to two decimals.

Table 51. Total outlays on medications: Summary of results

\begin{tabular}{|c|c|c|c|c|}
\hline $\begin{array}{l}\text { Study } \\
\text { Design/Risk of Bias }\end{array}$ & Study Arms & N Analyzed & $\begin{array}{l}\text { Outlays on } \\
\text { prescriptions }\end{array}$ & Results \\
\hline $\begin{array}{l}\text { Malone et al., } 2000^{40} \text {; } \\
\text { Ellis et al., } 2000^{41} ; \\
\text { Malone et al., } 2001^{43} \text {; } \\
\text { Ellis et al., } 2000^{42} \\
\text { RCT/Medium }\end{array}$ & $\begin{array}{l}\text { G1: Pharmaceutical } \\
\text { care } \\
\text { G2: Usual care }\end{array}$ & $\begin{array}{l}\text { G1: } 523 \\
\text { G2: } 531\end{array}$ & $\begin{array}{l}\text { Mean change in annual } \\
\text { drug costs in } \\
\text { (calculated from Denver } \\
\text { VAMC pharmacy } \\
\text { department, individual } \\
\text { sites, or the VA } \\
\text { Pharmacy } \\
\text { Benefits Management } \\
\text { group) }\end{array}$ & $\begin{array}{l}\text { Calculated mean difference: } \\
\$ 63.00 \\
95 \% \mathrm{Cl}:-\$ 5.08 \text { to } \$ 131.078 \text {; } \\
p=0.07 \\
\text { Calculated mean difference } \\
\text { per month: } \$ 63 / \$ 12=\$ 5.25\end{array}$ \\
\hline $\begin{array}{l}\text { Sellors et al., } 2003^{44} \\
\text { RCT/Medium }\end{array}$ & $\begin{array}{l}\text { G1: Pharmacist } \\
\text { consultation program } \\
\text { G2: Usual care }\end{array}$ & $\begin{array}{l}\text { G1: } 379 \\
\text { G2: } 409\end{array}$ & $\begin{array}{l}\text { Mean daily medication } \\
\text { costs per patient at } 5 \\
\text { months (assumed } \\
\text { CAD) }\end{array}$ & $\begin{array}{l}\text { Calculated mean difference: } \\
\$ 0.19 \text { (assumed CAD) } \\
95 \% \text { Cl: }-\$ 0.85 \text { to } \$ 1.23 \\
p=0.72\end{array}$ \\
\hline
\end{tabular}


Table 51. Total outlays on medications: Summary of results (continued)

\begin{tabular}{|c|c|c|c|c|}
\hline $\begin{array}{l}\text { Study } \\
\text { Design/Risk of Bias }\end{array}$ & Study Arms & N Analyzed & $\begin{array}{l}\text { Outlays on } \\
\text { prescriptions }\end{array}$ & Results \\
\hline $\begin{array}{l}\text { Williams, } 2004^{74} \\
\text { RCT/Medium }\end{array}$ & $\begin{array}{l}\text { G1: Modification of } \\
\text { patient's medication } \\
\text { regimen by an } \\
\text { interdisciplinary } \\
\text { medication adjustment } \\
\text { team } \\
\text { G2: Usual medical } \\
\text { care }\end{array}$ & $\begin{array}{l}\text { G1: } 57 \\
\text { G2: } 76\end{array}$ & $\begin{array}{l}\text { Average monthly } \\
\text { wholesale price of } \\
\text { prescription and } \\
\text { nonprescription drugs }\end{array}$ & $\begin{array}{l}\text { Reported mean difference: } \\
-\$ 20.16 \\
95 \% \mathrm{Cl}: \$ 5.78 \text { to } \$ 34.54 \\
\text { p: } 0.006\end{array}$ \\
\hline $\begin{array}{l}\text { Krska et al., } 2001^{73} \\
\text { RCT/High }\end{array}$ & $\begin{array}{l}\text { G1: Pharmacist-led } \\
\text { medication review } \\
\text { G2: Usual care } \\
\text { including identification } \\
\text { of pharmaceutical care } \\
\text { issues, but no plan }\end{array}$ & $\begin{array}{l}\text { G1: } 168 \\
\text { G2: } 164\end{array}$ & $\begin{array}{l}\text { Average monthly costs } \\
\text { of prescribed } \\
\text { medication per patient } \\
\text { in British pounds ( } £ \text { ) } \\
\text { (SD) at } 3 \text { months } \\
\text { (calculated using } \\
\text { information from } \\
\text { patients on actual use) }\end{array}$ & $\begin{array}{l}\text { Calculated mean difference: - } \\
£ .0 .19 \text {, } \\
95 \% \text { Cl:- } £ 6.69 \text { to } £ 6.49 \\
p=0.956 \text {. }\end{array}$ \\
\hline $\begin{array}{l}\text { Pai, } 2009^{59} ; \text { Pai, } \\
2009^{60} \\
\text { RCT/High }\end{array}$ & $\begin{array}{l}\text { G1: Pharmaceutical } \\
\text { care } \\
\text { G2: Usual care }\end{array}$ & $\begin{array}{l}\text { G1: NR } \\
\text { G2: NR }\end{array}$ & $\begin{array}{l}\text { Mean drug costs } \\
\text { (calculated from } \\
\text { average wholesale } \\
\text { price) over } 2 \text { years }\end{array}$ & $\begin{array}{l}\text { Pharmaceutical care reduced } \\
\text { mean drug costs by } \$ 6.21 \\
\text { compared with the usual care } \\
\text { group, } p=N S, \text { no absolute } \\
\text { costs or other details reported }\end{array}$ \\
\hline $\begin{array}{l}\text { Fox et al., } 2009^{31} \\
\text { Cohort/High }\end{array}$ & $\begin{array}{l}\text { G1: MTM program } \\
\text { (acceptors) } \\
\text { G2: Opt-out from MTM } \\
\text { program (refusers) }\end{array}$ & $\begin{array}{l}\text { G1: } 247 \\
\text { G2: } 50\end{array}$ & $\begin{array}{l}\text { Mean difference in } \\
\text { annual Medicare Part D } \\
\text { drug costs (patient } \\
\text { copayment + insurance } \\
\text { plan medication costs + } \\
\text { dispensing fee) }\end{array}$ & $\begin{array}{l}\text { Calculated mean difference: - } \\
\$ 27.78, \\
95 \% \mathrm{Cl}-\% 125.82 \text { to } \% 26.60 \\
p=0.57\end{array}$ \\
\hline $\begin{array}{l}\text { Pindolia et al., } 2009^{35} \\
\text { Cohort/High } \\
\end{array}$ & $\begin{array}{l}\text { G1: Telephone based } \\
\text { MTM program } \\
\text { (acceptors) } \\
\text { G2: Usual medical } \\
\text { care (refusers) }\end{array}$ & $\begin{array}{l}\text { G1: } 292 \\
\text { G2: } 1,081\end{array}$ & $\begin{array}{l}\text { Total annual } \\
\text { prescription drug cost } \\
\text { per health plan member } \\
\text { in USD }\end{array}$ & $\begin{array}{l}\text { Calculated mean difference: - } \\
62.22,95 \% \mathrm{Cl}-112.469 \text { to - } \\
11.971 ; p=0.015\end{array}$ \\
\hline $\begin{array}{l}\text { Staresinic et al., } \\
2007^{36} \\
\text { Cohort/High }\end{array}$ & $\begin{array}{l}\text { G1: MTP program } \\
\text { (acceptors) } \\
\text { G2: Usual care } \\
\text { (refusers) }\end{array}$ & $\begin{array}{l}\text { G1: } 282 \\
\text { G2: } 1,544\end{array}$ & $\begin{array}{l}\text { Total prescription cost } \\
\text { per MTM program } \\
\text { beneficiary per month } \\
\text { (gross drug } \\
\text { cost=ingredient cost } \\
\text { paid + dispensing fee + } \\
\text { sales tax per member- } \\
\text { months in Part D } \\
\text { contract) }\end{array}$ & $\begin{array}{l}\text { Participants spent less on } \\
\text { prescription medications on } \\
\text { average (described as per } \\
\text { member per month drug } \\
\text { spending) than } \\
\text { nonparticipants. Figure } \\
\text { provided suggested a } \\
\text { decrease in spending of } \\
\text { between } \$ 100 \text { and } \$ 150 \text { in the } \\
\text { intervention group, but exact } \\
\text { numbers not reported. }\end{array}$ \\
\hline
\end{tabular}


Table 51. Total outlays on medications: Summary of results (continued)

\begin{tabular}{|c|c|c|c|c|}
\hline $\begin{array}{l}\text { Study } \\
\text { Design/Risk of Bias }\end{array}$ & Study Arms & N Analyzed & $\begin{array}{l}\text { Outlays on } \\
\text { prescriptions }\end{array}$ & Results \\
\hline \multirow[t]{2}{*}{$\begin{array}{l}\text { Welch et al., } 2009^{37} \\
\text { Cohort/High }\end{array}$} & $\begin{array}{l}\text { G1: MTM program } \\
\text { provided to home- } \\
\text { based beneficiaries } \\
\text { G2: Opt-out among } \\
\text { home-based patients } \\
\text { eligible for MTM }\end{array}$ & $\begin{array}{l}\text { G1: } 459 \\
\text { G2: } 336\end{array}$ & $\begin{array}{l}\text { Mean change in } \\
\text { medication costs per } \\
\text { day at } 6 \text { months. } \\
\text { (Estimates come from } \\
\text { data on study } \\
\text { beneficiaries' } \\
\text { purchases } \\
\text { of ambulatory } \\
\text { prescription } \\
\text { medications) }\end{array}$ & $\begin{array}{l}\text { Difference in difference: } \$ 3.62 \text {, } \\
\text { SD NR, adjusted } p=0.203 \\
\text { NOTE: Age, sex, chronic } \\
\text { disease score, and preperiod } \\
\text { drug cost included in } \\
\text { multivariate regression } \\
\text { modeling for adjusted } P\end{array}$ \\
\hline & & & $\begin{array}{l}\text { Mean percentage } \\
\text { increase in medication } \\
\text { costs per day at } 6 \\
\text { months (No SD } \\
\text { reported,) }\end{array}$ & $\begin{array}{l}\text { Adjusted OR : } 1.495 \% \mathrm{Cl}: 1.1 \\
\text { to } 1.9 \\
\text { NOTE: Model adjusted for } \\
\text { age, sex, chronic disease } \\
\text { score, and baseline } \\
\text { medication cost }\end{array}$ \\
\hline $\begin{array}{l}\text { Winston et al., } \\
2009^{46} \\
\text { Cohort/High }\end{array}$ & $\begin{array}{l}\text { G1: Community } \\
\text { pharmacy MTM } \\
\text { G2: Pharmacist- } \\
\text { staffed, call-center- } \\
\text { based MTM } \\
\text { G3: Educational } \\
\text { mailings }\end{array}$ & $\begin{array}{l}\text { G1: } 21,336 \\
\text { G2: } 3,436 \\
\text { G3: } 49,021\end{array}$ & $\begin{array}{l}\text { Mean (SD) drug cost } \\
\text { per patient per month } \\
\text { after } 8 \text { months of } \\
\text { services (based on } \\
\text { drug claims processing } \\
\text { data, total allowed } \\
\text { charges, including } \\
\text { ingredient cost paid, } \\
\text { dispensing fee, and } \\
\text { sales tax, before } \\
\text { subtracting } \\
\text { any patient cost-sharing } \\
\text { amounts) }\end{array}$ & $\begin{array}{l}\text { Calculated mean difference for } \\
\text { G1 vs. } \mathrm{G} 3:-\$ 35.00 \text {, } \\
95 \% \mathrm{Cl}-43.390 \text { to }-26.610 \text {; } \\
\mathrm{P}<0.001 \\
\text { Calculated mean difference for } \\
\mathrm{G} 2 \text { vs. } \mathrm{G} 3:-15.0,95 \% \mathrm{Cl}- \\
33.411 \text { to } 3.411 ; \mathrm{P}=0.11\end{array}$ \\
\hline
\end{tabular}

Abbreviations: CAD = Canadian dollar; $\mathrm{CI}=$ confidence interval; MTMP = Medication Therapy Management Program; RCT= randomized controlled trial; $\mathrm{SMD}=$ standardized mean difference; USD = US dollar, VAMC = Veterans Affairs Medical Center

We did not pool the three medium risk-of-bias studies because of the heterogeneity of measures. $^{40-44,74}$ Two suggested an increase in outlays in the intervention $\operatorname{arm}^{40-44}$ (although estimates were imprecise and confidence intervals contained the null effect), and one suggested a reduction. ${ }^{74}$ The high risk-of-bias studies similarly demonstrated inconsistent results; some reported reduced outlays ${ }^{35,36,46}$ and others showed increased outlays ${ }^{31,37}$ or no effect ${ }^{59,60,73}$ following MTM.

Based on the lack of consistency, directness, and precision, we rated the evidence from three medium risk-of-bias trials as insufficient to evaluate the effect of MTM on total outlays on medications (Table 52).

Table 52. Total outlays on medications: Strength of evidence

\begin{tabular}{|c|c|c|c|c|c|}
\hline $\begin{array}{l}\text { Study } \\
\text { design }\end{array}$ & $\begin{array}{l}\text { Number of } \\
\text { Studies; } \\
\text { Subjects } \\
\text { (Analyzed) }\end{array}$ & $\begin{array}{l}\text { Study } \\
\text { Limitations }\end{array}$ & Consistency Directness Precision & $\begin{array}{l}\text { Findings and } \\
\text { Direction [Magnitude] } \\
\text { of Effect }\end{array}$ & $\begin{array}{l}\text { Strength of } \\
\text { Evidence }\end{array}$ \\
\hline$\overline{R C T}$ & $3 ; 2,083(1,975)$ & Medium & Inconsistent Indirect & $\begin{array}{l}\text { Mean difference varies } \\
\text { from }-26 \text { USD to }+5.25 \\
\text { USD per month }\end{array}$ & Insufficient \\
\hline
\end{tabular}

Abbreviations: $\mathrm{RCT}=$ randomized controlled trial; $\mathrm{USD}=\mathrm{US}$ dollar 


\section{Medication Costs: Combined Medication and Other Costs}

Three trials (two medium risk-of-bias; ${ }^{44,54}$ one high risk-of-bias ${ }^{51,52}$ ) and one NRCT (medium risk of bias $^{65}$ ) provided consistent evidence that MTM does not reduce combined medication and other costs (variably defined in each study) (Table 53). Studies did not report their results in sufficient detail to allow pooling. Based on available information, we judged the evidence to be insufficient to evaluate the effect of MTM on combined medication and other costs (Table 54).

Table 53. Medication and other costs: Summary of results

\begin{tabular}{|c|c|c|c|c|}
\hline $\begin{array}{l}\text { Study } \\
\text { Design/Risk of Bias }\end{array}$ & Study Arms & N Analyzed & $\begin{array}{l}\text { Medication and other } \\
\text { costs }\end{array}$ & Results \\
\hline \multirow[t]{2}{*}{$\begin{array}{l}\text { Sellors et al., } 2003^{44} \\
\text { RCT/Medium }\end{array}$} & $\begin{array}{l}\text { G1: Pharmacist } \\
\text { consultation program } \\
\text { G2: Usual care }\end{array}$ & $\begin{array}{l}\text { G1: } 379 \\
\text { G2: } 409\end{array}$ & $\begin{array}{l}\text { Mean cost of health } \\
\text { care resources per } \\
\text { patient, including all } \\
\text { hospital stays at } 5 \\
\text { months (CAD } \\
\text { assumed) }\end{array}$ & $\begin{array}{l}\text { Calculated mean difference: } \\
\$ 249.41 \text { (assumed CAD), } \\
\text { 95\% Cl: }-\$ 338.39 \text { to } \$ 837.21 \text {; } \\
p=0.406\end{array}$ \\
\hline & & & $\begin{array}{l}\text { Mean cost of health } \\
\text { care resources per } \\
\text { patient, including only } \\
\text { drug (i.e., medication)- } \\
\text { related hospital stays at } \\
5 \text { months (CAD } \\
\text { assumed) }\end{array}$ & $\begin{array}{l}\text { Calculated mean difference: - } \\
\$ 8.10 \text { (assumed CAD), } \\
95 \% \text { Cl: }-\$ 386.72 \text { to } 4350.52 ; \\
p=0.923\end{array}$ \\
\hline $\begin{array}{l}\text { Triller et al., } 2007^{54} \\
\text { RCT/Medium }\end{array}$ & $\begin{array}{l}\text { G1: Visiting nurse } \\
\text { association home visit } \\
\text { services plus } \\
\text { comprehensive } \\
\text { pharmaceutical care } \\
\text { services } \\
\text { G2: Visiting nurse } \\
\text { association home visit } \\
\text { services }\end{array}$ & $\begin{array}{l}\text { G1: NR } \\
\text { G2: NR }\end{array}$ & $\begin{array}{l}\text { Aggregate health } \\
\text { system costs (not } \\
\text { defined in detail) } \\
\text { Home care agency } \\
\text { costs (not defined in } \\
\text { detail) }\end{array}$ & $\begin{array}{l}\text { Values not reported, but } \\
\text { authors stated that costs did } \\
\text { not differ significantly between } \\
\text { the two groups. }\end{array}$ \\
\hline $\begin{array}{l}\text { Bernsten et al., } \\
2001^{51,52} \\
\text { RCT/High }\end{array}$ & $\begin{array}{l}\text { G1: Structured } \\
\text { community pharmacy- } \\
\text { based pharmaceutical } \\
\text { care program } \\
\text { G2: Usual community } \\
\text { pharmacy services }\end{array}$ & $\begin{array}{l}\text { Baseline } \\
\text { G1: } 867 \\
\text { G2: } 748 \\
6 \text { months } \\
\text { G1: NR } \\
\text { G2: NR } \\
\text { 12 months } \\
\text { G1: NR } \\
\text { G2: NR } \\
\text { 18 months } \\
\text { G1: NR } \\
\text { G2: NR }\end{array}$ & $\begin{array}{l}\text { Mean total cost per } \\
\text { patient including (1) } \\
\text { cost associated with } \\
\text { additional time spent by } \\
\text { pharmacists; (2) cost } \\
\text { associated with } \\
\text { contacts with GPs, } \\
\text { specialists and nurses; } \\
\text { and (3) cost of } \\
\text { hospitalizations and } \\
\text { drugs }\end{array}$ & $\begin{array}{l}\text { Cost data not pooled and } \\
\text { analyzed for costs because } \\
\text { health care systems differed } \\
\text { between } 7 \text { countries included in } \\
\text { the study. However, authors } \\
\text { reported no significant } \\
\text { between-group differences in } \\
\text { any country (p=NS) }\end{array}$ \\
\hline $\begin{array}{l}\text { Fischer et al., } 2002^{65} \\
\text { NRCT/Medium }\end{array}$ & $\begin{array}{l}\text { G1: Pharmaceutical } \\
\text { care } \\
\text { G2: Usual care }\end{array}$ & $\begin{array}{l}\text { G1: } 231 \\
\text { G2: } 444\end{array}$ & $\begin{array}{l}\text { Change in total charges } \\
\text { for inpatient care, } \\
\text { outpatient care, and } \\
\text { pharmacy charges }\end{array}$ & $\begin{array}{l}\text { G1: }-900 \\
\text { G2: }-2000 \\
95 \% \text { CI: NR } \\
\text { P: NS, no details reported } \\
\text { Calculated mean difference: } \\
\$ 1,100 .\end{array}$ \\
\hline
\end{tabular}

Abbreviations: $\mathrm{CAD}=$ Canadian dollar; $\mathrm{CI}=$ confidence interval; $\mathrm{GP}=$ general practitioner; $\mathrm{NR}=$ not reported, $\mathrm{NS}=$ not significant; RCT = randomized controlled trial. 
Table 54. Medication and other costs: Strength of evidence

\begin{tabular}{|c|c|c|c|c|c|c|c|}
\hline $\begin{array}{l}\text { Study } \\
\text { design }\end{array}$ & $\begin{array}{l}\text { Number of } \\
\text { Studies; } \\
\text { Subjects } \\
\text { (Analyzed) }\end{array}$ & $\begin{array}{l}\text { Study } \\
\text { Limitations }\end{array}$ & Consistency & Directness & Precision & $\begin{array}{l}\text { Findings and Direction } \\
\text { [Magnitude] of Effect }\end{array}$ & $\begin{array}{l}\text { Strength of } \\
\text { Evidence }\end{array}$ \\
\hline RCT & $\begin{array}{l}2 ;>889, \text { N NR } \\
\text { in } 1 \text { study } \\
(>779)\end{array}$ & Medium & Consistent & Indirect & Imprecise & Variable estimates & Insufficient \\
\hline NRCT & $1 ; 675(675)$ & Medium & $\begin{array}{l}\text { Consistency } \\
\text { unknown, single } \\
\text { study }\end{array}$ & Indirect & Imprecise & $\begin{array}{l}\text { Difference in mean } \\
\text { costs of } \$ 1100 \text { favoring } \\
\text { control group, but } \\
\text { results not statistically } \\
\text { significant. }\end{array}$ & Insufficient \\
\hline
\end{tabular}

Abbreviations: $\mathrm{N}=$ number; $\mathrm{NR}=$ not reported; $\mathrm{NRCT}=$ nonrandomized controlled trial; $\mathrm{RCT}=$ randomized controlled trial

\section{Number of Outpatient Visits}

Nine studies examined the effect of MTM interventions, when compared with usual care, on outpatient visits. These studies varied in geographic setting (seven Western European countries, ${ }^{51,52}$ the United States, ${ }^{40-43,45,55-57,63,65}$ the United Kingdom, ${ }^{73}$ Canada ${ }^{44}$ ), period of evaluation ( 3 months to 36 months), specific outcome measure (ranging from a focus on visits with physicians to total ambulatory care visits or contacts with physicians and nurses), and risk of bias. They are described in Table 55. No study indicated whether the intervention was specifically designed to increase or to decrease outpatient visits; as a result, the directionality of the results cannot be interpreted as a benefit or a harm.

Table 55. Number of outpatient visits: Summary of results

\begin{tabular}{|c|c|c|c|c|}
\hline $\begin{array}{l}\text { Study } \\
\text { Design/Risk of } \\
\text { Bias }\end{array}$ & Study Arms & N Analyzed & $\begin{array}{l}\text { Outcome and Time } \\
\text { Period }\end{array}$ & Results \\
\hline $\begin{array}{l}\text { Malone, } 2000^{40} ; \\
\text { Ellis, } 2000^{41} ; \\
\text { Malone, } 2001^{43} \text {; } \\
\text { Ellis, } 2000^{42} \\
\text { RCT/Medium }\end{array}$ & $\begin{array}{l}\text { G1: Pharmaceutical } \\
\text { care } \\
\text { G2: Usual care }\end{array}$ & $\begin{array}{l}\text { G1: } 523 \\
\text { G2: } 531\end{array}$ & $\begin{array}{l}\text { Mean change in number } \\
\text { of clinic visits (including } \\
\text { visits with the } \\
\text { pharmacists in the } \\
\text { intervention arm) over } \\
12 \text { months }\end{array}$ & $\begin{array}{l}\text { Calculated mean difference: } \\
2.0 \\
95 \% \mathrm{Cl}:-0.415 \text { to } 4.415, \\
p=0.104\end{array}$ \\
\hline $\begin{array}{l}\text { Sellors et al., } \\
2003^{44} \\
\text { RCT/Medium }\end{array}$ & $\begin{array}{l}\text { G1: Pharmacist } \\
\text { consultation program } \\
\text { G2: Usual care }\end{array}$ & $\begin{array}{l}\text { G1: } 379 \\
\text { G2: } 409\end{array}$ & $\begin{array}{l}\text { Number of clinic visits } \\
\text { over } 5 \text { moths }\end{array}$ & $\begin{array}{l}\text { Calculated mean difference: } \\
-0.02 \text {, } \\
95 \% \mathrm{Cl}:-1.274 \text { to } 1.234, \\
p=0.975\end{array}$ \\
\hline $\begin{array}{l}\text { Sidel, } 1990^{45} \\
\text { RCT/Medium }\end{array}$ & $\begin{array}{l}\text { G1: Patients received } \\
\text { at least } 2 \text { pharmacist } \\
\text { visits involving } \\
\text { medication review, } \\
\text { patient-specific } \\
\text { education and } \\
\text { counseling; follow-up } \\
\text { patient telephone calls } \\
\text { and contact of } \\
\text { physicians as needed } \\
\text { G2: Patients contacted } \\
\text { only to complete the } \\
\text { survey. }\end{array}$ & $\begin{array}{l}\text { G1: } 92 \\
\text { G2: } 104\end{array}$ & $\begin{array}{l}\text { Change in number of } \\
\text { ambulatory visits over } \\
\text { past } 3 \text { months, } \\
\text { measured at baseline } \\
\text { and again at } 36 \text { months }\end{array}$ & $\begin{array}{l}\text { Calculated mean difference: } \\
-1.41 \text {, } \\
95 \% \mathrm{Cl}:-2.98 \text { to } 0.160, \\
p=0.078\end{array}$ \\
\hline
\end{tabular}


Table 55. Number of outpatient visits: Summary of results (continued)

\begin{tabular}{|c|c|c|c|c|}
\hline $\begin{array}{l}\text { Study } \\
\text { Design/Risk of } \\
\text { Bias }\end{array}$ & Study Arms & N Analyzed & $\begin{array}{l}\text { Outcome and Time } \\
\text { Period }\end{array}$ & Results \\
\hline $\begin{array}{l}\text { Touchette et al., } \\
2012^{55} \\
\text { RCT/Medium }\end{array}$ & $\begin{array}{l}\text { G1: MTM basic } \\
\text { (comprehensive } \\
\text { medication review and } \\
\text { DRP assessment) } \\
\text { G2: MTM enhanced } \\
\text { (MTM plus } 2 \text { page } \\
\text { clinical summary } \\
\text { abstracted from } \\
\text { patient's medical chart). } \\
\text { G3: Usual care }\end{array}$ & $\begin{array}{l}\text { G1: } 183 \\
\text { G2: } 190 \\
\text { G3: } 183\end{array}$ & $\begin{array}{l}\text { 3-6 months } \\
\text { G1: } 183 \\
\text { G2: } 190 \\
\text { G3: } 183\end{array}$ & $\begin{array}{l}\text { G1 vs. G3 Calculated mean } \\
\text { difference: } 0.50,95 \% \mathrm{Cl} \text { - } \\
0.388 \text { to } 0.488, p=0.823 \\
\text { G2 vs. G3 Calculated mean } \\
\text { difference: }-0.50,95 \% \mathrm{Cl} \text { : - } \\
0.383 \text { to } 0.483, p=0.821\end{array}$ \\
\hline $\begin{array}{l}\text { Bernsten et al., } \\
2001^{51,52} \\
\text { RCT/High }\end{array}$ & $\begin{array}{l}\text { G1: Structured } \\
\text { community pharmacy- } \\
\text { based pharmaceutical } \\
\text { care program } \\
\text { G2: Usual community } \\
\text { pharmacy services }\end{array}$ & $\begin{array}{l}\text { G1: } 1024 \\
\text { G2: } 953\end{array}$ & $\begin{array}{l}\text { Mean number of } \\
\text { contacts with primary } \\
\text { care providers, including } \\
\text { home visits and office } \\
\text { appointments at } 6 \\
\text { months }\end{array}$ & $\begin{array}{l}\text { Calculated mean difference: } \\
0.120 \\
95 \% \mathrm{Cl}:-0.461 \text { to } 0.701 \\
p=0.686\end{array}$ \\
\hline $\begin{array}{l}\text { Krska et al., } 2001^{73} \\
\text { RCT/High }\end{array}$ & $\begin{array}{l}\text { G1: Pharmacist-led } \\
\text { medication review } \\
\text { G2: Usual care } \\
\text { including identification } \\
\text { of pharmaceutical care } \\
\text { issues, but no plan }\end{array}$ & $\begin{array}{l}\text { G1: NR } \\
\text { G2: NR }\end{array}$ & $\begin{array}{l}\text { Hospital clinic } \\
\text { attendance, use of } \\
\text { social services or } \\
\text { contacts with district } \\
\text { nurses and health } \\
\text { visitors before and after } \\
\text { the pharmacist review } \\
\end{array}$ & No differences; details NR \\
\hline $\begin{array}{l}\text { Fischer et al., } \\
2002^{65} \\
\text { NRCT/High }\end{array}$ & $\begin{array}{l}\text { G1: Pharmaceutical } \\
\text { care } \\
\text { G2: Usual care }\end{array}$ & $\begin{array}{l}\text { G1: } 231 \\
\text { G2: } 444\end{array}$ & $\begin{array}{l}\text { Changes in number of } \\
\text { clinic visits over } 1 \text { year }\end{array}$ & $\begin{array}{l}\text { Intention-to-treat analysis } \\
\text { Adjusted between-group } \\
\text { difference not significant, } \\
\text { details NR }\end{array}$ \\
\hline $\begin{array}{l}\text { Carter et al., } \\
1997^{56,57} \\
\text { Cohort/High }\end{array}$ & $\begin{array}{l}\text { G1: Pharmaceutical } \\
\text { care } \\
\text { G2: Usual care }\end{array}$ & $\begin{array}{l}\text { G1: } 25 \\
\text { G2: } 26\end{array}$ & $\begin{array}{l}\text { Number of distinct dates } \\
\text { of service over } 6 \text { months }\end{array}$ & $\begin{array}{l}\text { G1: } 2.2(2.4) \\
\text { G2: } 1.0(1.0) \\
95 \% \mathrm{Cl}: \mathrm{NR}, \mathrm{p}=0.07\end{array}$ \\
\hline $\begin{array}{l}\text { Chrischilles et al., } \\
2004^{63} \\
\text { Cohort/High }\end{array}$ & $\begin{array}{l}\text { G1: PCM-eligible } \\
\text { patients who received } \\
\text { PCM services } \\
\text { G2: PCM-eligible } \\
\text { patients who did not } \\
\text { receive PCM services }\end{array}$ & $\begin{array}{l}\text { G1: } 524 \\
\text { G2: } 1,687\end{array}$ & $\begin{array}{l}\text { Number of outpatient } \\
\text { facility claims at } 12 \\
\text { months }\end{array}$ & Results NR, $p=0.121$ \\
\hline
\end{tabular}

Abbreviations: $\mathrm{CI}=$ confidence interval; $\mathrm{DRP}$, drug-related problems; $\mathrm{MTM}=$ medication therapy management; $\mathrm{NR}=$ not reported; NRCT = nonrandomized controlled trial; $\mathrm{PCM}=$ pharmaceutical care management; $\mathrm{QOL}=$ quality of life; $\mathrm{RCT}=$ randomized controlled trial

Three RCTs (all medium risk of bias) provided sufficient data on outpatient visits within the first year to pool results. ${ }^{40-44,55}$ A meta-analysis of these studies, including results for the basic MTM arm for Touchette et al. (rather than the "enhanced MTM" arm), ${ }^{55}$ across outcomes from 5 to 12 months yielded an estimated standardized mean difference of 0.049 (95\% CI, -0.034 to $0.133, \mathrm{p}=0.247 ; \mathrm{I}^{2}=0$ ) (Appendix G-13). Including the results of the "enhanced MTM" arm instead of the basic MTM arm did not change the direction or precision or results (standardized mean difference: $0.27,95 \%$ CI: -0.036 to $0.91, p=0.40, \mathrm{I}^{2}=0$ ). Likewise, adding one trial with high risk of bias (stemming primarily from attrition bias ${ }^{51,52}$ ) to the meta-analysis did not alter the direction or precision of the estimate of effect (standardized difference in means: $0.032 ; 95 \%$ CI, -0.032 to $0.095, \mathrm{p}=0.326, \mathrm{I}^{2}=0$ ). A fifth study (medium risk-of-bias) found fewer outpatient visits in the intervention arm at 3 years, but confidence intervals spanned the null. ${ }^{45} \mathrm{~A}$ sixth medium risk-of-bias RCT noted "no differences in hospital clinic attendance, use of social services or contacts with district nurses and health visitors before and after the pharmacist 
review" but did not indicate whether this observation extended to the control arm and offered no statistics. ${ }^{73}$ The single nonrandomized controlled study found no differences between study arms in an intention-to-treat analysis. ${ }^{65}$

Two high risk-of-bias cohort studies, ${ }^{56,57,63}$ reported no statistically significant differences between study arms in the number of outpatient facility claims but offered no additional information.

Based on the lack of consistency and precision, we graded the body of evidence of medium risk-of-bias trials as insufficient to evaluate the effect of MTM interventions on outpatient resource utilization (Table 56).

Table 56. Number of outpatient visits: Strength of evidence

\begin{tabular}{|c|c|c|c|c|c|}
\hline $\begin{array}{l}\text { Study } \\
\text { design }\end{array}$ & $\begin{array}{l}\text { Number of } \\
\text { Studies; } \\
\text { Subjects } \\
\text { (Analyzed) }\end{array}$ & $\begin{array}{l}\text { Study } \\
\text { Limitations }\end{array}$ & Consistency Directness Precision & $\begin{array}{l}\text { Findings and Direction } \\
\text { [Magnitude] of Effect }\end{array}$ & $\begin{array}{l}\text { Strength of } \\
\text { Evidence }\end{array}$ \\
\hline$\overline{\mathrm{RCT}}$ & $3 ; 2227(2038)$ & Medium & Inconsistent Direct & $\begin{array}{l}\text { Standardized mean } \\
\text { difference: } 0.049 ; 95 \% \\
\mathrm{Cl},-0.034 \text { to } 0.133, \\
p=0.247 ; I^{2}=0\end{array}$ & Insufficient \\
\hline
\end{tabular}

Abbreviations: $\mathrm{RCT}=$ randomized controlled trial

\section{Cost of Outpatient Visits}

Four studies examined the effect of MTM interventions, when compared with usual care, on the costs of outpatient visits (Table 57). These studies included three set in the United States ${ }^{40-}$

43,56,57,63 and one set in Canada. ${ }^{44}$ The period of evaluation ranged from 5 months to 12 months. As with studies on the number of outpatient visits, no study indicated that the intervention was designed specifically to raise or lower the costs of outpatient visits; as a result, the directionality of the results cannot be interpreted as a benefit or a harm. As with other costs analyses, the data are in US dollars unless otherwise specified and rounded to nearest two decimals.

Two trials (medium risk-of-bias) offered inconsistent evidence on the effect of MTM interventions on outpatient costs. One U.S.-based VA study found a significantly smaller increase in annual costs of clinic visits in the intervention group than in the usual care group. ${ }^{40-43}$ The Canadian study found no significant differences by study arm. ${ }^{44}$ One cohort study (high risk of bias from selection bias) evaluated two measures of costs; the intervention arm had significantly higher costs for hypertension-related services than the usual care arm. ${ }^{56,57}$ Another US-based cohort study of the Medicaid program in Iowa (high risk of bias) found no statistically significant differences in cost of outpatient visits by intervention arm. ${ }^{63}$ 
Table 57. Costs of outpatient visits: Summary of results

\begin{tabular}{|c|c|c|c|c|}
\hline $\begin{array}{l}\text { Study } \\
\text { Design/Risk of } \\
\text { Bias }\end{array}$ & Study Arms & N Analyzed & $\begin{array}{l}\text { Outcome and Time } \\
\text { Period }\end{array}$ & Results \\
\hline $\begin{array}{l}\text { Malone, } 2000^{40} \\
\text { Ellis, } 2000^{41} ; \\
\text { Malone, } 2001^{43} \\
\text { Ellis, } 2000^{42} \\
\text { RCT/Medium }\end{array}$ & $\begin{array}{l}\text { G1: Pharmaceutical } \\
\text { care } \\
\text { G2: Usual care }\end{array}$ & $\begin{array}{l}\text { G1: } 523 \\
\text { G2: } 531\end{array}$ & $\begin{array}{l}\text { Mean change in annual } \\
\text { cost of clinic visits }\end{array}$ & $\begin{array}{l}\text { Calculated mean difference: } \\
-\$ 102.00 \\
95 \% \mathrm{Cl}:-\$ 187.81 \text { to } \\
-\$ 16.20 \\
p=0.02\end{array}$ \\
\hline \multirow[t]{3}{*}{$\begin{array}{l}\text { Sellors et al., } \\
2003^{44} \\
\text { RCT/Medium }\end{array}$} & \multirow[t]{3}{*}{$\begin{array}{l}\text { G1: Pharmacist } \\
\text { consultation program } \\
\text { G2: Usual care }\end{array}$} & \multirow[t]{3}{*}{$\begin{array}{l}\text { G1: } 379 \\
\text { G2: } 409\end{array}$} & $\begin{array}{l}\text { Mean cost of physician } \\
\text { visits (assumed CAD) at } \\
5 \text { months }\end{array}$ & $\begin{array}{l}\text { Calculated mean difference: } \\
\$ 5.66,95 \% \mathrm{Cl}:-\$ 24.22 \text { to } \\
\$ 35.54, p=0.71\end{array}$ \\
\hline & & & $\begin{array}{l}\text { Mean cost of clinic visits } \\
\text { (assumed CAD) at } 5 \\
\text { months }\end{array}$ & $\begin{array}{l}\text { Calculated mean difference: } \\
-\$ 2.13,95 \% \mathrm{Cl}:-\$ 20.46 \text { to } \\
\$ 16.20, p=0.82\end{array}$ \\
\hline & & & $\begin{array}{l}\text { Mean cost of other } \\
\text { health care services and } \\
\text { visits to health care } \\
\text { professionals (assumed } \\
\text { CAD) at } 5 \text { months }\end{array}$ & $\begin{array}{l}\text { Calculated mean difference: } \\
-\$ 4.70 \\
95 \% \mathrm{Cl}:-\$ 140.22 \text { to } \$ 130.82 ; \\
p=0.95\end{array}$ \\
\hline \multirow[t]{2}{*}{$\begin{array}{l}\text { Carter et al., } \\
1997^{56,57} \\
\text { Cohort/High }\end{array}$} & \multirow[t]{2}{*}{$\begin{array}{l}\text { G1: Pharmaceutical } \\
\text { care } \\
\text { G2: Usual care }\end{array}$} & $\begin{array}{l}\text { G1: } 25 \\
\text { G2: } 26\end{array}$ & $\begin{array}{l}\text { Hypertension-related } \\
\text { charges (SD) at } 6 \\
\text { months }\end{array}$ & $\begin{array}{l}\text { Calculated mean difference: } \\
\$ 70.00,95 \% \mathrm{Cl}: \$ 15.97 \text { to } \\
\$ 124.03, p=0.011\end{array}$ \\
\hline & & & $\begin{array}{l}\text { Mean visit charges at } 6 \\
\text { months }\end{array}$ & $\begin{array}{l}\text { Calculated mean difference: } \\
\$ 487.00,95 \% \mathrm{Cl}: \$ 44.87 \text { to } \\
\$ 929.14, p=0.031\end{array}$ \\
\hline $\begin{array}{l}\text { Chrischilles et al., } \\
2004^{63} \\
\text { Cohort/High }\end{array}$ & $\begin{array}{l}\text { G1: PCM-eligible } \\
\text { patients who received } \\
\text { PCM services } \\
\text { G2: PCM-eligible } \\
\text { patients who did not } \\
\text { receive PCM services }\end{array}$ & $\begin{array}{l}\text { G1: } 524 \\
\text { G2: } 1,687\end{array}$ & $\begin{array}{l}\text { Outpatient facility claims } \\
\text { at } 12 \text { months }\end{array}$ & Results NR, $p=0.107$ \\
\hline
\end{tabular}

Abbreviations: $\mathrm{CAD}=$ Canadian dollar; $\mathrm{CI}=$ confidence interval; $\mathrm{NR}=$ not reported; $\mathrm{RCT}=$ randomized controlled trial; $\mathrm{SD}=$ standard deviation

Based on the lack of consistency and precision, we graded the body of evidence from the two trials as being insufficient to evaluate the effect of MTM interventions on the costs of outpatient visits (Table 58).

Table 58. Costs of outpatient resource utilization: Strength of evidence

\begin{tabular}{|c|c|c|c|c|c|}
\hline $\begin{array}{l}\text { Study } \\
\text { Design }\end{array}$ & $\begin{array}{l}\text { Number of } \\
\text { Studies; Subjects } \\
\text { (Analyzed) }\end{array}$ & $\begin{array}{l}\text { Study } \\
\text { Limitations }\end{array}$ & Consistency Directness Precision & $\begin{array}{l}\text { Findings and } \\
\text { Direction } \\
\text { [Magnitude] of } \\
\text { Effect }\end{array}$ & $\begin{array}{l}\text { Strength of } \\
\text { Evidence }\end{array}$ \\
\hline $\mathrm{RCT}$ & $2 ; 1,943(1,842)$ & Medium & Inconsistent & Variable & Insufficient \\
\hline
\end{tabular}

Abbreviations: RCT= randomized controlled trial.

\section{Number of Laboratory Tests}

Understanding whether a change in the number and costs of laboratory tests as a result of an MTM intervention measures appropriate resource use requires knowledge of the goals of drug therapy. MTM could raise numbers and costs of laboratory tests by identifying patients who should be receiving more frequent laboratory monitoring or by starting patients on new drugs that require laboratory monitoring based on their clinical situation. However, MTM could also lower numbers and costs of laboratory tests if it produces better coordination of care and 
prevents duplicative testing. Included studies did not specify the expected direction of effect from MTM on the number and costs of laboratory tests.

Two trials (both medium risk of bias; one set in the United States ${ }^{40-43}$ the other in Canada ${ }^{44}$ ) reported on the number laboratory tests following MTM interventions (Table 59). The Canadian study included the number and costs of imaging procedures over a 5-month period; ${ }^{44}$ the USbased study did not specify the inclusion of imaging procedures and evaluated tests and costs over a 12-month period. The US-based found statistically significant differences; the Canadian study failed to find any significant differences.

Table 59. Number of laboratory tests: Summary of results

\begin{tabular}{|c|c|c|c|c|}
\hline $\begin{array}{l}\text { Study } \\
\text { Design/Risk of } \\
\text { bias }\end{array}$ & Study Arms & $\mathbf{N}$ analyzed & $\begin{array}{l}\text { Outcome and Time } \\
\text { Period }\end{array}$ & Results \\
\hline $\begin{array}{l}\text { Malone, } 2000^{40} \text {; } \\
\text { Ellis, 2000 } \\
\text { (interventions); } \\
\text { Malone, 2001 } \\
\text { (detailed QOL } \\
\text { outcomes); } \\
\text { Ellis, 2000 } \\
\text { RCT/Medium }\end{array}$ & $\begin{array}{l}\text { G1: Pharmaceutical } \\
\text { care } \\
\text { G2: Usual care }\end{array}$ & $\begin{array}{l}\text { G1: } 523 \\
\text { G2: } 531\end{array}$ & $\begin{array}{l}\text { Mean change in annual } \\
\text { number of laboratory } \\
\text { tests }\end{array}$ & $\begin{array}{l}\text { Calculated mean difference: } \\
-1.6,95 \% \mathrm{Cl}:-2.550 \text { to }-0.650 \text {, } \\
p=0.001\end{array}$ \\
\hline $\begin{array}{l}\text { Sellors et al., } \\
2003^{44} \\
\text { RCT/Medium }\end{array}$ & $\begin{array}{l}\text { G1: Pharmacist } \\
\text { consultation program } \\
\text { G2: Usual care }\end{array}$ & $\begin{array}{l}\text { G1: } 379 \\
\text { G2: } 409\end{array}$ & $\begin{array}{l}\text { Mean number of } \\
\text { laboratory tests and } \\
\text { imaging procedures at } 5 \\
\text { months }\end{array}$ & $\begin{array}{l}\text { Calculated mean difference: } \\
0.15,95 \% \mathrm{Cl}:-0.959 \text { to } 1.259 \text {, } \\
p=0.791\end{array}$ \\
\hline
\end{tabular}

Abbreviations: $\mathrm{RCT}=$ randomized controlled trial.

The small number of studies limits our ability to explore causes for the observed heterogeneity. Factors such as differences in health systems, period of evaluation, and definition of the outcome could explain differences in results. Based on lack of consistency, we graded the body of evidence from these two medium risk of bias trials as insufficient to evaluate either the effect of MTM interventions on the number of laboratory tests (Table 60).

Table 60 . Number of laboratory tests: Strength of evidence

\begin{tabular}{llllll}
\hline $\begin{array}{l}\text { Study } \\
\text { Design }\end{array}$ & $\begin{array}{l}\text { Number of } \\
\text { Studies; Subjects } \\
\text { (Analyzed) }\end{array}$ & $\begin{array}{l}\text { Study } \\
\text { Limitations }\end{array}$ & Consistency Directness Precision & $\begin{array}{l}\text { Findings and } \\
\text { [Magnitude] of } \\
\text { Effect }\end{array}$ & $\begin{array}{l}\text { Strength of } \\
\text { Evidence }\end{array}$ \\
\hline RCT & $2 ; 1,943(1,842)$ & Medium & Inconsistent & Indirect & Imprecise \\
& & & $\begin{array}{l}\text { Differences range } \\
\text { from }+0.15 \text { to }-1.6\end{array}$ & Insufficient \\
& & & tests & \\
\hline
\end{tabular}

Abbreviations: $\mathrm{RCT}=$ randomized controlled trial.

\section{Costs of Laboratory Tests}

The two studies reporting data on number of laboratory tests also provided information on costs. ${ }^{40-43}$ The challenges associated with interpreting evidence on number of laboratory tests apply to costs as well (Table 61). 
Table 61. Costs of laboratory tests: Summary of results

\begin{tabular}{|c|c|c|c|c|}
\hline $\begin{array}{l}\text { Study } \\
\text { Design/Risk of } \\
\text { Bias }\end{array}$ & Study Arms & N Analyzed & $\begin{array}{l}\text { Outcome and Time } \\
\text { Period }\end{array}$ & Results \\
\hline $\begin{array}{l}\text { Malone, } 2000^{40} ; \\
\text { Ellis, } 2000^{41} ; \\
\text { Malone, } 2001 ;{ }^{43} \\
\text { Ellis, } 2000^{42} \\
\text { RCT/Medium }\end{array}$ & $\begin{array}{l}\text { G1: Pharmaceutical } \\
\text { care } \\
\text { G2: Usual care }\end{array}$ & $\begin{array}{l}\text { G1: } 523 \\
\text { G2: } 531\end{array}$ & $\begin{array}{l}\text { Mean change in annual } \\
\text { costs for laboratory tests }\end{array}$ & $\begin{array}{l}\text { Calculated mean difference: } \\
-\$ 33.00 \\
95 \% \mathrm{Cl}:-\$ 65.96 \text { to }-\$ 0.04 \\
p=0.05\end{array}$ \\
\hline $\begin{array}{l}\text { Sellors et al., } \\
2003^{44} \\
\text { RCT/Medium }\end{array}$ & $\begin{array}{l}\text { G1: Pharmacist } \\
\text { consultation program } \\
\text { G2: Usual care }\end{array}$ & $\begin{array}{l}\text { G1: } 379 \\
\text { G2: } 409\end{array}$ & $\begin{array}{l}\text { Mean cost of all lab and } \\
\text { imaging procedures at } 5 \\
\text { months (assumed CAD) }\end{array}$ & $\begin{array}{l}\text { Calculated mean difference: } \\
\$ 6.24 \text { (assumed CAD) } \\
95 \% \mathrm{Cl}:-\$ 46.34 \text { to } \$ 58.88 \\
p=0.816\end{array}$ \\
\hline
\end{tabular}

Abbreviations: CAD $=$ Canadian dollar

Based on lack of consistency, we graded the body of evidence from these two medium risk of bias trials as insufficient to evaluate either the effect of MTM interventions on the costs of laboratory tests (Table 62).

Table 62. Costs of laboratory tests: Strength of evidence

\begin{tabular}{|c|c|c|c|c|c|c|c|}
\hline $\begin{array}{l}\text { Study } \\
\text { Design }\end{array}$ & $\begin{array}{l}\text { Number of } \\
\text { Studies; } \\
\text { Subjects } \\
\text { (Analyzed) }\end{array}$ & $\begin{array}{l}\text { Study } \\
\text { Limitations }\end{array}$ & Consistency & Directness & Precision & $\begin{array}{l}\text { Findings and } \\
\text { Direction } \\
\text { [Magnitude] of } \\
\text { Effect }\end{array}$ & $\begin{array}{l}\text { Strength } \\
\text { of } \\
\text { Evidence }\end{array}$ \\
\hline RCT & $\begin{array}{l}2 ; 1943 \\
(1842)\end{array}$ & Medium & Inconsistent & Indirect & Imprecise & $\begin{array}{l}\text { Differences } \\
\text { range from } \\
+0.62 \text { CAD to } \\
-33 \text { USD }\end{array}$ & Insufficient \\
\hline
\end{tabular}

Abbreviations: CAD, Canadian dollars; RCT $=$ randomized controlled trial; USD, US dollars.

\section{Emergency Department Visits}

Four studies in all reported changes in emergency department (ED) visits following MTM interventions: three trials (two medium risk of bias ${ }^{44,55}$ and one high risk-of-bias ${ }^{44,68}$ ) and one cohort study (medium risk of bias) ${ }^{37}$ (Table 63). With the exception of one arm from the Touchette et al. trial, ${ }^{55}$ the confidence intervals for the effects from the medium risk-of-bias studies spanned the null effect. ${ }^{37,44}$ One trial, rated high risk of bias for this outcome, reported a decline in ED visits in the intervention arm and no change in the control arm; ${ }^{68}$ it did not, however, provide patient-level means. As a result, we are unable to judge the variance within the sample. 
Table 63. Emergency department visits: Summary of results

\begin{tabular}{|c|c|c|c|c|}
\hline $\begin{array}{l}\text { Study } \\
\text { Design/Risk of } \\
\text { Bias }\end{array}$ & Study Arms & N Analyzed & $\begin{array}{l}\text { Outcome and Time } \\
\text { Period }\end{array}$ & Results \\
\hline $\begin{array}{l}\text { Sellors et al., } \\
2003^{44} \\
\text { RCT/Medium }\end{array}$ & $\begin{array}{l}\text { G1: Pharmacist } \\
\text { consultation program } \\
\text { G2: Usual care }\end{array}$ & $\begin{array}{l}\text { G1: } 379 \\
\text { G2: } 409\end{array}$ & $\begin{array}{l}\text { Mean number of ED or } \\
\text { urgent care visits and } \\
\text { ambulance use at } 5 \\
\text { months }\end{array}$ & $\begin{array}{l}\text { Calculated mean difference: } \\
-0.03 \\
95 \% \mathrm{Cl}:-0.113 \text { to } 0.053 p=0.48\end{array}$ \\
\hline $\begin{array}{l}\text { Touchette et al., } \\
2012^{55} \\
\text { RCT/Medium }\end{array}$ & $\begin{array}{l}\text { G1: MTM basic } \\
\text { (comprehensive } \\
\text { medication review and } \\
\text { DRP assessment) } \\
\text { G2: MTM enhanced } \\
\text { (MTM plus 2-page } \\
\text { clinical summary } \\
\text { abstracted from } \\
\text { patient's medical chart). } \\
\text { G3: Usual care }\end{array}$ & $\begin{array}{l}\text { G1: } 183 \\
\text { G2: } 190 \\
\text { G3: } 183\end{array}$ & $\begin{array}{l}\text { Mean number of ED } \\
\text { visits per participant } \\
\text { between 3-6 months } \\
\text { after intervention }\end{array}$ & $\begin{array}{l}\text { G1 vs. G3 calculated mean } \\
\text { difference: }-0.138 \\
95 \% \mathrm{Cl}:-0.258 \text { to }-0.018 \\
p=0.025 \\
\text { G2 vs. G3 calculated mean } \\
\text { difference: }-0.118 \\
95 \% \mathrm{Cl}:-0.242 \text { to } 0.006 \\
p=0.062\end{array}$ \\
\hline $\begin{array}{l}\text { Taylor et al., } \\
2003^{68} \\
\text { RCT/High }\end{array}$ & $\begin{array}{l}\text { G1: Pharmaceutical } \\
\text { care group } \\
\text { G2: Standard care }\end{array}$ & $\begin{array}{l}\text { G1: } 33 \\
\text { G2: } 36\end{array}$ & $\begin{array}{l}\text { Change in number of } \\
\text { ED visits from } 12 \\
\text { months before baseline } \\
\text { through } 12 \text { months after }\end{array}$ & $\begin{array}{l}\mathrm{G} 1:-12 \\
\mathrm{G} 2: 0 \\
p=0.044\end{array}$ \\
\hline $\begin{array}{l}\text { Welch et al., } \\
2009^{37} \\
\text { Cohort/medium }\end{array}$ & $\begin{array}{l}\text { G1: MTM program } \\
\text { provided to home- } \\
\text { based beneficiaries } \\
\text { G2: No-MTM control } \\
\text { group (voluntary opt- } \\
\text { out) }\end{array}$ & $\begin{array}{l}\text { G1: } 459 \\
\text { G2: } 336\end{array}$ & $\begin{array}{l}\text { Adjusted OR of ED visit } \\
\text { from } 6 \text { month before } \\
\text { MTM through } 6 \text { months } \\
\text { after MTM (adjusted for } \\
\text { age, sex, chronic } \\
\text { disease score, specific } \\
\text { baseline utilization) }\end{array}$ & $\begin{array}{l}\text { Reported adjusted OR: } 0.9 \\
95 \% \mathrm{Cl}: 0.6 \text { to } 1.3, \mathrm{p} \mathrm{NR}\end{array}$ \\
\hline
\end{tabular}

Abbreviations: $\mathrm{CI}=$ confidence interval; $\mathrm{DRP}=$ drug related problems; $\mathrm{ED}=$ emergency department; $\mathrm{MTM}=$ medication therapy management; $\mathrm{NR}=$ not reported; $\mathrm{OR}=$ odds ratio; $\mathrm{RCT}=$ randomized controlled trial.

Given the lack of consistency and precision, evidence is insufficient to draw conclusions about the effectiveness of MTM in reducing ED visits (Table 64).

Table 64. Emergency department visits: Strength of evidence

\begin{tabular}{|c|c|c|c|c|c|c|c|}
\hline $\begin{array}{l}\text { Study } \\
\text { Design }\end{array}$ & $\begin{array}{l}\text { Number of } \\
\text { Studies; } \\
\text { Subjects } \\
\text { (Analyzed) }\end{array}$ & $\begin{array}{l}\text { Study } \\
\text { Limitations }\end{array}$ & Consistency & Directness & Precision & $\begin{array}{l}\text { Findings and } \\
\text { Direction } \\
\text { [Magnitude] of } \\
\text { Effect }\end{array}$ & $\begin{array}{l}\text { Strength of } \\
\text { Evidence }\end{array}$ \\
\hline RCT & $\begin{array}{l}2 ; 1,526 \\
(1,344)\end{array}$ & Medium & Inconsistent & Direct & Imprecise & $\begin{array}{l}\text { Mean difference } \\
\text { ranges from }-0.03 \\
\text { to }-0.138\end{array}$ & Insufficient \\
\hline \multicolumn{2}{|c|}{ Observational 1; 904 (795) } & High & $\begin{array}{l}\text { Consistency } \\
\text { unknown-single } \\
\text { study }\end{array}$ & Direct & Imprecise & $\begin{array}{l}\text { Adjusted OR: } 0.9 \\
(0.6 \text { to } 1.3)\end{array}$ & Insufficient \\
\hline
\end{tabular}

Abbreviations: $\mathrm{OR}=$ odds ratio; $\mathrm{RCT}=$ randomized controlled trial.

\section{Emergency Department Costs}

One trial (medium risk of bias) ${ }^{44}$ and one cohort study (high risk of bias) ${ }^{63}$ reported on costs of ED visits following MTM interventions (Table 65). Despite differences in geographic setting and health care delivery systems (Canada ${ }^{44}$ and the United States ${ }^{63}$ ), period of evaluation ( 5 months, ${ }^{44}$ and 12 months ${ }^{63}$ ), and risk or bias, neither study demonstrated an effect of any MTMtype intervention (Table 65). ${ }^{44,63}$ Based on lack of precision, we graded the medium risk-of-bias study as being insufficient evidence to evaluate the effect of MTM interventions on the cost of ED visits (Table 66). 
Table 65. Costs of emergency department visits: Summary of results

\begin{tabular}{|c|c|c|c|c|c|c|}
\hline \multicolumn{3}{|l|}{ Study } & $\mathbf{N}$ analyzed & & & \\
\hline \multicolumn{2}{|c|}{$\begin{array}{l}\text { Design/Risk of } \\
\text { bias }\end{array}$} & \multicolumn{2}{|l|}{ Study Arms } & $\begin{array}{l}\text { Outcome and Time } \\
\text { Period }\end{array}$ & \multicolumn{2}{|l|}{ Results } \\
\hline \multicolumn{2}{|c|}{$\begin{array}{l}\text { Sellors et al., } \\
2003^{44} \\
\text { RCT/Medium }\end{array}$} & $\begin{array}{l}\text { G1: Pharmacist } \\
\text { consultation program } \\
\text { G2: Usual care }\end{array}$ & $\begin{array}{l}\text { G1: } 379 \\
\text { G2: } 409\end{array}$ & $\begin{array}{l}\text { Mean cost of ED or } \\
\text { urgent care visits and } \\
\text { ambulance use at } 5 \\
\text { months in } \$ \text { (assumed } \\
\text { CAD) (SE) }\end{array}$ & \multicolumn{2}{|c|}{$\begin{array}{l}\text { Calculated mean difference: } \\
-\$ 5.60 \text { (assumed CAD) } \\
95 \% \text { Cl:- } \$ 23.06 \text { to } \$ 11.86 \\
p=0.53\end{array}$} \\
\hline \multicolumn{2}{|c|}{$\begin{array}{l}\text { Chrischilles et } \\
\text { al., } 2004^{63} \\
\text { Cohort/High }\end{array}$} & \multicolumn{2}{|c|}{$\begin{array}{ll}\text { G1: PCM-eligible } & \text { G1: } 524 \\
\text { patients who received } & \text { G2: } 1,687 \\
\text { PCM services } & \\
\text { G2: PCM-eligible } & \\
\text { patients who did not } & \\
\text { receive PCM services } & \\
\end{array}$} & $\begin{array}{l}\text { Charges for ED claims } \\
\text { at } 12 \text { months }\end{array}$ & \multicolumn{2}{|l|}{$P=0.513$} \\
\hline \multicolumn{7}{|c|}{$\begin{array}{l}\text { Abbreviations: CAD: Canadian dollar, } \mathrm{CI}=\text { confidence interval; } \mathrm{ED}=\text { Emergency department; } \mathrm{NR}=\text { not reported; } \mathrm{PCM}= \\
\text { pharmaceutical care management; } \mathrm{SE}=\text { standard error }\end{array}$} \\
\hline $\begin{array}{l}\text { Study } \\
\text { Design }\end{array}$ & $\begin{array}{l}\text { Numbe } \\
\text { Studies } \\
\text { Subjec } \\
\text { (Analyz }\end{array}$ & $\begin{array}{l}\text { Study } \\
\text { Limitations }\end{array}$ & Consistency & Directness Precision & $\begin{array}{l}\text { Findings and } \\
\text { Direction } \\
\text { [Magnitude] of } \\
\text { Effect }\end{array}$ & $\begin{array}{l}\text { Strength of } \\
\text { Evidence }\end{array}$ \\
\hline$\overline{R C T}$ & $\begin{array}{l}1 ; 889 \\
(779)\end{array}$ & Medium & $\begin{array}{l}\text { Consistency } \\
\text { unknown-single } \\
\text { study }\end{array}$ & Imprecise & $\begin{array}{l}\text { Mean difference: } \\
-\$ 5.60 \text { (assumed } \\
\text { CAD) } \\
95 \% \mathrm{Cl}:-\$ 23.06 \text { to } \\
\$ 11.86, p=0.53\end{array}$ & Insufficient \\
\hline
\end{tabular}

Abbreviations: $\mathrm{CAD}=$ Canadian dollar; $\mathrm{RCT}=$ randomized controlled trial

\section{Hospitalizations}

Nine studies measured hospitalizations as an outcome following MTM interventions. ${ }^{37,40-}$ $44,51,52,54,55,59,60,62,73$ Of these, we have excluded data from one study in the analysis below because it reported total number of events by each intervention arm rather than by patients within intervention arm. As a result, we are unable to assess variance. ${ }^{73}$ We report on the mean number of hospitalizations, the risk of hospitalization, and the rates of hospitalization (Table 67).

Table 67. Hospitalizations: Mean number, risk and rates

\begin{tabular}{|c|c|c|c|c|}
\hline $\begin{array}{l}\text { Study } \\
\text { Design/Risk of } \\
\text { Bias }\end{array}$ & Study Arms & N Analyzed & $\begin{array}{l}\text { Outcome and Time } \\
\text { Period }\end{array}$ & Results \\
\hline $\begin{array}{l}\text { Malone, 2000 } \\
\text { Ellis, } 2000^{41} \\
\text { (interventions); } \\
\text { Malone, 2001 } \\
\text { (detailed QOL } \\
\text { outcomes); } \\
\text { Ellis, 2000 } \\
\text { RCT/Medium }\end{array}$ & $\begin{array}{l}\text { G1: Pharmaceutical } \\
\text { care } \\
\text { G2: Usual care }\end{array}$ & $\begin{array}{l}\text { G1: } 523 \\
\text { G2: } 531\end{array}$ & $\begin{array}{l}\text { Mean change in number } \\
\text { of hospitalizations }\end{array}$ & $\begin{array}{l}\text { Calculated mean difference: } \\
0.06,95 \% \mathrm{Cl}:-0.051 \text { to } 0.171 \text {, } \\
p=0.29\end{array}$ \\
\hline \multirow[t]{2}{*}{$\begin{array}{l}\text { Sellors et al., } \\
2003^{44} \\
\text { RCT/Medium }\end{array}$} & $\begin{array}{l}\text { G1: Pharmacist } \\
\text { consultation program } \\
\text { G2: Usual care }\end{array}$ & $\begin{array}{l}\text { G1: } 379 \\
\text { G2: } 409\end{array}$ & $\begin{array}{l}\text { Mean number of all- } \\
\text { cause hospitalizations }\end{array}$ & $\begin{array}{l}\text { Calculated mean difference: } \\
-0.03 \\
95 \% \mathrm{Cl}:-0.085 \text { to } 0.025 \\
p=0.289\end{array}$ \\
\hline & & & $\begin{array}{l}\text { Mean drug- } \\
\text { (medications) related } \\
\text { hospitalizations }\end{array}$ & $\begin{array}{l}\text { Calculated mean difference: } 0 \\
95 \% \mathrm{Cl}:-0.28 \text { to } 0.28 \\
p=1.0\end{array}$ \\
\hline
\end{tabular}


Table 67. Hospitalizations: Mean number, risk and rates (continued)

\begin{tabular}{|c|c|c|c|c|}
\hline $\begin{array}{l}\text { Study } \\
\text { Design/Risk of } \\
\text { Bias }\end{array}$ & Study Arms & N Analyzed & $\begin{array}{l}\text { Outcome and Time } \\
\text { Period }\end{array}$ & Results \\
\hline \multirow[t]{3}{*}{$\begin{array}{l}\text { Touchette et al., } \\
2012^{55} \\
\text { RCT/Medium }\end{array}$} & \multirow{3}{*}{$\begin{array}{l}\text { G1: MTM basic } \\
\text { (comprehensive } \\
\text { medication review and } \\
\text { DRP assessment) } \\
\text { G2: MTM enhanced } \\
\text { (MTM plus } 2 \text { page } \\
\text { clinical summary } \\
\text { abstracted from } \\
\text { patient's medical } \\
\text { chart). } \\
\text { G3: Usual care }\end{array}$} & $\begin{array}{l}\text { Time One } \\
\text { G1: } 180 \\
\text { G2: } 190 \\
\text { G3: } 193 \\
\text { Time Two } \\
\text { G1: } 183 \\
\text { G2: } 190\end{array}$ & $\begin{array}{l}\text { Percentage of } \\
\text { participants with at least } \\
\text { one hospital visit at } 3 \text { to } \\
6 \text { months }\end{array}$ & $\begin{array}{l}\text { G1 vs. G3: Calculated OR: } \\
2.069 \text {, } \\
95 \% \mathrm{Cl}: 1.104 \text { to } 3.878 \mathrm{p}=0.02 \\
\text { G2 vs. G3: Calculated OR: } \\
1.345 \\
95 \% \mathrm{Cl}:-0.9=693 \text { to } 2.609 \\
p=0.381\end{array}$ \\
\hline & & G3: 183 & $\begin{array}{l}\text { Mean number of } \\
\text { hospital visits per } \\
\text { participant }\end{array}$ & $\begin{array}{l}\text { G1 vs. G3: Calculated mean } \\
\text { difference: } 0.039 \\
95 \% \mathrm{Cl} \text { : }-0.047 \text { to } 0.125 \\
p=0.37\end{array}$ \\
\hline & & & & $\begin{array}{l}\text { G2 vs. G3: Calculated mean } \\
\text { difference: } 0.045,95 \% \mathrm{Cl} \text { - } \\
0.037 \text { to } 0.127, p=0.28\end{array}$ \\
\hline \multirow[t]{2}{*}{$\begin{array}{l}\text { Triller et al., } 2007^{54} \\
\text { RCT/Medium }\end{array}$} & \multirow{2}{*}{$\begin{array}{l}\text { G1: Visiting nurse } \\
\text { association home visit } \\
\text { services plus } \\
\text { comprehensive } \\
\text { pharmaceutical care } \\
\text { services } \\
\text { G2: Visiting nurse } \\
\text { association home visit } \\
\text { services }\end{array}$} & \multirow[t]{2}{*}{$\begin{array}{l}\text { G1: } 77 \\
\text { G2: } 77\end{array}$} & $\begin{array}{l}\text { Percentage with any } \\
\text { hospitalization, all } \\
\text { causes }\end{array}$ & $\begin{array}{l}\text { RR } 0.93, \\
\text { Calculated } 95 \% \mathrm{Cl}: 0.707 \text { to } \\
1.232 \\
\text { p: } 0.63\end{array}$ \\
\hline & & & $\begin{array}{l}\text { Percentage with any } \\
\text { hospitalization related to } \\
\text { heart failure }\end{array}$ & $\begin{array}{l}\text { RR: } 0.82, \\
\text { Calculated } 95 \% \text { Cl: } 0.581 \text { to } \\
1.581 \\
\text { p: } 0.26\end{array}$ \\
\hline $\begin{array}{l}\text { Bernsten et al., } \\
2001^{51,52} \\
\text { RCT/High }\end{array}$ & $\begin{array}{l}\text { G1: Structured } \\
\text { community pharmacy- } \\
\text { based pharmaceutical } \\
\text { care program } \\
\text { G2: Usual community } \\
\text { pharmacy services }\end{array}$ & $\begin{array}{l}\text { Baseline } \\
\text { G1: } 867 \\
\text { G2: } 748 \\
\text { 6 months } \\
\text { G1: NR } \\
\text { G2: NR } \\
\text { 12 months } \\
\text { G1: NR } \\
\text { G2: NR } \\
\text { 18 months } \\
\text { G1: NR } \\
\text { G2: NR }\end{array}$ & $\begin{array}{l}\text { Percentage with } \geq 1 \\
\text { hospitalization in the } \\
\text { prior } 18 \text { months }\end{array}$ & $\begin{array}{l}\text { Pooled sample } \\
\text { Baseline (during } 18 \text { months } \\
\text { before study) } \\
\text { Overall: NR } \\
\text { G1: } 41.7 \\
\text { G2: } 41.3 \\
p=N S \\
18 \text { months } \\
\text { Overall: NR } \\
\text { G1: } 35.6 \\
\text { G2: } 40.4 \\
p=N S \text {, cannot be calculated } \\
\text { without N }\end{array}$ \\
\hline \multirow[t]{2}{*}{$\begin{array}{l}\text { Pai, } 2009^{59} ; \text { Pai, } \\
2009^{60} \\
\text { RCT/High }\end{array}$} & \multirow[t]{2}{*}{$\begin{array}{l}\text { G1: Pharmaceutical } \\
\text { care } \\
\text { G2: Usual care }\end{array}$} & \multirow[t]{2}{*}{$\begin{array}{l}\text { G1: NR } \\
\text { G2: NR }\end{array}$} & $\begin{array}{l}\text { Mean number of all- } \\
\text { cause hospitalizations } \\
\text { over } 2 \text { years }\end{array}$ & $\begin{array}{l}\text { G1: } 1.8(2.4) \\
\text { G2: } 3.1(3) \\
95 \% \mathrm{Cl}: \mathrm{NR} \text {, cannot be } \\
\text { calculated without } \mathrm{N} \\
\text { p: } 0.02\end{array}$ \\
\hline & & & $\begin{array}{l}\text { Cumulative hospitalized } \\
\text { time in days (SD) }\end{array}$ & $\begin{array}{l}\text { Cumulative hospital time } \\
\text { G1: } 9.7 \text { days (14.7) } \\
\text { G2: } 15.5 \text { days }(16.3) \\
95 \% \text { Cl: NR, cannot be } \\
\text { calculated without } N \\
p=0.06\end{array}$ \\
\hline
\end{tabular}


Table 67. Hospitalizations: Mean number, risk and rates (continued)

\begin{tabular}{|c|c|c|c|c|}
\hline $\begin{array}{l}\text { Study } \\
\text { Design/Risk of } \\
\text { Bias }\end{array}$ & Study Arms & N Analyzed & $\begin{array}{l}\text { Outcome and Time } \\
\text { Period }\end{array}$ & Results \\
\hline $\begin{array}{l}\text { Roughead, } 2009^{62} \\
\text { Cohort/Medium }\end{array}$ & $\begin{array}{l}\text { G1: Collaborative } \\
\text { home-based } \\
\text { medication review } \\
\text { G2: No medication } \\
\text { review received }\end{array}$ & $\begin{array}{l}\text { G1: } 273 \\
\text { G2: } 5,444\end{array}$ & $\begin{array}{l}\text { Rate of hospitalization } \\
\text { for heart failure at any } \\
\text { time during study }\end{array}$ & $\begin{array}{l}\text { Adjusted HR (): } 0.5595 \% \mathrm{Cl} \text { : } \\
0.39 \text { to } 0.77 \\
\text { p: NR } \\
\text { NOTE: Model adjusted for age, } \\
\text { sex, comorbidity, SES, season, } \\
\text { region of residence, and } \\
\text { numbers of prescriptions, } \\
\text { prescribers, pharmacies, } \\
\text { changes in medications, } \\
\text { hospitalizations, occupational } \\
\text { therapy visits, and speech } \\
\text { therapy visits }\end{array}$ \\
\hline $\begin{array}{l}\text { Welch et al., } 2009^{37} \\
\text { Cohort } \\
\text { study/Medium }\end{array}$ & $\begin{array}{l}\text { G1: MTM program } \\
\text { provided to home- } \\
\text { based beneficiaries } \\
\text { G2: No-MTM control } \\
\text { group (voluntary opt- } \\
\text { out) }\end{array}$ & $\begin{array}{l}\text { G1: } 459 \\
\text { G2: } 336\end{array}$ & $\begin{array}{l}\text { Adjusted OR of } \\
\text { hospitalization from } 6 \\
\text { month before MTM } \\
\text { through } 6 \text { months after } \\
\text { (adjusted for age, sex, } \\
\text { chronic disease score, } \\
\text { specific baseline } \\
\text { utilization) }\end{array}$ & $\begin{array}{l}\text { Reported adjusted OR: } 1.4 \\
95 \% \mathrm{CI}) 1.1 \text { to } 2.0 \\
\text {; } \mathrm{p} \text { values NR } \\
\text { NOTE: Model adjusted for age, } \\
\text { sex, chronic disease score, } \\
\text { specific baseline utilization }\end{array}$ \\
\hline
\end{tabular}

Abbreviations: $\mathrm{CI}=$ confidence interval; DRP = drug-related problems; $\mathrm{HR}=$ hazard ratio MTM = medication therapy management, $\mathrm{NR}=$ not reported; $\mathrm{NS}=$ not significant; $\mathrm{OR}=$ odds ratio; $\mathrm{RCT}=$ randomized controlled trials; $\mathrm{RR}=$ relative risk; $\mathrm{SES}=$ socioeconomic status

Three trials (medium risk-of-bias) reported on the change in number of hospitalizations or mean number of hospitalizations following MTM interventions (Table 67). ${ }^{40-44,55}$ Using a random-effects model, we pooled these results for all-cause hospitalizations and obtained a mean difference of $0.038\left(95 \% \mathrm{CI},-0.005\right.$ to $\left.0.080 ; \mathrm{p}=0.085 ; \mathrm{I}^{2}=0\right)$ (Appendix G-14). We obtained similarly small effect sizes and wide confidence intervals spanning the null when including each arm of the Touchette et al. study separately ${ }^{55}$ or including the single high risk-of-bias trial. ${ }^{60}$ One study also provided data to calculate an effect size and confidence intervals for drug-related hospitalizations that also overlapped the null effect. ${ }^{44}$

Four studies (two medium-risk-of-bias RCTs, ${ }^{54,55}$ one high risk-of-bias RCT, ${ }^{51,52}$ and one medium risk-of-bias cohort study ${ }^{37}$ ) reported on the percent hospitalized following MTM (Table 67), based on percentages of patients hospitalized or odds or hazard ratios of hospitalization. Not all studies provided sufficient data to allow the generation of a summary estimate of effect with confidence intervals. The results are inconsistent; two studies suggested an higher hospitalizations with MTM rather than usual care ${ }^{37,55}$ and two suggested lower hospitalizations (but with confidence intervals overlapping the null). ${ }^{51,52,54}$ The inconsistency in results may be a consequence of the wide range of included populations, from a relatively homogenous group of home care patients with heart failure ${ }^{54}$ to veterans in ambulatory care..$^{51,52}$

One cohort study (medium risk-of-bias) reported a decreased rate of hospitalization for heart failure at any time during study. This study of home medications review was designed specifically to delay the next hospitalization among patients with heart failure in Australia. ${ }^{62}$

Based on consistent results from studies with medium study limitations that we rated as precise because of their narrow confidence intervals around the null, we rated MTM as having no effect on the mean number of hospitalizations (low strength of evidence, Table 68). We rated the evidence on the percent hospitalized as insufficient based on inconsistent and imprecise evidence 
(Table 69). By contrast, we rated the evidence on the rate of hospitalization as low based on a precise estimate from a large cohort study (Table 70); we note that the findings from a single study of a very specific intervention (home medicines review) of heart failure patients limits its applicability to patients with other morbidities and settings. Together, the lack of consistency across these measures of hospitalization likely reflects heterogeneity in numerous factors in this evidence base.

Table 68. Mean number of hospitalizations: Strength of evidence

\begin{tabular}{|c|c|c|c|c|c|c|c|}
\hline Study Design & $\begin{array}{l}\text { Number of } \\
\text { Studies; } \\
\text { Subjects } \\
\text { (Analyzed) }\end{array}$ & $\begin{array}{l}\text { Study } \\
\text { Limitations }\end{array}$ & Consistency & Directness & Precision & $\begin{array}{l}\text { Findings } \\
\text { and } \\
\text { Direction } \\
\text { [Magnitude] } \\
\text { of Effect }\end{array}$ & $\begin{array}{l}\text { Strength } \\
\text { of } \\
\text { Evidence }\end{array}$ \\
\hline$\overline{\mathrm{RCT}}$ & $\begin{array}{l}3 ; 2,580 \\
(2,398)\end{array}$ & Medium & Consistent & Direct & Precise & $\begin{array}{l}\text { Mean } \\
\text { difference of } \\
0.038(95 \% \\
\mathrm{Cl}-0.005 \text { to } \\
0.080\end{array}$ & $\begin{array}{l}\text { Low for no } \\
\text { benefit }\end{array}$ \\
\hline
\end{tabular}

Abbreviations: $\mathrm{CI}=$ confidence intervals; $\mathrm{RCT}=$ randomized controlled trial

Table 69. Percentage of patients hospitalized: Strength of evidence

\begin{tabular}{|c|c|c|c|c|c|c|c|}
\hline Study Design & $\begin{array}{l}\text { Number of } \\
\text { Studies; } \\
\text { Subjects } \\
\text { (Analyzed) }\end{array}$ & $\begin{array}{l}\text { Study } \\
\text { Limitations }\end{array}$ & Consistency & Directness & Precision & $\begin{array}{l}\text { Findings } \\
\text { and } \\
\text { Direction } \\
\text { [Magnitude] } \\
\text { of Effect }\end{array}$ & $\begin{array}{l}\text { Strength } \\
\text { of } \\
\text { Evidence }\end{array}$ \\
\hline$\overline{R C T}$ & $\begin{array}{l}2 ; 791 \\
(710)\end{array}$ & Medium & Inconsistent & Direct & Imprecise & $\begin{array}{l}\text { Direction and } \\
\text { magnitude } \\
\text { varies }\end{array}$ & Insufficient \\
\hline Cohort & $\begin{array}{l}1 ; 904 \\
(795)\end{array}$ & High & $\begin{array}{l}\text { Consistency } \\
\text { unknown, } \\
\text { single study }\end{array}$ & Direct & Precise & $\begin{array}{l}\text { Adjusted OR } \\
(95 \% \mathrm{Cl}): 1.4 \\
(1.1 \text { to } 2.0)\end{array}$ & Insufficient \\
\hline
\end{tabular}

Abbreviations: $\mathrm{CI}=$ confidence interval; $\mathrm{OR}=$ odds ratio; $\mathrm{RCT}=$ randomized controlled trial

Table 70. Rate of hospitalization: Strength of evidence

\begin{tabular}{|c|c|c|c|c|c|c|}
\hline Study Design & $\begin{array}{l}\text { Number of } \\
\text { Studies; } \\
\text { Subjects } \\
\text { (Analyzed) }\end{array}$ & $\begin{array}{l}\text { Study } \\
\text { Limitations }\end{array}$ & Consistency & Directness & Precision & $\begin{array}{l}\text { Findings and Strength } \\
\text { Direction of } \\
\text { [Magnitude] of Evidence } \\
\text { Effect }\end{array}$ \\
\hline Cohort & $\begin{array}{l}1 ; 5,717 \\
(5,717)\end{array}$ & High & $\begin{array}{l}\text { Consistency } \\
\text { unknown, } \\
\text { single study }\end{array}$ & Direct & Precise & $\begin{array}{l}\text { Adjusted HR Low } \\
(95 \% \mathrm{Cl}): 0.55 \\
(0.39 \text { to } 0.77)\end{array}$ \\
\hline
\end{tabular}

Abbreviations: $\mathrm{CI}=$ confidence interval; $\mathrm{HR}=$ hazard ratio

\section{Hospitalization Costs}

Two trials (medium risk-of-bias) ${ }^{40-44}$ and one cohort study (high risk-of-bias) ${ }^{63}$ reported changes in costs of hospitalization following MTM interventions (Table 71). Although two studies were set in the United States, one evaluated outcomes from Veteran Affairs Medical Centers ${ }^{40-43}$ and the other evaluated claims from the Iowa Medicaid program. ${ }^{63}$ The third study was set in Canada. ${ }^{44}$ The period of evaluation of outcomes ranged from 5 months ${ }^{44}$ to 12 months. ${ }^{40-43,63}$ All were consistent in demonstrating no effect of MTM interventions on the costs of hospitalization. Based on lack of consistency in direction of effect and lack of precision, we graded the body of evidence as being insufficient to evaluate the effect of MTM interventions on the cost of hospitalization (Table 72). 
Table 71. Costs of hospitalization: Summary of results

\begin{tabular}{|c|c|c|c|c|}
\hline $\begin{array}{l}\text { Study } \\
\text { Design/Risk of } \\
\text { Bias }\end{array}$ & Study Arms & N Analyzed & $\begin{array}{l}\text { Outcome and Time } \\
\text { Period }\end{array}$ & Results \\
\hline $\begin{array}{l}\text { Sellors et al., } \\
2003^{44} \\
\text { RCT/Medium }\end{array}$ & $\begin{array}{l}\text { G1: Pharmacist } \\
\text { consultation program } \\
\text { G2: Usual care }\end{array}$ & $\begin{array}{l}\text { G1: } 379 \\
\text { G2: } 409\end{array}$ & $\begin{array}{l}\text { Mean cost of all } \\
\text { admissions to hospital } \\
\text { (assumed CAD) over } \\
\text { what time period }\end{array}$ & $\begin{array}{l}\text { Calculated mean difference: } \\
\$ 159.74 \text { (assumed CAD) } \\
95 \% \text { Cl: }-\$ 281.99 \text { to } \$ 601.47 \\
p=0.478\end{array}$ \\
\hline $\begin{array}{l}\text { Malone, } 2000^{40} \\
\text { Ellis, } 2000^{41} \\
\text { Malone, } 2001^{43} \\
\text { (Ellis, } 2000^{42} \\
\text { RCT/Medium }\end{array}$ & $\begin{array}{l}\text { G1: Pharmaceutical } \\
\text { care } \\
\text { G2: Usual care }\end{array}$ & $\begin{array}{l}\text { G1: } 523 \\
\text { G2: } 531\end{array}$ & $\begin{array}{l}\text { Mean change in annual } \\
\text { hospitalization costs }\end{array}$ & $\begin{array}{l}\text { Calculated mean difference: } \\
-\$ 221.00 \\
95 \% \mathrm{Cl}:-\$ 566.33 \text { to } \$ 124.33 \\
p=-0.21\end{array}$ \\
\hline $\begin{array}{l}\text { Chrischilles et al., } \\
2004^{63} \\
\text { Cohort/High }\end{array}$ & $\begin{array}{l}\text { G1: PCM-eligible } \\
\text { patients who received } \\
\text { PCM services } \\
\text { G2: PCM-eligible } \\
\text { patients who did not } \\
\text { receive PCM services }\end{array}$ & $\begin{array}{l}\text { G1: } 524 \\
\text { G2: } 1,687\end{array}$ & $\begin{array}{l}\text { Inpatient claims within } 9 \\
\text { months of becoming } \\
\text { eligible for PCM }\end{array}$ & Results NR, $p=0.937$ \\
\hline
\end{tabular}

Abbreviations: $\mathrm{CAD}=$ Canadian dollar; $\mathrm{NR}=$ not reported; $\mathrm{PCM}=$ pharmaceutical care management; $\mathrm{RCT}=$ randomized controlled trial.

Table 72. Cost of hospitalization: Strength of evidence

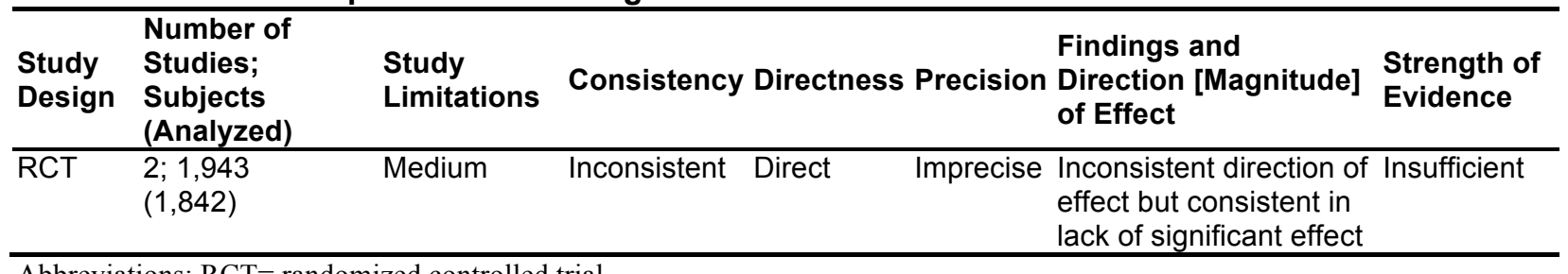

Abbreviations: $\mathrm{RCT}=$ randomized controlled trial.

\section{Hospital Length of Stay}

One trial (high risk of bias) ${ }^{59,60}$ reported that MTM interventions reduced length of hospital stay by 21 percent. Based on study limitations and lack of precision, we graded this outcome as having insufficient evidence to evaluate the effect of MTM interventions on the length of hospital visits (Table 73).

Table 73. Length of hospital stay: Strength of evidence

\begin{tabular}{|c|c|c|c|c|c|c|}
\hline $\begin{array}{l}\text { Study } \\
\text { Design }\end{array}$ & $\begin{array}{l}\text { Number of } \\
\text { Studies; } \\
\text { Subjects } \\
\text { (Analyzed) }\end{array}$ & $\begin{array}{l}\text { Study } \\
\text { Limitations }\end{array}$ & Consistency & Directness Precision & $\begin{array}{l}\text { Findings and } \\
\text { Direction } \\
\text { [Magnitude] of } \\
\text { Effect }\end{array}$ & $\begin{array}{l}\text { Strength of } \\
\text { Evidence }\end{array}$ \\
\hline RCT & $1 ; 107(46)$ & High & $\begin{array}{l}\text { Consistency } \\
\text { unknown-single } \\
\text { study }\end{array}$ & Imprecise & $\begin{array}{l}\text { MTM reduced } \\
\text { length of stay by } \\
21 \%\end{array}$ & Insufficient \\
\hline
\end{tabular}

Abbreviations: $\mathrm{RCT}=$ randomized controlled trial.

\section{Key Question 3: Outcomes of Medication Therapy Management by Intervention Features}

\section{Key Points}

- Studies do not routinely report outcomes of MTM by intervention features. 
- We found evidence on four intervention features informed by a single study for each feature: access to patient data, ${ }^{55}$ pharmacy intensity of adoption of the intervention, ${ }^{63}$ community pharmacy versus call-center pharmacy, ${ }^{46}$ and private versus Medicaid coverage of pharmaceutical care. ${ }^{47}$ With the exception of the investigation reporting on access to patient data, these studies had a high risk of bias.

- Evidence was insufficient on access to patient data, pharmacy intensity of adoption of the intervention, community pharmacy versus call-center pharmacy, and private versus Medicaid coverage of pharmaceutical care for most outcomes.

- MTM programs with pharmacist access to patient records reduces the number of adverse drug events (low strength of evidence) when compared basic MTM programs.

- Community pharmacists increase the generic dispensing ratio more than call-centerbased pharmacists (low strength of evidence).

\section{Detailed Synthesis: Intervention Features}

\section{Access to Patient Records}

A single trial (medium risk-of-bias) of 556 patients overall (373 in the two MTM arms) evaluated differences between two MTM intervention arms; one without access to patient records (denoted "basic" MTM and one specifically with such access in the form of a two-page clinical synopsis containing basic data on a patient's medical history, laboratory values, and current medications, including over-the-counter and herbal medications (denoted "enhanced MTM"). ${ }^{55}$ Table 74 provides the effect size and strength of evidence for the seven outcomes assessed in this trial. In all instances, we rated the trial as medium for study limitations and unknown for consistency; we do not repeat these ratings in the table. With the exception of mean number of adverse drug events, which suggested benefit for enhanced MTM when compared with basic MTM (low strength of evidence), we found insufficient evidence to evaluate the comparative effectiveness of the two arms.

Table 74. Access to patient records (basic MTM versus enhanced MTM): Strength of evidence

\begin{tabular}{|c|c|c|c|c|}
\hline Outcome & Directness & Precision & Findings and Direction [Magnitude] of Effect & $\begin{array}{l}\text { Strength of } \\
\text { Evidence }\end{array}$ \\
\hline $\begin{array}{l}\text { Percentage with } \geq 1 \\
A D E\end{array}$ & Direct & Imprecise & $\begin{array}{l}\text { Calculated OR: } 1.294,95 \% \text { Cl: } 0.768 \text { to } 2.180, \\
p=0.333\end{array}$ & Insufficient \\
\hline $\begin{array}{l}\text { Percentage with } \geq 1 \\
\text { emergency department } \\
\text { visit }\end{array}$ & Direct & Imprecise & $\begin{array}{l}\text { Calculated OR: } 1.222,95 \% \mathrm{Cl}: 0.795 \text { to } 1.878, \\
p=0.360\end{array}$ & Insufficient \\
\hline $\begin{array}{l}\text { Percentage with } \geq 1 \\
\text { hospitalization }\end{array}$ & Direct & Imprecise & $\begin{array}{l}\text { Calculated OR: } 1.539,95 \% \mathrm{Cl}: 0.862 \text { to } 2.746, \\
p=0.145\end{array}$ & Insufficient \\
\hline Mean number of $A D E s$ & Direct & Imprecise & $\begin{array}{l}\text { Calculated mean difference: } 0.346,95 \% \mathrm{Cl}: 0.112 \\
\text { to } 0.580, p=0.004\end{array}$ & \\
\hline $\begin{array}{l}\text { Mean number of } \\
\text { emergency department } \\
\text { visits }\end{array}$ & Direct & Imprecise & $\begin{array}{l}\text { Calculated mean difference: }-0.001,95 \% \mathrm{Cl} \text { : } \\
-0.119 \text { to } 0.117, p=0.987\end{array}$ & Insufficient \\
\hline $\begin{array}{l}\text { Mean number of } \\
\text { hospitalizations }\end{array}$ & Direct & Imprecise & $\begin{array}{l}\text { Calculated mean difference: } 0.055,95 \% \mathrm{Cl} \text { : } \\
-0.038 \text { to } 0.148, p=0.244\end{array}$ & Insufficient \\
\hline $\begin{array}{l}\text { Mean number of } \\
\text { physician visits }\end{array}$ & Indirect & Imprecise & $\begin{array}{l}\text { Calculated mean difference: } 0.100,95 \% \mathrm{Cl} \text { : } \\
-0.322 \text { to } 0.522, p=0.643\end{array}$ & Insufficient \\
\hline
\end{tabular}

Abbreviations: $\mathrm{ADE}=$ adverse drug event; $\mathrm{OR}=$ odds ratio. 


\section{Pharmacy Intensity of Adoption}

One cohort study (high risk of bias) of 2,211 patients evaluated eight outcomes based on pharmacy intensity of adoption of the MTM intervention (Table 75) ${ }^{63}$ Specifically, the authors categorized pharmacies that completed recommendations in at least one quarter into four groups: (1) for at least 50 percent of patients, high-intensity pharmacy (2) 25 to 49 percent as moderate intensity; (3) 1 to 24 percent as low intensity; and (4) no recommendations study as no intensity. For all outcomes, we rated this study as high for study limitations and unknown for consistency (not repeated in table). Outcomes for which we can infer a benefit or a harm from the effect are rated as direct outcomes. We found insufficient evidence to judge the effectiveness of MTM by intensity of adoption on all reported outcomes.

Table 75. Pharmacy intensity of adoption: Strength of evidence

\begin{tabular}{|c|c|c|c|}
\hline Outcome & Directness Precision & $\begin{array}{l}\text { Findings and Direction [Magnitude] } \\
\text { of Effect }\end{array}$ & $\begin{array}{l}\text { Strength of } \\
\text { Evidence }\end{array}$ \\
\hline $\begin{array}{l}\text { Number of emergency department } \\
\text { claims }\end{array}$ & Imprecise & Findings $N R, p=0.330$ & Insufficient \\
\hline Number of inpatient institutional claims & Imprecise & Findings $N R, p=0.839$ & Insufficient \\
\hline Number of outpatient facility claims & Imprecise & Findings $N R, p=0.112$ & Insufficient \\
\hline $\begin{array}{l}\text { Number of pharmacy, institutional, and } \\
\text { medical services }\end{array}$ & Imprecise & Findings $N R, p=0.616$ & Insufficient \\
\hline Emergency department claims & Imprecise & Findings $N R, p=0.652$ & Insufficient \\
\hline Inpatient institutional claims & Imprecise & Findings $N R, p=0.862$ & Insufficient \\
\hline Outpatient facility claims & Imprecise & Findings $N R, p=0.212$ & Insufficient \\
\hline $\begin{array}{l}\text { Pharmacy, institutional, and medical } \\
\text { services }\end{array}$ & Imprecise & Findings $N R, p=0.166$ & Insufficient \\
\hline
\end{tabular}

\section{Community Pharmacy Versus Call Center}

One large cohort study (high risk of bias) of the MirixiaPro platform (95,736 patients enrolled, 73,793 analyzed) compared patients using a community pharmacy, which included both face-toface and telephone interactions, with patients using a call center pharmacy (Table 76). ${ }^{46}$ The investigators measured three diverse outcomes. In all instances, we rated the study as high for study limitations and unknown for consistency (not repeated in table).Outcomes for which we can infer a benefit or a harm from the effect are rated as direct outcomes. We found insufficient evidence for drug cost and drug use outcomes, which we rated as indirect evidence with high study limitations. MTM delivered by community pharmacists increases the weighted generic dispensing ratio (GDR) when compared with MTM delivered by call-center pharmacists (low strength of evidence). The study defines the weighted GDR as the number of generic 30-day equivalent claims divided by the total number of claims, and then weighted for each patient by a factor equal to the individual's total prescription volume multiplied by a constant to hold sample size unchanged. 
Table 76. Community pharmacy versus call center: Strength of evidence

\begin{tabular}{|c|c|c|c|c|}
\hline Outcome & Directness & Precision & $\begin{array}{l}\text { Findings and Direction [Magnitude] of } \\
\text { Effect }\end{array}$ & $\begin{array}{l}\text { Strength of } \\
\text { Evidence }\end{array}$ \\
\hline $\begin{array}{l}\text { Drug cost per patient per month } \\
\text { (USD) }\end{array}$ & Indirect & Precise & $\begin{array}{l}\text { Calculated mean difference: }-20.0,95 \% \mathrm{Cl} \text { : } \\
-32.826 \text { to }-7.174, p=0.002\end{array}$ & Insufficient \\
\hline Drug use per patient per month & Indirect & Precise & $\begin{array}{l}\text { Calculated mean difference: }-0.370,95 \% \mathrm{Cl} \text { : } \\
-0.477 \text { to }-0.263, p<0.001\end{array}$ & Insufficient \\
\hline $\begin{array}{l}\text { Weighted generic dispensing } \\
\text { ratio }\end{array}$ & Direct & Precise & $\begin{array}{l}\text { Calculated mean difference: } 9.710,95 \% \mathrm{Cl} \text { : } \\
9.583 \text { to } 9.837, p<0.001\end{array}$ & Low \\
\hline
\end{tabular}

Abbreviations: $\mathrm{CI}=$ confidence interval; $\mathrm{USD}=$ United States dollar

\section{Type of Payer}

One cohort study (high risk of bias, $\mathrm{N}=615$ ) compared outcomes for patients with Medicaid and patients with private insurance (Table 77). ${ }^{47}$ The investigators reported on three diverse outcomes. In all instances, we rated the study as high for study limitations and unknown for consistency (not repeated for each outcome in the table). We found insufficient evidence to judge the effectiveness of MTM by type of payer on all reported outcomes.

Table 77. Type of payer: Strength of evidence

\begin{tabular}{lllll}
\hline Outcome & Directness Precision & $\begin{array}{l}\text { Findings and Direction [Magnitude] } \\
\text { of Effect }\end{array}$ & $\begin{array}{l}\text { Strength of } \\
\text { Evidence }\end{array}$ \\
\hline $\begin{array}{l}\text { Per-patient Medication } \\
\text { Appropriateness Index at followup }\end{array}$ & Direct & Precise & $\begin{array}{l}\text { Calculated mean difference: } 0.81, \\
95 \% \mathrm{Cl}:-1.303 \text { to } 2.923, p=0.452\end{array}$ & Insufficient \\
\hline $\begin{array}{l}\text { Proportion of patients for whom cost } \\
\text { was a problem at followup }\end{array}$ & Direct & Precise & $\begin{array}{l}\text { Calculated OR: } 1.498,95 \% \mathrm{Cl}: 0.807 \\
\text { to } 2.778, p=0.20\end{array}$ & Insufficient \\
\hline \begin{tabular}{l} 
Drug therapy problems identified \\
\hline
\end{tabular} & Direct & Precise & 2.6 in both arms, $\mathrm{p}=1.0$ & Insufficient \\
\hline
\end{tabular}

Abbreviations: $\mathrm{CI}=$ confidence interval; $\mathrm{OR}=$ odds ratio

\section{Key Question 4. Outcomes of MTM by Patient Characteristics}

We did not identify any studies that analyzed outcomes of MTM by patient characteristics.

\section{Key Question 5. Harms of Medication Therapy Management Interventions}

\section{Key Points}

- Studies do not routinely report harms that result from MTM interventions. One study reported on confusion from information received through an MTM intervention. Study limitations and lack of precision of these results suggested that evidence was insufficient to evaluate the effect of MTM interventions on harms.

\section{Detailed Synthesis: Confusion}

A single cohort study (high risk of bias) compared patients who accepted a home medicines review with those who did not have a home medicines review. ${ }^{61}$ The investigators reported a five-fold increase in the odds of being confused by information received ( 22 percent versus 5 percent; calculated OR, 5.57; 95\% CI, 1.03 to 30.1; $\mathrm{p}=0.04)$. However, the sample size does not meet optimal information size criteria, suggesting lack of precision of the results. 
Table 78. Confusion: Strength of evidence

\begin{tabular}{|c|c|c|c|c|c|c|}
\hline $\begin{array}{l}\text { Study } \\
\text { design }\end{array}$ & $\begin{array}{l}\text { Number of } \\
\text { Studies; } \\
\text { Subjects } \\
\text { (Analyzed) }\end{array}$ & $\begin{array}{l}\text { Study } \\
\text { Limitations }\end{array}$ & Consistency & Directness Precision & $\begin{array}{l}\text { Findings and } \\
\text { Direction } \\
\text { [Magnitude] of } \\
\text { Effect }\end{array}$ & $\begin{array}{l}\text { Strength of } \\
\text { Evidence }\end{array}$ \\
\hline Cohort & $1 ; 149(68)$ & High & $\begin{array}{l}\text { Consistency } \\
\text { unknown-single } \\
\text { study }\end{array}$ & Imprecise & $\begin{array}{l}\text { Calculated OR: } \\
5.57,95 \% \mathrm{Cl}: 1.03 \\
\text { to } 30.1, p=0.04\end{array}$ & Insufficient \\
\hline
\end{tabular}

Abbreviations: $\mathrm{CI}=$ confidence interval; $\mathrm{OR}=$ odds ratio

\section{Detailed Synthesis: Inconvenience}

A single cohort study (high risk of bias) compared pharmaceutical care with usual care. ${ }^{56,57}$ The investigators reported that patients in the intervention arm were less likely to agree or strongly agree with the statement that they were inconvenienced by monthly appointments with the pharmacists (40 percent versus 69 percent; calculated OR, $0.278 ; 95 \%$ CI, 0.088 to 0.875 ; $\mathrm{p}=0.029$ ). As with the information on confusion, the sample size does not meet optimal information size criteria, suggesting lack of precision of the results.

Table 79. Confusion: Strength of evidence

\begin{tabular}{|c|c|c|c|c|c|c|}
\hline $\begin{array}{l}\text { Study } \\
\text { Design }\end{array}$ & $\begin{array}{l}\text { Number of } \\
\text { Studies; } \\
\text { Subjects } \\
\text { (Analyzed) }\end{array}$ & $\begin{array}{l}\text { Study } \\
\text { Limitations }\end{array}$ & Consistency & Directness Precision & $\begin{array}{l}\text { Findings and } \\
\text { Direction } \\
\text { [Magnitude] of } \\
\text { Effect }\end{array}$ & $\begin{array}{l}\text { Strength of } \\
\text { Evidence }\end{array}$ \\
\hline Cohort & $1 ; 55(51)$ & High & $\begin{array}{l}\text { Consistency } \\
\text { unknown-single } \\
\text { study }\end{array}$ & Imprecise & $\begin{array}{l}\text { Calculated OR: } \\
0.278,95 \% \mathrm{Cl} \text { : } \\
0.088 \text { to } 0.875 \\
p=0.029\end{array}$ & Insufficient \\
\hline
\end{tabular}

Abbreviations: $\mathrm{CI}=$ confidence interval; $\mathrm{OR}=$ odds ratio 


\section{Discussion}

We conducted a systematic review of benefits and harms of medication therapy management (MTM) programs. Because of the wide variation in types of interventions classified as MTM, we first catalogued intervention components and implementation features of MTM interventions (Key Question [KQ] 1). We then evaluated the effect of MTM on intermediate, patient-centered, and resource utilization outcomes (KQ 2). We also reviewed the evidence to identify how these effects might vary by specific intervention components and features (KQ 3) and patient characteristics (KQ 4). Finally, we reviewed the evidence on harms associated with MTM (KQ 5).

Below, we summarize the main findings and strength of evidence, where applicable. We then discuss the findings in relationship to what is already known, applicability of the findings, implications for decisionmaking, limitations, research gaps, and conclusions.

This evidence base consisted of 36 studies (19 randomized controlled trials [RCTs] trials and 17 observational studies) reported in 42 articles. Most RCTs compared an MTM intervention with usual care rather than with a different active intervention; most observational studies were cohort studies. Numerous studies had methods problems that led us to rate them as having a medium or high risk of bias; only a few studies were of low risk of bias. When possible (enough studies similar in intervention, populations, and outcomes measured), we conducted metaanalyses of data from RCTs; in some cases, we did two sets, one with and one without the high risk-of-bias trials.

\section{Key Findings and Strength of Evidence}

\section{KQ 1: Intervention Components and Implementation Features}

Nearly two-thirds of included studies were broadly focused on patients with a wide-ranging collection of conditions; the remaining studies were narrowly focused on patients with a specific condition. All studies used a pharmacist as the interventionist. Services were provided face-toface in just over half of included studies. Included studies provided interventions in a variety of clinical settings, including community pharmacies, centralized pharmacies or pharmacy call centers, and outpatient medical clinics, and some used home visits; half of the narrowly focused interventions were delivered exclusively in an outpatient medical clinic.

Whether termed "pharmaceutical care" or "MTM," studies did not describe intervention components and features in a consistent manner or in sufficient detail. These drawbacks were especially prevalent for intervention intensity and frequency, method of patient enrollment for services, level of integration with usual care, and reimbursement characteristics for rendered MTM services. KQ 1 was descriptive in nature, so we did not grade strength of evidence.

\section{KQ 2: Overall Effectiveness}

Of the 36 studies included in this review, we rated 14 as high risk of bias overall; that is, concerns about randomization failure, confounding, or overall attrition increased the risk of bias for all outcomes. In addition, we rated some studies that were otherwise of low or medium risk of bias as high for individual outcomes, chiefly because of measurement bias. These instances are specified in the relevant results section in Chapter 3.

We rated the strength of evidence for each outcome from low- or medium risk-of-bias studies when available. MTM significantly improved medication appropriateness assessed in general 
(Table 80). However, we did not find evidence of benefit for any other intermediate outcomes on which we had data. No studies addressed either goals of therapy or patient engagement.

Table 80. Summary of findings and strength of evidence for intermediate outcomes of MTM interventions

\begin{tabular}{|c|c|c|c|}
\hline Intermediate Outcome & $\begin{array}{l}\text { Study Design: } \\
\text { No. Studies (N } \\
\text { Analyzed) }\end{array}$ & Results & $\begin{array}{l}\text { Strength of } \\
\text { Evidence }\end{array}$ \\
\hline Anticoagulation & RCT: $1(10)$ & Imprecise & Insufficient \\
\hline Hemoglobin A1C & RCT: 2 (102) & Inconsistent, imprecise & Insufficient \\
\hline Low density lipoprotein cholesterol & RCT: $1(38)$ & Imprecise & Insufficient \\
\hline $\begin{array}{l}\text { Hypertension: achieving blood } \\
\text { pressure goals }\end{array}$ & RCT: $1(44)$ & Imprecise & Insufficient \\
\hline $\begin{array}{l}\text { Hypertension: systolic blood } \\
\text { pressure }\end{array}$ & RCT: 1 (23) & Imprecise & Insufficient \\
\hline $\begin{array}{l}\text { Hypertension: diastolic blood } \\
\text { pressure }\end{array}$ & RCT: 1 (23) & Imprecise & Insufficient \\
\hline Drug therapy problems identified & $\begin{array}{l}\text { RCT: } 1 \text { (332) } \\
\text { Cohort: } 2 \text { (668) }\end{array}$ & Indirect, imprecise, high study limitations & Insufficient \\
\hline Drug therapy problems resolved & Cohort: $1(120)$ & Indirect, imprecise, high study limitations & Insufficient \\
\hline Medication adherence & $\begin{array}{l}\text { RCT: } 8(2,415) \\
\text { Cohort: } 2(1,493)\end{array}$ & Inconsistent, imprecise & Insufficient \\
\hline $\begin{array}{l}\text { Medication Appropriateness General } \\
\text { Index Scores }\end{array}$ & RCT: 1 (208) & $\begin{array}{l}\text { Improvement in MTM group from score of } \\
17.7 \text { to } 13.4 \text { and to } 12.8 \text { at } 3 \text { and } 12 \\
\text { months, respectively }\end{array}$ & Low for benefit \\
\hline Medication-specific appropriateness & RCT: 2 (261) & Indirect, imprecise, inconsistent & Insufficient \\
\hline Medication dosing & RCT: 2 (90) & Inconsistent, imprecise & Insufficient \\
\hline Goals of therapy & 0 & $\mathrm{NA}$ & NA \\
\hline Patient engagement & 0 & NA & NA \\
\hline
\end{tabular}

Abbreviations: $\mathrm{CI}=$ confidence interval, $\mathrm{MTM}=$ medication therapy management; $\mathrm{N}=$ number; $\mathrm{NA}=$ not applicable; $\mathrm{OR}=$ odds ratio; $\mathrm{RCT}=$ randomized controlled trial.

Similarly, we did not have evidence of benefit for most patient-centered outcomes (Table 81). MTM did not improve most measures of health-related quality of life (low strength of evidence for no benefit). We graded the "vitality" domain of the Medical Outcomes Study ShortForm (SF36) questionnaire as insufficient for this domain. For the SF-36, neither the other seven domains nor the two component scores (physical health, mental health) showed significant benefit from MTM interventions. The various patient satisfaction items also showed no impact from MTM programs (low strength of evidence for no benefit). 
Table 81. Summary of findings and strength of evidence for patient-centered outcomes of MTM interventions

\begin{tabular}{|c|c|c|c|}
\hline Patient-Centered Outcome & $\begin{array}{l}\text { Study Design: } \\
\text { No. Studies (N } \\
\text { Analyzed) }\end{array}$ & Results & $\begin{array}{l}\text { Strength of } \\
\text { Evidence }\end{array}$ \\
\hline Adverse drug events & RCT: 2 (806) & Inconsistent, imprecise & Insufficient \\
\hline $\begin{array}{l}\text { Cognitive, affective, and physical } \\
\text { function }\end{array}$ & RCT: 1 (133) & Imprecise & Insufficient \\
\hline Mortality & $\begin{array}{l}\text { RCT: } 2 \text { (335) } \\
\text { Cohort: } 1 \text { (795) }\end{array}$ & Inconsistent, imprecise & Insufficient \\
\hline Gastrointestinal bleeding events & Cohort: $1(1,373)$ & High study limitations & Insufficient \\
\hline $\begin{array}{l}\text { General health-related quality of life } \\
\text { domains other than vitality }\end{array}$ & RCT: $4(1,169)$ & $\begin{array}{l}\text { Variable mean difference with confidence } \\
\text { intervals consistently spanning the null } \\
\text { effect }\end{array}$ & $\begin{array}{l}\text { Low for no } \\
\text { benefit }\end{array}$ \\
\hline $\begin{array}{l}\text { General health-related quality of life } \\
\text { vitality domain }\end{array}$ & RCT: $4(1,169)$ & Imprecise & Insufficient \\
\hline $\begin{array}{l}\text { Condition-specific health-related } \\
\text { quality of life (diabetes) }\end{array}$ & RCT: 1 (73) & Imprecise & Insufficient \\
\hline Patient satisfaction & RCT: 3. $(1,625)$ & $\begin{array}{l}\text { No differences on } 17 \text { or } 21 \text { items of patient } \\
\text { satisfaction }\end{array}$ & $\begin{array}{l}\text { Low for no } \\
\text { benefit }\end{array}$ \\
\hline Activities of daily living & 0 & NA & $\mathrm{NA}$ \\
\hline Work or school absenteeism & 0 & NA & $\mathrm{NA}$ \\
\hline $\begin{array}{l}\text { Patient and caregiver participation in } \\
\text { medical care and decisionmaking }\end{array}$ & 0 & NA & NA \\
\hline \multicolumn{4}{|c|}{$\begin{array}{l}\text { Abbreviations: } \mathrm{CI}=\text { confidence interval, } \mathrm{MTM}=\text { medication therapy management; } \mathrm{N}=\text { number; } \mathrm{NA}=\text { not applicable; } \mathrm{RCT}= \\
\text { randomized controlled trial. }\end{array}$} \\
\hline \multicolumn{4}{|c|}{$\begin{array}{l}\text { Outcomes related to using health resources were similarly not much influenced by MTM } \\
\text { interventions (Table } 82 \text { ). Two exceptions may merit attention: (1) the use of generic medications } \\
\text { for patients receiving MTM from community pharmacy when compared with educational } \\
\text { mailings (low for benefit from the community pharmacy approach) and (2) the rate of } \\
\text { hospitalization among heart failure patients with home medicines review when compared with } \\
\text { usual care. When MTM was implemented in settings with a broad range of patients, it did not } \\
\text { reduce the number of hospitalizations (low strength of evidence). }\end{array}$} \\
\hline
\end{tabular}


Table 82. Summary of findings and strength of evidence for resource-utilization outcomes of MTM interventions

\begin{tabular}{|c|c|c|c|}
\hline Use of Resources Outcomes & $\begin{array}{l}\text { Study Design: } \\
\text { No. Studies (N } \\
\text { Analyzed) }\end{array}$ & Results & $\begin{array}{l}\text { Strength of } \\
\text { Evidence }\end{array}$ \\
\hline Number of high-risk medications & Cohort: $1(2,211)$ & High study limitations & Insufficient \\
\hline $\begin{array}{l}\text { Use of generic medications for call- } \\
\text { center pharmacy-based MTM vs. } \\
\text { educational mailings }\end{array}$ & $\begin{array}{l}2 ; 97,124 \\
(75,166)\end{array}$ & High study limitations, inconsistent & Insufficient \\
\hline $\begin{array}{l}\text { Use of generic medications for } \\
\text { community pharmacy-based MTM } \\
\text { vs. educational mailings }\end{array}$ & $\begin{array}{l}\text { Cohort: } 1 \\
(73,793)\end{array}$ & $\begin{array}{l}\text { Calculated mean difference in weighted } \\
\text { generic dispensing ratio: } 1.2(95 \% \mathrm{Cl} \text { : } \\
0.724 \text { to } 1.676 \text { to; } \mathrm{p}<0.001)\end{array}$ & $\begin{array}{l}\text { Low for benefit for } \\
\text { community } \\
\text { pharmacy }\end{array}$ \\
\hline $\begin{array}{l}\text { Medication costs: patient } \\
\text { copayments }\end{array}$ & NRCT: $1(1,626)$ & High study limitations, indirect & Insufficient \\
\hline $\begin{array}{l}\text { Medication costs: health plan } \\
\text { expenditures }\end{array}$ & $\begin{array}{l}\text { RCT: } 2 \text { (835); } \\
\text { NRCT \& Cohort: } \\
2(1,746)\end{array}$ & Indirect, imprecise & Insufficient \\
\hline Medication costs: total outlays & RCT: $3(1,975)$ & Inconsistent, indirect, imprecise & Insufficient \\
\hline $\begin{array}{l}\text { Medication costs: medication costs } \\
\text { plus other expenditures }\end{array}$ & $\begin{array}{l}\text { RCT: } 2 \text { (>779); } \\
\text { NRCT: } 1 \text { (675) }\end{array}$ & Indirect, imprecise & Insufficient \\
\hline Number of outpatient visits & RCT: $2(2,038)$ & Inconsistent, imprecise & Insufficient \\
\hline Outpatient costs & RCT: $2(1,842)$ & Inconsistent, indirect, imprecise & Insufficient \\
\hline Number of laboratory tests & RCT: $2(1,842)$ & Inconsistent, indirect, imprecise & Insufficient \\
\hline Costs of laboratory tests & RCT: $2(1,842)$ & Inconsistent, indirect, imprecise & Insufficient \\
\hline $\begin{array}{l}\text { Number of emergency department } \\
\text { visits }\end{array}$ & $\begin{array}{l}\text { RCT: } 2(1,344) \\
\text { Observational: } 1 \\
(795)\end{array}$ & Inconsistent, imprecise & Insufficient \\
\hline $\begin{array}{l}\text { Costs of emergency department } \\
\text { visits }\end{array}$ & RCT: 1 (779) & Imprecise & Insufficient \\
\hline Hospitalization: number & RCT: $2(2,398)$ & $\begin{array}{l}\text { Mean difference of } 0.038(95 \% \mathrm{Cl} \\
-0.005 \text { to } 0.080)\end{array}$ & Low for no benefit \\
\hline Hospitalization: percentage & RCT: 2 (710) & Inconsistent. Imprecise & Insufficient \\
\hline $\begin{array}{l}\text { Hospitalization: rate (patients with } \\
\text { heart failure and home medicine } \\
\text { review) }\end{array}$ & Cohort: $1(5,717)$ & $\begin{array}{l}\text { Adjusted HR }(95 \% \mathrm{Cl}): 0.55(0.39 \text { to } \\
0.77)\end{array}$ & Low for benefit \\
\hline Costs of hospitalization & RCT: $2(1,842)$ & Inconsistent, imprecise & Insufficient \\
\hline Length of hospital stay & RCT: $1(46)$ & High study limitations, imprecise & Insufficient \\
\hline
\end{tabular}

Over all three categories of outcomes, each of which had a substantial number of individual measures, MTM improved outcomes in only a couple of instances. Study limitations and lack of precision of the estimates of effects limited the strength of evidence considerably. As discussed later, even the minimal findings of effectiveness are at best only narrowly applicable.

\section{KQ 3: Effectiveness of MTM by Intervention Features}

We found evidence from one study each on four intervention features: (1) access of pharmacists to patient records,${ }^{55}(2)$ community pharmacy versus call center ${ }^{63}$ (3) level of intensity of intervention, ${ }^{46}$ and (4) type of payer (private vs. Medicaid) ${ }^{47}$ With the exception of the study on pharmacists' access to patient records, we rated these studies as high risk of bias. Evidence was insufficient for most outcomes for the first two intervention features, with two exceptions. First, MTM delivered by community pharmacists increased the weighted generic dispensing ratio when compared with call-center pharmacists (low strength of evidence). Second, enhanced MTM with pharmacists' access to patient records reduced the mean number of adverse 
drug events; this finding suggested benefit when compared with basic MTM (low strength of evidence). We found insufficient evidence for all outcomes for intensity of intervention and type of payer.

\section{KQ 4: Effectiveness of MTM by Patient Characteristics}

We did not identify any studies that analyzed outcomes of MTM by patient characteristics.

\section{KQ 5: Harms of MTM Interventions}

Lack of precision and the limitations of a single high risk-of-bias study meant that evidence was insufficient to judge whether MTM resulted in greater confusion ${ }^{61}$ or inconvenience $^{56,57}$ than usual care. We found no evidence on other prespecified harms, specifically including care fragmentation, patient decisional conflict, patient anxiety, increased (actual) adverse drug events, prescriber confusion, and prescriber dissatisfaction.

\section{Findings in Relation to What Is Already Known}

Our findings contrast with conclusions that Chisholm-Burns and colleagues reached in a recent systematic review. ${ }^{75}$ In that review, the authors concluded that "Pharmacist-provided direct patient care has favorable effects across various patient outcomes, health care settings, and disease states." 75 , p. 923 Several differences between the Chisholm-Burns review and the current review may account for the discrepant conclusions. First, the Chisholm-Burns review included all studies that cited evidence of pharmacist involvement in direct patient care. The interventions examined included chronic disease management and prospective and retrospective drug utilization review; we excluded these types of efforts because our intended focus was on the MTM intervention itself. Notably, the Chisholm-Burns review did not use the term "medication therapy management" to categorize the interventions in the articles they reviewed. Second, approximately 30 percent of the papers in the Chisholm-Burns review were conducted entirely in institutional settings. In contrast, we did not identify any studies within institutional settings that met our MTM intervention definition criteria. Third, the Chisholm-Burns review included a total of 298 articles and did not omit from their analyses studies with a high risk of bias; by contrast, we based our strength-of-evidence grades in this review on only those studies with no more than medium risk of bias.

The striking differences between the conclusions reached in these two reviews emphasize two important needs for both conceptual and practical efforts to systematically review MTM programs. The first is to create a systematic system for classifying the different types of direct patient care services that pharmacists can provide. The second is to develop consensus guidelines for describing intervention features in publications reporting findings from evaluation studies. Progress on these two steps would enable systematic reviews to differentiate better between different types of services and avoid the problem of overgeneralizing review results.

The Centers for Medicare \& Medicaid Services (CMS) supported a large evaluation of MTM programs that we were unable to include in this draft because of the timing of the release of that report. ${ }^{28}$ We will include it in our final report along with our update of the published and grey literature. Although we have not yet incorporated the findings of this large evaluation into our systematic review, we note that the report finds that MTM improved patient adherence to medication regimens and the quality of prescribing. Our review did not find sufficient evidence to evaluate the effect of MTM on improved adherence, but we did find low strength of evidence 
that MTM improves medication appropriateness, which is conceptually similar to "quality of prescribing." The discrepancy between the CMS findings regarding adherence and findings of studies included thus far in our review may reflect the greater precision that the CMS investigators might have had in their use of pharmaceutical prescription refill records to assess adherence when compared with other studies that primarily used self-report to assess adherence. The report also found some reduction in resource use, but these results were for patients with diabetes or congestive heart failure. Our review found that for patients with heart failure, MTM was likely to reduce hospitalization rates, but we found no effect on mean number of hospitalizations for broadly defined populations.

\section{Applicability of the Findings}

This body of evidence has significant clinical and methodological heterogeneity, which limits the ability to make any universal statements about effectiveness. ${ }^{76}$ However, the range of study designs, which includes RCTs, nonrandomized trials, and cohort studies, enhances the applicability of findings for real-world settings. Included studies ranged from relatively small interventions in single clinics provided by a single interventionist to evaluations of MTM services delivered on a large scale through integrated health systems or health plans as a Medicare Part D or other drug plan benefit. This diversity of studies enhanced the applicability of findings to a wide variety of settings, including outpatient clinics, community pharmacies, and centralized pharmacy call centers. A few studies conducted outside the United States included MTM as part of a home visits program; findings from this model may not be directly applicable within the United States.

The studies in this review are broadly applicable to a range of chronically ill, adult patient populations. The majority of interventions were directed at populations with multiple and common chronic conditions, such as diabetes, chronic heart failure, and hypertension. Several specifically targeted adults aged 65 years or older. Few studies reported sociodemographic characteristics beyond age and sex; thus, the applicability of findings to specific populations (e.g., rural, low socioeconomic status, cognitively impaired, uninsured) is unknown. The nature of the MTM intervention, which includes involving patients as active participants in the process, limits the extent to which findings can be generalized beyond patients who agreed to participate in such interventions. Patients who agree to participate may be systematically different from those who decline to be in such a program. For that reason, the impact of such interventions at a population or health-plan level may be limited by the degree of uptake among interested patients.

The intervention used across most studies can be characterized as complex and moderately resource intensive. Components involve identifying applicable patients; initially assessing patients; providing counseling, education, and care coordination; and following patients over time. These services were provided per protocol in some studies and as needed or ad hoc in others. Most studies described intervention components in terms of "pharmaceutical care model" components or Medicare Part D MTM program criteria, but few provided detailed descriptions or measurement of implementation fidelity.

The comparator arm in all studies was usual medical care. This does not typically include distinct MTM services by health care providers other than prescribing providers (not common for the time period covered by most of the studies). Models of collaborative health care delivery are evolving, and the changing roles and training of pharmacists increase the potential applicability of MTM interventions in future models of health care. 
The broad sets of outcomes evaluated across this body of evidence spanned a substantial range of both intermediate and health outcomes as well as outcomes related to resource use. Proximal and intermediate outcomes included number of drugs, identification of drug therapy problems, appropriateness of medication prescribing, and laboratory or biometric markers of disease control (e.g., hypertension, hemoglobin A1c, low-density lipoprotein cholesterol). Patient-centered outcomes focused on numerous measures of quality of life as well as adverse drug events. Many studies also reported outcomes involving health care resource use and expenditures (e.g., number and costs of hospitalizations, emergency department visits, outpatient visits).

Most studies did not, however, clearly indicate the expected, desired, or intended direction of effect on most resource use outcomes, making the applicability of using these interventions to reduce drug-related health care costs or expenditures difficult to assess. For example, whether one should expect the number of medications prescribed for heart failure to increase or decrease under the careful scrutiny of an MTM intervention is not clear.

The focus of outcome measurement in many studies was the short-term identification and characterization of drug therapy problems and their resolution; these endpoints are thought to be the outcomes most sensitive to change as a result of receiving MTM services. However, by design, because identification of drug therapy problems is a part of the MTM intervention itself, differences between the nature of the intervention and that of the control programs mean that measuring these outcomes cannot be as rigorous in a usual care comparison group as it is in the intervention group. In fact, many studies were able to measure only changes in this outcome in the intervention group. Hence, many studies failed to demonstrate a direct analytic link between the resolution of drug therapy problems as a result of MTM and impact on intermediate outcomes, patient-centered outcomes, and resource utilization. Thus, the applicability of studies that demonstrate an impact on the resolution of drug therapy problems is limited.

\section{Implications for Clinical Practice and Policymakers}

Although we found the evidence insufficient in general to draw definitive conclusions about the comparative effectiveness of MTM for most outcomes that we evaluated, our findings do suggest some implications for practice and policy. MTM is already in widespread practice and is now shaped in the United States largely by Medicare Part D policy: this presents both challenges and opportunities. MTM programs of the future, sponsored and administered by Part D drug benefit plans, may be less integrated into routine health care for Medicare beneficiaries than many of the pharmaceutical care interventions included in our review. We were unable to answer definitively whether level of integration matters for effectiveness, but policymakers may need to consider expectations about the impact that MTM might have on patient-centered outcomes and resource use in the context of other health care delivery transformation activities or quality improvement initiatives that are also occurring. More integration of MTM services with other activities may be effective; however, the more integrated MTM becomes within routine medical care, the more difficult it becomes to isolate it as a discrete intervention for evaluation.

Policymakers could thus consider whether MTM services should be positioned as a contributor to overall improvement in processes of care, health status, and costs or positioned as an intervention to which effects can be discretely attributed. Improvements in medication appropriateness or drug therapy regimens may not always translate into improvements in health or costs, and even if they do, secular improvements in other areas of quality improvement may make measuring outcomes attributable to MTM very challenging. 
Future training of MTM providers would benefit from a better understanding of which MTM components really matter. At the moment, such information is lacking. Policymakers and funders who wish to understand the comparative effectiveness of different MTM components could encourage rigorous program evaluation designs that fit within the context of the real-world implementation of these programs. For example, positive deviance analyses ${ }^{77}$ with rigorous measurement of implementation features or stepped wedge trial designs ${ }^{78}$ may be useful approaches.

A typical approach for evaluating complex interventions is to identify the "core" components for standardization, while allowing for flexibility for peripheral components or variations in implementation. In complex practice-based innovations, such flexibility may reflect desirable (or unavoidable) adaptations to local circumstances. Policy governing MTM programs may warrant modifications to permit investigators to conduct rigorous and innovative evaluative designs to identify core components or effectiveness-enhancing modifications. As future research and evaluation elucidates these components or enhancements, policy will need to evolve to keep pace with best practices.

Finally, considering both patients' and prescribers' perspectives in future design and delivery of MTM services may be needed. In our current analytic framework, MTM interventions require a significant element of engagement by both patients and prescribers if the interventions are to have a reasonable likelihood of improving outcomes. Although "opt in" strategies may increase the reach of such interventions, keeping patients (and their prescribing providers) engaged in the intervention over a reasonable amount of time may be the key to translating the potential of MTM interventions into actual improvements.

\section{Limitations of the Comparative Effectiveness Review Process}

The constraints for populations, interventions, and settings that we imposed on this systematic review may limit its applicability as discussed above. During topic refinement and based on technical expert panel inputs and public comment, we expanded the scope by removing an exclusion criterion that would have required MTM interventions to have been directed at a patient population with two or more chronic conditions. As a result, we did include studies that focused on one chronic condition. Because of the prevalence of certain chronic conditions in the adult population, and particularly among Medicare beneficiaries, we think this decision was sensible and permitted us to examine a broader evidence base than would otherwise have been the case.

Although we tried to distinguish MTM from disease or case management interventions, making this distinction was challenging. We created a threshold for what intervention components were required to be present for this distinction. Specifically, we elected to emphasize whether the intervention entailed a comprehensive review of all medications; for that reason, we did not constrain studies of interest to those that targeted a single medication or drug regimen or that focused on a single condition such as diabetes or hypertension.

As described in Chapter 2 on Methods, when we were unable to determine which medications the interventionist had reviewed, we wrote to the authors for additional information. We chose to pursue authors in an effort to permit us to use studies that had been designed as MTM but did not describe the comprehensive medication review component in detail.

Our approach may have been overly inclusive because it led us to include studies that addressed a single disease, as long as the pharmacist reviewed all medications. For example, 12 
of the 36 studies were relatively narrowly focused; four of these addressed patients with chronic heart failure and two addressed patients with either hypertension or hypertension and diabetes. The remaining six studies focused on post-transplant patients (kidney, lung), diabetes, glucocorticoid-induced osteoporosis, and hemodialysis. The fact that we drew the line at only one intervention component criterion resulted in an approach that was inclusive of these more narrowly focused (albeit often termed "MTM") studies and may render our results less applicable to MTM interventions targeted to patients with a wide range of chronic conditions.

Also based on feedback during the process of setting out the scope of this review, we chose to include interventions that were broader than the Medicare Part D MTM-defined interventions. Put another way, we broadened our view of patient populations and intervention criteria, and we allowed studies not conducted in the United States into the evidence base. This decision led us to include interventions described as "pharmaceutical care," which were generally based on the pharmaceutical care model as described and refined by Strand and associates; ${ }^{10}$ it also permitted us to examine investigations with elements of pharmaceutical care or MTM that did not specifically label the intervention as either MTM or pharmaceutical care. These studies were often described as "clinical pharmacist interventions."

Furthermore, all the non-U.S. studies involved interventions within single-payer health systems. Hence, the interventions in this review constitute a more heterogeneous group than if we had allowed only those labeled as Medicare Part D MTM programs. This is both a limitation and a strength. Although our approach makes results more challenging to interpret, it enhances our ability not to miss interventions that include MTM components but lack the descriptor term MTM.

Studies did not often explicitly describe certain MTM components. In cases when we could not determine whether investigators had provided certain MTM components (such as patient education and counseling, medication action plan, or coordination with other health care providers), we again contacted the authors to gain additional information that would allow us to make an informed decision. We were fairly permissive in interpreting the presence of the MTM intervention components other than comprehensive medication review (e.g., medication action plan). The main reason is that we recognized that terms describing some components have evolved over time and may have been absent from the lexicon in earlier years or implicitly conveyed by authors by simply using the terms "MTM" or pharmaceutical care to describe their intervention.

Our approach to categorizing interventions for KQ 1 relied primarily on the short descriptions in published manuscripts and those we were able to obtain via email inquiries. Their similarities or differences substituted for any overarching taxonomy, because none that we considered seemed to fit our purpose. Thus, we have introduced intervention labels that, admittedly, do not fully describe or account for clinical heterogeneity among interventions. This approach limits our ability to make definitive statements about the effectiveness of various intervention components. We believe that the clusters and categorizations we used are useful heuristics, but some may regard them more as hypothesis generating than as reflecting settled principles of classification.

Finally, our search process was complicated by having to ensure coverage of all terms that could be used to describe MTM interventions over time. Adding to this challenge was our effort to examine the gray literature, where we thought we might find studies tilted toward effectiveness and real-world program evaluation. As it turned out, studies of these types of interventions were not indexed similarly; for that reason, we needed to rely heavily on hand 
searches of citation lists from key background articles to identify possibly relevant studies for inclusion. Thus, we may have missed some studies that might have qualified for inclusion. Given the considerable diversity in the evidence base we did have, however, and the general lack of data supporting effectiveness of MTM, we do not think that any potential missed studies would have changed our conclusions in any material way. No meta-analyses included more than five studies; as a result, we did not examine included studies for publication bias quantitatively.

\section{Limitations of the Evidence}

As a body of evidence, the MTM literature evaluated in this review has measured numerous outcomes. As indicated in previous sections, very few outcomes, with the exception of harms, remain completely unexamined. Of the 36 studies in this review, we rated 22 as having medium or low risk of bias. The 36 studies included 19 trials and 3 nonrandomized controlled studies. In other words, the literature on this topic is not marked by failure to consider important outcomes; neither is it universally of high risk of bias, and it does not reflect pervasively weak designs.

Despite these advantages, we were unable to identify sufficient evidence on the majority of hypothesized outcomes of MTM. In several instances, our inability to rate evidence as higher than insufficient came from indirect, inconsistent, and imprecise evidence. The choice of outcome measures in this body of evidence limited our ability to come to conclusions in some instances. For example, some studies did not focus on changes that proponents might expect MTM services to produce. Because effective MTM can either increase or decrease expenditures or use of services based on the needs of the patient, studies that did not prespecify the expected direction of change had no way to interpret their results as an appropriate change. Studies that demonstrated inconsistent results in direction of change (i.e., some showing an increase in resource use and others showing a decrease) may well have been consistent in terms of appropriate change, but because they generally failed to establish a priori the direction in which they expected to find an effect, we rated such evidence as indirect and inconsistent.

Similarly, studies often used nonstandardized or idiosyncratic measures for outcomes such as adverse events, adherence, and expenditures or costs; this tendency limited our ability to metaanalyze results. When studies focused on specific outcomes, they were often significantly underpowered to detect differences between groups (that is, they did not meet optimal information size criteria). As a result, we rated several studies as imprecise.

MTM intervention studies are largely practice based and incorporate substantial heterogeneity in specific intervention elements and in patient populations targeted. Yet the evidence is sharply constrained in its ability to inform questions of the effectiveness of specific MTM components or intervention features (KQ 3 in our review) because study designs did not often capitalize on variants in MTM programs for a prospective evaluation of outcomes by those variants. Neither did they measure fidelity to intended MTM elements for post-hoc evaluation. Similarly, the relatively untargeted nature of the MTM interventions meant that, in many studies, only small numbers of patients had any one specific condition, and most studies did not measure patient characteristics beyond age and sex, thus limiting our ability to address KQ 4 in our review. For this reason, the evidence we identified for this review was most relevant for KQ 2.

\section{Research Gaps}

In many bodies of research, questions regarding the comparative effectiveness of specific intervention components or implementation features are best answered after clear evidence of the effectiveness of the intervention relative to usual care has been established. Our review largely 
indicates insufficient evidence on the primary question of effectiveness relative to usual care. By definition, this limited what we could say about comparative effectiveness.

Nonetheless, the widespread implementation of MTM coexists with the urgent need for actionable information for policy, program policies, and training. This clinical and policy environment means that new research cannot afford to address causal claims relative to usual care first, followed by comparative effectiveness of the intervention elements in a relatively controlled environment, and finally, program evaluation of real-world implementation, all in sequential order.

In choosing among various research goals, therefore, funders may wish to consider the relative value of new evidence on overall effectiveness, effectiveness of implementation features, and program implementation and accountability. Trial research in narrow clinical settings can address questions of effectiveness but may lack applicability to real-world implementation. Likewise, evaluations of real-world programs with variable fidelity to interventions can answer questions about process and implementation, but they offer limited information on effectiveness.

For new studies focusing on causal claims, a critical gap relates to the failure to specify the expected direction of effect. New research requires a strong theoretical foundation to help specify causal mechanisms and hypothesized effects. Without such an edifice, future research will continue to produce inconsistent and uninterpretable results.

Heightened attention to causal mechanisms will also help researchers convey their understanding of what outcomes these types of interventions are likely to influence. For instance, how should researchers wishing to establish direct causal links between MTM programs and outcomes evaluate distal outcomes such as patient-centered outcomes and resource utilization? This effort requires a better understanding of the relationship between proximal outcomes like "drug therapy problems identified and resolved" and distal outcomes. For instance, MTM may reduce outpatient visits to address side effects. MTM may also result in the need for further testing and evaluation for some patients, which could, in turn, result in more rather than fewer outpatient visits. Unless the nature of change resulting from MTM is specified in relation to goals of drug therapy, studies cannot assert benefit or harm. Further, drug therapy problems are diverse and may not all have the same causal relationship to health, quality of life, patient satisfaction, or resource use outcomes. Furthermore, a causal model of these distal outcomes may need to take into account the competing or complementary contributions of MTM, new models of health care delivery (e.g., patient-centered medical homes), and other quality improvement interventions.

Investigators embarking on new studies focusing on causal links between MTM and outcomes may wish to consider the limitations of studies based on secondary data from existing MTM programs that use opt-in/opt-out patient enrollment mechanisms. Although these studies may provide invaluable information on process measures such as patient engagement, underlying issues of confounding severely limit the validity of causal claims from such studies.

Regardless of the goal of their future research, investigators should consider issues of sample size to ensure precision of their results. This issue is particularly relevant when evaluating outcomes likely to occur in smaller subgroups of patients. Innovative designs (e.g., stepped wedge trials) can permit both rigor and adequate sample size within the context of real-world implementation. With careful attention to fidelity, such designs may also inform questions of the effectiveness of intervention components and implementation features. Such designs may also help inform our understanding of critical training elements for MTM service providers. 
Regarding research gaps for specific outcomes such as patient satisfaction, measures specific to the types of services provided through MTM (e.g., patient education about medications) or to the proximal outcomes that MTM is intended to achieve (e.g., reduced medication side effects, improved disease control) may offer better insights into the effects of MTM. Similarly, a medication-related instrument may better measure patients' concerns that are directly related to medication use (e.g., experience of side-effects, intrusiveness of the medication regimen) than generic tools.

\section{Conclusions}

We identified 36 studies (14 that we rated as high risk of bias) that offered information on a range of intermediate outcomes, patient-centered outcomes, and resource utilization. Evidence was insufficient on the effect of MTM on most outcomes. For a limited number of outcomes, we found enough evidence to show that MTM results in improvement when compared with usual care (low strength). Specifically, these outcomes include medication appropriateness, the rate of hospitalization among heart failure patients with home medicines review when compared with usual care, and the use of generic medications for patients receiving MTM from community pharmacies when compared with educational mailings. Similarly, we found sufficient evidence to conclude that MTM conferred no benefit for a limited number of outcomes. When MTM is implemented in settings with a broad range of patients, it does not reduce the number of hospitalizations (low strength of evidence). MTM does not improve most measures of healthrelated quality of life (low strength of evidence). We found evidence on four intervention components and intervention features: one study provided information on each feature and yielded insufficient evidence for most outcomes with two exceptions. MTM programs with pharmacist access to brief clinical summaries from the medical records reduce the mean number of adverse drug events when compared with basic MTM programs without such access (low strength of evidence). Community pharmacists increase the generic dispensing ratio more than call-center-based pharmacists (low strength of evidence). Similarly, the evidence on harms associated with MTM was limited to one study each on confusion and inconvenience and was rated as insufficient.

Investment in new research should be preceded by a careful consideration of goals of research. Studies focusing on causal claims require a strong theoretical foundation, an a priori statement of expected direction of effect that accounts for goals of therapy for each patient, and the use of designs that avoid confounding. Studies focusing on comparative effectiveness of intervention components and implementation features in real-world settings require a careful assessment of fidelity to the intervention components. 


\section{References}

1. Adams K, Corrigan E, eds. Priority areas for quality improvement. Washington, DC: Institute of Medicine of the National Academies, The National Academies Press; 2003.

2. Kohn LT, Corrigan J, Donaldson MS, eds. To Err is Human: Building A Safer Health System. Washington, DC: National Academy Press; 2000.

3. McGlynn EA, Asch SM, Adams J, et al. The quality of health care delivered to adults in the United States. N Engl J Med. 2003 Jun 26;348(26):2635-45. PMID: 12826639.

4. Zhan C, Arispe I, Kelley E, et al. Ambulatory care visits for treating adverse drug effects in the United States, 1995-2001. Jt Comm J Qual Patient Saf. 2005 Jul;31(7):372-8. PMID: 16130980.

5. Gurwitz JH, Rochon P, Food and Drug Administration. Improving the quality of medication use in elderly patients: a not-sosimple prescription. Arch Intern Med. 2002 Aug 12-26;162(15):1670-2. PMID: 12153368 .

6. Lazarou J, Pomeranz BH, Corey PN. Incidence of adverse drug reactions in hospitalized patients: a meta-analysis of prospective studies. JAMA. 1998 Apr 15;279(15):1200-5. PMID: 9555760.

7. Warholak TL, Nau DP. Quality and Safety in Pharmacy Practice. New York, NY: McGraw-Hill Medical; 2010.

8. Mahan D. Out-of-Bounds: Rising Prescription Drug Prices for Seniors. Families USA Foundation Publication No. 03-106. Washington, DC: July 2003.

9. Kaufman DW, Kelly JP, Rosenberg L, et al. Recent patterns of medication use in the ambulatory adult population of the United States: the Slone survey. JAMA. 2002 Jan 16;287(3):337-44. PMID: 11790213.

10. Pellegrino AN, Martin MT, Tilton JJ, et al. Medication therapy management services: definitions and outcomes. Drugs. 2009;69(4):393-406. PMID: 19323584.

11. Lewin Group. Medication therapy management services: a critical review. J
Am Pharm Assoc (2003). 2005 SepOct;45(5):580-7. PMID: 16295643.

12. 108th U.S. Congress. Medicare Prescription Drug, Improvement, and Modernization Act of 2003. 2003.

13. Department of Veterans Affairs, Veterans Health Administration. Pharmacy General Requirements. Washington, DC: Veterans Health Administration; 2008 April 17. http:/www.va.gov/vhapublications/ViewPu blication.asp?pub_ID=1678. Accessed February 20, 2013.

14. Tudor CG. Contract Year 2013 Medication Therapy Management (MTM) Program Guidance and Submission Instructions. Baltimore, MD: Centers for Medicare \& Medicaid Services; 2012. http:www.cms.gov/Medicare/PrescriptionDrug-

Coverage/PrescriptionDrugCovGenIn/Down loads/Memo-Contract-Year-2013Medication-Therapy-Management-MTMProgram-Submission-v041012.pdf.

15. Tudor CG. Contract Year 2014 Medication Therapy Management Program Guidance and Submission Instructions. Baltimore, MD: Centers for Medicare \& Medicaid Services; 2013

http://www.cms.gov/Medicare/PrescriptionDrug-

Coverage/PrescriptionDrugCovContra/Dow nloads/Memo-Contract-Year-2014Medication-Therapy-Management-MTMProgram-Submission-v040513.pdf.

16. Isetts BJ, Buffington DE. CPT code-change proposal: national data on pharmacists' medication therapy management services. Consult Pharm. 2007 Aug;22(8):684-9. PMID: 18203409.

17. American Medical Association. CPT changes 2006: an insider's view. Chicago, IL: American Medical Association; 2005. p. 309-12.

18. Hassol A, Shoemaker SJ. Exploratory Research on Medication Therapy Management. (Prepared by Abt Associates under Contract \#HHSM-500-200500181/TO\#3.) Baltimore, MD: Centers for Medicare \& Medicaid Services; July 8, 2008. http://www.cms.gov/Research- 
Statistics-Data-and-Systems/Statistics-

Trends-and-

Reports/Reports/downloads/blackwell.pdf.

19. Bodenheimer T, Berry-Millett R. Care management of patients with complex health care needs. Robert Wood Johnson Research Synthesis Report No. 19. San Francisco, CA: December 2009.

http://www.rwjf.org/content/dam/farm/repor ts/issue briefs/2009/rwjf49853/subassets/rw jf49853 1.

20. Agency for Healthcare Research and Quality. Evidence-based Practice Center Systematic Review Protocol. Project Title: Medication Therapy Management.

Rockville, MD: AHRQ; 2013 July 24. http://effectivehealthcare.ahrq.gov/ehc/prod ucts/516/1601/medication-therapymanagement-protocol-130724.pdf. Accessed September 7, 2013.

21. Moher D, Liberati A, Tetzlaff J, et al. Preferred reporting items for systematic reviews and meta-analyses: the PRISMA statement. J Clin Epidemiol. 2009 Oct;62(10):1006-12. PMID: 19631508.

22. Shea BJ, Grimshaw JM, Wells GA, et al. Development of AMSTAR: a measurement tool to assess the methodological quality of systematic reviews. BMC Med Res Methodol. 2007;7:10. PMID: 17302989.

23. Viswanathan M, Ansari MT, Berkman ND, et al. Assessing the Risk of Bias of Individual Studies in Systematic Reviews of Health Care Interventions. AHRQ Publication No. 12-EHC047-EF. Rockville, MD: Agency for Healthcare Research and Quality Methods Guide for Comparative Effectiveness Reviews; March 2012. www.effectivehealthcare.ahrq.gov/.

24. Higgins JP, Altman DG, Gotzsche PC, et al. The Cochrane Collaboration's tool for assessing risk of bias in randomised trials. BMJ. 2011;343:d5928. PMID: 22008217.

25. Viswanathan M, Berkman ND. Development of the RTI item bank on risk of bias and precision of observational studies. J Clin Epidemiol. 2012 Feb;65(2):163-78. PMID: 21959223.

26. West SL, Gartlehner G, Mansfield AJ, et al. Comparative effectiveness review methods: Clinical heterogeneity. Agency for Healthcare Research and Quality. Methods
Research Paper. AHRQ Publication No. 10EHC070-EF. Rockville, MD: September 2010. http://effectivehealthcare.ahrq.gov/.

27. Berkman ND, Lohr KN, Ansari M, et al. Grading the Strength of a Body of Evidence When Assessing Health Care Interventions for the Effective Health Care Program of the Agency for Healthcare Research and Quality: An Update. Methods Guide for Comparative Effectiveness Reviews (Prepared by the RTI-UNC Evidence-based Practice Center under Contract No. 2902007-10056-I) AHRQ Publication No. 13(14)-EHC130-EF. Rockville, MD: Agency for Healthcare Research and Quality; November 2013.

www.effectivehealthcare.ahrq.gov/reports/fi nal.cfm.

28. Guyatt GH, Oxman AD, Vist G, et al. GRADE guidelines: 4. Rating the quality of evidence--study limitations (risk of bias). J Clin Epidemiol. 2011 Apr;64(4):407-15. PMID: 21247734.

29. Owens DK, Lohr KN, Atkins D, et al. AHRQ series paper 5: grading the strength of a body of evidence when comparing medical interventions--Agency for Healthcare Research and Quality and the Effective Health-Care Program. J Clin Epidemiol. 2010 May;63(5):513-23. PMID: 19595577.

30. Atkins D, Chang SM, Gartlehner G, et al. Assessing applicability when comparing medical interventions: AHRQ and the Effective Health Care Program. J Clin Epidemiol. 2011 Nov;64(11):1198-207. PMID: 21463926.

31. Fox D, Ried LD, Klein GE, et al. A medication therapy management program's impact on low-density lipoprotein cholesterol goal attainment in Medicare Part D patients with diabetes. J Am Pharm Assoc (2003). 2009 Mar-Apr;49(2):192-9. PMID: 19289345.

32. Jeong EW, Gallagher MW, Fredriks D, et al. Impact of a pharmacist-managed medication therapy management (MTM) program. ASHP Midyear Clinical Meeting. 2007;42(DEC)PMID: 45-05344.

33. Moczygemba LR, Barner JC, Lawson KA, et al. Impact of telephone medication therapy management on medication and 
health-related problems, medication adherence, and Medicare Part D drug costs: a 6-month follow up. Am J Geriatr Pharmacother. 2011 Oct;9(5):328-38. PMID: 21865093.

34. Moczygemba LR, Barner JC, Gabrillo ER, et al. Development and implementation of a telephone medication therapy management program for Medicare beneficiaries. Am J Health Syst Pharm. 2008 Sep 1;65(17):1655-60. PMID: 18714113.

35. Pindolia VK, Stebelsky L, Romain TM, et al. Mitigation of medication mishaps via medication therapy management. Ann Pharmacother. 2009 Apr;43(4):611-20. PMID: 19336646.

36. Staresinic AG. Early experience with medication management - Description and preliminary outcomes of a Medicare Part D medication therapy management program. Journal of the Pharmacy Society of Wisconsin (USA). 2007(SEP-OCT):8-0. PMID: 45-01183.

37. Welch EK, Delate T, Chester EA, et al. Assessment of the impact of medication therapy management delivered to homebased Medicare beneficiaries. Ann Pharmacother. 2009 Apr;43(4):603-10. PMID: 19318600.

38. Christensen DB, Roth M, Trygstad T, et al. Evaluation of a pilot medication therapy management project within the North Carolina State Health Plan. J Am Pharm Assoc (2003). 2007 Jul-Aug;47(4):471-83. PMID: 17616493.

39. Jameson J, VanNoord G, Vanderwoud K. The impact of a pharmacotherapy consultation on the cost and outcome of medical therapy. J Fam Pract. 1995 Nov;41(5):469-72. PMID: 7595265.

40. Malone DC, Carter BL, Billups SJ, et al. An economic analysis of a randomized, controlled, multicenter study of clinical pharmacist interventions for high-risk veterans: the IMPROVE study. Impact of Managed Pharmaceutical Care Resource Utilization and Outcomes in Veterans Affairs Medical Centers. Pharmacotherapy. 2000 Oct;20(10):1149-58. PMID: 11034037.

41. Ellis SL, Billups SJ, Malone DC, et al. Types of interventions made by clinical pharmacists in the IMPROVE study. Impact of Managed Pharmaceutical Care on Resource Utilization and Outcomes in Veterans Affairs Medical Centers. Pharmacotherapy. 2000 Apr;20(4):429-35. PMID: 10772374.

42. Ellis SL, Carter BL, Malone DC, et al. Clinical and economic impact of ambulatory care clinical pharmacists in management of dyslipidemia in older adults: the IMPROVE study. Impact of Managed Pharmaceutical Care on Resource Utilization and Outcomes in Veterans Affairs Medical Centers. Pharmacotherapy. 2000 Dec;20(12):150816. PMID: 11130223.

43. Malone DC, Carter BL, Billups SJ, et al. Can clinical pharmacists affect SF-36 scores in veterans at high risk for medicationrelated problems? Med Care. 2001 Feb;39(2):113-22. PMID: 11176549.

44. Sellors J, Kaczorowski J, Sellors C, et al. A randomized controlled trial of a pharmacist consultation program for family physicians and their elderly patients. CMAJ. $2003 \mathrm{Jul}$ 8;169(1):17-22. PMID: 12847034.

45. Sidel VW, Beizer JL, Lisi-Fazio D, et al. Controlled study of the impact of educational home visits by pharmacists to high-risk older patients. J Community Health. 1990 Jun;15(3):163-74. PMID: 2195066.

46. Winston S, Lin YS. Impact on drug cost and use of Medicare part D of medication therapy management services delivered in 2007. J Am Pharm Assoc (2003). 2009 NovDec;49(6):813-20. PMID: 19858047.

47. Witry MJ, Doucette WR, Gainer KL. Evaluation of the pharmaceutical case management program implemented in a private sector health plan. J Am Pharm Assoc (2003). 2011 Sep-Oct;51(5):631-5. PMID: 21896463.

48. Chisholm MA, Mulloy LL, Jagadeesan M, et al. Effect of clinical pharmacy services on the blood pressure of African-American renal transplant patients. Ethn Dis. 2002 Summer;12(3):392-7. PMID: 12148711.

49. Gattis WA, Hasselblad V, Whellan DJ, et al. Reduction in heart failure events by the addition of a clinical pharmacist to the heart failure management team: results of the Pharmacist in Heart Failure Assessment 
Recommendation and Monitoring (PHARM) Study. Arch Intern Med. 1999 Sep 13;159(16):1939-45. PMID: 10493325.

50. McDonough RP, Doucette WR, Kumbera P, et al. An evaluation of managing and educating patients on the risk of glucocorticoid-induced osteoporosis. Value Health. 2005 Jan-Feb;8(1):24-31. PMID: 15841891 .

51. Bernsten C, Bjorkman I, Caramona M, et al. Improving the well-being of elderly patients via community pharmacy-based provision of pharmaceutical care: a multicentre study in seven European countries. Drugs Aging. 2001;18(1):63-77. PMID: 11232739.

52. Sturgess IK, McElnay JC, Hughes CM, et al. Community pharmacy based provision of pharmaceutical care to older patients. Pharm World Sci. 2003 Oct;25(5):218-26. PMID: 14584229 .

53. Harrison JJ, Wang J, Cervenko J, et al. Pilot study of a pharmaceutical care intervention in an outpatient lung transplant clinic. Clin Transplant. 2012 Mar-Apr;26(2):E149-57. PMID: 22507355.

54. Triller DM, Hamilton RA. Effect of pharmaceutical care services on outcomes for home care patients with heart failure. Am J Health Syst Pharm. 2007 Nov 1;64(21):2244-9. PMID: 17959576.

55. Touchette DR, Masica AL, Dolor RJ, et al. Safety-focused medication therapy management: a randomized controlled trial. J Am Pharm Assoc (2003). 2012 SepOct;52(5):603-12. PMID: 23023840.

56. Carter BL, Barnette DJ, Chrischilles E, et al. Evaluation of hypertensive patients after care provided by community pharmacists in a rural setting. Pharmacotherapy. 1997 NovDec;17(6):1274-85. PMID: 9399611.

57. Barnette DJ, Murphy CM, Carter BL. Clinical skill development for community pharmacists. J Am Pharm Assoc (Wash). 1996 Sep;NS36(9):573-80. PMID: 8824077.

58. Clifford RM, Batty KT, Davis TME, et al. A randomised controlled trial of a pharmaceutical care programme in high-risk diabetic patients in an outpatient clinic. Int $\mathrm{J}$ Pharm Pract. 2002;10(2):85-9.

59. Pai AB, Boyd A, Depczynski J, et al. Reduced drug use and hospitalization rates in patients undergoing hemodialysis who received pharmaceutical care: a 2-year, randomized, controlled study. Pharmacotherapy. 2009 Dec;29(12):143340. PMID: 19947803.

60. Pai AB, Boyd A, Chavez A, et al. Healthrelated quality of life is maintained in hemodialysis patients receiving pharmaceutical care: a 2-year randomized, controlled study. Hemodial Int. 2009 Jan;13(1):72-9. PMID: 19210281.

61. Blennerhassett JD, Cusack BM, Smith CD, et al. A novel medicines management pathway. Journal of Pharmacy Practice and Research (Australia). 2006;36(Mar):175-9. PMID: 44-00783.

62. Roughead EE, Barratt JD, Ramsay E, et al. The effectiveness of collaborative medicine reviews in delaying time to next hospitalization for patients with heart failure in the practice setting: results of a cohort study. Circ Heart Fail. 2009 Sep;2(5):424-8. PMID: 19808372.

63. Chrischilles EA, Carter BL, Lund BC, et al. Evaluation of the Iowa Medicaid pharmaceutical case management program. J Am Pharm Assoc (2003). 2004 MayJun;44(3):337-49. PMID: 15191244.

64. Isetts BJ, Schondelmeyer SW, Artz MB, et al. Clinical and economic outcomes of medication therapy management services: the Minnesota experience. J Am Pharm Assoc. 2008 Mar-Apr;48(2):203-11; 3 p following 11. PMID: 18359733.

65. Fischer LR, Defor TA, Cooper S, et al. Pharmaceutical care and health care utilization in an HMO. Eff Clin Pract. 2002 Mar-Apr;5(2):49-57. PMID: 11990212.

66. Fischer LR, Scott LM, Boonstra DM, et al. Pharmaceutical care for patients with chronic conditions. J Am Pharm Assoc (Wash). 2000 Mar-Apr;40(2):174-80. PMID: 10730021.

67. Hanlon JT, Weinberger M, Samsa GP, et al. A randomized, controlled trial of a clinical pharmacist intervention to improve inappropriate prescribing in elderly outpatients with polypharmacy. Am J Med. 1996 Apr;100(4):428-37. PMID: 8610730.

68. Taylor CT, Byrd DC, Krueger K. Improving primary care in rural Alabama with a 
pharmacy initiative. Am J Health Syst

Pharm. 2003 Jun 1;60(11):1123-9. PMID: 12816022 .

69. Volume CI, Farris KB, Kassam R, et al. Pharmaceutical care research and education project: patient outcomes. J Am Pharm Assoc (Wash). 2001 May-Jun;41(3):411-20. PMID: 11372906.

70. Kassam R, Farris KB, Burback L, et al. Pharmaceutical care research and education project: pharmacists' interventions. J Am Pharm Assoc (Wash). 2001 MayJun;41(3):401-10. PMID: 11372905.

71. Planas LG, Crosby KM, Mitchell KD, et al. Evaluation of a hypertension medication therapy management program in patients with diabetes. J Am Pharm Assoc (2003). 2009 Mar-Apr;49(2):164-70. PMID: 19289342.

72. Park JJ, Kelly P, Carter BL, et al. Comprehensive pharmaceutical care in the chain setting. J Am Pharm Assoc (Wash). 1996 Jul;NS36(7):443-51. PMID: 8840744.

73. Krska J, Cromarty JA, Arris F, et al. Pharmacist-led medication review in patients over 65: a randomized, controlled trial in primary care. Age Ageing. 2001 May;30(3):205-11. PMID: 11443021.
74. Williams ME, Pulliam CC, Hunter R, et al. The short-term effect of interdisciplinary medication review on function and cost in ambulatory elderly people. J Am Geriatr Soc. 2004 Jan;52(1):93-8. PMID: 14687321.

75. Chisholm-Burns MA, Kim Lee J, Spivey CA, et al. US pharmacists' effect as team members on patient care: systematic review and meta-analyses. Med Care. 2010 Oct;48(10):923-33. PMID: 20720510.

76. Atkins D, Chang S, Gartlehner G, et al. Assessing the applicability of studies when comparing medical interventions. Methods Guide for Comparative Effectiveness Reviews. AHRQ Publication No. 10(12)EHC063-EF. Rockville, MD: Agency for Healthcare Research and Quality.; 2011.

77. Bradley EH, Curry LA, Ramanadhan S, et al. Research in action: using positive deviance to improve quality of health care. Implement Sci. 2009;4:25. PMID: 19426507.

78. Brown CA, Lilford RJ. The stepped wedge trial design: A systematic review. BMC Med Res Methodol. 2006;6:54. PMID: 17092344. 


\section{Appendix A. Literature Search Strategies}

\section{Published Literature}

\section{PubMed. Total of 1521 records retrieved; 1425 records imported after removing}

duplicates.

PubMed search revision 6-27-13: added British terms for MTM to account for the MEDMAN study.

149 additional results; all imported

\begin{tabular}{lll}
\hline $\begin{array}{l}\text { Search } \\
\text { String }\end{array}$ & Search Terms & $\begin{array}{l}\text { Number of } \\
\text { Results }\end{array}$ \\
\hline$\# 1$ & $\begin{array}{l}\text { Search "medicine management"[tiab] OR "medicines management”[tiab] Filters: } \\
\text { Humans; English }\end{array}$ & 149 \\
\hline
\end{tabular}

PubMed search revision 2-27-13: search re-run while keeping "wildcard” search terms.

26 additional results; all imported

\begin{tabular}{|c|c|c|}
\hline $\begin{array}{l}\text { Search } \\
\text { String }\end{array}$ & Search Terms & $\begin{array}{l}\text { Number of } \\
\text { Results }\end{array}$ \\
\hline$\# 1$ & Search "Medication Therapy Management"[Mesh] & 475 \\
\hline \#2 & Search "medication therapy management" & 622 \\
\hline \#3 & Search "comprehensive medication review" & 18 \\
\hline$\# 4$ & Search "personal medication record" & 13 \\
\hline$\# 5$ & Search ("medication" AND "action plan") & 139 \\
\hline$\# 6$ & Search "medication therapy review" & 10 \\
\hline$\# 7$ & Search "Medication Reconciliation"[Mesh] & 168 \\
\hline$\# 8$ & Search (med ${ }^{*}$ AND reconciliation) & $\underline{27}$ \\
\hline$\# 9$ & Search "medication-related problems" & 197 \\
\hline$\# 10$ & Search MTMP & 31 \\
\hline \#11 & Search prescriber intervention* & 223 \\
\hline \#12 & Search "drug utilization management" & $\underline{5}$ \\
\hline \#13 & Search "chronic care improvement " & 13 \\
\hline \#14 & Search "drug therapy services" & 4 \\
\hline \#15 & Search ("utilization management strategies" OR "utilization management strategy") & 17 \\
\hline \#16 & Search "optimized treatment outcomes" & $\underline{6}$ \\
\hline \#17 & Search ((patient OR patients) AND "medication understanding") & 12 \\
\hline \#18 & Search ("drug therapy outcome" OR "drug therapy outcomes") & 33 \\
\hline \#19 & Search "medication counseling" & 122 \\
\hline \#20 & Search "pharmaceutical case management" & 11 \\
\hline \#21 & Search "drug therapy management" & 97 \\
\hline \#22 & Search ("drug therapy problem" OR "drug therapy problems") & 82 \\
\hline \#23 & $\begin{array}{l}\text { Search } \# 1 \text { or } \# 2 \text { or } \# 3 \text { or } \# 4 \text { or } \# 5 \text { or } \# 6 \text { or } \# 7 \text { or } \# 8 \text { or } \# 9 \text { or } \# 10 \text { or } \# 11 \text { or } \# 12 \text { or } \# 13 \text { or } \\
\# 14 \text { or } \# 15 \text { or } \# 16 \text { or } \# 17 \text { or } \# 18 \text { or } \# 19 \text { or } \# 20 \text { or } \# 21 \text { or } \# 22)\end{array}$ & 1694 \\
\hline \#24 & Search \#23 Filters: Humans & 1491 \\
\hline \#25 & Search \#23 Filters: Humans; English & 1387 \\
\hline \#26 & Search (\#25 AND (2012/10:2013/12[edat])) & 26 \\
\hline
\end{tabular}


PubMed search revision 2-18-13: updated final PubMed/Medline "specific" MTM-and-MTMcomponents search conducted on 11/26/12 by using Entrez date limit of October 2012 to February 2013, which is the date each record was entered into PubMed, as opposed to limiting by publication date.

17 additional results; all imported

\begin{tabular}{|c|c|c|}
\hline $\begin{array}{l}\text { Search } \\
\text { String }\end{array}$ & Search Terms & $\begin{array}{l}\text { Number of } \\
\text { Results }\end{array}$ \\
\hline$\# 1$ & Search "Medication Therapy Management"[Mesh] & 472 \\
\hline \#2 & Search "medication therapy management" & 621 \\
\hline \#3 & Search "comprehensive medication review" & 18 \\
\hline$\# 4$ & Search "personal medication record" & 13 \\
\hline$\# 5$ & Search ("medication" AND "action plan") & 139 \\
\hline$\# 6$ & Search "medication therapy review" & 10 \\
\hline \#7 & Search "Medication Reconciliation"[Mesh] & 162 \\
\hline \#8 & Search (med* AND reconciliation) & 27 \\
\hline$\# 9$ & Search "medication-related problems" & 197 \\
\hline$\# 10$ & Search MTMP & 31 \\
\hline$\# 11$ & Search prescriber intervention* & 223 \\
\hline$\# 12$ & Search "drug utilization management" & 5 \\
\hline \#13 & Search "chronic care improvement " & 13 \\
\hline$\# 14$ & Search "drug therapy services" & 4 \\
\hline \#15 & Search ("utilization management strategies" OR "utilization management strategy") & 17 \\
\hline \#16 & Search "optimized treatment outcomes" & 6 \\
\hline \#17 & Search ((patient OR patients) AND "medication understanding") & 12 \\
\hline \#18 & Search ("drug therapy outcome" OR "drug therapy outcomes") & 33 \\
\hline \#19 & Search "medication counseling" & 122 \\
\hline \#20 & Search "pharmaceutical case management" & 11 \\
\hline \#21 & Search "drug therapy management" & 97 \\
\hline \#22 & Search ("drug therapy problem" OR "drug therapy problems") & 82 \\
\hline \#23 & $\begin{array}{l}\text { Search } \# 1 \text { or } \# 2 \text { or } \# 3 \text { or } \# 4 \text { or } \# 5 \text { or } \# 6 \text { or } \# 7 \text { or } \# 8 \text { or } \# 9 \text { or } \# 10 \text { or } \# 11 \text { or } \# 12 \text { or } \# 13 \text { or } \\
\# 14 \text { or } \# 15 \text { or } \# 16 \text { or } \# 19 \text { or } \# 20 \text { or } \# 21 \text { or }\end{array}$ & 1687 \\
\hline \#24 & Search \#23 Filters: Humans & 1476 \\
\hline \#25 & Search \#23 Filters: Humans; English & 1372 \\
\hline \#26 & Search (\#25 AND (2012/10:2013/02[edat])) & 17 \\
\hline
\end{tabular}


PubMed primary search 11-26-12 - 1190 results, all imported

\begin{tabular}{|c|c|c|}
\hline $\begin{array}{l}\text { Search } \\
\text { String }\end{array}$ & Search Terms & $\begin{array}{l}\text { Number of } \\
\text { Results }\end{array}$ \\
\hline$\# 1$ & Search "Medication Therapy Management"[Mesh] & 433 \\
\hline \#2 & Search "medication therapy management" & 582 \\
\hline \#3 & Search "comprehensive medication review" & 17 \\
\hline$\# 4$ & Search "personal medication record" & 13 \\
\hline$\# 5$ & Search ("medication" AND "action plan") & 134 \\
\hline \#6 & Search "medication therapy review" & 10 \\
\hline \#7 & Search "Medication Reconciliation"[Mesh] & 135 \\
\hline \#8 & Search (med* AND reconciliation) & 27 \\
\hline \#9 & Search "medication-related problems" & 193 \\
\hline$\# 10$ & Search MTMP & 31 \\
\hline \#11 & Search prescriber intervention* & 217 \\
\hline$\# 12$ & Search "drug utilization management" & 5 \\
\hline \#13 & Search "chronic care improvement " & 13 \\
\hline \#14 & Search "drug therapy services" & 4 \\
\hline \#15 & Search ("utilization management strategies" OR "utilization management strategy") & 17 \\
\hline$\# 16$ & Search "optimized treatment outcomes" & 6 \\
\hline$\# 17$ & Search ((patient OR patients) AND "medication understanding") & 9 \\
\hline \#18 & Search ("drug therapy outcome" OR "drug therapy outcomes") & 33 \\
\hline \#19 & Search "medication counseling" & 120 \\
\hline \#20 & Search "pharmaceutical case management" & 11 \\
\hline \#21 & $\begin{array}{l}\text { Search } \# 1 \text { or } \# 2 \text { or } \# 3 \text { or } \# 4 \text { or } \# 5 \text { or } \# 6 \text { or } \# 7 \text { or } \# 8 \text { or } \# 9 \text { or } \# 10 \text { or } \# 11 \text { or } \# 12 \text { or } \# 13 \text { or } \\
\# 14 \text { or } \# 15 \text { or } \# 16 \text { or } \# 17 \text { or } \# 18 \text { or } \# 19 \text { or } \# 20)\end{array}$ & 1473 \\
\hline \#22 & $\begin{array}{l}\text { Search } \# 1 \text { or } \# 2 \text { or } \# 3 \text { or } \# 4 \text { or } \# 5 \text { or } \# 6 \text { or } \# 7 \text { or \#8 or \#9 or \#10 or \#11 or \#12 or \#13 or } \\
\# 14 \text { or } \# 15 \text { or \#16 or \#17 or \#18 or \#19 or \#20) Filters: Humans }\end{array}$ & 1280 \\
\hline \#23 & $\begin{array}{l}\text { Search (\#1 or \#2 or \#3 or \#4 or \#5 or \#6 or \#7 or \#8 or \#9 or \#10 or \#11 or \#12 or \#13 or } \\
\# 14 \text { or \#15 or \#16 or \#17 or \#18 or \#19 or \#20) Filters: Humans; English } \\
\text { MTM terms and specific component terms. }\end{array}$ & 1190 \\
\hline
\end{tabular}




\section{Cochrane Library. Total of 359 records retrieved; 250 imported after removing duplicates.}

\section{Cochrane Library search revision 6-27-13: added British terms for MTM to account for the MEDMAN study.}

21 additional results; all imported

\begin{tabular}{lll}
\hline $\begin{array}{l}\text { Search } \\
\text { String }\end{array}$ & Search Terms & $\begin{array}{l}\text { Number of } \\
\text { Results }\end{array}$ \\
\hline$\# 1$ & "medicine management":ti or "medicine management":ab or "medicines & 21 \\
& management":ti or "medicines management":ab & \\
\hline
\end{tabular}

Cochrane Library search revision 2-27-13: search re-run while removing "wildcard" search terms and conference papers and abstracts.

338 additional results; 337 imported

\begin{tabular}{|c|c|c|}
\hline $\begin{array}{l}\text { Search } \\
\text { String }\end{array}$ & Search Terms & $\begin{array}{l}\text { Number of } \\
\text { Results }\end{array}$ \\
\hline$\# 1$ & MeSH descriptor: [Medication Therapy Management] explode all trees & 19 \\
\hline \#2 & "medication therapy management" & 30 \\
\hline \#3 & "comprehensive medication review" & 3 \\
\hline \#4 & "personal medication record" & 1 \\
\hline$\# 5$ & "medication" and "action plan" & 81 \\
\hline \#6 & "medication therapy review" & 0 \\
\hline \#7 & MeSH descriptor: [Medication Reconciliation] explode all trees & 5 \\
\hline \#8 & "medication reconciliation" & 21 \\
\hline \#9 & "medication-related problems" & 32 \\
\hline$\# 10$ & MTMP & 0 \\
\hline \#11 & "prescriber intervention" or "prescriber interventions" & 0 \\
\hline$\# 12$ & "drug utilization management" & 0 \\
\hline \#13 & "chronic care improvement" & 0 \\
\hline \#14 & "drug therapy services" & 0 \\
\hline \#15 & "utilization management strategies" or "utilization management strategy" & 0 \\
\hline$\# 16$ & "optimized treatment outcomes" & 0 \\
\hline$\# 17$ & (patient or patients) and "medication understanding" & 3 \\
\hline \#18 & "drug therapy outcome" or "drug therapy outcomes" & 142 \\
\hline \#19 & "medication counseling" & 19 \\
\hline \#20 & "pharmaceutical case management" & 1 \\
\hline \#21 & "drug therapy problem" or "drug therapy problems" & 16 \\
\hline$\# 22$ & "drug therapy management" & 8 \\
\hline \#23 & $\begin{array}{l}\# 1 \text { or } \# 2 \text { or } \# 3 \text { or } \# 4 \text { or } \# 5 \text { or } \# 6 \text { or } \# 7 \text { or } \# 8 \text { or } \# 9 \text { or } \# 10 \text { or } \# 11 \text { or } \# 12 \text { or } \# 13 \text { or } \# 14 \text { or } \\
\# 15 \text { or } \# 16 \text { or } \# 17 \text { or } \# 18 \text { or } \# 19 \text { or } \# 20 \text { or } \# 21 \text { or } \# 22\end{array}$ & 338 \\
\hline \#24 & MeSH descriptor: [Congresses] explode all trees & 4 \\
\hline$\# 25$ & MeSH descriptor: [Congresses as Topic] explode all trees & 38 \\
\hline \#26 & congresses:pt & 45 \\
\hline \#27 & \#24 or \#25 or \#26 & 83 \\
\hline \#28 & \#23 not \#27 & 338 \\
\hline
\end{tabular}

Cochrane Library primary search 2-18-13: run concurrently with revised PubMed search, but eventually replaced with 2-27-13 search described above. 
534 additional results; 532 imported

\begin{tabular}{|c|c|c|}
\hline $\begin{array}{l}\text { Search } \\
\text { String }\end{array}$ & Search Terms & $\begin{array}{l}\text { Number of } \\
\text { Results }\end{array}$ \\
\hline \#1 & MeSH descriptor: [Medication Therapy Management] explode all trees & 19 \\
\hline \#2 & "medication therapy management" & 30 \\
\hline \#3 & "comprehensive medication review" & 3 \\
\hline \#4 & "personal medication record" & 1 \\
\hline$\# 5$ & "medication" and "action plan" & 81 \\
\hline \#6 & "medication therapy review" & 0 \\
\hline \#7 & MeSH descriptor: [Medication Reconciliation] explode all trees & 5 \\
\hline$\# 8$ & med $^{*}$ and reconciliation & 47 \\
\hline$\# 9$ & "medication-related problems" & 32 \\
\hline \#10 & MTMP & 0 \\
\hline \#11 & prescriber intervention* & 180 \\
\hline \#12 & "drug utilization management" & 0 \\
\hline \#13 & "chronic care improvement" & 0 \\
\hline \#14 & "drug therapy services" & 0 \\
\hline \#15 & "utilization management strategies" or "utilization management strategy" & 0 \\
\hline \#16 & "optimized treatment outcomes" & 0 \\
\hline \#17 & (patient or patients) and "medication understanding" & 3 \\
\hline \#18 & "drug therapy outcome" or "drug therapy outcomes" & 142 \\
\hline \#19 & "medication counseling" & 19 \\
\hline \#20 & "pharmaceutical case management" & 1 \\
\hline \#21 & "drug therapy problem" or "drug therapy problems" & 16 \\
\hline \#22 & "drug therapy management" & 8 \\
\hline \#23 & $\begin{array}{l}\# 1 \text { or } \# 2 \text { or } \# 3 \text { or } \# 4 \text { or } \# 5 \text { or } \# 6 \text { or } \# 7 \text { or } \# 8 \text { or } \# 9 \text { or } \# 10 \text { or } \# 11 \text { or } \# 12 \text { or } \# 13 \text { or } \# 14 \text { or } \\
\# 15 \text { or } \# 16 \text { or } \# 17 \text { or } \# 18 \text { or } \# 19 \text { or } \# 20 \text { or } \# 21 \text { or } \# 22\end{array}$ & 534 \\
\hline
\end{tabular}


International Pharmaceutical Abstracts (IPA): total of 684 records retrieved; 454 imported after removing duplicates.

IPA search revision 6-27-13: added British terms for MTM to account for the MEDMAN study. 19 additional results; 18 imported

\begin{tabular}{lllll}
\hline $\begin{array}{l}\text { Search } \\
\text { String }\end{array}$ & Search Terms & Limiters/Expanders & Last Run Via & $\begin{array}{l}\text { Number of } \\
\text { Results }\end{array}$ \\
\hline$\# 1$ & TI ( "medicine management" OR & Limiters - Language: & Interface - & 19 \\
& "medicines management" ) AND AB ( & English; Articles about & EBSCOhost & \\
& "medicine management" OR "medicines & Human Studies & Search Screen - \\
& management" ) & Search modes - & Advanced Search \\
& & Boolean/Phrase & Database - \\
& & & International \\
& & & Pharmaceutical \\
\end{tabular}

IPA search revision 2-27-13: search re-run while removing "wildcard" search terms.

673 additional results; 666 imported

\begin{tabular}{|c|c|c|c|c|}
\hline $\begin{array}{l}\text { Search } \\
\text { String }\end{array}$ & Search Terms & Limiters/Expanders & Last Run Via & $\begin{array}{l}\text { Number of } \\
\text { Results }\end{array}$ \\
\hline S22 & S21 & $\begin{array}{l}\text { Limiters - Language: } \\
\text { English; Articles about } \\
\text { Human Studies } \\
\text { Search modes - } \\
\text { Boolean/Phrase } \\
\end{array}$ & $\begin{array}{l}\text { Interface - EBSCOhost } \\
\text { Search Screen - Advanced } \\
\text { Search } \\
\text { Database - International } \\
\text { Pharmaceutical Abstracts }\end{array}$ & 673 \\
\hline S21 & $\begin{array}{l}\text { S1 OR S2 OR S3 OR S4 OR S5 } \\
\text { OR S6 OR S7 OR S8 OR S9 OR } \\
\text { S10 OR S11 OR S12 OR S13 OR } \\
\text { S14 OR S15 OR S16 OR S17 OR } \\
\text { S18 OR S19 OR S20 }\end{array}$ & $\begin{array}{l}\text { Search modes - } \\
\text { Boolean/Phrase }\end{array}$ & $\begin{array}{l}\text { Interface - EBSCOhost } \\
\text { Search Screen - Advanced } \\
\text { Search } \\
\text { Database - International } \\
\text { Pharmaceutical Abstracts }\end{array}$ & 1,558 \\
\hline S20 & "drug therapy management" & $\begin{array}{l}\text { Search modes - } \\
\text { Boolean/Phrase }\end{array}$ & $\begin{array}{l}\text { Interface - EBSCOhost } \\
\text { Search Screen - Advanced } \\
\text { Search } \\
\text { Database - International } \\
\text { Pharmaceutical Abstracts }\end{array}$ & 243 \\
\hline S19 & $\begin{array}{l}\text { "drug therapy problem" OR "drug } \\
\text { therapy problems" }\end{array}$ & $\begin{array}{l}\text { Search modes - } \\
\text { Boolean/Phrase }\end{array}$ & $\begin{array}{l}\text { Interface - EBSCOhost } \\
\text { Search Screen - Advanced } \\
\text { Search } \\
\text { Database - International } \\
\text { Pharmaceutical Abstracts }\end{array}$ & 145 \\
\hline$S 18$ & $\begin{array}{l}\text { "pharmaceutical case } \\
\text { management" }\end{array}$ & $\begin{array}{l}\text { Search modes - } \\
\text { Boolean/Phrase }\end{array}$ & $\begin{array}{l}\text { Interface - EBSCOhost } \\
\text { Search Screen - Advanced } \\
\text { Search } \\
\text { Database - International } \\
\text { Pharmaceutical Abstracts } \\
\end{array}$ & 14 \\
\hline S17 & "medication counseling" & $\begin{array}{l}\text { Search modes - } \\
\text { Boolean/Phrase }\end{array}$ & $\begin{array}{l}\text { Interface - EBSCOhost } \\
\text { Search Screen - Advanced } \\
\text { Search } \\
\text { Database - International } \\
\text { Pharmaceutical Abstracts }\end{array}$ & 232 \\
\hline$S 16$ & $\begin{array}{l}\text { "drug therapy outcome" OR "drug } \\
\text { therapy outcomes" }\end{array}$ & $\begin{array}{l}\text { Search modes - } \\
\text { Boolean/Phrase }\end{array}$ & $\begin{array}{l}\text { Interface - EBSCOhost } \\
\text { Search Screen - Advanced } \\
\text { Search } \\
\text { Database - International } \\
\text { Pharmaceutical Abstracts }\end{array}$ & 72 \\
\hline$\overline{S 15}$ & $\begin{array}{l}\text { (patient OR patients) AND } \\
\text { "medication understanding" }\end{array}$ & $\begin{array}{l}\text { Search modes - } \\
\text { Boolean/Phrase }\end{array}$ & $\begin{array}{l}\text { Interface - EBSCOhost } \\
\text { Search Screen - Advanced } \\
\text { Search } \\
\text { Database - International }\end{array}$ & 4 \\
\hline
\end{tabular}




\begin{tabular}{|c|c|c|c|c|}
\hline $\begin{array}{l}\text { Search } \\
\text { String }\end{array}$ & Search Terms & Limiters/Expanders & Last Run Via & $\begin{array}{l}\text { Number of } \\
\text { Results }\end{array}$ \\
\hline & & & Pharmaceutical Abstracts & \\
\hline$\overline{S 14}$ & "optimized treatment outcomes" & $\begin{array}{l}\text { Search modes - } \\
\text { Boolean/Phrase }\end{array}$ & $\begin{array}{l}\text { Interface - EBSCOhost } \\
\text { Search Screen - Advanced } \\
\text { Search } \\
\text { Database - International } \\
\text { Pharmaceutical Abstracts }\end{array}$ & 0 \\
\hline$S 13$ & $\begin{array}{l}\text { "utilization management } \\
\text { strategies" OR "utilization } \\
\text { management strategy" }\end{array}$ & $\begin{array}{l}\text { Search modes - } \\
\text { Boolean/Phrase }\end{array}$ & $\begin{array}{l}\text { Interface - EBSCOhost } \\
\text { Search Screen - Advanced } \\
\text { Search } \\
\text { Database - International } \\
\text { Pharmaceutical Abstracts }\end{array}$ & 4 \\
\hline$\overline{\mathrm{S} 12}$ & "drug therapy services" & $\begin{array}{l}\text { Search modes - } \\
\text { Boolean/Phrase }\end{array}$ & $\begin{array}{l}\text { Interface - EBSCOhost } \\
\text { Search Screen - Advanced } \\
\text { Search } \\
\text { Database - International } \\
\text { Pharmaceutical Abstracts }\end{array}$ & 2 \\
\hline$\overline{\mathrm{S} 11}$ & "chronic care improvement" & $\begin{array}{l}\text { Search modes - } \\
\text { Boolean/Phrase }\end{array}$ & $\begin{array}{l}\text { Interface - EBSCOhost } \\
\text { Search Screen - Advanced } \\
\text { Search } \\
\text { Database - International } \\
\text { Pharmaceutical Abstracts }\end{array}$ & 3 \\
\hline S10 & "drug utilization management" & $\begin{array}{l}\text { Search modes - } \\
\text { Boolean/Phrase }\end{array}$ & $\begin{array}{l}\text { Interface - EBSCOhost } \\
\text { Search Screen - Advanced } \\
\text { Search } \\
\text { Database - International } \\
\text { Pharmaceutical Abstracts }\end{array}$ & 16 \\
\hline S9 & $\begin{array}{l}\text { "prescriber intervention" OR } \\
\text { "prescriber interventions" }\end{array}$ & $\begin{array}{l}\text { Search modes - } \\
\text { Boolean/Phrase }\end{array}$ & $\begin{array}{l}\text { Interface - EBSCOhost } \\
\text { Search Screen - Advanced } \\
\text { Search } \\
\text { Database - International } \\
\text { Pharmaceutical Abstracts }\end{array}$ & 4 \\
\hline$S 8$ & MTMP & $\begin{array}{l}\text { Search modes - } \\
\text { Boolean/Phrase }\end{array}$ & $\begin{array}{l}\text { Interface - EBSCOhost } \\
\text { Search Screen - Advanced } \\
\text { Search } \\
\text { Database - International } \\
\text { Pharmaceutical Abstracts }\end{array}$ & 10 \\
\hline$\overline{\mathrm{S} 7}$ & "medication-related problems" & $\begin{array}{l}\text { Search modes - } \\
\text { Boolean/Phrase }\end{array}$ & $\begin{array}{l}\text { Interface - EBSCOhost } \\
\text { Search Screen - Advanced } \\
\text { Search } \\
\text { Database - International } \\
\text { Pharmaceutical Abstracts }\end{array}$ & 199 \\
\hline$\overline{S 6}$ & "medication reconciliation" & $\begin{array}{l}\text { Search modes - } \\
\text { Boolean/Phrase }\end{array}$ & $\begin{array}{l}\text { Interface - EBSCOhost } \\
\text { Search Screen - Advanced } \\
\text { Search } \\
\text { Database - International } \\
\text { Pharmaceutical Abstracts }\end{array}$ & 341 \\
\hline$\overline{\text { S5 }}$ & "medication therapy review" & $\begin{array}{l}\text { Search modes - } \\
\text { Boolean/Phrase }\end{array}$ & $\begin{array}{l}\text { Interface - EBSCOhost } \\
\text { Search Screen - Advanced } \\
\text { Search } \\
\text { Database - International } \\
\text { Pharmaceutical Abstracts }\end{array}$ & 10 \\
\hline$\overline{\mathrm{S} 4}$ & "medication" AND "action plan" & $\begin{array}{l}\text { Search modes - } \\
\text { Boolean/Phrase }\end{array}$ & $\begin{array}{l}\text { Interface - EBSCOhost } \\
\text { Search Screen - Advanced } \\
\text { Search } \\
\text { Database - International } \\
\text { Pharmaceutical Abstracts }\end{array}$ & 80 \\
\hline S3 & "personal medication record" & $\begin{array}{l}\text { Search modes - } \\
\text { Boolean/Phrase }\end{array}$ & $\begin{array}{l}\text { Interface - EBSCOhost } \\
\text { Search Screen - Advanced } \\
\text { Search }\end{array}$ & 12 \\
\hline
\end{tabular}




\begin{tabular}{|c|c|c|c|c|}
\hline $\begin{array}{l}\text { Search } \\
\text { String }\end{array}$ & Search Terms & Limiters/Expanders & Last Run Via & $\begin{array}{l}\text { Number of } \\
\text { Results }\end{array}$ \\
\hline & & & $\begin{array}{l}\text { Database - International } \\
\text { Pharmaceutical Abstracts }\end{array}$ & \\
\hline S2 & $\begin{array}{l}\text { "comprehensive medication } \\
\text { review" }\end{array}$ & $\begin{array}{l}\text { Search modes - } \\
\text { Boolean/Phrase }\end{array}$ & $\begin{array}{l}\text { Interface - EBSCOhost } \\
\text { Search Screen - Advanced } \\
\text { Search } \\
\text { Database - International } \\
\text { Pharmaceutical Abstracts }\end{array}$ & 12 \\
\hline$\overline{\mathrm{S} 1}$ & $\begin{array}{l}\text { "medication therapy } \\
\text { management" }\end{array}$ & $\begin{array}{l}\text { Search modes - } \\
\text { Boolean/Phrase }\end{array}$ & $\begin{array}{l}\text { Interface - EBSCOhost } \\
\text { Search Screen - Advanced } \\
\text { Search } \\
\text { Database - International } \\
\text { Pharmaceutical Abstracts }\end{array}$ & 289 \\
\hline
\end{tabular}


IPA primary search 2-18-13: run concurrently with revised PubMed search, but eventually replaced with 2-27-13 search described above.

\section{9 additional results; 679 imported}

\begin{tabular}{|c|c|c|c|}
\hline $\begin{array}{l}\text { Search } \\
\text { String }\end{array}$ & Search Terms & Limiters/Expanders & $\begin{array}{l}\text { Number of } \\
\text { Results }\end{array}$ \\
\hline S23 & $\begin{array}{l}\text { S1 OR S2 OR S3 OR S4 OR S5 OR S6 OR S7 OR S8 OR S9 } \\
\text { OR S10 OR S11 OR S12 OR S13 OR S14 OR S15 OR S16 OR } \\
\text { S17 OR S18 OR S19 OR S20 OR S21 }\end{array}$ & $\begin{array}{l}\text { Limiters - Language: } \\
\text { English; Articles about } \\
\text { Human Studies } \\
\text { Search modes - } \\
\text { Boolean/Phrase }\end{array}$ & 739 \\
\hline$\overline{S 22}$ & $\begin{array}{l}\text { S1 OR S2 OR S3 OR S4 OR S5 OR S6 OR S7 OR S8 OR S9 } \\
\text { OR S10 OR S11 OR S12 OR S13 OR S14 OR S15 OR S16 OR } \\
\text { S17 OR S18 OR S19 OR S20 OR S21 }\end{array}$ & $\begin{array}{l}\text { Search modes - } \\
\text { Boolean/Phrase }\end{array}$ & 1,803 \\
\hline$\overline{S 21}$ & "drug therapy management" & $\begin{array}{l}\text { Search modes - } \\
\text { Boolean/Phrase }\end{array}$ & 243 \\
\hline S20 & "drug therapy problem" OR "drug therapy problems" & $\begin{array}{l}\text { Search modes - } \\
\text { Boolean/Phrase }\end{array}$ & 145 \\
\hline S19 & "pharmaceutical case management" & $\begin{array}{l}\text { Search modes - } \\
\text { Boolean/Phrase }\end{array}$ & 14 \\
\hline$\overline{\mathrm{S} 18}$ & "medication counseling" & $\begin{array}{l}\text { Search modes - } \\
\text { Boolean/Phrase }\end{array}$ & 232 \\
\hline $\mathrm{S} 17$ & "drug therapy outcome" OR "drug therapy outcomes" & $\begin{array}{l}\text { Search modes - } \\
\text { Boolean/Phrase }\end{array}$ & 72 \\
\hline$\overline{S 16}$ & (patient OR patients) AND "medication understanding" & $\begin{array}{l}\text { Search modes - } \\
\text { Boolean/Phrase }\end{array}$ & 4 \\
\hline$\overline{\mathrm{S} 15}$ & "optimized treatment outcomes" & $\begin{array}{l}\text { Search modes - } \\
\text { Boolean/Phrase }\end{array}$ & 0 \\
\hline$\overline{\mathrm{S} 14}$ & $\begin{array}{l}\text { "utilization management strategies" OR "utilization management } \\
\text { strategy" }\end{array}$ & $\begin{array}{l}\text { Search modes - } \\
\text { Boolean/Phrase }\end{array}$ & 4 \\
\hline$\overline{\mathrm{S} 13}$ & "drug therapy services" & $\begin{array}{l}\text { Search modes - } \\
\text { Boolean/Phrase }\end{array}$ & 2 \\
\hline$\overline{\mathrm{S} 12}$ & "chronic care improvement" & $\begin{array}{l}\text { Search modes - } \\
\text { Boolean/Phrase }\end{array}$ & 3 \\
\hline S11 & "drug utilization management" & $\begin{array}{l}\text { Search modes - } \\
\text { Boolean/Phrase }\end{array}$ & 16 \\
\hline$S 10$ & prescriber intervention* & $\begin{array}{l}\text { Search modes - } \\
\text { Boolean/Phrase }\end{array}$ & 95 \\
\hline S9 & MTMP & $\begin{array}{l}\text { Search modes - } \\
\text { Boolean/Phrase }\end{array}$ & 10 \\
\hline$\overline{S 8}$ & "medication-related problems" & $\begin{array}{l}\text { Search modes - } \\
\text { Boolean/Phrase }\end{array}$ & 199 \\
\hline S7 & med* $^{*}$ AND reconciliation & $\begin{array}{l}\text { Search modes - } \\
\text { Boolean/Phrase }\end{array}$ & 508 \\
\hline$\overline{S 6}$ & "medication reconciliation" & $\begin{array}{l}\text { Search modes - } \\
\text { Boolean/Phrase }\end{array}$ & 341 \\
\hline$\overline{\mathrm{S} 5}$ & "medication therapy review" & $\begin{array}{l}\text { Search modes - } \\
\text { Boolean/Phrase }\end{array}$ & 10 \\
\hline S4 & "medication" AND "action plan" & $\begin{array}{l}\text { Search modes - } \\
\text { Boolean/Phrase }\end{array}$ & 80 \\
\hline S3 & "personal medication record" & $\begin{array}{l}\text { Search modes - } \\
\text { Boolean/Phrase }\end{array}$ & 12 \\
\hline$\overline{S 2}$ & "comprehensive medication review" & $\begin{array}{l}\text { Search modes - } \\
\text { Boolean/Phrase }\end{array}$ & 12 \\
\hline$\overline{\mathrm{S1}}$ & "medication therapy management" & $\begin{array}{l}\text { Search modes - } \\
\text { Boolean/Phrase }\end{array}$ & 289 \\
\hline
\end{tabular}




\section{Gray Literature}

Search revision 6-28-13: added British terms ("medicine management" OR "medicines management") for MTM to account for the MEDMAN study.

Total of 14 records retrieved, 13 imported after initial screening.

\begin{tabular}{|c|c|c|c|}
\hline Source & Search Terms & Limits or Adjustments & $\begin{array}{l}\text { Number of } \\
\text { Results } \\
\text { Retrieved } \\
\text { (Imported) }\end{array}$ \\
\hline $\begin{array}{l}\text { ClinicalTrials.gov } \\
\text { Expert Search } \\
\text { Strategy }\end{array}$ & $\begin{array}{l}\text { "medicine management" OR "medicines } \\
\text { management" }\end{array}$ & $\begin{array}{l}\text { [ALL-FIELDS] AND ( NOT } \\
\text { NOTEXT ) [FIRST-RECEIVED- } \\
\text { RESULTS-DATE] }\end{array}$ & $2(2)$ \\
\hline WHO ICTRP & $\begin{array}{l}\text { "medicine management" OR "medicines } \\
\text { management" }\end{array}$ & None & $10(10)$ \\
\hline $\begin{array}{l}\text { HSRProj Advanced } \\
\text { search }\end{array}$ & $\begin{array}{l}\text { "medicine management" OR "medicines } \\
\text { management" }\end{array}$ & None & 0 \\
\hline $\begin{array}{l}\text { NIH RePORTER } \\
\text { Advanced search }\end{array}$ & $\begin{array}{l}\text { "medicine management" OR "medicines } \\
\text { management" }\end{array}$ & None & 0 \\
\hline $\begin{array}{l}\text { DOPHER (Database } \\
\text { of Promoting Health } \\
\text { Effectiveness } \\
\text { Reviews) }\end{array}$ & $\begin{array}{l}\text { "medicine management" OR "medicines } \\
\text { management" }\end{array}$ & None & 0 \\
\hline $\begin{array}{l}\text { New York Academy of } \\
\text { Medicine Gray } \\
\text { Literature Report } \\
\text { (greylit.org) }\end{array}$ & $\begin{array}{l}\text { "medicine management" OR "medicines } \\
\text { management" }\end{array}$ & None & 0 \\
\hline CMS.gov & $\begin{array}{l}\text { "medicine management" OR "medicines } \\
\text { management" }\end{array}$ & $\begin{array}{l}\text { "allintitle", which limited results to } \\
\text { those in which "medication } \\
\text { therapy management" appeared } \\
\text { in title of retrieved websites }\end{array}$ & $2(1)$ \\
\hline
\end{tabular}


Primary searches 3-4-13: 750 records retrieved, 596 imported after removing duplicates.

\begin{tabular}{|c|c|c|c|}
\hline Source & Search Terms & $\begin{array}{l}\text { Limits or } \\
\text { Adjustments }\end{array}$ & $\begin{array}{l}\text { Number of Results } \\
\text { Retrieved (Imported) }\end{array}$ \\
\hline $\begin{array}{l}\text { ClinicalTrials.gov } \\
\text { Expert Search } \\
\text { Strategy }\end{array}$ & $\begin{array}{l}\text { ("medication therapy management" OR "comprehensive } \\
\text { medication review" OR "Medication Reconciliation" OR } \\
\text { "pharmaceutical case management" OR "drug therapy } \\
\text { management" OR "drug therapy problem" OR "drug therapy } \\
\text { problems" ) }\end{array}$ & $\begin{array}{l}\text { [ALL-FIELDS] } \\
\text { AND ( NOT } \\
\text { NOTEXT ) } \\
\text { [FIRST- } \\
\text { RECEIVED- } \\
\text { RESULTS- } \\
\text { DATE] }\end{array}$ & $119(119)$ \\
\hline WHO ICTRP & $\begin{array}{l}\text { Title search: medication therapy management OR } \\
\text { comprehensive medication review OR Medication } \\
\text { Reconciliation OR pharmaceutical case management OR } \\
\text { drug therapy management OR drug therapy problem OR } \\
\text { drug therapy problems } \\
\text { Intervention search: was either } 41,000+\text {, with the shorter } \\
\text { search (see Search Strings \#1c and \#1d), or no results for } \\
\text { "medication therapy management" by itself. }\end{array}$ & None & $\begin{array}{l}5 \text { (5) (Title search); } \\
0 \text { (Intervention search) }\end{array}$ \\
\hline $\begin{array}{l}\text { HSRProj } \\
\text { Advanced search }\end{array}$ & $\begin{array}{l}\text { "medication therapy management" OR "comprehensive } \\
\text { medication review" OR "personal medication record" OR } \\
\text { (medication AND "action plan") OR "medication therapy } \\
\text { review" OR "Medication Reconciliation" OR "medication- } \\
\text { related problems" OR "prescriber intervention" OR "drug } \\
\text { utilization management" OR "chronic care improvement" OR } \\
\text { "drug therapy services" OR "utilization management } \\
\text { strategies" OR "utilization management strategy" OR } \\
\text { "optimized treatment outcomes" OR ((patients OR patient) } \\
\text { AND "medication understanding") OR "drug therapy } \\
\text { outcome" OR "drug therapy outcomes" OR "medication } \\
\text { counseling" OR "pharmaceutical case management" OR } \\
\text { "drug therapy management" OR "drug therapy problem" OR } \\
\text { "drug therapy problems" [Limited to Ongoing/Completed } \\
\text { status] }\end{array}$ & None & $87(82)$ \\
\hline $\begin{array}{l}\text { NIH RePORTER } \\
\text { Advanced search }\end{array}$ & $\begin{array}{l}\text { medication therapy management OR comprehensive } \\
\text { medication review OR personal medication record OR } \\
\text { (medication AND action plan) OR medication therapy review } \\
\text { OR Medication Reconciliation OR medication-related } \\
\text { problems OR medication relation problems OR prescriber } \\
\text { intervention OR drug utilization management OR chronic } \\
\text { care improvement OR drug therapy services OR utilization } \\
\text { management strategies OR utilization management strategy } \\
\text { OR optimized treatment outcomes OR (patients OR patient) } \\
\text { AND medication understanding) OR drug therapy outcome } \\
\text { OR drug therapy outcomes OR medication counseling OR } \\
\text { pharmaceutical case management OR drug therapy } \\
\text { management OR drug therapy problem OR drug therapy } \\
\text { problems }\end{array}$ & None & $234(85)$ \\
\hline $\begin{array}{l}\text { DOPHER } \\
\text { (Database of } \\
\text { Promoting Health } \\
\text { Effectiveness } \\
\text { Reviews) }\end{array}$ & $\begin{array}{l}\text { 1) medication therapy management OR comprehensive } \\
\text { medication review OR personal medication record OR } \\
\text { (medication AND action plan) OR medication therapy review } \\
\text { OR Medication Reconciliation OR medication-related } \\
\text { problems OR medication relation problems OR prescriber } \\
\text { intervention OR drug utilization management OR chronic } \\
\text { care improvement OR drug therapy services OR utilization } \\
\text { management strategies OR utilization management strategy } \\
\text { OR optimized treatment outcomes OR (patients OR patient) } \\
\text { AND medication understanding) OR drug therapy outcome } \\
\text { OR drug therapy outcomes OR medication counseling OR } \\
\text { pharmaceutical case management OR drug therapy } \\
\text { management OR drug therapy problem OR drug therapy }\end{array}$ & None & 0 for all search strings \\
\hline
\end{tabular}




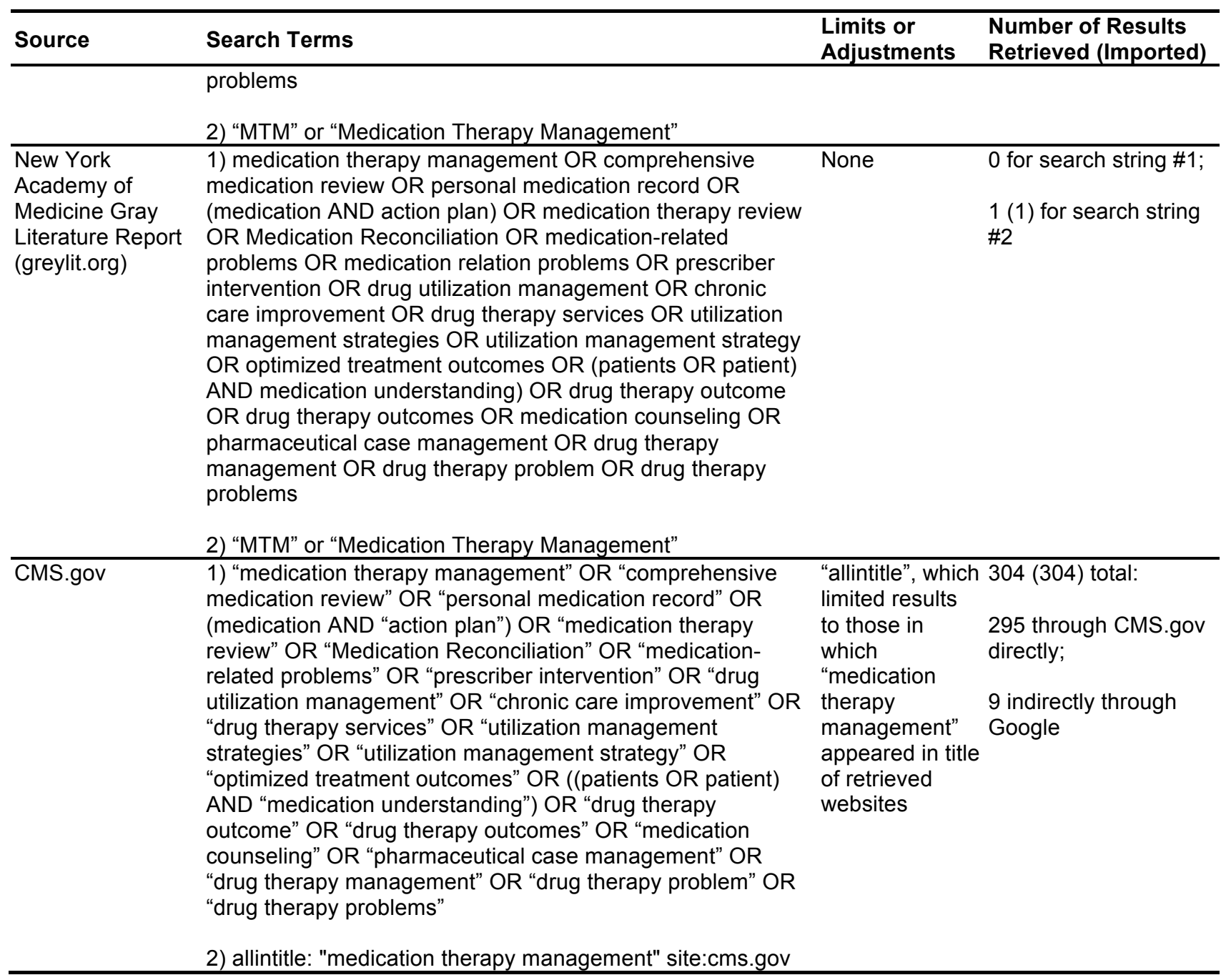




\section{Appendix B. Abstract and Full-Text Review Form Templates}

Abstract Review Form

\begin{tabular}{|c|c|c|c|c|c|c|c|c|c|}
\hline Ref ID & Author & Year & $\begin{array}{c}\text { Include or } \\
\text { Exclude? } \\
\text { (separate } \\
\text { exclusion } \\
\text { codes for } \\
\text { publication } \\
\text { type, PICOTS, } \\
\text { and study } \\
\text { design) }\end{array}$ & $\begin{array}{l}\text { If ineligible, is } \\
\text { manual review } \\
\text { or hand search } \\
\text { of full-text } \\
\text { needed? }\end{array}$ & $\begin{array}{l}\text { If ineligible, } \\
\text { potential } \\
\text { background } \\
\text { reference? }\end{array}$ & $\begin{array}{l}\text { NOTE: The } \\
\text { following } \\
\text { columns } \\
\text { apply only to } \\
\text { studies } \\
\text { meeting our } \\
\text { inclusion } \\
\text { criteria }\end{array}$ & $\begin{array}{c}\text { Study Design } \\
\text { (RCT, NRCT, } \\
\text { Other Study } \\
\text { Design) }\end{array}$ & $\begin{array}{c}\text { If "Other Study } \\
\text { Design", which } \\
\text { specific design does } \\
\text { it use? (Cohort, } \\
\text { Case-Control, } \\
\text { Nonconcurrent Time } \\
\text { Series, Other - } \\
\text { describe in } \\
\text { Comments column) }\end{array}$ & $\begin{array}{l}\text { Comments (e.g., if } \\
\text { reviewer included } \\
\text { an abstract due to a } \\
\text { lack of clarity within } \\
\text { the abstract) }\end{array}$ \\
\hline & & & & & & & & & \\
\hline
\end{tabular}

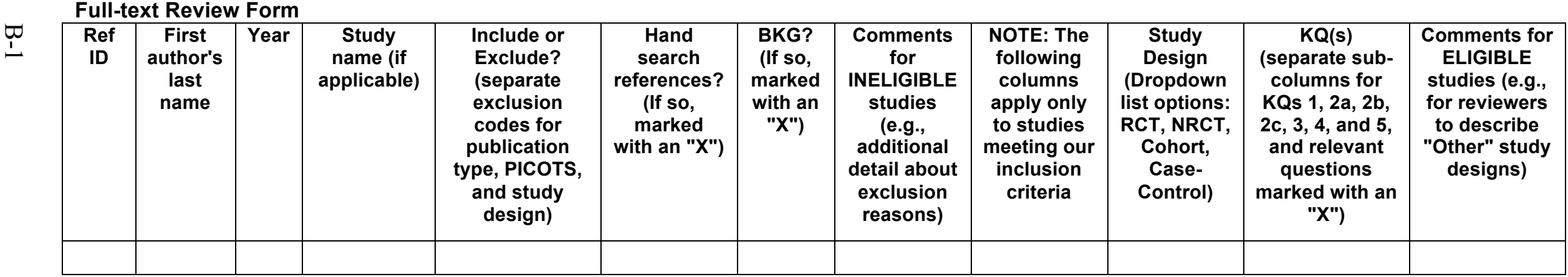




\section{Appendix C. List of Studies Excluded after Full-Text Level Review}

\section{Ineligible Publication Type $(n=71)$}

1. Author names not provided. MTM program increased statin use. Dis Manag Advis. 2008 Oct;14(10):suppl 1-3, 1. PMID: 19031586.

2. Author names not provided. What's expected for med reconciliation? OR Manager. 2008 Mar;24(3):21, 3. PMID: 18438074.

3. . Hospitals collaborate to reduce ED overuse. Hosp Case Manag. 2012 Oct;20(10):151-3. PMID: 23091842.

4. Altman JS. Medication therapy management and the new practitioner. Am J Health Syst Pharm. 2007 Mar 15;64(6):590-2. PMID: 17353567.

5. Avery AJ, Rodgers S, Cantrill JA, et al. Correction: Protocol for the PINCER trial: a cluster randomised trial comparing the effectiveness of a pharmacist-led IT-based intervention with simple feedback in reducing rates of clinically important errors in medicines management in general practices. Trials; 2010. p. 23.

6. Bates DW. Role of pharmacists in the medical home. Am J Health Syst Pharm. 2009 Jun 15;66(12):1116-8. PMID: 19498128.

7. Bell JS, Vaananen M, Ovaskainen H, et al. Providing patient care in community pharmacies: practice and research in Finland. Ann Pharmacother. 2007 Jun;41(6):1039-46. PMID: 17504836.

8. Bratcher CR, Bello E. Traditional or centralized models of diabetes care: the multidisciplinary diabetes team approach. J Fam Pract. 2011 Nov;60(11 Suppl):S6-11. PMID: 22336928.

9. Brown EV. Reconcilable differences. Health Manag Technol. 2008 Jan;29(1):12-4, 6. PMID: 18286931.

10. Brusig E, Davies W. PSTAC survey in 2006 was instrumental in obtaining permanent (category I) CPT codes for MTM services performed by pharmacists. J Manag Care Pharm. 2008 Jan-Feb;14(1):86-7. PMID: 18240887.
11. Butler CD. Medication therapy management for adverse drug event preventive care. J Am Pharm Assoc (2003). 2010 JulAug;50(4):449. PMID: 20621858.

12. Cahill JA, Manasse Jr HR. Medication therapy management programs: to optimize pharmacy outcomes. J Manag Care Pharm. 2005 Mar;11(2):179. PMID: 15766326.

13. Cantwell KM. Collaborating for successful medication therapy management programs. Am J Health Syst Pharm. 2005 Mar 15;62(6):583. PMID: 15757878.

14. Carter BL, Malone DC, Billups SJ, et al. Interpreting the findings of the IMPROVE study. Am J Health Syst Pharm. 2001 Jul 15;58(14):1330-7. PMID: 11471481.

15. Carter BL, Zillich AJ, Elliott WJ. How pharmacists can assist physicians with controlling blood pressure. J Clin Hypertens (Greenwich). 2003 Jan-Feb;5(1):31-7. PMID: 12556651.

16. Cauchi R. Medication therapy management: catching errors, saving lives and money. NCSL Legisbrief. 2010 Jan;18(4):1-2. PMID: 20196247.

17. Centers for Medicare \& Medicaid Services. Medicare Part D Medication Therapy Management (MTM) Programs: 2009 Fact Sheet. 2009.

18. Centers for Medicare \& Medicaid Services. 2010 Medicare Part D Medication Therapy Management (MTM) Programs Fact Sheet. 2010 .

19. Centers for Medicare \& Medicaid Services. 2012 Medicare Part D Medication Therapy Management (MTM) Programs Fact Sheet. 2012.

20. Cotterell CC, Dombroske L, Fischermann EA. Comprehensive drug-use evaluation program in a health maintenance organization. Am J Hosp Pharm. 1991 Aug;48(8):1712-7. PMID: 1897551. 
21. Degnin FD. Difficult patients, overmedication, and groupthink. J Clin Ethics. 2009 Spring;20(1):64-74. PMID: 19385324.

22. Dersch D. Patient assessment tool for pharmacists. Am J Health Syst Pharm. 2008 Apr 15;65(8):702-3. PMID: 18387897.

23. Dougherty L. Medicine management and infection prevention. Br J Nurs. 2011 Jul 28Aug 4;20(14):S3. PMID: 21841665.

24. Eden M, Avery A, Rodgers S, et al. Ensuring accuracy in routinely collected data in a cluster randomised controlled trial (RCT) comparing the effectiveness of a pharmacistled IT-based intervention with simple feedback in reducing rates of clinically important errors in medicines management: The PINCER Trial. International Journal of Pharmacy Practice; 2010. p. 60-1.

25. Epplen K, Marsden LM, Murphy C. Reimbursement for medication therapy management services. Am J Health Syst Pharm. 2008 May 15;65(10):906-8. PMID: 18463337.

26. Feldman PH, Totten AM, Foust J, et al. Medication management: evidence brief. Center for Home Care Policy \& Research. Home Healthc Nurse. 2009 Jun;27(6):37986. PMID: 19509525

27. Feldman SM. Pharmaceutical care and the consultant pharmacist. A model for drug therapy management. Am Pharm. 1995;Suppl:19-21. PMID: 7677061.

28. Figge HL. Interoperable health information exchange between medication therapy management services and the medical home. Am J Health Syst Pharm. 2010 Feb 1;67(3):190-1. PMID: 20101060.

29. Grantham D. Idaho behavioral health optimizes med management visits using telehealth. Behav Healthc. 2011 Oct;31(7):46-7. PMID: 22117294

30. Griffiths M. Medicines management. Nurs Stand. 2008 Oct 1-7;23(4):28. PMID: 19051531.

31. Griffiths M. Medicines management. Nurs Stand. 2009 Sep 23-29;24(3):28. PMID: 19856642.

32. Griffiths M. Medicines management. Nurs Stand. 2010 Nov 17-23;25(11):28. PMID: 21189818

33. Griffiths M. Medicines management. Clear criteria are needed for any dose adjustment.
Nurs Stand. 2010 Jan 13-19;24(19):28. PMID: 20175358.

34. Guirguis AB, Zingone MM. Medication therapy management and cost-related medication nonadherence. JAMA. 2008 Sep 10;300(10):1130; author reply -1. PMID: 18780839 .

35. Hunteman LM. Potential role of medication therapy management for bariatric surgery patients. J Am Pharm Assoc (2003). 2008 Jul-Aug;48(4):440-2. PMID: 18653412.

36. Jayaprakash A. ASCP reports. Consult Pharm. 2010 Feb;25(2):128. PMID: 20211826.

37. Kaboli PJ, Fernandes O. Medication reconciliation: moving forward. Arch Intern Med. 2012 Jul 23;172(14):1069-70. PMID: 22733283.

38. Klimek JS. MTM services standards improve patient safety. Medication therapy management safeguards, systems and services can reduce the total number of medication-related complications. Health Manag Technol. 2009 Jul;30(7):20-1. PMID: 19739561.

39. Kumbera P. A new addition to pharmacy's alphabet soup: MTMS. Pharmacy Times (USA). 2005;71(Mar):90. PMID: 43-01425.

40. Lau DT. Consumer medication management and error. Clin Ther. 2008 Nov;30(11):2156-8. PMID: 19108803.

41. Lemay G. Medication therapy management in community pharmacy practice. Med Health R I. 2012 Sep;95(9):281-2. PMID: 23094424.

42. McDonough RP. Medication therapy management and collaborative practice: How to make it work. p. 52.

43. McIntosh K. Medicines management. Nurs Times. 2008 Nov 4-10;104(44):18-20. PMID: 19014127.

44. McMahan R. Medication therapy management services: assessing year 1, gauging year 2. J Am Pharm Assoc (2003). 2006 NovDec;46(6):680-1. PMID: 17176682.

45. Mort J. Medication therapy management: a Medicare Part D benefit available to qualifying beneficiaries. S D Med. 2007 Mar;60(3):121. PMID: 17455583. 
46. Motz J. The implementation of MTM in community pharmacies - Communication and planning are key factors for success. Journal of the Pharmacy Society of Wisconsin (USA). 2007(SEP-OCT):11-2. PMID: 45-01241.

47. Osterberg L, Blaschke T. Adherence to medication. N Engl J Med. 2005 Aug 4;353(5):487-97. PMID: 16079372.

48. Reinke T. Medication therapy management program in N.C. saves $\$ 13$ million. Manag Care. 2011 Oct;20(10):17-8. PMID: 22111478 .

49. Riley D, Dodson P. Medicine management for the elderly. Tex Hosp. 1983 Apr;38(11):20. PMID: 10317312.

50. Rucker NL. Medicare Part D's Medication Therapy Management: Shifting from Neutral to Drive AARP Public Policy Institute. Washington, DC: 2012.

51. Schulz M. Pharmacist care for reactive airway disease. JAMA. 2003 Mar 5;289(9):1104; author reply 5-6. PMID: 12622576.

52. Smith SR, Clancy CM. Medication therapy management programs: forming a new cornerstone for quality and safety in medicare. Am J Med Qual. 2006 JulAug;21(4):276-9. PMID: 16849785.

53. Soller RW, Vogt E. Positioning patient counselling as a measurable cornerstone of medication therapy management. Int $\mathrm{J}$ Pharm Pract. 2010 Oct;18(5):249-51. PMID: 20840679.

54. Solow B. Medication therapy management--not just for seniors anymore. Manag Care. 2009 Oct;18(10):7. PMID: 19902682.

55. Spooner JJ. Medication therapy management programs: when will the outcomes come out? J Manag Care Pharm. 2007 Apr;13(3):276-7. PMID: 17407394.

56. Strom BL, Hennessy S. Pharmacist care and clinical outcomes for patients with reactive airways disease. JAMA. 2002 Oct 2;288(13):1642-3. PMID: 12350196.

57. Summers KH. Medication therapy management versus drug regimen review. J Manag Care Pharm. 2007 Jan-Feb;13(1):68-9. PMID: 17269840.

58. Taylor J, Davies P. The medicines management needs of carers during an episode of mental health crisis. J Psychiatr Ment Health Nurs. 2012 Mar;19(2):190-2. PMID: 22070543.

59. Thomas MC. A Practical Approach to Medication Reconciliation by Pharmacists in a Community Hospital Emergency Department. p. 41.

60. Thompson CA. State-paid medication therapy management services succeed. Am J Health Syst Pharm. 2008 Mar 15;65(6):490, 3, 8. PMID: 18319489.

61. Touchette DR. Improving adherence in the community and clinic pharmacy settings: an emerging opportunity. Pharmacotherapy. 2010 May;30(5):425-7. PMID: 20411993.

62. Traynor K. Maryland School of Pharmacy, XLHealth team up on medication management project. Am J Health Syst Pharm. 2010 Jun 1;67(11):868, 70. PMID: 20484207.

63. While A. Making the most of good medicines management. Br J Community Nurs. 2007 Sep;12(9):434. PMID: 18026009.

64. White B, Truax D. The long and winding road. Arriving at safe medication management in LTC setting. Adv Nurse Pract. 2007 Sep;15(9):28-35; quiz -6. PMID: 19998897.

65. Williams A. Medicine management. Nurs Mirror. 1984 Oct 3;159(12):i-iii, vi. PMID: 6567175.

66. Williams T, Acton C, Hicks RW. Deploying med reconciliation. Nurs Manage. 2008 Apr;39(4):54-7. PMID: 18391828.

67. Wilson J. Breaking down barriers. America's Pharmacist (USA). 2005;127(Sep):24-6. PMID: 43-01410.

68. Wong E. CDTM and smoking cessation. Hospital Pharmacy (USA). 1999;34(Nov):1295-303. PMID: 37-05144.

69. Young D. Pharmaceutical case management helps Iowa Medicaid patients. Am J Health Syst Pharm. 2003 Mar 1;60(5):414, 6. PMID: 12635443.

70. Zagaria MA. Medication therapy management services. US Pharmacist (USA). 2004;30(Apr):35-42. PMID: 42-10763.

71. Zellmer WA. Collaborative drug therapy management. Am J Health Syst Pharm. 1995 Aug 1;52(15):1732. PMID: 7583839. 


\section{Ineligible or No Intervention $(n=134)$}

1. Author name(s) not provided. Patients confirm that medication counseling helps. Am J Hosp Pharm. 1994 Jul 1;51(13):1606, 8. PMID: 7942886.

2. Author name(s) not provided. The MEDMAN study: a randomized controlled trial of community pharmacy-led medicines management for patients with coronary heart disease. Fam Pract. 2007 Apr;24(2):189200. PMID: 17272285.

3. Al-Ghamdi SA, Mahmoud MA, Alammari MA, et al. The outcome of pharmacist counseling at the time of hospital discharge: an observational nonrandomized study. Ann Saudi Med. 2012 Sep-Oct;32(5):492-7. PMID: 22871618.

4. Alsuwaidan S, Malone DC, Billups SJ, et al. Characteristics of ambulatory care clinics and pharmacists in Veterans Affairs medical centers. IMPROVE investigators. Impact of Managed Pharmaceutical Care on Resource Utilization and Outcomes in Veterans Affairs Medical Centers. Am J Health Syst Pharm. 1998 Jan 1;55(1):68-72. PMID: 9437478.

5. Asis ML, Greene R. A cost-effectiveness analysis of a peak flow-based asthma education and self-management plan in a high-cost population (Structured abstract). Journal of Asthma; 2004. p. 559-65.

6. Avery AJ, Rodgers S, Cantrill JA, et al. Protocol for the PINCER trial: a cluster randomised trial comparing the effectiveness of a pharmacist-led IT-based intervention with simple feedback in reducing rates of clinically important errors in medicines management in general practices. Trials. 2009;10:28. PMID: 19409095.

7. Bayoumi I, Howard M, Holbrook AM, et al. Interventions to improve medication reconciliation in primary care (Structured abstract). Annals of Pharmacotherapy; 2009. p. 1667-75.

8. Bellone JM, Barner JC, Lopez DA. Postdischarge interventions by pharmacists and impact on hospital readmission rates. J Am Pharm
Assoc (2003). 2012 May-Jun;52(3):358-62. PMID: 22618976.

9. Bertino J, Villa T, Corbett A, et al. Effects of optimal medication therapy management of depression in human immunodeficiency virus (HIV) infected patients on associated virologic and immunologic outcomes. Journal of Pharmacy Practice (USA). 2008;21(Jan):78. PMID: 45-14933.

10. Bilyeu KM, Gumm CJ, Fitzgerald JM, et al. Cultivating quality: Reducing the use of potentially inappropriate medications in older adults. Am J Nurs. 2011 Jan;111(1):47-52. PMID: 21191234.

11. Blakey SA, Hixson-Wallace JA. Clinical and economic effects of pharmacy services in a geriatric ambulatory clinic (Structured abstract). Pharmacotherapy; 2000. p. 1198203.

12. Brooks JM, Unni EJ, Klepser DG, et al. Factors affecting demand among older adults for medication therapy management services. Res Social Adm Pharm. 2008 Dec;4(4):30919. PMID: 19064238.

13. Brown DL, Wolff DJ, Smith LD, et al. Computersupported medication counseling for discharged patients. Am J Hosp Pharm. 1986 Sep;43(9):2232-5. PMID: 3766581.

14. Bunting BA, Cranor CW. The Asheville Project: long-term clinical, humanistic, and economic outcomes of a community-based medication therapy management program for asthma. J Am Pharm Assoc (2003). 2006 Mar-Apr;46(2):133-47. PMID: 16602223.

15. Bunting BA, Smith BH, Sutherland SE. The Asheville Project: clinical and economic outcomes of a community-based long-term medication therapy management program for hypertension and dyslipidemia. J Am Pharm Assoc (2003). 2008 JanFeb;48(1):23-31. PMID: 18192127.

16. Burnett KM, Scott MG, Fleming GF, et al. Effects of an integrated medicines management program on medication appropriateness in hospitalized patients. Am 
J Health Syst Pharm. 2009 May

1;66(9):854-9. PMID: 19386949.

17. Cadth. Medication reconciliation at discharge: a review of the clinical evidence and guidelines (Structured abstract). Health Technology Assessment Database: Canadian Agency for Drugs and Technologies in Health (CADTH); 2012.

18. Carter BL, Ardery G, Dawson JD, et al. Physician and pharmacist collaboration to improve blood pressure control. Arch Intern Med. 2009 Nov 23;169(21):1996-2002. PMID: 19933962.

19. Casteel C, Blalock SJ, Ferreri S, et al. Implementation of a community pharmacybased falls prevention program. Am J Geriatr Pharmacother. 2011 Oct;9(5):310-9 e2. PMID: 21925959.

20. Castro R, Leung J, Song J, et al. Outcomes of implementing a medication therapy management service for dialysis patients. $p$. 056 .

21. Ching MM, Chen T, Bounthavong $\mathrm{M}$. MEDICATION THERAPY MANAGEMENT PROGRAMS AT A VA MEDICAL FACILITY: EFFECTS ON BLOOD CHOLESTEROL IN HIGH RISK PATIENTS. p. 15.

22. Choe HM, Mitrovich S, Dubay D, et al. Proactive case management of high-risk patients with type 2 diabetes mellitus by a clinical pharmacist: a randomized controlled trial. Am J Manag Care. 2005 Apr;11(4):253-60. PMID: 15839185.

23. Christensen DB, Holmes G, Fassett WE, et al. Principal findings from the Washington State cognitive services demonstration project. Manag Care Interface. 1998 Jul;11(7):60-2, 4. PMID: 10181572.

24. Cioffi ST, Caron MF, Kalus JS, et al. Glycosylated hemoglobin, cardiovascular, and renal outcomes in a pharmacistmanaged clinic. Ann Pharmacother. 2004 May;38(5):771-5. PMID: 15031417.

25. Collins C, Kramer A, O'Day ME, et al. Evaluation of patient and provider satisfaction with a pharmacist-managed lipid clinic in a Veterans Affairs medical center. Am J Health Syst Pharm. 2006 Sep 15;63(18):1723-7. PMID: 16960256.
26. Cordina M, McElnay JC, Hughes CM. Assessment of a community pharmacybased program for patients with asthma. Pharmacotherapy. 2001 Oct;21(10):1196203. PMID: 11601666.

27. Couturaud F, Proust A, Frachon I, et al. Education and self-management: a one-year randomized trial in stable adult asthmatic patients. J Asthma. 2002 Sep;39(6):493-500. PMID: 12375708.

28. Cranor CW, Bunting BA, Christensen DB. The Asheville Project: long-term clinical and economic outcomes of a community pharmacy diabetes care program. J Am Pharm Assoc (Wash). 2003 MarApr;43(2):173-84. PMID: 12688435.

29. Curwain B. Hampshire medicines management project saves NHS over British pound sterling1 million. Pharmaceutical Journal (England). 2007;278(Feb):PM1-PM2. PMID: 44-11008.

30. Donohue JM, Zhang Y, Aiju M, et al. Impact of Medicare Part D on antidepressant treatment, medication choice, and adherence among older adults with depression. Am J Geriatr Psychiatry. 2011 Dec;19(12):989-97. PMID: 22123272.

31. Dudas V, Bookwalter T, Kerr KM, et al. The impact of follow-up telephone calls to patients after hospitalization. Dis Mon. 2002 Apr;48(4):239-48. PMID: 12021756.

32. Emmerton L, Shaw J, Kheir N. Asthma management by New Zealand pharmacists: a pharmaceutical care demonstration project. J Clin Pharm Ther. 2003 Oct;28(5):395-402. PMID: 14632964.

33. Epplen K, Dusing-Wiest M, Freedlund J, et al. Stepwise approach to implementing ambulatory clinical pharmacy services. Am J Health Syst Pharm. 2007 May 1;64(9):945-51. PMID: 17468149.

34. Ermer M, Harder S. Medication review of community-dwelling seniors using intensified home-care service. Int J Clin Pharmacol Ther. 2011 Mar;49(3):179-84. PMID: 21329619.

35. Farris KB, Ganther-Urmie JM, Fang G, et al. Population-based medication reviews: a descriptive analysis of the medication issues identified in a medicare not-for-profit prescription discount program. Ann 
Pharmacother. 2004 Nov;38(11):1823-9. PMID: 15479770.

36. Finley PR, Rens HR, Pont JT, et al. Impact of a collaborative care model on depression in a primary care setting: a randomized controlled trial. Pharmacotherapy. 2003 Sep;23(9):1175-85. PMID: 14524649.

37. Fisher JE, Zhang Y, Sketris I, et al. The effect of an educational intervention on meperidine use in Nova Scotia, Canada: a time series analysis. Pharmacoepidemiol Drug Saf. 2012 Feb;21(2):177-83. PMID: 22081471.

38. Fletcher J, Hogg W, Farrell B, et al. Effect of nurse practitioner and pharmacist counseling on inappropriate medication use in family practice. Can Fam Physician. 2012 Aug;58(8):862-8. PMID: 22893340.

39. Garcao JA, Cabrita J. Evaluation of a pharmaceutical care program for hypertensive patients in rural Portugal. J Am Pharm Assoc (Wash). 2002 NovDec;42(6):858-64. PMID: 12482009.

40. Gardella JE, Cardwell TB, Nnadi M. Improving medication safety with accurate preadmission medication lists and postdischarge education. Jt Comm J Qual Patient Saf. 2012 Oct;38(10):452-8. PMID: 23130391 .

41. Garrett DG, Bluml BM. Patient self-management program for diabetes: first-year clinical, humanistic, and economic outcomes. J Am Pharm Assoc (2003). 2005 MarApr;45(2):130-7. PMID: 15868754.

42. Geletko SM, Poulakos MN. Pharmaceutical services in an HIV clinic. Am J Health Syst Pharm. 2002 Apr 15;59(8):709-13. PMID: 11977854.

43. Gizzi L, Slain D, Hare J, et al. An assessment of a pharmacy-based enhancement to the hospital medication reconciliation process.

44. Goode JV, Swiger K, Bluml BM. Regional osteoporosis screening, referral, and monitoring program in community pharmacies: findings from Project ImPACT: Osteoporosis. J Am Pharm Assoc (2003). 2004 Mar-Apr;44(2):152-60. PMID: 15098849 .

45. Gourley GA, Portner TS, Gourley DR, et al. Humanistic outcomes in the hypertension and COPD arms of a multicenter outcomes study. Journal of the American
Pharmaceutical Association

(Washington,D.C. : 1996); 1998. p. 586-97.

46. Gruffydd-Jones K, Hollinghurst S, Ward S, et al. Targeted routine asthma care in general practice using telephone triage (Structured abstract). British Journal of General Practice; 2005. p. 918-23.

47. Hata M, Klotz R, Sylvies R, et al. Medication therapy management services provided by student pharmacists. Am J Pharm Educ. 2012 Apr 10;76(3):51. PMID: 22544968.

48. Hellstrom LM, Bondesson A, Hoglund P, et al. Impact of the Lund Integrated Medicines Management (LIMM) model on medication appropriateness and drug-related hospital revisits. Eur J Clin Pharmacol. 2011 Jul;67(7):741-52. PMID: 21318595.

49. Hemens BJ, Holbrook A, Tonkin M, et al. Computerized clinical decision support systems for drug prescribing and management: a decision-maker-researcher partnership systematic review. Implement Sci. 2011;6:89. PMID: 21824383.

50. Hogan EG, Leal S, Slack M, et al. Comparison of pharmacist led collaborative drug therapy management to standard physician provided therapy for type 2 diabetes mellitus. ASHP Midyear Clinical Meeting. 2005;40:P518E. PMID: 43-01390.

51. Holtorf AP, McAdam-Marx C, Schaaf D, et al. Systematic review on quality control for drug management programs: is quality reported in the literature? BMC Health Serv Res. 2009;9:38. PMID: 19243591.

52. Humphries TL, Carroll N, Chester EA, et al. Evaluation of an electronic critical drug interaction program coupled with active pharmacist intervention. Ann Pharmacother. 2007 Dec;41(12):1979-85. PMID: 17986517.

53. Hussein M, Benner JS, Lee D, et al. Propensity score matching in the evaluation of drug therapy management programs: an illustrative analysis of a program for patients with hepatitis C virus. Qual Manag Health Care. 2010 Jan-Mar;19(1):25-33. PMID: 20042931.

54. Isetts BJ, Brummel AR, de Oliveira DR, et al. Managing drug-related morbidity and mortality in the patient-centered medical 
home. Med Care. 2012 Nov;50(11):9971001. PMID: 23047790.

55. Isetts BJ, Schondelmeyer SW, Heaton AH, et al. Effects of collaborative drug therapy management on patients' perceptions of care and health-related quality of life. Res Social Adm Pharm. 2006 Mar;2(1):129-42. PMID: 17138505.

56. Jaber LA, Halapy H, Fernet M, et al. Evaluation of a pharmaceutical care model on diabetes management. Ann Pharmacother. 1996 Mar;30(3):238-43. PMID: 8833557.

57. Jack BW, Chetty VK, Anthony D, et al. A reengineered hospital discharge program to decrease rehospitalization: a randomized trial. Annals of Internal Medicine; 2009. p. 178-87.

58. Jackevicius CA, Li P, Tu JV. Prevalence, predictors, and outcomes of primary nonadherence after acute myocardial infarction. Circulation. 2008 Feb 26;117(8):1028-36. PMID: 18299512.

59. Jameson JP, VanNoord GR. Pharmacotherapy consultation on polypharmacy patients in ambulatory care. Ann Pharmacother. 2001 Jul-Aug;35(7-8):835-40. PMID: 11485129.

60. Kimberlin CL, Berardo DH, Pendergast JF, et al. Effects of an education program for community pharmacists on detecting drugrelated problems in elderly patients. Med Care. 1993 May;31(5):451-68. PMID: 8501992 .

61. Kocarnik BM, Liu CF, Wong ES, et al. Does the presence of a pharmacist in primary care clinics improve diabetes medication adherence? BMC Health Serv Res. 2012;12:391. PMID: 23148570.

62. Krass I, Taylor SJ, Smith C, et al. Impact on medication use and adherence of Australian pharmacists' diabetes care services. J Am Pharm Assoc (2003). 2005 JanFeb;45(1):33-40. PMID: 15730115.

63. Kuzuya M, Hirakawa Y, Suzuki Y, et al. Association between unmet needs for medication support and all-cause hospitalization in community-dwelling disabled elderly people. J Am Geriatr Soc. 2008 May;56(5):881-6. PMID: 18384585.

64. LaMarr B, Valdez C, Driscoll K, et al. Influence of pharmacist intervention on prescribing of angiotensin-converting-enzyme inhibitors, angiotensin II-receptor blockers, and aspirin for diabetic patients. Am J Health Syst Pharm. 2010 Feb 15;67(4):290-4. PMID: 20133534.

65. Lapane KL, Hughes CM, Christian JB, et al. Evaluation of the fleetwood model of longterm care pharmacy. J Am Med Dir Assoc. 2011 Jun;12(5):355-63. PMID: 21450170.

66. Ledwidge M, Barry M, Cahill J, et al. Is multidisciplinary care of heart failure costbeneficial when combined with optimal medical care? (Structured abstract). European Journal of Heart Failure; 2003. p. 381-9.

67. Lee JK, Grace KA, Taylor AJ. Effect of a pharmacy care program on medication adherence and persistence, blood pressure, and low-density lipoprotein cholesterol: a randomized controlled trial. JAMA. 2006 Dec 6;296(21):2563-71. PMID: 17101639.

68. Li X, Mao M, Ping Q. Effect of pharmaceutical care programs on glycemic control in patients with diabetes mellitus: a metaanalysis of randomized controlled trials (Structured abstract). Journal of Pharmacy Technology; 2010. p. 255-63.

69. Linton A, Bacon TA, Trice S, et al. Results from a mailed promotion of medication reviews among Department of Defense beneficiaries receiving 10 or more chronic medications. J Manag Care Pharm. 2010 Oct;16(8):578-92. PMID: 20866163.

70. Lipton HL, Bero LA, Bird JA, et al. The impact of clinical pharmacists' consultations on physicians' geriatric drug prescribing. A randomized controlled trial. Med Care. 1992 Jul;30(7):646-58. PMID: 1614233.

71. Ma CB, Nakasato SK. Effectiveness of an educational program to improve patient understanding of and compliance with drug therapies used to treat HIV disease. ASHP Midyear Clinical Meeting. 1991;26(Dec):PR. PMID: 29-00124.

72. Malaeb DN, Aoun J. Physician community pharmacist collaborative care in diabetes management. p. P.

73. McAdam-Marx C, Schaaf DT, Holtorf AP, et al. Systematic analysis of outcomes evaluations applied to drug management programs. Am J Manag Care. 2008 Nov; 14(11 Suppl):SP36-45. PMID: 18991480. 
74. McAllister D, Schommer JC, McAuley JW, et al. Comparison of skilled nursing and assisted living residents to determine potential benefits of pharmacist intervention. Consultant Pharmacist (USA). 2000;15(Nov):1110-6. PMID: 38-05993.

75. McCall N, Cromwell J, Urato C. Evaluation of Medicare Care Management for High Cost Beneficiaries (CMHCB) Demonstration: VillageHealth's Key to Better Health (KTBH) (Prepared by RTI International under CMS Contract No. \#500-000024/TO\#25). Baltimore, MD: Services CfMM; 2010.

76. McCombs JS, Liu G, Shi J, et al. The Kaiser Permanente/USC Patient Consultation Study: change in use and cost of health care services. Am J Health Syst Pharm. 1998 Dec 1;55(23):2485-99. PMID: 9853633.

77. McCord AD. Clinical impact of a pharmacistmanaged diabetes mellitus drug therapy management service. Pharmacotherapy. 2006 Feb;26(2):248-53. PMID: 16466329.

78. McDonough RP, Doucette WR. Drug therapy management: an empirical report of drug therapy problems, pharmacists' interventions, and results of pharmacists' actions. J Am Pharm Assoc (2003). 2003 Jul-Aug;43(4):511-8. PMID: 12952316.

79. McFarland M, Davis K, Wallace J, et al. Use of home telehealth monitoring with active medication therapy management by clinical pharmacists in veterans with poorly controlled type 2 diabetes mellitus. Pharmacotherapy. 2012 May;32(5):420-6. PMID: 22488512.

80. McKibbon KA, Lokker C, Handler SM, et al. The effectiveness of integrated health information technologies across the phases of medication management: a systematic review of randomized controlled trials. J Am Med Inform Assoc. 2012 Jan-Feb;19(1):2230. PMID: 21852412.

81. McLean W, Gillis J, Waller R. The BC Community Pharmacy Asthma Study: A study of clinical, economic and holistic outcomes influenced by an asthma care protocol provided by specially trained community pharmacists in British Columbia. Can Respir J. 2003 MayJun;10(4):195-202. PMID: 12851665.
82. Midlov P, Bahrani L, Seyfali M, et al. The effect of medication reconciliation in elderly patients at hospital discharge. Int J Clin Pharm. 2012 Feb;34(1):113-9. PMID: 22207271.

83. Mueller SK, Sponsler KC, Kripalani S, et al. Hospital-based medication reconciliation practices: a systematic review. Arch Intern Med. 2012 Jul 23;172(14):1057-69. PMID: 22733210 .

84. Murray MD, Young J, Hoke S, et al. Pharmacist intervention to improve medication adherence in heart failure: a randomized trial. Ann Intern Med. 2007 May 15;146(10):714-25. PMID: 17502632.

85. Nau DP, Pacholski AM. Impact of pharmacy care services on patients' perceptions of health care quality for diabetes. J Am Pharm Assoc (2003). 2007 May-Jun;47(3):358-65. PMID: 17510030 .

86. Nola KM, Gourley DR, Portner TS, et al. Clinical and humanistic outcomes of a lipid management program in the community pharmacy setting. Journal of the American Pharmaceutical Association (Washington,D.C. : 1996); 2000. p. 166-73.

87. Pakes GE. Group medication counseling conducted by a pharmacist for severely disturbed clients. Hosp Community Psychiatry. 1979 Apr;30(4):237-8. PMID: 422130.

88. Patterson SM, Hughes C, Kerse N, et al. Interventions to improve the appropriate use of polypharmacy for older people. Cochrane Database Syst Rev. 2012;5:CD008165. PMID: 22592727.

89. Pecora PG, Flint NB, Aleman MA. Improving disease control in patients with hypertension and/or diabetes mellitus with the implementation of ambulatory clinical pharmacy services. ASHP Midyear Clinical Meeting. 1998;33(Dec):P-E. PMID: 3513312 .

90. Ponnusankar S, Surulivelrajan M, Anandamoorthy N, et al. Assessment of impact of medication counseling on patients' medication knowledge and compliance in an outpatient clinic in South India. Patient Educ Couns. 2004 Jul;54(1):55-60. PMID: 15210260. 
91. Powers MF. Recently approved medicare provisions for prescription drugs and medication management services. Journal of Pharmacy Technology (USA). 2005;21(Feb):63-8. PMID: 42-10642.

92. Raimondi GA, Menga G, Botas C, et al. Decline of outpatient asthma management in Argentina. Respirology. 2008 Jan;13(1):134-7. PMID: 18197924.

93. Roughead EE, Barratt JD, Ramsay E, et al. Collaborative home medicines review delays time to next hospitalization for warfarin associated bleeding in Australian war veterans. J Clin Pharm Ther. 2011 Feb;36(1):27-32. PMID: 21108651.

94. Rozenfeld V, Pflomm J, Singh KK, et al. Assessing the impact of medication consultations with a Medication Event Monitoring System. Hospital Pharmacy; 1999. p. 539-49.

95. Sadler MA, Force RW. Pharmacy services in an ambulatory FFS setting can increase physician revenue. Formulary (USA). 2002;37(Feb):97-01. PMID: 39-13925.

96. Saini B, Filipovska J, Bosnic-Anticevich S, et al. An evaluation of a community pharmacybased rural asthma management service. Aust J Rural Health. 2008 Apr;16(2):100-8. PMID: 18318852.

97. Saini B, Krass I, Armour C. Development, implementation, and evaluation of a community pharmacy-based asthma care model (Structured abstract). Annals of Pharmacotherapy; 2004. p. 1954-60.

98. Salvador CH, Ruiz-Sanchez A, Gonzalez de Mingo MA, et al. Evaluation of a telemedicine-based service for the follow-up and monitoring of patients treated with oral anticoagulant therapy. IEEE Trans Inf Technol Biomed. 2008 Nov;12(6):696-706. PMID: 19000948.

99. Schulz M, Verheyen F, Muhlig S, et al. Pharmaceutical care services for asthma patients: a controlled intervention study. J Clin Pharmacol. 2001 Jun;41(6):668-76. PMID: 11402636.

100. Schulz RM, Porter C, Lane M, et al. Impact of a medication management system on nursing home admission rate in a communitydwelling nursing home-eligible Medicaid population. Am J Geriatr Pharmacother. 2011 Feb;9(1):69-79. PMID: 21459310.

101. Scott A, Tinelli M, Bond C. Costs of a community pharmacist-led medicines management service for patients with coronary heart disease in England: healthcare system and patient perspectives. Pharmacoeconomics. 2007;25(5):397-411. PMID: 17488138.

102. Shah C, Coyne T. Medicines management programme for non-medical prescribers. Nurs Manag (Harrow). 2012 Dec;19(8):347. PMID: 23285791.

103. Simon GE, Ludman EJ, Operskalski BH. Randomized trial of a telephone care management program for outpatients starting antidepressant treatment. Psychiatr Serv. 2006 Oct;57(10):1441-5. PMID: 17035563.

104. Simpson SH, Johnson JA, Tsuyuki RT. Economic impact of community pharmacist intervention in cholesterol risk management: an evaluation of the study of cardiovascular risk intervention by pharmacists. Pharmacotherapy. 2001 May;21(5):627-35. PMID: 11349751.

105. Sklenicka JM, Proffitt LS, Dare D. The role of a geriatric clinical pharmacist in improving medication safety in outpatient clinics. ASHP Midyear Clinical Meeting. 2005;40:P-D. PMID: 43-01370.

106. Smith SR, Catellier DJ, Conlisk EA, et al. Effect on health outcomes of a community-based medication therapy management program for seniors with limited incomes. Am J Health Syst Pharm. 2006 Feb 15;63(4):3729. PMID: 16452523.

107. Solomon DK, Portner TS, Bass GE, et al. Clinical and economic outcomes in the hypertension and COPD arms of a multicenter outcomes study. Journal of the American Pharmaceutical Association (Washington,D.C. : 1996); 1998. p. 574-85.

108. Stang P, Morris L, Kempf J, et al. The coprescription of contraindicated drugs with statins: continuing potential for increased risk of adverse events. Am J Ther. 2007 JanFeb;14(1):30-40. PMID: 17303973.

109. Stell R, Bonollo M, Fiddes K, et al. Successful integration of a clinical pharmacist into a disease management unit. Journal of 
Pharmacy Practice and Research (Australia). 2008;38(Feb):132-6. PMID: 45-17110.

110. Stemer G, Lemmens-Gruber R. Clinical pharmacy services and solid organ transplantation: a literature review (Structured abstract). Pharmacy World and Science; 2010. p. 7-18.

111. Stewart S, Pearson S, Luke CG, et al. Effects of home-based intervention on unplanned readmissions and out-of-hospital deaths (Structured abstract). Journal of the American Geriatrics Society; 1998. p. 17480.

112. Stockl KM, Shin JS, Gong S, et al. Improving patient self-management of multiple sclerosis through a disease therapy management program. Am J Manag Care. 2010 Feb;16(2):139-44. PMID: 20148619.

113. Swims MP, Reed P. A pharmacotherapy clinic in a neurologic practice. Journal of Pharmacy Technology (USA). 2006;22(May):254-60. PMID: 44-00819.

114. Tarn YH, Chou SF, Wang TY. Drug regimen review at nursing homes in Taiwan (CPS-O013). International Pharmaceutical Federation World Congress. 2002;62:78. PMID: 40-01282.

115. Tett SE, Higgins GM, Armour CL. Impact of pharmacist interventions on medication management by the elderly: a review of the literature. Ann Pharmacother. 1993 Jan;27(1):80-6. PMID: 8431627.

116. Tinelli M, Bond C, Blenkinsopp A, et al. Patient evaluation of a community pharmacy medications management service. Ann Pharmacother. 2007 Dec;41(12):1962-70. PMID: 17971403.

117. Trask SJ, Szabo SA. Polypharmacy intervention program in a tertiary care VA medical center. ASHP Midyear Clinical Meeting. 1991;26(Dec):P-R. PMID: 29-00493.

118. Trygstad TK, Christensen D, Garmise J, et al. Pharmacist response to alerts generated from Medicaid pharmacy claims in a long-term care setting: results from the North Carolina polypharmacy initiative. J Manag Care Pharm. 2005 Sep;11(7):575-83. PMID: 16137215.

119. Trygstad TK, Christensen DB, Wegner SE, et al. Analysis of the North Carolina long-term care polypharmacy initiative: a multiple- cohort approach using propensity-score matching for both evaluation and targeting. Clin Ther. 2009 Sep;31(9):2018-37. PMID: 19843492.

120. Tsuyuki RT, Johnson JA, Teo KK, et al. A randomized trial of the effect of community pharmacist intervention on cholesterol risk management: the Study of Cardiovascular Risk Intervention by Pharmacists (SCRIP). Arch Intern Med. 2002 May 27;162(10):1149-55. PMID: 12020186.

121. Vinks TH, Egberts TC, de Lange TM, et al. Pharmacist-based medication review reduces potential drug-related problems in the elderly: the SMOG controlled trial. Drugs Aging. 2009;26(2):123-33. PMID: 19220069.

122. Vuong T, Marriott JL, Kong DCM, et al. Implementation of a community liaison pharmacy service: a randomised controlled trial. International Journal of Pharmacy Practice; 2008. p. 127-35.

123. Walker S, Willey CW. Impact on drug costs and utilization of a clinical pharmacist in a multisite primary care medical group. $\mathrm{J}$ Manag Care Pharm. 2004 JulAug;10(4):345-54. PMID: 15298532.

124. Ward MA, Xu Y. Pharmacist-provided telephonic medication therapy management in an MAPD plan. Am J Manag Care. 2011 Oct;17(10):e399-409. PMID: 21999720.

125. Weinberger M, Murray MD, Marrero DG, et al. Effectiveness of pharmacist care for patients with reactive airways disease: a randomized controlled trial. JAMA. 2002 Oct 2;288(13):1594-602. PMID: 12350190.

126. Westfall GR, Narducci WA. A communitypharmacy-based callback program for antibiotic therapy. J Am Pharm Assoc (Wash). 1997 May-Jun;NS37(3):330-4. PMID: 9170810.

127. Wilk JE, West JC, Rae DS, et al. Medicare Part $D$ prescription drug benefits and administrative burden in the care of dually eligible psychiatric patients. Psychiatr Serv. 2008 Jan;59(1):34-9. PMID: 18182537.

128. Williford SL, Johnson DF. Impact of pharmacist counseling on medication knowledge and compliance. Mil Med. 1995

Nov;160(11):561-4. PMID: 8538891. 
129. Wolfe SC, Schirm V. Medication counseling for the elderly: effects on knowledge and compliance after hospital discharge. Geriatr Nurs. 1992 May-Jun;13(3):134-8. PMID: 1319935.

130. Wong MC, Kong AP, So WY, et al. Pharmacoepidemiological profiles of oral hypoglycemic agents among 28,773 Chinese patients with diabetes. Diabetes Res Clin Pract. 2012 Jun;96(3):319-25. PMID: 22305941.

131. Wu JC, Chin M, Pitcher K, et al. IDENTIFYING HOSPITALIZATION RISK FACTORS TO PRIORITIZE MEDICATION THERAPY MANAGEMENT ENROLLMENT. p. 19.

132. Yamamura K, Osada T, Yano K, et al. Evaluation of pharmacist-managed

\section{Ineligible Population $(\mathbf{n}=\mathbf{9})$}

1. Borgsdorf LR, Miano JS, Knapp KK. Pharmacistmanaged medication review in a managed care system. Am J Hosp Pharm. 1994 Mar 15;51(6):772-7. PMID: 8010315.

2. Lee KL, Peng YL, Chou JL, et al. Economic evaluation of therapeutic drug monitoring services in renal transplant recipients treated with cyclosporine (Structured abstract). Transplantation Proceedings; 2000. p. 18016.

3. Lee M, Kemp JA, Canning A, et al. A randomized controlled trial of an enhanced patient compliance program for Helicobacter pylori therapy. Arch Intern Med. 1999 Oct 25;159(19):2312-6. PMID: 10547171.

4. Lin KE, Lei EX, Chretien SD. Pharmacotherapy assessment and education for newly enrolled patients in a primary care clinic. ASHP Midyear Clinical Meeting. 2001;36(Dec):PE. PMID: 38-12877.

5. Mortimer C, Emmerton L, Lum E. The impact of an aged care pharmacist in a department of emergency medicine. J Eval Clin Pract. 2011 Jun;17(3):478-85. PMID: 21040247.

\section{Ineligible Study Design $(n=44)$}

1. Atkinson WL, Frey D. Integration of a medication management model into outcome-based quality improvement: a pilot program in a anticoagulation classroom. ASHP Midyear Clinical Meeting. 2006;41(Dec)PMID: 4406505 .

133. Zillich AJ, Jaynes HA, Snyder ME, et al. Evaluation of specialized medication packaging combined with medication therapy management: adherence, outcomes, and costs among Medicaid patients. Med Care. 2012 Jun;50(6):485-93. PMID: 22498687.

134. Zillich AJ, Sutherland JM, Kumbera PA, et al. Hypertension outcomes through blood pressure monitoring and evaluation by pharmacists (HOME study). J Gen Intern Med. 2005 Dec;20(12):1091-6. PMID: 16423096.

6. Ramalho de Oliveira D, Brummel AR, Miller DB. Medication therapy management: 10 years of experience in a large integrated health care system. J Manag Care Pharm. 2010 Apr;16(3):185-95. PMID: 20331323.

7. Roughead EE, Semple SJ, Vitry AI. Pharmaceutical care services: a systematic review of published studies, 1990 to 2003 , examining effectiveness in improving patient outcomes (Structured abstract). International Journal of Pharmacy Practice; 2005. p. 53-70.

8. Sperling S, Neal K, Hales K, et al. A quality improvement project to reduce falls and improve medication management. Home Health Care Serv Q. 2005;24(1-2):13-28. PMID: 16236656.

9. Stergachis A, Gardner JS, Anderson MT, et al. Improving pediatric asthma outcomes in the community setting: does pharmaceutical care make a difference? J Am Pharm Assoc (Wash). 2002 Sep-Oct;42(5):743-52. PMID: 12269709.

rural propriety home healthcare agency. Home Health Care Serv Q. 2005;24(1-2):2945. PMID: 16236657. 
2. Chisholm MA, Reinhardt BO, Vollenweider LJ, et al. Effect of pharmaceutical care services on renal transplant patients' blood glucose levels. ASHP Midyear Clinical Meeting. 1999;34(Dec):P-E. PMID: 36-12509.

3. Chisholm MA, Spivey CA, Mulloy LL. Effects of a medication assistance program with medication therapy management on the health of renal transplant recipients. Am J Health Syst Pharm. 2007 Jul 15;64(14):1506-12. PMID: 17617501.

4. Christensen DB, Campbell WH, Madsen S, et al. Documenting outpatient problem intervention activities of pharmacists in an HMO. Med Care. 1981 Jan;19(1):104-17. PMID: 7464303.

5. Coast-Senior EA, Kroner BA, Kelley CL, et al. Management of patients with type 2 diabetes by pharmacists in primary care clinics. Ann Pharmacother. 1998 Jun;32(6):636-41. PMID: 9640480.

6. Doucette WR, McDonough RP, Klepser D, et al. Comprehensive medication therapy management: identifying and resolving drug-related issues in a community pharmacy. Clin Ther. 2005 Jul;27(7):110411. PMID: 16154490.

7. Doucette WR, Witry MJ, Alkhateeb F, et al. Attitudes of Medicare beneficiaries toward pharmacist-provided medication therapy management activities as part of the Medicare Part D benefit. J Am Pharm Assoc (2003). 2007 Nov-Dec;47(6):758-62. PMID: 18032140 .

8. Downie G, Cromarty E, Ellis G, et al. Assessment of medicine management in people ages 75 years or over living in the community in Grampian, Scotland. ASHP Midyear Clinical Meeting. 2001;36(Dec):P-E. PMID: 38-12661.

9. Farris KB, Kumbera P, Halterman T, et al. Outcomes-based pharmacist reimbursement: Reimbursing pharmacists for cognitive services - (Part 1 of a 2-part series). Journal of Managed Care Pharmacy (USA). 2002;8(May):383-93. PMID: 40-02496.

10. Frew EJ, Kluettgens B, Gilday N, et al. The impact of a medicines management service on patients with cystic fibrosis. p. 686 .

11. Gilbert AL, Roughead EE, Beilby J, et al. Collaborative medication management services: improving patient care. Med J Aust. 2002 Aug 19;177(4):189-92. PMID: 12175322.

12. Hall DL, Pater KS. Implementation of a Medication Therapy Management Program in a Hospital-Based Outpatient Pharmacy. p. 512.

13. Hardin HC, Hall AM, Roane TE, et al. An Advanced Pharmacy Practice Experience in a Student-Staffed Medication Therapy Management Call Center. p. NIL.

14. Harris IM, Westberg SM, Frakes MJ, et al. Outcomes of medication therapy review in a family medicine clinic. J Am Pharm Assoc (2003). 2009 Sep-Oct;49(5):623-7. PMID: 19748869.

15. Hassol A, Shoemaker SJ. Exploratory Research on Medication Therapy Management. (Prepared by Abt Associates under Contract \#HHSM-500-2005-00181/TO\#3.) Baltimore, MD: Centers for Medicare \& Medicaid Services; July 8, 2008. http://www.cms.gov/Research-StatisticsData-and-Systems/Statistics-Trends-andReports/Reports/downloads/blackwell.pdf.

16. Hong N, Jackson L, Pisupati R. Evaluation of clinical pharmacy services in an ambulatory care HIV/AIDS Clinic. p. 056.

17. Isetts BJ. Evaluating Effectiveness of the Minnesota Medication Therapy Management Care Program: Final Report University of Minnesota College of Pharmacy. Minneapolis, MN: 2007.

18. Isetts BJ, Brown LM, Schondelmeyer SW, et al. Quality assessment of a collaborative approach for decreasing drug-related morbidity and achieving therapeutic goals. Arch Intern Med. 2003 Aug 1125;163(15):1813-20. PMID: 12912717.

19. Johnson CL, Nicholas A, Divine H, et al. Outcomes from DiabetesCARE: a pharmacist-provided diabetes management service. J Am Pharm Assoc (2003). 2008 Nov-Dec;48(6):722-30. PMID: 19019800.

20. Johnston AM, Doane K, Phipps S, et al. Outcomes of pharmacists' cognitive services in the long-term care setting. Consult Pharm. 1996;11:41-50.

21. Lam A. Practice innovations: Delivering medication therapy management services via 
videoconference interviews. Consultant pharmacist; 2011. p. 764-73.

22. Lam A, Odegard PS, Gardner J. School of pharmacy-based medication therapy management program: development and initial experience. J Am Pharm Assoc (2003). 2012 May-Jun;52(3):398-404. PMID: 22618981.

23. Lam S, Ruby CM. Impact of an interdisciplinary team on drug therapy outcomes in a geriatric clinic. Am J Health Syst Pharm. 2005 Mar 15;62(6):626-9. PMID: 15757885.

24. Leikola SN, Virolainen J, Tuomainen L, et al. Comprehensive medication reviews for elderly patients: findings and recommendations to physicians. J Am Pharm Assoc (2003). 2012 SepOct;52(5):630-3. PMID: 23023843.

25. Maack B, Miller DR, Johnson T, et al. Economic impact of a pharmacy resident in an assisted living facility-based medication therapy management program. Ann Pharmacother. 2008 Nov;42(11):1613-20. PMID: 18940921.

26. Meindl CL, Duncan AL, Achusim LE, et al. Impact of a clinical pharmacist in an internal medicine resident clinic. ASHP Midyear Clinical Meeting. 1998;33(Dec):P-R. PMID: 35-12686.

27. Michaels NM, Jenkins GF, Pruss DL, et al. Retrospective analysis of community pharmacists' recommendations in the North Carolina Medicaid medication therapy management program. J Am Pharm Assoc (2003). 2010 May-Jun;50(3):347-53. PMID: 20452907.

28. Monte SV, Slazak EM, Albanese NP, et al. Clinical and economic impact of a diabetes clinical pharmacy service program in a university and primary care-based collaboration model. J Am Pharm Assoc (2003). 2009 Mar-Apr;49(2):200-8. PMID: 19289346.

29. Oyetayo OO, James C, Martinez A, et al. The Hispanic Diabetes Management Program: Impact of community pharmacists on clinical outcomes. J Am Pharm Assoc (2003). 2011 Sep-Oct;51(5):623-6. PMID: 21896461 .

30. Padiyara RS, Rabi SM. Physician perceptions of pharmacist provision of outpatient medication therapy management services. J Am Pharm Assoc (2003). 2006 NovDec;46(6):660, 3. PMID: 17176679.

31. Parks PM. A patient medication counseling group in an adult psychiatric facility. Hosp Pharm. 1977 Feb;12(2):63-4. PMID: 10305432.

32. Perera PN, Guy MC, Sweaney AM, et al. Evaluation of prescriber responses to pharmacist recommendations communicated by fax in a medication therapy management program (MTMP). J Manag Care Pharm. 2011 Jun;17(5):345-54. PMID: 21657804.

33. Pinto SL, Bechtol RA, Partha G. Evaluation of outcomes of a medication therapy management program for patients with diabetes. p. 519.

34. Singhal PK, Raisch DW, Gupchup GV. The impact of pharmaceutical services in community and ambulatory care settings: evidence and recommendations for future research. Ann Pharmacother. 1999 Dec;33(12):1336-55. PMID: 10630834.

35. Smith CP, Christensen DB. Identification and clarification of drug therapy problems by Indian health service pharmacists. Ann Pharmacother. 1996 Feb;30(2):119-24. PMID: 8835041.

36. Stebbins MR, Kaufman DJ, Lipton HL. The PRICE clinic for low-income elderly: a managed care model for implementing pharmacist-directed services. J Manag Care Pharm. 2005 May;11(4):333-41. PMID: 15871644.

37. Strand LM, Cipolle RJ, Morley PC, et al. The impact of pharmaceutical care practice on the practitioner and the patient in the ambulatory practice setting: twenty-five years of experience. Curr Pharm Des. 2004;10(31):3987-4001. PMID: 15579084.

38. Stratton TP, Cernohous T, Hager K, et al. College of pharmacy-based medication therapy management program for a university system. J Am Pharm Assoc (2003). 2012 Sep-Oct;52(5):653-60. PMID: 23023847.

39. Thomas R, Kilbey C. Complex medicines management. Nurs Times. 2011 Mar 17;107(8):20-2. PMID: 21667662.

40. Truong AC. Evaluating the impact of a medication teaching clinic in a hospital based outpatient pharmacy. ASHP Midyear 
Clinical Meeting. 2006;41(Dec)PMID: 4406528.

41. VanHaaren A. Pharmaceutical care in a multi-site medical group practice: reaching goals in the treatment of hyperlipidemia and diabetes.

ASHP Midyear Clinical Meeting. 2006;41(Dec)PMID: 44-06529.

42. Walberg SJ, Haight R. Discharge follow-up with a pharmacist study: Incorporating pharmacist-provided discharge education and medication reconciliation with an outpatient follow-up service for patients with psychiatric illness discharged to an outpatient day treatment program. Journal of
Pharmacy Practice (USA). 2008;21(Jan):756. PMID: 45-15441.

43. Watkins JL, Landgraf A, Barnett CM, et al. Evaluation of pharmacist-provided medication therapy management services in an oncology ambulatory setting. J Am Pharm Assoc (2003). 2012 MarApr;52(2):170-4. PMID: 22370379.

44. Zierler-Brown S, Coll RE, Llewellyn K. Implementation and evaluation of a pharmacist-managed ambulatory, indigent care practice, focused on cardiovascular disease (CVD) prevention and management. ASHP Midyear Clinical Meeting. 2005;40:P-R. PMID: 43-00015

Pharm. 2009 Jan-Feb;15(1):32-41. PMID: 19125548.

4. Lapane KL, Hughes CM. Pharmacotherapy interventions undertaken by pharmacists in the Fleetwood phase III study: the role of process control. Ann Pharmacother. 2006 Sep;40(9):1522-6. PMID: 16882872.

2. Carter BL, Malone DC, Ellis SL, et al. Antihypertensive Drug Utilization in Hypertensive Veterans With Complex Medication Profiles. J Clin Hypertens (Greenwich). 2000 May;2(3):172-80. PMID: 11416643 .

3. Hirsch JD, Rosenquist A, Best BM, et al. Evaluation of the first year of a pilot program in community pharmacy: HIV/AIDS medication therapy management for Medi-Cal beneficiaries. J Manag Care

\section{Ineligible Outcomes $(\mathbf{n}=\mathbf{8})$}

1. Berjis M, Ho MI, Gray DR. Evaluation of patient outcome and pharmaceutical care in an HIV clinic. ASHP Midyear Clinical Meeting. 1996;31(Dec):P-E. PMID: 33-13178.

2. Billups SJ, Okano G, Malone D, et al. Assessing the structure and process for providing pharmaceutical care in Veterans Affairs medical centers. Am J Health Syst Pharm. 2000 Jan 1;57(1):29-39. PMID: 10630554.

3. Carter BL, Malone DC, Valuck RJ, et al. The IMPROVE study: background and study design. Impact of Managed Pharmaceutical Care on Resource Utilization and Outcomes in Veterans Affairs Medical Centers. Am J Health Syst Pharm. 1998 Jan 1;55(1):62-7. PMID: 9437477.

4. Kucukarslan SN, Hagan AM, Shimp LA, et al. Integrating medication therapy management in the primary care medical home: A review of randomized controlled trials. Am J Health Syst Pharm. 2011 Feb 15;68(4):335-45. PMID: 21289329. 
5. Lee E, Braund R, Tordoff J. Examining the first year of Medicines Use Review services provided by pharmacists in New Zealand: 2008. N Z Med J. 2009 Apr 24;122(1293):3566. PMID: 19448788.

6. Nkansah N, Mostovetsky O, Yu C, et al. Effect of outpatient pharmacists' non-dispensing roles on patient outcomes and prescribing patterns. Cochrane Database Syst Rev. 2010(7):CD000336. PMID: 20614422.

\section{Ineligible Setting $(\mathbf{n}=7)$}

1. Bergkvist A, Midlov P, Hoglund P, et al. Improved quality in the hospital discharge summary reduces medication errors--LIMM: Landskrona Integrated Medicines Management. Eur J Clin Pharmacol. 2009 Oct;65(10):1037-46. PMID: 19557400.

2. Bergkvist A, Midlov P, Hoglund P, et al. A multiintervention approach on drug therapy can lead to a more appropriate drug use in the elderly. LIMM-Landskrona Integrated Medicines Management. J Eval Clin Pract. 2009 Aug;15(4):660-7. PMID: 19674217.

3. Blozik E, Born AM, Stuck AE, et al. Reduction of inappropriate medications among older nursing-home residents: a nurse-led, pre/post-design, intervention study. Drugs Aging. 2010 Dec 1;27(12):1009-17. PMID: 21087070 .

4. Carey N, Courtenay M, James J, et al. An evaluation of a Diabetes Specialist Nurse prescriber on the system of delivering medicines to patients with diabetes. J Clin
7. Roberts S, Gainsbrugh R. Medication therapy management and collaborative drug therapy management. J Manag Care Pharm. 2010 Jan-Feb;16(1):67-8. PMID: 20044847.

8. Williams BR, Lopez S. Reaching the homebound elderly: the Prescription Intervention and Lifelong Learning (PILL) program. Home Health Care Serv Q. 2005;24(1-2):61-72. PMID: 16236659.

Nurs. 2008 Jun;17(12):1635-44. PMID: 18482124.

5. Karapinar-Carkit F, Borgsteede SD, Zoer J, et al. The effect of the $\mathrm{COACH}$ program (Continuity Of Appropriate pharmacotherapy, patient Counselling and information transfer in Healthcare) on readmission rates in a multicultural population of internal medicine patients. BMC Health Serv Res. 2010;10:39. PMID: 20156368.

6. Kripalani S, Roumie CL, Dalal AK, et al. Effect of a pharmacist intervention on clinically important medication errors after hospital discharge: a randomized trial. Ann Intern Med. 2012 Jul 3;157(1):1-10. PMID: 22751755.

7. Schnipper JL, Roumie CL, Cawthon C, et al. Rationale and design of the Pharmacist Intervention for Low Literacy in Cardiovascular Disease (PILL-CVD) study. Circulation. Cardiovascular quality and outcomes; 2010. p. 212-9. 


\section{Appendix D. List of Studies on Hold}

\section{Articles awaiting author response for final inclusion/exclusion decision $(n=7)$}

1. Borges AP, Guidoni CM, Ferreira LD, et al. The pharmaceutical care of patients with type 2 diabetes mellitus. Pharm World Sci. 2010 Dec;32(6):730-6. PMID: 20734138.

2. Borges AP, Guidoni CM, Freitas O, et al. Economic evaluation of outpatients with type 2 diabetes mellitus assisted by a pharmaceutical care service. Arq Bras Endocrinol Metabol. 2011 Dec;55(9):68691. PMID: 22231970.

3. Bucci C, Jackevicius C, McFarlane K, et al. Pharmacist's contribution in a heart function clinic: patient perception and medication appropriateness. Can J Cardiol. 2003 Mar 31;19(4):391-6. PMID: 12704485.

4. Hirsch JD, Gonzales M, Rosenquist A, et al. Antiretroviral therapy adherence, medication use, and health care costs during 3 years of a community pharmacy medication therapy management program for Medi-Cal beneficiaries with HIV/AIDS. J Manag Care Pharm. 2011 Apr;17(3):213-23. PMID: 21434698
5. Kliethermes MA. Adherence in an MTM clinic. 2009. p. 204.

6. Marrufo G, Dixit A, Perlroth D, et al. Medication Therapy Management in a Chronically Ill Population: Interim Report (Prepared by Acumen LLC under Contract \#HHSM-5002011-00012I/TOT\#0001.) Baltimore, MD: Services CfMM; 2013.

7. Nguyen J, Matsuoka B, Morodomi L, et al. EVALUATING THE MEDICATION THERAPY MANAGEMENT PROGRAM IMPACT. 2011. p. 11. 


\section{Appendix E. Evidence Tables}

Table E1. Study and patient-level characteristics (continued)

\begin{tabular}{|c|c|c|c|c|c|c|c|c|c|c|c|}
\hline $\begin{array}{l}\text { Author, } \\
\text { Year } \\
\text { Trial } \\
\text { Name }^{a}\end{array}$ & $\begin{array}{l}\text { Interventions } \\
\text { and } \\
\text { Comparator } \\
\text { Descriptions }\end{array}$ & $\begin{array}{l}\text { Intervention } \\
\text { Goal }\end{array}$ & Inclusion Criteria & $\begin{array}{l}\text { Exclusion } \\
\text { Criteria }\end{array}$ & $\begin{array}{l}\text { Study } \\
\text { Design }\end{array}$ & $\begin{array}{l}\text { Study } \\
\text { Duration }\end{array}$ & $\begin{array}{l}\text { Funding } \\
\text { Source(s) }\end{array}$ & $\begin{array}{l}\text { Baseline } \\
\% \text { Rural }\end{array}$ & $\begin{array}{l}\text { Baseline } \\
\text { Age - Mean } \\
\text { (SD) or } \\
\text { Median } \\
\text { (Range) }\end{array}$ & $\begin{array}{l}\text { Baseline \% } \\
\text { Female }\end{array}$ & $\begin{array}{l}\text { Race/ } \\
\text { Ethnicity \% }\end{array}$ \\
\hline $\begin{array}{l}\text { Bernsten } \\
\text { et al., } \\
2001^{1} \text {; } \\
\text { Sturgess } \\
\text { et al., } \\
2003^{2}\end{array}$ & $\begin{array}{l}\text { G1: Structured } \\
\text { community } \\
\text { pharmacy- } \\
\text { based } \\
\text { pharmaceutica } \\
\text { I care program } \\
\text { G2: Normal } \\
\text { pharmaceutica } \\
\text { I Usual } \\
\text { community } \\
\text { pharmacy } \\
\text { services }\end{array}$ & $\begin{array}{l}\text { To identify } \\
\text { actual and } \\
\text { potential } \\
\text { DRPs using a } \\
\text { structured } \\
\text { approach, } \\
\text { and to } \\
\text { resolve those } \\
\text { problems in } \\
\text { collaboration } \\
\text { with PCPs } \\
\text { using } \\
\text { pharmacy- } \\
\text { based } \\
\text { interventions }\end{array}$ & $\begin{array}{l}\text { 1) Aged } \geq 65 \\
\text { 2) Taking } \geq 4 \\
\text { prescribed } \\
\text { medications } \\
\text { 3) Oriented with } \\
\text { respect to self, time, } \\
\text { and place } \\
\text { 4) Community- } \\
\text { dwelling } \\
\text { 5) Regular visitors to } \\
\text { recruited community } \\
\text { pharmacy }\end{array}$ & $\begin{array}{l}\text { Houseboun } \\
\text { d or } \\
\text { resident in } \\
\text { nursing/ } \\
\text { residential } \\
\text { home }\end{array}$ & $\begin{array}{l}\text { RCT: } \\
\text { cluster- } \\
\text { rando- } \\
\text { mized }\end{array}$ & 18 & $\begin{array}{l}\text { Multiple } \\
\text { (Government, } \\
\text { foundation, } \\
\text { professional } \\
\text { organizations, } \\
\text { pharmaceutical } \\
\text { companies) }\end{array}$ & NR & $\begin{array}{l}\text { Pooled } \\
\text { sample } \\
\text { Median (IQR) } \\
\text { Overall: NR } \\
\text { G1: } 74(8) \\
\text { G2: } 74(8) \\
\text { Northern } \\
\text { Ireland } \\
\text { Mean (SD) } \\
\text { Overall: NR } \\
\text { G1: } 73.1(5.0) \\
\text { G2: } 74.2(6.3)\end{array}$ & $\begin{array}{l}\text { Pooled } \\
\text { sample } \\
\text { Overall: NR } \\
\text { G1: } 57.9 \\
\text { G2: } 57.3 \\
\text { Northern } \\
\text { Ireland } \\
\text { Overall: NR } \\
\text { G1: } 63.6 \\
\text { G2: } 61.0\end{array}$ & NR \\
\hline
\end{tabular}


Table E1. Study and patient-level characteristics (continued)

\begin{tabular}{|c|c|c|c|c|c|c|c|c|c|c|c|}
\hline $\begin{array}{l}\text { Author, } \\
\text { Year } \\
\text { Trial } \\
\text { Name }^{\text {a }}\end{array}$ & $\begin{array}{l}\text { Interventions } \\
\text { and } \\
\text { Comparator } \\
\text { Descriptions }\end{array}$ & $\begin{array}{l}\text { Intervention } \\
\text { Goal }\end{array}$ & Inclusion Criteria & $\begin{array}{l}\text { Exclusion } \\
\text { Criteria }\end{array}$ & $\begin{array}{l}\text { Study } \\
\text { Design }\end{array}$ & $\begin{array}{l}\text { Study } \\
\text { Duration }\end{array}$ & $\begin{array}{l}\text { Funding } \\
\text { Source(s) }\end{array}$ & $\begin{array}{l}\text { Baseline } \\
\text { \% Rural }\end{array}$ & $\begin{array}{l}\text { Baseline } \\
\text { Age - Mean } \\
\text { (SD) or } \\
\text { Median } \\
\text { (Range) }\end{array}$ & $\begin{array}{l}\text { Baseline \% } \\
\text { Female }\end{array}$ & $\begin{array}{l}\text { Race/ } \\
\text { Ethnicity \% }\end{array}$ \\
\hline $\begin{array}{l}\text { Blenner- } \\
\text { hassett et } \\
\text { al., } 2007^{3}\end{array}$ & $\begin{array}{l}\text { Implementation } \\
\text { of a Home } \\
\text { Medication } \\
\text { Review (HMR) } \\
\text { into a chronic } \\
\text { heart failure } \\
\text { collaborative } \\
\text { care model. } \\
\text { HMRs were } \\
\text { conducted by } \\
\text { accredited } \\
\text { pharmacists. } \\
\text { G2: No HMR }\end{array}$ & $\begin{array}{l}\text { To help } \\
\text { implementati } \\
\text { on of home } \\
\text { medicine } \\
\text { review and } \\
\text { improve } \\
\text { medication } \\
\text { management }\end{array}$ & $\begin{array}{l}\text { (1) Patient lives at } \\
\text { home or in } \\
\text { unfunded self-care; } \\
\text { (2) At risk for } \\
\text { medication } \\
\text { mismanagement } \\
\text { (e.g., taking five or } \\
\text { more medications; } \\
\text { suspected non- } \\
\text { compliance; taking } \\
\text { more than 12 } \\
\text { doses per day; } \\
\text { difficulty managing } \\
\text { because of literacy, } \\
\text { language, } \\
\text { dexterity, impaired } \\
\text { vision, confusion or } \\
\text { cognitive } \\
\text { difficulties; many } \\
\text { changes to their } \\
\text { medication } \\
\text { regimen; attending } \\
\text { multiple doctors; } \\
\text { taking medications } \\
\text { with a narrow } \\
\text { therapeutic index; } \\
\text { recent discharge } \\
\text { from hospital; } \\
\text { symptoms } \\
\text { suggestive of an } \\
\text { adverse drug } \\
\text { reaction; } \\
\text { and other (e.g. loss } \\
\text { of spouse, } \\
\text { recurrent falls). }\end{array}$ & NR & Cohort & NR & $\begin{array}{l}\text { Professional } \\
\text { organization }\end{array}$ & NR & $\begin{array}{l}\text { Overall: NR } \\
\text { G1: } 80.6 \\
\text { G2: } 79.9\end{array}$ & $\begin{array}{l}\text { Overall: NR } \\
\text { G1: } 59 \\
\text { G2: } 46\end{array}$ & $\overline{N R}$ \\
\hline
\end{tabular}


Table E1. Study and patient-level characteristics (continued)

\begin{tabular}{|c|c|c|c|c|c|c|c|c|c|c|c|}
\hline $\begin{array}{l}\text { Author, } \\
\text { Year } \\
\text { Trial } \\
\text { Name }^{a}\end{array}$ & $\begin{array}{l}\text { Interventions } \\
\text { and } \\
\text { Comparator } \\
\text { Descriptions }\end{array}$ & $\begin{array}{l}\text { Intervention } \\
\text { Goal }\end{array}$ & $\begin{array}{l}\text { Inclusion } \\
\text { Criteria }\end{array}$ & $\begin{array}{l}\text { Exclusion } \\
\text { Criteria }\end{array}$ & $\begin{array}{l}\text { Study } \\
\text { Design }\end{array}$ & $\begin{array}{l}\text { Study } \\
\text { Duration }\end{array}$ & $\begin{array}{l}\text { Funding } \\
\text { Source(s) }\end{array}$ & $\begin{array}{l}\text { Baseline } \\
\% \text { Rural }\end{array}$ & $\begin{array}{l}\text { Baseline } \\
\text { Age - Mean } \\
\text { (SD) or } \\
\text { Median } \\
\text { (Range) }\end{array}$ & $\begin{array}{l}\text { Baseline \% } \\
\text { Female }\end{array}$ & $\begin{array}{l}\text { Race/ } \\
\text { Ethnicity \% }\end{array}$ \\
\hline $\begin{array}{l}\text { Carter et } \\
\text { al., } 1997^{4} \text {; } \\
\text { Barnette, } \\
\text { Murphy, } \\
\text { and } \\
\text { Carter, } \\
1996^{5}\end{array}$ & $\begin{array}{l}\text { G1: } \\
\text { Pharmaceutical } \\
\text { care provided } \\
\text { by pharmacists } \\
\text { within an } \\
\text { interdisciplinary } \\
\text { practice model. } \\
\text { Patient } \\
\text { education } \\
\text { (lifestyle, risk } \\
\text { factor } \\
\text { modifications, } \\
\text { and drug } \\
\text { therapy) was } \\
\text { standardized. } \\
\text { G2: Usual care }\end{array}$ & $\begin{array}{l}\text { To train } \\
\text { community } \\
\text { pharmacists } \\
\text { to provide } \\
\text { HTN } \\
\text { monitoring } \\
\text { and direct } \\
\text { consultation } \\
\text { to physicians } \\
\text { and nurses }\end{array}$ & $\begin{array}{l}\text { (1) Greater than } \\
18 \text { years of age, } \\
\text { with essential } \\
\text { HTN (one of the } \\
\text { following: } \\
\text { average diastolic } \\
\text { blood pressure } \\
90 \mathrm{~mm} \text { Hg or } \\
\text { above, average } \\
\text { systolic blood } \\
\text { pressure } 140 \\
\text { mm Hg or above, } \\
\text { or current } \\
\text { therapy with } \\
\text { antihypertensive } \\
\text { drugs [controlled } \\
\text { or uncontrolled } \\
\text { blood pressure]); } \\
\text { (2) Receiving } \\
\text { care from a } \\
\text { physician in the } \\
\text { medical center or } \\
\text { annex and } \\
\text { prescriptions } \\
\text { from the clinic } \\
\text { pharmacy }\end{array}$ & $\begin{array}{l}(1) \\
\text { Secondary } \\
\text { causes of } \\
\text { HTN; (2) } \\
\text { Unwilling or } \\
\text { unable to } \\
\text { return to } \\
\text { clinic } \\
\text { pharmacy for } \\
\text { scheduled } \\
\text { appointment; } \\
\text { (3) Spouse or } \\
\text { sibling } \\
\text { enrolled in } \\
\text { study; (4) BP } \\
>210 \text { mm Hg } \\
\text { systolic or } \\
>115 \text { mm Hg } \\
\text { diastolic; (5) } \\
\text { Serious } \\
\text { complicating } \\
\text { disease so } \\
\text { disabling that } \\
\text { BP control } \\
\text { was } \\
\text { secondary or } \\
\text { minor } \\
\text { concern (e.g., } \\
\text { terminal } \\
\text { cancer, New } \\
\text { York Heart } \\
\text { Association } \\
\text { class III or IV } \\
\text { CHF) }\end{array}$ & Cohort & 6 months & Unspecified & $\begin{array}{l}\text { Overall: } \\
\text { NR, but } \\
\text { likely } \\
100 \% \\
\text { rural } \\
\text { G1: NR } \\
\text { G2: NR }\end{array}$ & $\begin{array}{l}\text { Mean (range) } \\
\text { Overall: NR } \\
\text { G1: } 67.3 \text { (47- } \\
\text { 80) } \\
\text { G2: } 68.5 \text { (40- } \\
\text { 92) }\end{array}$ & NR & NR \\
\hline
\end{tabular}


Table E1. Study and patient-level characteristics (continued)

\begin{tabular}{|c|c|c|c|c|c|c|c|c|c|c|c|}
\hline $\begin{array}{l}\text { Author, } \\
\text { Year } \\
\text { Trial } \\
\text { Name }^{\text {a }}\end{array}$ & $\begin{array}{l}\text { Interventions } \\
\text { and Comparator } \\
\text { Descriptions }\end{array}$ & $\begin{array}{l}\text { Interventio } \\
\text { n Goal }\end{array}$ & Inclusion Criteria & $\begin{array}{l}\text { Exclusion } \\
\text { Criteria }\end{array}$ & $\begin{array}{l}\text { Study } \\
\text { Design }\end{array}$ & $\begin{array}{l}\text { Study } \\
\text { Duration }\end{array}$ & $\begin{array}{l}\text { Funding } \\
\text { Source(s) }\end{array}$ & $\begin{array}{l}\text { Baseline } \\
\% \text { Rural }\end{array}$ & $\begin{array}{l}\text { Baseline } \\
\text { Age - Mean } \\
\text { (SD) or } \\
\text { Median } \\
\text { (Range) }\end{array}$ & $\begin{array}{l}\text { Baseline \% } \\
\text { Female }\end{array}$ & $\begin{array}{l}\text { Race/ } \\
\text { Ethnicity \% }\end{array}$ \\
\hline $\begin{array}{l}\text { Chisholm } \\
\text { et al., } \\
2002^{6}\end{array}$ & $\begin{array}{l}\text { G1: Clinical } \\
\text { pharmacy } \\
\text { services, including } \\
\text { reviewing } \\
\text { patients' } \\
\text { medication } \\
\text { therapy with } \\
\text { emphasis on } \\
\text { controlling BP, } \\
\text { and preventing or } \\
\text { resolving DTPs. } \\
\text { Pharmacists } \\
\text { counseled } \\
\text { patients about } \\
\text { their regimen, } \\
\text { including desired } \\
\text { clinical responses } \\
\text { and possible } \\
\text { adverse reactions. } \\
\text { G2: Routine } \\
\text { transplant clinic } \\
\text { services, but } \\
\text { without clinical } \\
\text { pharmacist } \\
\text { interaction. } \\
\text { Routine clinical } \\
\text { services here } \\
\text { entailed meeting a } \\
\text { renal transplant } \\
\text { clinic team that } \\
\text { consisted of } 2 \\
\text { nephrologists, a } \\
\text { clinical } \\
\text { pharmacist, PAs } \\
\text { and a nurse. }\end{array}$ & $\begin{array}{l}\text { To improve } \\
\text { blood } \\
\text { pressure } \\
\text { control } \\
\text { among } \\
\text { African- } \\
\text { American } \\
\text { renal } \\
\text { transplant } \\
\text { patients. } \\
\end{array}$ & $\begin{array}{l}\text { African-American } \\
\text { patients who } \\
\text { received a renal } \\
\text { transplant at the } \\
\text { Medical College } \\
\text { of Georgia (MCG) } \\
\text { from November } \\
1996 \text { through } \\
\text { March } 1998 \text { and } \\
\text { met the following } \\
\text { criteria: } 1 \text { ) be a } \\
\text { minimum of } 1 \\
\text { years of age; } 2 \text { ) } \\
\text { have received } \\
\text { only one renal } \\
\text { transplant } \\
\text { (primary renal } \\
\text { transplant); and } \\
3 \text { ) have received } \\
\text { post-transplant } \\
\text { care at the MCG } \\
\text { renal transplant } \\
\text { clinic. Patients } \\
\text { were included in } \\
\text { the study } \\
\text { regardless of } \\
\text { whether or not } \\
\text { they had HTN. }\end{array}$ & $\begin{array}{l}\text { See } \\
\text { inclusion } \\
\text { criteria }\end{array}$ & $\begin{array}{l}\text { RCT: } \\
\text { parallel, } \\
\text { not } \\
\text { clustere } \\
\text { d }\end{array}$ & $\begin{array}{l}\text { Approxi } \\
\text { mately } \\
30 \\
\text { months }\end{array}$ & $\begin{array}{l}\text { Foundation or } \\
\text { non-profit }\end{array}$ & NR & $\begin{array}{l}\text { Overall: NR } \\
\text { G1: } 51(16.8) \\
\text { G2: } 47(12.7)\end{array}$ & $\begin{array}{l}\text { Overall: NR } \\
\text { G1: } 38.5 \\
\text { G2: } 30.0\end{array}$ & $\begin{array}{l}\text { Overall: } \\
100 \% \\
\text { African- } \\
\text { American }\end{array}$ \\
\hline
\end{tabular}


Table E1. Study and patient-level characteristics (continued)

\begin{tabular}{|c|c|c|c|c|c|c|c|c|c|c|c|}
\hline $\begin{array}{l}\text { Author, } \\
\text { Year } \\
\text { Trial } \\
\text { Name }^{a}\end{array}$ & $\begin{array}{l}\text { Interventions } \\
\text { and } \\
\text { Comparator } \\
\text { Descriptions }\end{array}$ & $\begin{array}{l}\text { Intervention } \\
\text { Goal }\end{array}$ & Inclusion Criteria & $\begin{array}{l}\text { Exclusion } \\
\text { Criteria }\end{array}$ & $\begin{array}{l}\text { Study } \\
\text { Design }\end{array}$ & $\begin{array}{l}\text { Study } \\
\text { Duration }\end{array}$ & $\begin{array}{l}\text { Funding } \\
\text { Source(s) }\end{array}$ & $\begin{array}{l}\text { Baseline } \\
\% \text { Rural }\end{array}$ & $\begin{array}{l}\text { Baseline } \\
\text { Age - Mean } \\
\text { (SD) or } \\
\text { Median } \\
\text { (Range) } \\
\end{array}$ & $\begin{array}{l}\text { Baseline \% } \\
\text { Female }\end{array}$ & $\begin{array}{l}\text { Race/ } \\
\text { Ethnicity \% }\end{array}$ \\
\hline $\begin{array}{l}\text { Chris- } \\
\text { chilles et } \\
\text { al., } 2004^{7}\end{array}$ & $\begin{array}{l}\text { G1: PCM } \\
\text { provided by } \\
\text { pharmacists } \\
\text { G2: Did not } \\
\text { receive PCM } \\
\text { services }\end{array}$ & $\begin{array}{l}\text { Avoid } \\
\text { adverse drug } \\
\text { events and } \\
\text { the health } \\
\text { system costs } \\
\text { associated } \\
\text { with these } \\
\text { adverse } \\
\text { events in a } \\
\text { Medicaid } \\
\text { population at } \\
\text { high risk for } \\
\text { adverse } \\
\text { effects }\end{array}$ & $\begin{array}{l}\text { Noninstitutionalized } \\
\text { lowa Medicaid } \\
\text { patients taking four } \\
\text { or more long-term } \\
\text { medications, } \\
\text { including at least } \\
\text { one medication } \\
\text { representing } 1 \text { of } \\
\text { specified } 12 \\
\text { diseases, were } \\
\text { eligible (the } 12 \\
\text { diseases were } \\
\text { congestive heart } \\
\text { disease, ischemic } \\
\text { heart disease, } \\
\text { diabetes mellitus, } \\
\text { HTN, } \\
\text { hyperlipidemia, } \\
\text { asthma, } \\
\text { depression, atrial } \\
\text { fibrillation, } \\
\text { osteoarthritis, } \\
\text { gastroesophageal } \\
\text { reflux, pep- tic } \\
\text { ulcer disease, and } \\
\text { chronic obstructive } \\
\text { pulmonary } \\
\text { disease) with } \\
\text { pharmacy claims at } \\
\text { one (or more) of } \\
\text { the } 117 \\
\text { participating } \\
\text { pharmacies. }\end{array}$ & $\begin{array}{l}\text { All patients } \\
\text { who were } \\
\text { not } \\
\text { continuousl } \\
\text { y eligible } \\
\text { for } \\
\text { Medicaid } \\
\text { from } 6 \\
\text { months } \\
\text { before } \\
\text { through } 12 \\
\text { months } \\
\text { after the } \\
\text { date on } \\
\text { which they } \\
\text { became } \\
\text { eligible for } \\
\text { PCM. }\end{array}$ & 列 & $\begin{array}{l}21 \\
\text { months }\end{array}$ & $\begin{array}{l}\text { Multiple } \\
\text { (Government } \\
\text { and foundation } \\
\text { funding) }\end{array}$ & NR & $\begin{array}{l}\text { Overall: } 52.5 \\
(20.2) \\
\text { G1: } 54.1(0.8) \\
\text { G2: } 48.4(0.5)\end{array}$ & $\begin{array}{l}\text { Overall: } 71.4 \\
\text { G1: } 80.0 \\
\text { G2: } 69.3\end{array}$ & $\begin{array}{l}\text { Overall: NR } \\
\text { White } \\
\text { G1: } 89.1 \\
\text { G2: } 90.0 \\
\text { Black } \\
\text { G1: } 5.9 \\
\text { G2: } 5.5 \\
\text { Other } \\
\text { G1: } 1.0 \\
\text { G2: } 2.1 \\
\text { Unknown } \\
\text { G1: } 4.0 \\
\text { G2: } 2.4\end{array}$ \\
\hline
\end{tabular}


Table E1. Study and patient-level characteristics (continued)

\begin{tabular}{|c|c|c|c|c|c|c|c|c|c|c|c|}
\hline $\begin{array}{l}\text { Author, } \\
\text { Year } \\
\text { Trial } \\
\text { Name }^{a}\end{array}$ & $\begin{array}{l}\text { Interventions } \\
\text { and } \\
\text { Comparator } \\
\text { Descriptions }\end{array}$ & $\begin{array}{l}\text { Intervention } \\
\text { Goal }\end{array}$ & Inclusion Criteria & $\begin{array}{l}\text { Exclusion } \\
\text { Criteria }\end{array}$ & $\begin{array}{l}\text { Study } \\
\text { Design }\end{array}$ & $\begin{array}{l}\text { Study } \\
\text { Duration }\end{array}$ & $\begin{array}{l}\text { Funding } \\
\text { Source(s) }\end{array}$ & $\begin{array}{l}\text { Baseline } \\
\% \text { Rural }\end{array}$ & $\begin{array}{l}\text { Baseline } \\
\text { Age - Mean } \\
\text { (SD) or } \\
\text { Median } \\
\text { (Range) }\end{array}$ & $\begin{array}{l}\text { Baseline \% } \\
\text { Female }\end{array}$ & $\begin{array}{l}\text { Race/ } \\
\text { Ethnicity \% }\end{array}$ \\
\hline $\begin{array}{l}\text { Christen- } \\
\text { sen et al., } \\
2007^{8}\end{array}$ & $\begin{array}{l}\text { G1: MTM } \\
\text { services } \\
\text { designed by a } \\
\text { health plan for } \\
\text { its beneficiaries } \\
\text { and provided by } \\
\text { either } \\
\text { community } \\
\text { pharmacists or } \\
\text { medical clinic- } \\
\text { based } \\
\text { pharmacists. } \\
\text { G2: Patients } \\
\text { from same } \\
\text { counties as G1 } \\
\text { who did not } \\
\text { receive } \\
\text { intervention } \\
\text { (control group } \\
\text { 1) } \\
\text { G3: Patients } \\
\text { from a different } \\
\text { county than G1 } \\
\text { who did not } \\
\text { receive } \\
\text { intervention } \\
\text { (control group } \\
\text { 2) }\end{array}$ & $\begin{array}{l}\text { To assess } \\
\text { the feasibility } \\
\text { of a } \\
\text { pharmacist } \\
\text { based } \\
\text { medication } \\
\text { therapy } \\
\text { management } \\
\text { service for } \\
\text { North } \\
\text { Carolina } \\
\text { State Health } \\
\text { Plan } \\
\text { enrollees. } \\
\end{array}$ & $\begin{array}{l}\text { (1) Residence in } \\
\text { Orange or Durham } \\
\text { County, NC; } \\
\text { (2) Among the } \\
1,000 \text { highest } \\
\text { number of } \\
\text { prescriptions used } \\
\text { during the first } 6 \\
\text { months of } 2004 \text {. }\end{array}$ & NR & NRCT & 6 months & $\begin{array}{l}\text { Multiple (Third- } \\
\text { party payor and } \\
\text { foundation) }\end{array}$ & NR & $\begin{array}{l}\text { G1: } 67.7 \\
(11.4) \\
\text { G2: } 67.6 \\
(12.2) \\
\text { G3: } \\
66.0(12.1)\end{array}$ & $\begin{array}{l}\text { G1: } 62.3 \\
\text { G2: } 68.9 \\
\text { G3: } 71.3\end{array}$ & NR \\
\hline
\end{tabular}


Table E1. Study and patient-level characteristics (continued)

\begin{tabular}{|c|c|c|c|c|c|c|c|c|c|c|c|}
\hline $\begin{array}{l}\text { Author, } \\
\text { Year } \\
\text { Trial } \\
\text { Name }^{a}\end{array}$ & $\begin{array}{l}\text { Interventions } \\
\text { and } \\
\text { Comparator } \\
\text { Descriptions }\end{array}$ & $\begin{array}{l}\text { Intervention } \\
\text { Goal }\end{array}$ & Inclusion Criteria & $\begin{array}{l}\text { Exclusion } \\
\text { Criteria }\end{array}$ & $\begin{array}{l}\text { Study } \\
\text { Design }\end{array}$ & $\begin{array}{l}\text { Study } \\
\text { Duration }\end{array}$ & $\begin{array}{l}\text { Funding } \\
\text { Source(s) }\end{array}$ & $\begin{array}{l}\text { Baseline } \\
\% \text { Rural }\end{array}$ & $\begin{array}{l}\text { Baseline } \\
\text { Age - Mean } \\
\text { (SD) or } \\
\text { Median } \\
\text { (Range) }\end{array}$ & $\begin{array}{l}\text { Baseline \% } \\
\text { Female }\end{array}$ & $\begin{array}{l}\text { Race/ } \\
\text { Ethnicity \% }\end{array}$ \\
\hline $\begin{array}{l}\text { Clifford et } \\
\text { al., } 2002^{9}\end{array}$ & $\begin{array}{l}\text { G1: } \\
\text { Pharmaceutical } \\
\text { care provided } \\
\text { by a clinical } \\
\text { pharmacist, } \\
\text { which included } \\
\text { a } \\
\text { comprehensive } \\
\text { review relating } \\
\text { to } \\
\text { pharmacothera } \\
\text { py and } \\
\text { diabetes, use of } \\
\text { proprietary and } \\
\text { non-proprietary } \\
\text { medications, } \\
\text { such as } \\
\text { complementary } \\
\text { medicines, and } \\
\text { identification of } \\
\text { drug therapy } \\
\text { problems. } \\
\text { G2: Standard } \\
\text { outpatient care } \\
\text { for diabetes }\end{array}$ & $\begin{array}{l}\text { To improve } \\
\text { glycemic } \\
\text { control in } \\
\text { diabetic } \\
\text { patients } \\
\text { without } \\
\text { adversely } \\
\text { affecting QOL } \\
\text { or satisfaction } \\
\text { with health } \\
\text { care provided } \\
\text { : }\end{array}$ & $\begin{array}{l}\text { Adult patients } \geq 18 \\
\text { years with type } 1 \text { or } \\
\text { type } 2 \text { diabetes } \\
\text { and at least one of } \\
\text { the following } \\
\text { features indicating } \\
\text { high risk for } \\
\text { development of } \\
\text { diabetes } \\
\text { complications: } \\
\text { 1) Random blood } \\
\text { glucose levels }>11 \\
\text { mmol/L on } \geq 2 \\
\text { occasions in } \\
\text { tertiary care setting } \\
\text { within previous } 12 \\
\text { months; } \\
\text { 2) HbA1C }>8 \% \text { on } \\
\geq 2 \text { occasions in } \\
\text { previous } 12 \\
\text { months; } \\
3 \text { ) HTN (SBP }>160 \\
\text { mm Hg and/or DBP } \\
>90 \text { mm Hg) and/or } \\
\text { taking drug } \\
\text { therapy; } \\
4 \text { ) Dyslipidemia } \\
\text { (total serum } \\
\text { cholesterol }>5.5 \\
\text { mmol/L and/or } \\
\text { serum triglycerides } \\
>4.0 \text { mmol/L); } \\
5) \text { Polypharmacy } \\
\text { (>3 drugs) }\end{array}$ & $\begin{array}{l}\text { See } \\
\text { inclusion } \\
\text { criteria }\end{array}$ & $\begin{array}{l}\text { RCT: } \\
\text { parallel, } \\
\text { not } \\
\text { clustere } \\
\text { d }\end{array}$ & 6 months & $\begin{array}{l}\text { Multiple } \\
\text { (Pharmaceutica } \\
\text { I, professional } \\
\text { organization) }\end{array}$ & NR & $\begin{array}{l}\text { Overall: NR } \\
\text { G1: } 60(12) \\
\text { G2: } 61(12) \\
p=N S\end{array}$ & $\begin{array}{l}\text { Overall: NR } \\
\text { G1: } 42 \\
\text { G2: } 52 \\
p=N S\end{array}$ & NR \\
\hline
\end{tabular}


Table E1. Study and patient-level characteristics (continued)

\begin{tabular}{|c|c|c|c|c|c|c|c|c|c|c|c|}
\hline $\begin{array}{l}\text { Author, } \\
\text { Year } \\
\text { Trial } \\
\text { Name }^{a}\end{array}$ & $\begin{array}{l}\text { Interventions and } \\
\text { Comparator } \\
\text { Descriptions }\end{array}$ & $\begin{array}{l}\text { Interventio } \\
\text { n Goal }\end{array}$ & Inclusion Criteria & $\begin{array}{l}\text { Exclusion } \\
\text { Criteria }\end{array}$ & $\begin{array}{l}\text { Study } \\
\text { Design }\end{array}$ & $\begin{array}{l}\text { Study } \\
\text { Duration }\end{array}$ & $\begin{array}{l}\text { Funding } \\
\text { Source(s) }\end{array}$ & $\begin{array}{l}\text { Baseline } \\
\text { \% Rural }\end{array}$ & $\begin{array}{l}\text { Baseline } \\
\text { Age - Mean } \\
\text { (SD) or } \\
\text { Median } \\
\text { (Range) } \\
\end{array}$ & $\begin{array}{l}\text { Baseline \% } \\
\text { Female }\end{array}$ & $\begin{array}{l}\text { Race/ } \\
\text { Ethnicity \% }\end{array}$ \\
\hline $\begin{array}{l}\text { Fischer et } \\
\text { al., } 2000^{10}\end{array}$ & $\begin{array}{l}\text { G1: } \\
\text { Pharmaceutical } \\
\text { care based on } \\
\text { Encara Practice } \\
\text { System provided } \\
\text { by onsite health } \\
\text { maintenance } \\
\text { organization staff } \\
\text { pharmacists } \\
\text { G2: Standard } \\
\text { community } \\
\text { pharmacy practice } \\
\text { G3: Patients at } \\
\text { eligible clinics who } \\
\text { declined to receive } \\
\text { intervention but } \\
\text { were included in } \\
\text { some analyses. }\end{array}$ & $\begin{array}{l}\text { (1) To } \\
\text { improve the } \\
\text { amount of } \\
\text { information } \\
\text { patients } \\
\text { received; (2) } \\
\text { To improve } \\
\text { the way } \\
\text { patients self- } \\
\text { administer } \\
\text { medication; } \\
\text { (3) To } \\
\text { enhance } \\
\text { awareness } \\
\text { of side } \\
\text { effects. }\end{array}$ & $\begin{array}{l}\text { (1) HMO enrollees } \\
\text { enrolled in a } \\
\text { participating clinic; } \\
\text { (2) Had asthma, } \\
\text { COPD or heart } \\
\text { disease identified } \\
\text { via pharmacy or } \\
\text { hospital data base } \\
\text { medication } \\
\text { records. }\end{array}$ & NR & NRCT & & $\begin{array}{l}\text { Foundation or } \\
\text { non-profit }\end{array}$ & NR & $\begin{array}{l}\text { Overall: NR } \\
\text { G1: } 67.2 \\
\text { G2: } 68.3 \\
\text { G3: } 58.9\end{array}$ & $\begin{array}{l}\text { Overall: NR } \\
\text { G1: } 54 \\
\text { G2: } 52 \\
\text { G3: } 50\end{array}$ & $\begin{array}{l}\text { \% White } \\
\text { Overall: NR } \\
\text { G1: } 98 \\
\text { G2: } 96 \\
\text { G3: } 92\end{array}$ \\
\hline $\begin{array}{l}\text { Fischer et } \\
\text { al., } 2002^{11}\end{array}$ & $\begin{array}{l}\text { Pharmaceutical } \\
\text { care based on } \\
\text { Encara Practice } \\
\text { System provided } \\
\text { by pharmacists. } \\
\text { Pharmacist- } \\
\text { physician } \\
\text { communication } \\
\text { about pharmacist- } \\
\text { identified DTPs. } \\
\text { G2: Usual care } \\
\text { with no additional } \\
\text { interventions }\end{array}$ & $\begin{array}{l}\text { To assess } \\
\text { whether } \\
\text { pharmaceuti } \\
\text { cal care } \\
\text { program } \\
\text { decreases } \\
\text { health care } \\
\text { utilization, } \\
\text { medication } \\
\text { use, or } \\
\text { charges }\end{array}$ & $\begin{array}{l}\text { (1) Age } \geq 18 ; \text { (2) } \\
\text { Enrolled in } \\
\text { participating HMO } \\
\text { for } \geq 2 \text { years with } \\
\text { active prescriptions } \\
\text { treating heart or } \\
\text { lung disease; (3) } \\
\text { Obtained } \\
\text { prescriptions from } \\
\text { participating } \\
\text { pharmacy; (4) Must } \\
\text { have filled } \\
\text { prescriptions for } \\
\text { one of several pre- } \\
\text { specified } \\
\text { medication types } \\
\text { for heart or lung } \\
\text { disease in } 6 \\
\text { months before } \\
\text { study }\end{array}$ & $\begin{array}{l}\text { Died, } \\
\text { disenrolled, } \\
\text { or } \\
\text { discontinued } \\
\text { pharmacy } \\
\text { benefit } \\
\text { before end } \\
\text { of study } \\
\text { period } \\
\end{array}$ & NRCT & $\begin{array}{l}2 \text { years } \\
\text { (1997- } \\
98) \text { [one } \\
\text { year } \\
\text { before } \\
\text { interventi } \\
\text { on } \\
\text { initiation } \\
\text { and one } \\
\text { year } \\
\text { after] }\end{array}$ & $\begin{array}{l}\text { Multiple } \\
\text { (Pharmaceu- } \\
\text { tical } \\
\text { companies, } \\
\text { third-party } \\
\text { payors) }\end{array}$ & NR & $\begin{array}{l}\text { Overall: NR } \\
\text { G1: } 57 \\
\text { G2: } 58\end{array}$ & $\begin{array}{l}\text { Overall: NR } \\
\text { G1: } 50 \\
\text { G2: } 51\end{array}$ & NR \\
\hline
\end{tabular}


Table E1. Study and patient-level characteristics (continued)

\begin{tabular}{|c|c|c|c|c|c|c|c|c|c|c|c|}
\hline $\begin{array}{l}\text { Author, } \\
\text { Year } \\
\text { Trial } \\
\text { Name }^{a}\end{array}$ & $\begin{array}{l}\text { Interventions } \\
\text { and } \\
\text { Comparator } \\
\text { Descriptions }\end{array}$ & $\begin{array}{l}\text { Intervention } \\
\text { Goal }\end{array}$ & Inclusion Criteria & $\begin{array}{l}\text { Exclusion } \\
\text { Criteria }\end{array}$ & $\begin{array}{l}\text { Study } \\
\text { Design }\end{array}$ & $\begin{array}{l}\text { Study } \\
\text { Duration }\end{array}$ & $\begin{array}{l}\text { Funding } \\
\text { Source(s) }\end{array}$ & $\begin{array}{l}\text { Baseline } \\
\% \text { Rural }\end{array}$ & $\begin{array}{l}\text { Baseline } \\
\text { Age - Mean } \\
\text { (SD) or } \\
\text { Median } \\
\text { (Range) }\end{array}$ & $\begin{array}{l}\text { Baseline \% } \\
\text { Female }\end{array}$ & $\begin{array}{l}\text { Race/ } \\
\text { Ethnicity \% }\end{array}$ \\
\hline $\begin{array}{l}\text { Fox et al., } \\
2009^{12}\end{array}$ & $\begin{array}{l}\text { G1: Florida } \\
\text { Health Care } \\
\text { Plans MTM } \\
\text { program, } \\
\text { consisting of a } \\
\text { medication } \\
\text { therapy review } \\
\text { and evaluation } \\
\text { by a clinical } \\
\text { pharmacist that } \\
\text { was } \\
\text { documented } \\
\text { and sent to the } \\
\text { patient's } \\
\text { physician } \\
\text { through health } \\
\text { plan review } \\
\text { G2: Opt-out } \\
\text { from MTM } \\
\text { program }\end{array}$ & $\begin{array}{l}\text { To reduce } \\
\text { LDL-C and } \\
\text { improve } \\
\text { HEDIS goal } \\
\text { attainment } \\
\text { among } \\
\text { patients with } \\
\text { diabetes on } \\
\text { lipid-lowering } \\
\text { medications }\end{array}$ & $\begin{array}{l}\text { FHCP enrollees } \\
\text { who: } \\
\text { 1) Were Medicare } \\
\text { Part D members; } \\
\text { 2) Were diagnosed } \\
\text { with } \geq 3 \text { chronic } \\
\text { diseases; } \\
\text { 3) Used } \geq 4 \\
\text { maintenance } \\
\text { medications; } \\
\text { 4) Were likely to } \\
\text { have Part D } \\
\text { medication costs } \\
\geq \$ 4000 \text { per year; } \\
\text { 5) Were eligible for } \\
\text { inclusion in } 2008 \\
\text { HEDIS } \\
\text { comprehensive } \\
\text { diabetes care } \\
\text { (CDC) } \\
\text { administrative } \\
\text { dataset }\end{array}$ & $\begin{array}{l}\text { None } \\
\text { specified }\end{array}$ & Cohort & $\begin{array}{l}21 \\
\text { months }\end{array}$ & Unspecified & NR & $\begin{array}{l}\text { Overall: NR } \\
\text { G1: } 67.6(7.2) \\
\text { G2: } 68.3(6.1)\end{array}$ & $\begin{array}{l}\text { Overall: NR } \\
\text { G1: } 45.5 \\
\text { G2: } 57.9\end{array}$ & NR \\
\hline
\end{tabular}


Table E1. Study and patient-level characteristics (continued)

\begin{tabular}{|c|c|c|c|c|c|c|c|c|c|c|c|}
\hline $\begin{array}{l}\text { Author, } \\
\text { Year } \\
\text { Trial } \\
\text { Name }^{\text {a }}\end{array}$ & $\begin{array}{l}\text { Interventions } \\
\text { and Comparator } \\
\text { Descriptions }\end{array}$ & $\begin{array}{l}\text { Intervention } \\
\text { Goal }\end{array}$ & $\begin{array}{l}\text { Inclusion } \\
\text { Criteria }\end{array}$ & $\begin{array}{l}\text { Exclusion } \\
\text { Criteria }\end{array}$ & $\begin{array}{l}\text { Study } \\
\text { Design }\end{array}$ & $\begin{array}{l}\text { Study } \\
\text { Duration }\end{array}$ & $\begin{array}{l}\text { Funding } \\
\text { Source(s) }\end{array}$ & $\begin{array}{l}\text { Baseline } \\
\% \text { Rural }\end{array}$ & $\begin{array}{l}\text { Baseline } \\
\text { Age - Mean } \\
\text { (SD) or } \\
\text { Median } \\
\text { (Range) }\end{array}$ & $\begin{array}{l}\text { Baseline \% } \\
\text { Female }\end{array}$ & $\begin{array}{l}\text { Race/ } \\
\text { Ethnicity \% }\end{array}$ \\
\hline $\begin{array}{l}\text { Gattis et } \\
\text { al., 1999 } \\
\end{array}$ & $\begin{array}{l}\text { G1: Clinical } \\
\text { pharmacy } \\
\text { services, including } \\
\text { an assessment of } \\
\text { prescribed } \\
\text { regimen, } \\
\text { compliance, and } \\
\text { adverse effects, } \\
\text { and symptoms } \\
\text { and response to } \\
\text { therapy. Providing } \\
\text { patient education } \\
\text { about the purpose } \\
\text { of each drug and } \\
\text { reinforcing } \\
\text { adherence. } \\
\text { Detailed written } \\
\text { information was } \\
\text { also provided to } \\
\text { patients. } \\
\text { G2: Usual medical } \\
\text { care }\end{array}$ & $\begin{array}{l}\text { To improve } \\
\text { outcomes in } \\
\text { outpatients } \\
\text { with heart } \\
\text { failure. }\end{array}$ & $\begin{array}{l}\text { Patients with a } \\
\text { diagnosis of } \\
\text { heart failure } \\
\text { with LVEF < } \\
45 \% \text { in a } \\
\text { general } \\
\text { cardiology clinic } \\
\text { at a University } \\
\text { Medical Center. }\end{array}$ & $\begin{array}{l}\text { Life } \\
\text { expectancy < } \\
6 \text { months; } \\
\text { currently } \\
\text { participating } \\
\text { in a drug trial, } \\
\text { primary } \\
\text { residence } \\
\text { was a skilled } \\
\text { nursing } \\
\text { facility, } \\
\text { marked } \\
\text { dementia or } \\
\text { other } \\
\text { psychological } \\
\text { disorder that } \\
\text { prevented } \\
\text { participation } \\
\text { in patient } \\
\text { education or } \\
\text { follow-up. }\end{array}$ & $\begin{array}{l}\text { RCT: } \\
\text { parallel, } \\
\text { not } \\
\text { clustered } \\
\end{array}$ & $\begin{array}{l}24 \\
\text { weeks }\end{array}$ & $\begin{array}{l}\text { Multiple } \\
\text { (Foundation } \\
\text { and } \\
\text { academic) }\end{array}$ & NR & $\begin{array}{l}\text { Overall: } \\
\text { G1: } 71.5 \\
(25 \%: 60 \\
75 \%: 77) \\
\text { G2: } 63.0 \\
(25 \%: 55, \\
75 \%: 72)\end{array}$ & $\begin{array}{l}\text { Overall: NR } \\
\text { G1: } 31 \\
\text { G2: } 33\end{array}$ & $\begin{array}{l}\text { White } \\
\text { Overall: NR } \\
\text { G1: } 80 \\
\text { G2: } 79\end{array}$ \\
\hline
\end{tabular}


Table E1. Study and patient-level characteristics (continued)

\begin{tabular}{|c|c|c|c|c|c|c|c|c|c|c|c|}
\hline $\begin{array}{l}\text { Author, } \\
\text { Year } \\
\text { Trial } \\
\text { Name }^{a}\end{array}$ & $\begin{array}{l}\text { Interventions } \\
\text { and } \\
\text { Comparator } \\
\text { Descriptions }\end{array}$ & $\begin{array}{l}\text { Intervention } \\
\text { Goal }\end{array}$ & $\begin{array}{l}\text { Inclusion } \\
\text { Criteria }\end{array}$ & $\begin{array}{l}\text { Exclusion } \\
\text { Criteria }\end{array}$ & $\begin{array}{l}\text { Study } \\
\text { Design }\end{array}$ & $\begin{array}{l}\text { Study } \\
\text { Duration }\end{array}$ & $\begin{array}{l}\text { Funding } \\
\text { Source(s) }\end{array}$ & $\begin{array}{l}\text { Baseline } \\
\% \text { Rural }\end{array}$ & $\begin{array}{l}\text { Baseline } \\
\text { Age - Mean } \\
\text { (SD) or } \\
\text { Median } \\
\text { (Range) }\end{array}$ & $\begin{array}{l}\text { Baseline \% } \\
\text { Female }\end{array}$ & $\begin{array}{l}\text { Race/ } \\
\text { Ethnicity \% }\end{array}$ \\
\hline $\begin{array}{l}\text { Hanlon et } \\
\text { al., } 1996^{14}\end{array}$ & $\begin{array}{l}\text { G1: } \\
\text { Pharmaceutical } \\
\text { care provided } \\
\text { by a clinical } \\
\text { pharmacist } \\
\text { G2: Usual care } \\
\text { in the General } \\
\text { Medicine Clinic }\end{array}$ & $\begin{array}{l}\text { To evaluate the } \\
\text { effect of } \\
\text { sustained } \\
\text { clinical } \\
\text { pharmacist } \\
\text { interventions } \\
\text { involving elderly } \\
\text { outpatients with } \\
\text { polypharmacy } \\
\text { and their } \\
\text { primary care } \\
\text { physicians on: } \\
\text { prescribing } \\
\text { appropriateness } \\
\text {, health-related } \\
\text { quality of life, } \\
\text { adverse drug } \\
\text { events, } \\
\text { medication } \\
\text { compliance and } \\
\text { knowledge, } \\
\text { number of } \\
\text { medications } \\
\text { used, patient } \\
\text { satisfaction, } \\
\text { and physician } \\
\text { receptivity }\end{array}$ & $\begin{array}{l}\text { (1) Age } \geq 65 ;(2) \\
\text { Had evidence of } \\
\text { polypharmacy } \\
\text { operationally } \\
\text { defined as } \\
\text { prescribed } 5+ \\
\text { regularly } \\
\text { scheduled } \\
\text { medications by a } \\
\text { VA physician; (3) } \\
\text { Receiving } \\
\text { primary care in } \\
\text { the General } \\
\text { Medicine Clinic. } \\
\end{array}$ & $\begin{array}{l}\text { 1) Nursing } \\
\text { home } \\
\text { residence; } \\
\text { 2) Patients } \\
\text { with } \\
\text { cognitive } \\
\text { impairment } \\
\text {, as } \\
\text { determined } \\
\text { by the } \\
\text { Mental } \\
\text { Status } \\
\text { Questionna } \\
\text { ire; 3) No } \\
\text { caregiver } \\
\text { available to } \\
\text { be involved } \\
\text { in the } \\
\text { intervention }\end{array}$ & $\begin{array}{l}\text { RCT: } \\
\text { parallel, } \\
\text { not } \\
\text { clustere } \\
\text { d }\end{array}$ & One year & r Government & NR & $\begin{array}{l}\text { Overall: NR } \\
\text { G1: } 69.7(3.5) \\
\text { G2: } 69.9(4.1)\end{array}$ & $\begin{array}{l}\text { Overall: NR } \\
\text { G1: } 1.9 \\
\text { G2: } 0\end{array}$ & $\begin{array}{l}\text { White } \\
\text { Overall: NR } \\
\text { G1: } 79 \\
\text { G2: } 74.8\end{array}$ \\
\hline
\end{tabular}


Table E1. Study and patient-level characteristics (continued)

\begin{tabular}{|c|c|c|c|c|c|c|c|c|c|c|c|}
\hline $\begin{array}{l}\text { Author, } \\
\text { Year } \\
\text { Trial } \\
\text { Name }^{a}\end{array}$ & $\begin{array}{l}\text { Interventions } \\
\text { and } \\
\text { Comparator } \\
\text { Descriptions }\end{array}$ & $\begin{array}{l}\text { Interventio } \\
\text { n Goal }\end{array}$ & Inclusion Criteria & $\begin{array}{l}\text { Exclusion } \\
\text { Criteria }\end{array}$ & $\begin{array}{l}\text { Study } \\
\text { Design }\end{array}$ & $\begin{array}{l}\text { Study } \\
\text { Duration }\end{array}$ & $\begin{array}{l}\text { Funding } \\
\text { Source(s) }\end{array}$ & $\begin{array}{l}\text { Baseline } \\
\text { \% Rural }\end{array}$ & $\begin{array}{l}\text { Baseline } \\
\text { Age - Mean } \\
\text { (SD) or } \\
\text { Median } \\
\text { (Range) }\end{array}$ & $\begin{array}{l}\text { Baseline \% } \\
\text { Female }\end{array}$ & $\begin{array}{l}\text { Race/ } \\
\text { Ethnicity \% }\end{array}$ \\
\hline $\begin{array}{l}\text { Harrison } \\
\text { et al., } \\
2012^{15}\end{array}$ & $\begin{array}{l}\text { G1: } \\
\text { Pharmaceutical } \\
\text { care provided by } \\
\text { a clinical } \\
\text { pharmacist for } \\
\text { the purpose of } \\
\text { identifying and } \\
\text { resolving actual } \\
\text { and potential } \\
\text { drug therapy } \\
\text { problems, } \\
\text { medication } \\
\text { teaching, } \\
\text { adherence } \\
\text { optimization, } \\
\text { medication } \\
\text { reconciliation, } \\
\text { and provision of } \\
\text { drug information. } \\
\text { G2: } \\
\text { Retrospective } \\
\text { historical control } \\
\text { of matched } \\
\text { patients who } \\
\text { received } \\
\text { standard care at } \\
\text { a routine medical } \\
\text { visit within } 8 \\
\text { months prior to } \\
\text { study period }\end{array}$ & $\begin{array}{l}\text { Primarily } \\
\text { focused on } \\
\text { reducing } \\
\text { DTPs, but } \\
\text { study also } \\
\text { assessed } \\
\text { recommend } \\
\text { ations made } \\
\text { and patient } \\
\text { satisfaction } \\
\text { with service. } \\
\end{array}$ & $\begin{array}{l}\text { (1) All new lung } \\
\text { transplant } \\
\text { recipients; (2) } \\
\text { Referrals for } \\
\text { medication-related } \\
\text { concerns were also } \\
\text { accepted } \\
\text { from } \\
\text { interprofessional } \\
\text { team or at patient's } \\
\text { request; } \\
\text { (3) Control group } \\
\text { patients identified } \\
\text { from the } 8 \text { months } \\
\text { before study period } \\
\text { were matched with } \\
\text { study patients for } \\
\text { time post- } \\
\text { transplant. }\end{array}$ & NR & Cohort & NR & Unfunded & NR & $\begin{array}{l}\text { Mean or } \\
\text { median age } \\
\text { NR. Only } \% \\
\text { within } 3 \\
\text { specified } \\
\text { ranges } \\
\text { reported. } \\
\text { Ages } 18-39 \\
\text { G1: } 12 \% \\
\text { G2: } 30 \% \\
\text { Ages } 40-59 \\
\text { G1: } 51 \% \\
\text { G2: } 47 \% \\
\text { Ages }>60 \\
\text { G1: } 37 \% \\
\text { G2: } 23 \%\end{array}$ & $\begin{array}{l}\text { Overall: } 44 \\
\text { G1: } 44 \\
\text { G2: } 44\end{array}$ & NR \\
\hline
\end{tabular}


Table E1. Study and patient-level characteristics (continued)

\begin{tabular}{|c|c|c|c|c|c|c|c|c|c|c|c|}
\hline $\begin{array}{l}\text { Author, } \\
\text { Year } \\
\text { Trial } \\
\text { Name }^{\text {a }}\end{array}$ & $\begin{array}{l}\text { Interventions } \\
\text { and } \\
\text { Comparator } \\
\text { Descriptions }\end{array}$ & $\begin{array}{l}\text { Intervention } \\
\text { Goal }\end{array}$ & Inclusion Criteria & $\begin{array}{l}\text { Exclusion } \\
\text { Criteria }\end{array}$ & $\begin{array}{l}\text { Study } \\
\text { Design }\end{array}$ & $\begin{array}{l}\text { Study } \\
\text { Duration }\end{array}$ & $\begin{array}{l}\text { Funding } \\
\text { Source(s) }\end{array}$ & $\begin{array}{l}\text { Baseline } \\
\text { \% Rural }\end{array}$ & $\begin{array}{l}\text { Baseline } \\
\text { Age - Mean } \\
\text { (SD) or } \\
\text { Median } \\
\text { (Range) }\end{array}$ & $\begin{array}{l}\text { Baseline \% } \\
\text { Female }\end{array}$ & $\begin{array}{l}\text { Race/ } \\
\text { Ethnicity \% }\end{array}$ \\
\hline $\begin{array}{l}\text { Isetts et } \\
\text { al., } 2008^{16}\end{array}$ & $\begin{array}{l}\text { G1: MTM } \\
\text { services } \\
\text { provided by } \\
\text { staff } \\
\text { pharmacists, } \\
\text { including the } \\
\text { establishment } \\
\text { of goals of } \\
\text { therapy, in } \\
\text { collaboration } \\
\text { with primary } \\
\text { care providers. } \\
\text { G2: Usual } \\
\text { medical care } \\
\text { without MTM }\end{array}$ & $\begin{array}{l}\text { (1) To } \\
\text { provide MTM } \\
\text { services to } \\
\text { patients; (2) } \\
\text { to measure } \\
\text { clinical } \\
\text { effects } \\
\text { associated } \\
\text { with MTM, (3) } \\
\text { to measure } \\
\text { percent of } \\
\text { patients } \\
\text { achieving } \\
\text { goals for HTN } \\
\text { and } \\
\text { hyperlipidemi } \\
\text { a in MTM vs. } \\
\text { comparison; } \\
\text { and 4) to } \\
\text { compare } \\
\text { patients' total } \\
\text { health } \\
\text { expenditures } \\
\text { for the year } \\
\text { before and } \\
\text { after MTM }\end{array}$ & $\begin{array}{l}\text { Patients in } \\
\text { intervention } \\
\text { group: } 1 \text { ) Enrolled } \\
\text { in Blue Plus } \\
\text { insurance product } \\
\text { of Blue Cross } \\
\text { BlueShield of } \\
\text { Minnesota; (2) } \\
\text { Age } \geq 18 \text { years; } \\
\text { (3) Receiving } \\
\text { medical care at } \\
\text { one of } 6 \text { clinics in } \\
\text { Fairview, MN } \\
\text { where MTM } \\
\text { services provided; } \\
\text { (4) Diagnosed } \\
\text { with } \geq 1 \text { of } 12 \\
\text { study medical } \\
\text { conditions, (5) } \geq 2 \\
\text { health care claims } \\
\text { related to } 12 \\
\text { study conditions } \\
\text { in } 6 \text {-month period } \\
\text { before the start of } \\
\text { the study. }\end{array}$ & NR & Cohort & 12 & Academic & NR & $\begin{array}{l}\text { Overall: NR } \\
\text { G1: } 14 \% \\
\text { were age } 65 \\
\text { or older } \\
\text { G2: NR }\end{array}$ & $\begin{array}{l}\text { Overall: NR } \\
\text { G1: } 66 \\
\text { G2: NR }\end{array}$ & NR \\
\hline
\end{tabular}


Table E1. Study and patient-level characteristics (continued)

\begin{tabular}{|c|c|c|c|c|c|c|c|c|c|c|c|}
\hline $\begin{array}{l}\text { Author, } \\
\text { Year } \\
\text { Trial } \\
\text { Name }^{\text {a }}\end{array}$ & $\begin{array}{l}\text { Interventions } \\
\text { and } \\
\text { Comparator } \\
\text { Descriptions }\end{array}$ & $\begin{array}{l}\text { Intervention } \\
\text { Goal }\end{array}$ & Inclusion Criteria & $\begin{array}{l}\text { Exclusion } \\
\text { Criteria }\end{array}$ & $\begin{array}{l}\text { Study } \\
\text { Design }\end{array}$ & $\begin{array}{l}\text { Study } \\
\text { Duration }\end{array}$ & $\begin{array}{l}\text { Funding } \\
\text { Source(s) }\end{array}$ & $\begin{array}{l}\text { Baseline } \\
\% \text { Rural }\end{array}$ & $\begin{array}{l}\text { Baseline } \\
\text { Age - Mean } \\
\text { (SD) or } \\
\text { Median } \\
\text { (Range) }\end{array}$ & $\begin{array}{l}\text { Baseline \% } \\
\text { Female }\end{array}$ & $\begin{array}{l}\text { Race/ } \\
\text { Ethnicity \% }\end{array}$ \\
\hline $\begin{array}{l}\text { Jameson, } \\
\text { VanNoord, } \\
\text { and } \\
\text { Vanderwo } \\
\text { ud, } 1995^{17}\end{array}$ & $\begin{array}{l}\text { Pharmacothera } \\
\text { py consultation } \\
\text { and followup } \\
\text { provided by } \\
\text { clinical } \\
\text { ambulatory care } \\
\text { pharmacist. } \\
\text { G2: Standard } \\
\text { office-based } \\
\text { primary care. } \\
\end{array}$ & $\begin{array}{l}\text { To simplify the } \\
\text { pharmacologic } \\
\text { regimen, } \\
\text { improve } \\
\text { effectiveness } \\
\text { of the regimen, } \\
\text { and decrease } \\
\text { side effects. } \\
\text { Secondary } \\
\text { goal to } \\
\text { decrease cost } \\
\text { without } \\
\text { adversely } \\
\text { affecting the } \\
\text { first three } \\
\text { goals. } \\
\text { Improved } \\
\text { clinical } \\
\text { outcomes } \\
\text { (decreased } \\
\text { number of } \\
\text { medications, } \\
\text { decreased } \\
\text { number of } \\
\text { doses per day, } \\
\text { monthly cost } \\
\text { of } \\
\text { medications, } \\
\text { patient self- } \\
\text { reported } \\
\text { compliance, } \\
\text { drug regimen } \\
\text { convenience, } \\
\text { fewer side } \\
\text { effects and } \\
\text { problems). }\end{array}$ & $\begin{array}{l}\text { All patients at a } \\
\text { Family Health } \\
\text { Center seen } \\
\text { during a } 1 \text { year } \\
\text { period with } 2 \text { or } \\
\text { more risk factors: } \\
5 \text { or more } \\
\text { medications, } 12 \text { or } \\
\text { more daily doses, } \\
4 \text { or more } \\
\text { medication } \\
\text { changes in last } 12 \\
\text { months, more } \\
\text { than } 3 \text { disease } \\
\text { states, } \\
\text { documented } \\
\text { medication } \\
\text { noncompliance, } \\
\text { medications that } \\
\text { require } \\
\text { therapeutic } \\
\text { monitoring. }\end{array}$ & $\begin{array}{l}\text { Active } \\
\text { alcohol or } \\
\text { illicit drug } \\
\text { use, } \\
\text { unwilling or } \\
\text { unable to } \\
\text { return for a } \\
\text { pharmacot } \\
\text { herapy } \\
\text { consultatio } \\
\text { n, } \\
\text { medication } \\
\text { regimen } \\
\text { primarily } \\
\text { managed } \\
\text { by an } \\
\text { outside } \\
\text { provider, } \\
\text { terminally } \\
\text { ill, less } \\
\text { than } 18 \\
\text { years of } \\
\text { age. }\end{array}$ & $\begin{array}{l}\text { RCT: } \\
\text { parallel, } \\
\text { not } \\
\text { clustere } \\
\text { d }\end{array}$ & 6 months & $\begin{array}{l}\text { Multiple } \\
\text { (Foundation or } \\
\text { non-profit, } \\
\text { academic, and } \\
\text { pharmaceutical) }\end{array}$ & NR & $\begin{array}{l}\text { Overall: } 60.5 \\
\text { G1: NR } \\
\text { G2: NR }\end{array}$ & $\begin{array}{l}\text { Overall: } 80 \\
\text { G1: NR } \\
\text { G2: NR }\end{array}$ & $\begin{array}{l}\text { African } \\
\text { American } \\
\text { Overall: } 28 \\
\text { G1: NR } \\
\text { G2: NR }\end{array}$ \\
\hline
\end{tabular}


Table E1. Study and patient-level characteristics (continued)

\begin{tabular}{|c|c|c|c|c|c|c|c|c|c|c|c|}
\hline $\begin{array}{l}\text { Author, } \\
\text { Year } \\
\text { Trial } \\
\text { Name }^{a}\end{array}$ & $\begin{array}{l}\text { Interventions } \\
\text { and } \\
\text { Comparator } \\
\text { Descriptions }\end{array}$ & $\begin{array}{l}\text { Intervention } \\
\text { Goal }\end{array}$ & Inclusion Criteria & $\begin{array}{l}\text { Exclusion } \\
\text { Criteria }\end{array}$ & $\begin{array}{l}\text { Study } \\
\text { Design }\end{array}$ & $\begin{array}{l}\text { Study } \\
\text { Duration }\end{array}$ & $\begin{array}{l}\text { Funding } \\
\text { Source(s) }\end{array}$ & $\begin{array}{l}\text { Baseline } \\
\% \text { Rural }\end{array}$ & $\begin{array}{l}\text { Baseline } \\
\text { Age - Mean } \\
\text { (SD) or } \\
\text { Median } \\
\text { (Range) } \\
\end{array}$ & $\begin{array}{l}\text { Baseline \% } \\
\text { Female }\end{array}$ & $\begin{array}{l}\text { Race/ } \\
\text { Ethnicity \% }\end{array}$ \\
\hline $\begin{array}{l}\text { Jeong et } \\
\text { al., } 2007^{18}\end{array}$ & $\begin{array}{l}\text { G1: } \\
\text { Pharmacist- } \\
\text { managed } \\
\text { MTMP provided } \\
\text { by ambulatory } \\
\text { care } \\
\text { pharmacists } \\
\text { and healthcare } \\
\text { support staff } \\
\text { G2: Eligible for } \\
\text { Part D MTMP } \\
\text { but declined } \\
\text { enrollment } \\
\text { G3: Patients } \\
\text { without Part D } \\
\text { as their primary } \\
\text { drug benefit }\end{array}$ & NR & $\begin{array}{l}\text { Patients who: } \\
\text { 1) Were likely to } \\
\text { incur }>\$ 4,000 \text { in } \\
\text { drug costs per year } \\
\text { 2) Received } \geq 2 \\
\text { Part D medications } \\
\text { 3) Had } \geq 2 \text { chronic } \\
\text { conditions } \\
\text { 4) Had a diagnosis } \\
\text { of hyperlipidemia, } \\
\text { diabetes, or CAD } \\
\text { for LDL-C analysis } \\
\text { 5) Had a diagnosis } \\
\text { of diabetes for } \\
\text { HbA1c analysis } \\
6 \text { ) Had a lab (LDL- } \\
\mathrm{C} \text { or HbA1c) within } \\
6 \text { months before } \\
\text { and } 6 \text { months after } \\
\text { index date }\end{array}$ & $\begin{array}{l}\text { See } \\
\text { inclusion } \\
\text { criteria }\end{array}$ & Cohort & $\begin{array}{l}12 \\
\text { months }\end{array}$ & $\begin{array}{l}\text { Integrated } \\
\text { health care } \\
\text { system (Kaiser } \\
\text { Permanente) }\end{array}$ & NR & $\begin{array}{l}\text { Overall: NR } \\
\text { G1: } 72(10) \\
\text { G2: } 71(11) \\
\text { G3: } 74(7)\end{array}$ & $\begin{array}{l}\text { Overall: NR } \\
\text { G1: } 53 \\
\text { G2: } 55 \\
\text { G3: } 52\end{array}$ & NR \\
\hline $\begin{array}{l}\text { Krska et } \\
\text { al., } 2001^{19}\end{array}$ & $\begin{array}{l}\text { G1: Medication } \\
\text { reviews led by } \\
\text { clinically-trained } \\
\text { pharmacists. } \\
\text { G2: Usual care } \\
\text { involving } \\
\text { interviews and } \\
\text { identification of } \\
\text { pharmaceutical } \\
\text { care issues but } \\
\text { with no } \\
\text { pharmaceutical } \\
\text { care plan } \\
\text { implemented. }\end{array}$ & $\begin{array}{l}\text { To study the } \\
\text { effect of } \\
\text { medication } \\
\text { review led by } \\
\text { a pharmacist } \\
\text { on resolution } \\
\text { of } \\
\text { pharmaceutic } \\
\text { al care issues } \\
\text { medicine } \\
\text { costs, use of } \\
\text { health and } \\
\text { social } \\
\text { services and } \\
\text { health-related } \\
\text { quality of life }\end{array}$ & $\begin{array}{l}\text { Patients aged at } \\
\text { least } 65 \text { years with } \\
\text { at least two chronic } \\
\text { disease states, } \\
\text { taking at least } 4 \\
\text { prescribed } \\
\text { medicines regularly } \\
\end{array}$ & $\begin{array}{l}\text { Dementia, } \\
\text { being } \\
\text { considered } \\
\text { by the GP } \\
\text { to be } \\
\text { unable to } \\
\text { cope with } \\
\text { the study }\end{array}$ & $\begin{array}{l}\text { RCT: } \\
\text { parallel, } \\
\text { not } \\
\text { clustere } \\
\text { d }\end{array}$ & 3 months & Government & NR & $\begin{array}{l}\text { Overall: NR } \\
\text { G1: } 74.8(6.2) \\
\text { G2: } 75.2 \\
(6.6) \\
p=0.972\end{array}$ & $\begin{array}{l}\text { Overall: NR } \\
\text { G1: } 56.5 \\
\text { G2: } 64.6 \\
p=0.132\end{array}$ & NR \\
\hline
\end{tabular}


Table E1. Study and patient-level characteristics (continued)

\begin{tabular}{|c|c|c|c|c|c|c|c|c|c|c|c|}
\hline $\begin{array}{l}\text { Author, } \\
\text { Year } \\
\text { Trial } \\
\text { Name }^{a}\end{array}$ & $\begin{array}{l}\text { Interventions } \\
\text { and } \\
\text { Comparator } \\
\text { Descriptions }\end{array}$ & $\begin{array}{l}\text { Intervention } \\
\text { Goal }\end{array}$ & Inclusion Criteria & $\begin{array}{l}\text { Exclusion } \\
\text { Criteria }\end{array}$ & $\begin{array}{l}\text { Study } \\
\text { Design }\end{array}$ & $\begin{array}{l}\text { Study } \\
\text { Duration }\end{array}$ & $\begin{array}{l}\text { Funding } \\
\text { Source(s) }\end{array}$ & $\begin{array}{l}\text { Baseline } \\
\% \text { Rural }\end{array}$ & $\begin{array}{l}\text { Baseline } \\
\text { Age - Mean } \\
\text { (SD) or } \\
\text { Median } \\
\text { (Range) }\end{array}$ & $\begin{array}{l}\text { Baseline \% } \\
\text { Female }\end{array}$ & $\begin{array}{l}\text { Race/ } \\
\text { Ethnicity \% }\end{array}$ \\
\hline $\begin{array}{l}\text { Malone et } \\
\text { al., } 2000^{20} \text {; } \\
\text { Ellis et al., } \\
2000^{21} \text {; } \\
\text { Malone et } \\
\text { al., } 2001^{22} \text {; } \\
\text { Ellis et al., } \\
2000^{23} \\
\text { IMPROVE }\end{array}$ & $\begin{array}{l}\text { G1: } \\
\text { Pharmaceutical } \\
\text { care provided } \\
\text { by clinical } \\
\text { pharmacists } \\
\text { practicing } \\
\text { according to } \\
\text { scope of } \\
\text { practice within } \\
\text { their respective } \\
\text { health care } \\
\text { facilities. } \\
\text { G2: Usual care } \\
\text { without } \\
\text { pharmaceutical } \\
\text { care }\end{array}$ & $\begin{array}{l}\text { Determine if } \\
\text { clinical } \\
\text { pharmacists } \\
\text { could change } \\
\text { resource use } \\
\text { and } \\
\text { humanistic } \\
\text { outcomes } \\
\text { among } \\
\text { Veterans } \\
\text { identified at } \\
\text { high risk for } \\
\text { medication- } \\
\text { related } \\
\text { problems. }\end{array}$ & $\begin{array}{l}\text { 1) High risk for } \\
\text { drug-related } \\
\text { problems (were } \\
\text { taking } 5 \text { or more } \\
\text { drugs, } 12 \text { or more } \\
\text { doses/day, had } 3 \\
\text { or more chronic } \\
\text { medical conditions, } \\
4 \text { or more } \\
\text { changes in their } \\
\text { drug regimen over } \\
\text { the past year, } \\
\text { history of } \\
\text { nonadherence or } \\
\text { taking an agent } \\
\text { that required } \\
\text { therapeutic drug } \\
\text { monitoring); } 2 \text { ) } \\
\text { Received care at } \\
\text { the VA within the } \\
\text { past } 12 \text { months } \\
\text { and anticipated } \\
\text { continued VA care } \\
\text { for the duration of } \\
\text { the study; } 3 \text { ) Lived } \\
\text { close/had } \\
\text { transportation to } \\
\text { VA. }\end{array}$ & $\begin{array}{l}\text { 1) } \\
\text { Participatio } \\
\mathrm{n} \text { in a } \\
\text { pharmacist } \\
\text { managed } \\
\text { clinic within } \\
\text { previous 12 } \\
\text { months; 2) } \\
\text { Terminal } \\
\text { condition/p } \\
\text { oor life } \\
\text { expectancy } \\
\text {; 3) } \\
\text { Required } \\
\text { mental } \\
\text { health } \\
\text { services; 4) } \\
\text { Poor } \\
\text { spoken or } \\
\text { written } \\
\text { English; 5) } \\
\text { Visually } \\
\text { impaired. }\end{array}$ & $\begin{array}{l}\text { RCT: } \\
\text { parallel, } \\
\text { not } \\
\text { clustere } \\
\text { d } \\
\\
\end{array}$ & $\begin{array}{l}12 \\
\text { months }\end{array}$ & Pharmaceutical & $\begin{array}{l}\text { Overall: } \\
67(10.1) \\
\text { G1: } 66.8 \\
(10.2) \\
\text { G2: } 66.6 \\
(10.0)\end{array}$ & $\begin{array}{l}\text { Overall: NR } \\
\text { G1: } 3.6 \\
\text { G2: } 3.8\end{array}$ & NR & NR \\
\hline
\end{tabular}


Table E1. Study and patient-level characteristics (continued)

\begin{tabular}{|c|c|c|c|c|c|c|c|c|c|c|c|}
\hline $\begin{array}{l}\text { Author, } \\
\text { Year } \\
\text { Trial } \\
\text { Name }^{a}\end{array}$ & $\begin{array}{l}\text { Interventions } \\
\text { and } \\
\text { Comparator } \\
\text { Descriptions }\end{array}$ & $\begin{array}{l}\text { Intervention } \\
\text { Goal }\end{array}$ & Inclusion Criteria & $\begin{array}{l}\text { Exclusion } \\
\text { Criteria }\end{array}$ & $\begin{array}{l}\text { Study } \\
\text { Design }\end{array}$ & $\begin{array}{l}\text { Study } \\
\text { Duration }\end{array}$ & $\begin{array}{l}\text { Funding } \\
\text { Source(s) }\end{array}$ & $\begin{array}{l}\text { Baseline } \\
\% \text { Rural }\end{array}$ & $\begin{array}{l}\text { Baseline } \\
\text { Age - Mean } \\
\text { (SD) or } \\
\text { Median } \\
\text { (Range) }\end{array}$ & $\begin{array}{l}\text { Baseline \% } \\
\text { Female }\end{array}$ & $\begin{array}{l}\text { Race/ } \\
\text { Ethnicity \% }\end{array}$ \\
\hline $\begin{array}{l}\text { McDonoug } \\
\text { h et al., } \\
2005^{24}\end{array}$ & $\begin{array}{l}\text { G1: } \\
\text { Pharmaceutical } \\
\text { care provided } \\
\text { by community } \\
\text { pharmacists. } \\
\text { Drug therapy } \\
\text { monitoring } \\
\text { focused on } 5 \\
\text { drug therapy } \\
\text { problems: } \\
\text { appropriateness } \\
\text { of does, proper } \\
\text { regimen, } \\
\text { potential } \\
\text { interactions, } \\
\text { nonadherence, } \\
\text { and adverse } \\
\text { effects. Patient } \\
\text { education also } \\
\text { provided. } \\
\text { G2: Usual care }\end{array}$ & $\begin{array}{l}\text { To reduce } \\
\text { risk of } \\
\text { glucocorticoid } \\
\text { osteoporosis. }\end{array}$ & $\begin{array}{l}\text { Patients } 18 \text { years } \\
\text { of age or older who } \\
\text { had been on the } \\
\text { equivalent of at } \\
\text { least } 7.5 \mathrm{mg} \text { of } \\
\text { prednisone for at } \\
\text { least } 6 \text { months }\end{array}$ & NR & $\begin{array}{l}\mathrm{RCT}: \\
\text { cluster- } \\
\text { randomi } \\
\text { zed }\end{array}$ & 9 months & $\begin{array}{l}\text { Multiple } \\
\text { (Pharmaceutica } \\
\text { I company and } \\
\text { academic) }\end{array}$ & NR & NR & $\begin{array}{l}\text { Overall: NR } \\
\text { G1: } 57.7 \\
\text { G2: } 74.3\end{array}$ & $\begin{array}{l}\text { Caucasian } \\
\text { or Asian } \\
\text { Overall: NR } \\
\text { G1: } 92.3 \\
\text { G2: } 84.3\end{array}$ \\
\hline
\end{tabular}


Table E1. Study and patient-level characteristics (continued)

\begin{tabular}{|c|c|c|c|c|c|c|c|c|c|c|c|}
\hline $\begin{array}{l}\text { Author, } \\
\text { Year } \\
\text { Trial } \\
\text { Name }^{a}\end{array}$ & $\begin{array}{l}\text { Interventions } \\
\text { and } \\
\text { Comparator } \\
\text { Descriptions }\end{array}$ & $\begin{array}{l}\text { Intervention } \\
\text { Goal }\end{array}$ & Inclusion Criteria & $\begin{array}{l}\text { Exclusion } \\
\text { Criteria }\end{array}$ & $\begin{array}{l}\text { Study } \\
\text { Design }\end{array}$ & $\begin{array}{l}\text { Study } \\
\text { Duration }\end{array}$ & $\begin{array}{l}\text { Funding } \\
\text { Source(s) }\end{array}$ & $\begin{array}{l}\text { Baseline } \\
\% \text { Rural }\end{array}$ & $\begin{array}{l}\text { Baseline } \\
\text { Age - Mean } \\
\text { (SD) or } \\
\text { Median } \\
\text { (Range) } \\
\end{array}$ & $\begin{array}{l}\text { Baseline \% } \\
\text { Female }\end{array}$ & $\begin{array}{l}\text { Race/ } \\
\text { Ethnicity \% }\end{array}$ \\
\hline $\begin{array}{l}\text { Moczygem } \\
\text { ba et al., } \\
2011^{25} \text {; } \\
\text { Moczygem } \\
\text { ba et al., } \\
2008^{26}\end{array}$ & $\begin{array}{l}\text { G1: Opt-in } \\
\text { telephone- } \\
\text { based MTM } \\
\text { program, in } \\
\text { which MTM } \\
\text { services } \\
\text { provided by } \\
\text { clinical } \\
\text { pharmacists or } \\
\text { a managed } \\
\text { care pharmacy } \\
\text { resident based } \\
\text { on the } \\
\text { American } \\
\text { Pharmacists } \\
\text { Association and } \\
\text { National } \\
\text { Association of } \\
\text { Chain Drug } \\
\text { Stores } \\
\text { Foundation } \\
\text { MTM } \\
\text { framework. } \\
\text { G2: No-MTM } \\
\text { control group }\end{array}$ & $\begin{array}{l}\text { To identify } \\
\text { and resolve } \\
\text { medication } \\
\text { and health- } \\
\text { related } \\
\text { problems }\end{array}$ & $\begin{array}{l}\text { Medicare Part } D \\
\text { beneficiaries of the } \\
\text { Scott } \& \text { White } \\
\text { Health Plan with: } \\
\text { 1) } \geq 2 \text { chronic } \\
\text { diseases } \\
\text { 2) } \geq 2 \text { Part } D \text { drugs } \\
\text { 3) } \geq \$ 4000 \text { in Part } D \\
\text { drug costs } \\
\text { 4) Received } \geq 1 \\
\text { MTM consultation }\end{array}$ & $\begin{array}{l}\text { Patients } \\
\geq 90 \text { years } \\
\text { of age due } \\
\text { to patient } \\
\text { privacy } \\
\text { concerns } \\
\end{array}$ & Cohort & 9 & $\begin{array}{l}\text { Foundation or } \\
\text { non-profit }\end{array}$ & NR & $\begin{array}{l}\text { Mean (SD) } \\
\text { Overall: NR } \\
\text { G1: 71.2 (7.5) } \\
\text { (range: } 53- \\
\text { 86) } \\
\text { G2: } 73.9(8.0) \\
\text { (range: } 46- \\
88) \\
\text { p: } 0.06\end{array}$ & $\begin{array}{l}\text { Overall: NR } \\
\text { G1: } 48.3 \\
\text { G2: } 71.7 \\
\text { p: } 0.009\end{array}$ & $\begin{array}{l}\text { White } \\
\text { Overall: NR } \\
\text { G1: } 78.3 \\
\text { G2: } 91.7 \\
\text { p: } 0.29\end{array}$ \\
\hline
\end{tabular}


Table E1. Study and patient-level characteristics (continued)

\begin{tabular}{|c|c|c|c|c|c|c|c|c|c|c|c|}
\hline $\begin{array}{l}\text { Author, } \\
\text { Year } \\
\text { Trial } \\
\text { Name }^{a}\end{array}$ & $\begin{array}{l}\text { Interventions } \\
\text { and } \\
\text { Comparator } \\
\text { Descriptions }\end{array}$ & $\begin{array}{l}\text { Intervention } \\
\text { Goal }\end{array}$ & Inclusion Criteria & $\begin{array}{l}\text { Exclusion } \\
\text { Criteria }\end{array}$ & $\begin{array}{l}\text { Study } \\
\text { Design }\end{array}$ & $\begin{array}{l}\text { Study } \\
\text { Duration }\end{array}$ & $\begin{array}{l}\text { Funding } \\
\text { Source(s) }\end{array}$ & $\begin{array}{l}\text { Baseline } \\
\% \text { Rural }\end{array}$ & $\begin{array}{l}\text { Baseline } \\
\text { Age - Mean } \\
\text { (SD) or } \\
\text { Median } \\
\text { (Range) } \\
\end{array}$ & $\begin{array}{l}\text { Baseline \% } \\
\text { Female }\end{array}$ & $\begin{array}{l}\text { Race/ } \\
\text { Ethnicity \% }\end{array}$ \\
\hline $\begin{array}{l}\text { Pai et al., } \\
2009^{27} \text {; } \\
\text { Pai et al., } \\
2009^{28}\end{array}$ & $\begin{array}{l}\text { G1: } \\
\text { Pharmaceutical } \\
\text { care including } \\
\text { drug therapy } \\
\text { reviews } \\
\text { conducted by a } \\
\text { nephrology- } \\
\text { trained clinical } \\
\text { pharmacist with } \\
\text { the patient. Also } \\
\text { included patient } \\
\text { and health care } \\
\text { provider } \\
\text { education. } \\
\text { G2: Standard of } \\
\text { care, consisting } \\
\text { of brief therapy } \\
\text { reviews } \\
\text { conducted by a } \\
\text { nurse }\end{array}$ & $\begin{array}{l}\text { To } \\
\text { investigate } \\
\text { the impact of } \\
\text { a } \\
\text { pharmaceutic } \\
\text { al care } \\
\text { program } \\
\text { managed by } \\
\text { clinical } \\
\text { pharmacists } \\
\text { on drug use, } \\
\text { drug costs, } \\
\text { hospitalizatio } \\
n \text { rates, and } \\
\text { drug-related } \\
\text { problems } \\
\text { (DRPs) in } \\
\text { ambulatory } \\
\text { patients } \\
\text { undergoing } \\
\text { hemodialysis. }\end{array}$ & $\begin{array}{l}\text { To participate in } \\
\text { the study, patients } \\
\text { had to speak } \\
\text { English, and be } \\
\text { older than } 18 \text { years } \\
\text { and undergoing a } \\
\text { stable } \\
\text { hemodialysis } \\
\text { regimen for at least } \\
3 \text { months. Informed } \\
\text { consent was } \\
\text { obtained from each } \\
\text { patient before } \\
\text { starting the study, } \\
\text { with a consent rate } \\
\text { of } 70 \% \text {. }\end{array}$ & $\begin{array}{l}\text { If patients } \\
\text { elected not } \\
\text { to consent } \\
\text { or were } \\
\text { unable to } \\
\text { provide } \\
\text { informed } \\
\text { consent, } \\
\text { they } \\
\text { continued } \\
\text { to receive } \\
\text { the care } \\
\text { that their } \\
\text { shift was } \\
\text { assigned; } \\
\text { however, } \\
\text { no data } \\
\text { from these } \\
\text { patients } \\
\text { were } \\
\text { collected or } \\
\text { analyzed. }\end{array}$ & $\begin{array}{l}\text { RCT: } \\
\text { cluster- } \\
\text { randomi } \\
\text { zed }\end{array}$ & 2 years & $\begin{array}{l}\text { Foundation or } \\
\text { non-profit }\end{array}$ & NR & $\begin{array}{l}\text { Overall: } 59.0 \\
(15.0) \\
\text { G1: } 56.3(15) \\
\text { G2: } 60.5 \\
(14.7)\end{array}$ & $\begin{array}{l}\text { Overall: } \\
48.1 \% \\
\text { G1: } 38.6 \% \\
\text { G2: } 59.6 \%\end{array}$ & $\begin{array}{l}\text { Caucasian } \\
\text { Overall: } \\
\text { 27.9\% } \\
\text { G1: } 22.8 \% \\
\text { G2: } 34.0 \% \\
\text { Hispanic } \\
\text { Overall: } \\
\text { 30.8\% } \\
\text { G1: } 29.8 \% \\
\text { G2: } 31.9 \% \\
\text { Native } \\
\text { American } \\
\text { Overall: } 17.3 \\
\text { G1: } 22.8 \% \\
\text { G2: } 10.6 \% \\
\text { Other } \\
\text { Overall: } \\
\text { 24.0\% } \\
\text { G1: } 24.6 \% \\
\text { G2: } 23.4 \%\end{array}$ \\
\hline $\begin{array}{l}\text { Park et al. } \\
1996^{29}\end{array}$ & $\begin{array}{l}\text { G1: } \\
\text { Comprehensive } \\
\text { pharmaceutical } \\
\text { services } \\
\text { including drug } \\
\text { therapy } \\
\text { monitoring and } \\
\text { patient } \\
\text { education } \\
\text { provided by a } \\
\text { community } \\
\text { pharmacy } \\
\text { resident. } \\
\text { G2: Usual care }\end{array}$ & $\begin{array}{l}\text { Improve } \\
\text { blood } \\
\text { pressure and } \\
\text { quality of life } \\
\text { for patients } \\
\text { with HTN. }\end{array}$ & $\begin{array}{l}\text { Patients with HTN } \\
\text { either currently } \\
\text { taking anti- } \\
\text { hypertensive } \\
\text { medication or with } \\
\text { a BP }>140 / 90 \text {. }\end{array}$ & $\begin{array}{l}\text { Bedridden; } \\
\text { non- } \\
\text { English } \\
\text { speaking; } \\
\text { had } \\
\text { another } \\
\text { family } \\
\text { member } \\
\text { enrolled in } \\
\text { the study. }\end{array}$ & $\begin{array}{l}\text { RCT: } \\
\text { parallel, } \\
\text { not } \\
\text { clustere } \\
\text { d }\end{array}$ & 4 months & Unspecified & NR & $\begin{array}{l}\text { Overall: NR } \\
\text { G1: } 57.3 \\
\text { (range 29-82) } \\
\text { G2: 63.0 } \\
\text { (Range 23- } \\
\text { 88) }\end{array}$ & $\begin{array}{l}\text { Overall: NR } \\
\text { G1: } 44 \\
\text { G2: } 41\end{array}$ & $\begin{array}{l}\text { \% white } \\
\text { G1: } 81 \\
\text { G2: } 69\end{array}$ \\
\hline
\end{tabular}


Table E1. Study and patient-level characteristics (continued)

\begin{tabular}{|c|c|c|c|c|c|c|c|c|c|c|c|}
\hline $\begin{array}{l}\text { Author, } \\
\text { Year } \\
\text { Trial } \\
\text { Name }^{\text {a }}\end{array}$ & $\begin{array}{l}\text { Interventions } \\
\text { and } \\
\text { Comparator } \\
\text { Descriptions }\end{array}$ & $\begin{array}{l}\text { Intervention } \\
\text { Goal }\end{array}$ & Inclusion Criteria & $\begin{array}{l}\text { Exclusion } \\
\text { Criteria }\end{array}$ & $\begin{array}{l}\text { Study } \\
\text { Design }\end{array}$ & $\begin{array}{l}\text { Study } \\
\text { Duration }\end{array}$ & $\begin{array}{l}\text { Funding } \\
\text { Source(s) }\end{array}$ & $\begin{array}{l}\text { Baseline } \\
\text { \% Rural }\end{array}$ & $\begin{array}{l}\text { Baseline } \\
\text { Age - Mean } \\
\text { (SD) or } \\
\text { Median } \\
\text { (Range) }\end{array}$ & $\begin{array}{l}\text { Baseline \% } \\
\text { Female }\end{array}$ & $\begin{array}{l}\text { Race/ } \\
\text { Ethnicity \% }\end{array}$ \\
\hline $\begin{array}{l}\text { Pindolia et } \\
\text { al., } 2009^{30}\end{array}$ & $\begin{array}{l}\text { G1: Telephone- } \\
\text { based MTM } \\
\text { services } \\
\text { provided as part } \\
\text { of a Medicare } \\
\text { Part D MTM } \\
\text { program by } \\
\text { pharmacy care } \\
\text { management } \\
\text { clinical } \\
\text { pharmacists } \\
\text { (acceptors). } \\
\text { G2: Usual } \\
\text { medical care } \\
\text { (refusers) } \\
\end{array}$ & $\begin{array}{l}\text { To 1) ensure } \\
\text { that safest, } \\
\text { most } \\
\text { efficacious, } \\
\text { and cost- } \\
\text { effective drug } \\
\text { therapy is } \\
\text { provided by } \\
\text { collaborating } \\
\text { with } \\
\text { physicians } \\
\text { and } \\
\text { patients/care } \\
\text { givers in the } \\
\text { development } \\
\text { of an optimal } \\
\text { drug regimen } \\
\text { that meets } \\
\text { both medical } \\
\text { and patient } \\
\text { needs; (2) } \\
\text { educate } \\
\text { patients on all } \\
\text { aspects of } \\
\text { their drug } \\
\text { therapy; and } \\
\text { (3) improve } \\
\text { adherence to } \\
\text { drug therapy } \\
\text { regimens }\end{array}$ & $\begin{array}{l}\text { In 2006: } \\
\text { 1) Diagnosed with } \\
2 \text { of } 26 \text { selected } \\
\text { chronic diseases; } \\
\text { 2) Filled } \geq 2 \\
\text { prescriptions as } \\
\text { identified by } \\
\text { pharmacy claims } \\
\text { data; } \\
\text { 3) Likely to incur } \\
\text { annual costs of } \\
\geq \$ 4000 \text { for all } \\
\text { Medicare Part D- } \\
\text { covered } \\
\text { medications based } \\
\text { on quarterly } \\
\text { prescription drug } \\
\text { expenditures of } \\
\$ 1000 \\
\text { In } 2007 \text { : } \\
\text { 1) Diagnosed with } \\
3 \text { of } 21 \text { selected } \\
\text { I chronic diseases; } \\
2 \text { ) Filled } \geq 4 \\
\text { prescriptions as } \\
\text { identified by } \\
\text { pharmacy claims } \\
\text { data; } \\
3 \text { ) Likely to incur } \\
\text { annual costs of } \\
\geq \$ 4000 \text { for all } \\
\text { Medicare Part D- } \\
\text { covered } \\
\text { medications based } \\
\text { on monthly } \\
\text { prescription drug } \\
\text { expenditures of } \\
\$ 334\end{array}$ & NR & Cohort & 2 years & Unspecified & NR & $\begin{array}{l}2006 \\
\text { Mean (SD) } \\
\text { [range] } \\
\text { Overall: NR } \\
\text { G1: } 73.5(9.7) \\
{[42-92]} \\
\text { G2: } 74.2(9.8) \\
{[32-96]} \\
\text { p: } 0.229 \\
2007 \\
\text { Mean (SD) } \\
\text { [range] } \\
\text { Overall: NR } \\
\text { G1: } 73.0(9.1) \\
\text { [39-93] } \\
\text { G2: } 73.9(9.8) \\
\text { [33-98] } \\
\text { p: } 0.168\end{array}$ & $\begin{array}{l}\text { 2006 } \\
\text { Overall: NR } \\
\text { G1: } 64 \\
\text { G2: } 60 \\
\text { p: } 0.175 \\
\text { 2007 } \\
\text { Overall: NR } \\
\text { G1: } 54 \\
\text { G2: } 63 \\
\text { p: } 0.01\end{array}$ & NR \\
\hline
\end{tabular}


Table E1. Study and patient-level characteristics (continued)

\begin{tabular}{|c|c|c|c|c|c|c|c|c|c|c|c|}
\hline $\begin{array}{l}\text { Author, } \\
\text { Year } \\
\text { Trial } \\
\text { Name }^{a}\end{array}$ & $\begin{array}{l}\text { Interventions } \\
\text { and } \\
\text { Comparator } \\
\text { Descriptions }\end{array}$ & $\begin{array}{l}\text { Intervention } \\
\text { Goal }\end{array}$ & Inclusion Criteria & $\begin{array}{l}\text { Exclusion } \\
\text { Criteria }\end{array}$ & $\begin{array}{l}\text { Study } \\
\text { Design }\end{array}$ & $\begin{array}{l}\text { Study } \\
\text { Duration }\end{array}$ & $\begin{array}{l}\text { Funding } \\
\text { Source(s) }\end{array}$ & $\begin{array}{l}\text { Baseline } \\
\% \text { Rural }\end{array}$ & $\begin{array}{l}\text { Baseline } \\
\text { Age - Mean } \\
\text { (SD) or } \\
\text { Median } \\
\text { (Range) }\end{array}$ & $\begin{array}{l}\text { Baseline \% } \\
\text { Female }\end{array}$ & $\begin{array}{l}\text { Race/ } \\
\text { Ethnicity \% }\end{array}$ \\
\hline $\begin{array}{l}\text { Planas et } \\
\text { al., } 2009^{31}\end{array}$ & $\begin{array}{l}\text { G1: MTM } \\
\text { services } \\
\text { provided by } \\
\text { community } \\
\text { pharmacists. } \\
\text { Also included } \\
\text { patient } \\
\text { education on } \\
\text { diet and lifestyle } \\
\text { modifications to } \\
\text { lower blood } \\
\text { pressure. } \\
\text { G2: No MTM } \\
\text { received, but } \\
\text { only informed of } \\
\text { blood pressure } \\
\text { goals for } \\
\text { patients with } \\
\text { diabetes }\end{array}$ & $\begin{array}{l}\text { To improve } \\
\text { BP and } \\
\text { antihypertens } \\
\text { ive } \\
\text { medication } \\
\text { adherence in } \\
\text { patients with } \\
\text { both diabetes } \\
\text { and HTN }\end{array}$ & $\begin{array}{l}\text { Members of } \\
\text { managed care } \\
\text { organization } \\
\text { (MCO) already } \\
\text { enrolled in } \\
\text { concurrent study of } \\
\text { community } \\
\text { pharmacy-based } \\
\text { diabetes } \\
\text { management } \\
\text { program. } \\
\text { Criteria for } \\
\text { diabetes study: } \\
\text { (1) Lack of } \\
\text { diabetes control } \\
\text { (i.e., most recent } \\
\text { A1C within last } 6 \\
\text { months }>7.0 \% \text { ) } \\
\text { (2) } \geq 18 \text { years old } \\
\text { (3) Currently } \\
\text { insured by MCO } \\
\text { (4) Able and willing } \\
\text { to come to periodic } \\
\text { visits during a } 9- \\
\text { month period } \\
\text { Criteria for HTN } \\
\text { study: } \\
\text { (1) Present at } \\
\text { baseline diabetes } \\
\text { study visit } \\
\text { (2) BP } \geq 130 / 80 \text { mm } \\
\text { Hg or currently } \\
\text { taking } \\
\text { antihypertensive } \\
\text { therapy }\end{array}$ & $\begin{array}{l}\text { (1) } \\
\text { Pregnant; } \\
\text { (2) } \\
\text { Currently } \\
\text { enrolled in } \\
\text { another } \\
\text { diabetes } \\
\text { program } \\
\end{array}$ & $\begin{array}{l}\text { RCT: } \\
\text { parallel, } \\
\text { not } \\
\text { clustere } \\
\text { d }\end{array}$ & 9 months & $\begin{array}{l}\text { Multiple } \\
\text { (Foundation } \\
\text { and pharmacy } \\
\text { chain) }\end{array}$ & NR & $\begin{array}{l}\text { Overall: NR } \\
\text { G1: } 64.2 \\
(10.5) \\
\text { G2: } 65.2 \\
(14.1)\end{array}$ & $\begin{array}{l}\text { Overall: NR } \\
\text { G1: } 65.6 \\
\text { G2: } 60.0\end{array}$ & $\begin{array}{l}\text { White } \\
\text { Overall: NR } \\
\text { G1: } 75.0 \\
\text { G2: } 90.0 \\
\text { Black } \\
\text { Overall: NR } \\
\text { G1: } 21.9 \\
\text { G2: } 10.0 \\
\text { Hispanic } \\
\text { Overall: NR } \\
\text { G1: } 3.1 \\
\text { G2: } 0\end{array}$ \\
\hline
\end{tabular}


Table E1. Study and patient-level characteristics (continued)

\begin{tabular}{|c|c|c|c|c|c|c|c|c|c|c|c|}
\hline $\begin{array}{l}\text { Author, } \\
\text { Year } \\
\text { Trial } \\
\text { Name }^{a}\end{array}$ & $\begin{array}{l}\text { Interventions } \\
\text { and } \\
\text { Comparator } \\
\text { Descriptions }\end{array}$ & $\begin{array}{l}\text { Intervention } \\
\text { Goal }\end{array}$ & Inclusion Criteria & $\begin{array}{l}\text { Exclusion } \\
\text { Criteria }\end{array}$ & $\begin{array}{l}\text { Study } \\
\text { Design }\end{array}$ & $\begin{array}{l}\text { Study } \\
\text { Duration }\end{array}$ & $\begin{array}{l}\text { Funding } \\
\text { Source(s) }\end{array}$ & $\begin{array}{l}\text { Baseline } \\
\% \text { Rural }\end{array}$ & $\begin{array}{l}\text { Baseline } \\
\text { Age - Mean } \\
\text { (SD) or } \\
\text { Median } \\
\text { (Range) }\end{array}$ & $\begin{array}{l}\text { Baseline \% } \\
\text { Female }\end{array}$ & $\begin{array}{l}\text { Race/ } \\
\text { Ethnicity \% }\end{array}$ \\
\hline $\begin{array}{l}\text { Roughead } \\
\text { et al., } \\
2009^{32}\end{array}$ & $\begin{array}{l}\text { G1: Home } \\
\text { Medication } \\
\text { Reviews } \\
\text { (HMR), a } \\
\text { collaborative } \\
\text { model of } \\
\text { pharmaceutical } \\
\text { care, conducted } \\
\text { by accredited } \\
\text { pharmacists. } \\
\text { G2: No } \\
\text { medication } \\
\text { review received }\end{array}$ & $\begin{array}{l}\text { To reduce } \\
\text { time to next } \\
\text { hospitalizatio } \\
\text { n for heart } \\
\text { failure (HF) } \\
\text { among } \\
\text { Australian } \\
\text { war veterans } \\
\text { and war } \\
\text { widows with } \\
\text { HF }\end{array}$ & $\begin{array}{l}\text { Community- } \\
\text { dwelling elderly } \\
\text { who: } \\
\text { 1) Had all health } \\
\text { services fully } \\
\text { subsidized by } \\
\text { Australian } \\
\text { Government's } \\
\text { Department of } \\
\text { Veterans' Affairs } \\
\text { (DVA); } \\
\text { 2) Were dispensed } \\
\text { beta-blocker } \\
\text { subsidized for HF } \\
\text { in } 6 \text { months before } \\
\text { the HMR; } \\
\text { 3) Were aged } \geq 65 \\
\text { years at the time of } \\
\text { home review }\end{array}$ & $\begin{array}{l}\text { 1) } \\
\text { Residents } \\
\text { in aged- } \\
\text { care } \\
\text { facilities }\end{array}$ & Cohort & $\begin{array}{l}40 \\
\text { months }\end{array}$ & Government & $\begin{array}{l}\text { Region of } \\
\text { residence } \\
\text { Remote } \\
\text { Overall: NR } \\
\text { G1: } 0 \\
\text { G2: } 1 \\
\text { Outer } \\
\text { regional } \\
\text { Overall: NR } \\
\text { G1: } 12 \\
\text { G2: } 9 \\
\text { Inner } \\
\text { regional } \\
\text { Overall: NR } \\
\text { G1: } 29 \\
\text { G2: } 31\end{array}$ & $\begin{array}{l}\text { Median (SD) } \\
\text { Overall: } \\
\text { G1: } 81.6(4.8) \\
\text { G2: } 81.6(4.8) \\
\end{array}$ & 更 & NR \\
\hline $\begin{array}{l}\text { Sellors et } \\
\text { al., } 2003^{33}\end{array}$ & $\begin{array}{l}\text { G1: Clinical } \\
\text { pharmacist } \\
\text { consultations } \\
\text { provided to } \\
\text { family } \\
\text { physicians and } \\
\text { their patients by } \\
\text { community } \\
\text { pharmacists. } \\
\text { G2: Usual care } \\
\text { for family } \\
\text { physicians and } \\
\text { their patients } \\
\text { from matched } \\
\text { postal codes }\end{array}$ & $\begin{array}{l}\text { Reducing } \\
\text { regimen } \\
\text { complexity } \\
\text { and } \\
\text { improving } \\
\text { patient } \\
\text { outcomes }\end{array}$ & $\begin{array}{l}\text { 1) Community } \\
\text { dwelling; } \\
\text { 2) } 65 \text { years or } \\
\text { older; 3) taking } 5 \text { or } \\
\text { more medications; } \\
\text { 4) had been seen } \\
\text { by their physician } \\
\text { within the past } 12 \\
\text { months; } 5 \text { ) had no } \\
\text { evidence of } \\
\text { cognitive } \\
\text { impairment; and } 6 \text { ) } \\
\text { could understand } \\
\text { English }\end{array}$ & $\begin{array}{l}\text { 1) Had } \\
\text { planned } \\
\text { surgery, 2) } \\
\text { were on a } \\
\text { nursing } \\
\text { home } \\
\text { waiting } \\
\text { list or 3) } \\
\text { were } \\
\text { receiving } \\
\text { palliative } \\
\text { care }\end{array}$ & $\begin{array}{l}\text { RCT: } \\
\text { cluster- } \\
\text { randomi } \\
\text { zed }\end{array}$ & 3 months & $\begin{array}{l}\text { Multiple } \\
\text { (Government } \\
\text { and hospital) }\end{array}$ & NR & $\begin{array}{l}\text { Overall: NR } \\
\text { G1: } 74.0(6.1) \\
\text { G2: } 74.0(6.0)\end{array}$ & $\begin{array}{l}\text { Overall: NR } \\
\text { G1: } 277 \\
(64.3) \\
\text { G2: } 281 \\
(61.4)\end{array}$ & NR \\
\hline
\end{tabular}


Table E1. Study and patient-level characteristics (continued)

\begin{tabular}{|c|c|c|c|c|c|c|c|c|c|c|c|}
\hline $\begin{array}{l}\text { Author, } \\
\text { Year } \\
\text { Trial } \\
\text { Name }^{a}\end{array}$ & $\begin{array}{l}\text { Interventions } \\
\text { and } \\
\text { Comparator } \\
\text { Descriptions }\end{array}$ & $\begin{array}{l}\text { Intervention } \\
\text { Goal }\end{array}$ & Inclusion Criteria & $\begin{array}{l}\text { Exclusion } \\
\text { Criteria }\end{array}$ & $\begin{array}{l}\text { Study } \\
\text { Design }\end{array}$ & $\begin{array}{l}\text { Study } \\
\text { Duration }\end{array}$ & $\begin{array}{l}\text { Funding } \\
\text { Source(s) }\end{array}$ & $\begin{array}{l}\text { Baseline } \\
\% \text { Rural }\end{array}$ & $\begin{array}{l}\text { Baseline } \\
\text { Age - Mean } \\
\text { (SD) or } \\
\text { Median } \\
\text { (Range) }\end{array}$ & $\begin{array}{l}\text { Baseline \% } \\
\text { Female }\end{array}$ & $\begin{array}{l}\text { Race/ } \\
\text { Ethnicity \% }\end{array}$ \\
\hline $\begin{array}{l}\text { Sidel et } \\
\text { al., } 1990^{34}\end{array}$ & $\begin{array}{l}\text { G1: Home visits } \\
\text { by pharmacists } \\
\text { and, when } \\
\text { needed, } \\
\text { consultations } \\
\text { with physicians } \\
\text { to identify and } \\
\text { correct } \\
\text { problems } \\
\text { associated with } \\
\text { medication use. } \\
\text { G2: Standard } \\
\text { care without } \\
\text { any visits or } \\
\text { information } \\
\text { provided to G1. }\end{array}$ & $\begin{array}{l}\text { To determine } \\
\text { the } \\
\text { prevalence of } \\
\text { use of } \\
\text { prescription } \\
\text { and OTC } \\
\text { medications } \\
\text { and home } \\
\text { remedies, to } \\
\text { characterize } \\
\text { medication } \\
\text { taking } \\
\text { behaviors } \\
\text { and } \\
\text { practices, } \\
\text { and to assess } \\
\text { the impact of } \\
\text { in-home } \\
\text { pharmacist } \\
\text { intervention } \\
\text { in identifying } \\
\text { and } \\
\text { correcting } \\
\text { problems with } \\
\text { medication } \\
\text { use }\end{array}$ & $\begin{array}{l}\text { (1) All Medicare } \\
\text { recipients } 65 \text { years } \\
\text { or older living in the } \\
\text { Norwood area } \\
\text { eligible; (2) } \\
\text { Patients who were } \\
\text { considered "high } \\
\text { risk" by baseline } \\
\text { RAP questionnaire } \\
\end{array}$ & $\begin{array}{l}\text { (1) Patients } \\
\text { considered } \\
\text { reluctant or } \\
\text { difficult; (2) } \\
\text { Those who } \\
\text { died or } \\
\text { moved } \\
\text { during } \\
\text { identificatio } \\
\mathrm{n} \text { and } \\
\text { assignment }\end{array}$ & $\begin{array}{l}\text { RCT: } \\
\text { parallel, } \\
\text { not } \\
\text { clustere } \\
\text { d }\end{array}$ & $\begin{array}{l}\text { 6-11 } \\
\text { months }\end{array}$ & Government & Overall: 0 & $\begin{array}{l}\text { 65-74 years } \\
\text { G1: } 48.4 \% \\
\text { G2: } 48.1 \% \\
75-84 \text { years } \\
\text { G1: } 38.5 \% \\
\text { G2: } 41.4 \% \\
85 \text { years and } \\
\text { older } \\
\text { G1: } 13.2 \% \\
\text { G2: } 10.6 \%\end{array}$ & $\begin{array}{l}\text { Overall: NR } \\
\text { G1: } 76.9 \\
\text { G2: } 77.9\end{array}$ & $\begin{array}{l}\text { Non-White } \\
\text { G1: } 7.7 \\
\text { G2: } 6.7 \\
\text { Hispanic } \\
\text { G1: } 4.4 \\
\text { G2: } 7.7\end{array}$ \\
\hline
\end{tabular}


Table E1. Study and patient-level characteristics (continued)

\begin{tabular}{|c|c|c|c|c|c|c|c|c|c|c|c|}
\hline $\begin{array}{l}\text { Author, } \\
\text { Year } \\
\text { Trial } \\
\text { Name }^{a}\end{array}$ & $\begin{array}{l}\text { Interventions } \\
\text { and } \\
\text { Comparator } \\
\text { Descriptions }\end{array}$ & $\begin{array}{l}\text { Intervention } \\
\text { Goal }\end{array}$ & Inclusion Criteria & $\begin{array}{l}\text { Exclusion } \\
\text { Criteria }\end{array}$ & $\begin{array}{l}\text { Study } \\
\text { Design }\end{array}$ & $\begin{array}{l}\text { Study } \\
\text { Duration }\end{array}$ & $\begin{array}{l}\text { Funding } \\
\text { Source(s) }\end{array}$ & $\begin{array}{l}\text { Baseline } \\
\% \text { Rural }\end{array}$ & $\begin{array}{l}\text { Baseline } \\
\text { Age - Mean } \\
\text { (SD) or } \\
\text { Median } \\
\text { (Range) }\end{array}$ & $\begin{array}{l}\text { Baseline \% } \\
\text { Female }\end{array}$ & $\begin{array}{l}\text { Race/ } \\
\text { Ethnicity \% }\end{array}$ \\
\hline $\begin{array}{l}\text { Staresinic } \\
\text { et al., } \\
2007^{35}\end{array}$ & $\begin{array}{l}\text { G1: MTM } \\
\text { services } \\
\text { provided as part } \\
\text { of a Medicare } \\
\text { Part D MTM } \\
\text { program by an } \\
\text { MTM } \\
\text { Coordinator } \\
\text { (non-clinical } \\
\text { staff) and a } \\
\text { pharmacist } \\
\text { G2: Usual care } \\
\text { provided to } \\
\text { MTM-eligible } \\
\text { enrollees who } \\
\text { chose not to } \\
\text { participate }\end{array}$ & $\begin{array}{l}\text { To ensure } \\
\text { that drugs } \\
\text { prescribed to } \\
\text { beneficiaries } \\
\text { are } \\
\text { appropriately } \\
\text { used to } \\
\text { optimize } \\
\text { therapeutic } \\
\text { outcomes } \\
\text { and lower the } \\
\text { risks of } \\
\text { adverse drug } \\
\text { events and } \\
\text { drug } \\
\text { interactions. }\end{array}$ & $\begin{array}{l}\text { (1) PDP } \\
\text { beneficiaries who } \\
\text { fill at least two Part } \\
D \text { covered drugs } \\
\text { for } \geq 2 \text { chronic } \\
\text { diseases of } \\
\text { interest, including, } \\
\text { but not limited to, } \\
\text { asthma, CD, CHF, } \\
\text { diabetes, } \\
\text { dyslipidemia, and } \\
\text { HTN (specific } \\
\text { disease states } \\
\text { varied by PDP); (2) } \\
\text { Additional } \\
\text { independent } \\
\text { eligibility criteria } \\
\text { included an } \\
\text { extrapolated } \\
\text { annual drug cost } \\
\text { (set by the } \\
\text { Secretary of } \\
\text { DHHS) of } \$ 4000 \text { or } \\
\text { more by the end of } \\
\text { the plan year. } \\
\text { Long-term care } \\
\text { residents eligible. }\end{array}$ & $\begin{array}{l}\text { CMS- } \\
\text { mandated } \\
\text { exclusions } \\
\text { including } \\
\text { any one of } \\
\text { following: } \\
\text { use of OTC } \\
\text { drugs, } \\
\text { vitamins, } \\
\text { drugs for } \\
\text { cosmetic } \\
\text { use, } \\
\text { medication } \\
\text { s to treat } \\
\text { cold or } \\
\text { cough } \\
\text { symptoms, } \\
\text { fertility } \\
\text { agents, } \\
\text { DESI } \\
\text { drugs, and } \\
\text { drugs not } \\
\text { covered } \\
\text { under Part } \\
\text { D. }\end{array}$ & Cohort & NR & Unspecified & NR & $\begin{array}{l}<45 \text { years } \\
\text { Overall: } 6.8 \% \\
\text { G1: } 2.1 \% \\
\text { G2: } 7.7 \% \\
45-64 \text { years } \\
\text { Overall: } \\
29.2 \% \\
\text { G1: } 25.9 \% \\
\text { G2: } 29.9 \% \\
\geq 65 \text { years } \\
\text { Overall: } \\
63.9 \% \\
\text { G1: } 72.0 \% \\
\text { G2: } 62.4 \%\end{array}$ & $\begin{array}{l}\text { Overall: } 61.3 \\
\text { G1: } 58.2 \\
\text { G2: } 61.9\end{array}$ & \\
\hline
\end{tabular}


Table E1. Study and patient-level characteristics (continued)

\begin{tabular}{|c|c|c|c|c|c|c|c|c|c|c|c|}
\hline $\begin{array}{l}\text { Author, } \\
\text { Year } \\
\text { Trial } \\
\text { Name }^{a}\end{array}$ & $\begin{array}{l}\text { Interventions } \\
\text { and } \\
\text { Comparator } \\
\text { Descriptions }\end{array}$ & $\begin{array}{l}\text { Intervention } \\
\text { Goal }\end{array}$ & Inclusion Criteria & $\begin{array}{l}\text { Exclusion } \\
\text { Criteria }\end{array}$ & $\begin{array}{l}\text { Study } \\
\text { Design }\end{array}$ & $\begin{array}{l}\text { Study } \\
\text { Duration }\end{array}$ & $\begin{array}{l}\text { Funding } \\
\text { Source(s) }\end{array}$ & $\begin{array}{l}\text { Baseline } \\
\% \text { Rural }\end{array}$ & $\begin{array}{l}\text { Baseline } \\
\text { Age - Mean } \\
\text { (SD) or } \\
\text { Median } \\
\text { (Range) } \\
\end{array}$ & $\begin{array}{l}\text { Baseline \% } \\
\text { Female }\end{array}$ & $\begin{array}{l}\text { Racel } \\
\text { Ethnicity \% }\end{array}$ \\
\hline $\begin{array}{l}\text { Taylor, } \\
\text { Byrd, and } \\
\text { Krueger, } \\
2003^{36}\end{array}$ & $\begin{array}{l}\text { G1: } \\
\text { Pharmaceutical } \\
\text { care provided } \\
\text { by pharmacists } \\
\text { G2: Standard } \\
\text { care without } \\
\text { advice or } \\
\text { recommendatio } \\
\text { ns given to } \\
\text { patients or } \\
\text { physicians }\end{array}$ & $\begin{array}{l}\text { Prevention, } \\
\text { detection and } \\
\text { resolution of } \\
\text { medication- } \\
\text { related } \\
\text { problems in } \\
\text { high-risk } \\
\text { patients in a } \\
\text { rural } \\
\text { community. } \\
\end{array}$ & $\begin{array}{l}\text { (1) Age } \geq 18 \text { years; } \\
\text { (2) Receiving care } \\
\text { at participating } \\
\text { clinic; }(3) \text { Identified } \\
\text { as high risk for } \\
\text { medication-related } \\
\text { adverse event } \\
\text { (defined as } \geq 3 \text { of } \\
\text { following risk } \\
\text { factors: } 5+ \\
\text { medications, } 12+ \\
\text { doses per day, } 4+ \\
\text { medications } \\
\text { changes in the } \\
\text { previous year, } 3+ \\
\text { concurrent } \\
\text { diseases, history of } \\
\text { medication } \\
\text { noncompliance, } \\
\text { presence of drugs } \\
\text { that require } \\
\text { therapeutic } \\
\text { monitoring) }\end{array}$ & $\begin{array}{l}\text { (1) } \\
\text { Significant } \\
\text { cognitive } \\
\text { impairment } \\
\text {; (2) History } \\
\text { of missed } \\
\text { office visits; } \\
\text { (3) } \\
\text { Scheduling } \\
\text { conflicts; } \\
\text { (4) Life } \\
\text { expectancy } \\
\text { of <1 year } \\
\end{array}$ & $\begin{array}{l}\text { RCT: } \\
\text { parallel, } \\
\text { not } \\
\text { clustere } \\
\text { d }\end{array}$ & $\begin{array}{l}12 \\
\text { months }\end{array}$ & $\begin{array}{l}\text { Foundation or } \\
\text { non-profit }\end{array}$ & $\begin{array}{l}\text { Overall: } \\
100\end{array}$ & $\begin{array}{l}\text { Overall: NR } \\
\text { G1: } 64.4 \\
(13.7) \\
\text { G2: } 66.7 \\
(12.3)\end{array}$ & $\begin{array}{l}\text { Overall: NR } \\
\text { G1: } 63.6 \\
\text { G2: } 72.2\end{array}$ & $\begin{array}{l}\text { White } \\
\text { Overall: NR } \\
\text { G1: } 60.6 \\
\text { G2: } 61.1\end{array}$ \\
\hline
\end{tabular}


Table E1. Study and patient-level characteristics (continued)

\begin{tabular}{|c|c|c|c|c|c|c|c|c|c|c|c|}
\hline $\begin{array}{l}\text { Author, } \\
\text { Year } \\
\text { Trial } \\
\text { Name }^{a}\end{array}$ & $\begin{array}{l}\text { Interventions } \\
\text { and } \\
\text { Comparator } \\
\text { Descriptions }\end{array}$ & $\begin{array}{l}\text { Intervention } \\
\text { Goal }\end{array}$ & Inclusion Criteria & $\begin{array}{l}\text { Exclusion } \\
\text { Criteria }\end{array}$ & $\begin{array}{l}\text { Study } \\
\text { Design }\end{array}$ & $\begin{array}{l}\text { Study } \\
\text { Duration }\end{array}$ & $\begin{array}{l}\text { Funding } \\
\text { Source(s) }\end{array}$ & $\begin{array}{l}\text { Baseline } \\
\% \text { Rural }\end{array}$ & $\begin{array}{l}\text { Baseline } \\
\text { Age - Mean } \\
\text { (SD) or } \\
\text { Median } \\
\text { (Range) }\end{array}$ & $\begin{array}{l}\text { Baseline \% } \\
\text { Female }\end{array}$ & $\begin{array}{l}\text { Racel } \\
\text { Ethnicity \% }\end{array}$ \\
\hline $\begin{array}{l}\text { Touchette } \\
\text { et al., } \\
2012^{37}\end{array}$ & $\begin{array}{l}\text { G1: MTM basic } \\
\text { (comprehensive } \\
\text { medication } \\
\text { review and } \\
\text { DRP } \\
\text { assessment) } \\
\text { G2: MTM } \\
\text { enhanced } \\
\text { (MTM plus 2- } \\
\text { page clinical } \\
\text { summary } \\
\text { abstracted from } \\
\text { patient's } \\
\text { medical chart) } \\
\text { G3: Usual care, } \\
\text { consisting of } \\
\text { medication } \\
\text { counseling per } \\
\text { clinic's normal } \\
\text { routine but no } \\
\text { formal MTM } \\
\text { from a study } \\
\text { pharmacist }\end{array}$ & $\begin{array}{l}\text { To improve } \\
\text { the safety of } \\
\text { medication by } \\
\text { reducing } \\
\text { ADEs and } \\
\text { DRPs; Also } \\
\text { to reduce } \\
\text { health care, } \\
\text { especially ED } \\
\text { visits. }\end{array}$ & $\begin{array}{l}\text { 1) Age } \geq 65 \text { years; } \\
\text { (2) Primary use of } \\
\text { English for written } \\
\text { and oral } \\
\text { communication; ( } 3 \text { ) } \\
\text { Telephone access } \\
\text { for the study's } \\
\text { duration; (4) } \\
\text { Presence of } \geq 3 \\
\text { comorbid chronic } \\
\text { conditions } \\
\text { associated with } \\
\text { increased health } \\
\text { care use; (5) }>2 \\
\text { visits to clinic } \\
\text { provider during } \\
\text { previous year; (6) } \\
>6 \text { chronic } \\
\text { prescription } \\
\text { medications during } \\
6 \text { months before } \\
\text { enrollment; (7) }>1 \\
\text { recent situations } \\
\text { placing patient at } \\
\text { higher risk of DRP } \\
\text { (i.e., } \geq 3 \text { different } \\
\text { health care } \\
\text { providers visited in } \\
\text { the last } 12 \text { months; } \\
\text { any medication } \\
\text { change, new } \\
\text { physician visit, ED } \\
\text { visit, } \\
\text { hospitalization; or } \\
\text { invasive procedure } \\
\text { [requiring stopping } \\
\text { medications] in } \\
\text { previous } 30 \text { days). }\end{array}$ & $\begin{array}{l}\text { 1) } \\
\text { Presence } \\
\text { of a } \\
\text { terminal } \\
\text { condition } \\
\text { with life } \\
\text { expectancy } \\
\text { of } 6 \\
\text { months or } \\
\text { less; } \\
\text { 2) Previous } \\
\text { enrollment } \\
\text { in MTM } \\
\text { program } \\
\text { involving } \\
\text { comprehen } \\
\text { sive } \\
\text { medication } \\
\text { review in } \\
\text { previous } 12 \\
\text { months. }\end{array}$ & $\begin{array}{l}\text { RCT: } \\
\text { parallel, } \\
\text { not } \\
\text { clustere } \\
\text { d } \\
\\
\end{array}$ & 6 months & Government & NR & $\begin{array}{l}\text { Overall: } 74.6 \\
(6.7) \\
\text { G1: } 74.5(6.6) \\
\text { G2: } 74.8(6.8) \\
\text { G3: } 74.6(6.8)\end{array}$ & $\begin{array}{l}\text { Overall: } 66.2 \\
\text { G1: } 63.0 \\
\text { G2: } 67.0 \\
\text { G3: } 68.3\end{array}$ & $\begin{array}{l}\text { Black } \\
\text { Overall: } 51.2 \\
\text { G1: } 48.3 \\
\text { G2: } 49.1 \\
\text { G3: } 56.3 \\
\text { Hispanic } \\
\text { Overall: } 4.4 \\
\text { G1: } 6.2 \\
\text { G2: } 2.3 \\
\text { G3: } 4.8 \\
\text { Asian } \\
\text { Overall: } 0.8 \\
\text { G1: } 0.5 \\
\text { G2: } 0.9 \\
\text { G3: } 1.0 \\
\text { American } \\
\text { Indian } \\
\text { Overall: } 0.3 \\
\text { G1: } 0 \\
\text { G2: } 0 \\
\text { G3: } 1.0\end{array}$ \\
\hline
\end{tabular}


Table E1. Study and patient-level characteristics (continued)

\begin{tabular}{|c|c|c|c|c|c|c|c|c|c|c|c|}
\hline $\begin{array}{l}\text { Author, } \\
\text { Year } \\
\text { Trial } \\
\text { Name }^{a}\end{array}$ & $\begin{array}{l}\text { Interventions } \\
\text { and } \\
\text { Comparator } \\
\text { Descriptions }\end{array}$ & $\begin{array}{l}\text { Intervention } \\
\text { Goal }\end{array}$ & Inclusion Criteria & $\begin{array}{l}\text { Exclusion } \\
\text { Criteria }\end{array}$ & $\begin{array}{l}\text { Study } \\
\text { Design }\end{array}$ & $\begin{array}{l}\text { Study } \\
\text { Duration }\end{array}$ & $\begin{array}{l}\text { Funding } \\
\text { Source(s) }\end{array}$ & $\begin{array}{l}\text { Baseline } \\
\% \text { Rural }\end{array}$ & $\begin{array}{l}\text { Baseline } \\
\text { Age - Mean } \\
\text { (SD) or } \\
\text { Median } \\
\text { (Range) }\end{array}$ & $\begin{array}{l}\text { Baseline \% } \\
\text { Female }\end{array}$ & $\begin{array}{l}\text { Race/ } \\
\text { Ethnicity \% }\end{array}$ \\
\hline $\begin{array}{l}\text { Triller and } \\
\text { Hamilton, } \\
2007^{38}\end{array}$ & $\begin{array}{l}\text { G1: Visiting } \\
\text { nurse } \\
\text { association } \\
\text { home visit } \\
\text { services plus } \\
\text { comprehensive } \\
\text { pharmaceutical } \\
\text { care services } \\
\text { G2: Visiting } \\
\text { nurse } \\
\text { association } \\
\text { home visit } \\
\text { services only }\end{array}$ & $\begin{array}{l}\text { Study } \\
\text { conducted to } \\
\text { determine } \\
\text { whether } \\
\text { comprehensi } \\
\text { ve } \\
\text { pharmaceutic } \\
\text { al care } \\
\text { services for } \\
\text { home care } \\
\text { patients with } \\
\text { heart failure } \\
\text { who were } \\
\text { receiving } \\
\text { visiting nurse } \\
\text { association } \\
\text { services } \\
\text { could } \\
\text { significantly } \\
\text { reduce the } \\
\text { rate of all- } \\
\text { cause } \\
\text { hospitalizatio } \\
\text { n or death. }\end{array}$ & $\begin{array}{l}\text { (1) Patients with } \\
\text { primary or } \\
\text { secondary } \\
\text { diagnosis of HF } \\
\text { documented in the } \\
\text { medical record or } \\
\text { billing system who } \\
\text { were referred from } \\
\text { Northeast Health } \\
\text { inpatient facilities } \\
\text { to Eddy VNA for } \\
\text { skilled nursing } \\
\text { services; (2) Age } \\
\geq 21 \text { years; (3) } \\
\text { Residence in } \\
\text { Albany, Rensselaer } \\
\text { or Saratoga } \\
\text { county; (4) Patients } \\
\text { must have received } \\
\text { at least } 3 \text { days of } \\
\text { care from VNA and } \\
\geq 1 \text { pharmacist to } \\
\text { be included in final } \\
\text { study analysis; (5) } \\
\text { Non-English } \\
\text { speaking patients } \\
\text { included only if } \\
\text { adequate } \\
\text { translation services } \\
\text { available from } \\
\text { family or friends. }\end{array}$ & $\begin{array}{l}\text { (1) } \\
\text { Individuals } \\
\text { without } \\
\text { telephone } \\
\text { service; (2) } \\
\text { Individuals, } \\
\text { who, due to } \\
\text { disability or } \\
\text { illness, } \\
\text { lacked the } \\
\text { mental } \\
\text { capacity to } \\
\text { provide } \\
\text { informed } \\
\text { consent } \\
\end{array}$ & $\begin{array}{l}\text { RCT: } \\
\text { parallel, } \\
\text { not } \\
\text { clustere } \\
\text { d }\end{array}$ & 24 & $\begin{array}{l}\text { Foundation or } \\
\text { non-profit }\end{array}$ & NR & $\begin{array}{l}\text { Overall: NR } \\
\text { G1: } 81.3(9.3) \\
\text { G2: } 78.1 \\
(11.2)\end{array}$ & $\begin{array}{l}\text { Overall: NR } \\
\text { G1: } 73 \\
\text { G2: } 72\end{array}$ & $\begin{array}{l}\text { White } \\
\text { Overall: NR } \\
\text { G1: } 97 \\
\text { G2: } 88\end{array}$ \\
\hline
\end{tabular}


Table E1. Study and patient-level characteristics (continued)

\begin{tabular}{|c|c|c|c|c|c|c|c|c|c|c|c|}
\hline $\begin{array}{l}\text { Author, } \\
\text { Year } \\
\text { Trial } \\
\text { Name }^{a}\end{array}$ & $\begin{array}{l}\text { Interventions } \\
\text { and } \\
\text { Comparator } \\
\text { Descriptions }\end{array}$ & $\begin{array}{l}\text { Intervention } \\
\text { Goal }\end{array}$ & Inclusion Criteria & $\begin{array}{l}\text { Exclusio } \\
\text { n Criteria }\end{array}$ & $\begin{array}{l}\text { Study } \\
\text { Design }\end{array}$ & $\begin{array}{l}\text { Study } \\
\text { Duration }\end{array}$ & $\begin{array}{l}\text { Funding } \\
\text { Source(s) }\end{array}$ & $\begin{array}{l}\text { Baseline } \\
\% \text { Rural }\end{array}$ & $\begin{array}{l}\text { Baseline } \\
\text { Age - Mean } \\
\text { (SD) or } \\
\text { Median } \\
\text { (Range) }\end{array}$ & $\begin{array}{l}\text { Baseline \% } \\
\text { Female }\end{array}$ & $\begin{array}{l}\text { Race/ } \\
\text { Ethnicity } \\
\%\end{array}$ \\
\hline $\begin{array}{l}\text { Volume et } \\
\text { al., } 2001^{39} \text {; } \\
\text { Kassam et } \\
\text { al., } 2001^{40} \\
\text { PREP }\end{array}$ & $\begin{array}{l}\text { G1: } \\
\text { Comprehensive } \\
\text { pharmaceutical } \\
\text { care services } \\
\text { using a nine- } \\
\text { step process as } \\
\text { defined by } \\
\text { Hepler and } \\
\text { Strand provided } \\
\text { by community } \\
\text { pharmacists. } \\
\text { G2: Traditional } \\
\text { pharmacy care }\end{array}$ & $\begin{array}{l}\text { Authors } \\
\text { describe the } \\
\text { goal of } \\
\text { pharmaceutic } \\
\text { al care as the } \\
\text { "improvement } \\
\text { of patient } \\
\text { outcomes } \\
\text { and quality of } \\
\text { life." They } \\
\text { add study } \\
\text { objective to } \\
\text { be to } \\
\text { describe } \\
\text { changes in } \\
\text { intermediate } \\
\text { and primary } \\
\text { outcomes } \\
\text { after the } \\
\text { provision of } \\
\text { pharmaceutic } \\
\text { al care. }\end{array}$ & $\begin{array}{l}\text { Pharmacies: 1) } \\
\text { Participation of } \\
\text { pharmacists working }>8 \\
\text { hours a week } \\
\text { dispensing medications; } \\
\text { 2) Agreement to } \\
\text { participate in practice } \\
\text { enhancement program; } \\
\text { 3) Agreement to } \\
\text { conform with } \\
\text { professional standards } \\
\text { developed by Alberta } \\
\text { Pharmaceutical } \\
\text { Association; } 4 \text { ) Alberta } \\
\text { Blue Cross Billings } \\
\text { represented at least } \\
\text { one-third of pharmacy } \\
\text { billings; 5) Located } \\
\leq 200 \text { miles of } \\
\text { Edmonton. } \\
\text { Patients: } 1 \text { ) }>65 \text { years; } \\
\text { 2) Prescription } \\
\text { medication coverage } \\
\text { under Alberta Health } \\
\text { and Wellness' Senior } \\
\text { Health Plan; } 3 \text { ) Use } \geq 3 \\
\text { medications } \\
\text { concurrently; } 4 \text { ) } \\
\text { Residing in Alberta for } \\
\text { 12 of } 15 \text { study months; } \\
5) \text { Agree to receive } \\
\text { prescription } \\
\text { medications only from } \\
\text { study pharmacy during } \\
\text { study period }\end{array}$ & $\begin{array}{l}\text { Patients: } \\
\text { 1) } \\
\text { Individual } \\
\text { s with } \\
\text {; terminal } \\
\text { disease; } \\
\text { 2) Could } \\
\text { not } \\
\text { communic } \\
\text { ate in } \\
\text { English; } \\
\text { 3) Could } \\
\text { not } \\
\text { complete } \\
\text { telephone } \\
\text { interviews } \\
\text {. }\end{array}$ & $\begin{array}{l}\text { RCT: } \\
\text { cluster- } \\
\text { randomi } \\
\text { zed }\end{array}$ & $\begin{array}{l}12 \text { to } 13 \\
\text { months }\end{array}$ & $\begin{array}{l}\text { Multiple } \\
\text { (Government, } \\
\text { foundation, } \\
\text { and } \\
\text { pharmaceutic } \\
\text { al) }\end{array}$ & NR & $\begin{array}{l}\text { Mean (SD) } \\
\text { Overall: } 74 \\
\text { (NR) } \\
\text { G1: } 73.9(6.1) \\
\text { G2: } 73.2(6.1)\end{array}$ & $\begin{array}{l}\text { Mean (SD) } \\
\text { Overall: NR } \\
\text { G1: } 63.5 \\
\text { G2: } 69.6\end{array}$ & NR \\
\hline
\end{tabular}


Table E1. Study and patient-level characteristics (continued)

\begin{tabular}{|c|c|c|c|c|c|c|c|c|c|c|c|}
\hline $\begin{array}{l}\text { Author, } \\
\text { Year } \\
\text { Trial } \\
\text { Name }^{a}\end{array}$ & $\begin{array}{l}\text { Interventions } \\
\text { and } \\
\text { Comparator } \\
\text { Descriptions }\end{array}$ & $\begin{array}{l}\text { Intervention } \\
\text { Goal }\end{array}$ & Inclusion Criteria & $\begin{array}{l}\text { Exclusion } \\
\text { Criteria }\end{array}$ & $\begin{array}{l}\text { Study } \\
\text { Design }\end{array}$ & $\begin{array}{l}\text { Study } \\
\text { Duration }\end{array}$ & $\begin{array}{l}\text { Funding } \\
\text { Source(s) }\end{array}$ & $\begin{array}{l}\text { Baseline } \\
\% \text { Rural }\end{array}$ & $\begin{array}{l}\text { Baseline } \\
\text { Age - Mean } \\
\text { (SD) or } \\
\text { Median } \\
\text { (Range) }\end{array}$ & $\begin{array}{l}\text { Baseline \% } \\
\text { Female }\end{array}$ & $\begin{array}{l}\text { Race/ } \\
\text { Ethnicity \% }\end{array}$ \\
\hline $\begin{array}{l}\text { Welch et } \\
\text { al., } 2009^{41}\end{array}$ & $\begin{array}{l}\text { G1: MTM } \\
\text { program } \\
\text { provided to } \\
\text { home-based } \\
\text { beneficiaries as } \\
\text { part of a } \\
\text { Medicare Part } \\
\text { D MTM } \\
\text { program } \\
\text { G2: No-MTM } \\
\text { control group } \\
\text { (voluntary opt- } \\
\text { out) }\end{array}$ & $\begin{array}{l}\text { To reduce } \\
\text { mortality, } \\
\text { inpatient } \\
\text { hospitalizatio } \\
\text { ns, ED visits, } \\
\text { and Part D- } \\
\text { covered } \\
\text { medication } \\
\text { costs }\end{array}$ & $\begin{array}{l}\text { 1) MTM-eligible } \\
\text { Kaiser Permanente } \\
\text { Colorado (KPCO) } \\
\text { beneficiaries; } \\
\text { 1) Had } \geq 2 \text { chronic } \\
\text { conditions, one of } \\
\text { which was } \\
\text { considered high } \\
\text { risk; } \\
\text { 2) Receiving } 5 \text { or } \\
\text { more Part D- } \\
\text { covered } \\
\text { medications; } \\
\text { 3) Likely to incur at } \\
\text { least } \$ 4000 \text { in total } \\
\text { costs for Part D- } \\
\text { covered } \\
\text { medications. }\end{array}$ & $\begin{array}{l}\text { KPCO } \\
\text { beneficiarie } \\
\mathrm{s} \text { with end- } \\
\text { stage renal } \\
\text { disease } \\
\text { (ESRD) }\end{array}$ & Cohort & 180 days & $\begin{array}{l}\text { Integrated } \\
\text { health care } \\
\text { system (Kaiser } \\
\text { Permanente } \\
\text { Colorado) }\end{array}$ & NR & $\begin{array}{l}\text { Mean (SD) } \\
\text { Overall: NR } \\
\text { G1: } 68.8 \\
(10.7) \\
\text { G2: } 68.9 \\
(11.3) \\
p=0.949\end{array}$ & $\begin{array}{l}\text { Overall: NR } \\
\text { G1: } 56.6 \\
\text { G2: } 54.5 \\
p=0.541\end{array}$ & NR \\
\hline
\end{tabular}


Table E1. Study and patient-level characteristics (continued)

\begin{tabular}{|c|c|c|c|c|c|c|c|c|c|c|c|}
\hline $\begin{array}{l}\text { Author, } \\
\text { Year } \\
\text { Trial } \\
\text { Name }^{a}\end{array}$ & $\begin{array}{l}\text { Interventions } \\
\text { and } \\
\text { Comparator } \\
\text { Descriptions }\end{array}$ & $\begin{array}{l}\text { Intervention } \\
\text { Goal }\end{array}$ & Inclusion Criteria & $\begin{array}{l}\text { Exclusion } \\
\text { Criteria }\end{array}$ & $\begin{array}{l}\text { Study } \\
\text { Design }\end{array}$ & $\begin{array}{l}\text { Study } \\
\text { Duration }\end{array}$ & $\begin{array}{l}\text { Funding } \\
\text { Source(s) }\end{array}$ & $\begin{array}{l}\text { Baseline } \\
\% \text { Rural }\end{array}$ & $\begin{array}{l}\text { Baseline } \\
\text { Age - Mean } \\
\text { (SD) or } \\
\text { Median } \\
\text { (Range) }\end{array}$ & $\begin{array}{l}\text { Baseline \% } \\
\text { Female }\end{array}$ & $\begin{array}{l}\text { Race/ } \\
\text { Ethnicity \% }\end{array}$ \\
\hline $\begin{array}{l}\text { Williams et } \\
\text { al., } 2004^{42}\end{array}$ & $\begin{array}{l}\text { G1: Medication } \\
\text { review and } \\
\text { optimization of } \\
\text { patient's } \\
\text { medication } \\
\text { regimen } \\
\text { conducted by } \\
\text { an } \\
\text { interdisciplinary } \\
\text { medication } \\
\text { adjustment } \\
\text { team in addition } \\
\text { to usual } \\
\text { medical care } \\
\text { and "Bound for } \\
\text { Health" booklet. } \\
\text { G2: Usual } \\
\text { medical care } \\
\text { plus provision } \\
\text { of "Bound for } \\
\text { Health" booklet }\end{array}$ & $\begin{array}{l}\text { To promote } \\
\text { regimen } \\
\text { changes to } \\
\text { simplify } \\
\text { regimens in } \\
\text { elders taking } \\
\text { multiple } \\
\text { medications } \\
\text { and to see } \\
\text { whether } \\
\text { these } \\
\text { changes } \\
\text { improved } \\
\text { functioning. } \\
\end{array}$ & $\begin{array}{l}\text { 1) Age } \geq 65 \text { years; } \\
\text { 2) Cognitively } \\
\text { intact (no evidence } \\
\text { of dementia or } \\
\text { cognitive } \\
\text { dysfunction in the } \\
\text { medical record); } 3 \text { ) } \\
\text { Minimum of } 5 \\
\text { prescription } \\
\text { medications, of } \\
\text { which } 2 \text { had to be } \\
\text { potentially } \\
\text { problematic for } \\
\text { geriatric patients. }\end{array}$ & NR & $\begin{array}{l}\text { RCT: } \\
\text { parallel, } \\
\text { not } \\
\text { clustere } \\
\text { d }\end{array}$ & 6 weeks & Unspecified & NR & $\begin{array}{l}\text { G1: } 73.5(5.9) \\
\text { G2: } 73.9(5.6)\end{array}$ & $\begin{array}{l}\text { G1: } 65.1 \\
\text { G2: } 50.6\end{array}$ & $\begin{array}{l}\text { White } \\
\text { G1: } 79.4 \\
\text { G2: } 76.6 \\
\text { Non-White } \\
\text { G1: } 20.6 \\
\text { G2: } 23.4\end{array}$ \\
\hline
\end{tabular}


Table E1. Study and patient-level characteristics (continued)

\begin{tabular}{|c|c|c|c|c|c|c|c|c|c|c|c|}
\hline $\begin{array}{l}\text { Author, } \\
\text { Year } \\
\text { Trial } \\
\text { Name }^{a}\end{array}$ & $\begin{array}{l}\text { Interventions } \\
\text { and } \\
\text { Comparator } \\
\text { Descriptions }\end{array}$ & $\begin{array}{l}\text { Intervention } \\
\text { Goal }\end{array}$ & Inclusion Criteria & $\begin{array}{l}\text { Exclusion } \\
\text { Criteria }\end{array}$ & $\begin{array}{l}\text { Study } \\
\text { Design }\end{array}$ & $\begin{array}{l}\text { Study } \\
\text { Duration }\end{array}$ & $\begin{array}{l}\text { Funding } \\
\text { Source(s) }\end{array}$ & $\begin{array}{l}\text { Baseline } \\
\% \text { Rural }\end{array}$ & $\begin{array}{l}\text { Baseline } \\
\text { Age - Mean } \\
\text { (SD) or } \\
\text { Median } \\
\text { (Range) }\end{array}$ & $\begin{array}{l}\text { Baseline \% } \\
\text { Female }\end{array}$ & $\begin{array}{l}\text { Race/ } \\
\text { Ethnicity \% }\end{array}$ \\
\hline $\begin{array}{l}\text { Winston } \\
\text { and Lin, } \\
2009^{43}\end{array}$ & $\begin{array}{l}\text { G1: MTM } \\
\text { provided in a } \\
\text { community } \\
\text { pharmacy (i.e., } \\
\text { care in face-to- } \\
\text { face meetings } \\
\text { or by } \\
\text { telephone) as } \\
\text { part of a } \\
\text { Medicare Part } \\
\text { D MTM } \\
\text { program } \\
\text { G2: MTM } \\
\text { provided by } \\
\text { pharmacist- } \\
\text { staffed call } \\
\text { centers as part } \\
\text { of a Medicare } \\
\text { Part D MTM } \\
\text { program } \\
\text { G3: Educational } \\
\text { mailings (i.e., } \\
\text { mailed letter } \\
\text { containing } \\
\text { patient-specific } \\
\text { medication } \\
\text { related } \\
\text { information, } \\
\text { personal } \\
\text { medication } \\
\text { record, and tips } \\
\text { to save money } \\
\text { on } \\
\text { prescriptions) }\end{array}$ & $\begin{array}{l}\text { Describe } \\
\text { experiences } \\
\text { with MTM } \\
\text { services } \\
\text { delivered to } \\
\text { beneficiaries } \\
\text { of Mirixa's } \\
\text { health plan } \\
\text { clients } \\
\end{array}$ & $\begin{array}{l}\text { Patients who } \\
\text { qualified for MTM } \\
\text { services between } \\
\text { April 1, } 2007 \text { and } \\
\text { June } 30,2007 . \\
\text { MTM qualification } \\
\text { determined by } \\
\text { each participating } \\
\text { health plan; } \\
\text { generally patients } \\
\text { who had increased } \\
\text { cardiovascular risk } \\
\text { due to diabetes } \\
\text { and HTN and/or } \\
\text { dyslipidemia }\end{array}$ & $\begin{array}{l}\text { (1) Patients } \\
\text { whose } \\
\text { coverage } \\
\text { was } \\
\text { discontinue } \\
\text { d for any } \\
\text { reason; (2) } \\
\text { Patients } \\
\text { who } \\
\text { received } \\
\text { additional } \\
\text { pharmacist } \\
\text {-provided } \\
\text { services } \\
\text { (i.e., } \\
\text { formulary } \\
\text { review or } \\
\text { other MTM) } \\
\text { during } \\
\text { intervention } \\
\text { period. }\end{array}$ & Cohort & Unclear & $\begin{array}{l}\text { Private MTM } \\
\text { and pharmacy- } \\
\text { delivered } \\
\text { service provider }\end{array}$ & NR & $\begin{array}{l}\text { Overall: NR } \\
\text { G1: } 67.4 \\
(13.1) \\
\text { G2: } 67.8 \\
(12.8) \\
\text { G3: } 66.5 \\
(13.4)\end{array}$ & $\begin{array}{l}\text { Overall: NR } \\
\text { G1: } 70.4 \\
\text { G2: } 70.5 \\
\text { G3: } 69.5\end{array}$ & NR \\
\hline
\end{tabular}


Table E1. Study and patient-level characteristics (continued)

\begin{tabular}{|c|c|c|c|c|c|c|c|c|c|c|c|}
\hline $\begin{array}{l}\text { Author, } \\
\text { Year } \\
\text { Trial } \\
\text { Name }^{a}\end{array}$ & $\begin{array}{l}\text { Interventions } \\
\text { and } \\
\text { Comparator } \\
\text { Descriptions }\end{array}$ & $\begin{array}{l}\text { Intervention } \\
\text { Goal }\end{array}$ & Inclusion Criteria & $\begin{array}{l}\text { Exclusion } \\
\text { Criteria }\end{array}$ & $\begin{array}{l}\text { Study } \\
\text { Design }\end{array}$ & $\begin{array}{l}\text { Study } \\
\text { Duration }\end{array}$ & $\begin{array}{l}\text { Funding } \\
\text { Source(s) }\end{array}$ & $\begin{array}{l}\text { Baseline } \\
\% \text { Rural }\end{array}$ & $\begin{array}{l}\text { Baseline } \\
\text { Age - Mean } \\
\text { (SD) or } \\
\text { Median } \\
\text { (Range) }\end{array}$ & $\begin{array}{l}\text { Baseline \% } \\
\text { Female }\end{array}$ & $\begin{array}{l}\text { Race/ } \\
\text { Ethnicity \% }\end{array}$ \\
\hline $\begin{array}{l}\text { Witry, } \\
\text { Doucette, } \\
\text { and } \\
\text { Gainer, } \\
2011^{44}\end{array}$ & $\begin{array}{l}\text { G1: PCM } \\
\text { provided by } \\
\text { community } \\
\text { pharmacists to } \\
\text { lowa Medicaid } \\
\text { enrollees } \\
\text { G2: PCM } \\
\text { provided by } \\
\text { community } \\
\text { pharmacists to } \\
\text { patients with } \\
\text { private } \\
\text { individual-group } \\
\text { insurance }\end{array}$ & $\begin{array}{l}\text { To decrease } \\
\text { the risk of } \\
\text { DRPs }\end{array}$ & $\begin{array}{l}\text { (1) Patients } \geq 1 \\
\text { chronic condition } \\
\text { (i.e., who filled a } \\
\text { medication } \\
\text { commonly used to } \\
\text { treat } 1 \text { of } 12 \\
\text { chronic conditions, } \\
\text { as defined by } \\
\text { Medicaid PCM } \\
\text { program, at least } \\
\text { twice during } 3 \\
\text { months prior to } \\
\text { screening date); } \\
\text { (2) Must have filled } \\
\geq 4 \text { unique, } \\
\text { nontopical } \\
\text { medications during } \\
3 \text { months prior to } \\
\text { screening date; } \\
\text { (3) Patrons of } \\
\text { study pharmacies, } \\
\text { meaning that } \geq 50 \% \\
\text { of patients' } \\
\text { prescription claims } \\
\text { were paid to those } \\
\text { pharmacies }\end{array}$ & $\begin{array}{l}\text { See } \\
\text { inclusion } \\
\text { criteria }\end{array}$ & Cohort & $\begin{array}{l}21 \\
\text { months }\end{array}$ & $\begin{array}{l}\text { Foundation or } \\
\text { non-profit }\end{array}$ & NR & $\begin{array}{l}\text { Mean (SD) } \\
\text { Overall: NR } \\
\text { G1: } 54.1(0.8) \\
\text { G2: } 58.9 \\
(7.51)\end{array}$ & $\begin{array}{l}\text { Mean (SD) } \\
\text { Overall: NR } \\
\text { G1: } 80 \\
\text { G2: } 68.1\end{array}$ & NR \\
\hline
\end{tabular}

Abbreviations: CHF = chronic heart failure; CMR = comprehensive medication review; CMS = Centers for Medicare and Medicaid Services; DHHS = Department of Health and Human Services; $\mathrm{DRP}=$ drug-related problem; $\mathrm{ED}=$ emergency department; $\mathrm{ESRD}=$ end-stage renal disease; $\mathrm{GP}=$ general practitioner; $\mathrm{HF}=$ heart failure; $\mathrm{HMR}=\mathrm{home}$ medication review; HTN = hypertension; $\mathrm{KPCO}=$ Kaiser Permanente Colorado; $\mathrm{mm} \mathrm{Hg}=$ milligrams mercury; MTM = medication therapy management; NR = not reported; NSAIDS = non-steroidal anti-inflammatory drugs; OTC = over-the-counter; PCM = pharmaceutical case management; PREP = Pharmaceutical Care Research and Education Project; $\mathrm{RCT}=$ randomized controlled trial; $\mathrm{SD}=$ standard deviation; $\mathrm{US}=$ United States; VNA = visiting nurse agency. 
Table E2. Other patient-level and clinical characteristics

\begin{tabular}{|c|c|c|c|c|c|c|c|}
\hline $\begin{array}{l}\text { Author, Year } \\
\text { Trial Name }^{a}\end{array}$ & $\begin{array}{l}\text { Interventions } \\
\text { and Comparator } \\
\text { Descriptions }\end{array}$ & $\begin{array}{l}\text { Study } \\
\text { Design }\end{array}$ & $\begin{array}{l}\text { Other Baseline } \\
\text { Characteristics }\end{array}$ & $\begin{array}{l}\text { Measure of Co- } \\
\text { Morbidity }\end{array}$ & $\begin{array}{l}\text { Diagnosed } \\
\text { Conditions or } \\
\text { Diseases (\%) }\end{array}$ & $\begin{array}{l}\text { Baseline Number } \\
\text { of Prescribed } \\
\text { Medications }\end{array}$ & $\begin{array}{l}\text { Other Patient } \\
\text { Clinical } \\
\text { Characteristics }\end{array}$ \\
\hline $\begin{array}{l}\text { Bernsten et al., } \\
2001^{1} \text {; } \\
\text { Sturgess et al., } \\
2003^{2}\end{array}$ & $\begin{array}{l}\text { G1: Structured } \\
\text { community } \\
\text { pharmacy-based } \\
\text { pharmaceutical } \\
\text { care program } \\
\text { G2: Normal } \\
\text { pharmaceutical } \\
\text { Usual community } \\
\text { pharmacy } \\
\text { services }\end{array}$ & $\begin{array}{l}\text { RCT: } \\
\text { cluster- } \\
\text { randomiz } \\
\text { ed }\end{array}$ & $\begin{array}{l}\text { Pooled sample } \\
\text { Patients living alone (\%) } \\
\text { Overall: NR } \\
\text { G1: } 37.2 \\
\text { G2: } 37.7 \\
\text { Patients requiring help with } \\
\text { daily activities (\%) } \\
\text { Overall: NR } \\
\text { G1: } 50.9 \\
\text { G2: } 47.4 \\
\text { Northern Ireland } \\
\text { Patients living alone (\%) } \\
\text { Overall: NR } \\
\text { G1: } 30.9 \\
\text { G2: } 26.9 \\
\text { Patients requiring help with } \\
\text { daily activities (\%) } \\
\text { Overall: NR } \\
\text { G1: } 43.1 \\
\text { G2: } 55.7\end{array}$ & NR & NR & $\begin{array}{l}\text { Pooled sample } \\
\text { Overall: NR } \\
\text { G1: } 7.1(2.5) \\
\text { G2: } 7.0(2.5) \\
\text { p=NS } \\
\text { Northern Ireland } \\
\text { Overall: NR } \\
\text { G1: } 5.9(1.9) \\
\text { G2: } 6.7(1.9) \\
\text { P<0.05 }\end{array}$ & \\
\hline $\begin{array}{l}\text { Blennerhassett } \\
\text { et al., } 2007^{3}\end{array}$ & $\begin{array}{l}\text { Implementation of } \\
\text { a Home } \\
\text { Medication } \\
\text { Review (HMR) } \\
\text { into a chronic } \\
\text { heart failure } \\
\text { collaborative care } \\
\text { model. HMRs } \\
\text { were conducted } \\
\text { by accredited } \\
\text { pharmacists. } \\
\text { G2: No HMR }\end{array}$ & Cohort & $\begin{array}{l}\text { Smoking Status - Never (\%) } \\
\text { Overall: NR } \\
\text { G1: } 52 \\
\text { G2: } 42 \\
\text { Smoking Status - Ex (\%) } \\
\text { Overall: NR } \\
\text { G1: } 28 \\
\text { G2: } 20 \\
\text { Smoking Status - Current } \\
\text { (\%) } \\
\text { G1: } 3 \\
\text { G2: } 5\end{array}$ & NR & $\begin{array}{l}\text { Atrial Fibrillation } \\
\text { Overall: NR } \\
\text { G1: } 32 \\
\text { G2: } 12 \\
\text { Cerebrovascular } \\
\text { Accident/Transient } \\
\text { Ischemic Attack } \\
\text { Overall: NR } \\
\text { G1: } 18 \\
\text { G2: } 5 \\
\text { Diabetes: } \\
\text { Overall: NR } \\
\text { G1: } 27 \\
\text { G2: } 15 \\
\text { Chronic Heart } \\
\text { Failure } \\
\text { Overall: } 100 \\
\text { G1: } 100 \\
\text { G2: } 100 \\
\end{array}$ & NR & $\begin{array}{l}\text { Manage own } \\
\text { medications } \\
\text { G1: } 36 \% \\
\text { G2: } 71 \% \\
p=0.016\end{array}$ \\
\hline
\end{tabular}


Table E2. Other patient-level and clinical characteristics (continued)

\begin{tabular}{|c|c|c|c|c|c|c|c|}
\hline $\begin{array}{l}\text { Author, Year } \\
\text { Trial Name }^{a}\end{array}$ & $\begin{array}{l}\text { Interventions } \\
\text { and Comparator } \\
\text { Descriptions } \\
\end{array}$ & $\begin{array}{l}\text { Study } \\
\text { Design }\end{array}$ & $\begin{array}{l}\text { Other Baseline } \\
\text { Characteristics }\end{array}$ & $\begin{array}{l}\text { Measure of Co- } \\
\text { Morbidity }\end{array}$ & $\begin{array}{l}\text { Diagnosed } \\
\text { Conditions or } \\
\text { Diseases (\%) } \\
\end{array}$ & $\begin{array}{l}\text { Baseline Number } \\
\text { of Prescribed } \\
\text { Medications } \\
\end{array}$ & $\begin{array}{l}\text { Other Patient } \\
\text { Clinical } \\
\text { Characteristics }\end{array}$ \\
\hline $\begin{array}{l}\text { Carter et al., } \\
1997^{4} \text {; } \\
\text { Barnette et al., } \\
1996^{5}\end{array}$ & $\begin{array}{l}\text { G1: } \\
\text { Pharmaceutical } \\
\text { care provided by } \\
\text { pharmacists } \\
\text { within an } \\
\text { interdisciplinary } \\
\text { practice model. } \\
\text { Patient education } \\
\text { (lifestyle, risk } \\
\text { factor } \\
\text { modifications, and } \\
\text { drug therapy) was } \\
\text { standardized. } \\
\text { G2: Usual care }\end{array}$ & Cohort & NR & $\begin{array}{l}\mathrm{N} \text { of comorbid } \\
\text { conditions } \\
\text { Overall: NR } \\
\text { G1: } 3.5(2.4) \\
\text { G2: } 3.2(2.0) \\
\mathrm{p}=0.47\end{array}$ & $\begin{array}{l}\text { No. }(\%) \text { with } \\
\text { controlled blood } \\
\text { pressure at baseline }\end{array}$ & $\begin{array}{l}\text { Overall: NR } \\
\text { G1: } 13(52) \\
\text { G2: } 14(54)\end{array}$ & \\
\hline
\end{tabular}


Table E2. Other patient-level and clinical characteristics (continued)

\begin{tabular}{|c|c|c|c|c|c|c|c|}
\hline $\begin{array}{l}\text { Author, Year } \\
\text { Trial Name }^{a}\end{array}$ & $\begin{array}{l}\text { Interventions and } \\
\text { Comparator } \\
\text { Descriptions }\end{array}$ & $\begin{array}{l}\text { Study } \\
\text { Design }\end{array}$ & $\begin{array}{l}\text { Other Baseline } \\
\text { Characteristics }\end{array}$ & $\begin{array}{l}\text { Measure of Co- } \\
\text { Morbidity }\end{array}$ & $\begin{array}{l}\text { Diagnosed } \\
\text { Conditions or } \\
\text { Diseases (\%) }\end{array}$ & $\begin{array}{l}\text { Baseline Number } \\
\text { of Prescribed } \\
\text { Medications }\end{array}$ & $\begin{array}{l}\text { Other Patient } \\
\text { Clinical } \\
\text { Characteristics }\end{array}$ \\
\hline $\begin{array}{l}\text { Chisholm et al., } \\
2002^{6}\end{array}$ & $\begin{array}{l}\text { G1: Clinical } \\
\text { pharmacy services, } \\
\text { including reviewing } \\
\text { patients' medication } \\
\text { therapy, with an } \\
\text { emphasis on } \\
\text { controlling blood } \\
\text { pressure, and } \\
\text { preventing or } \\
\text { resolving drug } \\
\text { therapy problems. } \\
\text { Pharmacists } \\
\text { counseled patients } \\
\text { about their regimen, } \\
\text { including desired } \\
\text { clinical responses } \\
\text { and possible } \\
\text { adverse reactions. } \\
\text { G2: Routine clinic } \\
\text { services, but without } \\
\text { clinical pharmacist } \\
\text { interaction. Routine } \\
\text { clinical services } \\
\text { here entailed } \\
\text { meeting a renal } \\
\text { transplant clinic } \\
\text { team that consisted } \\
\text { of } 2 \text { nephrologists, a } \\
\text { clinical pharmacist, } \\
\text { PAs and a nurse. }\end{array}$ & $\begin{array}{l}\text { RCT: } \\
\text { parallel, not } \\
\text { clustered }\end{array}$ & NR & NR & $\begin{array}{l}\text { Hypertension (\%) } \\
\text { Overall: NR } \\
\text { G1: } 92 \\
\text { G2: } 90\end{array}$ & $\mathrm{NR}$ & NR \\
\hline
\end{tabular}


Table E2. Other patient-level and clinical characteristics (continued)

\begin{tabular}{|c|c|c|c|c|c|c|c|}
\hline $\begin{array}{l}\text { Author, Year } \\
\text { Trial Name }^{\mathrm{a}}\end{array}$ & $\begin{array}{l}\text { Interventions } \\
\text { and Comparator } \\
\text { Descriptions }\end{array}$ & $\begin{array}{l}\text { Study } \\
\text { Design }\end{array}$ & $\begin{array}{l}\text { Other Baseline } \\
\text { Characteristics }\end{array}$ & $\begin{array}{l}\text { Measure of Co- } \\
\text { Morbidity }\end{array}$ & $\begin{array}{l}\text { Diagnosed } \\
\text { Conditions or } \\
\text { Diseases (\%) }\end{array}$ & $\begin{array}{l}\text { Baseline Number } \\
\text { of Prescribed } \\
\text { Medications }\end{array}$ & $\begin{array}{l}\text { Other Patient } \\
\text { Clinical } \\
\text { Characteristics }\end{array}$ \\
\hline $\begin{array}{l}\text { Chrischilles et } \\
\text { al., } 2004^{7}\end{array}$ & $\begin{array}{l}\text { G1: PCM } \\
\text { provided by } \\
\text { pharmacists } \\
\text { G2: Did not } \\
\text { receive PCM } \\
\text { services }\end{array}$ & Cohort & NR & NR & NR & $\begin{array}{l}\text { Overall: NR } \\
\text { G1: } 7.5(0.2) \\
\text { G2: } 6.9(0.1)\end{array}$ & NR \\
\hline $\begin{array}{l}\text { Christensen et } \\
\text { al., } 2007^{8}\end{array}$ & $\begin{array}{l}\text { G1: MTM services } \\
\text { designed by a } \\
\text { health plan for its } \\
\text { beneficiaries and } \\
\text { provided by either } \\
\text { community } \\
\text { pharmacists or } \\
\text { medical clinic- } \\
\text { based } \\
\text { pharmacists. } \\
\text { G2: Patients from } \\
\text { same counties as } \\
\text { G1 who did not } \\
\text { receive } \\
\text { intervention } \\
\text { (control group 1) } \\
\text { G3: Patients from } \\
\text { a different county } \\
\text { than G1 who did } \\
\text { not receive } \\
\text { intervention } \\
\text { (control group 2) }\end{array}$ & NRCT & NR & NR & $\begin{array}{l}\text { Patients younger } \\
\text { than } 65: \\
\text { Hypertension } \\
\text { G1: } 48.1 \\
\text { G2: } 47.9 \\
\text { G3: } 46.4 \\
\text { >1 Condition } \\
\text { G1: } 42.8 \\
\text { G2: } 34.0 \\
\text { G3: } 38.3 \\
\text { Diabetes } \\
\text { G1: } 37 \\
\text { G2: } 31.7 \\
\text { G3: } 37.7 \\
\text { Patients older than } \\
\text { 65: } \\
\text { Hypertension } \\
\text { G1: } 62.5 \\
\text { G2: } 41.5 \\
\text { G3: } 48.5 \\
\text { Cardiovascular } \\
\text { Disease } \\
\text { G1: } 55.0 \\
\text { G2: } 48.4 \\
\text { G3: } 50.2 \\
\text { >1 Condition } \\
\text { G1: } 46.3 \\
\text { G2: } 39.7 \\
\text { G3: } 39.9 \\
\text { Diabetes } \\
\text { G1: } 45.0 \\
\text { G2: } 36.8 \\
\text { G3: } 33.7 \\
\text { (a) }\end{array}$ & $\begin{array}{l}\text { Patients younger } \\
\text { than } 65: \\
\text { G1: } 40.3(15.3) \\
\text { G2: } 37.2(17.5) \\
\text { G3: } 36.9(17.3) \\
\text { Patients older than } \\
65: \\
\text { G1: } 41.7(16.3) \\
\text { G2: } 38.4(16.3) \\
\text { G3: } 41.7(16.2)\end{array}$ & $\begin{array}{l}\text { Differences in \% } \\
\text { with selected } \\
\text { conditions and in } \\
\text { number of baseline } \\
\text { medications were } \\
\text { not significant } \\
\text { among the three } \\
\text { groups. }\end{array}$ \\
\hline
\end{tabular}


Table E2. Other patient-level and clinical characteristics (continued)

\begin{tabular}{|c|c|c|c|c|c|c|c|}
\hline $\begin{array}{l}\text { Author, Year } \\
\text { Trial Name }^{a}\end{array}$ & $\begin{array}{l}\text { Interventions } \\
\text { and Comparator } \\
\text { Descriptions }\end{array}$ & $\begin{array}{l}\text { Study } \\
\text { Design }\end{array}$ & $\begin{array}{l}\text { Other Baseline } \\
\text { Characteristics }\end{array}$ & $\begin{array}{l}\text { Measure of Co- } \\
\text { Morbidity }\end{array}$ & $\begin{array}{l}\text { Diagnosed } \\
\text { Conditions or } \\
\text { Diseases (\%) }\end{array}$ & $\begin{array}{l}\text { Baseline Number } \\
\text { of Prescribed } \\
\text { Medications }\end{array}$ & $\begin{array}{l}\text { Other Patient } \\
\text { Clinical } \\
\text { Characteristics }\end{array}$ \\
\hline $\begin{array}{l}\text { Clifford et al., } \\
2002^{9}\end{array}$ & $\begin{array}{l}\text { G1: } \\
\text { Pharmaceutical } \\
\text { care provided by } \\
\text { a clinical } \\
\text { pharmacist, } \\
\text { which included a } \\
\text { comprehensive } \\
\text { review relating to } \\
\text { pharmacotherapy } \\
\text { and diabetes, use } \\
\text { of proprietary and } \\
\text { non-proprietary } \\
\text { medications, such } \\
\text { as complementary } \\
\text { medicines, and } \\
\text { identification of } \\
\text { drug therapy } \\
\text { problems. } \\
\text { G2: Standard } \\
\text { outpatient care for } \\
\text { diabetes }\end{array}$ & $\begin{array}{l}\text { RCT: } \\
\text { parallel, } \\
\text { not } \\
\text { clustered } \\
\end{array}$ & NR & NR & $\begin{array}{l}\text { Type } 1 \text { or } 2 \\
\text { Diabetes } \\
\text { Overall: } 100 \\
\text { G1: } 100 \\
\text { G2: } 100 \\
\text { Type } 1 \text { Diabetes } \\
\text { Overall: NR } \\
\text { G1: } 29.2 \\
\text { G2: } 20.0 \\
\text { Type } 2 \text { Diabetes } \\
\text { Overall: NR } \\
\text { G1: } 70.8 \\
\text { G2: } 80.0 \\
\text { Hypertension: NR } \\
\text { Dyslipidemia: NR }\end{array}$ & NR & \\
\hline
\end{tabular}


Table E2. Other patient-level and clinical characteristics (continued)

\begin{tabular}{|c|c|c|c|c|c|c|c|}
\hline $\begin{array}{l}\text { Author, Year } \\
\text { Trial Name }^{a}\end{array}$ & $\begin{array}{l}\text { Interventions } \\
\text { and Comparator } \\
\text { Descriptions }\end{array}$ & $\begin{array}{l}\text { Study } \\
\text { Design }\end{array}$ & $\begin{array}{l}\text { Other Baseline } \\
\text { Characteristics }\end{array}$ & $\begin{array}{l}\text { Measure of Co- } \\
\text { Morbidity }\end{array}$ & $\begin{array}{l}\text { Diagnosed } \\
\text { Conditions or } \\
\text { Diseases (\%) } \\
\end{array}$ & $\begin{array}{l}\text { Baseline Number } \\
\text { of Prescribed } \\
\text { Medications } \\
\end{array}$ & $\begin{array}{l}\text { Other Patient } \\
\text { Clinical } \\
\text { Characteristics }\end{array}$ \\
\hline $\begin{array}{l}\text { Fischer et al., } \\
2000^{10}\end{array}$ & $\begin{array}{l}\text { G1: } \\
\text { Pharmaceutical } \\
\text { care based on the } \\
\text { Encara Practice } \\
\text { System provided } \\
\text { by onsite health } \\
\text { maintenance } \\
\text { organization staff } \\
\text { pharmacists. } \\
\text { G2: Standard } \\
\text { Community } \\
\text { Pharmacy } \\
\text { Practice } \\
\text { G3: A set of } \\
\text { refusers surveyed } \\
\text { and included in } \\
\text { some analyses } \\
\text { among those who } \\
\text { were at eligible } \\
\text { clinics but initially } \\
\text { declined to } \\
\text { participate. }\end{array}$ & NRCT & $\begin{array}{l}\text { \% Married } \\
\text { Overall: NR } \\
\text { G1: } 68 \\
\text { G2: } 71 \\
\text { G3: } 72 \\
\text { \% Education < HS } \\
\text { Overall: NR } \\
\text { G1: } 9 \\
\text { G2: } 18 \\
\text { G3: } 20 \\
\text { \% Income < 10K } \\
\text { Overall: NR } \\
\text { G1: } 3 \\
\text { G2: } 9 \\
\text { G3: } 9\end{array}$ & $\begin{array}{l}\text { \% in Fair or Poor } \\
\text { Health } \\
\text { Overall: NR } \\
\text { G1: } 28 \\
\text { G2: } 26 \\
\text { G3: } 35\end{array}$ & $\begin{array}{l}\text { \% Heart/HTN } \\
\text { problems } \\
\text { Overall: NR } \\
\text { G1: } 68 \\
\text { G2: } 61 \\
\text { G3: } 65 \\
\% \text { Asthma/Lung } \\
\text { Problems } \\
\text { Overall: NR } \\
\text { G1: } 49 \\
\text { G2: } 52 \\
\text { G3: } 42\end{array}$ & $\begin{array}{l}\text { Overall: NR } \\
\text { G1: } 5.2 \\
\text { G2: } 4.6 \\
\text { G3: } 4.3\end{array}$ & $\begin{array}{l}\text { Mean N non- } \\
\text { prescription meds: } \\
\text { Overall: NR } \\
\text { G1: } 2.2 \\
\text { G2: } 1.8 \\
\text { G3: } 1.7\end{array}$ \\
\hline $\begin{array}{l}\text { Fischer et al., } \\
2002^{11}\end{array}$ & $\begin{array}{l}\text { Pharmaceutical } \\
\text { care based on the } \\
\text { Encara Practice } \\
\text { System provided } \\
\text { by pharmacists. } \\
\text { Communication of } \\
\text { pharmacist with } \\
\text { the patient's } \\
\text { physician about } \\
\text { drug therapy } \\
\text { problems } \\
\text { identified by the } \\
\text { pharmacist. } \\
\text { G2: Usual care } \\
\text { with no additional } \\
\text { interventions. }\end{array}$ & NRCT & $\begin{array}{l}\text { Annual health care charges } \\
\text { Overall: NR } \\
\text { G1: } \$ 9,600 \\
\text { G2: } \$ 11,000\end{array}$ & $\begin{array}{l}\text { Charlson Index } \\
\text { G1: } 1.2 \\
\text { G2: } 1.3\end{array}$ & $\begin{array}{l}\text { Heart disease (\%) } \\
\text { Overall: NR } \\
\text { G1: } 43 \\
\text { G2: } 40\end{array}$ & $\begin{array}{l}\text { Overall: NR } \\
\text { G1: } 9.1 \\
\text { G2: } 9.4\end{array}$ & NR \\
\hline
\end{tabular}


Table E2. Other patient-level and clinical characteristics (continued)

\begin{tabular}{|c|c|c|c|c|c|c|c|}
\hline $\begin{array}{l}\text { Author, Year } \\
\text { Trial Name }^{a}\end{array}$ & $\begin{array}{l}\text { Interventions } \\
\text { and Comparator } \\
\text { Descriptions }\end{array}$ & $\begin{array}{l}\text { Study } \\
\text { Design }\end{array}$ & $\begin{array}{l}\text { Other Baseline } \\
\text { Characteristics }\end{array}$ & $\begin{array}{l}\text { Measure of Co- } \\
\text { Morbidity }\end{array}$ & $\begin{array}{l}\text { Diagnosed } \\
\text { Conditions or } \\
\text { Diseases (\%) }\end{array}$ & $\begin{array}{l}\text { Baseline Number } \\
\text { of Prescribed } \\
\text { Medications }\end{array}$ & $\begin{array}{l}\text { Other Patient } \\
\text { Clinical } \\
\text { Characteristics }\end{array}$ \\
\hline $\begin{array}{l}\text { Fox et al., } \\
2009^{12}\end{array}$ & $\begin{array}{l}\text { G1: Florida Health } \\
\text { Care Plans MTM } \\
\text { program, } \\
\text { consisting of a } \\
\text { medication } \\
\text { therapy review } \\
\text { and evaluation by } \\
\text { a clinical } \\
\text { pharmacist that } \\
\text { was documented } \\
\text { and sent to the } \\
\text { patient's physician } \\
\text { through health } \\
\text { plan review } \\
\text { G2: Opt-out from } \\
\text { MTM program }\end{array}$ & Cohort & NR & NR & Diabetes: 100 & $\begin{array}{l}\text { Number of } \\
\text { prescriptions per } \\
\text { member per month } \\
\text { (PMPM) in } 2007 \\
\text { Overall: NR } \\
\text { G1: } 9.4 \\
\text { G2: } 8.8\end{array}$ & None \\
\hline $\begin{array}{l}\text { Gattis et al., } \\
1999^{13}\end{array}$ & $\begin{array}{l}\text { G1: Clinical } \\
\text { pharmacy } \\
\text { services, including } \\
\text { an assessment of } \\
\text { prescribed } \\
\text { regimen, } \\
\text { compliance, and } \\
\text { adverse effects, } \\
\text { and symptoms } \\
\text { and response to } \\
\text { therapy. Providing } \\
\text { patient education } \\
\text { about the purpose } \\
\text { of each drug and } \\
\text { reinforcing } \\
\text { adherence. } \\
\text { Detailed written } \\
\text { information was } \\
\text { also provided to } \\
\text { patients. } \\
\text { G2: Usual medical } \\
\text { care }\end{array}$ & $\begin{array}{l}\text { RCT: } \\
\text { parallel, } \\
\text { not } \\
\text { clustered } \\
\end{array}$ & NR & NR & $\begin{array}{l}\text { Heart Failure } \\
\text { Overall: } 100 \\
\text { G1: } 100 \\
\text { G2: } 100\end{array}$ & $\begin{array}{l}\text { Overall: NR } \\
\text { G1: } 6.5(25 \%: 5 \text {, } \\
75 \%: 8) \\
\text { G2: } 6(25 \%: 4.5 \text {, } \\
75 \%: 8)\end{array}$ & NR \\
\hline
\end{tabular}


Table E2. Other patient-level and clinical characteristics (continued)

\begin{tabular}{|c|c|c|c|c|c|c|c|}
\hline $\begin{array}{l}\text { Author, Year } \\
\text { Trial Name }\end{array}$ & $\begin{array}{l}\text { Interventions } \\
\text { and Comparator } \\
\text { Descriptions }\end{array}$ & $\begin{array}{l}\text { Study } \\
\text { Design }\end{array}$ & $\begin{array}{l}\text { Other Baseline } \\
\text { Characteristics }\end{array}$ & $\begin{array}{l}\text { Measure of Co- } \\
\text { Morbidity }\end{array}$ & $\begin{array}{l}\text { Diagnosed } \\
\text { Conditions or } \\
\text { Diseases (\%) } \\
\end{array}$ & $\begin{array}{l}\text { Baseline Number } \\
\text { of Prescribed } \\
\text { Medications }\end{array}$ & $\begin{array}{l}\text { Other Patient } \\
\text { Clinical } \\
\text { Characteristics } \\
\end{array}$ \\
\hline $\begin{array}{l}\text { Hanlon et al., } \\
1996^{14}\end{array}$ & $\begin{array}{l}\text { G1: } \\
\text { Pharmaceutical } \\
\text { care provided by } \\
\text { a clinical } \\
\text { pharmacist } \\
\text { G2: Usual care in } \\
\text { the General } \\
\text { Medicine Clinic }\end{array}$ & $\begin{array}{l}\text { RCT: } \\
\text { parallel, } \\
\text { not } \\
\text { clustered } \\
\end{array}$ & $\begin{array}{l}\text { Married (\%) } \\
\text { Overall: NR } \\
\text { G1: } 65.7 \\
\text { G2: } 85.4 \\
\text { Mean years of education } \\
\text { (SD) } \\
\text { Overall: NR } \\
\text { G1: } 10.2(3.8) \\
\text { G2: } 9.9(4.2)\end{array}$ & $\begin{array}{l}\mathrm{N} \text { of chronic } \\
\text { conditions } \\
\text { Overall: NR } \\
\text { G1: } 9.2(3.7) \\
\text { G2: } 9.0(3.0)\end{array}$ & NR & $\begin{array}{l}\text { Overall: NR } \\
\text { G1: } 7.6(2.8) \\
\text { G2: } 8.2(2.7) \\
\text { These were limited } \\
\text { to medications } \\
\text { prescribed by a VA } \\
\text { physician. }\end{array}$ & $\begin{array}{l}\% \text { of medications for } \\
\text { which compliant } \\
\text { Overall: NR } \\
\text { G1: } 73 \% \\
\text { G2: } 74 \%\end{array}$ \\
\hline $\begin{array}{l}\text { Harrison et al., } \\
2012^{15}\end{array}$ & $\begin{array}{l}\text { G1: } \\
\text { Pharmaceutical } \\
\text { care, provided by } \\
\text { clinical pharmacist } \\
\text { for purpose of } \\
\text { identifying and } \\
\text { resolving actual } \\
\text { and potential } \\
\text { DTPs, medication } \\
\text { teaching, } \\
\text { adherence } \\
\text { optimization, } \\
\text { medication } \\
\text { reconciliation, and } \\
\text { provision of drug } \\
\text { information. } \\
\text { G2: Retrospective } \\
\text { historical control } \\
\text { of matched } \\
\text { patients who } \\
\text { received standard } \\
\text { care at a routine } \\
\text { medical visit } \\
\text { within } 8 \text { months } \\
\text { prior to study } \\
\text { period }\end{array}$ & Cohort & & NR & NR & NR & $\begin{array}{l}\text { Authors provide } \\
\text { information on } \\
\text { cause of need for } \\
\text { lung transplant, but } \\
\text { not a list of } \\
\text { comorbidities. }\end{array}$ \\
\hline
\end{tabular}


Table E2. Other patient-level and clinical characteristics (continued)

\begin{tabular}{|c|c|c|c|c|c|c|c|}
\hline $\begin{array}{l}\text { Author, Year } \\
\text { Trial Name }\end{array}$ & $\begin{array}{l}\text { Interventions } \\
\text { and Comparator } \\
\text { Descriptions }\end{array}$ & $\begin{array}{l}\text { Study } \\
\text { Design }\end{array}$ & $\begin{array}{l}\text { Other Baseline } \\
\text { Characteristics }\end{array}$ & $\begin{array}{l}\text { Measure of Co- } \\
\text { Morbidity }\end{array}$ & $\begin{array}{l}\text { Diagnosed } \\
\text { Conditions or } \\
\text { Diseases (\%) }\end{array}$ & $\begin{array}{l}\text { Baseline Number } \\
\text { of Prescribed } \\
\text { Medications }\end{array}$ & $\begin{array}{l}\text { Other Patient } \\
\text { Clinical } \\
\text { Characteristics }\end{array}$ \\
\hline $\begin{array}{l}\text { Isetts et al., } \\
2008^{16}\end{array}$ & $\begin{array}{l}\text { G1: MTM services } \\
\text { provided by staff } \\
\text { pharmacists, } \\
\text { including the } \\
\text { establishment of } \\
\text { goals of therapy, } \\
\text { in collaboration } \\
\text { with primary care } \\
\text { providers. } \\
\text { G2: Usual medical } \\
\text { care without MTM }\end{array}$ & Cohort & NR & $\begin{array}{l}\text { Mean Number of } \\
\text { Conditions } \\
\text { Overall: NR } \\
\text { G1: } 6.4 \text { (NR) } \\
\text { G2: NR }\end{array}$ & NR & $\begin{array}{l}\text { Overall: NR } \\
\text { G1: } 14 \% \text { were age } \\
65 \text { or older } \\
\text { G2: NR }\end{array}$ & $\begin{array}{l}\text { These variables } \\
\text { were not reported } \\
\text { for the HEDIS } \\
\text { comparison group } \\
\text { other than a } \\
\text { statement that says } \\
\text { "...were similar to } \\
\text { intervention group } \\
\text { patients in terms of } \\
\text { age, gender, and } \\
\text { presence of study } \\
\text { medical conditions." } \\
\text { (bottom of page } \\
\text { 205) }\end{array}$ \\
\hline $\begin{array}{l}\text { Jameson, } \\
\text { VanNoord, and } \\
\text { Vanderwoud, } \\
1995^{17}\end{array}$ & $\begin{array}{l}\text { Pharmacotherapy } \\
\text { consultation and } \\
\text { followup provided } \\
\text { by clinical } \\
\text { ambulatory care } \\
\text { pharmacist. } \\
\text { G2: Standard } \\
\text { office-based } \\
\text { primary care. }\end{array}$ & $\begin{array}{l}\text { RCT: } \\
\text { parallel, } \\
\text { not } \\
\text { clustered } \\
\end{array}$ & NR & $\begin{array}{l}\text { More than } 3 \text { chronic } \\
\text { diseases Overall: } \\
\text { NR } \\
\text { G1: } 70 \% \\
\text { G2: } 76 \%\end{array}$ & NR & $\begin{array}{l}5 \text { or more long-term } \\
\text { medications (\%) } \\
\text { Overall: NR } \\
\text { G1: } 89 \\
\text { G2: } 90\end{array}$ & NR \\
\hline $\begin{array}{l}\text { Jeong et al., } \\
2007^{18}\end{array}$ & $\begin{array}{l}\text { G1: Pharmacist- } \\
\text { managed MTMP } \\
\text { provided by } \\
\text { ambulatory care } \\
\text { pharmacists and } \\
\text { healthcare } \\
\text { support staff } \\
\text { G2: Eligible for } \\
\text { Part D MTMP but } \\
\text { declined } \\
\text { enrollment } \\
\text { G3: Patients } \\
\text { without Part D as } \\
\text { their primary drug } \\
\text { benefit }\end{array}$ & Cohort & NR & NR & $\begin{array}{l}\text { Hyperlipidemia } \\
\text { Overall: NR } \\
\text { G1: } 65.1 \\
\text { G2: } 63.1 \\
\text { G3: } 58.6 \\
\text { CAD } \\
\text { Overall: NR } \\
\text { G1: } 28.3 \\
\text { G2: } 24.8 \\
\text { G3: } 26.1 \\
\text { Diabetes } \\
\text { Overall: NR } \\
\text { G1: } 54.8 \\
\text { G2: } 48.1 \\
\text { G3: } 46.9\end{array}$ & NR & NR \\
\hline
\end{tabular}


Table E2. Other patient-level and clinical characteristics (continued)

\begin{tabular}{|c|c|c|c|c|c|c|c|}
\hline $\begin{array}{l}\text { Author, Year } \\
\text { Trial Name }^{a}\end{array}$ & $\begin{array}{l}\text { Interventions } \\
\text { and Comparator } \\
\text { Descriptions }\end{array}$ & $\begin{array}{l}\text { Study } \\
\text { Design }\end{array}$ & $\begin{array}{l}\text { Other Baseline } \\
\text { Characteristics }\end{array}$ & $\begin{array}{l}\text { Measure of Co- } \\
\text { Morbidity }\end{array}$ & $\begin{array}{l}\text { Diagnosed } \\
\text { Conditions or } \\
\text { Diseases (\%) }\end{array}$ & $\begin{array}{l}\text { Baseline Number } \\
\text { of Prescribed } \\
\text { Medications }\end{array}$ & $\begin{array}{l}\text { Other Patient } \\
\text { Clinical } \\
\text { Characteristics }\end{array}$ \\
\hline $\begin{array}{l}\text { Krska et al., } \\
2001^{19}\end{array}$ & $\begin{array}{l}\text { G1: Medication } \\
\text { reviews led by } \\
\text { clinically-trained } \\
\text { pharmacists. } \\
\text { G2: Usual care } \\
\text { involving } \\
\text { interviews and } \\
\text { identification of } \\
\text { pharmaceutical } \\
\text { care issues but } \\
\text { with no } \\
\text { pharmaceutical } \\
\text { care plan } \\
\text { implemented. }\end{array}$ & $\begin{array}{l}\text { RCT: } \\
\text { parallel, } \\
\text { not } \\
\text { clustered }\end{array}$ & NR & $\begin{array}{l}\text { Overall: NR } \\
\text { G1: } 3.9(1.4) \\
\text { G2: } 3.8(1.4) \\
p=0.968\end{array}$ & NR & $\begin{array}{l}\text { Repeat medicines } \\
\text { on computer records } \\
\text { Overall: NR } \\
\text { G1: } 7.4(2.7) \\
\text { G2: } 7.7(2.8) \\
\text { p: } 0.951\end{array}$ & NR \\
\hline $\begin{array}{l}\text { Malone et al., } \\
2000^{20} ; \\
\text { Ellis et al., } \\
2000^{21} \text {; } \\
\text { Malone et al., } \\
2001^{22} \text {; } \\
\text { Ellis et al., } \\
2000^{23} \\
\text { IMPROVE }\end{array}$ & $\begin{array}{l}\text { G1: } \\
\text { Pharmaceutical } \\
\text { care provided by } \\
\text { clinical } \\
\text { pharmacists } \\
\text { practicing } \\
\text { according to } \\
\text { scope of practice } \\
\text { within their } \\
\text { respective health } \\
\text { care facilities. } \\
\text { G2: Usual care } \\
\text { without } \\
\text { pharmaceutical } \\
\text { care }\end{array}$ & $\begin{array}{l}\text { RCT: } \\
\text { parallel, } \\
\text { not } \\
\text { clustered }\end{array}$ & $\begin{array}{l}\text { \% Married } \\
\text { Overall: NR } \\
\text { G1: } 68.5 \\
\text { G2: } 67.8\end{array}$ & $\begin{array}{l}\text { Mean number of } \\
\text { chronic conditions } \\
\text { Overall: NR } \\
\text { G1: } 4.0(2.0) \\
\text { G2: } 3.8(1.9)\end{array}$ & $\begin{array}{l}\text { Hypertension } \\
\text { Overall: NR } \\
\text { G1: } 68.5 \\
\text { G2: } 66.5 \\
\text { Angina } \\
\text { Overall: NR } \\
\text { G1: } 46.1 \\
\text { G2: } 46.7 \\
\text { Hyperlipidemia } \\
\text { Overall: NR } \\
\text { G1: } 39.8 \\
\text { G2: } 43.1\end{array}$ & $\begin{array}{l}\text { Overall: NR } \\
\text { G1: } 8.4(4.4) \\
\text { G2: } 8.0(4.0)\end{array}$ & NR \\
\hline
\end{tabular}


Table E2. Other patient-level and clinical characteristics (continued)

\begin{tabular}{|c|c|c|c|c|c|c|c|}
\hline $\begin{array}{l}\text { Author, Year } \\
\text { Trial Name }\end{array}$ & $\begin{array}{l}\text { Interventions } \\
\text { and Comparator } \\
\text { Descriptions }\end{array}$ & $\begin{array}{l}\text { Study } \\
\text { Design }\end{array}$ & $\begin{array}{l}\text { Other Baseline } \\
\text { Characteristics }\end{array}$ & $\begin{array}{l}\text { Measure of Co- } \\
\text { Morbidity }\end{array}$ & $\begin{array}{l}\text { Diagnosed } \\
\text { Conditions or } \\
\text { Diseases (\%) } \\
\end{array}$ & $\begin{array}{l}\text { Baseline Number } \\
\text { of Prescribed } \\
\text { Medications }\end{array}$ & $\begin{array}{l}\text { Other Patient } \\
\text { Clinical } \\
\text { Characteristics }\end{array}$ \\
\hline $\begin{array}{l}\text { McDonough et } \\
\text { al., } 2005^{24}\end{array}$ & $\begin{array}{l}\text { G1: } \\
\text { Pharmaceutical } \\
\text { care provided by } \\
\text { community } \\
\text { pharmacists. Drug } \\
\text { therapy } \\
\text { monitoring } \\
\text { focused on } 5 \text { drug } \\
\text { therapy problems: } \\
\text { appropriateness } \\
\text { of does, proper } \\
\text { regimen, potential } \\
\text { interactions, } \\
\text { nonadherence, } \\
\text { and adverse } \\
\text { effects. Patient } \\
\text { education also } \\
\text { provided. } \\
\text { G2: Usual care }\end{array}$ & $\begin{array}{l}\text { RCT: } \\
\text { cluster- } \\
\text { randomiz } \\
\text { ed }\end{array}$ & NR & NR & NR & $\begin{array}{l}\text { Overall: } \\
\text { G1: } 5.6(3.1) \\
\text { G2: } 7.0(3.2)\end{array}$ & $\begin{array}{l}\text { At baseline, the } \\
\text { treatment group was } \\
\text { significantly less } \\
\text { likely to report } \\
\text { alcohol use and } \\
\text { more likely to be } \\
\text { post-menopausal. }\end{array}$ \\
\hline $\begin{array}{l}\text { Moczygemba et } \\
\text { al., } 2011^{25} \text {; } \\
\text { Moczygemba et } \\
\text { al., } 2008^{26}\end{array}$ & $\begin{array}{l}\text { G1: Opt-in } \\
\text { telephone-based } \\
\text { MTM program, in } \\
\text { which MTM } \\
\text { services provided } \\
\text { by clinical } \\
\text { pharmacists or a } \\
\text { managed care } \\
\text { pharmacy } \\
\text { resident based on } \\
\text { the American } \\
\text { Pharmacists } \\
\text { Association and } \\
\text { National } \\
\text { Association of } \\
\text { Chain Drug } \\
\text { Stores Foundation } \\
\text { MTM framework. } \\
\text { G2: No-MTM } \\
\text { control group }\end{array}$ & Cohort & NR & $\begin{array}{l}\text { Number of chronic } \\
\text { dx } \\
\text { Mean (SD) } \\
\text { Overall: NR } \\
\text { G1: } 6.5(2.3) \\
\text { G2: } 7.0(2.1) \\
\text { p: } 0.18\end{array}$ & $\begin{array}{l}\text { Hypertension } \\
\text { Overall: NR } \\
\text { G1: } 95 \\
\text { G2: } 95 \\
\text { Dyslipidemia } \\
\text { Overall: NR } \\
\text { G1: } 77 \\
\text { G2: } 87 \\
\text { Diabetes } \\
\text { Overall: NR } \\
\text { G1: } 55 \\
\text { G2: } 60\end{array}$ & $\begin{array}{l}\text { Mean (SD) } \\
\text { Overall: NR } \\
\text { G1: } 13.0(3.2) \\
\text { G2: } 13.2(3.4)\end{array}$ & $\begin{array}{l}\text { Medication Regimen } \\
\text { Complexity Index } \\
\text { (MRCI) } \\
\text { Mean (range) } \\
\text { Overall: NR } \\
\text { G1: } 21.5(8-43) \\
\text { G2: } 22.8(9-43) \\
\text { p: } 0.32\end{array}$ \\
\hline
\end{tabular}


Table E2. Other patient-level and clinical characteristics (continued)

\begin{tabular}{|c|c|c|c|c|c|c|c|}
\hline $\begin{array}{l}\text { Author, Year } \\
\text { Trial Name }\end{array}$ & $\begin{array}{l}\text { Interventions } \\
\text { and Comparator } \\
\text { Descriptions }\end{array}$ & $\begin{array}{l}\text { Study } \\
\text { Design }\end{array}$ & $\begin{array}{l}\text { Other Baseline } \\
\text { Characteristics }\end{array}$ & $\begin{array}{l}\text { Measure of Co- } \\
\text { Morbidity }\end{array}$ & $\begin{array}{l}\text { Diagnosed } \\
\text { Conditions or } \\
\text { Diseases (\%) }\end{array}$ & $\begin{array}{l}\text { Baseline Number } \\
\text { of Prescribed } \\
\text { Medications }\end{array}$ & $\begin{array}{l}\text { Other Patient } \\
\text { Clinical } \\
\text { Characteristics }\end{array}$ \\
\hline $\begin{array}{l}\text { Pai et al., } \\
2009^{27} \text {; Pai et } \\
\text { al., } 2009^{28}\end{array}$ & $\begin{array}{l}\text { G1: } \\
\text { Pharmaceutical } \\
\text { care including } \\
\text { drug therapy } \\
\text { reviews } \\
\text { conducted by a } \\
\text { nephrology- } \\
\text { trained clinical } \\
\text { pharmacist with } \\
\text { the patient. Also } \\
\text { included patient } \\
\text { and health care } \\
\text { provider } \\
\text { education. } \\
\text { G2: Standard of } \\
\text { care, consisting of } \\
\text { brief therapy } \\
\text { reviews } \\
\text { conducted by a } \\
\text { nurse }\end{array}$ & $\begin{array}{l}\text { RCT: } \\
\text { cluster- } \\
\text { randomiz } \\
\text { ed }\end{array}$ & $\begin{array}{l}\text { Mean Time on Hemodialysis } \\
\text { in years (SD) } \\
\text { Overall: } 2.6(2.0) \\
\text { G1: } 2.8(1.8) \\
\text { G2: } 2.4(2.2)\end{array}$ & NR & $\begin{array}{l}\text { ESRD etiology - } \\
\text { Diabetes mellitus } \\
\text { Overall: } 43.3 \\
\text { G1: } 38.6 \\
\text { G2: } 48.9 \\
\text { ESRD etiology - } \\
\text { Hypertension } \\
\text { Overall: } 28.9 \\
\text { G1: } 31.6 \\
\text { G2: } 25.5 \\
\text { ESRD etiology - } \\
\text { Other } \\
\text { Overall: } 27.9 \\
\text { G1: } 29.8 \\
\text { G2: } 25.5\end{array}$ & $\begin{array}{l}\text { Overall: } 10(4) \\
\text { G1: } 10(4) \\
\text { G2: } 10(4)\end{array}$ & NR \\
\hline $\begin{array}{l}\text { Park et al., } \\
1996^{29}\end{array}$ & $\begin{array}{l}\text { G1: } \\
\text { Comprehensive } \\
\text { pharmaceutical } \\
\text { services including } \\
\text { drug therapy } \\
\text { monitoring and } \\
\text { patient education } \\
\text { provided by a } \\
\text { community } \\
\text { pharmacy } \\
\text { resident. } \\
\text { G2: Usual care }\end{array}$ & $\begin{array}{l}\text { RCT: } \\
\text { parallel, } \\
\text { not } \\
\text { clustered }\end{array}$ & NR & NR & NR & NR & $\begin{array}{l}\text { Mean number of } \\
\text { antihypertensives } \\
\text { Overall: NR } \\
\text { G1: } 1.4 \\
\text { G2: } 1.3\end{array}$ \\
\hline
\end{tabular}


Table E2. Other patient-level and clinical characteristics (continued)

\begin{tabular}{|c|c|c|c|c|c|c|c|}
\hline $\begin{array}{l}\text { Author, Year } \\
\text { Trial Name }^{\mathrm{a}}\end{array}$ & $\begin{array}{l}\text { Interventions } \\
\text { and Comparator } \\
\text { Descriptions }\end{array}$ & $\begin{array}{l}\text { Study } \\
\text { Design }\end{array}$ & $\begin{array}{l}\text { Other Baseline } \\
\text { Characteristics }\end{array}$ & $\begin{array}{l}\text { Measure of Co- } \\
\text { Morbidity }\end{array}$ & $\begin{array}{l}\text { Diagnosed } \\
\text { Conditions or } \\
\text { Diseases (\%) }\end{array}$ & $\begin{array}{l}\text { Baseline Number } \\
\text { of Prescribed } \\
\text { Medications }\end{array}$ & $\begin{array}{l}\text { Other Patient } \\
\text { Clinical } \\
\text { Characteristics }\end{array}$ \\
\hline $\begin{array}{l}\text { Pindolia et al., } \\
2009^{30}\end{array}$ & $\begin{array}{l}\text { G1: Telephone- } \\
\text { based MTM } \\
\text { services provided } \\
\text { as part of a } \\
\text { Medicare Part D } \\
\text { MTM program by } \\
\text { pharmacy care } \\
\text { management } \\
\text { clinical } \\
\text { pharmacists } \\
\text { (acceptors). } \\
\text { G2: Usual medical } \\
\text { care (opt-out) }\end{array}$ & Cohort & $\begin{array}{l}\text { 2006 Part D type (\%) } \\
\text { Donut hole } \\
\text { G1: } 68 \\
\text { G2: } 60 \\
\text { Nondonut hole or coverage } \\
\text { gap } \\
\text { G1: } 6 \\
\text { G2: } 8 \\
\text { Low income subsidy } \\
\text { G1: } 18 \\
\text { G2: } 24 \\
\text { Institutionalized } \\
\text { G1: } 8 \\
\text { G2: } 7 \\
\text { Overall p: } 0.054 \\
\text { 2007 Part D type (\%) } \\
\text { Donut hole } \\
\text { G1: } 93 \\
\text { G2: } 63 \\
\text { Nondonut hole or coverage } \\
\text { gap } \\
\text { G1: } 1 \\
\text { G2: } 9 \\
\text { Low income subsidy } \\
\text { G1: } 6 \\
\text { G2: } 26 \\
\text { Institutionalized } \\
\text { G1: } 0 \\
\text { G2: } 2 \\
\text { Overall p: } 0.001\end{array}$ & $\begin{array}{l}2006 \\
\mathrm{~N} \text { of qualifying } \\
\text { diseases (mean, } \\
\text { SD) } \\
\text { Overall: NR } \\
\text { G1: } 5.9(2.2) \\
\text { G2: } 5.6(2.1) \\
\text { p: } 0.047 \\
2007 \\
\mathrm{~N} \text { of qualifying } \\
\text { diseases (mean, } \\
\text { SD) } \\
\text { Overall: NR } \\
\text { G1: } 5.8(2.0) \\
\text { G2: } 5.9(2.0) \\
\text { Overall p: } 0.701\end{array}$ & NR & $\begin{array}{l}\text { 2006 } \\
\text { Unique prescriptions } \\
\text { filled (mean, SD) } \\
\text { Overall: NR } \\
\text { G1: } 16.7(7.2) \\
\text { G2: } 14.8(6.1) \\
\text { p: } 0.001 \\
\text { 2007 } \\
\text { Unique prescriptions } \\
\text { filled (mean, SD) } \\
\text { Overall: NR } \\
\text { G1: } 14.4(6.2) \\
\text { G2: } 14.9(6.2) \\
\text { p: } 0.223\end{array}$ & NR \\
\hline
\end{tabular}


Table E2. Other patient-level and clinical characteristics (continued)

\begin{tabular}{|c|c|c|c|c|c|c|c|}
\hline $\begin{array}{l}\text { Author, Year } \\
\text { Trial Name }^{a}\end{array}$ & $\begin{array}{l}\text { Interventions } \\
\text { and Comparator } \\
\text { Descriptions }\end{array}$ & $\begin{array}{l}\text { Study } \\
\text { Design }\end{array}$ & $\begin{array}{l}\text { Other Baseline } \\
\text { Characteristics }\end{array}$ & $\begin{array}{l}\text { Measure of Co- } \\
\text { Morbidity }\end{array}$ & $\begin{array}{l}\text { Diagnosed } \\
\text { Conditions or } \\
\text { Diseases (\%) }\end{array}$ & $\begin{array}{l}\text { Baseline Number } \\
\text { of Prescribed } \\
\text { Medications }\end{array}$ & $\begin{array}{l}\text { Other Patient } \\
\text { Clinical } \\
\text { Characteristics }\end{array}$ \\
\hline $\begin{array}{l}\text { Planas et al., } \\
2009^{31}\end{array}$ & $\begin{array}{l}\text { G1: MTM services } \\
\text { provided by } \\
\text { community } \\
\text { pharmacists. Also } \\
\text { included patient } \\
\text { education on diet } \\
\text { and lifestyle } \\
\text { modifications to } \\
\text { lower blood } \\
\text { pressure. } \\
\text { G2: No MTM } \\
\text { received, but only } \\
\text { informed of blood } \\
\text { pressure goals for } \\
\text { patients with } \\
\text { diabetes }\end{array}$ & $\begin{array}{l}\text { RCT: } \\
\text { parallel, } \\
\text { not } \\
\text { clustered } \\
\end{array}$ & $\begin{array}{l}\text { Overweight }\left(25-29.9 \mathrm{~kg} / \mathrm{m}^{2}\right) \text {, } \\
\text { \%: } \\
\text { Overall: NR } \\
\text { G1: } 15.6 \\
\text { G2: } 42.1 \\
\text { p: NR } \\
\text { Obese }\left(\geq 30 \mathrm{~kg} / \mathrm{m}^{2}\right), \% \text { : } \\
\text { Overall: NR } \\
\text { G1: } 68.8 \\
\text { G2: } 47.4 \\
\text { p: NR }\end{array}$ & NR & $\begin{array}{l}\text { Hypertension: } 100 \\
\text { Diabetes: } 100\end{array}$ & NR & $\mathrm{NR}$ \\
\hline $\begin{array}{l}\text { Roughead et al., } \\
2009^{32}\end{array}$ & $\begin{array}{l}\text { G1: Home } \\
\text { Medication } \\
\text { Reviews (HMR), a } \\
\text { collaborative } \\
\text { model of } \\
\text { pharmaceutical } \\
\text { care, conducted } \\
\text { by accredited } \\
\text { pharmacists. } \\
\text { G2: No } \\
\text { medication review } \\
\text { received }\end{array}$ & Cohort & $\begin{array}{l}\text { Socioeconomic index of } \\
\text { disadvantage (\%) } \\
\text { Lowest disadvantage } \\
\text { Overall: NR } \\
\text { G1: } 31 \\
\text { G2: } 25 \\
\text { Medium/low disadvantage } \\
\text { Overall: NR } \\
\text { G1: } 25 \\
\text { G2: } 25 \\
\text { Medium/high disadvantage } \\
\text { Overall: NR } \\
\text { G1: } 24 \\
\text { G2: } 25 \\
\text { Highest disadvantage } \\
\text { Overall: NR } \\
\text { G1: } 20 \\
\text { G2: } 25 \\
\text { Overall p: } 0.01\end{array}$ & $\begin{array}{l}\mathrm{N} \text { of co-morbidities } \\
\text { (median, SD) } \\
\text { Overall: NR } \\
\text { G1: } 8(2) \\
\text { G2: } 7(2) \\
\text { p: }<0.0001\end{array}$ & NR & $\begin{array}{l}\mathrm{N} \text { (range) of } \\
\text { prescriptions in last } \\
\text { year } \\
\text { Overall: NR } \\
\text { G1: } 95(69-123) \\
\text { G2: } 76(54-104) \\
\text { p: }<0.0001\end{array}$ & $\begin{array}{l}\text { Changes in } \\
\text { medicines during 6- } \\
\text { month period in } \\
\text { previous year (N, } \\
\text { SD) } \\
\text { Overall: NR } \\
\text { G1: } 3(2-6) \\
\text { G2: } 3(1-5) \\
\text { p: }<0.0001\end{array}$ \\
\hline
\end{tabular}


Table E2. Other patient-level and clinical characteristics (continued)

\begin{tabular}{|c|c|c|c|c|c|c|c|}
\hline $\begin{array}{l}\text { Author, Year } \\
\text { Trial Name }^{a}\end{array}$ & $\begin{array}{l}\text { Interventions } \\
\text { and Comparator } \\
\text { Descriptions }\end{array}$ & $\begin{array}{l}\text { Study } \\
\text { Design }\end{array}$ & $\begin{array}{l}\text { Other Baseline } \\
\text { Characteristics }\end{array}$ & $\begin{array}{l}\text { Measure of Co- } \\
\text { Morbidity }\end{array}$ & $\begin{array}{l}\text { Diagnosed } \\
\text { Conditions or } \\
\text { Diseases (\%) }\end{array}$ & $\begin{array}{l}\text { Baseline Number } \\
\text { of Prescribed } \\
\text { Medications }\end{array}$ & $\begin{array}{l}\text { Other Patient } \\
\text { Clinical } \\
\text { Characteristics }\end{array}$ \\
\hline $\begin{array}{l}\text { Sellors et al., } \\
2003^{33}\end{array}$ & $\begin{array}{l}\text { G1: Clinical } \\
\text { pharmacist } \\
\text { consultations } \\
\text { provided to family } \\
\text { physicians and } \\
\text { their patients by } \\
\text { community } \\
\text { pharmacists. } \\
\text { G2: Usual care for } \\
\text { family physicians } \\
\text { and their patients } \\
\text { from matched } \\
\text { postal codes. }\end{array}$ & $\begin{array}{l}\text { RCT: } \\
\text { cluster- } \\
\text { randomiz } \\
\text { ed } \\
\\
\end{array}$ & $\begin{array}{l}\text { Education: highest level } \\
\text { attained (\%) } \\
\text { Elementary School } \\
\text { Overall: NR } \\
\text { G1: } 26.9 \\
\text { G2: } 24.1 \\
\text { High school graduate } \\
\text { Overall: NR } \\
\text { G1: } 50.8 \\
\text { G2: } 51.0 \\
\text { Some college } \\
\text { Overall: NR } \\
\text { G1: } 22.2 \\
\text { G2: } 24.9 \\
\% \text { married FPL/ common-law } \\
\text { spouse } \\
\text { Overall: NR } \\
\text { G1: } 58.2 \\
\text { G2: } 63.1\end{array}$ & NR & $\begin{array}{l}\text { Hypertension } \\
\text { G1: } 54.3 \\
\text { G2: } 55.7 \\
\text { Osteoarthritis } \\
\text { G1: } 46.4 \\
\text { G2: } 48.3 \\
\text { IHD } \\
\text { G1: } 36.0 \\
\text { G2: } 38.0\end{array}$ & NR & NR \\
\hline
\end{tabular}


Table E2. Other patient-level and clinical characteristics (continued)

\begin{tabular}{|c|c|c|c|c|c|c|c|}
\hline $\begin{array}{l}\text { Author, Year } \\
\text { Trial Name }^{a}\end{array}$ & $\begin{array}{l}\text { Interventions } \\
\text { and Comparator } \\
\text { Descriptions } \\
\end{array}$ & $\begin{array}{l}\text { Study } \\
\text { Design }\end{array}$ & $\begin{array}{l}\text { Other Baseline } \\
\text { Characteristics }\end{array}$ & $\begin{array}{l}\text { Measure of Co- } \\
\text { Morbidity }\end{array}$ & $\begin{array}{l}\text { Diagnosed } \\
\text { Conditions or } \\
\text { Diseases (\%) } \\
\end{array}$ & $\begin{array}{l}\text { Baseline Number } \\
\text { of Prescribed } \\
\text { Medications }\end{array}$ & $\begin{array}{l}\text { Other Patient } \\
\text { Clinical } \\
\text { Characteristics }\end{array}$ \\
\hline $\begin{array}{l}\text { Sidel et al., } \\
1990^{34}\end{array}$ & $\begin{array}{l}\text { G1: Home visits } \\
\text { by pharmacists } \\
\text { and, when } \\
\text { needed, } \\
\text { consultations with } \\
\text { physicians to } \\
\text { identify and } \\
\text { correct problems } \\
\text { associated with } \\
\text { medication use. } \\
\text { G2: Standard care } \\
\text { without any visits } \\
\text { or information } \\
\text { provided to G1. }\end{array}$ & $\begin{array}{l}\text { RCT: } \\
\text { parallel, } \\
\text { not } \\
\text { clustered } \\
\end{array}$ & $\begin{array}{l}\text { Income }(\%) \\
\text { Overall: NR } \\
\text { Under } \$ 5000 \\
\text { G1: } 23.3 \\
\text { G2: } 22.2 \\
\$ 5000 \text { \$15000 } \\
\text { G1: } 61.0 \\
\text { G2: } 63.3 \\
>\$ 15000 \\
\text { G1: } 15.9 \\
\text { G2: } 14.4 \\
\text { Education: \% with } 9 \text { or more } \\
\text { years } \\
\text { Overall: NR } \\
\text { G1: } 62.2 \\
\text { G2: } 54.8 \\
\% \text { with Self-Assessed Health } \\
\text { Fair or Poor } \\
\text { Overall: NR } \\
\text { G1: } 44.0 \\
\text { G2: } 42.7 \\
\% \text { with Problems with } \\
\text { Activities of Daily Living } \\
\text { Overall: NR } \\
\text { G1: } 33.0 \\
\text { G2: } 34.6 \\
\% \text { with Symptoms of } \\
\text { Depression } \\
\text { Overall: NR } \\
\text { G1: } 10.8 \\
\text { G2: } 22.6 \\
\% \text { with Cognitive Impairment } \\
\text { Overall: NR } \\
\text { G1: } 15.4 \\
\text { G2: } 21.4\end{array}$ & $\begin{array}{l}\text { Number of medical } \\
\text { conditions }(\%) \\
\text { Overall: NR } \\
\text { None } \\
\text { G1: } 3.3 \\
\text { G2: } 2.9 \\
\frac{1-3}{G 1:} 58.2 \\
\text { G2: } 70.2 \\
4 \text { or more } \\
\text { G1: } 38.5 \\
\text { G2: } 29.9\end{array}$ & NR & $\begin{array}{l}\text { Overall: } 65.3 \% \\
\text { (mean 2.4, range 1- } \\
\text { 10) } \\
\text { G1: NR } \\
\text { G2: NR }\end{array}$ & $\mathrm{NR}$ \\
\hline
\end{tabular}


Table E2. Other patient-level and clinical characteristics (continued)

\begin{tabular}{|c|c|c|c|c|c|c|c|}
\hline $\begin{array}{l}\text { Author, Year } \\
\text { Trial Name }^{a}\end{array}$ & $\begin{array}{l}\text { Interventions } \\
\text { and Comparator } \\
\text { Descriptions }\end{array}$ & $\begin{array}{l}\text { Study } \\
\text { Design }\end{array}$ & $\begin{array}{l}\text { Other Baseline } \\
\text { Characteristics }\end{array}$ & $\begin{array}{l}\text { Measure of Co- } \\
\text { Morbidity }\end{array}$ & $\begin{array}{l}\text { Diagnosed } \\
\text { Conditions or } \\
\text { Diseases (\%) }\end{array}$ & $\begin{array}{l}\text { Baseline Number } \\
\text { of Prescribed } \\
\text { Medications }\end{array}$ & $\begin{array}{l}\text { Other Patient } \\
\text { Clinical } \\
\text { Characteristics }\end{array}$ \\
\hline $\begin{array}{l}\text { Staresinic et al., } \\
2007^{35}\end{array}$ & $\begin{array}{l}\text { G1: MTM services } \\
\text { provided as part } \\
\text { of a Medicare Part } \\
\text { D MTM program } \\
\text { by an MTM } \\
\text { Coordinator (non- } \\
\text { clinical staff) and } \\
\text { a pharmacist } \\
\text { G2: Usual care } \\
\text { provided to MTM- } \\
\text { eligible enrollees } \\
\text { who chose not to } \\
\text { participate }\end{array}$ & Cohort & $\begin{array}{l}\text { Dual eligible (\%) } \\
\text { G1: } 6 \\
\text { G2: } 25\end{array}$ & NR & $\begin{array}{l}\text { Hypertension/CHF } \\
\text { Overall: } 96.1 \\
\text { G1: } 96.5 \\
\text { G2: } 96.0 \\
\text { Hyperlipidemia } \\
\text { Overall: } 70.7 \\
\text { G1: } 75.9 \\
\text { G2: } 69.8 \\
\text { Diabetes } \\
\text { Overall: } 51.2 \\
\text { G1: } 51.4 \\
\text { G2: } 51.1\end{array}$ & NR & NR \\
\hline $\begin{array}{l}\text { Taylor, Byrd, } \\
\text { and Krueger, } \\
2003^{36}\end{array}$ & $\begin{array}{l}\text { G1: } \\
\text { Pharmaceutical } \\
\text { care provided by } \\
\text { pharmacists } \\
\text { G2: Standard care } \\
\text { without advice or } \\
\text { recommendations } \\
\text { given to patients } \\
\text { or physicians }\end{array}$ & $\begin{array}{l}\text { RCT: } \\
\text { parallel, } \\
\text { not } \\
\text { clustered } \\
\end{array}$ & $\begin{array}{l}\text { Median years of education } \\
\text { (Range) } \\
\text { Overall: NR } \\
\text { G1: } 12(4-16) \\
\text { G2: } 12(8-16) \\
\text { No insurance coverage for } \\
\text { Rx medications } \\
\text { Overall: } 17 \% \\
\text { G1: NR } \\
\text { G2: NR } \\
\text { Marital status: \% married } \\
\text { Overall: NR } \\
\text { G1: } 75.8 \\
\text { G2: } 72.2\end{array}$ & NR & $\begin{array}{l}\text { Hypertension: } \\
\text { Overall: } 51 \\
\text { Dyslipidemia: } \\
\text { Overall: } 40 \\
\text { Diabetes Mellitus: } \\
\text { Overall: } 27\end{array}$ & $\begin{array}{l}\text { Mean } \mathrm{N} \text { of } \\
\text { medications (SD) } \\
\text { Overall: NR } \\
\text { G1: } 6.3(2.2) \\
\text { G2: } 5.7(1.7)\end{array}$ & NR \\
\hline
\end{tabular}


Table E2. Other patient-level and clinical characteristics (continued)

\begin{tabular}{|c|c|c|c|c|c|c|c|}
\hline $\begin{array}{l}\text { Author, Year } \\
\text { Trial Name }\end{array}$ & $\begin{array}{l}\text { Interventions } \\
\text { and Comparator } \\
\text { Descriptions }\end{array}$ & $\begin{array}{l}\text { Study } \\
\text { Design }\end{array}$ & $\begin{array}{l}\text { Other Baseline } \\
\text { Characteristics }\end{array}$ & $\begin{array}{l}\text { Measure of Co- } \\
\text { Morbidity }\end{array}$ & $\begin{array}{l}\text { Diagnosed } \\
\text { Conditions or } \\
\text { Diseases (\%) }\end{array}$ & $\begin{array}{l}\text { Baseline Number } \\
\text { of Prescribed } \\
\text { Medications }\end{array}$ & $\begin{array}{l}\text { Other Patient } \\
\text { Clinical } \\
\text { Characteristics }\end{array}$ \\
\hline $\begin{array}{l}\text { Touchette et al., } \\
2012^{37}\end{array}$ & $\begin{array}{l}\text { G1: MTM basic } \\
\text { (comprehensive } \\
\text { medication review } \\
\text { and DRP } \\
\text { assessment) } \\
\text { G2: MTM } \\
\text { enhanced (MTM } \\
\text { plus 2-page } \\
\text { clinical summary } \\
\text { abstracted from } \\
\text { patient's medical } \\
\text { chart) } \\
\text { G3: Usual care, } \\
\text { consisting of } \\
\text { medication } \\
\text { counseling per } \\
\text { clinic's normal } \\
\text { routine but no } \\
\text { formal MTM from } \\
\text { a study } \\
\text { pharmacist }\end{array}$ & $\begin{array}{l}\text { RCT: } \\
\text { parallel, } \\
\text { not } \\
\text { clustered }\end{array}$ & NR & $\begin{array}{l}\text { Number of } \\
\text { comorbidities } \\
\text { Overall: } 4.9(1.6) \\
\text { G1: } 5.0(1.6) \\
\text { G2: } 5.0(1.6) \\
\text { G3: } 4.9(1.6)\end{array}$ & $\begin{array}{l}\text { Hypertension } \\
\text { Overall: } 90.9 \\
\text { G1: } 89.6 \\
\text { G2: } 90.8 \\
\text { G3: } 92.3 \\
\text { Dyslipidemia } \\
\text { Overall: } 77.7 \\
\text { G1: } 76.3 \\
\text { G2: } 80.7 \\
\text { G3: } 76.0 \\
\text { Arthritis } \\
\text { Overall: } 70.2 \\
\text { G1: } 68.2 \\
\text { G2: } 73.4 \\
\text { G3: } 68.8\end{array}$ & $\begin{array}{l}\text { Mean (SD) } \\
\text { Overall: } 8.0(2.4) \\
\text { G1: } 8.2(2.6) \\
\text { G2: } 7.7(2.3) \\
\text { G3: } 8.0(2.3)\end{array}$ & NR \\
\hline $\begin{array}{l}\text { Triller and } \\
\text { Hamilton, } \\
2007^{38}\end{array}$ & $\begin{array}{l}\text { G1: Visiting nurse } \\
\text { association home } \\
\text { visit services plus } \\
\text { comprehensive } \\
\text { pharmaceutical } \\
\text { care services } \\
\text { G2: Visiting nurse } \\
\text { association home } \\
\text { visit services only }\end{array}$ & $\begin{array}{l}\text { RCT: } \\
\text { parallel, } \\
\text { not } \\
\text { clustered } \\
\end{array}$ & NR & NR & $\begin{array}{l}\text { Primary or } \\
\text { secondary diagnosis } \\
\text { of heart failure } \\
\text { Overall: } 100\end{array}$ & NR & NR \\
\hline
\end{tabular}


Table E2. Other patient-level and clinical characteristics (continued)

\begin{tabular}{|c|c|c|c|c|c|c|c|}
\hline $\begin{array}{l}\text { Author, Year } \\
\text { Trial Name }^{a}\end{array}$ & $\begin{array}{l}\text { Interventions } \\
\text { and Comparator } \\
\text { Descriptions }\end{array}$ & $\begin{array}{l}\text { Study } \\
\text { Design }\end{array}$ & $\begin{array}{l}\text { Other Baseline } \\
\text { Characteristics }\end{array}$ & $\begin{array}{l}\text { Measure of Co- } \\
\text { Morbidity }\end{array}$ & $\begin{array}{l}\text { Diagnosed } \\
\text { Conditions or } \\
\text { Diseases (\%) }\end{array}$ & $\begin{array}{l}\text { Baseline Number } \\
\text { of Prescribed } \\
\text { Medications }\end{array}$ & $\begin{array}{l}\text { Other Patient } \\
\text { Clinical } \\
\text { Characteristics }\end{array}$ \\
\hline $\begin{array}{l}\text { Volume et al., } \\
2001^{39} ; \text { Kassam } \\
\text { et al., } 2001^{40} \\
\text { PREP } \\
\text { (Pharmaceutical } \\
\text { Care Research } \\
\text { and Education } \\
\text { Project) }\end{array}$ & $\begin{array}{l}\text { G1: } \\
\text { Comprehensive } \\
\text { pharmaceutical } \\
\text { care services } \\
\text { using a nine-step } \\
\text { process as } \\
\text { defined by Hepler } \\
\text { and Strand } \\
\text { provided by } \\
\text { community } \\
\text { pharmacists. } \\
\text { G2: Traditional } \\
\text { pharmacy care }\end{array}$ & $\begin{array}{l}\text { RCT: } \\
\text { cluster- } \\
\text { randomiz } \\
\text { ed }\end{array}$ & $\begin{array}{l}\text { All Overall \%'s NR } \\
\text { Education (\%) } \\
\text { Some high school } \\
\text { G1: } 46 \\
\text { G2: } 50 \\
\text { Completed high school } \\
\text { G1: } 17 \\
\text { G2: } 18 \\
\text { Some trade school/college } \\
\text { G1: } 19 \\
\text { G2: } 17 \\
\text { Completed college } \\
\text { G1: } 17 \\
\text { G2: } 14 \\
\text { Annual income (\%, CAD) } \\
<\$ 20,000 \\
\text { G1: } 40 \\
\text { G2: } 40 \\
\$ 20,000-\$ 39,000 \\
\text { G1: } 40 \\
\text { G2: } 43 \\
\$ 40,000 \text { - } \$ 59,000 \\
\text { G1: } 11 \\
\text { G2: } 11 \\
\geq \$ 60,000 \\
\text { G1: } 8 \\
\text { G2: } 5 \\
\text { Living situation (\%) } \\
\text { Live alone } \\
\text { G1: } 34 \\
\text { G2: } 29 \\
\text { Live with spouse/partner } \\
\text { G1: } 57 \\
\text { G2: } 61 \\
\text { Live with other relative } \\
\text { G1: } 7 \\
\text { G2: } 6 \\
\text { Live with unrelated person } \\
\text { G1: } 2 \\
\text { G2: } 2\end{array}$ & $\begin{array}{l}\text { Mean number of } \\
\text { conditions (SD) } \\
\text { G1: } 3.3(1.7) \text { based } \\
\text { on study interview } \\
\text { and } 10(4.8) \text { based } \\
\text { on data collected by } \\
\text { treatment } \\
\text { pharmacist. } \\
\text { G2: NR }\end{array}$ & NR & $\begin{array}{l}\text { Mean (SD) } \\
\text { Overall: NR } \\
\text { G1: } 4.7(2.8) \\
\text { G2: } 3.9(2.5) \\
p<0.05\end{array}$ & NR \\
\hline
\end{tabular}


Table E2. Other patient-level and clinical characteristics (continued)

\begin{tabular}{|c|c|c|c|c|c|c|c|}
\hline $\begin{array}{l}\text { Author, Year } \\
\text { Trial Name }^{a}\end{array}$ & $\begin{array}{l}\text { Interventions } \\
\text { and Comparator } \\
\text { Descriptions }\end{array}$ & $\begin{array}{l}\text { Study } \\
\text { Design }\end{array}$ & $\begin{array}{l}\text { Other Baseline } \\
\text { Characteristics }\end{array}$ & $\begin{array}{l}\text { Measure of Co- } \\
\text { Morbidity }\end{array}$ & $\begin{array}{l}\text { Diagnosed } \\
\text { Conditions or } \\
\text { Diseases (\%) } \\
\end{array}$ & $\begin{array}{l}\text { Baseline Number } \\
\text { of Prescribed } \\
\text { Medications }\end{array}$ & $\begin{array}{l}\text { Other Patient } \\
\text { Clinical } \\
\text { Characteristics }\end{array}$ \\
\hline $\begin{array}{l}\text { Welch et al., } \\
2009^{41}\end{array}$ & $\begin{array}{l}\text { G1: MTM program } \\
\text { provided to home- } \\
\text { based } \\
\text { beneficiaries as } \\
\text { part of a Medicare } \\
\text { Part D MTM } \\
\text { program } \\
\text { G2: No-MTM } \\
\text { control group } \\
\text { (voluntary opt-out) }\end{array}$ & Cohort & NR & NR & NR & $\mathrm{NR}$ & $\begin{array}{l}\text { Mean Chronic } \\
\text { Disease Score (SD) } \\
\text { (ranges from 0-35, } \\
\text { with larger scores } \\
\text { indicating increasing } \\
\text { burden of chronic } \\
\text { diseases under } \\
\text { treatment) } \\
\text { Overall: NR } \\
\text { G1: } 8.8 \text { (3.1) } \\
\text { G2: } 8.2 \text { (3.5) } \\
\text { p: } 0.016 \\
\text { NOTE: Difference } \\
\text { represents, on } \\
\text { average, less than } \\
\text { one additional } \\
\text { chronic disease per } \\
\text { patient } \\
\text { Median (IQR) } \\
\text { baseline medication } \\
\text { cost (\$) } \\
\text { G1: } 3149 \text { (2378 to } \\
4806) \\
\text { G2: } 3186 \text { (2363 to } \\
5123 \text { ) } \\
\text { Mean baseline } \\
\text { medication cost ( } \$ \text { ) } \\
\text { (no SD reported) } \\
\text { G1: } 4465 \\
\text { G2: } 5197 \\
\text { p: } 0.525\end{array}$ \\
\hline
\end{tabular}


Table E2. Other patient-level and clinical characteristics (continued)

\begin{tabular}{|c|c|c|c|c|c|c|c|}
\hline $\begin{array}{l}\text { Author, Year } \\
\text { Trial Name }\end{array}$ & $\begin{array}{l}\text { Interventions } \\
\text { and Comparator } \\
\text { Descriptions }\end{array}$ & $\begin{array}{l}\text { Study } \\
\text { Design }\end{array}$ & $\begin{array}{l}\text { Other Baseline } \\
\text { Characteristics }\end{array}$ & $\begin{array}{l}\text { Measure of Co- } \\
\text { Morbidity }\end{array}$ & $\begin{array}{l}\text { Diagnosed } \\
\text { Conditions or } \\
\text { Diseases (\%) } \\
\end{array}$ & $\begin{array}{l}\text { Baseline Number } \\
\text { of Prescribed } \\
\text { Medications }\end{array}$ & $\begin{array}{l}\text { Other Patient } \\
\text { Clinical } \\
\text { Characteristics }\end{array}$ \\
\hline $\begin{array}{l}\text { Williams et al., } \\
2004^{42}\end{array}$ & $\begin{array}{l}\text { G1: Medication } \\
\text { review and } \\
\text { optimization of } \\
\text { patient's } \\
\text { medication } \\
\text { regimen } \\
\text { conducted by an } \\
\text { interdisciplinary } \\
\text { medication } \\
\text { adjustment team } \\
\text { in addition to } \\
\text { usual medical } \\
\text { care and "Bound } \\
\text { for Health" } \\
\text { booklet. } \\
\text { G2: Usual medical } \\
\text { care plus } \\
\text { provision of } \\
\text { "Bound for } \\
\text { Health" booklet }\end{array}$ & $\begin{array}{l}\text { RCT: } \\
\text { parallel, } \\
\text { not } \\
\text { clustered } \\
\end{array}$ & $\begin{array}{l}\text { Education (\%) } \\
\text { Had not completed high } \\
\text { school } \\
\text { G1: } 33.3 \\
\text { G2: } 32.5 \\
\text { High school or some college } \\
\text { G1: } 25.4 \\
\text { G2: } 19.5 \\
\text { Completed college } \\
\text { G1: } 41.3 \\
\text { G2: } 48.1 \\
\text { Marital status (\%) } \\
\text { Married } \\
\text { G1: } 47.6 \\
\text { G2: } 53.2 \\
\text { Living Alone } \\
\text { G1: } 38.1 \\
\text { G2: } 33.8\end{array}$ & NR & NR & $\begin{array}{l}\text { G1: } 6.6(1.8) \\
\text { G2: } 7.7(2.3)\end{array}$ & $\begin{array}{l}\text { Baseline number of } \\
\text { non-prescription } \\
\text { drugs } \\
\text { G1: } 5.1(3.1) \\
\text { G2: } 4.6(2.5)\end{array}$ \\
\hline
\end{tabular}


Table E2. Other patient-level and clinical characteristics (continued)

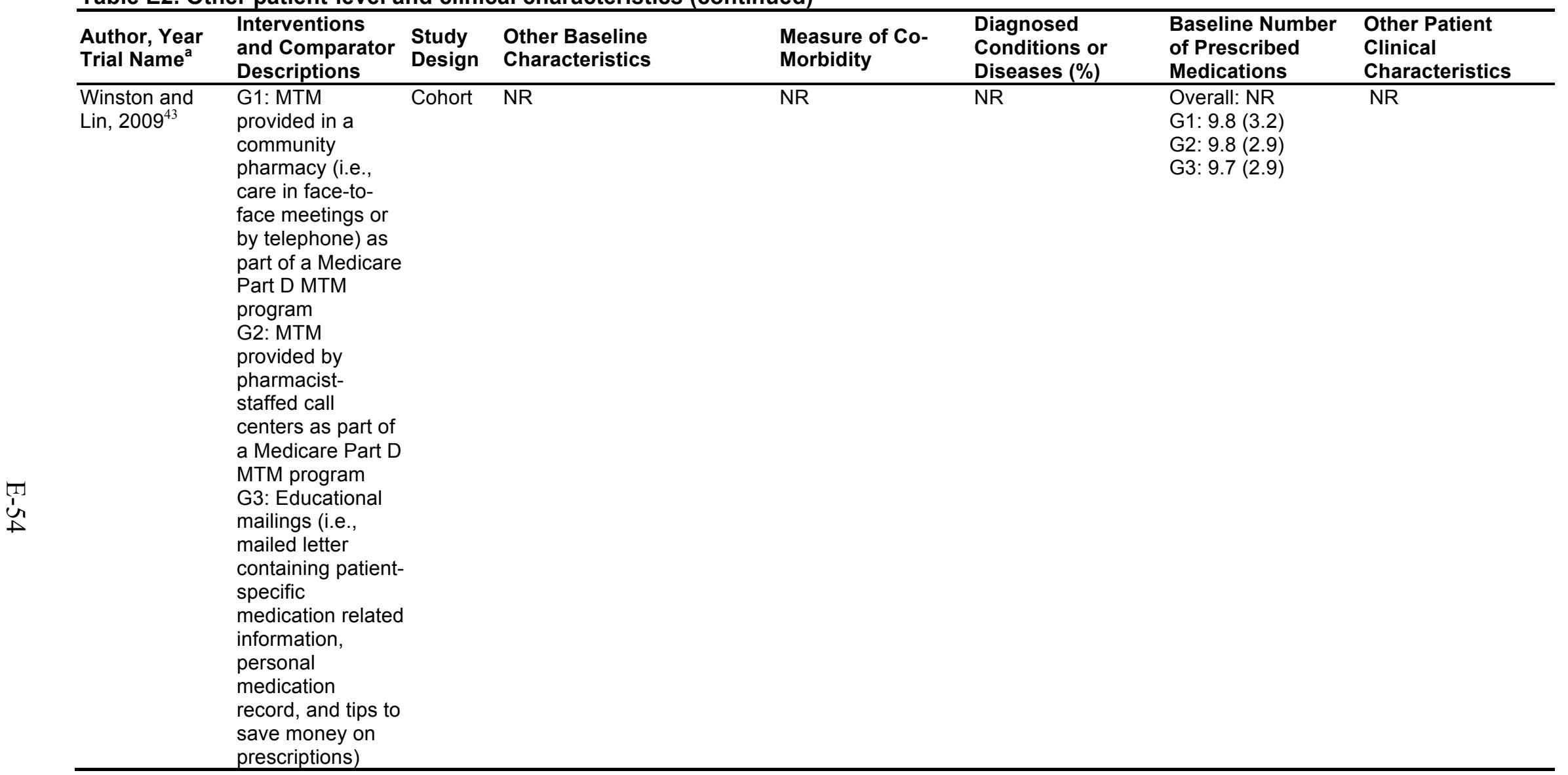


Table E2. Other patient-level and clinical characteristics (continued)

\begin{tabular}{|c|c|c|c|c|c|c|c|}
\hline $\begin{array}{l}\text { Author, Year } \\
\text { Trial Name }^{\mathrm{a}}\end{array}$ & $\begin{array}{l}\text { Interventions } \\
\text { and Comparator } \\
\text { Descriptions }\end{array}$ & $\begin{array}{l}\text { Study } \\
\text { Design }\end{array}$ & $\begin{array}{l}\text { Other Baseline } \\
\text { Characteristics }\end{array}$ & $\begin{array}{l}\text { Measure of Co- } \\
\text { Morbidity }\end{array}$ & $\begin{array}{l}\text { Diagnosed } \\
\text { Conditions or } \\
\text { Diseases (\%) } \\
\end{array}$ & $\begin{array}{l}\text { Baseline Number } \\
\text { of Prescribed } \\
\text { Medications }\end{array}$ & $\begin{array}{l}\text { Other Patient } \\
\text { Clinical } \\
\text { Characteristics }\end{array}$ \\
\hline $\begin{array}{l}\text { Witry, Doucette, } \\
\text { and Gainer, } \\
2011^{44}\end{array}$ & $\begin{array}{l}\text { G1: PCM } \\
\text { provided by } \\
\text { community } \\
\text { pharmacists to } \\
\text { lowa Medicaid } \\
\text { enrollees } \\
\text { G2: PCM } \\
\text { provided by } \\
\text { community } \\
\text { pharmacists to } \\
\text { patients with } \\
\text { private individual- } \\
\text { group insurance }\end{array}$ & Cohort & NR & NR & NR & $\begin{array}{l}\text { Mean (SD) } \\
\text { Overall: NR } \\
\text { G1: } 7.9(3.8) \\
\text { G2: } 4.7(2.2) \\
\text { p: }<0.001\end{array}$ & $\mathrm{NR}$ \\
\hline
\end{tabular}

Abbreviations: $\mathrm{BMI}=$ body mass index; $\mathrm{CMR}=$ comprehensive medication review; $\mathrm{DRP}=$ drug regimen problem; $\mathrm{DTP}=\mathrm{drug}$ therapy problem; $\mathrm{dx}=$ diagnosis; $\mathrm{G}=$ group;

HEDIS = Healthcare Effectiveness Data and Information Set; HMR = home medication review; ITT = intention-to-treat; LTFU = lost to follow-up; MTM = medication therapy

management; MTMP = medication therapy management program; $\mathrm{N}=$ sample or group size; $\mathrm{NA}=$ not applicable; $\mathrm{NR}=$ not reported; $\mathrm{NRCT}=$ non-randomized controlled trial;

$\mathrm{OR}=$ odds ratio $\mathrm{PA}=$ physician assistant $\mathrm{PCM}=$ pharmaceutical case management; $\mathrm{PDP}=$ prescription drug plan; $\mathrm{RCT}=$ randomized controlled trial; $\mathrm{SD}=$ standard deviation; 
Table E3. Key Question 1: Components and features of medication therapy management interventions: Broadly focused studies

\section{Author, Year}

State (Province) or

\section{Country}

Bernsten et al., $2001^{1} ; \quad$ Intervention: Structured pharmaceutical care

Sturgess et at., $2003^{2}$

7 European countries: Denmark, Germany, The Netherlands, Northern Ireland, Portugal, Republic details regarding pharmacist access to clinica of Ireland, and Sweden

simplifying drug regimens in collaboration with the patient's general practitioner was structured using drug use profiles, however specific details regarding the communication between pharmacist and physicians was NR.

Chrischilles et al., 2004 ${ }^{7} \quad$ Intervention: Pharmaceutical case management Claims data or pharmacy
provided by pharmacists.

lowa, US
Level of Integration with Usual Care: Pharmacist access to clinical information in medical record

NR. Pharmacist written communication with physicians about problems identified. A collaboratively determined action plan can be implemented by the pharmacist without requiring a patient visit to a physician.
Setting: Community pharmacy.

Method of Identifying Patients for Receipt of

Personal recruitment by pharmacists within pharmacy, or via GP

records

prescription profile records

Mode of delivery: Face-toface

Frequency and interval of follow-up: Initial

consultations with followup contacts as needed and routine re-assessments every 6 months by design.
Health plan intervention that ncluded a payment reform to allow for reimbursement of multiple participating pharmacies and providers across different systems.

Reimbursement characteristics: provided as a Medicaid benefit using state and federal matching 
Table E3. Key Question 1: Components and features of medication therapy management interventions: Broadly focused studies (continued)

Author, Year

State (Province) or

Intervention and Level of Integration with Usual Care

\section{Country}

Christensen et al., $2007^{8} \quad$ Intervention: Medication therapy management services designed by a health plan for its

North Carolina, US beneficiaries and provided by either community pharmacists or medical clinic-based pharmacists.

Level of Integration with Usual Care: Pharmacist access to clinical information in the medical record NR. Pharmacist contacted prescribing physicians to discuss drug therapy problems and implemented any resulting approved action plan. Encara Practice System provided by onsite Midwest, US health maintenance organization staff pharmacists.

Level of Integration with Usual Care: Pharmacist access to clinical information in the medical record NR. Information to and consultation with physicians on behalf of patients mentioned but specific operational details NR.

\section{Method of Identifying \\ Patients for Receipt of} MTM Services

Eligible patients identified through claims data, then recruited through a letter sent inviting them to participate.

\section{Setting, Mode of}

Delivery, Frequency and Interval of Followup

Setting: Some patients received services within

their medical clinic, some received services within a community pharmacy setting.

Mode of delivery: Face-to face and telephone

Frequency and interval of follow-up: study designed as one initial visit and one follow-up, $37.5 \%$ of

enrolled patients received follow-up contact.

\section{Claims data or pharmacy Setting: Pharmacies}

prescription profile records located within clinics to identify eligible participants who were then Mode of delivery: Face-toinvited by letter.

\section{Health maintenance}$$
\text { (eter. }
$$

face organization with clinics and on-site pharmacies.

Reimbursement for services: NR
Health plan intervention involving multiple health

Reimbursement characteristics: Pharmacists compensated through studyrelated funding (e.g., grant).

Frequency and interval of

follow-up: NR 
Table E3. Key Question 1: Components and features of medication therapy management interventions: Broadly focused studies (continued)

Author, Year

State (Province) or

Intervention and Level of Integration with Usual Care

Country

Fischer et al., $2002^{1}$

Minnesota, US

Intervention: Pharmaceutical care based on the
Encara Practice System provided by
Encara Practice System provided by

pharmacists.

Level of Integration with Usual Care: Pharmacist access to clinical information in the medical

record NR. Communication with the patient's physician about drug therapy problems identified, but specific operational details NR.

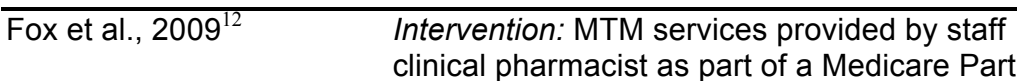

Florida, US

clinical pharmacist as part of a Medicare Part

evel of Integration with Usual Care: Pharmacist

\section{Method of Identifying \\ Patients for Receipt of MTM Services}

\section{Setting, Mode of}

Delivery, Frequency and Health Care System and Interval of Followup

Claims data or pharmacy

profle records located within clinics and

free-standing pharmacies

participants.
Mode of delivery: Face-toface

Frequency and interval of

follow-up: With each

prescription refill (as

designed); actual

frequency and interval NR had access to clinical information in the medical record, including laboratory data. Pharmacist documented findings on a form, which was sent to the patient's physician.
Claims data or pharmacy to target eligible participants. pharmacies used.

Mode of delivery: Primarily Plan.

telephone, supplemented

by mailed written

Setting: Health plan Mixed-staff model health

pharmacy, unclear whether maintenance organization

a single centralized center that combines pharmacist

or outpatient clinic-based services, primary care, and

specialty medical care with a

Reimbursement

materials.

characteristics: Medicare

Part D drug benefit

Frequency and interval of

follow-up: Initial

consultation, and up to 3

follow-up contacts if a drug

therapy problem identified

or based on clinical need. 
Table E3. Key Question 1: Components and features of medication therapy management interventions: Broadly focused studies (continued)

Author, Year

State (Province) or

Intervention and Level of Integration with Usual Care

Country

Hanlon et al., $1996^{14}$

North Carolina, US

Level of Integration with Usual Care:
Pharmacists had access to clinical in

Pharmacists had access to clinical information

in medical record. Pharmacist recommendations

were presented orally and in writing to the

patient's primary physician, pharmacist

reinforced and amplified the primary physician's

instructions.

\begin{tabular}{ll}
\hline Isetts et al., $2008^{16}$ & Intervention: Medication therapy management \\
services provided by staff pharmacists, including
\end{tabular}

Minnesota, US the establishment of goals of therapy.

Level of Integration with Usual Care: Pharmacists had access to clinical information

in medical record. Pharmacist urgently consulted with primary care provider for potentially harmful drug therapy problems, but details regarding routine communication were NR.

\section{Method of Identifying \\ Patients for Receipt of \\ MTM Services}

\section{Setting, Mode of}

Delivery, Frequency and Health Care System and Interval of Followup

$\begin{array}{ll}\text { Computerized and manual } & \text { Setting: Outpatient medica } \\ \text { chart audits to identify } & \text { clinic }\end{array}$

eligible subjects

Mode of delivery: Face-to-

face

\section{Reimbursement}

characteristics: NR

Frequency and Interval of

Follow-up: NR

Claims data or pharmacy Setting: Integrated health prescription profile records care delivery system. used to identify eligible

participants who were then Mode of delivery: Face-toinvited by letter and face

Integrated health system with an established pharmaceutical care

provider referral.

program involving

Frequency and Interval of specific pharmaceutical care Follow-up: NR (at least 1 documentation system. follow-up visit was required for inclusion in the evaluation)
Reimbursement characteristics: Costs of providing services were funded through research grants, demonstration projects, third-party payer pilot programs, and internal health system funding. 
Table E3. Key Question 1: Components and features of medication therapy management interventions: Broadly focused studies (continued)

Author, Year

State (Province) or

Intervention and Level of Integration with Usual Care

\section{Method of Identifying \\ Patients for Receipt of}

Setting, Mode of

Setting, Mode of
Delivery, Frequency and Care System and

\section{Country}

Jameson, VanNoord, and

Vanderwoud, $1995^{17}$

Intervention: Pharmacotherapy consultation and followup provided by clinical ambulatory care

Interval of Followup Services

(Medting: Outpatient medical pharmacist.

至

Single family health clinic

Michigan, US

were randomly screened

that was part of a family

Level of Integration with Usual Care: Pharmacist medication outcomes. access to clinical information in the medical

record was NR. Pharmacist met with treating

physician to discuss findings and new regimen

was developed collaboratively with the

physician.

Jeong et al., $2007^{18}$

California, US

bे

Intervention: MTM services provided by

ambulatory care pharmacists and health care

support staff.

Level of Integration with Usual Care: Pharmacist access to clinical information in the medical record was NR. Specific details regarding communication with treating physician NR.

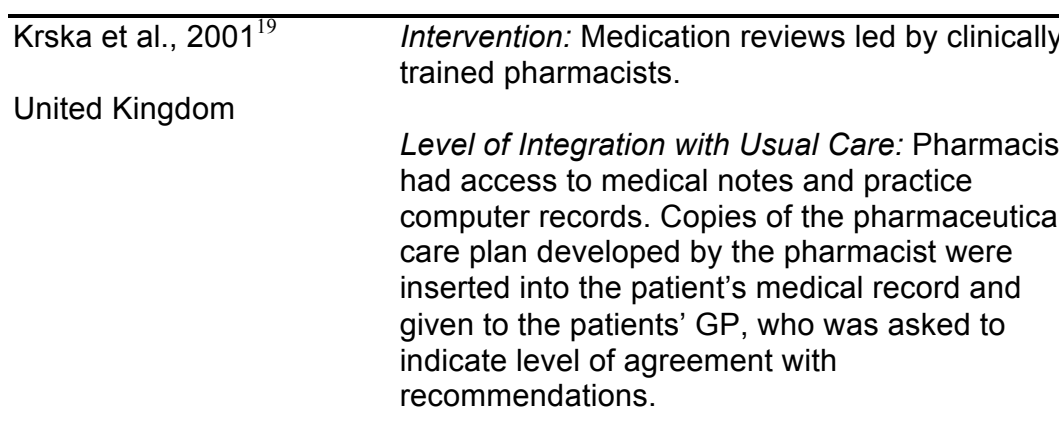

for risks of adverse

Mode of Delivery: Face-to- program.

face and telephone

\section{Reimbursement}

Frequency and interval of characteristics: NR

Follow-up: 1 initial visit and

1 follow-up visit 1 month

later (by design); actual

frequency and interval of follow-up NR.

Setting: Integrated health Integrated health care

care delivery system

delivery system providing

Medicare Part D MTM

Mode of delivery: Primarily services to eligible

telephone

beneficiaries

Frequency and interval of

Follow-up: NR

Reimbursement characteristics: Medicare

Part D drug benefit.

Provider referral required Setting: Home visits

but enrollment limited to 70

patients from each

Mode of Delivery: Initial

General medicine clinics that were part of a single payer

health care system.

consultation was face-to-

face; follow-up consultation Reimbursement

NR

characteristics: NR

Frequency and Interval of

Follow-up: Two contacts, 3

months apart as designed;

actual frequency and

interval NR 
Table E3. Key Question 1: Components and features of medication therapy management interventions: Broadly focused studies (continued)

\begin{tabular}{|c|c|c|c|c|}
\hline $\begin{array}{l}\text { Author, Year } \\
\text { State (Province) or } \\
\text { Country }\end{array}$ & $\begin{array}{l}\text { Intervention and Level of Integration with } \\
\text { Usual Care }\end{array}$ & $\begin{array}{l}\text { Method of Identifying } \\
\text { Patients for Receipt of } \\
\text { MTM Services }\end{array}$ & $\begin{array}{l}\text { Setting, Mode of } \\
\text { Delivery, Frequency and } \\
\text { Interval of Followup }\end{array}$ & $\begin{array}{l}\text { Health Care System and } \\
\text { Reimbursement Context }\end{array}$ \\
\hline $\begin{array}{l}\text { Malone et al., } 2000^{20} ; \\
\text { Ellis et al., } 2000^{21} \\
\text { (interventions); } \\
\text { Malone et al., } 2001^{22} \\
\text { (detailed QOL outcomes); } \\
\text { Ellis et al., } 2000^{23} \\
\text { (dyslipidemia subgroup } \\
\text { intermediate and utilization } \\
\text { outcomes) } \\
\text { Multiple states, US }\end{array}$ & $\begin{array}{l}\text { Intervention: Pharmaceutical care provided by } \\
\text { clinical pharmacists practicing according to } \\
\text { scope of practice within their respective health } \\
\text { care facilities. } \\
\text { Level of Integration with Usual Care: Pharmacist } \\
\text { had access to medical record information. } \\
\text { Pharmacist communication with primary care } \\
\text { physician or other prescribers NR. }\end{array}$ & $\begin{array}{l}\text { Pharmacy prescription } \\
\text { records to identify patients } \\
\text { at high risk for drug-related } \\
\text { problems. }\end{array}$ & $\begin{array}{l}\text { Setting: Outpatient medical } \\
\text { clinic } \\
\text { Mode of Delivery: Face-to- } \\
\text { face }(76.6 \% \text { of contacts) } \\
\text { and telephone }(23.4 \%) \\
\text { Frequency and Interval of } \\
\text { Follow-Up: At least } 3 \text { visits } \\
\text { over } 12 \text { months as } \\
\text { designed. Actual } \\
\text { frequency: mean (SD) } \\
\text { number of visits was } 3.5 \\
\text { (2.3). } 27.7 \% \text { did not } \\
\text { complete the minimum } \\
\text { number of visits (3) as } \\
\text { designed }\end{array}$ & $\begin{array}{l}\text { Multiple Veterans Health } \\
\text { Administration Medical } \\
\text { Centers with established } \\
\text { ambulatory clinical } \\
\text { pharmacy services } \\
\text { Reimbursement } \\
\text { characteristics: Services } \\
\text { provided as part of patient's } \\
\text { VHA health care benefits }\end{array}$ \\
\hline $\begin{array}{l}\text { Moczygemba et al., } 2011^{25} \\
\text { Moczygemba et al., } 2008^{26} \\
\text { Texas, US }\end{array}$ & $\begin{array}{l}\text { Intervention: Medication therapy management } \\
\text { services provided by clinical pharmacists or a } \\
\text { managed care pharmacy resident based on the } \\
\text { American Pharmacists Association and National } \\
\text { Association of Chain Drug Stores Foundation } \\
\text { MTM framework. } \\
\text { Level of Integration with Usual Care: } \\
\text { Pharmacist had access to medical record } \\
\text { information, including laboratory information. } \\
\text { Patients are encouraged to share the Personal } \\
\text { Medication Record and Medication Action Plan } \\
\text { developed in the course of MTM consultation } \\
\text { with their health care providers and patients are } \\
\text { requested to contact their physicians regarding } \\
\text { pharmacist's recommendations. Copies are kept } \\
\text { in the patient's MTM file, but no MTM } \\
\text { documentation goes back to the patient's } \\
\text { medical record. }\end{array}$ & $\begin{array}{l}\text { Eligible patients are } \\
\text { identified quarterly and } \\
\text { mailed MTM Program } \\
\text { information and instructions } \\
\text { for opting in to the } \\
\text { program. }\end{array}$ & $\begin{array}{l}\text { Setting: centralized MTM } \\
\text { program } \\
\text { Mode of Delivery: } \\
\text { Telephone } \\
\text { Frequency and Interval of } \\
\text { Follow-Up: one initial } \\
\text { consultation by design with } \\
\text { follow-up scheduled as } \\
\text { needed. Actual frequency } \\
\text { and interval of follow-up } \\
\text { NR. }\end{array}$ & $\begin{array}{l}\text { Health plan intervention } \\
\text { provided by a regional } \\
\text { Medicare Part D MTM } \\
\text { Provider. } \\
\text { Reimbursement } \\
\text { characteristics: Medicare } \\
\text { Part D drug benefit. }\end{array}$ \\
\hline
\end{tabular}


Table E3. Key Question 1: Components and features of medication therapy management interventions: Broadly focused studies (continued)

\begin{tabular}{|c|c|c|c|c|}
\hline $\begin{array}{l}\text { Author, Year } \\
\text { State (Province) or } \\
\text { Country }\end{array}$ & $\begin{array}{l}\text { Intervention and Level of Integration with } \\
\text { Usual Care }\end{array}$ & $\begin{array}{l}\text { Method of Identifying } \\
\text { Patients for Receipt of } \\
\text { MTM Services }\end{array}$ & $\begin{array}{l}\text { Setting, Mode of } \\
\text { Delivery, Frequency and } \\
\text { Interval of Followup }\end{array}$ & $\begin{array}{l}\text { Health Care System and } \\
\text { Reimbursement Context }\end{array}$ \\
\hline $\begin{array}{l}\text { Pindolia et al., } 2009^{30} \\
\text { Michigan, US }\end{array}$ & $\begin{array}{l}\text { Intervention: Medication therapy management } \\
\text { services provided as part of a Medicare Part D } \\
\text { MTM program by pharmacy care management } \\
\text { clinical pharmacists. } \\
\text { Level of Integration with Usual Care: } \\
\text { Pharmacists had access to clinical information } \\
\text { in the medical record. Communications with } \\
\text { physicians were by telephone, face-to-face, or } \\
\text { e-mail. }\end{array}$ & $\begin{array}{l}\text { Monthly query of clinical } \\
\text { care management systems } \\
\text { for eligible patients with } \\
\text { subsequent letter mailed } \\
\text { and follow-up phone call to } \\
\text { enroll patients. }\end{array}$ & $\begin{array}{l}\text { Setting: Integrated } \\
\text { healthcare delivery system } \\
\text { Mode of Delivery: } \\
\text { telephone } \\
\text { Frequency and Interval of } \\
\text { Follow-Up: NR }\end{array}$ & $\begin{array}{l}\text { Health plan intervention } \\
\text { within an Integrated health } \\
\text { system with an established } \\
\text { pharmaceutical care } \\
\text { program. } \\
\text { Reimbursement } \\
\text { characteristics: Medicare } \\
\text { Part D drug benefit. }\end{array}$ \\
\hline $\begin{array}{l}\text { Sellors et al., } 2003^{33} \\
\text { Ontario, Canada }\end{array}$ & $\begin{array}{l}\text { Intervention: Clinical pharmacy consultations } \\
\text { provided to patients by community pharmacists. } \\
\text { Level of Integration with Usual Care: } \\
\text { Pharmacist access to clinical information in } \\
\text { medical record was NR. Pharmacists provided a } \\
\text { consultation letter to physician and } \\
\text { subsequently met with physician to review the } \\
\text { letter. They met again in } 3 \text { months to discuss } \\
\text { progress in implementing recommendations. }\end{array}$ & $\begin{array}{l}\text { About } 20 \text { randomly chosen } \\
\text { eligible senior citizens per } \\
\text { practice were recruited by } \\
\text { the office staff of the } \\
\text { practice, selection process } \\
\text { for recruitment not } \\
\text { reported. }\end{array}$ & $\begin{array}{l}\text { Setting: Outpatient medical } \\
\text { clinic } \\
\text { Mode of Delivery: Face-to- } \\
\text { face and telephone } \\
\text { Frequency and Interval of } \\
\text { Follow-Up: Initial contact } \\
\text { plus } 2 \text { follow-up contacts at } \\
1 \text { and } 3 \text { months as } \\
\text { designed. Actual frequency } \\
\text { and interval of contact NR. }\end{array}$ & $\begin{array}{l}\text { Family medicine practice } \\
\text { settings within a single- } \\
\text { payer health care system. } \\
\text { Reimbursement } \\
\text { characteristics: NR }\end{array}$ \\
\hline $\begin{array}{l}\text { Sidel et al., } 1990^{34} \\
\text { New York, US }\end{array}$ & $\begin{array}{l}\text { Intervention: Home visits by pharmacists to } \\
\text { identify and correct problems associated with } \\
\text { medication use. } \\
\text { Level of Integration with Usual Care: Pharmacist } \\
\text { access to clinical information in medical record } \\
\text { was NR. No information about communication } \\
\text { with providers was reported. }\end{array}$ & $\begin{array}{l}\text { Study population identified } \\
\text { from a combination of the } \\
\text { following: Medicare } \\
\text { recipients living in the } \\
\text { region, Senior Centers, } \\
\text { houses of worship, Meals- } \\
\text { on-Wheels, hospital } \\
\text { admissions records and } \\
\text { voter registration rolls. }\end{array}$ & $\begin{array}{l}\text { Setting: Community setting } \\
\text { Mode of delivery: home } \\
\text { visits and telephone } \\
\text { Frequency and Interval of } \\
\text { Follow-Up: } 2 \text { visits over a } \\
12 \text { month period as } \\
\text { designed. Actual frequency } \\
\text { and interval of follow-up } \\
\text { NR. }\end{array}$ & $\begin{array}{l}\text { Implemented outside the } \\
\text { health care system through } \\
\text { a multidisciplinary research } \\
\text { program on aging. } \\
\text { Reimbursement } \\
\text { characteristics: NR }\end{array}$ \\
\hline
\end{tabular}


Table E3. Key Question 1: Components and features of medication therapy management interventions: Broadly focused studies (continued)

\begin{tabular}{|c|c|}
\hline Author, Year & \\
\hline $\begin{array}{l}\text { State (Province) or } \\
\text { Country }\end{array}$ & $\begin{array}{l}\text { Intervention and Level of Integration with } \\
\text { Usual Care }\end{array}$ \\
\hline Staresinic, $2007^{35}$ & $\begin{array}{l}\text { Intervention: Medication therapy management } \\
\text { services provided as part of a Medicare Part } D\end{array}$ \\
\hline Wisconsin, US & $\begin{array}{l}\text { MTM program by an MTM Coordinator (non- } \\
\text { clinical staff) and a pharmacist. }\end{array}$ \\
\hline & $\begin{array}{l}\text { Level of Integration with Usual Care: } \\
\text { Pharmacists request lab data from participants; } \\
\text { access to clinical information in medical records } \\
\text { was NR. Pharmacists send a tailored letter by } \\
\text { fax to each of the patient's health care } \\
\text { providers. }\end{array}$ \\
\hline
\end{tabular}

\section{Method of Identifying \\ Patients for Receipt of MTM Services}

Setting, Mode of
Delivery, Frequen

Interval of Followup

Pharmacy claims based Setting: centralized MTM

algorithm identifies eligible program

beneficiaries with invitation

letters mailed within 2

Mode of Delivery:

weeks of identifying

telephone

eligibility.

Frequency and Interval of

Follow-Up: One initial

contact and one follow-up

contact at 3 months as

designed. Actual frequency

and interval of follow-up

NR.

Taylor, Byrd, and Krueger, Intervention: Pharmaceutical care provided by $2003^{36}$

pharmacists.

Patients were identified by

the participating

pharmacists through

Setting

clinic

Level of Integration with Usual Care: Pharmacist manual evaluation of clinic Mode access to clinical information in medical record medical records and review face

was NR, but visits with pharmacist occurred 20 of computerized medical

minutes before seeing the physician in the same records in physician

clinic. Recommendations to physicians were

offices.

communicated through discussions or progress

notes.
Frequency and Interval of

Follow-Up: Before each

scheduled physician visit

by design. Actual

frequency and follow-up

interval NR.
Health Care System and Reimbursement Context

Health plan intervention provided by a regional Medicare Part D MTM Provider

Reimbursement Raracteristics: Medicare Part D drug benefit.

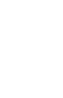


Table E3. Key Question 1: Components and features of medication therapy management interventions: Broadly focused studies (continued)

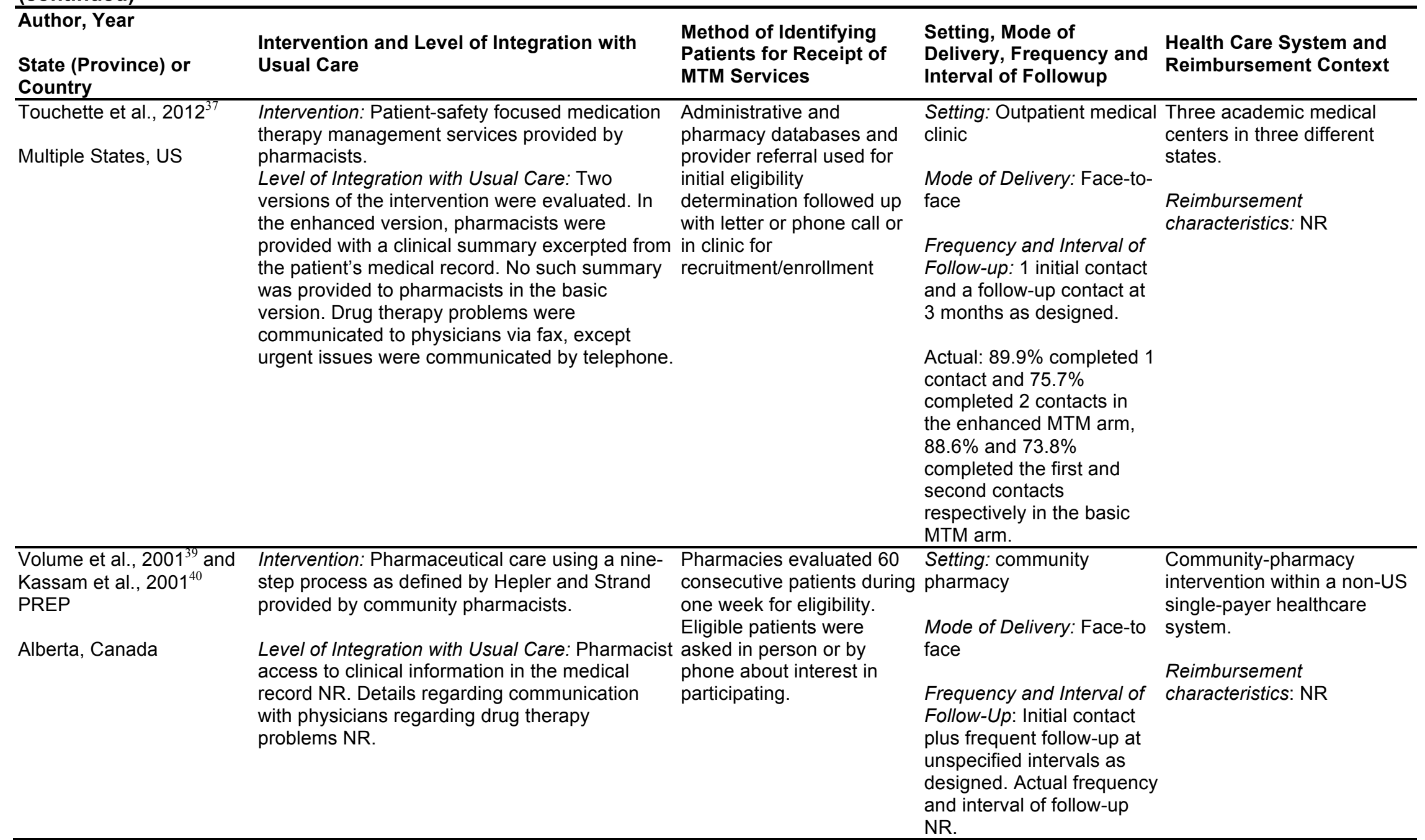


Table E3. Key Question 1: Components and features of medication therapy management interventions: Broadly focused studies (continued)

Author, Year

\section{State (Province) or \\ Country}

Welch et al., $2009^{41}$

Colorado, US

\section{Intervention and Level of Integration with} Usual Care

Intervention: Medication therapy management services provided by clinical pharmacists as pa of a Medicare Part D MTM program.

Level of Integration with Usual Care:

Pharmacists had access to clinical information

in the medical record. Pharmacists forwarded copies of consultation notes to providers and also placed a copy in the patient's medical record.

\section{Method of Identifying \\ Patients for Receipt of \\ MTM Services}

Medicare beneficiaries

identified as eligible usin
computerized system.

\section{Mode of Delivery: \\ telephone}

Frequency and interval of Follow-Up: Initial consult with follow-up depending

on clinical situation as

designed. Actual frequency

and interval of follow-up

NR.

Williams et al., $2004^{42}$

North Carolina, US provided by a consulting pharmacist.

Patients were recruited

from practices and through clinic community print and radio

\section{Intervention: Medication review and optimization}

Setting: Outpatient medical

\author{
Mode
}

Level of Integration with Usual Care: Pharmacist advertisements and mass
had access to clinical information in the medical mailings, and presentations face record. A MAT comprised of a physician, nurse, to community groups.

and consultant pharmacist met to discuss

pharmacy recommendations.

Frequency and Interval of

Follow-Up: Initial contact with follow-up contact as needed as designed.

Actual frequency and

interval of follow-up contact NR

Winston and Lin, 2009 ${ }^{43}$ Intervention: Medication therapy management services provided by either community pharmacists or call center pharmacists as part

Health plan used pharmacy Setting: community prescription profile records pharmacy and centralized identify eligible patients.

Multiple States, US of a Medicare Part D MTM Program. Information on eligible patients was

Level of Integration with Usual Care: Pharmacist communicated to access to clinical information in the medical record was NR. Pharmacist contacted

prescribers on behalf of the patients by phone or fax for medication adjustments related to cost or pharmacies by fax or email.
Health Care System and Reimbursement Context

Group-model health maintenance organization using a centralized clinical pharmacy call center.

Reimbursement Reracteristics: Medicare Part D drug benefit. safety.

Method of Delivery: Faceto-face or telephone Health plan intervention provided by a national Medicare Part D MTM provider.

Reimbursement characteristics: Medicare Frequency and Interval of Part D drug benefit Follow-up: NR

medicine clinic of

Reimbursement

characteristics: NR 
Table E3. Key Question 1: Components and features of medication therapy management interventions: Broadly focused studies (continued)

\begin{tabular}{|c|c|c|c|c|}
\hline $\begin{array}{l}\text { Author, Year } \\
\text { State (Province) or } \\
\text { Country }\end{array}$ & $\begin{array}{l}\text { Intervention and Level of Integration with } \\
\text { Usual Care }\end{array}$ & $\begin{array}{l}\text { Method of Identifying } \\
\text { Patients for Receipt of } \\
\text { MTM Services }\end{array}$ & $\begin{array}{l}\text { Setting, Mode of } \\
\text { Delivery, Frequency and } \\
\text { Interval of Follow-up }\end{array}$ & $\begin{array}{l}\text { Health Care System and } \\
\text { Reimbursement Context }\end{array}$ \\
\hline $\begin{array}{l}\text { Witry, Doucette, and } \\
\text { Gainer, } 2011^{44}\end{array}$ & $\begin{array}{l}\text { Intervention: Pharmaceutical case management } \\
\text { provided by community pharmacists. }\end{array}$ & $\begin{array}{l}\text { Health plan used pharmacy } \\
\text { prescription profile records } \\
\text { identify eligible patients. }\end{array}$ & $\begin{array}{l}\text { Setting: community } \\
\text { pharmacy }\end{array}$ & $\begin{array}{l}\text { Health plan intervention } \\
\text { executed by pharmacies that } \\
\text { had previously participated }\end{array}$ \\
\hline lowa, US & $\begin{array}{l}\text { Level of integration with Usual Care: Pharmacist } \\
\text { access to clinical information in the medical } \\
\text { record was NR. Pharmacists faxed a one-page } \\
\text { summary of findings to physician. }\end{array}$ & $\begin{array}{l}\text { This was sent to each } \\
\text { pharmacy. The health plan } \\
\text { also sent letters to inform } \\
\text { eligible patients about the } \\
\text { PCM service benefit, and } \\
\text { an article about PCM } \\
\text { appeared in the health plan } \\
\text { newsletter. Pharmacies } \\
\text { also sent letters and } \\
\text { telephoned eligible } \\
\text { patients. }\end{array}$ & $\begin{array}{l}\text { Mode of Delivery: Face-to- } \\
\text { face and telephone } \\
\text { Frequency and Interval of } \\
\text { Follow-Up: Initial contact } \\
\text { with additional follow-up } \\
\text { contacts as needed as } \\
\text { designed. Actual frequency } \\
\text { and interval of follow-up: } \\
46 \% \text { received } 1 \text { contact, } \\
24 \% \text { received } 2 \text { contacts, } \\
16 \% \text { received } 3 \\
\text { contacts, } 13 \% \text { received } 4 \text { or } \\
\text { more contacts }\end{array}$ & $\begin{array}{l}\text { in a similar intervention } \\
\text { sponsored by Medicaid. } \\
\text { Reimbursement } \\
\text { characteristics: Participating } \\
\text { pharmacies were } \\
\text { reimbursed for services } \\
\text { provided by study grant. }\end{array}$ \\
\hline
\end{tabular}

Abbreviations: GP = general practitioner; MAT = medication adjustment team; MTM = Medication Therapy Management; NR = not reported; PCM = pharmaceutical case management; PREP = Pharmaceutical Care Research and Education Project; QOL = quality of life; SD = standard deviation; US = United States; VHA = Veterans Health Administration. 


\section{Table E4. Key Question 1: Components and features of medication therapy management interventions: Narrow focused studies}

$\begin{array}{ll}\text { Author, Year } & \begin{array}{l}\text { Special Focus, Intervention, and Integration } \\ \text { with Usual Care }\end{array} \\ \text { Country/ Region } & \end{array}$

Country/ Region

Blennerhassett et Focus: Chronic heart failure

al., $2007^{3}$

Australia

Intervention: Implementation of a HMR into a chronic heart failure collaborative care model. HMRs were conducted by accredited pharmacists.

Level of integration with usual care: General practitioner (GP) provided pharmacist with diagnosis, current medications, relevant test results and medical history. Pharmacist submitted a written and verbal report to the GP for assistance in developing or revising a

management plan.

Carter et al., $1997^{4}$
Barnette, Murphy,
and Carter, $1996^{5}$

Illinois, US pharmacists within an interdisciplinary practice

\section{Focus: Hypertension}

Intervention: Pharmaceutical care provided by model. Patient education (lifestyle, risk factor modifications, and drug therapy) was standardized.

Level of Integration with Usual Care: The pharmacist had access to patients' medical records, diagnostic data, and laboratory data, and had face-to-face interaction with the clinic physicians and nurses.
Method of Identifying Patients for Receipt of MTM Services

Patients with admission and Setting: Community

Delivery, Frequency

Health Care System and

and interval of follow- Reimbursement Contexts up (as reported).

discharge related to $\mathrm{CHF}$

identified as eligible to

receive services under a

broader Home Medication

Review benefit (not

necessarily specific to

CHF).

Mode of Delivery: Home Reimbursement characteristics: visits

Frequency and Interval

of Follow-up: NR

Single payer health care system be claimed up to once per year.

Patients were identified
through a computerized
profile review, details NR

Setting: Outpatient

primary care clinic

Rural medical clinic co-located owned pharmacy.

Mode of delivery: Face-

to-face

Reimbursement characteristics:

Frequency and Interval

of Follow-up: Monthly

contacts for 6 months as

designed. Actual

frequency and interval

of follow-up NR. 
Table E4. Key Question 1: Components and features of medication therapy management interventions: narrow focused studies (continued)

\begin{tabular}{|c|c|c|c|c|}
\hline $\begin{array}{l}\text { Author, Year } \\
\text { Country/ Region }\end{array}$ & $\begin{array}{l}\text { Special Focus, Intervention, and Integration } \\
\text { with Usual Care }\end{array}$ & $\begin{array}{l}\text { Method of Identifying } \\
\text { Patients for Receipt of } \\
\text { MTM Services }\end{array}$ & $\begin{array}{l}\text { Setting, Mode of } \\
\text { Delivery, Frequency } \\
\text { and interval of follow- } \\
\text { up (as reported). }\end{array}$ & $\begin{array}{l}\text { Health Care System and } \\
\text { Reimbursement Contexts }\end{array}$ \\
\hline $\begin{array}{l}\text { Chisholm et al., } \\
2002^{6} \\
\text { Georgia, US }\end{array}$ & $\begin{array}{l}\text { Focus: Postkidney transplantation } \\
\text { Intervention: Clinical pharmacy services, including } \\
\text { reviewing patients' medication therapy, with an } \\
\text { emphasis on controlling blood pressure and } \\
\text { preventing or resolving drug therapy problems. } \\
\text { Pharmacists counseled patients about their } \\
\text { regimen, including desired clinical responses and } \\
\text { possible adverse reactions } \\
\text { Level of integration with usual care: Pharmacist } \\
\text { had access to physical examination findings and } \\
\text { medical and medication history. Pharmacists } \\
\text { were embedded within the clinic and provided } \\
\text { recommendations to attending nephrologists. }\end{array}$ & $\begin{array}{l}\text { All African-American } \\
\text { patients after a primary } \\
\text { kidney transplant receiving } \\
\text { care in transplant clinic. }\end{array}$ & $\begin{array}{l}\text { Setting: Outpatient } \\
\text { transplant clinic } \\
\text { Mode of delivery: Face- } \\
\text { to-face for patients } \\
\text { within } 8 \text { months of } \\
\text { transplant, and } \\
\text { telephone for patients } \\
\text { more than } 8 \text { months } \\
\text { post-transplant } \\
\text { Frequency and Interval } \\
\text { of Follow-up: Monthly as } \\
\text { designed. Actual } \\
\text { frequency and interval } \\
\text { NR. }\end{array}$ & $\begin{array}{l}\text { Transplant clinic of an academic } \\
\text { medical center. } \\
\text { Reimbursement characteristics: } \\
\text { NR. }\end{array}$ \\
\hline $\begin{array}{l}\text { Clifford et al., } 2002^{9} \\
\text { Australia }\end{array}$ & $\begin{array}{l}\text { Focus: Diabetes } \\
\text { Intervention: Pharmaceutical care provided by a } \\
\text { clinical pharmacist, which included a } \\
\text { comprehensive review relating to } \\
\text { pharmacotherapy and diabetes, use of proprietary } \\
\text { and non-proprietary medications, such as } \\
\text { complementary medicines, and identification of } \\
\text { drug therapy problems. } \\
\text { Level of Integration with Usual Care: Pharmacist } \\
\text { had access to patient's case notes. } \\
\text { Pharmaceutical care was provided in cooperation } \\
\text { with the patient's diabetes physicians and other } \\
\text { diabetes health team members. }\end{array}$ & $\begin{array}{l}\text { Medical records were } \\
\text { screened for eligible } \\
\text { patients. Eligible patients } \\
\text { were telephoned about their } \\
\text { willingness to participate. }\end{array}$ & $\begin{array}{l}\text { Setting: Outpatient } \\
\text { hospital diabetes clinic } \\
\text { Mode of Delivery: Face- } \\
\text { to-face } \\
\text { Frequency and Interval } \\
\text { of Follow-Up: Initial visit } \\
\text { followed by follow-up } \\
\text { visits at } 6 \text { week intervals } \\
\text { for } 6 \text { months as } \\
\text { designed. Actual } \\
\text { frequency and interval } \\
\text { of follow-up NR. }\end{array}$ & $\begin{array}{l}\text { Non-US, single payer health } \\
\text { care system } \\
\text { Reimbursement characteristics: } \\
\text { NR }\end{array}$ \\
\hline
\end{tabular}


Table E4. Key Question 1: Components and features of medication therapy management interventions: narrow focused studies (continued)

\begin{tabular}{|c|c|c|c|c|}
\hline $\begin{array}{l}\text { Author, Year } \\
\text { Country/ Region }\end{array}$ & $\begin{array}{l}\text { Special Focus, Intervention, and Integration } \\
\text { with Usual Care }\end{array}$ & $\begin{array}{l}\text { Method of Identifying } \\
\text { Patients for Receipt of } \\
\text { MTM Services }\end{array}$ & $\begin{array}{l}\text { Setting, Mode of } \\
\text { Delivery, Frequency } \\
\text { and interval of follow- } \\
\text { up (as reported). }\end{array}$ & $\begin{array}{l}\text { Health Care System and } \\
\text { Reimbursement Contexts }\end{array}$ \\
\hline $\begin{array}{l}\text { Gattis et al., } 1999^{13} \\
\text { North Carolina, US }\end{array}$ & $\begin{array}{l}\text { Focus: Chronic heart failure } \\
\text { Intervention: Clinical pharmacy services, including } \\
\text { an assessment of prescribed regimen, } \\
\text { compliance, and adverse effects, and symptoms } \\
\text { and response to therapy. Providing patient } \\
\text { education about the purpose of each drug and } \\
\text { reinforcing adherence. Detailed written } \\
\text { information was also provided to patients. } \\
\text { Level of integration with usual care: Pharmacist } \\
\text { had access to patient medical records and } \\
\text { verbally recommendations regarding optimization } \\
\text { of therapy with attending physician. }\end{array}$ & $\begin{array}{l}\text { Patients seen in a general } \\
\text { cardiology clinic meeting } \\
\text { inclusion criteria were } \\
\text { recruited for enrollment. }\end{array}$ & $\begin{array}{l}\text { Setting: Outpatient } \\
\text { cardiology clinic } \\
\text { Mode of Delivery: Initial } \\
\text { visit was face-to-face } \\
\text { and follow-up visits were } \\
\text { by telephone } \\
\text { Frequency and Interval } \\
\text { of Follow-Up: } 3 \text { visits, } \\
\text { baseline, two weeks, } \\
\text { and } 24 \text { weeks by } \\
\text { design. Actual } \\
\text { frequency and interval } \\
\text { of follow-up NR. }\end{array}$ & $\begin{array}{l}\text { Single clinic within an academic } \\
\text { medical center. } \\
\text { Reimbursement characteristics: } \\
\text { NR }\end{array}$ \\
\hline $\begin{array}{l}\text { Harrison et al., } \\
2012^{15} \\
\text { Ontario, Canada }\end{array}$ & $\begin{array}{l}\text { Focus: Post-lung transplant } \\
\text { Intervention: Pharmaceutical care provided by a } \\
\text { clinical pharmacist for identifying and resolving } \\
\text { actual and potential drug therapy problems, } \\
\text { medication teaching, adherence optimization, } \\
\text { medication reconciliation, and provision of drug } \\
\text { information. } \\
\text { Level of integration with usual care: } \\
\text { Patients seen separately by pharmacist at a } \\
\text { routine clinic visit after transplant. Pharmacist } \\
\text { documentation of pharmaceutical care was in the } \\
\text { electronic chart. Drug therapy recommendations } \\
\text { were made verbally or through electronic } \\
\text { communication to clinic physicians. }\end{array}$ & $\begin{array}{l}\text { Combination of provider } \\
\text { referral, patient self-referral, } \\
\text { and routine evaluation for } \\
\text { enrollment at post- } \\
\text { transplant outpatient } \\
\text { followup. }\end{array}$ & $\begin{array}{l}\text { Setting: Outpatient } \\
\text { transplant clinic } \\
\text { Mode of Delivery: Face- } \\
\text { to-face } \\
\text { Frequency and Interval } \\
\text { of Follow-up: One initial } \\
\text { visit with additional } \\
\text { follow-up visits as } \\
\text { needed by design. } \\
\text { Actual frequency and } \\
\text { interval of follow-up: } \\
93 \% \text { received } 1 \text { visit. }\end{array}$ & $\begin{array}{l}\text { Transplant clinic of an Academic } \\
\text { Medical Center. } \\
\text { Reimbursement characteristics: } \\
\text { NR }\end{array}$ \\
\hline
\end{tabular}


Table E4. Key Question 1: Components and features of medication therapy management interventions: narrow focused studies (continued)

\begin{tabular}{|c|c|c|c|c|}
\hline $\begin{array}{l}\text { Author, Year } \\
\text { Country/ Region }\end{array}$ & $\begin{array}{l}\text { Special Focus, Intervention, and Integration } \\
\text { with Usual Care }\end{array}$ & $\begin{array}{l}\text { Method of Identifying } \\
\text { Patients for Receipt of } \\
\text { MTM Services }\end{array}$ & $\begin{array}{l}\text { Setting, Mode of } \\
\text { Delivery, Frequency } \\
\text { and interval of follow- } \\
\text { up (as reported). }\end{array}$ & $\begin{array}{l}\text { Health Care System and } \\
\text { Reimbursement Contexts }\end{array}$ \\
\hline $\begin{array}{l}\text { McDonough et al., } \\
2005^{24} \\
\text { lowa, US }\end{array}$ & $\begin{array}{l}\text { Focus: Glucocorticoid-induced osteoporosis } \\
\text { Intervention: Pharmaceutical care provided by } \\
\text { community pharmacists. Drug therapy monitoring } \\
\text { focused on } 5 \text { drug therapy problems: } \\
\text { appropriateness of does, proper regimen, } \\
\text { potential interactions, nonadherence, and adverse } \\
\text { effects. Patient education was also provided. } \\
\text { Level of Integration With Usual Care: Pharmacist } \\
\text { access to patient medical records NR. A } \\
\text { standardized physician communication form was } \\
\text { used by pharmacists to communicate information } \\
\text { to prescribing physicians. }\end{array}$ & $\begin{array}{l}\text { Claims data or pharmacy } \\
\text { prescription profile records } \\
\text { used to identify eligible } \\
\text { patients who were then } \\
\text { contacted by mail or } \\
\text { telephone to participate. }\end{array}$ & $\begin{array}{l}\text { Setting: community } \\
\text { pharmacy } \\
\text { Mode of Delivery: NR } \\
\text { Frequency and Interval } \\
\text { of Follow-Up: NR }\end{array}$ & $\begin{array}{l}\text { Network of independent and } \\
\text { retail chain pharmacies. Some } \\
\text { pharmacies located within a } \\
\text { clinic, while others are } \\
\text { freestanding. } \\
\text { Reimbursement characteristics: } \\
\text { Pharmacists were reimbursed } \\
\text { using a web-based claims } \\
\text { system, but entity providing } \\
\text { reimbursement was NR. }\end{array}$ \\
\hline $\begin{array}{l}\text { Pai et al., } 2009^{27} \\
\text { Pai et al., } 2009^{28}\end{array}$ & $\begin{array}{l}\text { Focus: Hemodialysis } \\
\text { Intervention: Pharmaceutical care including drug }\end{array}$ & $\begin{array}{l}\text { Patients on stable } \\
\text { hemodialysis regimen for } \\
\text { the previous } 3 \text { months were }\end{array}$ & $\begin{array}{l}\text { Setting: Outpatient } \\
\text { hemodialysis clinic }\end{array}$ & $\begin{array}{l}\text { University-affiliated outpatient } \\
\text { dialysis clinic }\end{array}$ \\
\hline New Mexico, US & $\begin{array}{l}\text { therapy reviews conducted by a nephrology- } \\
\text { trained clinical pharmacist with the patient. Also } \\
\text { included patient and health care provider } \\
\text { education. } \\
\text { Level of Integration with Usual Care: The } \\
\text { pharmacist had access to patient's medical record } \\
\text { and laboratory data. The pharmacists provided } \\
\text { cognitive services during weekly rounds and } \\
\text { during monthly formal reviews of the patients with } \\
\text { the multidisciplinary health care team. }\end{array}$ & $\begin{array}{l}\text { approached for } \\
\text { participation. }\end{array}$ & $\begin{array}{l}\text { Mode of Delivery: Face- } \\
\text { to-face } \\
\text { Frequency and Interval } \\
\text { of Follow-Up: Every } 8 \\
\text { weeks for two years by } \\
\text { design. Actual } \\
\text { frequency and interval } \\
\text { of follow-up NR. }\end{array}$ & $\begin{array}{l}\text { Reimbursement characteristics: } \\
\text { NR }\end{array}$ \\
\hline
\end{tabular}


Table E4. Key Question 1: Components and features of medication therapy management interventions: narrow focused studies (continued)

\begin{tabular}{|c|c|c|c|c|}
\hline $\begin{array}{l}\text { Author, Year } \\
\text { Country/ Region }\end{array}$ & $\begin{array}{l}\text { Special Focus, Intervention, and Integration } \\
\text { with Usual Care }\end{array}$ & $\begin{array}{l}\text { Method of Identifying } \\
\text { Patients for Receipt of } \\
\text { MTM Services }\end{array}$ & $\begin{array}{l}\text { Setting, Mode of } \\
\text { Delivery, Frequency } \\
\text { and interval of follow- } \\
\text { up (as reported). }\end{array}$ & $\begin{array}{l}\text { Health Care System and } \\
\text { Reimbursement Contexts }\end{array}$ \\
\hline $\begin{array}{l}\text { Park et al., } 1996^{29} \\
\text { Wisconsin and }\end{array}$ & $\begin{array}{l}\text { Focus: Hypertension } \\
\text { Intervention: Comprehensive pharmaceutical }\end{array}$ & $\begin{array}{l}\text { Claims data or pharmacy } \\
\text { prescription profile records }\end{array}$ & $\begin{array}{l}\text { Setting: Community } \\
\text { pharmacy }\end{array}$ & $\begin{array}{l}\text { Chain pharmacy with community } \\
\text { pharmacy residents. }\end{array}$ \\
\hline
\end{tabular}

Wisconsin and Intervention: Comprehensive pharmaceutical

Illinois, US $\quad \begin{aligned} & \text { services including drug therapy monitoring } \\ & \text { patient education provided by a community }\end{aligned}$

pharmacy resident.

Level of Integration with Usual Care: Pharmacists access to patient medical records, or labs or vital signs from clinic was NR. Communication with provider was via fax or mail after each pharmacist visit, unless urgency required telephone

Mode of Delivery: Face- Reimbursement characteristics:

to-face

NR

Frequency and Interval

of Follow-up: 4 visits

scheduled 1 month

apart by design. Actual

frequency and interval

communication.

of follow-up NR.

Intervention: Medication therapy management services provided by community pharmacists.

Also included patient education on diet and

lifestyle modifications to lower blood pressure.

Level of integration with usual care: Pharmacist access to patient medical records, or labs, or vital signs from clinic was NR. Providers were contacted via fax or telephone when drug th recommendations.

Three methods were used: Setting: Community

managed care organization pharmacy

identification of patients with

uncontrolled diabetes

through lab data screening, to-face

screening for uncontrolled

diabetes at a health fair for employees sponsored by

the managed care

organization, provider referral of patients with uncontrolled diabetes.

design. Actual

of follow-up NR.
Services provided through a

collaboration between a

managed care organization and

Mode of Delivery: Face- a regional retail chain pharmacy.

Frequency and Interval NR

of Follow-up: Monthly

visits for 9 months by

frequency and interval 
Table E4. Key Question 1: Components and features of medication therapy management interventions: narrow focused studies (continued)

\begin{tabular}{|c|c|c|c|c|}
\hline $\begin{array}{l}\text { Author, Year } \\
\text { Country/ Region }\end{array}$ & $\begin{array}{l}\text { Special Focus, Intervention, and Integration } \\
\text { with Usual Care }\end{array}$ & $\begin{array}{l}\text { Method of Identifying } \\
\text { Patients for Receipt of } \\
\text { MTM Services }\end{array}$ & $\begin{array}{l}\text { Setting, Mode of } \\
\text { Delivery, Frequency } \\
\text { and interval of follow- } \\
\text { up (as reported). }\end{array}$ & $\begin{array}{l}\text { Health Care System and } \\
\text { Reimbursement Contexts }\end{array}$ \\
\hline $\begin{array}{l}\text { Roughead et al., } \\
2009^{32} \\
\text { Australia }\end{array}$ & $\begin{array}{l}\text { Focus: Chronic heart failure } \\
\text { Intervention: HMR, a collaborative model of } \\
\text { pharmaceutical care. HMRs are conducted by } \\
\text { accredited pharmacists. } \\
\text { Level of Integration with Usual Care: NR for this } \\
\text { study specifically, but the HMR model is that the } \\
\text { GP provides pharmacist with diagnosis, current } \\
\text { medications, relevant test results and medical } \\
\text { history. Pharmacist conducts the HMR and } \\
\text { submits a written and verbal report to the GP for } \\
\text { assistance in developing or revising a } \\
\text { management plan. }\end{array}$ & $\begin{array}{l}\text { HMRs are conducted upon } \\
\text { request of a provider. } \\
\text { Claims data or pharmacy } \\
\text { prescription profile records }\end{array}$ & $\begin{array}{l}\text { Setting: Outpatient clinic } \\
\text { and home visits } \\
\text { Mode of Delivery: Face- } \\
\text { to-face } \\
\text { Frequency and Interval } \\
\text { of Follow-Up: NR }\end{array}$ & $\begin{array}{l}\text { Non-US, single payer health } \\
\text { care system } \\
\text { Reimbursement characteristics: } \\
\text { services reimbursed through } \\
\text { payer's home medicine review } \\
\text { benefit. }\end{array}$ \\
\hline $\begin{array}{l}\text { Triller and Hamilton, } \\
2007^{38} \\
\text { New York, US }\end{array}$ & $\begin{array}{l}\text { Focus: Heart failure } \\
\text { Intervention: Comprehensive pharmaceutical care } \\
\text { services provided by pharmacist. } \\
\text { Level of integration with usual care: Pharmacists } \\
\text { had access to patient medical records and } \\
\text { laboratory results. Pharmaceutical care services } \\
\text { were coordinated and provided alongside visiting } \\
\text { nurse services. Recommendations to physicians } \\
\text { were communicated via fax or telephone, } \\
\text { depending on the urgency of the situation. }\end{array}$ & $\begin{array}{l}\text { Enrollment during a } \\
\text { transition in care from } \\
\text { inpatient to home. }\end{array}$ & $\begin{array}{l}\text { Setting: Home visit } \\
\text { Mode of delivery: Face- } \\
\text { to-face } \\
\text { Frequency and interval } \\
\text { of Follow-up: Initial visit } \\
\text { plus additional follow-up } \\
\text { visits at } 7-10 \text { days and } \\
18-21 \text { days, provided } \\
\text { patient was still } \\
\text { receiving visiting nurse } \\
\text { services by design. } \\
\text { Actual frequency and } \\
\text { interval of follow-up: } \\
53 \% \text { received all } 3 \\
\text { visits, } 31 \% \text { received } 2 \\
\text { visits, } 12 \% \text { received } 1 \\
\text { visit, and } 4 \% \text { received } 4 \\
\text { visits. }\end{array}$ & $\begin{array}{l}\text { Integrated health system and } \\
\text { associated Visiting Nurse } \\
\text { Association. } \\
\text { Reimbursement characteristics: } \\
\text { NR }\end{array}$ \\
\hline
\end{tabular}

Abbreviations: $\mathrm{CHF}=$ chronic heart failure; $\mathrm{GP}=$ general practitioner; $\mathrm{HMR}=$ home medication review; MTM = medication therapy management; NR = not reported; US =

United States. 
Table E5. Hemoglobin A1c: Summary of results

\begin{tabular}{|c|c|c|c|c|}
\hline $\begin{array}{l}\text { Study } \\
\text { Design/Risk of Bias }\end{array}$ & Study Arms & N Analyzed & Outcome and Time Period & Results \\
\hline $\begin{array}{l}\text { Clifford et al. } 2002^{9} \\
\text { RCT/Medium }\end{array}$ & $\begin{array}{l}\text { G1: Pharmaceutical care } \\
\text { G2: Standard care }\end{array}$ & $\begin{array}{l}\text { G1: } 48 \\
\text { G2: } 25\end{array}$ & Mean (SD) HbA1c at six months. & $\begin{array}{l}\text { Baseline: } \\
\text { G1: } 8.4(1.4) \\
\text { G2: } 8.5(1.6) \\
\text { p: NS } \\
6 \text { months } \\
\text { G1: } 8.2(1.5) \\
\text { G2: } 8.1(1.6) \\
\text { Calculated mean difference: } \\
-0.2,95 \% \mathrm{Cl},-0.93 \text { to } 0.53 \text { (assuming pre- } \\
\text { post correlation of } 0.5) \\
\text { p=0.207 }\end{array}$ \\
\hline $\begin{array}{l}\text { Taylor, Byrd, and Krueger, } \\
2003^{36} \\
\text { RCT/Medium }\end{array}$ & $\begin{array}{l}\text { G1: Pharmaceutical care } \\
\text { G2: Standard care }\end{array}$ & $\begin{array}{l}\text { G1: } 13 \\
\text { G2: } 16 \\
\text { (Study included } \\
\text { more subjects, but } \\
\text { this outcome was } \\
\text { only assessed } \\
\text { among patients with } \\
\text { diabetes within each } \\
\text { study arm) }\end{array}$ & $\begin{array}{l}\text { Percent with } \mathrm{HbA} 1 \mathrm{c} \text { at goal } \\
\text { (defined as less than or equal to } \\
7.5 \% \text { ) at baseline and at } 12 \\
\text { months. }\end{array}$ & $\begin{array}{l}\text { Baseline: } \\
\text { G1: } 23.1 \\
\text { G2: } 56.3 \\
\text { p=0.071 } \\
\text { Calculated OR: } 0.2 \\
95 \% \mathrm{Cl}, 0.05 \text { to } 1.19 \\
\text { Follow-up } \\
\text { G1: } 100 \\
\text { G2: } 26.7 \\
\text { p=0.001 } \\
\text { Calculated OR: } 56.5 \\
\text { 95\% Cl, } 2.81 \text { to } 1,133.91 \\
\text { p=0.008 }\end{array}$ \\
\hline
\end{tabular}


Table E5. Hemoglobin A1c: Summary of results (continued)

\begin{tabular}{|c|c|c|c|c|}
\hline $\begin{array}{l}\text { Study } \\
\text { Design/Risk of Bias }\end{array}$ & Study Arms & N Analyzed & Outcome and Time Period & Results \\
\hline $\begin{array}{l}\text { Pindolia et al., } 2009^{30} \\
\text { Cohort study/High }\end{array}$ & $\begin{array}{l}\text { G1: Opted in to a telephone } \\
\text { based MTM Program } \\
\text { G2: Usual medical care (opted } \\
\text { out of MTM program) }\end{array}$ & $\begin{array}{l}\text { G1: NR } \\
\text { G2: NR } \\
\text { (Was only assessed } \\
\text { among patients with } \\
\text { DM in each study } \\
\text { arm and N for this } \\
\text { outcome was not } \\
\text { reported) }\end{array}$ & $\begin{array}{l}\text { Change in percent of patients with } \\
\mathrm{HbA} 1 \mathrm{c} \text { less than } 7 \text { at } 6 \text { months }\end{array}$ & $\begin{array}{l}\text { G1: }+3 \\
\text { G2: }+7 \\
\text { Between-group p: inferred to be NS, exact } p \\
\text { NR } \\
\text { Within-group p: NR }\end{array}$ \\
\hline $\begin{array}{l}\text { Jeong et al., } 2007^{18} \\
\text { Cohort study/High }\end{array}$ & $\begin{array}{l}\text { G1: Participants in Part D } \\
\text { Medicare MTM program } \\
\text { G2: Control subjects eligible for } \\
\text { Part D MTM program but } \\
\text { declined enrollment } \\
\text { G3: Control subjects without Part } \\
\text { D Medicare as their primary drug } \\
\text { benefit }\end{array}$ & $\begin{array}{l}\text { G1: } 1,211 \\
\text { G2: } 1,000 \\
\text { G3: } 743 \\
\text { (Study included } \\
\text { more subjects but } \\
\text { this outcome was } \\
\text { assessed among } \\
\text { only patients with } \\
\text { diabetes within } \\
\text { each study arm) }\end{array}$ & $\begin{array}{l}\text { Mean change (SD) in } \mathrm{HbA} 1 \mathrm{c} \text { at } \\
\text { six months }\end{array}$ & $\begin{array}{l}\text { G1: }-0.05(1.0) \\
\text { G2: }-0.01(1.0) \\
\text { Calculated mean difference of } \mathrm{G} 1 \text { vs. G2: } \\
0.04,95 \% \mathrm{Cl},-0.04 \text { to } 0.13 ; p=0.337 \\
\text { G3: }-0.05(1.0) \\
\text { Calculated mean difference of } \mathrm{G} 1 \text { vs. G3: } \\
0.004,95 \% \mathrm{Cl},-0.09 \text { to } 0.10 ; p=0.931 \\
\text { Author reported overall } p=0.74\end{array}$ \\
\hline
\end{tabular}

Abbreviations: $\mathrm{CI}=$ confidence interval; DM = diabetes mellitus; $\mathrm{G}=$ group; HbA1C = hemoglobin A1C or glycosylated hemoglobin; MTM = Medication Therapy Management; $\mathrm{N}=$ number; $\mathrm{NR}=$ not reported; $\mathrm{NS}=$ not significant; $\mathrm{RCT}=$ randomized controlled trial; $\mathrm{SD}=$ standard deviation; vs. = versus 
Table E6. LDL cholesterol: Summary of results

\begin{tabular}{|c|c|c|c|c|}
\hline $\begin{array}{l}\text { Study } \\
\text { Design/Risk of Bias }\end{array}$ & Study Arms & N Analyzed & Outcome and Time Period & Results \\
\hline \multirow[t]{2}{*}{$\begin{array}{l}\text { Taylor, Byrd, and } \\
\text { Krueger, } 2003^{36} \\
\text { RCT/Medium }\end{array}$} & $\begin{array}{l}\text { G1: Pharmaceutical care } \\
\text { G2: Standard care }\end{array}$ & $\begin{array}{l}\text { Baseline } \\
\text { G1: } 19 \\
\text { G2: } 19 \\
\text { (Was only assessed } \\
\text { among patients with } \\
\text { dyslipidemia in each } \\
\text { study arm) }\end{array}$ & $\begin{array}{l}\text { Percent of patients at LDL-C goal } \\
\text { based on ATPIII criteria at } 12 \\
\text { months. }\end{array}$ & $\begin{array}{l}\text { Baseline: } \\
\text { G1: } 10.5 \\
\text { G2: } 15.8 \\
\text { Calculated OR: } 0.6 ; 95 \% \mathrm{Cl}, 0.09 \text { to } \\
4.25 ; p=0.631\end{array}$ \\
\hline & & $\begin{array}{l}\text { Follow-up ( } \mathrm{N} \text { inferred } \\
\text { from percent in results) } \\
\text { G1: } 18 \\
\text { G2: } 17\end{array}$ & & $\begin{array}{l}\text { Follow-up } \\
\text { G1: } 77.8 \\
\text { G2: } 5.9 \\
\text { Calculated OR: } 56.0 ; 95 \% \mathrm{Cl}, 5.58 \text { to } \\
561.75 ; \text { p: } 0.001\end{array}$ \\
\hline $\begin{array}{l}\text { Isetts et al., } 2008^{16} \\
\text { Cohort study/High }\end{array}$ & $\begin{array}{l}\text { G1: MTM services provided by } \\
\text { health plan in existing medical } \\
\text { care clinics in collaboration with } \\
\text { primary care providers. } \\
\text { G2: Usual medical care without } \\
\text { MTM }\end{array}$ & $\begin{array}{l}\text { G1: } 128 \\
\text { G2: } 126\end{array}$ & $\begin{array}{l}\text { Percent of patients meeting } \\
\text { HEDIS measures related to } \\
\text { cholesterol control after } \\
\text { cardiovascular event at } 12 \\
\text { months. }\end{array}$ & $\begin{array}{l}\text { G1: } 52 \\
\text { G2: } 30 \\
\text { Calculated OR: } 2.5 ; 95 \% \text { Cl, } 1.52 \text { to } 4.26 \text {; } \\
\text { p: } 0.001\end{array}$ \\
\hline $\begin{array}{l}\text { Pindolia et al., } 2009^{30} \\
\text { Cohort study/High }\end{array}$ & $\begin{array}{l}\text { G1: Opted in to a telephone } \\
\text { based MTM Program } \\
\text { G2: Usual medical care (opted } \\
\text { out of MTM program) }\end{array}$ & $\begin{array}{l}\text { G1: NR } \\
\text { G2: NR } \\
\text { (Was only assessed } \\
\text { among patients with } \\
\text { coronary artery disease } \\
\text { in each study arm) }\end{array}$ & $\begin{array}{l}\text { Change in percent of patients } \\
\text { with LDL-C less than } 100 \mathrm{mg} / \mathrm{dl} \\
\text { at } 6 \text { months. }\end{array}$ & $\begin{array}{l}\text { G1: }-5 \\
\text { G2: }+7 \\
\text { p: NR and could not be calculated }\end{array}$ \\
\hline \multirow[t]{2}{*}{$\begin{array}{l}\text { Fox et al. } 2009^{12} \\
\text { Cohort study/High }\end{array}$} & $\begin{array}{l}\text { G1: MTM program, provided } \\
\text { through a health plan } \\
\text { G2: Usual medical care (eligible } \\
\text { but opt-out from MTM program) }\end{array}$ & $\begin{array}{l}\text { G1: } 255 \\
\text { G2: } 56 \\
\\
\end{array}$ & $\begin{array}{l}\text { Percent of patients with diabetes } \\
\text { with LDL-C less than } 100 \mathrm{mg} / \mathrm{dl} \\
\text { at } 12 \text { to } 24 \text { months. } \\
\text { Mean (SD) LDL-C at } 12 \text { to } 24 \\
\text { months. }\end{array}$ & $\begin{array}{l}\text { G1: } 69 \\
\text { G2: } 50 \\
\text { OR: } 2.2 ; 95 \% \mathrm{Cl}, 1.24 \text { to } 4.01 \text {; Calculated } \\
p=0.008\end{array}$ \\
\hline & & $\begin{array}{l}\text { G1: } 215 \\
\text { G2: } 46\end{array}$ & & $\begin{array}{l}\mathrm{G} 1: 83.4(31.1) \\
\mathrm{G} 2: 90.8(31.0) \\
\text { Calculated mean difference: }-7.4 \\
95 \% \mathrm{Cl},-17.30 \text { to } 2.50 \\
p=0.33 \text { as reported by study authors, } \\
\mathrm{p}=0.143 \text { as calculated }\end{array}$ \\
\hline
\end{tabular}




\section{Table E6. LDL cholesterol: Summary of results (continued)}

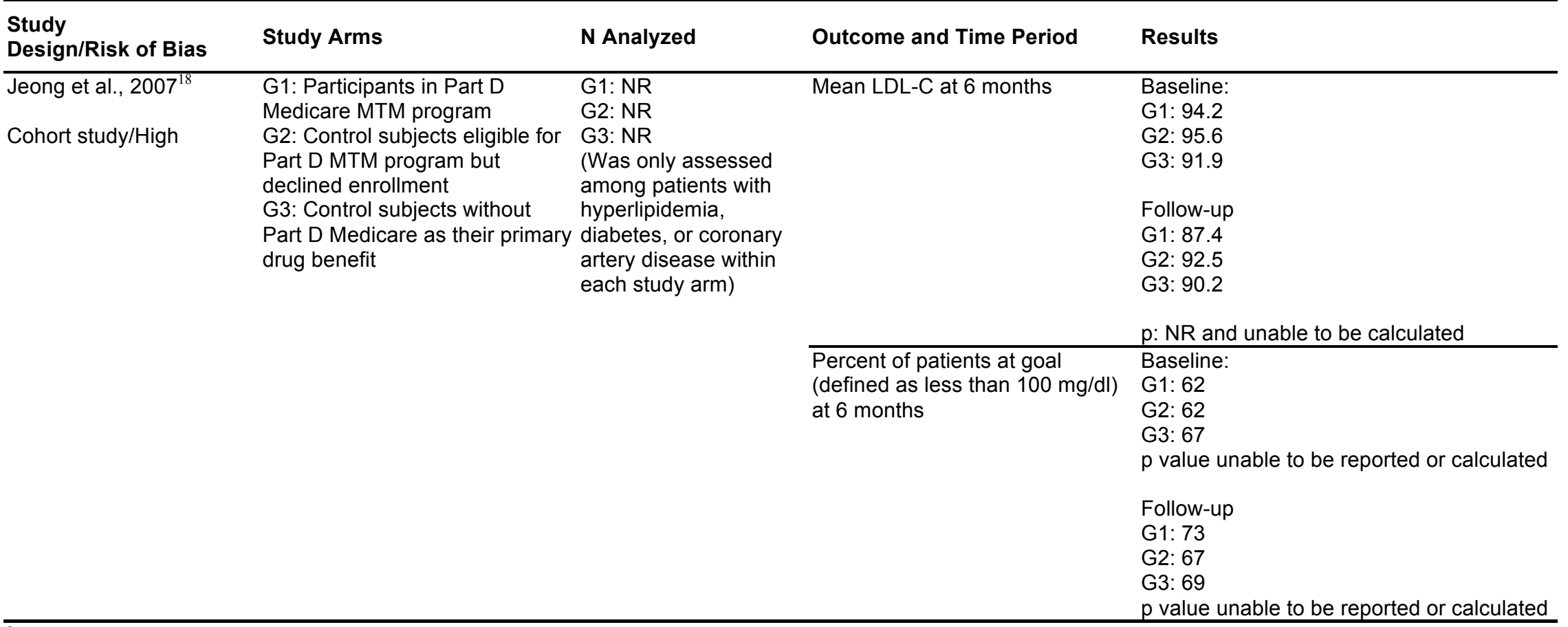

${ }^{a} p$ values reported as $<0.001$ for G1 vs. G2 and G1 vs. G3, but unclear whether these refer to between-group differences at followup in LDL-C, between group differences in LDL-C change, or to between-group differences in change in percent at LDL-C goal. Calculated mean differences and OR were unable to be calculated due to absence of SD and number analyzed.

Abbreviations: ATPIII=Adult Treatment Panel III (Expert Panel on Detection; Evaluation; and Treatment of High Blood Cholesterol); CI = confidence interval; G = group; HEDIS= Healthcare Effectiveness Data and Information Set; LDL-C $=$ low density lipoprotein cholesterol; $\mathrm{mg} / \mathrm{dl}=$ milligrams per deciliter; MTM = Medication Therapy Management; $\mathrm{N}=$ number; $\mathrm{NR}=$ not reported; $\mathrm{OR}=$ odds ratio; $\mathrm{RCT}=$ randomized controlled trial; $\mathrm{SD}=$ standard deviation; vs. $=$ versus. 
Table E7. Blood pressure: Summary of results

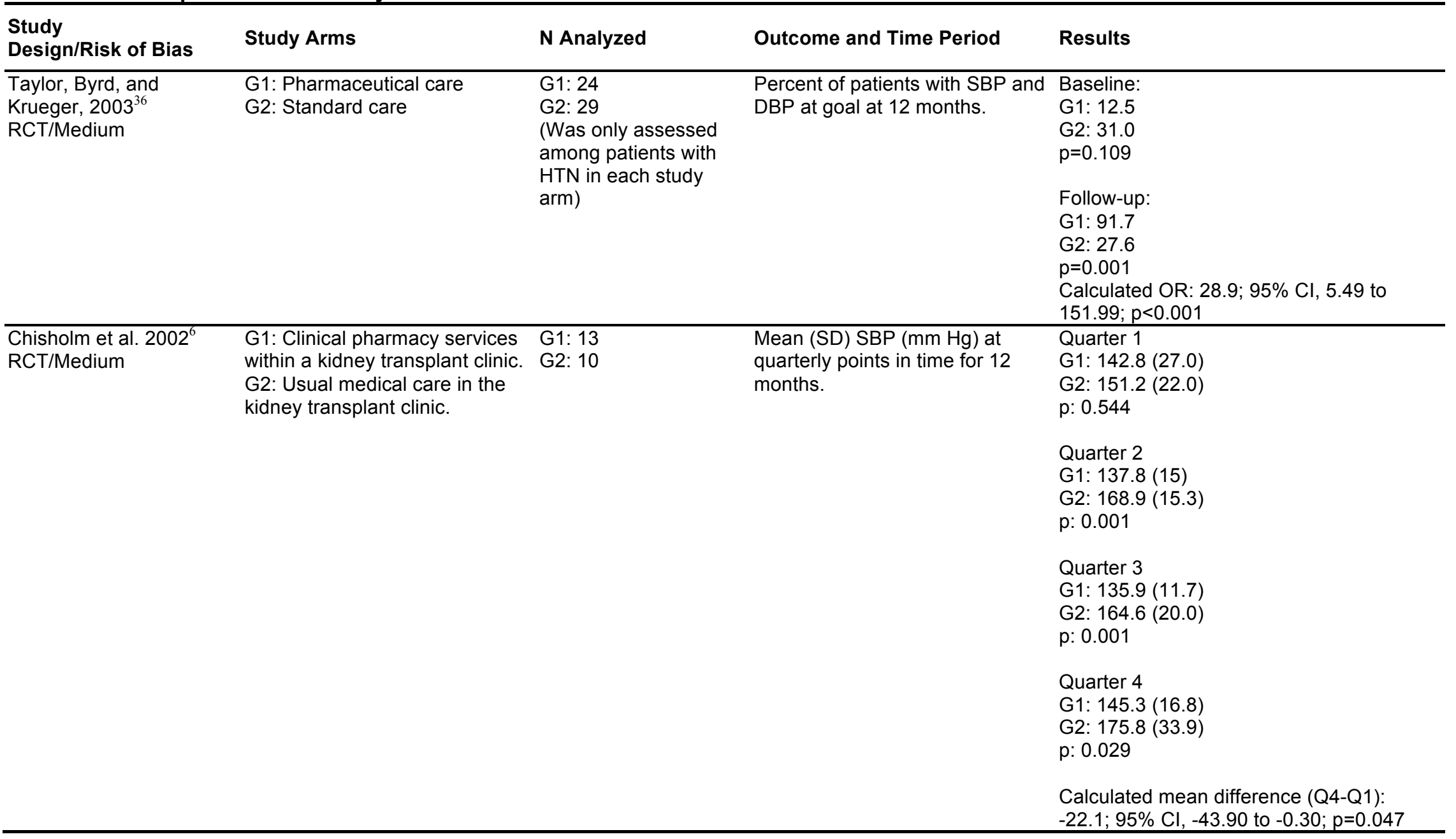


Table E7. Blood pressure: Summary of results (continued)

Study $\quad$ Study Arms N Analyzed

Design/Risk of Bias

Study Arms

N Analyzed

Outcome and Time Period

Results

Chisholm et al. $2002^{6} \quad$ Mean (SD) DBP (mm Hg) at

(continued)

quarterly points in time for 12

Quarter 1

months.

G1: $81.7(12.8)$

G2: $78(15.5)$

p: 0.611

Quarter 2

G1: $76.0(11.8)$

G2: $84.9(6.1)$

p: 0.036

Quarter 3

G1: $71.4(13)$

G2: $78.3(9.5)$

p: 0.205

Quarter 4

G1: $77.0(10.2)$

G2: $91.8(12.0)$

p: 0.020

Calculated mean difference (Q4-Q1):

$-18.5 ; 95 \% \mathrm{Cl},-29.04$ to $-7.96 ; p=0.001$ 
Table E7. Blood pressure: Summary of results (continued)

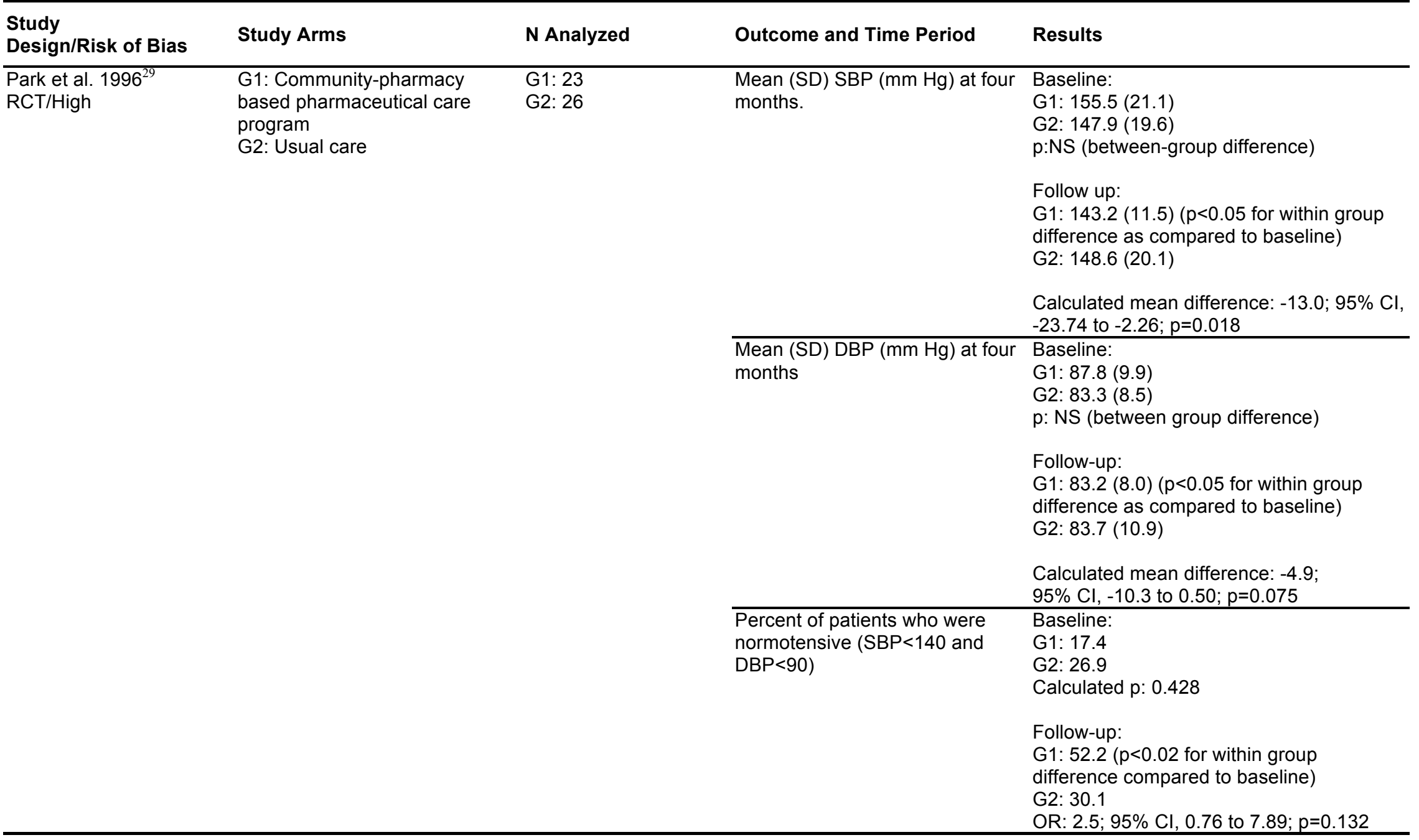


Table E7. Blood pressure: Summary of results (continued)

\begin{tabular}{|c|c|c|c|c|}
\hline $\begin{array}{l}\text { Study } \\
\text { Design/Risk of Bias }\end{array}$ & Study Arms & N Analyzed & Outcome and Time Period & Results \\
\hline \multirow[t]{4}{*}{$\begin{array}{l}\text { Planas et al. } 2009^{31} \\
\text { RCT/High }\end{array}$} & \multirow{4}{*}{$\begin{array}{l}\text { G1: Community pharmacy- } \\
\text { based hypertension MTM } \\
\text { program for patients with } \\
\text { diabetes } \\
\text { G2: Control group (BP recorded, } \\
\text { informed of BP goals at three } \\
\text { time during study) }\end{array}$} & G1: 25 & $\begin{array}{l}\text { Mean (SD) change in SBP }(\mathrm{mm} \\
\mathrm{Hg}) \text { at nine months }\end{array}$ & $\begin{array}{l}\text { G1: }-17.3 \\
\text { G2: } 2.7 \\
\text { Between-group difference }(95 \% \mathrm{Cl}):-20.0 \\
(-32.7 \text { to }-7.4) \\
\text { p: } 0.003\end{array}$ \\
\hline & & & $\begin{array}{l}\text { Percent of patients at BP goal at } \\
\text { nine months. }\end{array}$ & $\begin{array}{l}\text { Baseline } \\
\text { G1: } 16.0 \\
\text { G2: } 20.0 \\
\text { Calculated p: } 0.714\end{array}$ \\
\hline & & & & $\begin{array}{l}9 \text { months } \\
\text { G1: } 48.0 \\
\text { G2: } 6.7 \\
\text { p: } 0.007\end{array}$ \\
\hline & & & $\begin{array}{l}\text { OR for intervention group } \\
\text { participant achieving BP goal } \\
\text { relative to control group. }(95 \% \mathrm{Cl})\end{array}$ & $\begin{array}{l}\text { OR : } 12.9(1.5 \text { to } 113.8) \\
p: 0.021\end{array}$ \\
\hline
\end{tabular}


Table E7. Blood pressure: Summary of results (continued)

\begin{tabular}{|c|c|c|c|c|}
\hline $\begin{array}{l}\text { Study } \\
\text { Design/Risk of Bias }\end{array}$ & Study Arms & N Analyzed & Outcome and Time Period & Results \\
\hline \multirow{7}{*}{$\begin{array}{l}\text { Carter et al., } \\
1997^{4} \\
\text { Barnette, Murphy, and } \\
\text { Carter, } 1996^{5} \\
\text { Cohort study/High }\end{array}$} & $\begin{array}{l}\text { G1: Pharmacy-based } \\
\text { pharmaceutical care } \\
\text { G2: usual medical care }\end{array}$ & $\begin{array}{l}\text { G1: } 25 \\
\text { G2: } 26\end{array}$ & $\begin{array}{l}\text { Mean (SD) SBP }(\mathrm{mm} \mathrm{Hg}) \text { at } 6 \\
\text { months }\end{array}$ & $\begin{array}{l}\text { Baseline } \\
\text { G1: } 151(21) \\
\text { G2: } 145(19) \\
\text { p: } 0.29\end{array}$ \\
\hline & & & & $\begin{array}{l}\text { Follow-up } \\
\text { G1: } 140(14) \\
\text { G2: } 143(20) \\
\text { Calculated Mean Difference: }-9.0 \\
95 \% \mathrm{Cl},-19.45 \text { to } 1.45 ; p=0.0914\end{array}$ \\
\hline & & & $\begin{array}{l}\text { Mean (SD) DBP }(\mathrm{mm} \mathrm{Hg}) \text { at } 6 \\
\text { months. }\end{array}$ & $\begin{array}{l}\text { Baseline } \\
\text { G1: } 82(9) \\
\text { G2: } 80(9) \\
\text { p: NS }\end{array}$ \\
\hline & & & & $\begin{array}{l}\text { Follow-up } \\
\text { G1: } 80(8) \\
\text { G2: } 79(10) \\
\text { Calculated SMD, }-1.0 ; \\
95 \% \mathrm{Cl},-5.98 \text { to } 3.98 ; p=0.694\end{array}$ \\
\hline & & & $\begin{array}{l}\text { Percent with blood pressure } \\
\text { control }\end{array}$ & $\begin{array}{l}\text { Baseline: } \\
\text { G1: } 52 \\
\text { G2: } 54 \\
\text { Calculated } p=0.90\end{array}$ \\
\hline & & & & $\begin{array}{l}\text { Follow-up: } \\
\text { G1: } 68 \\
\text { G2: } 58\end{array}$ \\
\hline & & & & $\begin{array}{l}\text { Calculated OR: } 1.6 ; \\
95 \% \mathrm{Cl}, 0.50 \text { to } 4.90 ; p=0.448\end{array}$ \\
\hline
\end{tabular}


Table E7. Blood pressure: Summary of results (continued)

\begin{tabular}{|c|c|c|c|c|}
\hline $\begin{array}{l}\text { Study } \\
\text { Design/Risk of Bias }\end{array}$ & Study Arms & N Analyzed & Outcome and Time Period & Results \\
\hline $\begin{array}{l}\text { Isetts et al., } 2008^{16} \\
\text { Cohort study/High }\end{array}$ & $\begin{array}{l}\text { G1: MTM services provided by } \\
\text { health plan in existing medical } \\
\text { care clinics in collaboration with } \\
\text { primary care providers. } \\
\text { G2: Usual medical care without } \\
\text { MTM }\end{array}$ & $\begin{array}{l}\text { G1: } 128 \\
\text { G2: } 126\end{array}$ & $\begin{array}{l}\text { Percent of patients meeting } \\
\text { HEDIS measures related to } \\
\text { hypertension management at } 12 \\
\text { months. }\end{array}$ & $\begin{array}{l}\text { G1: } 71 \\
\text { G2: } 59 \\
\text { Calculated OR: } 1.7 ; \\
95 \% \mathrm{Cl}, 1.03 \text { to } 2.91 ; p=0.04\end{array}$ \\
\hline
\end{tabular}

Abbreviations: $\mathrm{BP}=$ blood pressure; $\mathrm{CI}=$ confidence interval; DBP = diastolic blood pressure; $\mathrm{G}=$ group; HEDIS = Healthcare Effectiveness Data and Information Set; HTN = hypertension; $\mathrm{mm} \mathrm{Hg}=$ millimeter mercury; MTM = Medication Therapy Management; NR= not reported; OR = odds ratio; $\mathrm{Q}=$ quarter; RCT= randomized controlled trial; $\mathrm{SBP}$ = systolic blood pressure; $\mathrm{SD}=$ standard deviation; $\mathrm{SMD}=$ standardized mean difference 
Table E8. Drug therapy problems identified: Summary of results

\begin{tabular}{|c|c|c|c|c|}
\hline $\begin{array}{l}\text { Study } \\
\text { Design/Risk of Bias }\end{array}$ & Study Arms & N Analyzed & Outcome and Time Period & Results \\
\hline $\begin{array}{l}\text { Krska et al., } 2001^{19} \\
\text { RCT/High }\end{array}$ & $\begin{array}{l}\text { G1: Pharmacist-led medication } \\
\text { review } \\
\text { G2: Usual care including } \\
\text { identification of pharmaceutical } \\
\text { care issues, but no plan }\end{array}$ & $\begin{array}{l}\text { G1: } 168 \\
\text { G2: } 164\end{array}$ & $\begin{array}{l}\text { Drug therapy problems identified } \\
\text { for each study arm at } 3 \text { months }\end{array}$ & $\begin{array}{l}\text { G1: } 1206 \\
\text { G2: } 1380\end{array}$ \\
\hline $\begin{array}{l}\text { Harrison et al., } 2012^{15} \\
\text { Cohort/High }\end{array}$ & $\begin{array}{l}\text { G1: Pharmaceutical care } \\
\text { G2: Standard care }\end{array}$ & $\begin{array}{l}\text { G1: } 43 \\
\text { G2: } 43\end{array}$ & $\begin{array}{l}\text { Drug therapy problems identified } \\
\text { per visit, follow-up } 2 \text { weeks after } \\
\text { intervention }\end{array}$ & $\begin{array}{l}\text { Baseline } \\
\text { G1: } 0.5(0.6) \\
\text { G2: NA } \\
\text { Follow-up } \\
\text { G1: } 1.1(1.3) \\
\text { G2: } 0.7(0.8) \\
\mathrm{p}=0.19 \text { for pharmaceutical care vs. } \\
\text { standard care, not controlling for } \\
\text { differences between } \mathrm{G} 1 \text { at baseline and } \\
\mathrm{G} 2\end{array}$ \\
\hline $\begin{array}{l}\text { Welch et al., } 2009^{41} \\
\text { Cohort/High }\end{array}$ & $\begin{array}{l}\text { G1: MTM program provided to } \\
\text { home-based beneficiaries } \\
\text { G2: No-MTM control group } \\
\text { (voluntary opt-out) }\end{array}$ & $\begin{array}{l}\text { G1: } 459 \\
\text { G2: } 123\end{array}$ & $\begin{array}{l}\text { At least } 1 \text { potential drug therapy } \\
\text { problem during MTM process }\end{array}$ & $\begin{array}{l}\text { G1: } 89.8 \% \\
\text { G2: } 83.7 \% \\
\text { Calculated } p=0.062\end{array}$ \\
\hline
\end{tabular}

difference. 
Table E9. Drug therapy problems resolved: Summary of results

\begin{tabular}{|c|c|c|c|c|}
\hline $\begin{array}{l}\text { Study } \\
\text { Design/Risk of Bias }\end{array}$ & Study Arms & N Analyzed & Outcome and Time Period & Results \\
\hline $\begin{array}{l}\text { Krska et al., } 2001^{19} \\
\text { RCT/High }\end{array}$ & $\begin{array}{l}\text { G1: Pharmacist-led medication } \\
\text { review } \\
\text { G2: Usual care including } \\
\text { identification of pharmaceutical } \\
\text { care issues, but no plan }\end{array}$ & $\begin{array}{l}\text { G1: } 168 \\
\text { G2: } 164\end{array}$ & $\begin{array}{l}\text { Drug therapy problems wholly or } \\
\text { partially resolved at } 3 \text { months }\end{array}$ & $\begin{array}{l}\text { G1: } 998 \\
\text { G2: } 569\end{array}$ \\
\hline $\begin{array}{l}\text { Bernsten et al., } 2001^{1,2} \\
\text { RCT/High }\end{array}$ & $\begin{array}{l}\text { G1: Structured community } \\
\text { pharmacy-based } \\
\text { pharmaceutical care program } \\
\text { G2: Usual community pharmacy } \\
\text { services }\end{array}$ & $\begin{array}{l}\text { Baseline } \\
\text { G1: } 1290 \\
\text { G2: } 1164 \\
\text { 6 months } \\
\text { G1: } 1024 \\
\text { G2: } 953 \\
\text { 12 months } \\
\text { G1: } 863 \\
\text { G2: } 764 \\
\text { 18 months } \\
\text { G1: } 704 \\
\text { G2: } 636\end{array}$ & $\begin{array}{l}\text { Number of changes in therapy at } \\
\text { baseline, } 6,12 \text {, and } 18 \text { months }\end{array}$ & $\begin{array}{l}\text { Baseline } \\
\mathrm{G} 1: 1.1(1.3) \\
\mathrm{G} 2: 0.9(1.2) \\
\mathrm{p}:<0.05 \\
6 \text { months } \\
\mathrm{G} 1: 1.5(1.8) \\
\mathrm{G} 2: 1.1(1.4) \\
\mathrm{p}:<0.05 \\
12 \text { months } \\
\mathrm{G} 1: 1.3(1.6) \\
\mathrm{G} 2: 1.2(1.5) \\
\mathrm{p}: \mathrm{NS} \\
18 \text { months } \\
\mathrm{G} 1: 1.4(1.5) \\
\mathrm{G} 2: 1.4(1.4) \\
\mathrm{p}: \mathrm{NS}\end{array}$ \\
\hline $\begin{array}{l}\text { Moczygemba et al., } 2011^{25} \\
\text { Moczygemba et al., } 2008^{26} \\
\text { Cohort/Medium }\end{array}$ & $\begin{array}{l}\text { G1: Opt-in telephone MTM } \\
\text { program } \\
\text { G2: No-MTM control group }\end{array}$ & $\begin{array}{l}\text { G1: } 60 \\
\text { G2: } 60\end{array}$ & $\begin{array}{l}\text { Medication and health-related } \\
\text { problems identified at baseline } \\
\text { and } 6 \text { months }\end{array}$ & $\begin{array}{l}\text { Mean (SD) } \\
\text { Baseline } \\
\text { G1: } 4.8(2.7) \\
\text { G2: } 9.2(2.9) \\
6 \text { month } \\
\text { G1: } 2.5(2.0) \\
\text { G2: } 7.9(3.0) \\
\text { Calculated mean difference: }-1.0(95 \% \mathrm{Cl} \text {, } \\
-1.97 \text { to }-0.03), p=0.4\end{array}$ \\
\hline
\end{tabular}

Abbreviations: $\mathrm{CI}$ = confidence interval; G = group; MTM = Medication Therapy Management; NS = not significant; $\mathrm{RCT}$ = randomized controlled trial; $\mathrm{SD}=$ standard deviation; $\mathrm{SMD}=$ standardized mean difference. 
Table E10. Medication adherence: Summary of results

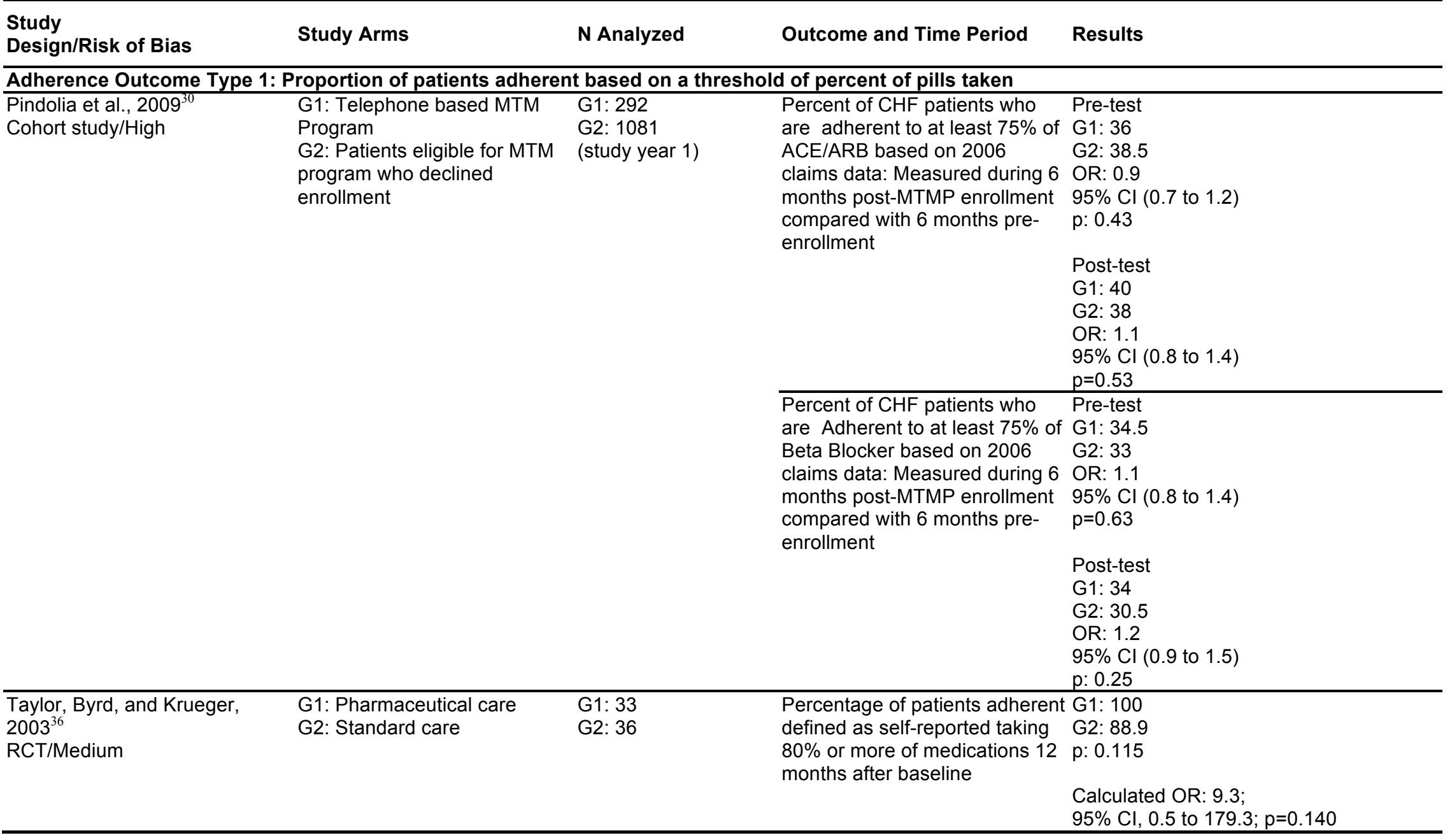


Table E10. Medication adherence: Summary of results (continued)

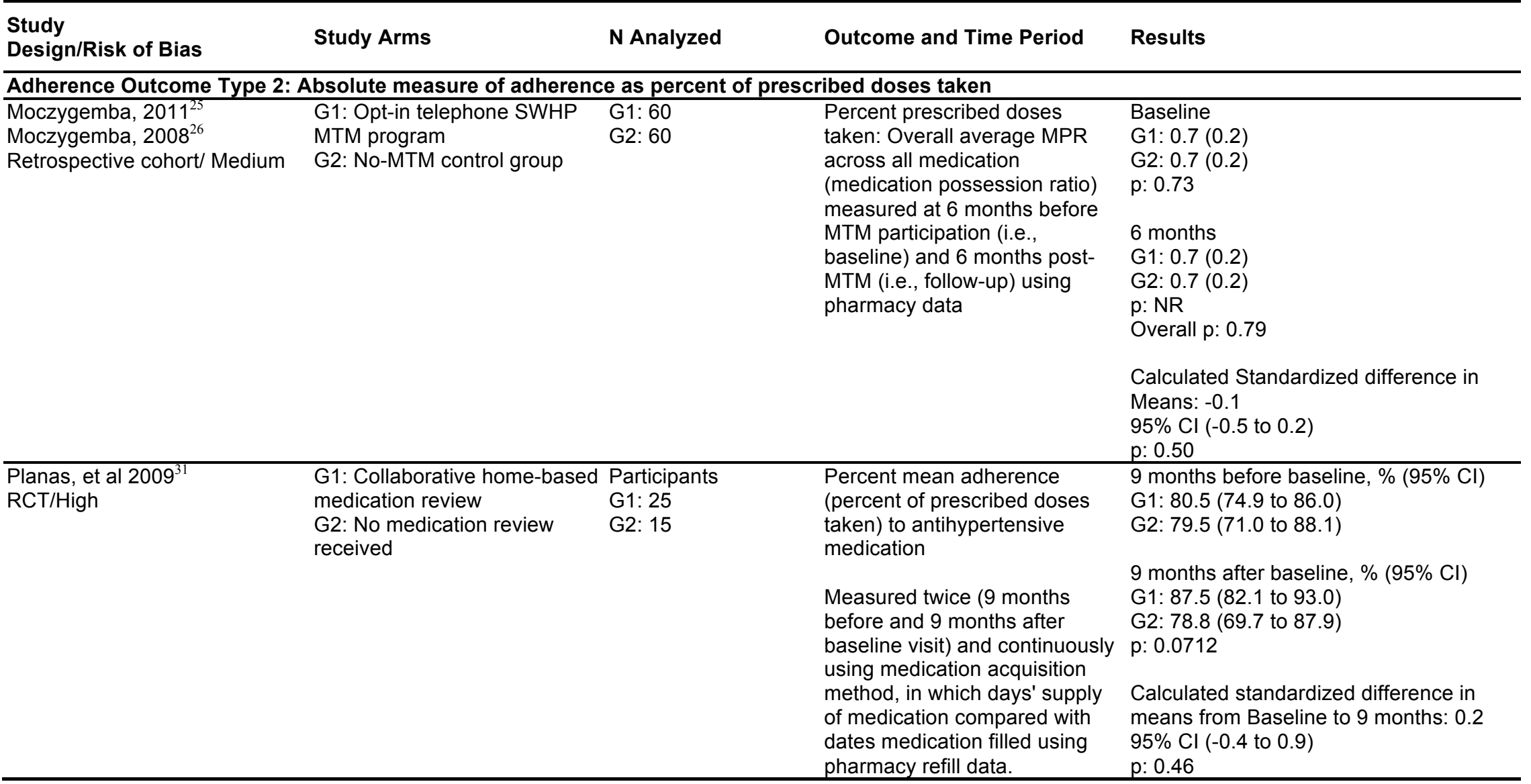


Table E10. Medication adherence: Summary of results (continued)

\begin{tabular}{|c|c|c|c|c|}
\hline $\begin{array}{l}\text { Study } \\
\text { Design/Risk of Bias }\end{array}$ & Study Arms & N Analyzed & Outcome and Time Period & Results \\
\hline $\begin{array}{l}\text { Park et al., } 1996^{29} \\
\text { RCT/High }\end{array}$ & $\begin{array}{l}\text { G1: Comprehensive } \\
\text { pharmaceutical care } \\
\text { G2: Usual care }\end{array}$ & $\begin{array}{l}\text { Visit 1 } \\
\text { G1: } 7 \\
\text { G2: } 5 \\
\text { Visit } 2 \\
\text { G1: } 21 \\
\text { G2: } 23 \\
\\
\text { Visit } 3 \\
\text { G1: } 23 \\
\text { G2: } 20 \\
\\
\text { Visit } 4 \\
\text { G1: } 21 \\
\text { G2: } 22\end{array}$ & $\begin{array}{l}\text { Mean percent compliance } \\
\text { (percent of prescribed pills } \\
\text { taken) from pharmacist report } \\
\text { of pill counts } \\
4 \text { month timeframe }\end{array}$ & $\begin{array}{l}\text { Baseline/Visit } 1 \\
\text { G1: } 87.4(0.9) \\
\text { G2: } 87.8(13.7) \\
\text { Visit } 2 \\
\text { G1: } 96.7(4) \\
\text { G2: } 86.0(20.7) \\
\text { p=0.025 } \\
\text { Visit } 3 \\
\text { G1: } 97.2(4.4) \\
\text { G2: } 86.7(23.1) \\
p=0.037 \\
\text { Visit } 4 \\
\text { G1: } 86.8(28.7) \\
\text { G2: } 89.1(21.8) \\
\text { Calculated standardized difference in } \\
\text { means for change from baseline to } \\
\text { Visit } 4:-0.1 \\
95 \% \text { I (-0.7 to 0.5) } \\
\text { p=0.77 }\end{array}$ \\
\hline
\end{tabular}


Table E10. Medication adherence: Summary of results (continued)

\begin{tabular}{|c|c|c|c|c|}
\hline $\begin{array}{l}\text { Study } \\
\text { Design/Risk of Bias }\end{array}$ & Study Arms & N Analyzed & Outcome and Time Period & Results \\
\hline \multicolumn{5}{|c|}{ Adherence Outcome Type 3: Self-reported Adherence using Morisky Scale } \\
\hline $\begin{array}{l}\text { Bernsten, } 2001^{1} ; \\
\text { Sturgess, } 2003^{2} \\
\text { RCT/ High (pooled data) }\end{array}$ & $\begin{array}{l}\text { G1: Structured community } \\
\text { pharmacy-based } \\
\text { pharmaceutical care program } \\
\text { G2: Normal pharmaceutical } \\
\text { Usual community pharmacy } \\
\text { services }\end{array}$ & $\begin{array}{l}\text { Pooled sample } \\
\text { (excluding The } \\
\text { Netherlands because } \\
\text { no baseline } \\
\text { adherence data } \\
\text { collected) } \\
\text { Baseline } \\
\text { G1: } 867 \\
\text { G2: } 748 \\
\text { 6 months } \\
\text { G1: NR } \\
\text { G2: NR } \\
\text { 12 months } \\
\text { G1: NR } \\
\text { G2: NR } \\
\text { 18 months } \\
\text { G1: } 792 \\
\text { G2: } 758\end{array}$ & $\begin{array}{l}\text { Medication adherence: self- } \\
\text { reported as assessed by } \\
\text { Morisky Scale } \\
\text { (Note: Percent of participants } \\
\text { who we adherent defined as } \\
\text { patients responded that they } \\
\text { "never" experienced any } \\
\text { aspects of non-compliance on } \\
\text { the 4-item 4-point scale) }\end{array}$ & $\begin{array}{l}\text { Pooled sample (percent adherent) } \\
\text { Baseline } \\
\text { G1: } 33.9 \\
\text { G2: } 38.6 \\
\text { OR: } 0.8 \\
\text { Calculated } 95 \% \mathrm{Cl}(0.7 \text { to } 1.0) \\
\text { p: } 0.049 \\
\text { 6 months } \\
\text { G1: } 38.5 \\
\text { G2: } 36.6 \\
\text { p: NR } \\
\text { 12 months } \\
\text { G1: } 43.8 \\
\text { G2: } 37.3 \\
\text { p: NR } \\
\text { 18 months } \\
\text { G1: } 38.2 \\
\text { G2: } 39.4 \\
\text { OR: } 1.1 \\
95 \% \text { CI (0.9 to } 1.3) \\
\text { p=0.440101 } \\
\text { Percent changing from nonadherent to } \\
\text { adherent over } 18 \text { months } \\
\text { G1: } 15.2 \\
\text { G2: } 12.2 \\
\text { p: } 0.028\end{array}$ \\
\hline
\end{tabular}


Table E10. Medication adherence: Summary of results (continued)

\begin{tabular}{|c|c|c|c|c|}
\hline $\begin{array}{l}\text { Study } \\
\text { Design/Risk of Bias }\end{array}$ & Study Arms & N Analyzed & Outcome and Time Period & Results \\
\hline $\begin{array}{l}\text { Volume et al., } 2001{\text { (PREP })^{39}} \text {; } \\
\text { Kassam et al., } 2001^{40} \\
\text { RCT/Medium }\end{array}$ & $\begin{array}{l}\text { G1: Comprehensive } \\
\text { pharmaceutical care services } \\
\text { G2: Traditional pharmacy care }\end{array}$ & $\begin{array}{l}\text { T1: } N=363 \\
\mathrm{G} 1: 159 \\
\mathrm{G} 2: 204 \\
\text { T2: } \mathrm{N}=317 \\
\text { T3: } \mathrm{N}=292 \\
\\
\text { Estimated by group } \\
\text { based on overall } \\
\text { retention } \\
\mathrm{G} 1: 127 \\
\mathrm{G} 2: 163\end{array}$ & $\begin{array}{l}\text { Self-reported adherence using } \\
\text { the } 4 \text {-item } 2 \text {-point Morisky } \\
\text { Scale where summary score is } \\
0-4 \text { with lower scores being } \\
\text { better adherence } \\
\text { Time } 1 \text { (Baseline), Time } 2 \text { (mid- } \\
\text { point, } 6 \text { to } 7 \text { months after } \\
\text { intervention) and Time } 3 \text { ( } 12 \text { to } \\
13 \text { months after intervention) }\end{array}$ & $\begin{array}{l}\text { Mean Adherence Scale Score } \\
\text { Time 1: } \\
\text { G1: } 0.5(0.8) \\
\text { G2: } 0.6(0.9) \\
\text { p: NS } \\
\text { Calculated standardized difference in } \\
\text { means: }-0.1 \\
\text { 95\% Cl }(-0.3 \text { to } 0.1) \\
\text { p=0.208957 } \\
\text { Time } 2: \\
\text { G1: } 0.5(0.7) \\
\text { G2: } 0.6(0.8) \\
\text { p:NS } \\
\text { Time } 3: \\
\text { G1: } 0.6(0.8) \\
\text { G2: } 0.5(0.7) \\
\text { p: NS } \\
\text { Calculated standardized difference in } \\
\text { means: }-0.13 \\
\text { 95\% Cl (-0.11 to } 0.36) \\
\text { p=0.289285 }\end{array}$ \\
\hline
\end{tabular}


Table E10. Medication adherence: Summary of results (continued)

\begin{tabular}{|c|c|c|c|c|}
\hline $\begin{array}{l}\text { Study } \\
\text { Design/Risk of Bias }\end{array}$ & Study Arms & N Analyzed & Outcome and Time Period & Results \\
\hline \multirow[t]{3}{*}{$\begin{array}{l}\text { Jameson, VanNoord, and } \\
\text { Vanderwoud, } 1995^{17} \\
\text { RCT/High (medium for study } \\
\text { overall by high for adherence } \\
\text { due to poor measure) }\end{array}$} & \multirow[t]{3}{*}{$\begin{array}{l}\text { G1: Consultation with a clinical } \\
\text { pharmacist within a primary } \\
\text { care office. } \\
\text { G2: Standard medical care at } \\
\text { the primary care office. }\end{array}$} & $\begin{array}{l}\text { G1: } 27 \\
\text { G2: } 29\end{array}$ & $\begin{array}{l}\text { Self-reported composite } \\
\text { "understanding and } \\
\text { compliance" } 0-12 \text { score at } \\
\text { baseline and } 6 \text { months } \\
\text { (no further information on } \\
\text { measure used) } \\
\end{array}$ & $\begin{array}{l}\text { Baseline Means Scale Score (SD not } \\
\text { reported) } \\
\text { G1: } 2.3 \\
\text { G2: } 2.3 \\
\text { p: NS }\end{array}$ \\
\hline & & & $\begin{array}{l}\text { Change in self-reported } \\
\text { composite score over } 6 \text { months } \\
\text { with negative score } \\
\text { representing improvement }\end{array}$ & $\begin{array}{l}6 \text { months } \\
\text { G1: } 0.6 \\
\text { G2: } 2.1 \\
\text { p: NS }\end{array}$ \\
\hline & & & & $\begin{array}{l}\text { G1: }-1.6 \\
\text { G2: }-0.2 \\
\mathrm{p}: \mathrm{NS}\end{array}$ \\
\hline
\end{tabular}


Table E10. Medication adherence: Summary of results (continued)

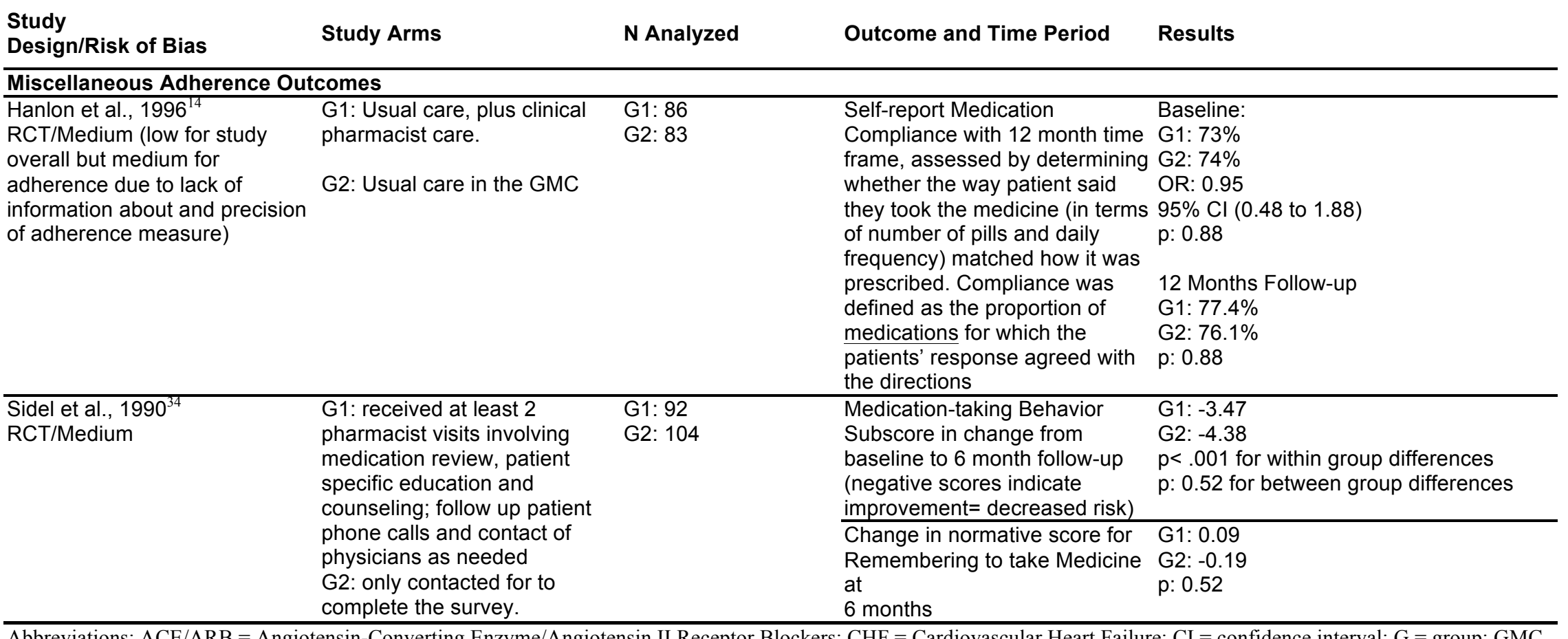

Abbreviations: ACE/ARB = Angiotensin-Converting Enzyme/Angiotensin II Receptor Blockers; CHF = Cardiovascular Heart Failure; CI = confidence interval; G = group; GMC

$=$ General Medicine Clinic; MPR = medication possession ratio; MTM = Medication Therapy Management; MTMP = Medication Therapy Management Program; NR = not

reported; NS = not sufficient; OR = odds ratio; PREP = Pharmaceutical Care Research and Education Project; RCT= randomized controlled trial; SD = standard deviation; $\mathrm{SWHP}$

$=$ Scott $\&$ White Health Plan; $\mathrm{T}=$ time. 
Table E11. Medication appropriateness scales: Summary of results

\begin{tabular}{|c|c|c|c|c|}
\hline $\begin{array}{l}\text { Study } \\
\text { Design/Risk of Bias }\end{array}$ & Study Arms & N Analyzed & Outcome and Time Period & Results \\
\hline $\begin{array}{l}\text { Hanlon et al., } 1996^{14} \\
\text { RCT/Low }\end{array}$ & $\begin{array}{l}\text { G1: Usual care, plus clinical } \\
\text { pharmacist care. } \\
\text { G2: Usual care in the GMC }\end{array}$ & $\begin{array}{l}\text { G1: } 105 \\
\text { G2: } 103\end{array}$ & $\begin{array}{l}\text { Change in covariate-adjusted } \\
\text { Medication Appropriateness } \\
\text { Index } \\
\text { Assessed at baseline, } 3,12 \\
\text { months by blinded research } \\
\text { pharmacist }\end{array}$ & 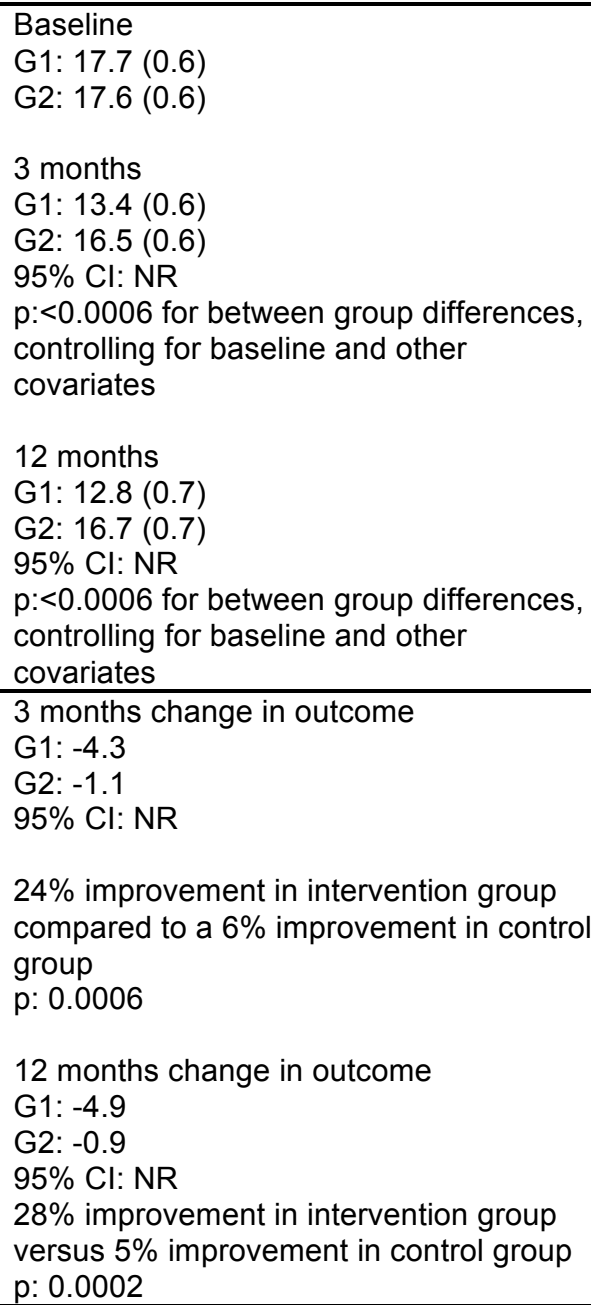 \\
\hline
\end{tabular}


Table E11. Medication appropriateness scales: Summary of results (continued)

\begin{tabular}{|c|c|c|c|c|}
\hline $\begin{array}{l}\text { Study } \\
\text { Design/Risk of Bias }\end{array}$ & Study Arms & N Analyzed & Outcome and Time Period & Results \\
\hline \multirow[t]{3}{*}{$\begin{array}{l}\text { Carter et al., } 1997^{4} ; \\
\text { Barnette, Murphy, and } \\
\text { Carter, } 1996^{5} \\
\text { Cohort study/High }\end{array}$} & $\begin{array}{l}\text { G1: Pharmacy-based } \\
\text { pharmaceutical care } \\
\text { G2: Usual medical care }\end{array}$ & $\begin{array}{l}\text { G1: } 25 \\
\text { G2: } 26\end{array}$ & $\begin{array}{l}\text { Appropriateness of BP regimen } \\
\text { A blinded review panel of three } \\
\text { evaluated cases in random order } \\
\text { on a visual analog scale, using } \\
\text { medical records. The } \\
\text { investigators averaged and } \\
\text { converted scores to a numerical } \\
\text { value by measuring the distance } \\
\text { from the best option. Score } \\
\text { arranged from } 0-16.2 \text {. Higher } \\
\text { scores are more positive. }\end{array}$ & $\begin{array}{l}\text { BP Regimen } \\
\text { Baseline } \\
\text { G1: } 8.7(4.7) \\
\text { G2: } 10.3(4.8) \\
\text { Follow-up } \\
\text { G1: } 10.9(4.5) \\
\text { G2: } 10.1(5.2) \\
\text { p for change scores NR }\end{array}$ \\
\hline & & & Appropriateness of daily dosage & $\begin{array}{l}\text { Appropriateness of daily dosage } \\
\text { Baseline } \\
\text { G1: } 11.6(4.5) \\
\text { G2: } 12.6(4.5) \\
\text { Follow-up } \\
\text { G1: } 13.4(3.7) \\
\text { G2: } 13.2(4.1) \\
\text { p for change scores NR }\end{array}$ \\
\hline & & & $\begin{array}{l}\text { Appropriateness of dosing } \\
\text { interval }\end{array}$ & $\begin{array}{l}\text { Appropriateness of dosing interval } \\
\text { Baseline } \\
\text { G1: } 13.8(4.3) \\
\text { G2: } 13.4(4.6) \\
\text { Follow-up } \\
\text { G1: } 15.1(2.3) \\
\text { G2: } 13.8(4.1) \\
\text { p for change scores NR }\end{array}$ \\
\hline
\end{tabular}


Table E12. Medication appropriateness for individual medications: Summary of results

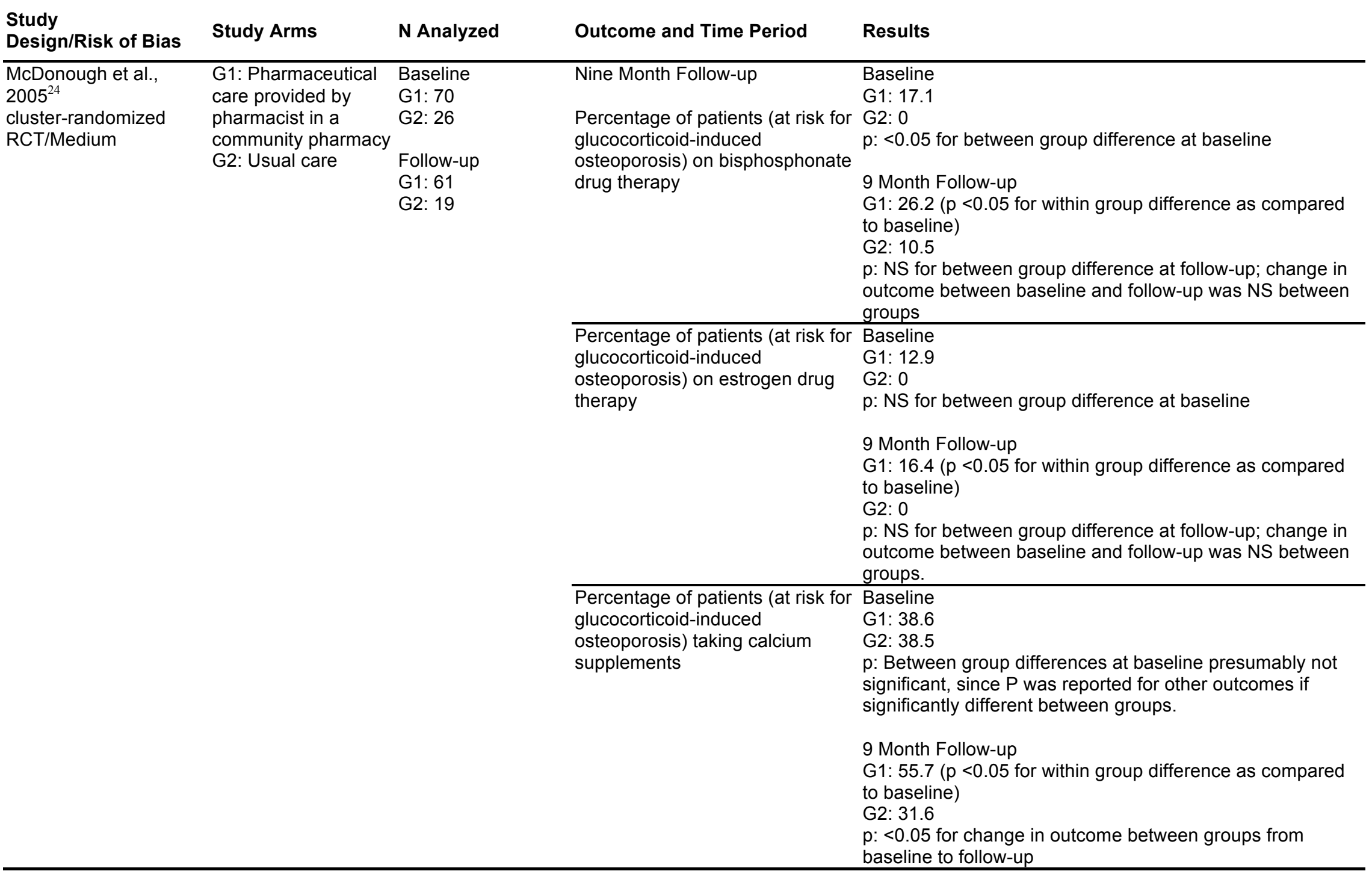


Table E12. Medication appropriateness for individual medications: Summary of results (continued)

\begin{tabular}{|c|c|c|c|c|}
\hline $\begin{array}{l}\text { Study } \\
\text { Design/Risk of Bias }\end{array}$ & Study Arms & N Analyzed & Outcome and Time Period & Results \\
\hline $\begin{array}{l}\text { Gattis et al., } 1999^{13} \\
\text { RCT/Medium }\end{array}$ & $\begin{array}{l}\text { G1: Clinical } \\
\text { pharmacist } \\
\text { intervention } \\
\text { G2: Usual medical } \\
\text { care }\end{array}$ & $\begin{array}{l}\text { G1: } 90 \\
\text { G2: } 91\end{array}$ & $\begin{array}{l}6 \text { month follow-up } \\
\text { Percent receiving an ACEI at } \\
\text { follow-up } \\
\text { Fraction of target ACEI dose at } \\
\text { follow up }\end{array}$ & $\begin{array}{l}\text { G1: } 87 \\
\text { G2: } 79 \\
\text { p: } 0.18 \\
\text { G1: } 1(25 \%: 0.5,75 \%: 1) \\
\text { G2: } 0.5(25 \% 0.188,75 \%: 1) \\
\text { 95\% Cl: NR } \\
\text { p: }<0.001\end{array}$ \\
\hline & & $\begin{array}{l}\text { G1: } 12 \\
\text { G2: } 19\end{array}$ & $\begin{array}{l}\text { Of those NOT on an ACEI at } \\
\text { follow-up, percentage receiving } \\
\text { alternative drug therapy }\end{array}$ & $\begin{array}{l}\mathrm{G} 1: 75 \\
\mathrm{G} 2: 26 \\
\mathrm{p}: 0.02\end{array}$ \\
\hline
\end{tabular}

Abbreviations: ACEI = Angiotensin-Converting Enzyme Inhibitors; $\mathrm{CI}=$ confidence interval; $\mathrm{G}=$ group; $\mathrm{NR}=$ not reported; RCT = randomized controlled trial 
Table E13. Medication Appropriateness Index Item 1 (Is there an indication for the drug?): Summary of results

\begin{tabular}{|c|c|c|c|c|}
\hline $\begin{array}{l}\text { Study } \\
\text { Design/Risk of Bias }\end{array}$ & Study Arms & N Analyzed & Outcome and Time Period & Results \\
\hline $\begin{array}{l}\text { Hanlon et al., } 1996^{14} \\
\text { RCT/Low }\end{array}$ & $\begin{array}{l}\text { G1: Usual care, plus clinical } \\
\text { pharmacist care. } \\
\text { G2: Usual care in the GMC }\end{array}$ & $\begin{array}{l}\text { N participants } \\
\text { G1: } 105 \\
\text { G2: } 103 \\
\text { Number of } \\
\text { prescriptions: } \\
\text { Baseline } \\
\text { G1: } 798 \\
\text { G2: } 846 \\
\text { 12 months } \\
\text { G1: } 734 \\
\text { G2: } 847\end{array}$ & $\begin{array}{l}\text { Percent Prescriptions } \\
\text { Inappropriate } \\
\text { Assessed at baseline, } 3,12 \\
\text { months by blinded research } \\
\text { pharmacist }\end{array}$ & $\begin{array}{l}\text { Baseline } \\
\text { G1: } 10.5 \\
\text { G2: } 12.4 \\
\\
3 \text { months } \\
\text { G1: } 8.1 \\
\text { G2: } 10.5 \\
\text { p: NR } \\
\text { 12 months } \\
\text { G1: } 6.0 \\
\text { G2: } 9.7 \\
\text { p: NR }\end{array}$ \\
\hline $\begin{array}{l}\text { Taylor, Byrd, and Krueger, } \\
2003^{36} \\
\text { RCT/Medium }\end{array}$ & $\begin{array}{l}\text { G1: Pharmaceutical care } \\
\text { G2: Standard care }\end{array}$ & $\begin{array}{l}\text { N participants } \\
\text { G1: } 33 \\
\text { G2: } 36 \\
\text { Number of } \\
\text { prescriptions: } \\
\text { Baseline } \\
\text { G1: } 210 \\
\text { G2: } 207 \\
\text { 12 months } \\
\text { G1: } 155 \\
\text { G2: } 224\end{array}$ & $\begin{array}{l}\text { Percent Prescriptions } \\
\text { Inappropriate } \\
\text { Assessed at baseline, } 12 \text { months } \\
\text { by blinded research pharmacist }\end{array}$ & $\begin{array}{l}\text { Baseline } \\
\text { G1: } 33.3 \\
\text { G2: } 46.8 \\
\text { 12 months } \\
\text { G1: } 16.1 \\
\text { G2: } 48.2\end{array}$ \\
\hline
\end{tabular}

Abbreviations: $\mathrm{CI}=$ confidence interval; diff = difference; $\mathrm{G}=$ group; $\mathrm{GMC}=$ General Medicine Clinic; $\mathrm{N}=$ number; $\mathrm{NR}=$ not reported; $\mathrm{RCT}=$ randomized controlled trial; $\mathrm{SE}=$ standard error; $\mathrm{Std}=$ standard 
Table E14. Medication Appropriateness Index Item 2 (Is the medication effective for the condition?): Summary of results

\begin{tabular}{|c|c|c|c|c|}
\hline $\begin{array}{l}\text { Study } \\
\text { Design/Risk of Bias }\end{array}$ & Study Arms & N Analyzed & Outcome and Time Period & Results \\
\hline $\begin{array}{l}\text { Hanlon et al., } 1996^{14} \\
\text { RCT/Low }\end{array}$ & $\begin{array}{l}\text { G1: Usual care, plus clinical } \\
\text { pharmacist care. } \\
\text { G2: Usual care in the GMC }\end{array}$ & $\begin{array}{l}\text { N participants } \\
\text { G1: } 105 \\
\text { G2: } 103 \\
\text { Number of } \\
\text { prescriptions: } \\
\text { Baseline } \\
\text { G1: } 798 \\
\text { G2: } 846 \\
\text { 12 months } \\
\text { G1: } 734 \\
\text { G2: } 847 \\
\end{array}$ & $\begin{array}{l}\text { Percent Prescriptions } \\
\text { Inappropriate } \\
\text { Assessed at baseline, 3, } 12 \\
\text { months by blinded research } \\
\text { pharmacist }\end{array}$ & $\begin{array}{l}\text { Baseline } \\
\text { G1: } 4.5 \\
\text { G2: } 4.9 \\
\\
3 \text { months } \\
\text { G1: } 3.6 \\
\text { G2: } 4.9 \\
\text { p: NR } \\
\text { 12 months } \\
\text { G1: } 3.4 \\
\text { G2: } 4.9 \\
\text { p: NR } \\
\end{array}$ \\
\hline $\begin{array}{l}\text { Taylor, Byrd, and Krueger, } \\
2003^{36} \\
\text { RCT/Medium }\end{array}$ & $\begin{array}{l}\text { G1: Pharmaceutical care } \\
\text { G2: Standard care }\end{array}$ & $\begin{array}{l}\text { N participants } \\
\text { G1: } 33 \\
\text { G2: } 36 \\
\text { Number of } \\
\text { prescriptions: } \\
\text { Baseline } \\
\text { G1: } 210 \\
\text { G2: } 207 \\
\text { 12 months } \\
\text { G1: } 155 \\
\text { G2: } 224\end{array}$ & $\begin{array}{l}\text { Percent Prescriptions } \\
\text { Inappropriate } \\
\text { Assessed at baseline, } 12 \text { months } \\
\text { by blinded research pharmacist }\end{array}$ & $\begin{array}{l}\text { Baseline } \\
\text { G1: } 29.1 \\
\text { G2: } 44.9 \\
\\
12 \text { months } \\
\text { G1: } 13.6 \\
\text { G2: } 44.6\end{array}$ \\
\hline
\end{tabular}

Abbreviations: $\mathrm{CI}=$ confidence interval; diff $=$ difference; $\mathrm{G}=$ group; $\mathrm{GMC}=$ General Medicine Clinic; $\mathrm{N}=$ number; $\mathrm{NR}=$ not reported; $\mathrm{RCT}=$ randomized controlled trial; $\mathrm{SE}=$ standard error; $\mathrm{Std}=$ standard 
Table E15. Medication appropriateness index Item 3 (Is the dosage correct?): Summary of results

\begin{tabular}{|c|c|c|c|c|}
\hline $\begin{array}{l}\text { Study } \\
\text { Design/Risk of Bias }\end{array}$ & Study Arms & N Analyzed & Outcome and Time Period & Results \\
\hline $\begin{array}{l}\text { Hanlon et al., } 1996^{14} \\
\text { RCT/Low }\end{array}$ & $\begin{array}{l}\text { G1: Usual care, plus clinical } \\
\text { pharmacist care. } \\
\text { G2: Usual care in the GMC }\end{array}$ & $\begin{array}{l}\text { N participants } \\
\text { G1: } 105 \\
\text { G2: } 103 \\
\text { Number of } \\
\text { prescriptions: } \\
\text { Baseline } \\
\text { G1: } 798 \\
\text { G2: } 846 \\
\text { 12 months } \\
\text { G1: } 734 \\
\text { G2: } 847\end{array}$ & $\begin{array}{l}\text { Percent Prescriptions } \\
\text { Inappropriate } \\
\text { Assessed at baseline, } 3,12 \\
\text { months by blinded research } \\
\text { pharmacist }\end{array}$ & $\begin{array}{l}\text { Baseline } \\
\text { G1: } 17.4 \\
\text { G2: } 17.3 \\
\\
3 \text { months } \\
\text { G1: } 13.1 \\
\text { G2: } 18.2 \\
\text { p: NR } \\
\text { 12 months } \\
\text { G1: } 15.0 \\
\text { G2: } 20.4 \\
\text { p: NR }\end{array}$ \\
\hline $\begin{array}{l}\text { Taylor, Byrd, and Krueger, } \\
2003^{36} \\
\text { RCT/Medium }\end{array}$ & $\begin{array}{l}\text { G1: Pharmaceutical care } \\
\text { G2: Standard care }\end{array}$ & $\begin{array}{l}\text { N participants } \\
\text { G1: } 33 \\
\text { G2: } 36 \\
\text { Number of } \\
\text { prescriptions: } \\
\text { Baseline } \\
\text { G1: } 210 \\
\text { G2: } 207 \\
\text { 12 months } \\
\text { G1: } 155 \\
\text { G2: } 224\end{array}$ & $\begin{array}{l}\text { Percent Prescriptions } \\
\text { Inappropriate } \\
\text { Assessed at baseline, } 12 \text { months } \\
\text { by blinded research pharmacist }\end{array}$ & $\begin{array}{l}\text { Baseline } \\
\text { G1: } 63.3 \\
\text { G2: } 62.3 \\
\text { 12 months } \\
\text { G1: } 12.9 \\
\text { G2: } 63.8\end{array}$ \\
\hline
\end{tabular}

Abbreviations: $\mathrm{CI}=$ confidence interval; diff = difference; $\mathrm{G}=$ group; $\mathrm{GMC}=$ General Medicine Clinic; $\mathrm{N}=$ number; $\mathrm{NR}=$ not reported; $\mathrm{RCT}=$ randomized controlled trial; $\mathrm{SE}=$ standard error; Std $=$ standard 
Table E16. Medication Appropriateness Index Item 4 (Are the directions correct?): Summary of results

\begin{tabular}{|c|c|c|c|c|}
\hline $\begin{array}{l}\text { Study } \\
\text { Design/Risk of Bias }\end{array}$ & Study Arms & N Analyzed & Outcome and Time Period & Results \\
\hline $\begin{array}{l}\text { Hanlon et al., } 1996^{14} \\
\text { RCT/Low }\end{array}$ & $\begin{array}{l}\text { G1: Usual care, plus clinical } \\
\text { pharmacist care. } \\
\text { G2: Usual care in the GMC }\end{array}$ & $\begin{array}{l}\text { N participants } \\
\text { G1: } 105 \\
\text { G2: } 103 \\
\text { Number of } \\
\text { prescriptions: } \\
\text { Baseline } \\
\text { G1: } 798 \\
\text { G2: } 846 \\
\text { 12 months } \\
\text { G1: } 734 \\
\text { G2: } 847\end{array}$ & $\begin{array}{l}\text { Percent Prescriptions } \\
\text { Inappropriate } \\
\text { Assessed at baseline, 3, } 12 \\
\text { months by blinded research } \\
\text { pharmacist }\end{array}$ & $\begin{array}{l}\text { Baseline } \\
\text { G1: } 32.7 \\
\text { G2: } 32.2 \\
\\
3 \text { months } \\
\text { G1: } 28.1 \\
\text { G2: } 32.6 \\
\text { p: NR } \\
\text { 12 months } \\
\text { G1: } 27.5 \\
\text { G2: } 29.9 \\
\text { p: NR }\end{array}$ \\
\hline $\begin{array}{l}\text { Taylor, Byrd, and Krueger, } \\
2003^{36} \\
\text { RCT/Medium }\end{array}$ & $\begin{array}{l}\text { G1: Pharmaceutical care } \\
\text { G2: Standard care }\end{array}$ & $\begin{array}{l}\text { N participants } \\
\text { G1: } 33 \\
\text { G2: } 36 \\
\text { Number of } \\
\text { prescriptions: } \\
\text { Baseline } \\
\text { G1: } 210 \\
\text { G2: } 207 \\
\text { 12 months } \\
\text { G1: } 155 \\
\text { G2: } 224\end{array}$ & $\begin{array}{l}\text { Percent Prescriptions } \\
\text { Inappropriate } \\
\text { Assessed at baseline, } 12 \text { months } \\
\text { by blinded research pharmacist }\end{array}$ & $\begin{array}{l}\text { Baseline } \\
\text { G1: } 70.5 \\
\text { G2: } 64.3 \\
\\
12 \text { months } \\
\text { G1: } 29.7 \\
\text { G2: } 56.7\end{array}$ \\
\hline
\end{tabular}

Abbreviations: $\mathrm{CI}=$ confidence interval; diff = difference; $\mathrm{G}=$ group; $\mathrm{GMC}=$ General Medicine Clinic; $\mathrm{N}=$ number; $\mathrm{NR}=$ not reported; $\mathrm{RCT}=$ randomized controlled trial; $\mathrm{SE}=$ standard error; Std $=$ standard 
Table E17. Medication Appropriateness Index Item 5 (Are the directions practical?): Summary of results

\begin{tabular}{|c|c|c|c|c|}
\hline $\begin{array}{l}\text { Study } \\
\text { Design/Risk of Bias }\end{array}$ & Study Arms & N Analyzed & Outcome and Time Period & Results \\
\hline $\begin{array}{l}\text { Hanlon et al., } 1996^{14} \\
\text { RCT/Low }\end{array}$ & $\begin{array}{l}\text { G1: Usual care, plus clinical } \\
\text { pharmacist care. } \\
\text { G2: Usual care in the General } \\
\text { Medicine Clinic (GMC) }\end{array}$ & $\begin{array}{l}\text { N participants } \\
\text { G1: } 105 \\
\text { G2: } 103 \\
\text { Number of } \\
\text { prescriptions: } \\
\text { Baseline } \\
\text { G1: } 798 \\
\text { G2: } 846 \\
\text { 12 months } \\
\text { G1: } 734 \\
\text { G2: } 847\end{array}$ & $\begin{array}{l}\text { Percent Prescriptions } \\
\text { Inappropriate } \\
\text { Assessed at baseline, } 3,12 \\
\text { months by blinded research } \\
\text { pharmacist }\end{array}$ & $\begin{array}{l}\text { Baseline } \\
\text { G1: } 20.7 \\
\text { G2: } 20.0 \\
3 \text { months } \\
\text { G1: } 15.8 \\
\text { G2: } 18.9 \\
\text { p: NR } \\
\text { 12 months } \\
\text { G1: } 15.3 \\
\text { G2: } 21.2 \\
\text { p: NR }\end{array}$ \\
\hline $\begin{array}{l}\text { Taylor, Byrd, and Krueger, } \\
2003^{36} \\
\text { RCT/Medium }\end{array}$ & $\begin{array}{l}\text { G1: Pharmaceutical care } \\
\text { G2: Standard care }\end{array}$ & $\begin{array}{l}\text { N participants } \\
\text { G1: } 33 \\
\text { G2: } 36 \\
\text { Number of } \\
\text { prescriptions: } \\
\text { Baseline } \\
\text { G1: } 210 \\
\text { G2: } 207 \\
\text { 12 months } \\
\text { G1: } 155 \\
\text { G2: } 224\end{array}$ & $\begin{array}{l}\text { Percent Prescriptions } \\
\text { Inappropriate } \\
\text { Assessed at baseline, } 12 \text { months } \\
\text { by blinded research pharmacist }\end{array}$ & $\begin{array}{l}\text { Baseline } \\
\text { G1: } 61.0 \\
\text { G2: } 57.0 \\
\text { 12 months } \\
\text { G1: } 29.7 \\
\text { G2: } 56.7\end{array}$ \\
\hline
\end{tabular}

Abbreviations: $\mathrm{CI}=$ confidence interval; diff = difference; $\mathrm{G}=$ group; $\mathrm{GMC}=$ General Medicine Clinic; $\mathrm{N}=$ number; $\mathrm{NR}=$ not reported; $\mathrm{RCT}=$ randomized controlled trial; $\mathrm{SE}=$ standard error; $\mathrm{Std}=$ standard 
Table E18. Medication Appropriateness Index Item 6 (Are there clinically significant drug-drug interactions?): Summary of results

\begin{tabular}{|c|c|c|c|c|}
\hline $\begin{array}{l}\text { Study } \\
\text { Design/Risk of Bias }\end{array}$ & Study Arms & N Analyzed & Outcome and Time Period & Results \\
\hline $\begin{array}{l}\text { Hanlon et al., } 1996^{14} \\
\text { RCT/Low }\end{array}$ & $\begin{array}{l}\text { G1: Usual care, plus clinical } \\
\text { pharmacist care. } \\
\text { G2: Usual care in the GMC }\end{array}$ & $\begin{array}{l}\text { N participants } \\
\text { G1: } 105 \\
\text { G2: } 103 \\
\text { Number of } \\
\text { prescriptions: } \\
\text { Baseline } \\
\text { G1: } 798 \\
\text { G2: } 846 \\
\text { 12 months } \\
\text { G1: } 734 \\
\text { G2: } 847\end{array}$ & $\begin{array}{l}\text { Percent Prescriptions } \\
\text { Inappropriate } \\
\text { Assessed at baseline, } 3,12 \\
\text { months by blinded research } \\
\text { pharmacist }\end{array}$ & $\begin{array}{l}\text { Baseline } \\
\text { G1: } 0 \\
\text { G2: } 0 \\
3 \text { months } \\
\text { G1: } 0 \\
\text { G2: } 0.1 \\
\text { p: NR } \\
\text { 12 months } \\
\text { G1: } 0 \\
\text { G2: } 0.1\end{array}$ \\
\hline $\begin{array}{l}\text { Taylor, Byrd, and Krueger, } \\
2003^{36} \\
\text { RCT/Medium }\end{array}$ & $\begin{array}{l}\text { G1: Pharmaceutical care } \\
\text { G2: Standard care }\end{array}$ & $\begin{array}{l}\text { N participants } \\
\text { G1: } 33 \\
\text { G2: } 36 \\
\text { Number of } \\
\text { prescriptions: } \\
\text { Baseline } \\
\text { G1: } 210 \\
\text { G2: } 207 \\
\text { 12 months } \\
\text { G1: } 155 \\
\text { G2: } 224\end{array}$ & $\begin{array}{l}\text { Percent Prescriptions } \\
\text { Inappropriate } \\
\text { Assessed at baseline, } 12 \text { months } \\
\text { by blinded research pharmacist }\end{array}$ & $\begin{array}{l}\text { Baseline } \\
\text { G1: } 22.9 \\
\text { G2: } 17.9 \\
\\
12 \text { months } \\
\text { G1: } 5.8 \\
\text { G2: } 22.8\end{array}$ \\
\hline
\end{tabular}

Abbreviations: $\mathrm{CI}=$ confidence interval; diff = difference; $\mathrm{G}=$ group; $\mathrm{GMC}=$ General Medicine Clinic; $\mathrm{N}=$ number; $\mathrm{NR}=$ not reported; $\mathrm{RCT}=$ randomized controlled trial; $\mathrm{SE}=$ standard error; Std $=$ standard 
Table E19. Medication Appropriateness Index Item 7 (Are there clinically significant drug-disease interactions?): Summary of results

\begin{tabular}{|c|c|c|c|c|}
\hline $\begin{array}{l}\text { Study } \\
\text { Design/Risk of Bias }\end{array}$ & Study Arms & N Analyzed & Outcome and Time Period & Results \\
\hline $\begin{array}{l}\text { Hanlon et al., } 1996^{14} \\
\text { RCT/Low }\end{array}$ & $\begin{array}{l}\text { G1: Usual care, plus clinical } \\
\text { pharmacist care. } \\
\text { G2: Usual care in the GMC }\end{array}$ & $\begin{array}{l}\text { N participants } \\
\text { G1: } 105 \\
\text { G2: } 103 \\
\text { Number of } \\
\text { prescriptions: } \\
\text { Baseline } \\
\text { G1: } 798 \\
\text { G2: } 846 \\
\text { 12 months } \\
\text { G1: } 734 \\
\text { G2: } 847\end{array}$ & $\begin{array}{l}\text { Percent Prescriptions } \\
\text { Inappropriate } \\
\text { Assessed at baseline, } 3,12 \\
\text { months by blinded research } \\
\text { pharmacist }\end{array}$ & $\begin{array}{l}\text { Baseline } \\
\text { G1: } 1.9 \\
\text { G2: } 1.0 \\
\text { 3 months } \\
\text { G1: } 2.0 \\
\text { G2: } 0.7 \\
\text { p: NR } \\
\text { 12 months } \\
\text { G1: } 1.9 \\
\text { G2: } 1.1 \\
\text { p: NR }\end{array}$ \\
\hline $\begin{array}{l}\text { Taylor, Byrd, and Krueger, } \\
2003^{36} \\
\text { RCT/Medium }\end{array}$ & $\begin{array}{l}\text { G1: Pharmaceutical care } \\
\text { G2: Standard care }\end{array}$ & $\begin{array}{l}\text { N participants } \\
\text { G1: } 33 \\
\text { G2: } 36 \\
\text { Number of } \\
\text { prescriptions: } \\
\text { Baseline } \\
\text { G1: } 210 \\
\text { G2: } 207 \\
\text { 12 months } \\
\text { G1: } 155 \\
\text { G2: } 224\end{array}$ & $\begin{array}{l}\text { Percent Prescriptions } \\
\text { Inappropriate } \\
\text { Assessed at baseline, } 12 \text { months } \\
\text { by blinded research pharmacist }\end{array}$ & $\begin{array}{l}\text { Baseline } \\
\text { G1: } 18.6 \\
\text { G2: } 21.3 \\
\text { 12 months } \\
\text { G1: } 9.0 \\
\text { G2: } 19.6\end{array}$ \\
\hline
\end{tabular}

Abbreviations: $\mathrm{CI}=$ confidence interval; diff = difference; $\mathrm{G}=$ group; $\mathrm{GMC}=$ General Medicine Clinic; $\mathrm{N}=$ number; $\mathrm{NR}=$ not reported; $\mathrm{RCT}=$ randomized controlled trial; $\mathrm{SE}=$ standard error; Std $=$ standard 
Table E20. Medication Appropriateness Index Item 8 (Is there unnecessary duplication with other drugs?): Summary of results

\begin{tabular}{|c|c|c|c|c|}
\hline $\begin{array}{l}\text { Study } \\
\text { Design/Risk of Bias }\end{array}$ & Study Arms & N Analyzed & Outcome and Time Period & Results \\
\hline $\begin{array}{l}\text { Hanlon et al., } 1996^{14} \\
\text { RCT/Low }\end{array}$ & $\begin{array}{l}\text { G1: Usual care, plus clinical } \\
\text { pharmacist care. } \\
\text { G2: Usual care in the General } \\
\text { Medicine Clinic (GMC) }\end{array}$ & $\begin{array}{l}\text { N participants } \\
\text { G1: } 105 \\
\text { G2: } 103 \\
\text { Number of } \\
\text { prescriptions: } \\
\text { Baseline } \\
\text { G1: } 798 \\
\text { G2: } 846 \\
\text { 12 months } \\
\text { G1: } 734 \\
\text { G2: } 847\end{array}$ & $\begin{array}{l}\text { Percent Prescription } \\
\text { Inappropriate } \\
\text { Assessed at baseline, } 3,12 \\
\text { months by blinded research } \\
\text { pharmacist }\end{array}$ & $\begin{array}{l}\text { Baseline } \\
\text { G1: } 4.9 \\
\text { G2: } 6.4 \\
\text { 3 months } \\
\text { G1: } 3.0 \\
\text { G2: } 5.9 \\
\text { p: NR } \\
\text { 12 months } \\
\text { G1: } 4.9 \\
\text { G2: } 8.2 \\
\text { p: NR }\end{array}$ \\
\hline $\begin{array}{l}\text { Taylor, Byrd, and Krueger, } \\
2003^{36} \\
\text { RCT/Medium }\end{array}$ & $\begin{array}{l}\text { G1: Pharmaceutical care } \\
\text { G2: Standard care }\end{array}$ & $\begin{array}{l}\text { N participants } \\
\text { G1: } 33 \\
\text { G2: } 36 \\
\text { Number of } \\
\text { prescriptions: } \\
\text { Baseline } \\
\text { G1: } 210 \\
\text { G2: } 207 \\
\text { 12 months } \\
\text { G1: } 155 \\
\text { G2: } 224\end{array}$ & $\begin{array}{l}\text { Percent Prescriptions } \\
\text { Inappropriate } \\
\text { Assessed at baseline, } 12 \text { months } \\
\text { by blinded research pharmacist }\end{array}$ & $\begin{array}{l}\text { Baseline } \\
\text { G1: } 11.9 \\
\text { G2: } 6.8 \\
\text { 12 months } \\
\text { G1: } 4.5 \\
\text { G2: } 7.6\end{array}$ \\
\hline
\end{tabular}

Abbreviations: $\mathrm{CI}=$ confidence interval; diff = difference; $\mathrm{G}=$ group; $\mathrm{GMC}=$ General Medicine Clinic; $\mathrm{N}=$ number; $\mathrm{NR}=$ not reported; RCT = randomized controlled trial; $\mathrm{SE}=$ standard error; Std $=$ standard 
Table E21. Medication Appropriateness Index Item 9 (Is the duration of therapy acceptable?): Summary of results

\begin{tabular}{|c|c|c|c|c|}
\hline $\begin{array}{l}\text { Study } \\
\text { Design/Risk of Bias }\end{array}$ & Study Arms & N Analyzed & Outcome and Time Period & Results \\
\hline $\begin{array}{l}\text { Hanlon et al., } 1996^{14} \\
\text { RCT/Low }\end{array}$ & $\begin{array}{l}\text { G1: Usual care, plus clinical } \\
\text { pharmacist care. } \\
\text { G2: Usual care in the GMC }\end{array}$ & $\begin{array}{l}\text { N participants } \\
\text { G1: } 105 \\
\text { G2: } 103 \\
\text { Number of } \\
\text { prescriptions: } \\
\text { Baseline } \\
\text { G1: } 798 \\
\text { G2: } 846 \\
\text { 12 months } \\
\text { G1: } 734 \\
\text { G2: } 847\end{array}$ & $\begin{array}{l}\text { Percent Prescriptions } \\
\text { Inappropriate } \\
\text { Assessed at baseline, 3, } 12 \\
\text { months by blinded research } \\
\text { pharmacist }\end{array}$ & $\begin{array}{l}\text { Baseline } \\
\text { G1: } 15.4 \\
\text { G2: } 17.5 \\
\text { 3 months } \\
\text { G1: } 11.8 \\
\text { G2: } 14.9 \\
\text { p: NR } \\
\text { 12 months } \\
\text { G1: } 10.1 \\
\text { G2: } 14.9 \\
\text { p: NR } \\
\end{array}$ \\
\hline $\begin{array}{l}\text { Taylor, Byrd, and Krueger, } \\
2003^{36} \\
\text { RCT/Medium }\end{array}$ & $\begin{array}{l}\text { G1: Pharmaceutical care } \\
\text { G2: Standard care }\end{array}$ & $\begin{array}{l}\text { N participants } \\
\text { G1: } 33 \\
\text { G2: } 36 \\
\text { Number of } \\
\text { prescriptions: } \\
\text { Baseline } \\
\text { G1: } 210 \\
\text { G2: } 207 \\
\text { 12 months } \\
\text { G1: } 155 \\
\text { G2: } 224\end{array}$ & $\begin{array}{l}\text { Percent Prescriptions } \\
\text { Inappropriate } \\
\text { Assessed at baseline, } 12 \text { months } \\
\text { by blinded research pharmacist }\end{array}$ & $\begin{array}{l}\text { Baseline } \\
\text { G1: } 35.2 \\
\text { G2: } 48.8 \\
\\
12 \text { months } \\
\text { G1: } 18.1 \\
\text { G2: } 49.1\end{array}$ \\
\hline
\end{tabular}

Abbreviations: $\mathrm{CI}=$ confidence interval; diff $=$ difference; $\mathrm{G}=$ group; $\mathrm{GMC}=$ General Medicine Clinic; $\mathrm{N}=$ number; $\mathrm{NR}=$ not reported; $\mathrm{RCT}=$ randomized controlled trial; $\mathrm{SE}=$ standard error; $\mathrm{Std}=$ standard 
Table E22. Medication Appropriateness Index Item 10 (Is this drug the least expensive alternative compared with others of equal utility?): Summary of results

\begin{tabular}{|c|c|c|c|c|}
\hline $\begin{array}{l}\text { Study } \\
\text { Design/Risk of Bias }\end{array}$ & Study Arms & N Analyzed & Outcome and Time Period & Results \\
\hline $\begin{array}{l}\text { Hanlon et al., } 1996^{14} \\
\text { RCT/Low }\end{array}$ & $\begin{array}{l}\text { G1: Usual care, plus clinical } \\
\text { pharmacist care. } \\
\text { G2: Usual care in the GMC }\end{array}$ & $\begin{array}{l}\text { G1: } 105 \\
\text { G2: } 103\end{array}$ & $\begin{array}{l}\text { Percent Prescriptions } \\
\text { Inappropriate } \\
\text { Assessed at baseline, } 3,12 \\
\text { months by blinded research } \\
\text { pharmacist }\end{array}$ & $\begin{array}{l}\text { Baseline } \\
\text { G1: } 29.2 \\
\text { G2: } 30.3 \\
\\
3 \text { months } \\
\text { G1: } 25.6 \\
\text { G2: } 27.7 \\
\text { p: NR } \\
\text { 12 months } \\
\text { G1: } 25.3 \\
\text { G2: } 28.2 \\
\text { p: NR }\end{array}$ \\
\hline $\begin{array}{l}\text { Taylor, Byrd, and Krueger, } \\
2003^{36} \\
\text { RCT/Medium }\end{array}$ & $\begin{array}{l}\text { G1: Pharmaceutical care } \\
\text { G2: Standard care }\end{array}$ & $\begin{array}{l}\text { Baseline } \\
\text { G1: } 210 \\
\text { G2: } 207 \\
\\
12 \text { months } \\
\text { G1: } 155 \\
\text { G2: } 224\end{array}$ & $\begin{array}{l}\text { Percent Prescriptions } \\
\text { Inappropriate } \\
\text { Assessed at baseline, } 12 \text { months } \\
\text { by blinded research pharmacist }\end{array}$ & $\begin{array}{l}\text { Baseline } \\
\text { G1: } 50.0 \\
\text { G2: } 62.3 \\
\\
12 \text { months } \\
\text { G1: } 38.7 \\
\text { G2: } 60.3\end{array}$ \\
\hline
\end{tabular}

Abbreviations: $\mathrm{CI}=$ confidence interval; diff = difference; $\mathrm{G}=$ group; $\mathrm{GMC}=$ General Medicine Clinic; $\mathrm{NR}=$ not reported; RCT = randomized controlled trial; $\mathrm{SE}=$ standard error; Std $=$ standard 
Table E23. Medication dosing: Summary of results

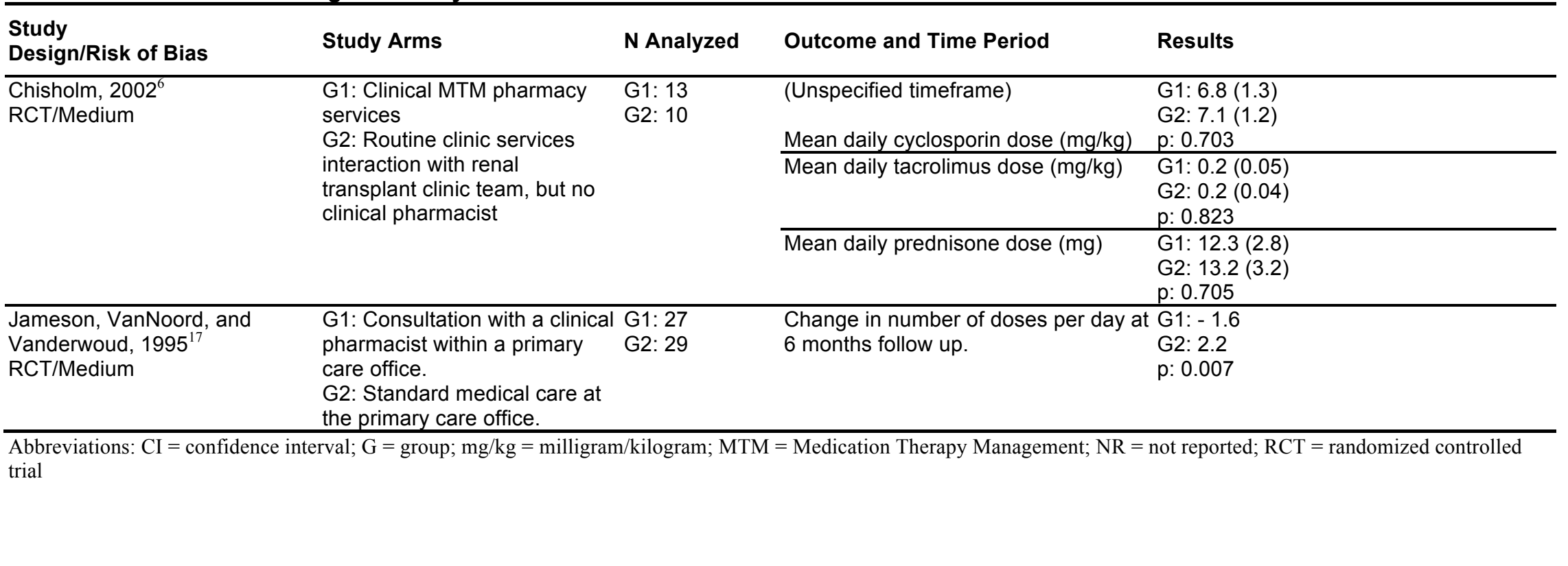


Table E24. Adverse events: Summary of results

\begin{tabular}{|c|c|c|c|c|}
\hline $\begin{array}{l}\text { Study } \\
\text { Design/Risk of Bias }\end{array}$ & Study Arms & N Analyzed & Outcome and Time Period & Results \\
\hline $\begin{array}{l}\text { Hanlon et al., } 1996^{14} \\
\text { RCT/Low }\end{array}$ & $\begin{array}{l}\text { G1: Clinical pharmacist care } \\
\text { within a general medicine clinic. } \\
\text { G2: Usual care }\end{array}$ & $\begin{array}{l}\text { G1: } 86 \\
\text { G2: } 83\end{array}$ & $\begin{array}{l}\text { Percent with an ADE at } 12 \\
\text { months }\end{array}$ & $\begin{array}{l}\text { G1: } 30.2 \\
\text { G2: } 40.0 \\
p=0.19 \\
\text { Calculated OR: } 0.6 \\
95 \% \mathrm{Cl}, 0.37 \text { to } 1.15 \\
p=0.014\end{array}$ \\
\hline \multirow[t]{5}{*}{$\begin{array}{l}\text { Touchette et al., } 2012^{37} \\
\text { RCT/Low }\end{array}$} & $\begin{array}{l}\text { G1: Basic MTM services (with } \\
\text { medication information gleaned } \\
\text { through patient interview) } \\
\text { G2: Enhanced MTM services } \\
\text { (pharmacist provided with } 2 \\
\text { page clinical summary from }\end{array}$ & $\begin{array}{l}\text { G1: } 211 \\
\text { G2: } 218 \\
\text { G3: } 208\end{array}$ & $\begin{array}{l}\text { Percent of patients with an ADE } \\
\text { between } 0 \text { and } 3 \text { months and OR }\end{array}$ & $\begin{array}{l}\text { G1: } 42.2 \\
\text { G2: } 27.9 \\
\text { G3: } 33.7 \\
\text { G1 vs. G3: OR: } 1.6 \\
\text { (p=0.078) } \\
\text { G2 vs. G3: OR: } 0.7 \\
(p=0.278)\end{array}$ \\
\hline & $\begin{array}{l}\text { patient medical record). } \\
\text { G3: Usual pharmacy care }\end{array}$ & & $\begin{array}{l}\text { Percent of patients with an ADE } \\
\text { between } 3 \text { and } 6 \text { months and OR }\end{array}$ & $\begin{array}{l}\text { G1: } 36.1 \\
\text { G2: } 31.1 \\
\text { G3: } 34.4 \\
\text { G1 vs. G3: OR: } 1.1 \\
\text { ( } p=0.717 \text { ) } \\
\text { G2 vs. G3: OR: } 0.9 \\
(p=0.672)\end{array}$ \\
\hline & & & $\begin{array}{l}\text { Mean number (SD) of ADEs per } \\
\text { patient between } 0 \text { and } 3 \text { months }\end{array}$ & $\begin{array}{l}\text { G1: } 0.8(1.1) \\
\text { G2: } 0.5(1.2) \\
\text { G3: } 0.6(1.2) \\
\text { G1 vs. G3: Calculated SMD, } 0.2 \\
95 \% \mathrm{Cl},-0.03 \text { to } 0.36 \\
\mathrm{p}=0.110\end{array}$ \\
\hline & & & & $\begin{array}{l}\text { G2 vs. G3: Calculated SMD, }-0.01 \text {; } \\
95 \% \mathrm{Cl},-0.20 \text { to } 0.18 \\
p=0.916\end{array}$ \\
\hline & & & $\begin{array}{l}\text { Mean number (SD) of ADEs per } \\
\text { patient between } 3 \text { and } 6 \text { months }\end{array}$ & $\begin{array}{l}\text { G1: } 0.8(1.4) \\
\text { G2: } 0.5(0.8) \\
\text { G3: } 0.5(0.9) \\
\text { G1 vs. G3: Calculated SMD, } 0.2 ; \\
95 \% \mathrm{Cl}, 0.05 \text { to } 0.43 \\
\text { p=0.041 } \\
\text { G2 vs. G3: Calculated SMD, }-0.1 ; \\
95 \% \mathrm{Cl},-0.26 \text { to } 0.12 \\
\text { p=0.479 }\end{array}$ \\
\hline
\end{tabular}


Table E24. Adverse events: Summary of results (continued)

\begin{tabular}{|c|c|c|c|c|}
\hline $\begin{array}{l}\text { Study } \\
\text { Design/Risk of Bias }\end{array}$ & Study Arms & N Analyzed & Outcome and Time Period & Results \\
\hline $\begin{array}{l}\text { Fischer et al., } 2000^{10} \\
\text { NRCT/High }\end{array}$ & $\begin{array}{l}\text { G1: Comprehensive drug } \\
\text { therapy management program } \\
\text { G2: Standard community } \\
\text { pharmacy practice }\end{array}$ & $\begin{array}{l}\text { G1: } 201 \\
\text { G2: } 368\end{array}$ & $\begin{array}{l}\text { OR for likelihood of reporting side } \\
\text { effects or problems due to } \\
\text { prescription medication }(95 \% \mathrm{Cl})\end{array}$ & 1.8 (1.20 to 2.80$)$ \\
\hline $\begin{array}{l}\text { Taylor, Byrd, and Krueger, } \\
2003^{36} \\
\text { RCT/High }\end{array}$ & $\begin{array}{l}\text { G1: Pharmaceutical care } \\
\text { G2: Standard care }\end{array}$ & $\begin{array}{l}\text { G1: } 33 \\
\text { G2: } 36\end{array}$ & $\begin{array}{l}\text { Percent of patients with at least } \\
\text { one medication misadventure at } \\
12 \text { months }\end{array}$ & $\begin{array}{l}\mathrm{G} 1: 2.8^{\mathrm{a}}(\mathrm{N}=4) \\
\mathrm{G} 2: 3.0^{\mathrm{a}}(\mathrm{N}=3) \\
\text { Calculated } \mathrm{OR} \text { based on reported percent: } \\
0.93 ; \\
95 \% \mathrm{Cl}, 0.056 \text { to } 15.603 \\
\text { p: } 0.0961 \\
\text { Calculated } \mathrm{OR} \text { based on reported } \mathrm{N}: 1.5 \\
(95 \% \mathrm{Cl}, 0.31 \text { to } 7.34), \mathrm{p}=0.606\end{array}$ \\
\hline $\begin{array}{l}\text { Jameson, VanNoord, and } \\
\text { Vanderwoud, } 1995^{17} \\
\text { RCT/High }\end{array}$ & $\begin{array}{l}\text { G1: Consultation with a clinical } \\
\text { pharmacist within a primary care } \\
\text { office. } \\
\text { G2: Standard medical care at the } \\
\text { primary care office. }\end{array}$ & $\begin{array}{l}\text { G1: } 27 \\
\text { G2: } 29\end{array}$ & $\begin{array}{l}\text { Change in mean medication side } \\
\text { effect score at six months. }\end{array}$ & $\begin{array}{l}\text { G1: }-3.7 \\
\text { G2: }-1.9 \\
\text { p: NS and unable to calculate. }\end{array}$ \\
\hline
\end{tabular}

$\stackrel{1}{\infty} \frac{\text { primary care office. }}{{ }^{a} \text { The percent reported by authors cannot be generated based on the reported } \mathrm{N} \text { and the reported number of events. }}$

Abbreviations: $\mathrm{ADE}=$ adverse drug event; $\mathrm{CI}=$ confidence interval; $\mathrm{DRP}=$ drug-related problems; $\mathrm{G}=$ group MTM = Medication Therapy Management; $\mathrm{N}=$ number; $\mathrm{NRCT}=$ nonrandomized controlled trial; NS = not significant; $\mathrm{NS}=$ not sufficient; $\mathrm{OR}=$ odds ratio; $\mathrm{RCT}=$ randomized controlled trial; $\mathrm{SD}=$ standard deviation; $\mathrm{SMD}=$ standardized mean difference; vs. = versus. 


\section{Table E25. All-cause mortality: Summary of results}

\begin{tabular}{|c|c|c|c|c|}
\hline $\begin{array}{l}\text { Study } \\
\text { Design/Risk of Bias }\end{array}$ & Study Arms & N Analyzed & Outcome and Time Period & Results \\
\hline $\begin{array}{l}\text { Triller and Hamilton, } \\
2007^{38} \\
\text { RCT/Medium }\end{array}$ & $\begin{array}{l}\text { G1: Visiting nurse association } \\
\text { home visit services plus } \\
\text { comprehensive pharmaceutical } \\
\text { care services } \\
\text { G2: Visiting nurse association } \\
\text { home visit services }\end{array}$ & $\begin{array}{l}\text { G1: } 77 \\
\text { G2: } 77\end{array}$ & $\begin{array}{l}\text { RR for all-cause mortality within } \\
180 \text { days }\end{array}$ & $\begin{array}{l}\text { G1: } 22 \% \\
\text { G2: } 18 \% \\
\text { RR: } 1.21 \text { (Calculated } 95 \% \mathrm{Cl}, 0.65 \text { to } 2.30 \text { ) } \\
p=0.67\end{array}$ \\
\hline $\begin{array}{l}\text { Gattis et al., } 1992 \\
\text { [\#2564\} } \\
\text { RCT/Medium }\end{array}$ & $\begin{array}{l}\text { G1: Clinical pharmacist } \\
\text { intervention in addition to usual } \\
\text { medical care } \\
\text { G2: Usual medical care }\end{array}$ & $\begin{array}{l}\text { G1: } 90 \\
\text { G2: } 91\end{array}$ & $\begin{array}{l}\text { OR for all-cause mortality within } 6 \\
\text { months } \\
(95 \% \mathrm{Cl})\end{array}$ & $\begin{array}{l}\text { G1: } 3.3 \% \\
\text { G2: } 5.5 \% \\
\text { OR: } 0.6(0.12 \text { to } 2.49) \\
p=0.48\end{array}$ \\
\hline $\begin{array}{l}\text { Welch et al., } 2009^{41} \\
\text { Cohort study/Medium }\end{array}$ & $\begin{array}{l}\text { G1: MTM program provided to } \\
\text { home-based beneficiaries } \\
\text { G2: No-MTM control group (opt- } \\
\text { out) }\end{array}$ & $\begin{array}{l}\text { G1: } 459 \\
\text { G2: } 336\end{array}$ & $\begin{array}{l}\text { Adjusted OR for all-cause } \\
\text { mortality, within } 6 \text { months } \\
\text { (adjusted for age, sex, chronic } \\
\text { disease score, specific baseline } \\
\text { utilization) } \\
(95 \% \mathrm{Cl})\end{array}$ & $\begin{array}{l}\mathrm{G} 1: 4.1 \% \\
\mathrm{G} 2: 7.4 \% \\
\text { Adjusted OR: } 0.5 \text { (0.3 to } 0.9) \\
\mathrm{p}=0.044\end{array}$ \\
\hline
\end{tabular}


Table E26. Self-reported health status: Summary of results

\begin{tabular}{|c|c|c|c|c|}
\hline $\begin{array}{l}\text { Study } \\
\text { Design/Risk of Bias }\end{array}$ & Study Arms & N Analyzed & Outcome and Time Period & Results \\
\hline $\begin{array}{l}\text { Malone et al., } 2000^{20} ; \\
\text { Ellis et al., } 2000^{21} ; \\
\text { Malone et al, } 2001^{22} \\
\text { Ellis et al., } 2000^{23} \\
\text { RCT/Medium }\end{array}$ & $\begin{array}{l}\text { G1: Pharmaceutical care } \\
\text { provided by clinical pharmacists } \\
\text { within ambulatory VA clinics } \\
\text { G2: Usual care (i.e., no } \\
\text { pharmaceutical care) }\end{array}$ & $\begin{array}{l}\text { G1: } 447 \\
\text { G2: } 484\end{array}$ & $\begin{array}{l}\text { SF-36 Physical Functioning } \\
\text { Domain (change from baseline) }\end{array}$ & $\begin{array}{l}\text { 6-Month Follow-up } \\
\text { G1: -4.9 (1.0 SE) } \\
\text { G2: }-3.4 \text { (0.9 SE) } \\
\text { 12-Month Follow-up } \\
\text { G1: -5.3 (1.0 SE) } \\
\text { G2: }-6.1 \text { (1.0 SE) } \\
p=0.412\end{array}$ \\
\hline $\begin{array}{l}\text { Taylor, Byrd, and Krueger, } \\
2003^{36} \\
\text { RCT/Medium }\end{array}$ & $\begin{array}{l}\text { G1: Pharmaceutical care } \\
\text { provided by pharmacist in } \\
\text { conjunction with an outpatient } \\
\text { physician office visit } \\
\text { G2: Standard care. }\end{array}$ & $\begin{array}{l}\text { G1: } 33 \\
\text { G2: } 36\end{array}$ & $\begin{array}{l}\text { SF-36 Physical Functioning } \\
\text { Domain }\end{array}$ & $\begin{array}{l}\text { Baseline } \\
\text { G1: } 62.0(29.4) \\
\text { G2: } 61.9(24.3) \\
\text { 12-Month Follow-up } \\
\text { G1: } 68.6(24.0) \\
\text { G2: } 56.1(27.5) \\
\text { p: NS }\end{array}$ \\
\hline $\begin{array}{l}\text { Hanlon et al., } 1996^{14} \\
\text { RCT/Low }\end{array}$ & $\begin{array}{l}\text { G1: Usual care at outpatient } \\
\text { clinic, plus clinical pharmacist } \\
\text { care. } \\
\text { G2: Usual care at outpatient } \\
\text { clinic }\end{array}$ & $\begin{array}{l}\text { G1: } 86 \\
\text { G2: } 83\end{array}$ & $\begin{array}{l}\text { SF-36 Physical Functioning } \\
\text { Domain }\end{array}$ & $\begin{array}{l}\text { Baseline: } \\
\text { G1: } 48.0(2.7) \\
\text { G2: } 45.3(2.7) \\
\text { 12-Month Follow-up } \\
\text { G1: } 44.1(2.0) \\
\text { G2: } 42.2(2.0) \\
p=0.99\end{array}$ \\
\hline $\begin{array}{l}\text { Bernsten et al., } 2001^{1} \text {; } \\
\text { Sturgess et al., } 2003^{2} \\
\text { RCT, Cluster- } \\
\text { Randomized/High }\end{array}$ & $\begin{array}{l}\text { G1: Structured community } \\
\text { pharmacy-based } \\
\text { pharmaceutical care program } \\
\text { G2: Usual community pharmacy } \\
\text { services }\end{array}$ & $\begin{array}{l}\text { Baseline } \\
\text { G1: } 1290 \\
\text { G2: } 1164 \\
\text { 18 months } \\
\text { G1: } 704 \\
\text { G2: } 636\end{array}$ & $\begin{array}{l}\text { SF-36 Physical Functioning } \\
\text { Domain (Change between } \\
\text { Baseline and 18-Month Follow- } \\
\text { Up) }\end{array}$ & $\begin{array}{l}\text { G1: }-1.0 \\
\text { G2: }-0.7 \\
\mathrm{p}: \mathrm{NS}\end{array}$ \\
\hline
\end{tabular}


Table E26. Self-reported health status: Summary of results (continued)

\begin{tabular}{|c|c|c|c|c|}
\hline $\begin{array}{l}\text { Study } \\
\text { Design/Risk of Bias }\end{array}$ & Study Arms & N Analyzed & Outcome and Time Period & Results \\
\hline $\begin{array}{l}\text { Carter et al.,1997 }{ }^{4} \text {; } \\
\text { Barnette, Murphy, and Carter, } \\
1996^{5} \\
\text { Cohort/High }\end{array}$ & $\begin{array}{l}\text { G1: Pharmaceutical care } \\
\text { G2: Usual care with patients } \\
\text { seen by pharmacists who did } \\
\text { not participate in the intensive } \\
\text { skills development program }\end{array}$ & $\begin{array}{l}\text { G1: } 25 \\
\text { G2: } 26\end{array}$ & $\begin{array}{l}\text { SF-36 Physical Functioning } \\
\text { Domain }\end{array}$ & $\begin{array}{l}\text { Baseline } \\
\text { G1: } 61.5 \\
\text { G2: } 66.5 \\
\text { 6-Month Follow-up } \\
\text { G1: } 70.7 \\
\text { G2: } 67.7 \\
\text { p=NR }\end{array}$ \\
\hline $\begin{array}{l}\text { Park et al, } 1996^{29} \\
\text { RCT/High }\end{array}$ & $\begin{array}{l}\text { G1: comprehensive } \\
\text { pharmaceutical care } \\
\text { G2: usual care }\end{array}$ & $\begin{array}{l}\text { G1: } 23 \\
\text { G2: } 26\end{array}$ & $\begin{array}{l}\text { SF-36 Physical Functioning } \\
\text { Domain }\end{array}$ & $\begin{array}{l}\text { Baseline } \\
\text { G1: } 77.0(26.1) \\
\text { G2: } 66.3(29.1) \\
\text { 4-Month Follow-up } \\
\text { G1: } 77.8(30.4) \\
\text { G2: } 70.2(29.2) \\
\text { p=NS }\end{array}$ \\
\hline $\begin{array}{l}\text { Sellors et al., } 2003^{33} \\
\text { RCT-Cluster } \\
\text { randomized/Medium }\end{array}$ & $\begin{array}{l}\text { G1: Pharmacists conducted } \\
\text { face-to-face medication reviews } \\
\text { with the patients and then gave } \\
\text { written recommendations to the } \\
\text { physicians to resolve any drug- } \\
\text { related problems. } \\
\text { G2: Usual Care for Family } \\
\text { Physicians and their Patients } \\
\text { from matched postal codes. }\end{array}$ & $\begin{array}{l}\text { Baseline } \\
\text { G1: } 379 \\
\text { G2: } 409\end{array}$ & $\begin{array}{l}\text { SF-36 Physical Functioning } \\
\text { Domain }\end{array}$ & $\begin{array}{l}\text { Baseline } \\
\text { G1: } 55.6(95 \% \mathrm{Cl}, 55.5 \text { to } 56.0) \\
\text { G2: } 54.2(95 \% \mathrm{Cl}, 48.0 \text { to } 54.4) \\
\text { 5-Month Follow-up } \\
\text { G1: } 55.0(95 \% \mathrm{Cl}, 54.6 \text { to } 55.3) \\
\text { G2: } 55.0(95 \% \mathrm{Cl}, 54.8 \text { to } 55.2) \\
\text { p: } 0.93\end{array}$ \\
\hline $\begin{array}{l}\text { Krska et al, } 2001^{19} \\
\text { RCT/Medium }\end{array}$ & $\begin{array}{l}\text { G1: Pharmacist-led medication } \\
\text { review } \\
\text { G2: Usual care involving } \\
\text { interviews and identification of } \\
\text { PCls but with no } \\
\text { pharmaceutical care plan } \\
\text { implemented. }\end{array}$ & $\begin{array}{l}\text { Baseline } \\
\text { G1: } 168 \\
\text { G2: } 164 \\
\text { (Not clear if all } \\
\text { were included in } \\
\text { analyses) }\end{array}$ & $\begin{array}{l}\text { SF-36 Physical Functioning } \\
\text { Domain }\end{array}$ & $\begin{array}{l}\text { G1: NR } \\
\text { G2: NR } \\
\mathrm{p}: \mathrm{NS}\end{array}$ \\
\hline
\end{tabular}


Table E26. Self-reported health status: Summary of results (continued)

\begin{tabular}{|c|c|c|c|c|}
\hline $\begin{array}{l}\text { Study } \\
\text { Design/Risk of Bias }\end{array}$ & Study Arms & N Analyzed & Outcome and Time Period & Results \\
\hline $\begin{array}{l}\text { Malone et al., } 2000^{20} ; \\
\text { Ellis et al., } 2000^{21} ; \\
\text { Malone et al, } 2001^{22} \\
\text { Ellis et al., } 2000^{23} \\
\text { RCT/Medium }\end{array}$ & $\begin{array}{l}\text { G1: Pharmaceutical care } \\
\text { provided by clinical pharmacists } \\
\text { within ambulatory VA clinics } \\
\text { G2: Usual care (i.e., no } \\
\text { pharmaceutical care) }\end{array}$ & $\begin{array}{l}\text { G1: } 447 \\
\text { G2: } 484\end{array}$ & $\begin{array}{l}\text { SF-36 Role Physical Domain } \\
\text { (change from baseline) }\end{array}$ & $\begin{array}{l}\text { 6-Month Follow-up } \\
\text { G1: }-3.5 \text { (1.8 SE) } \\
\text { G2: }-4.3 \text { (2.1 SE) } \\
\text { 12-Month Follow-up } \\
\text { G1: }-4.3(2.0 \mathrm{SE}) \\
\text { G2: }-8.2 \text { (2.00 SE) } \\
\text { p=0.245 }\end{array}$ \\
\hline $\begin{array}{l}\text { Taylor, Byrd, and Krueger, } \\
2003^{36} \\
\text { RCT/Medium }\end{array}$ & $\begin{array}{l}\text { G1: Pharmaceutical care } \\
\text { provided by pharmacist in } \\
\text { conjunction with an outpatient } \\
\text { physician office visit } \\
\text { G2: Standard care. }\end{array}$ & $\begin{array}{l}\text { G1: } 33 \\
\text { G2: } 36\end{array}$ & SF-36 Role Physical Domain & $\begin{array}{l}\text { Baseline } \\
\text { G1: } 50.8(42.2) \\
\text { G2: } 47.9(42.8) \\
\text { 12-Month Follow-up } \\
\text { G1: } 68.2(42.1) \\
\text { G2: } 52.8(42.2) \\
\text { 95\% Cl: NR } \\
\text { p=NS }\end{array}$ \\
\hline $\begin{array}{l}\text { Hanlon et al., } 1996^{14} \\
\text { RCT/Low }\end{array}$ & $\begin{array}{l}\text { G1: Usual care at outpatient } \\
\text { clinic, plus clinical pharmacist } \\
\text { care. } \\
\text { G2: Usual care at outpatient } \\
\text { clinic }\end{array}$ & $\begin{array}{l}\text { G1: } 86 \\
\text { G2: } 83\end{array}$ & SF-36 Role Physical Domain & $\begin{array}{l}\text { Baseline: } \\
\text { G1: } 38.3(3.2) \\
\text { G2: } 36.5(3.2) \\
\text { 12-Month Follow-up } \\
\text { G1: } 38.6(3.2) \\
\text { G2: } 32.3(3.7) \\
\text { p=0.99 }\end{array}$ \\
\hline $\begin{array}{l}\text { Bernsten et al., } 2001^{1} \text {; } \\
\text { Sturgess et al., } 2003^{2} \\
\text { RCT, Cluster- } \\
\text { Randomized/High }\end{array}$ & $\begin{array}{l}\text { G1: Structured community } \\
\text { pharmacy-based } \\
\text { pharmaceutical care program } \\
\text { G2: Usual community pharmacy } \\
\text { services }\end{array}$ & $\begin{array}{l}\text { Baseline } \\
\text { G1: } 1290 \\
\text { G2: } 1164 \\
\text { 18 months } \\
\text { G1: } 704 \\
\text { G2: } 636\end{array}$ & $\begin{array}{l}\text { SF-36 Role Physical Domain } \\
\text { (Change between Baseline and } \\
\text { 18-Month Follow-Up) }\end{array}$ & $\begin{array}{l}\text { G1: }-1.1 \\
\text { G2: }-0.3 \\
p=N S\end{array}$ \\
\hline
\end{tabular}


Table E26. Self-reported health status: Summary of results (continued)

\begin{tabular}{|c|c|c|c|c|}
\hline $\begin{array}{l}\text { Study } \\
\text { Design/Risk of Bias }\end{array}$ & Study Arms & N Analyzed & Outcome and Time Period & Results \\
\hline $\begin{array}{l}\text { Carter et al.,1997 }{ }^{4} \text {, } \\
\text { Barnette, Murphy, and Carter, } \\
1996^{5} \\
\text { Cohort/High }\end{array}$ & $\begin{array}{l}\text { G1: Pharmaceutical care } \\
\text { G2: Usual care with patients } \\
\text { seen by pharmacists who did } \\
\text { not participate in the intensive } \\
\text { skills development program }\end{array}$ & $\begin{array}{l}\text { G1: } 25 \\
\text { G2: } 26\end{array}$ & SF-36 Role Physical Domain & $\begin{array}{l}\text { Baseline } \\
\text { G1: } 54.3 \\
\text { G2: } 63.5 \\
\text { 6-Month Follow-up } \\
\text { G1: } 74.0 \\
\text { G2: } 62.5 \\
\text { p=NR }\end{array}$ \\
\hline $\begin{array}{l}\text { Park et al, } 1996^{29} \\
\text { RCT/High }\end{array}$ & $\begin{array}{l}\text { G1: comprehensive } \\
\text { pharmaceutical care } \\
\text { G2: usual care }\end{array}$ & $\begin{array}{l}\text { G1: } 23 \\
\text { G2: } 26\end{array}$ & SF-36 Role Physical Domain & $\begin{array}{l}\text { Baseline } \\
\text { G1: } 85.9(30.0) \\
\text { G2: } 77.9(31.1) \\
\text { 4-Month Follow-up } \\
\text { G1: } 85.2(31.5) \\
\text { G2: } 73.1(40.6) \\
\text { p=NS }\end{array}$ \\
\hline $\begin{array}{l}\text { Sellors et al., } 2003^{33} \\
\text { RCT-Cluster } \\
\text { randomized/Medium }\end{array}$ & $\begin{array}{l}\text { G1: Pharmacists conducted } \\
\text { face-to-face medication reviews } \\
\text { with the patients and then gave } \\
\text { written recommendations to the } \\
\text { physicians to resolve any drug- } \\
\text { related problems. } \\
\text { G2: Usual Care for Family } \\
\text { Physicians and their Patients } \\
\text { from matched postal codes. }\end{array}$ & $\begin{array}{l}\text { Baseline } \\
\text { G1: } 379 \\
\text { G2: } 409\end{array}$ & SF-36 Role Physical Domain & $\begin{array}{l}\text { Baseline } \\
\text { G1: } 53.8(95 \% \mathrm{Cl}, 53.1 \text { to } 54.6) \\
\text { G2: } 55.0(54.5 \text { to } 55.5) \\
\text { Exit } \\
\text { G1: } 48.5(95 \% \mathrm{Cl}, 47.8 \text { to } 49.3) \\
\text { G2: } 52.1(95 \% \mathrm{Cl}, 41.6 \text { to } 42.6) \\
\text { p: } 0.65\end{array}$ \\
\hline $\begin{array}{l}\text { Krska et al, } 2001^{19} \\
\text { RCT/Medium }\end{array}$ & $\begin{array}{l}\text { G1: Pharmacist-led medication } \\
\text { review } \\
\text { G2: Usual care involving } \\
\text { interviews and identification of } \\
\text { PCls but with no } \\
\text { pharmaceutical care plan } \\
\text { implemented. }\end{array}$ & $\begin{array}{l}\text { Baseline } \\
\text { G1: } 168 \\
\text { G2: } 164 \\
\text { (Not clear if all } \\
\text { were included in } \\
\text { analyses) }\end{array}$ & SF-36 Role Physical Domain & $\begin{array}{l}\text { G1: NR } \\
\text { G2: NR } \\
\text { p: NS }\end{array}$ \\
\hline
\end{tabular}


Table E26. Self-reported health status: Summary of results (continued)

\begin{tabular}{|c|c|c|c|c|}
\hline $\begin{array}{l}\text { Study } \\
\text { Design/Risk of Bias }\end{array}$ & Study Arms & N Analyzed & Outcome and Time Period & Results \\
\hline $\begin{array}{l}\text { Malone et al., } 2000^{20} ; \\
\text { Ellis et al., } 2000^{21} ; \\
\text { Malone et al, } 2001^{22} \\
\text { Ellis et al., } 2000^{23} \\
\text { RCT/Medium }\end{array}$ & $\begin{array}{l}\text { G1: Pharmaceutical care } \\
\text { provided by clinical pharmacists } \\
\text { within ambulatory VA clinics } \\
\text { G2: Usual care (i.e., no } \\
\text { pharmaceutical care) }\end{array}$ & $\begin{array}{l}\text { G1: } 447 \\
\text { G2: } 484\end{array}$ & $\begin{array}{l}\text { SF-36 Bodily Pain Domain } \\
\text { (change from baseline) }\end{array}$ & $\begin{array}{l}\text { 6-Month Follow-up } \\
\text { G1: -0.8 (1.0 SE) } \\
\text { G2: }-3.3 \text { (0.9 SE) } \\
\text { 12-Month Follow-up } \\
\text { G1: }-0.3 \text { (1.0 SE) } \\
\text { G2: }-4.8 \text { (1.0 SE) } \\
p=0.004\end{array}$ \\
\hline $\begin{array}{l}\text { Taylor, Byrd, and Krueger, } \\
2003^{36} \\
\text { RCT/Medium }\end{array}$ & $\begin{array}{l}\text { G1: Pharmaceutical care } \\
\text { provided by pharmacist in } \\
\text { conjunction with an outpatient } \\
\text { physician office visit } \\
\text { G2: Standard care. }\end{array}$ & $\begin{array}{l}\text { G1: } 33 \\
\text { G2: } 36\end{array}$ & SF-36 Bodily Pain Domain & $\begin{array}{l}\text { Baseline } \\
\text { G1: } 60.0(27.0) \\
\text { G2: } 65.4(23.0) \\
\text { 12-Month Follow-up } \\
\text { G1: } 68.5(22.3) \\
\text { G2: } 63.1(25.8) \\
\text { p=NS }\end{array}$ \\
\hline $\begin{array}{l}\text { Hanlon et al., } 1996^{14} \\
\text { RCT/Low }\end{array}$ & $\begin{array}{l}\text { G1: Usual care at outpatient } \\
\text { clinic, plus clinical pharmacist } \\
\text { care. } \\
\text { G2: Usual care at outpatient } \\
\text { clinic }\end{array}$ & $\begin{array}{l}\text { G1: } 86 \\
\text { G2: } 83\end{array}$ & SF-36 Bodily Pain Domain & $\begin{array}{l}\text { Baseline } \\
\text { G1: } 45.0(2.8) \\
\text { G2: } 42.2(2.8) \\
\text { 12-Month Follow-up } \\
\text { G1: } 43.6(2.7) \\
\text { G2: } 41.7(2.7) \\
\text { p=0.99 }\end{array}$ \\
\hline $\begin{array}{l}\text { Bernsten et al., } 2001^{1} \text {; } \\
\text { Sturgess et al., } 2003^{2} \\
\text { RCT, Cluster- } \\
\text { Randomized/High }\end{array}$ & $\begin{array}{l}\text { G1: Structured community } \\
\text { pharmacy-based } \\
\text { pharmaceutical care program } \\
\text { G2: Usual community pharmacy } \\
\text { services }\end{array}$ & $\begin{array}{l}\text { Baseline } \\
\text { G1: } 1290 \\
\text { G2: } 1164 \\
\text { 18 months } \\
\text { G1: } 704 \\
\text { G2: } 636\end{array}$ & $\begin{array}{l}\text { SF-36 Bodily Pain Domain } \\
\text { (Change between Baseline and } \\
\text { 18-Month Follow-Up) }\end{array}$ & $\begin{array}{l}\text { G1: }-0.06 \\
\text { G2: }+0.53 \\
p=N S\end{array}$ \\
\hline
\end{tabular}


Table E26. Self-reported health status: Summary of results (continued)

\begin{tabular}{|c|c|c|c|c|}
\hline $\begin{array}{l}\text { Study } \\
\text { Design/Risk of Bias }\end{array}$ & Study Arms & N Analyzed & Outcome and Time Period & Results \\
\hline $\begin{array}{l}\text { Carter et al.,1997, } \\
\text { Barnette, Murphy, and Carter, } \\
1996^{5} \\
\text { Cohort/High }\end{array}$ & $\begin{array}{l}\text { G1: Pharmaceutical care } \\
\text { G2: Usual care with patients } \\
\text { seen by pharmacists who did } \\
\text { not participate in the intensive } \\
\text { skills development program }\end{array}$ & $\begin{array}{l}\text { G1: } 25 \\
\text { G2: } 26\end{array}$ & SF-36 Bodily Pain Domain & $\begin{array}{l}\text { Baseline } \\
\text { G1: } 58.4 \\
\text { G2: } 76.7 \\
\text { 6-Month Follow-up } \\
\text { G1: } 71.1 \\
\text { G2: } 74.7 \\
\text { p=NR }\end{array}$ \\
\hline $\begin{array}{l}\text { Park et al, } 1996^{29} \\
\text { RCT/High }\end{array}$ & $\begin{array}{l}\text { G1: comprehensive } \\
\text { pharmaceutical care } \\
\text { G2: usual care }\end{array}$ & $\begin{array}{l}\text { G1: } 23 \\
\text { G2: } 26\end{array}$ & SF-36 Bodily Pain & $\begin{array}{l}\text { Baseline } \\
\text { G1: } 77.4(19.0) \\
\text { G2: } 73.1(21.3) \\
\text { 4-Month Follow-up } \\
\text { G1: } 80.5(22.9) \\
\text { G2: } 73.7(19.0) \\
\text { p=NS }\end{array}$ \\
\hline $\begin{array}{l}\text { Sellors et al., } 2003^{33} \\
\text { RCT-Cluster } \\
\text { randomized/Medium }\end{array}$ & $\begin{array}{l}\text { G1: Pharmacists conducted } \\
\text { face-to-face medication reviews } \\
\text { with the patients and then gave } \\
\text { written recommendations to the } \\
\text { physicians to resolve any drug- } \\
\text { related problems. } \\
\text { G2: Usual Care for Family } \\
\text { Physicians and their Patients } \\
\text { from matched postal codes. }\end{array}$ & $\begin{array}{l}\text { Baseline } \\
\text { G1: } 379 \\
\text { G2: } 409\end{array}$ & SF-36 Bodily Pain & $\begin{array}{l}\text { Baseline } \\
\text { G1: } 60.5(95 \% \mathrm{Cl}, 60.2 \text { to } 60.8) \\
\text { G2: } 60.8(95 \% \mathrm{Cl}, 60.6 \text { to } 61.0) \\
\text { 5-Month Follow-up } \\
\text { G1: } 56.6(95 \% \mathrm{Cl}, 56.4 \text { to } 56.8) \\
\text { G2: } 59.0(95 \% \mathrm{Cl}, 58.8 \text { to } 59.2) \\
\text { p: } 0.65\end{array}$ \\
\hline $\begin{array}{l}\text { Krska et al, } 2001^{19} \\
\text { RCT/Medium }\end{array}$ & $\begin{array}{l}\text { G1: Pharmacist-led medication } \\
\text { review } \\
\text { G2: Usual care involving } \\
\text { interviews and identification of } \\
\text { PCls but with no } \\
\text { pharmaceutical care plan } \\
\text { implemented. }\end{array}$ & $\begin{array}{l}\text { Baseline } \\
\text { G1: } 168 \\
\text { G2: } 164 \\
\text { (Not clear if all } \\
\text { were included in } \\
\text { analyses) }\end{array}$ & SF-36 Bodily Pain & $\begin{array}{l}\text { G1: NR } \\
\text { G2: NR } \\
\text { p: NS }\end{array}$ \\
\hline
\end{tabular}


Table E26. Self-reported health status: Summary of results (continued)

\begin{tabular}{|c|c|c|c|c|}
\hline $\begin{array}{l}\text { Study } \\
\text { Design/Risk of Bias }\end{array}$ & Study Arms & N Analyzed & Outcome and Time Period & Results \\
\hline $\begin{array}{l}\text { Malone et al., } 2000^{20} \\
\text { Ellis et al., } 2000^{21} ; \\
\text { Malone et al, } 2001^{22} ; \\
\text { Ellis et al., } 2000^{23} \\
\text { RCT/Medium }\end{array}$ & $\begin{array}{l}\text { G1: Pharmaceutical care } \\
\text { provided by clinical pharmacists } \\
\text { within ambulatory VA clinics } \\
\text { G2: Usual care (i.e., no } \\
\text { pharmaceutical care) }\end{array}$ & $\begin{array}{l}\text { G1: } 447 \\
\text { G2: } 484\end{array}$ & $\begin{array}{l}\text { SF-36 General Health Perception } \\
\text { Domain (change from baseline) }\end{array}$ & $\begin{array}{l}\text { 6-Month Follow-up } \\
\text { G1: -1.6 (0.8 SE) } \\
\text { G2: }-2.2 \text { (0.7 SE) } \\
\text { 12-Month Follow-up } \\
\text { G1: }-2.4(0.8 \mathrm{SE}) \\
\text { G2: }-5.3(0.8 \mathrm{SE}) \\
\text { 95\% Cl: NR } \\
\text { p=0.026 }\end{array}$ \\
\hline $\begin{array}{l}\text { Taylor, Byrd, and Krueger, } \\
2003^{36} \\
\text { RCT/Medium }\end{array}$ & $\begin{array}{l}\text { G1: Pharmaceutical care } \\
\text { provided by pharmacist in } \\
\text { conjunction with an outpatient } \\
\text { physician office visit } \\
\text { G2: Standard care. }\end{array}$ & $\begin{array}{l}\text { G1: } 33 \\
\text { G2: } 36\end{array}$ & $\begin{array}{l}\text { SF-36 General Health Perception } \\
\text { Domain }\end{array}$ & $\begin{array}{l}\text { Baseline } \\
\text { G1: } 50.8(19.5) \\
\text { G2: } 49.9(19.8) \\
\text { 12-Month Follow-up } \\
\text { G1: } 57.0(19.6) \\
\text { G2: } 50.1(15.9) \\
\text { p: NS }\end{array}$ \\
\hline $\begin{array}{l}\text { Hanlon et al., } 1996^{14} \\
\text { RCT/Low }\end{array}$ & $\begin{array}{l}\text { G1: Usual care at outpatient } \\
\text { clinic, plus clinical pharmacist } \\
\text { care. } \\
\text { G2: Usual care at outpatient } \\
\text { clinic }\end{array}$ & $\begin{array}{l}\text { G1: } 86 \\
\text { G2: } 83\end{array}$ & $\begin{array}{l}\text { SF-36 General Health Perception } \\
\text { Domain }\end{array}$ & $\begin{array}{l}\text { Baseline } \\
\text { G1: } 34.9(2.1) \\
\text { G2: } 34.2(2.1) \\
\text { 12-Month Follow-up } \\
\text { G1: } 37.4(1.6) \\
\text { G2: } 35.2(1.7) \\
\text { p=0.99 }\end{array}$ \\
\hline $\begin{array}{l}\text { Bernsten et al., } 2001^{1} \text {; } \\
\text { Sturgess et al., } 2003^{2} \\
\text { RCT, Cluster- } \\
\text { Randomized/High }\end{array}$ & $\begin{array}{l}\text { G1: Structured community } \\
\text { pharmacy-based } \\
\text { pharmaceutical care program } \\
\text { G2: Usual community pharmacy } \\
\text { services }\end{array}$ & $\begin{array}{l}\text { Baseline } \\
\text { G1: } 1290 \\
\text { G2: } 1164 \\
\text { 18 months } \\
\text { G1: } 704 \\
\text { G2: } 636\end{array}$ & $\begin{array}{l}\text { SF-36 General Health Perception } \\
\text { Domain (Change between } \\
\text { Baseline and 18-Month Follow- } \\
\text { Up) }\end{array}$ & $\begin{array}{l}\text { G1: +0.28 } \\
\text { G2: }-0.66 \\
\text { p: NS }\end{array}$ \\
\hline
\end{tabular}


Table E26. Self-reported health status: Summary of results (continued)

\begin{tabular}{|c|c|c|c|c|}
\hline $\begin{array}{l}\text { Study } \\
\text { Design/Risk of Bias }\end{array}$ & Study Arms & N Analyzed & Outcome and Time Period & Results \\
\hline $\begin{array}{l}\text { Carter et al.,1997 }{ }^{4} \text {; } \\
\text { Barnette, Murphy, and Carter, } \\
1996^{5} \\
\text { Cohort/High }\end{array}$ & $\begin{array}{l}\text { G1: Pharmaceutical care } \\
\text { G2: Usual care with patients } \\
\text { seen by pharmacists who did } \\
\text { not participate in the intensive } \\
\text { skills development program }\end{array}$ & $\begin{array}{l}\text { G1: } 25 \\
\text { G2: } 26\end{array}$ & $\begin{array}{l}\text { SF-36 General Health Perception } \\
\text { Domain }\end{array}$ & $\begin{array}{l}\text { Baseline } \\
\text { G1: } 58.2 \\
\text { G2: } 61.2 \\
\text { 6-Month Follow-up } \\
\text { G1: } 58.7 \\
\text { G2: } 64.0 \\
\text { p=NR }\end{array}$ \\
\hline $\begin{array}{l}\text { Park et al, } 1996^{29} \\
\text { RCT/High }\end{array}$ & $\begin{array}{l}\text { G1: comprehensive } \\
\text { pharmaceutical care } \\
\text { G2: usual care }\end{array}$ & $\begin{array}{l}\text { G1: } 23 \\
\text { G2: } 26\end{array}$ & $\begin{array}{l}\text { SF-36 General Health Perception } \\
\text { Domain } \\
\end{array}$ & $\begin{array}{l}\text { Baseline } \\
\text { G1: } 67.8(18.7) \\
\text { G2: } 59.5(15.1) \\
\text { 4-Month Follow-up } \\
\text { G1: } 72.3(13.1) \\
\text { G2: } 64.7(19.0) \\
\text { p: NS }\end{array}$ \\
\hline $\begin{array}{l}\text { Sellors et al., } 2003^{33} \\
\text { RCT-Cluster } \\
\text { randomized/Medium }\end{array}$ & $\begin{array}{l}\text { G1: Pharmacists conducted } \\
\text { face-to-face medication reviews } \\
\text { with the patients and then gave } \\
\text { written recommendations to the } \\
\text { physicians to resolve any drug- } \\
\text { related problems. } \\
\text { G2: Usual Care for Family } \\
\text { Physicians and their Patients } \\
\text { from matched postal codes. }\end{array}$ & $\begin{array}{l}\text { Baseline } \\
\text { G1: } 379 \\
\text { G2: } 409\end{array}$ & $\begin{array}{l}\text { SF-36 General Health Perception } \\
\text { Domain }\end{array}$ & $\begin{array}{l}\text { Baseline } \\
\text { G1: } 62.2(95 \% \mathrm{Cl}, 61.9 \text { to } 62.6) \\
\text { G2: } 65.0(95 \% \mathrm{Cl}, 64.8 \text { to } 65.2) \\
\text { 5-Month Follow-up } \\
\text { G1: } 60.5(95 \% \mathrm{Cl}, 60.3 \text { to } 60.7) \\
\text { G2: } 60.8(95 \% \mathrm{Cl}, 60.6 \text { to } 61.0) \\
\text { p: } 0.17\end{array}$ \\
\hline $\begin{array}{l}\text { Krska et al, } 2001^{19} \\
\text { RCT/Medium }\end{array}$ & $\begin{array}{l}\text { G1: Pharmacist-led medication } \\
\text { review } \\
\text { G2: Usual care involving } \\
\text { interviews and identification of } \\
\text { PCls but with no } \\
\text { pharmaceutical care plan } \\
\text { implemented. }\end{array}$ & $\begin{array}{l}\text { Baseline } \\
\text { G1: } 168 \\
\text { G2: } 164 \\
\text { (Not clear if all } \\
\text { were included in } \\
\text { analyses) }\end{array}$ & $\begin{array}{l}\text { SF-36 General Health Perception } \\
\text { Domain }\end{array}$ & $\begin{array}{l}\text { G1: NR } \\
\text { G2: NR } \\
\mathrm{p}: \mathrm{NS}\end{array}$ \\
\hline
\end{tabular}


Table E26. Self-reported health status: Summary of results (continued)

\begin{tabular}{|c|c|c|c|c|}
\hline $\begin{array}{l}\text { Study } \\
\text { Design/Risk of Bias }\end{array}$ & Study Arms & N Analyzed & Outcome and Time Period & Results \\
\hline $\begin{array}{l}\text { Park et al, } 1996^{29} \\
\text { RCT/High }\end{array}$ & $\begin{array}{l}\text { G1: comprehensive } \\
\text { pharmaceutical care } \\
\text { G2: usual care }\end{array}$ & $\begin{array}{l}\text { G1: } 23 \\
\text { G2: } 26\end{array}$ & $\begin{array}{l}\text { SF-36 Social Functioning } \\
\text { Domain }\end{array}$ & $\begin{array}{l}\text { Baseline } \\
\text { G1: } 88.6(16.8) \\
\text { G2: } 81.3(18.5) \\
\text { 4-Month Follow-up } \\
\text { G1: } 90.2(15.5) \\
\text { G2: } 81.0(19.1) \\
\text { p: NS }\end{array}$ \\
\hline $\begin{array}{l}\text { Sellors et al., } 2003^{33} \\
\text { RCT-Cluster } \\
\text { randomized/Medium }\end{array}$ & $\begin{array}{l}\text { G1: Pharmacists conducted } \\
\text { face-to-face medication reviews } \\
\text { with the patients and then gave } \\
\text { written recommendations to the } \\
\text { physicians to resolve any drug- } \\
\text { related problems. } \\
\text { G2: Usual Care for Family } \\
\text { Physicians and their Patients } \\
\text { from matched postal codes. }\end{array}$ & $\begin{array}{l}\text { Baseline } \\
\text { G1: } 379 \\
\text { G2: } 409 \\
\end{array}$ & $\begin{array}{l}\text { SF-36 Social Functioning } \\
\text { Domain }\end{array}$ & $\begin{array}{l}\text { Baseline } \\
\text { G1: } 79.2(95 \% \mathrm{Cl}, 79.0 \text { to } 79.4) \\
\text { G2: } 81.9(95 \% \mathrm{Cl}, 81.8 \text { to } 82.0) \\
\text { 5-Month Follow-up } \\
\text { G1: } 75.4(95 \% \mathrm{Cl}, 75.1 \text { to } 75.8) \\
\text { G2: } 77.5(95 \% \mathrm{Cl}, 77.3 \text { to } 77.7) \\
\text { p: } 0.34\end{array}$ \\
\hline $\begin{array}{l}\text { Krska et al, } 2001^{19} \\
\text { RCT/Medium }\end{array}$ & $\begin{array}{l}\text { G1: Pharmacist-led medication } \\
\text { review } \\
\text { G2: Usual care involving } \\
\text { interviews and identification of } \\
\text { PCls but with no } \\
\text { pharmaceutical care plan } \\
\text { implemented. }\end{array}$ & $\begin{array}{l}\text { Baseline } \\
\text { G1: } 168 \\
\text { G2: } 164 \\
\text { (Not clear if all } \\
\text { were included in } \\
\text { analyses) }\end{array}$ & $\begin{array}{l}\text { SF-36 Social Functioning } \\
\text { Domain }\end{array}$ & $\begin{array}{l}\text { G1: NR } \\
\text { G2: NR } \\
\mathrm{p}: \mathrm{NS}\end{array}$ \\
\hline $\begin{array}{l}\text { Malone et al., } 2000^{20} ; \\
\text { Ellis et al., } 2000^{21} ; \\
\text { Malone et al, } 2001^{22} \text {; } \\
\text { Ellis et al., } 2000^{23} \\
\text { RCT/Medium }\end{array}$ & $\begin{array}{l}\text { G1: Pharmaceutical care } \\
\text { provided by clinical pharmacists } \\
\text { within ambulatory VA clinics } \\
\text { G2: Usual care (i.e., no } \\
\text { pharmaceutical care) }\end{array}$ & $\begin{array}{l}\text { G1: } 447 \\
\text { G2: } 484\end{array}$ & $\begin{array}{l}\text { SF-36 Role Emotional Domain } \\
\text { (change from baseline) }\end{array}$ & $\begin{array}{l}\text { 6-Month Follow-up } \\
\text { G1: }-2.6 \text { (2.2 SE) } \\
\text { G2: }-3.4 \text { (1.9 SE) } \\
\text { 12-Month Follow-up } \\
\text { G1: }-0.3 \text { (2.3 SE) } \\
\text { G2: }-7.4 \text { (2.3 SE) } \\
p=0.065\end{array}$ \\
\hline
\end{tabular}


Table E26. Self-reported health status: Summary of results (continued)

\begin{tabular}{|c|c|c|c|c|}
\hline $\begin{array}{l}\text { Study } \\
\text { Design/Risk of Bias }\end{array}$ & Study Arms & N Analyzed & Outcome and Time Period & Results \\
\hline $\begin{array}{l}\text { Taylor, Byrd, and Krueger, } \\
2003^{36} \\
\text { RCT/Medium }\end{array}$ & $\begin{array}{l}\text { G1: Pharmaceutical care } \\
\text { provided by pharmacist in } \\
\text { conjunction with an outpatient } \\
\text { physician office visit } \\
\text { G2: Standard care. }\end{array}$ & $\begin{array}{l}\text { G1: } 33 \\
\text { G2: } 36\end{array}$ & SF-36 Role Emotional Domain & $\begin{array}{l}\text { Baseline } \\
\text { G1: } 59.6(44.7) \\
\text { G2: } 69.4(45.3) \\
\text { 12-Month Follow-up } \\
\text { G1: } 82.8(36.4) \\
\text { G2: } 65.8(45.4) \\
\text { p: NS }\end{array}$ \\
\hline $\begin{array}{l}\text { Hanlon et al., } 1996^{14} \\
\text { RCT/Low }\end{array}$ & $\begin{array}{l}\text { G1: Usual care at outpatient } \\
\text { clinic, plus clinical pharmacist } \\
\text { care. } \\
\text { G2: Usual care at outpatient } \\
\text { clinic }\end{array}$ & $\begin{array}{l}\text { G1: } 86 \\
\text { G2: } 83\end{array}$ & SF-36 Role Emotional Domain & $\begin{array}{l}\text { Baseline: } \\
\text { G1: } 73.0(4.1) \\
\text { G2: } 68.1(4.1) \\
\text { 12-Month Follow-up } \\
\text { G1: } 66.4(1.8) \\
\text { G2: } 67.0(3.9) \\
\text { p=0.99 }\end{array}$ \\
\hline $\begin{array}{l}\text { Bernsten et al., } 2001^{1} \text {; } \\
\text { Sturgess et al., } 2003^{2} \\
\text { RCT, Cluster- } \\
\text { Randomized/High }\end{array}$ & $\begin{array}{l}\text { G1: Structured community } \\
\text { pharmacy-based } \\
\text { pharmaceutical care program } \\
\text { G2: Usual community pharmacy } \\
\text { services }\end{array}$ & $\begin{array}{l}\text { Baseline } \\
\text { G1: } 1290 \\
\text { G2: } 1164 \\
\text { 18 months } \\
\text { G1: } 704 \\
\text { G2: } 636\end{array}$ & $\begin{array}{l}\text { SF-36 Role Emotional Domain } \\
\text { (Change between Baseline and } \\
\text { 18-Month Follow-Up) }\end{array}$ & $\begin{array}{l}\text { G1: }+0.2 \\
\text { G2: }-2.9 \\
\mathrm{p}: \mathrm{NS}\end{array}$ \\
\hline $\begin{array}{l}\text { Carter et al., } 1997^{4}, \\
\text { Barnette, Murphy, and Carter, } \\
1996^{5} \\
\text { Cohort/High }\end{array}$ & $\begin{array}{l}\text { G1: Pharmaceutical care } \\
\text { G2: Usual care with patients } \\
\text { seen by pharmacists who did } \\
\text { not participate in the intensive } \\
\text { skills development program }\end{array}$ & $\begin{array}{l}\text { G1: } 25 \\
\text { G2: } 26\end{array}$ & SF-36 Role Emotional Domain & $\begin{array}{l}\text { Baseline } \\
\text { G1: } 50.0 \\
\text { G2: } 69.4 \\
\text { 6-Month Follow-up } \\
\text { G1: } 63.9 \\
\text { G2: } 65.3 \\
p=N R\end{array}$ \\
\hline
\end{tabular}


Table E26. Self-reported health status: Summary of results (continued)

\begin{tabular}{|c|c|c|c|c|}
\hline $\begin{array}{l}\text { Study } \\
\text { Design/Risk of Bias }\end{array}$ & Study Arms & N Analyzed & Outcome and Time Period & Results \\
\hline $\begin{array}{l}\text { Park et al, } 1996^{29} \\
\text { RCT/High }\end{array}$ & $\begin{array}{l}\text { G1: comprehensive } \\
\text { pharmaceutical care } \\
\text { G2: usual care }\end{array}$ & $\begin{array}{l}\text { G1: } 23 \\
\text { G2: } 26\end{array}$ & SF-36 Role Emotional Domain & $\begin{array}{l}\text { Baseline } \\
\text { G1: } 88.4(25.8) \\
\text { G2: } 88.5(28.2) \\
\text { 4-Month Follow-up } \\
\text { G1: } 92.8(24.5) \\
\text { G2: } 78.2(29.7) \\
\text { p: NS }\end{array}$ \\
\hline $\begin{array}{l}\text { Sellors et al., } 2003^{33} \\
\text { RCT-Cluster } \\
\text { randomized/Medium }\end{array}$ & $\begin{array}{l}\text { G1Pharmacists conducted face- } \\
\text { to-face medication reviews with } \\
\text { the patients and then gave } \\
\text { written recommendations to the } \\
\text { physicians to resolve any drug- } \\
\text { related problems. } \\
\text { G2: Usual Care for Family } \\
\text { Physicians and their Patients } \\
\text { from matched postal codes. }\end{array}$ & $\begin{array}{l}\text { Baseline } \\
\text { G1: } 379 \\
\text { G2: } 409\end{array}$ & SF-36 Role Emotional Domain & $\begin{array}{l}\text { Baseline } \\
\text { G1: } 71.8(95 \% \mathrm{Cl}, 70.9 \text { to } 72.7) \\
\text { G2: } 74.9(95 \% \mathrm{Cl}, 74.5 \text { to } 75.2) \\
\text { 5-Month Follow-up } \\
\text { G1: } 66.4(95 \% \mathrm{Cl}, 65.7 \text { to } 67.0) \\
\text { G2: } 72.7(95 \% \mathrm{Cl}, 72.1 \text { to } 73.2) \\
\text { p: } 0.80\end{array}$ \\
\hline $\begin{array}{l}\text { Krska et al, } 2001^{19} \\
\text { RCT/Medium }\end{array}$ & $\begin{array}{l}\text { G1: Pharmacist-led medication } \\
\text { review } \\
\text { G2: Usual care involving } \\
\text { interviews and identification of } \\
\text { PCls but with no } \\
\text { pharmaceutical care plan } \\
\text { implemented. }\end{array}$ & $\begin{array}{l}\text { Baseline } \\
\text { G1: } 168 \\
\text { G2: } 164 \\
\text { (Not clear if all } \\
\text { were included in } \\
\text { analyses) }\end{array}$ & SF-36 Role Emotional Domain & $\begin{array}{l}\text { G1: NR } \\
\text { G2: NR } \\
\mathrm{p}: \mathrm{NS}\end{array}$ \\
\hline $\begin{array}{l}\text { Malone et al., } 2000^{20} ; \\
\text { Ellis et al., } 2000^{21} ; \\
\text { Malone et al, } 2001^{22} \text {; } \\
\text { Ellis et al., } 2000^{23} \\
\text { RCT/Medium }\end{array}$ & $\begin{array}{l}\text { G1: Pharmaceutical care } \\
\text { provided by clinical pharmacists } \\
\text { within ambulatory VA clinics } \\
\text { G2: Usual care (i.e., no } \\
\text { pharmaceutical care) }\end{array}$ & $\begin{array}{l}\text { G1: } 447 \\
\text { G2: } 484\end{array}$ & $\begin{array}{l}\text { SF-36 Mental Health Domain } \\
\text { (change from baseline) }\end{array}$ & $\begin{array}{l}\text { 6-Month Follow-Up } \\
\text { G1: }-0.5 \text { (0.8 SE) } \\
\text { G2: }-1.4 \text { (0.7 SE) } \\
\text { 12-Month Follow-up } \\
\text { G1: } 0.1 \text { (0.8 SE) } \\
\text { G2: }-2.3 \text { (0.8 SE) } \\
\text { p=0.029 }\end{array}$ \\
\hline
\end{tabular}


Table E26. Self-reported health status: Summary of results (continued)

\begin{tabular}{|c|c|c|c|c|}
\hline $\begin{array}{l}\text { Study } \\
\text { Design/Risk of Bias }\end{array}$ & Study Arms & N Analyzed & Outcome and Time Period & Results \\
\hline $\begin{array}{l}\text { Taylor, Byrd, and Krueger, } \\
2003^{36} \\
\text { RCT/Medium }\end{array}$ & $\begin{array}{l}\text { G1: Pharmaceutical care } \\
\text { provided by pharmacist in } \\
\text { conjunction with an outpatient } \\
\text { physician office visit } \\
\text { G2: Standard care. }\end{array}$ & $\begin{array}{l}\text { G1: } 33 \\
\text { G2: } 36\end{array}$ & SF-36 Mental Health Domain & $\begin{array}{l}\text { Baseline } \\
\text { G1: } 72.0(17.4) \\
\text { G2: } 69.0(18.6) \\
\text { 12-Month Follow-up } \\
\text { G1: } 73.1(21.2) \\
\text { G2: } 72.3(17.1) \\
\text { p=NS }\end{array}$ \\
\hline $\begin{array}{l}\text { Hanlon et al., } 1996^{14} \\
\text { RCT/Low }\end{array}$ & $\begin{array}{l}\text { G1: Usual care at outpatient } \\
\text { clinic, plus clinical pharmacist } \\
\text { care. } \\
\text { G2: Usual care at outpatient } \\
\text { clinic }\end{array}$ & $\begin{array}{l}\text { G1: } 86 \\
\text { G2: } 83\end{array}$ & SF-36 Mental Health Domain & $\begin{array}{l}\text { Baseline: } \\
\text { G1: } 61.0(2.5) \\
\text { G2: } 63.5(2.5) \\
\text { 12-Month Follow-up } \\
\text { G1: } 61.1(1.8) \\
\text { G2: } 60.4(1.8) \\
\text { p=0.99 }\end{array}$ \\
\hline $\begin{array}{l}\text { Bernsten et al., } 2001^{1} \text {; } \\
\text { Sturgess et al., } 2003^{2} \\
\text { RCT, Cluster- } \\
\text { Randomized/High }\end{array}$ & $\begin{array}{l}\text { G1: Structured community } \\
\text { pharmacy-based } \\
\text { pharmaceutical care program } \\
\text { G2: Usual community pharmacy } \\
\text { services }\end{array}$ & $\begin{array}{l}\text { Baseline } \\
\text { G1: } 1290 \\
\text { G2: } 1164 \\
\text { 18 months } \\
\text { G1: } 704 \\
\text { G2: } 636\end{array}$ & $\begin{array}{l}\text { SF-36 Mental Health Domain } \\
\text { (Change between Baseline and } \\
\text { 18-Month Follow-Up) }\end{array}$ & $\begin{array}{l}\text { G1: }-0.8 \\
\text { G2: }-1.3 \\
p=N S\end{array}$ \\
\hline $\begin{array}{l}\text { Carter et al., } 1997^{4} ; \\
\text { Barnette, Murphy, and Carter, } \\
1996^{5} \\
\text { Cohort/High }\end{array}$ & $\begin{array}{l}\text { G1: Pharmaceutical care } \\
\text { G2: Usual care with patients } \\
\text { seen by pharmacists who did } \\
\text { not participate in the intensive } \\
\text { skills development program }\end{array}$ & $\begin{array}{l}\text { G1: } 25 \\
\text { G2: } 26\end{array}$ & SF-36 Mental Health Domain & $\begin{array}{l}\text { Baseline } \\
\text { G1: } 73.4 \\
\text { G2: } 75.5 \\
\text { 6-Month Follow-up } \\
\text { G1: } 71.0 \\
\text { G2: } 75.7 \\
\text { p: NR }\end{array}$ \\
\hline
\end{tabular}


Table E26. Self-reported health status: Summary of results (continued)

\begin{tabular}{|c|c|c|c|c|}
\hline $\begin{array}{l}\text { Study } \\
\text { Design/Risk of Bias }\end{array}$ & Study Arms & N Analyzed & Outcome and Time Period & Results \\
\hline $\begin{array}{l}\text { Park et al, } 1996^{29} \\
\text { RCT/High }\end{array}$ & $\begin{array}{l}\text { G1: comprehensive } \\
\text { pharmaceutical care } \\
\text { G2: usual care }\end{array}$ & $\begin{array}{l}\text { G1: } 23 \\
\text { G2: } 26\end{array}$ & SF-36 Mental Health Domain & $\begin{array}{l}\text { Baseline } \\
\text { G1: } 77.0(14.6) \\
\text { G2: } 73.1(21.3) \\
\text { 4-Month Follow-Up } \\
\text { G1: } 80.2(14.6) \\
\text { G2: } 73.7(19.0) \\
\text { p=NS }\end{array}$ \\
\hline $\begin{array}{l}\text { Sellors et al., } 2003^{33} \\
\text { RCT-Cluster } \\
\text { randomized/Medium }\end{array}$ & $\begin{array}{l}\text { G1: Pharmacists conducted } \\
\text { face-to-face medication reviews } \\
\text { with the patients and then gave } \\
\text { written recommendations to the } \\
\text { physicians to resolve any drug- } \\
\text { related problems. } \\
\text { G2: Usual Care for Family } \\
\text { Physicians and their Patients } \\
\text { from matched postal codes. }\end{array}$ & $\begin{array}{l}\text { Baseline } \\
\text { G1: } 379 \\
\text { G2: } 409\end{array}$ & SF-36 Mental Health Domain & $\begin{array}{l}\text { Baseline } \\
\text { G1: } 75.2(95 \% \mathrm{Cl}, 75.1 \text { to } 75.3) \\
\text { G2: } 76.7(95 \% \mathrm{Cl}, 75.8 \text { to } 77.6) \\
\text { 5 Month Follow-Up } \\
\text { G1: } 74.2(95 \% \mathrm{Cl}, 74.0 \text { to } 74.3) \\
\text { G2: } 74.7(95 \% \mathrm{Cl}: 74.7 \text { to } 74.8) \\
\text { p: } 0.49\end{array}$ \\
\hline $\begin{array}{l}\text { Krska et al, } 2001^{19} \\
\text { RCT/Medium }\end{array}$ & $\begin{array}{l}\text { G1: Pharmacist-led medication } \\
\text { review } \\
\text { G2: Usual care involving } \\
\text { interviews and identification of } \\
\text { PCls but with no } \\
\text { pharmaceutical care plan } \\
\text { implemented. }\end{array}$ & $\begin{array}{l}\text { Baseline } \\
\text { G1: } 168 \\
\text { G2: } 164 \\
\text { (Not clear if all } \\
\text { were included in } \\
\text { analyses) }\end{array}$ & SF-36 Mental Health Domain & $\begin{array}{l}\text { G1: NR } \\
\text { G2: NR } \\
\mathrm{p}: \mathrm{NS}\end{array}$ \\
\hline $\begin{array}{l}\text { Bernsten et al., } 2001^{1} \text {; } \\
\text { Sturgess et al., } 2003^{2} \\
\text { RCT, Cluster- } \\
\text { Randomized/High }\end{array}$ & $\begin{array}{l}\text { G1: Structured community } \\
\text { pharmacy-based } \\
\text { pharmaceutical care program } \\
\text { G2: Usual community pharmacy } \\
\text { services }\end{array}$ & $\begin{array}{l}\text { Baseline } \\
\text { G1: } 1290 \\
\text { G2: } 1164 \\
18 \text { months } \\
\text { G1: } 704 \\
\text { G2: } 636\end{array}$ & $\begin{array}{l}\text { SF-36 Mental Health Domain } \\
\text { (Change between Baseline and } \\
\text { 18-Month Follow-Up) }\end{array}$ & $\begin{array}{l}\text { G1: }-0.8 \\
\text { G2: }-1.3 \\
p=N S\end{array}$ \\
\hline
\end{tabular}


Table E26. Self-reported health status: Summary of results (continued)

\begin{tabular}{|c|c|c|c|c|}
\hline $\begin{array}{l}\text { Study } \\
\text { Design/Risk of Bias }\end{array}$ & Study Arms & N Analyzed & Outcome and Time Period & Results \\
\hline $\begin{array}{l}\text { Carter et al.,1997 }{ }^{4} \text {; } \\
\text { Barnette, Murphy, and Carter, } \\
1996^{5} \\
\text { Cohort/High }\end{array}$ & $\begin{array}{l}\text { G1: Pharmaceutical care } \\
\text { G2: Usual care with patients } \\
\text { seen by pharmacists who did } \\
\text { not participate in the intensive } \\
\text { skills development program }\end{array}$ & $\begin{array}{l}\text { G1: } 25 \\
\text { G2: } 26\end{array}$ & SF-36 Mental Health Domain & $\begin{array}{l}\text { Baseline } \\
\text { G1: } 73.4 \\
\text { G2: } 75.5 \\
\text { 6-Month Follow-Up } \\
\text { G1: } 71.0 \\
\text { G2: } 75.7 \\
\text { p:NR }\end{array}$ \\
\hline $\begin{array}{l}\text { Park et al, } 1996^{29} \\
\text { RCT/High }\end{array}$ & $\begin{array}{l}\text { G1: comprehensive } \\
\text { pharmaceutical care } \\
\text { G2: usual care }\end{array}$ & $\begin{array}{l}\text { G1: } 23 \\
\text { G2: } 26\end{array}$ & SF-36 Mental Health Domain & $\begin{array}{l}\text { Baseline } \\
\text { G1: } 77.0(14.6) \\
\text { G2: } 73.1(21.3) \\
\text { 4-Month Follow-Up } \\
\text { G1: } 80.2(14.6) \\
\text { G2: } 73.7(19.0) \\
\text { p=NS }\end{array}$ \\
\hline $\begin{array}{l}\text { Sellors et al., } 2003^{33} \\
\text { RCT-Cluster } \\
\text { randomized/Medium }\end{array}$ & $\begin{array}{l}\text { G1: Pharmacists conducted } \\
\text { face-to-face medication reviews } \\
\text { with the patients and then gave } \\
\text { written recommendations to the } \\
\text { physicians to resolve any drug- } \\
\text { related problems. } \\
\text { G2: Usual Care for Family } \\
\text { Physicians and their Patients } \\
\text { from matched postal codes. }\end{array}$ & $\begin{array}{l}\text { Baseline } \\
\text { G1: } 379 \\
\text { G2: } 409\end{array}$ & SF-36 Mental Health Domain & $\begin{array}{l}\text { Baseline } \\
\text { G1: } 75.2(95 \% \mathrm{Cl}, 75.1 \text { to } 75.3) \\
\text { G2: } 76.7(95 \% \mathrm{Cl}, 75.8 \text { to } 77.6) \\
5 \text { Month Follow-Up } \\
\text { G1: } 74.2(95 \% \mathrm{Cl}, 74.0 \text { to } 74.3) \\
\text { G2: } 74.795 \% \mathrm{Cl},(74.7 \text { to } 74.8) \\
\text { p: } 0.49\end{array}$ \\
\hline $\begin{array}{l}\text { Krska et al, } 2001^{19} \\
\text { RCT/Medium }\end{array}$ & $\begin{array}{l}\text { G1: Pharmacist-led medication } \\
\text { review } \\
\text { G2: Usual care involving } \\
\text { interviews and identification of } \\
\text { PCls but with no } \\
\text { pharmaceutical care plan } \\
\text { implemented. }\end{array}$ & $\begin{array}{l}\text { Baseline } \\
\text { G1: } 168 \\
\text { G2: } 164 \\
\text { (Not clear if all } \\
\text { were included in } \\
\text { analyses) }\end{array}$ & SF-36 Mental Health Domain & $\begin{array}{l}\text { G1: NR } \\
\text { G2: NR } \\
\mathrm{p}: \mathrm{NS}\end{array}$ \\
\hline
\end{tabular}


Table E26. Self-reported health status: Summary of results (continued)

\begin{tabular}{|c|c|c|c|c|}
\hline $\begin{array}{l}\text { Study } \\
\text { Design/Risk of Bias }\end{array}$ & Study Arms & N Analyzed & Outcome and Time Period & Results \\
\hline $\begin{array}{l}\text { Malone et al., } 2000^{20} ; \\
\text { Ellis et al., } 2000^{21} ; \\
\text { Malone et al, } 2001^{22} ; \\
\text { Ellis et al., } 2000^{23} \\
\text { RCT/Medium }\end{array}$ & $\begin{array}{l}\text { G1: Pharmaceutical care } \\
\text { provided by clinical pharmacists } \\
\text { within ambulatory VA clinics } \\
\text { G2: Usual care (i.e., no } \\
\text { pharmaceutical care) }\end{array}$ & $\begin{array}{l}\mathrm{G} 1: 447 \\
\mathrm{G} 2: 484\end{array}$ & $\begin{array}{l}\text { SF-36 Change in Health (change } \\
\text { from baseline) }\end{array}$ & $\begin{array}{l}\text { 6-Month Follow-Up } \\
\text { G1: }-1.1(1.3) \\
\text { G2: }-4.8(1.3) \\
\text { 12-Month Follow-Up } \\
\text { G1: }-2.4(1.5 \text { SE) } \\
\text { G2: }-6.3(1.3 \text { SE) } \\
\text { 95\% Cl: NR } \\
\text { p=0.004 }\end{array}$ \\
\hline $\begin{array}{l}\text { Triller and Hamilton, } 2007^{38} \\
\text { RCT/Medium }\end{array}$ & $\begin{array}{l}\text { G1: Visiting nurse association } \\
\text { home visit services plus } \\
\text { comprehensive pharmaceutical } \\
\text { care services } \\
\text { G2: Visiting nurse association } \\
\text { home visit services }\end{array}$ & $\begin{array}{l}\text { G1: } 77 \\
\text { G2: } 77\end{array}$ & $\begin{array}{l}\text { SF }-12 \text { assessed at } 30,90, \text { and } \\
180 \text { day follow ups }\end{array}$ & $\begin{array}{l}\text { Values not reported, but results state that } \\
\text { values did not significantly differ between } \\
\text { the two groups. }\end{array}$ \\
\hline $\begin{array}{l}\text { Sellors et al., } 2003^{33} \\
\text { RCT-Cluster } \\
\text { randomized/Medium }\end{array}$ & $\begin{array}{l}\text { G1: Pharmacists conducted } \\
\text { face-to-face medication reviews } \\
\text { with the patients and then gave } \\
\text { written recommendations to the } \\
\text { physicians to resolve any drug- } \\
\text { related problems. } \\
\text { G2: Usual Care for Family } \\
\text { Physicians and their Patients } \\
\text { from matched postal codes. }\end{array}$ & $\begin{array}{l}\text { Baseline } \\
\text { G1: } 379 \\
\text { G2: } 409\end{array}$ & $\begin{array}{l}\text { SF-36 Question 1: Overall } \\
\text { Health Rating }\end{array}$ & $\begin{array}{l}\text { Baseline } \\
\text { G1: } 3.3(95 \% \mathrm{Cl}, 3.3 \text { to } 3.3) \\
\text { G2: } 3.4(95 \% \mathrm{Cl}, 3.3 \text { to } 3.4) \\
\text { 5-Month Follow-Up } \\
\text { G1: } 3.2(95 \% \mathrm{Cl}, 3.2 \text { to } 3.3) \\
\text { G2: } 3.2(95 \% \mathrm{Cl}, 3.2 \text { to } 3.3) \\
\text { p: } 0.35\end{array}$ \\
\hline $\begin{array}{l}\text { Sellors et al., } 2003^{33} \\
\text { RCT-Cluster } \\
\text { randomized/Medium }\end{array}$ & $\begin{array}{l}\text { G1: Pharmacists conducted } \\
\text { face-to-face medication reviews } \\
\text { with the patients and then gave } \\
\text { written recommendations to the } \\
\text { physicians to resolve any drug- } \\
\text { related problems. } \\
\text { G2: Usual Care for Family } \\
\text { Physicians and their Patients } \\
\text { from matched postal codes. }\end{array}$ & $\begin{array}{l}\text { Baseline } \\
\text { G1: } 379 \\
\text { G2: } 409\end{array}$ & SF-36 Physical Component & $\begin{array}{l}\text { Baseline } \\
\text { G1: } 39.1(95 \% \mathrm{Cl}, 37.2 \text { to } 41.0) \\
\text { G2: } 38.9(95 \% \mathrm{Cl}, 37.7 \text { to } 40.1) \\
\text { 5-Month Follow-Up } \\
\text { G1: } 37.9(95 \% \mathrm{Cl}, 36.6 \text { to } 39.2) \\
\text { G2: } 38.4(95 \% \mathrm{Cl}, 37.2 \text { to } 39.7) \\
\text { p: } 0.30\end{array}$ \\
\hline
\end{tabular}


Table E26. Self-reported health status: Summary of results (continued)

\begin{tabular}{|c|c|c|c|c|}
\hline $\begin{array}{l}\text { Study } \\
\text { Design/Risk of Bias }\end{array}$ & Study Arms & N Analyzed & Outcome and Time Period & Results \\
\hline $\begin{array}{l}\text { Volume et al., 2001,39,40 } \\
\text { RCT-Cluster } \\
\text { Randomized/Medium }\end{array}$ & $\begin{array}{l}\text { G1: Comprehensive } \\
\text { pharmaceutical care services } \\
\text { G2: Traditional pharmacy care }\end{array}$ & $\begin{array}{l}\text { Time 1: } \\
\text { N= 363 } \\
\text { G1: } 159 \\
\text { G2: } 204 \\
\text { Time 2: } \\
\text { N=317 } \\
\text { G1: NR } \\
\text { G2: NR } \\
\text { Time 3: } \\
\text { N = 292 } \\
\text { G1: NR } \\
\text { G2: NR }\end{array}$ & SF-36 Physical Component & $\begin{array}{l}\text { Time 1: } \\
\text { G1: } 38.4(12.7) \\
\text { G2: } 40.1(11.9) \\
\text { 6-7 Month Follow-Up } \\
\text { G1: } 38.0(11.9) \\
\text { G2: } 39.2(11.6) \\
\text { 12-13 Month Follow-Up } \\
\text { G1: } 36.9(11.6) \\
\text { G2: } 38.4(11.4) \\
\text { p= NS (Between group comparisons at } \\
\text { follow-up assessments) }\end{array}$ \\
\hline $\begin{array}{l}\text { Sellors et al., } 2003^{33} \\
\text { RCT-Cluster } \\
\text { randomized/Medium }\end{array}$ & $\begin{array}{l}\text { G1: Pharmacists conducted } \\
\text { face-to-face medication reviews } \\
\text { with the patients and then gave } \\
\text { written recommendations to the } \\
\text { physicians to resolve any drug- } \\
\text { related problems. } \\
\text { G2: Usual Care for Family } \\
\text { Physicians and their Patients } \\
\text { from matched postal codes. }\end{array}$ & $\begin{array}{l}\text { Baseline } \\
\text { G1: } 379 \\
\text { G2: } 409\end{array}$ & SF-36 Mental Component & $\begin{array}{l}\text { Baseline } \\
\text { G1: } 52.2(95 \% \mathrm{Cl}, 50.8 \text { to } 53.5) \\
\text { G2: } 53.4(95 \% \mathrm{Cl}, 52.6 \text { to } 54.3) \\
\text { 5-Month Follow-Up } \\
\text { G1: } 51.0(95 \% \mathrm{Cl}, 49.7 \text { to } 52.4) \\
\text { G2: } 52.2(95 \% \mathrm{Cl}, 51.2 \text { to } 53.2) \\
\text { p: } 0.65\end{array}$ \\
\hline
\end{tabular}


Table E26. Self-reported health status: Summary of results (continued)

\begin{tabular}{|c|c|c|c|c|}
\hline $\begin{array}{l}\text { Study } \\
\text { Design/Risk of Bias }\end{array}$ & Study Arms & N Analyzed & Outcome and Time Period & Results \\
\hline $\begin{array}{l}\text { Volume et al., 2001, } \\
\text { RCT-Cluster } \\
\text { Randomized/Medium }\end{array}$ & $\begin{array}{l}\text { G1: Comprehensive } \\
\text { pharmaceutical care services } \\
\text { G2: Traditional pharmacy care }\end{array}$ & $\begin{array}{l}\text { Time 1: } \\
N=363 \\
\text { G1: } 159 \\
\text { G2: } 204 \\
\text { Time 2: } \\
N=317 \\
\text { G1: NR } \\
\text { G2: NR } \\
\text { Time 3: } \\
\text { N= 292 } \\
\text { G1: NR } \\
\text { G2: NR }\end{array}$ & SF-36 Mental Component & $\begin{array}{l}\text { Time 1: } \\
\text { G1: } 55.1(8.7) \\
\text { G2: } 53.2(9.3) \\
\text { 6-7 Month Follow-Up } \\
\text { G1: } 55.9(9.1) \\
\text { G2: } 54.4(9.3) \\
\text { 12-13 Month Follow-Up } \\
\text { G1: } 56.1 \text { (8.3) } \\
\text { G2: } 54.6 \text { (8.7) } \\
\text { p= NS (Between group comparisons at } \\
\text { follow-up assessments) }\end{array}$ \\
\hline $\begin{array}{l}\text { Williams et al., } 2004^{42} \\
\text { RCT/Medium }\end{array}$ & $\begin{array}{l}\text { G1: Modification of patient's } \\
\text { medication regimen by an } \\
\text { interdisciplinary team in addition } \\
\text { to usual care and "Bound for } \\
\text { Health" booklet. } \\
\text { G2: Usual care plus provision of } \\
\text { "Bound for Health" booklet }\end{array}$ & $\begin{array}{l}\text { G1: } 57 \\
\text { G2: } 76\end{array}$ & SF-36 Overall Score & $\begin{array}{l}\text { Baseline: } \\
\text { G1: } 61.8(17.8) \\
\text { G2: } 63.3(16.5) \\
\text { 6-Week Follow-Up: } \\
\text { G1: } 65.5(18.9) \\
\text { G2: } 65.7(17.0) \\
\text { p=NS }\end{array}$ \\
\hline
\end{tabular}

Abbreviations: $\mathrm{G}$ = group; $\mathrm{N}$ = number; $\mathrm{NR}=$ not reported; $\mathrm{CI}$ = confidence interval; $\mathrm{NS}$ = not sufficient; PCIs = pharmaceutical care issues; RCT= randomized controlled trial; $\mathrm{SE}=$ standard error; SF-36 = multi-purpose, short-form health survey with only 36 questions; VA = Veteran's Administration 
Table E27. Condition-specific quality-of-life: Summary of results

\begin{tabular}{|c|c|c|c|c|}
\hline $\begin{array}{l}\text { Study } \\
\text { Design/Risk of Bias }\end{array}$ & Study Arms & N Analyzed & Outcome and Time Period & Results \\
\hline $\begin{array}{l}\text { Pai et al., } 2009^{27} ; \\
\text { Pai et al., } 2009^{28} \\
\text { RCT/High }\end{array}$ & $\begin{array}{l}\text { G1: Pharmaceutical care, } \\
\text { consisting of one-on-one care, } \\
\text { with in-depth drug therapy } \\
\text { reviews conducted by a clinical } \\
\text { pharmacist } \\
\text { G2: Standard of care, } \\
\text { consisting of brief therapy } \\
\text { reviews conducted by a nurse }\end{array}$ & $\begin{array}{l}\text { Baseline } \\
\text { G1: } 61 \\
\text { G2: } 44 \\
\text { Year 1: } \\
\text { G1: } 44 \\
\text { G2: } 36 \\
\text { Year 2: } \\
\text { G1: } 24 \\
\text { G2: } 32\end{array}$ & $\begin{array}{l}\text { Renal Quality of Life Profile } \\
\text { (Increased score indicates } \\
\text { worsening of HRQOL, maximum } \\
\text { score=172) }\end{array}$ & $\begin{array}{l}\text { Total Score } \\
\text { Baseline } \\
\text { G1: } 71.9(40) \\
\text { G2: } 74.5(33.5) \\
\text { Y1 } \\
\text { G1: } 71.4(33.6) \\
\text { G2: } 87.5(30.4) \\
\text { Y2 } \\
\text { G1: } 56.5(32.6) \\
\text { G2: } 68.8(35.8) \\
\text { p<0.05 for G1 vs. G2 for } Y 1 \text {; }\end{array}$ \\
\hline $\begin{array}{l}\text { Clifford et al., } 2002^{9} \\
\text { RCT/Medium }\end{array}$ & $\begin{array}{l}\text { G1: Collaborative } \\
\text { pharmaceutical care program } \\
\text { G2: Standard outpatient care } \\
\text { for diabetes }\end{array}$ & $\begin{array}{l}\text { G1: } 48 \\
\text { G2: } 25\end{array}$ & $\begin{array}{l}\text { Diabetes Quality of Life } \\
\text { instrument } \\
\text { Scale of } 1-5, \text { with higher scores } \\
\text { indicating greater dissatisfaction, } \\
\text { worry, or impact of diabetes }\end{array}$ & $\begin{array}{l}\text { Baseline } \\
\text { G1: } 2.0(0.6) \\
\text { G2: } 1.9(0.5) \\
\text { p: NS } \\
\text { 6-Month Follow-Up } \\
\text { G1: } 1.9(0.5) \\
\text { G2: } 1.9(0.4) \\
\text { p>0.15 }\end{array}$ \\
\hline
\end{tabular}
vs. $=$ versus 
Table E28. Patient satisfaction: Summary of results

\begin{tabular}{|c|c|c|c|c|}
\hline $\begin{array}{l}\text { Study } \\
\text { Design/Risk of Bias }\end{array}$ & Study Arms & N Analyzed & Outcome and Time Period & Results \\
\hline $\begin{array}{l}\text { Hanlon et al., } 1996^{14} \\
\text { RCT/Low }\end{array}$ & $\begin{array}{l}\text { G1: Usual care at outpatient } \\
\text { clinic, plus clinical pharmacist } \\
\text { care. } \\
\text { G2: Usual care at outpatient } \\
\text { clinic }\end{array}$ & $\begin{array}{l}\text { G1: } 86 \\
\text { G2: } 83\end{array}$ & $\begin{array}{l}\text { General health care satisfaction } \\
\text { at 12-Month Follow Up } \\
\text { (Higher scores indicate greater } \\
\text { dissatisfaction) }\end{array}$ & $\begin{array}{l}\text { G1: } 1.5(0.7) \\
\text { G2: } 1.6(0.8) \\
p=0.70\end{array}$ \\
\hline $\begin{array}{l}\text { Hanlon et al., } 1996^{14} \\
\text { RCT/Low }\end{array}$ & $\begin{array}{l}\text { G1: Usual care at outpatient } \\
\text { clinic, plus clinical pharmacist } \\
\text { care. } \\
\text { G2: Usual care at outpatient } \\
\text { clinic }\end{array}$ & $\begin{array}{l}\text { G1: } 86 \\
\text { G2: } 83\end{array}$ & $\begin{array}{l}\text { Pharmacy-related health care } \\
\text { satisfaction at 12-month Follow- } \\
\text { Up } \\
\text { (Higher scores indicate greater } \\
\text { dissatisfaction) }\end{array}$ & $\begin{array}{l}\mathrm{G} 1: 5.2(1.5) \\
\mathrm{G} 2: 5.4(1.7) \\
\mathrm{p}=0.52\end{array}$ \\
\hline $\begin{array}{l}\text { Malone et al., } 2000^{20} ; \\
\text { Ellis et al., } 2000^{21} ; \\
\text { Malone et al, } 2001^{22} \text {; } \\
\text { Ellis et al., } 2000^{23} \\
\text { RCT/Medium }\end{array}$ & $\begin{array}{l}\text { G1: Pharmaceutical care } \\
\text { provided by clinical pharmacists } \\
\text { within ambulatory VA clinics } \\
\text { G2: Usual care (i.e., no } \\
\text { pharmaceutical care) }\end{array}$ & $\begin{array}{l}\text { G1: } 447 \\
\text { G2: } 484\end{array}$ & $\begin{array}{l}\text { Patient satisfaction with primary } \\
\text { health care provider } \\
\text { (Higher scores indicate greater } \\
\text { satisfaction) }\end{array}$ & $\begin{array}{l}\text { G1: } \\
\text { Time 1: } 51.9(7.5) \\
\text { Time 2: } 51.7(7.3) \\
\text { G2: } \\
\text { Time 1: } 51.9(7.5) \\
\text { Time 2: NR } \\
\text { p=NS }\end{array}$ \\
\hline $\begin{array}{l}\text { Bernsten et al., } 2001^{1} \text {; } \\
\text { Sturgess et al., } 2003^{2} \\
\text { RCT, Cluster- } \\
\text { Randomized/High }\end{array}$ & $\begin{array}{l}\text { G1: Structured community } \\
\text { pharmacy-based } \\
\text { pharmaceutical care program } \\
\text { G2: Usual community pharmacy } \\
\text { services }\end{array}$ & $\begin{array}{l}\text { Baseline } \\
\text { G1: } 1290 \\
\text { G2: } 1164 \\
6 \text { months } \\
\text { G1: } 1024 \\
\text { G2: } 953 \\
\text { 12 months } \\
\text { G1: } 863 \\
\text { G2: } 764 \\
\text { 18 months } \\
\text { G1: } 704 \\
\text { G2: } 636\end{array}$ & $\begin{array}{l}\% \text { rating pharmacy services } \\
\text { provided as "excellent" }\end{array}$ & $\begin{array}{l}\text { Baseline } \\
\text { G1: } 66.2 \\
\text { G2: } 68.2 \\
\text { p: NR } \\
6 \text { months } \\
\text { G1: } 72.8 \\
\text { G2: } 63.7 \\
\text { p: }<0.05 \\
\text { 12 months } \\
\text { G1: } 73.4 \\
\text { G2: } 71.2 \\
\text { p: NR } \\
\text { 18 months } \\
\text { G1: } 73.8 \\
\text { G2: } 64.6 \\
\text { p: }<0.05\end{array}$ \\
\hline
\end{tabular}


Table E28. Patient satisfaction: Summary of results (continued)

\begin{tabular}{|c|c|c|c|}
\hline $\begin{array}{l}\text { Study } \\
\text { Design/Risk of Bias }\end{array}$ & Study Arms & N Analyzed & Outcome and Time Period \\
\hline $\begin{array}{l}\text { Carter et al.,19974; } \\
\text { Barnette, Murphy, and Carter, } \\
1996^{5} \\
\text { Cohort/High }\end{array}$ & $\begin{array}{l}\text { G1: Pharmaceutical care } \\
\text { G2: Usual care with patients } \\
\text { seen by pharmacists who did } \\
\text { not participate in the intensive } \\
\text { skills development program }\end{array}$ & $\begin{array}{l}\text { G1: } 25 \\
\text { G2: } 26\end{array}$ & $\begin{array}{ll}\text { Percent of patients agreeing or } & \mathrm{G} 1: 100 \\
\text { strongly agreeing with statement, } \mathrm{G} 2: 96 \\
\text { "I am very satisfied with the } & \text { p: } 0.065 \\
\text { pharmacy services I receive," } \\
\text { collected at } 6 \text { months }\end{array}$ \\
\hline $\begin{array}{l}\text { Carter et al.,1997 } \\
\text { Barnette, Murphy, and Carter, } \\
1996^{5} \\
\text { Cohort/High }\end{array}$ & $\begin{array}{l}\text { G1: Pharmaceutical care } \\
\text { G2: Usual care with patients } \\
\text { seen by pharmacists who did } \\
\text { not participate in the intensive } \\
\text { skills development program }\end{array}$ & $\begin{array}{l}\text { G1: } 25 \\
\text { G2: } 26\end{array}$ & $\begin{array}{l}\text { Percent of patients agreeing or } \mathrm{G} 1: 100 \\
\text { strongly agreeing with statement, G2: } 80 \\
\text { "Overall, the program provided a } \mathrm{p}: 0.0018 \\
\text { valuable service to me," } \\
\text { collected at } 6 \text { months }\end{array}$ \\
\hline $\begin{array}{l}\text { Carter et al.,1997 } \\
\text { Barnette, Murphy, and Carter, } \\
1996^{5} \\
\text { Cohort/High }\end{array}$ & $\begin{array}{l}\text { G1: Pharmaceutical care } \\
\text { G2: Usual care with patients } \\
\text { seen by pharmacists who did } \\
\text { not participate in the intensive } \\
\text { skills development program }\end{array}$ & $\begin{array}{l}\text { G1: } 25 \\
\text { G2: } 26\end{array}$ & $\begin{array}{l}\text { Percent of patients agreeing or } \mathrm{G} 1: 100 \\
\text { strongly agreeing with statement, G2: } 88 \\
\text { "The quality of information } \\
\text { provided to me by the } 0.012 \\
\text { pharmacist was excellent," } \\
\text { collected at } 6 \text { months }\end{array}$ \\
\hline $\begin{array}{l}\text { Carter et al., } 1997^{4} ; \\
\text { Barnette, Murphy, and Carter, } \\
1996^{5} \\
\text { Cohort/High }\end{array}$ & $\begin{array}{l}\text { G1: Pharmaceutical care } \\
\text { G2: Usual care with patients } \\
\text { seen by pharmacists who did } \\
\text { not participate in the intensive } \\
\text { skills development program }\end{array}$ & $\begin{array}{l}\text { G1: } 25 \\
\text { G2: } 26\end{array}$ & $\begin{array}{l}\text { Percent of patients agreeing or } \mathrm{G} 1: 100 \\
\text { strongly agreeing with statement, G2: } 83 \\
\text { "My participation in this program p: } 0.011 \\
\text { helped me to understand high } \\
\text { blood pressure better," collected } \\
\text { at } 6 \text { months }\end{array}$ \\
\hline $\begin{array}{l}\text { Carter et al., } 1997^{4} ; \\
\text { Barnette, Murphy, and Carter, } \\
1996^{5} \\
\text { Cohort/High }\end{array}$ & $\begin{array}{l}\text { G1: Pharmaceutical care } \\
\text { G2: Usual care with patients } \\
\text { seen by pharmacists who did } \\
\text { not participate in the intensive } \\
\text { skills development program }\end{array}$ & $\begin{array}{l}\text { G1: } 25 \\
\text { G2: } 26\end{array}$ & $\begin{array}{l}\text { Percent of patients agreeing or } \mathrm{G} 1: 96 \\
\text { strongly agreeing with statement, G2: } 96 \\
\text { "The area was private enough p: } 0.036 \\
\text { for me to feel comfortable talking } \\
\text { about my high blood pressure," } \\
\text { collected at } 6 \text { months }\end{array}$ \\
\hline $\begin{array}{l}\text { Carter et al., } 1997^{4} ; \\
\text { Barnette, Murphy, and Carter, } \\
1996^{5} \\
\text { Cohort/High }\end{array}$ & $\begin{array}{l}\text { G1: Pharmaceutical care } \\
\text { G2: Usual care with patients } \\
\text { seen by pharmacists who did } \\
\text { not participate in the intensive } \\
\text { skills development program }\end{array}$ & $\begin{array}{l}\text { G1: } 25 \\
\text { G2: } 26\end{array}$ & $\begin{array}{l}\text { Percent of patients agreeing or } \mathrm{G} 1: 100 \\
\text { strongly agreeing with statement, G2: } 96 \\
\text { "I felt comfortable talking with the p: } 0.052 \\
\text { pharmacist about my health } \\
\text { problems," collected at } 6 \text { months }\end{array}$ \\
\hline
\end{tabular}


Table E28. Patient satisfaction: Summary of results (continued)

\begin{tabular}{|c|c|c|c|c|}
\hline $\begin{array}{l}\text { Study } \\
\text { Design/Risk of Bias }\end{array}$ & Study Arms & N Analyzed & Outcome and Time Period & Results \\
\hline $\begin{array}{l}\text { Carter et al., } 1997^{4} ; \\
\text { Barnette, Murphy, and Carter, } \\
1996^{5} \\
\text { Cohort/High }\end{array}$ & $\begin{array}{l}\text { G1: Pharmaceutical care } \\
\text { G2: Usual care with patients } \\
\text { seen by pharmacists who did } \\
\text { not participate in the intensive } \\
\text { skills development program }\end{array}$ & $\begin{array}{l}\text { G1: } 25 \\
\text { G2: } 26\end{array}$ & $\begin{array}{l}\text { Percent of patients agreeing or } \\
\text { strongly agreeing with statement, } \\
\text { "I am confident the pharmacist is } \\
\text { able to help me control my high } \\
\text { blood pressure," collected at } 6 \\
\text { months }\end{array}$ & $\begin{array}{l}\text { G1: } 100 \\
\text { G2: } 92 \\
\text { p: } 0.340\end{array}$ \\
\hline $\begin{array}{l}\text { Carter et al., } 1997^{4} ; \\
\text { Barnette, Murphy, and Carter, } \\
1996^{5} \\
\text { Cohort/High }\end{array}$ & $\begin{array}{l}\text { G1: Pharmaceutical care } \\
\text { G2: Usual care with patients } \\
\text { seen by pharmacists who did } \\
\text { not participate in the intensive } \\
\text { skills development program }\end{array}$ & $\begin{array}{l}\text { G1: } 25 \\
\text { G2: } 26\end{array}$ & $\begin{array}{l}\text { Percent of patients agreeing or } \\
\text { strongly agreeing with statement, } \\
\text { "I am confident the information } \\
\text { provided by the pharmacist to } \\
\text { the physician improved my } \\
\text { health care," collected at } 6 \\
\text { months }\end{array}$ & $\begin{array}{l}\text { G1: } 87 \\
\text { G2: } 83 \\
p: 0.325\end{array}$ \\
\hline $\begin{array}{l}\text { Carter et al.,1997 }{ }^{4} ; \\
\text { Barnette, Murphy, and Carter, } \\
1996^{5} \\
\text { Cohort/High }\end{array}$ & $\begin{array}{l}\text { G1: Pharmaceutical care } \\
\text { G2: Usual care with patients } \\
\text { seen by pharmacists who did } \\
\text { not participate in the intensive } \\
\text { skills development program }\end{array}$ & $\begin{array}{l}\text { G1: } 25 \\
\text { G2: } 26\end{array}$ & $\begin{array}{l}\text { Percent of patients agreeing or } \\
\text { strongly agreeing with statement, } \\
\text { "There are things about the high } \\
\text { blood pressure program that } \\
\text { could be better," collected at } 6 \\
\text { months }\end{array}$ & $\begin{array}{l}\text { G1: } 9 \\
\text { G2: } 0 \\
\mathrm{p}: 0.157 \\
\end{array}$ \\
\hline $\begin{array}{l}\text { Carter et al.,1997 }{ }^{4} ; \\
\text { Barnette, Murphy, and Carter, } \\
1996^{5} \\
\text { Cohort/High }\end{array}$ & $\begin{array}{l}\text { G1: Pharmaceutical care } \\
\text { G2: Usual care with patients } \\
\text { seen by pharmacists who did } \\
\text { not participate in the intensive } \\
\text { skills development program }\end{array}$ & $\begin{array}{l}\text { G1: } 25 \\
\text { G2: } 26\end{array}$ & $\begin{array}{l}\text { Percent of patients agreeing or } \\
\text { strongly agreeing with statement, } \\
\text { "I am very willing to continue to } \\
\text { see the pharmacist for help with } \\
\text { my high blood pressure control," } \\
\text { collected at } 6 \text { months }\end{array}$ & $\begin{array}{l}\text { G1: } 95 \\
\text { G2: } 88 \\
\text { p: } 0.459 \\
\end{array}$ \\
\hline $\begin{array}{l}\text { Carter et al., } 1997^{4} ; \\
\text { Barnette, Murphy, and Carter, } \\
1996^{5} \\
\text { Cohort/High }\end{array}$ & $\begin{array}{l}\text { G1: Pharmaceutical care } \\
\text { G2: Usual care with patients } \\
\text { seen by pharmacists who did } \\
\text { not participate in the intensive } \\
\text { skills development program }\end{array}$ & $\begin{array}{l}\text { G1: } 25 \\
\text { G2: } 26\end{array}$ & $\begin{array}{l}\text { Percent of patients agreeing or } \\
\text { strongly agreeing with statement, } \\
\text { "I think the pharmacist should } \\
\text { provide this type of service for } \\
\text { everyone," collected at } 6 \text { months }\end{array}$ & $\begin{array}{l}\text { G1: } 77 \\
\text { G2: } 75 \\
\text { p: } 0.890\end{array}$ \\
\hline $\begin{array}{l}\text { Carter et al., } 1997^{4} ; \\
\text { Barnette, Murphy, and Carter, } \\
1996^{5} \\
\text { Cohort/High }\end{array}$ & $\begin{array}{l}\text { G1: Pharmaceutical care } \\
\text { G2: Usual care with patients } \\
\text { seen by pharmacists who did } \\
\text { not participate in the intensive } \\
\text { skills development program }\end{array}$ & $\begin{array}{l}\text { G1: } 25 \\
\text { G2: } 26\end{array}$ & $\begin{array}{l}\text { Percent of patients agreeing or } \\
\text { strongly agreeing with statement, } \\
\text { "I think the pharmacist should be } \\
\text { paid for this type of service," } \\
\text { collected at } 6 \text { months }\end{array}$ & $\begin{array}{l}\text { G1: } 91 \\
\text { G2: } 82 \\
\text { p: } 0.379\end{array}$ \\
\hline
\end{tabular}


Table E28. Patient satisfaction: Summary of results (continued)

\begin{tabular}{|c|c|c|c|c|}
\hline $\begin{array}{l}\text { Study } \\
\text { Design/Risk of Bias }\end{array}$ & Study Arms & N Analyzed & Outcome and Time Period & Results \\
\hline $\begin{array}{l}\text { Volume et al., } 2001^{39} ; \\
\text { Kassam et al., } 2001^{40} \\
\text { RCT-Cluster } \\
\text { Randomized/Medium }\end{array}$ & $\begin{array}{l}\text { G1: Comprehensive } \\
\text { pharmaceutical care services } \\
\text { G2: Traditional pharmacy care }\end{array}$ & $\begin{array}{l}\text { Time 1: } \\
\text { N=363 } \\
\text { G1: } 159 \\
\text { G2: } 204 \\
\text { Time 2: } \\
\text { N=317 } \\
\text { G1: NR } \\
\text { G2: NR } \\
\text { Time 3: } \\
\text { N = 292 } \\
\text { G1: NR } \\
\text { G2: NR }\end{array}$ & $\begin{array}{l}\text { General satisfaction } \\
\text { (Higher numbers reflect greater } \\
\text { dissatisfaction) }\end{array}$ & $\begin{array}{l}\text { Time 1: } \\
\text { G1: } 1.59(0.77) \\
\text { G2: } 1.56(0.73) \\
\text { Time 2: } \\
\text { G1: } 1.51(0.84) \\
\text { G2: } 1.57(0.72) \\
\text { Time 3: } \\
\text { G1: } 1.53(0.77) \\
\text { G2: } 1.62(0.88) \\
\text { p= NS for all between-group differences }\end{array}$ \\
\hline $\begin{array}{l}\text { Volume et al., } 2001^{39} ; \\
\text { Kassam et al., } 2001^{40} \\
\text { RCT-Cluster } \\
\text { Randomized/Medium }\end{array}$ & $\begin{array}{l}\text { G1: Comprehensive } \\
\text { pharmaceutical care services } \\
\text { G2: Traditional pharmacy care }\end{array}$ & $\begin{array}{l}\text { Time 1: } \\
\text { N=363 } \\
\text { G1: } 159 \\
\text { G2: } 204 \\
\text { Time 2: } \\
\text { N=317 } \\
\text { G1: NR } \\
\text { G2: NR } \\
\text { Time 3: } \\
\text { N = 292 } \\
\text { G1: NR } \\
\text { G2: NR }\end{array}$ & $\begin{array}{l}\text { Interpersonal skills } \\
\text { (Higher numbers reflect greater } \\
\text { dissatisfaction) }\end{array}$ & $\begin{array}{l}\text { Time 1: } \\
\text { G1: } 1.36(0.48) \\
\text { G2: } 1.37(0.53 \\
\text { Time 2: } \\
\text { G1: } 1.37(0.59) \\
\text { G2: } 1.35(0.57) \\
\text { Time 3: } \\
\text { G1: } 1.31(0.50) \\
\text { G2: } 1.45(0.72) \\
p=\text { NS for all between-group differences }\end{array}$ \\
\hline
\end{tabular}


Table E28. Patient satisfaction: Summary of results (continued)

\begin{tabular}{|c|c|c|c|c|}
\hline $\begin{array}{l}\text { Study } \\
\text { Design/Risk of Bias }\end{array}$ & Study Arms & N Analyzed & Outcome and Time Period & Results \\
\hline $\begin{array}{l}\text { Volume et al., } 2001^{39} \text {; } \\
\text { Kassam et al., } 2001^{40} \\
\text { RCT-Cluster } \\
\text { Randomized/Medium }\end{array}$ & $\begin{array}{l}\text { G1: Comprehensive } \\
\text { pharmaceutical care services } \\
\text { G2: Traditional pharmacy care }\end{array}$ & $\begin{array}{l}\text { Time 1: } \\
\text { N= 363 } \\
\text { G1: } 159 \\
\text { G2: } 204 \\
\\
\text { Time 2: } \\
\text { N = 317 } \\
\text { G1: NR } \\
\text { G2: NR } \\
\text { Time 3: } \\
\text { N = 292 } \\
\text { G1: NR } \\
\text { G2: NR }\end{array}$ & $\begin{array}{l}\text { Evaluation and goal setting } \\
\text { (Higher numbers reflect greater } \\
\text { dissatisfaction) }\end{array}$ & $\begin{array}{l}\text { Time 1: } \\
\text { G1: } 2.58(1.12) \\
\text { G2: } 2.74(1.09) \\
\text { Time 2: } \\
\text { G1: } 2.46(0.98) \\
\text { G2: } 2.98(1.24) \\
\text { Time } 3: \\
\text { G1: } 2.49(1.10) \\
\text { G2: } 2.90(1.08) \\
\text { p<0.05 for between-group differences in } \\
\text { score changes from Time } 1 \text { to Time } 2 \text { and } \\
\text { Time } 1 \text { to Time } 3\end{array}$ \\
\hline $\begin{array}{l}\text { Volume et al., } 2001^{39} \text {; } \\
\text { Kassam et al., } 2001^{40} \\
\text { RCT-Cluster } \\
\text { Randomized/Medium }\end{array}$ & $\begin{array}{l}\text { G1: Comprehensive } \\
\text { pharmaceutical care services } \\
\text { G2: Traditional pharmacy care }\end{array}$ & $\begin{array}{l}\text { Time 1: } \\
\text { N = 363 } \\
\text { G1: } 159 \\
\text { G2: } 204 \\
\text { Time 2: } \\
\text { N = 317 } \\
\text { G1: NR } \\
\text { G2: NR } \\
\text { Time 3: } \\
\text { N = 292 } \\
\text { G1: NR } \\
\text { G2: NR }\end{array}$ & $\begin{array}{l}\text { Trust } \\
\text { (Higher numbers reflect greater } \\
\text { dissatisfaction) }\end{array}$ & $\begin{array}{l}\text { Time 1: } \\
\text { G1: } 1.62(0.66) \\
\text { G2: } 1.46(0.57) \\
\text { Time 2: } \\
\text { G1: } 1.40(0.54) \\
\text { G2: } 1.39(0.58) \\
\text { Time 3: } \\
\text { G1: } 1.43(0.58) \\
\text { G2: } 1.51(0.75) \\
\text { p<0.05 for between-group differences in } \\
\text { score changes from Time } 1 \text { to Time } 2 \\
\text { p<0.05 for group x measure interaction } \\
\text { over all three time periods }\end{array}$ \\
\hline
\end{tabular}


Table E28. Patient satisfaction: Summary of results (continued)

\begin{tabular}{|c|c|c|c|c|}
\hline $\begin{array}{l}\text { Study } \\
\text { Design/Risk of Bias }\end{array}$ & Study Arms & N Analyzed & Outcome and Time Period & Results \\
\hline $\begin{array}{l}\text { Volume et al., } 2001^{39} \text {; } \\
\text { Kassam et al., } 2001^{40} \\
\text { RCT-Cluster } \\
\text { Randomized/Medium }\end{array}$ & $\begin{array}{l}\text { G1: Comprehensive } \\
\text { pharmaceutical care services } \\
\text { G2: Traditional pharmacy care }\end{array}$ & $\begin{array}{l}\text { Time 1: } \\
\text { N=363 } \\
\text { G1: } 159 \\
\text { G2: } 204 \\
\text { Time 2: } \\
\text { N=317 } \\
\text { G1: NR } \\
\text { G2: NR } \\
\text { Time 3: } \\
\text { N = 292 } \\
\text { G1: NR } \\
\text { G2: NR }\end{array}$ & $\begin{array}{l}\text { Helping patients } \\
\text { (Higher numbers reflect greater } \\
\text { dissatisfaction) }\end{array}$ & $\begin{array}{l}\text { Time 1: } \\
\text { G1: } 2.25(1.31) \\
\text { G2: } 2.22(1.14) \\
\text { Time 2: } \\
\text { G1: } 1.98(1.17) \\
\text { G2: } 2.23(1.15) \\
\text { Time 3: } \\
\text { G1: } 2.07(1.22) \\
\text { G2: } 2.37(1.21) \\
\text { p= NS for all between-group differences }\end{array}$ \\
\hline $\begin{array}{l}\text { Volume et al., } 2001^{39} \text {; } \\
\text { Kassam et al., } 2001^{40} \\
\text { RCT-Cluster } \\
\text { Randomized/Medium }\end{array}$ & $\begin{array}{l}\text { G1: Comprehensive } \\
\text { pharmaceutical care services } \\
\text { G2: Traditional pharmacy care }\end{array}$ & $\begin{array}{l}\text { Time 1: } \\
\text { N=363 } \\
\text { G1: } 159 \\
\text { G2: } 204 \\
\text { Time 2: } \\
\text { N=317 } \\
\text { G1: NR } \\
\text { G2: NR } \\
\text { Time 3: } \\
\text { N = 292 } \\
\text { G1: NR } \\
\text { G2: NR }\end{array}$ & $\begin{array}{l}\text { Explanation } \\
\text { (Higher numbers reflect greater } \\
\text { dissatisfaction) }\end{array}$ & $\begin{array}{l}\text { Time 1: } \\
\text { G1: } 1.34(0.55) \\
\text { G2: } 1.34(0.63) \\
\text { Time 2: } \\
\text { G1: } 1.39(0.67) \\
\text { G2: } 1.30(0.56) \\
\text { Time 3: } \\
\text { G1: } 1.38(0.73) \\
\text { G2: } 1.35(0.61) \\
\mathrm{p}=\text { NS for all between-group differences }\end{array}$ \\
\hline
\end{tabular}


Table E28. Patient satisfaction: Summary of results (continued)

\begin{tabular}{|c|c|c|c|c|}
\hline $\begin{array}{l}\text { Study } \\
\text { Design/Risk of Bias }\end{array}$ & Study Arms & N Analyzed & Outcome and Time Period & Results \\
\hline $\begin{array}{l}\text { Volume et al., } 2001^{39} \text {; } \\
\text { Kassam et al., } 2001^{40} \\
\text { RCT-Cluster } \\
\text { Randomized/Medium }\end{array}$ & $\begin{array}{l}\text { G1: Comprehensive } \\
\text { pharmaceutical care services } \\
\text { G2: Traditional pharmacy care }\end{array}$ & $\begin{array}{l}\text { Time 1: } \\
\text { N=363 } \\
\text { G1: } 159 \\
\text { G2: } 204 \\
\text { Time 2: } \\
\text { N=317 } \\
\text { G1: NR } \\
\text { G2: NR } \\
\text { Time 3: } \\
\text { N = 292 } \\
\text { G1: NR } \\
\text { G2: NR }\end{array}$ & $\begin{array}{l}\text { Pharmacy finances } \\
\text { (Higher numbers reflect greater } \\
\text { dissatisfaction) }\end{array}$ & $\begin{array}{l}\text { Time 1: } \\
\text { G1: } 3.08(1.82) \\
\text { G2: } 2.85(1.80) \\
\text { Time 2: } \\
\text { G1: } 2.89(1.89) \\
\text { G2: } 2.86(1.75) \\
\text { Time 3: } \\
\text { G1: } 3.08(1.80) \\
\text { G2: } 3.16(1.88) \\
\text { p= NS for all between-group differences }\end{array}$ \\
\hline $\begin{array}{l}\text { Volume et al., } 2001^{39} \text {; } \\
\text { Kassam et al., } 2001^{40} \\
\text { RCT-Cluster } \\
\text { Randomized/Medium }\end{array}$ & $\begin{array}{l}\text { G1: Comprehensive } \\
\text { pharmaceutical care services } \\
\text { G2: Traditional pharmacy care }\end{array}$ & $\begin{array}{l}\text { Time 1: } \\
\text { N=363 } \\
\text { G1: } 159 \\
\text { G2: } 204 \\
\text { Time 2: } \\
\text { N=317 } \\
\text { G1: NR } \\
\text { G2: NR } \\
\text { Time 3: } \\
\text { N = 292 } \\
\text { G1: NR } \\
\text { G2: NR }\end{array}$ & $\begin{array}{l}\text { Drug plan finances } \\
\text { (Higher numbers reflect greater } \\
\text { dissatisfaction) }\end{array}$ & $\begin{array}{l}\text { Time 1: } \\
\text { G1: } 3.31(1.70) \\
\text { G2: } 3.41(1.75) \\
\text { Time 2: } \\
\text { G1: } 3.45(1.96) \\
\text { G2: } 3.39(1.83) \\
\text { Time 3: } \\
\text { G1: } 3.65(1.67) \\
\text { G2: } 3.56(1.83) \\
\text { p= NS for all between-group differences }\end{array}$ \\
\hline
\end{tabular}


Table E28. Patient satisfaction: Summary of results (continued)

\begin{tabular}{|c|c|c|c|c|}
\hline $\begin{array}{l}\text { Study } \\
\text { Design/Risk of Bias }\end{array}$ & Study Arms & N Analyzed & Outcome and Time Period & Results \\
\hline $\begin{array}{l}\text { Volume et al., } 2001^{39} \text {; } \\
\text { Kassam et al., } 2001^{40} \\
\text { RCT-Cluster } \\
\text { Randomized/Medium }\end{array}$ & $\begin{array}{l}\text { G1: Comprehensive } \\
\text { pharmaceutical care services } \\
\text { G2: Traditional pharmacy care }\end{array}$ & $\begin{array}{l}\text { Time 1: } \\
\text { N = 363 } \\
\text { G1: } 159 \\
\text { G2: } 204 \\
\text { Time 2: } \\
\text { N=317 } \\
\text { G1: NR } \\
\text { G2: NR } \\
\text { Time 3: } \\
\text { N = 292 } \\
\text { G1: NR } \\
\text { G2: NR }\end{array}$ & $\begin{array}{l}\text { Communicates with doctor } \\
\text { (Higher numbers reflect greater } \\
\text { dissatisfaction) }\end{array}$ & $\begin{array}{l}\text { Time 1: } \\
\text { G1: } 1.50(0.77) \\
\text { G2: } 1.60(0.89) \\
\text { Time 2: } \\
\text { G1: } 1.36(0.63) \\
\text { G2: } 1.72(1.00) \\
\text { Time 3: } \\
\text { G1: } 1.36(0.65) \\
\text { G2: } 1.74(0.97) \\
\text { p<0.05 for between-group differences in } \\
\text { score changes from Time } 1 \text { to Time } 3\end{array}$ \\
\hline
\end{tabular}


Table E29. Use of generic medications: Summary of results

\begin{tabular}{|c|c|c|c|c|}
\hline \multicolumn{2}{|l|}{ Study } & \multicolumn{3}{|l|}{ N Analyzed } \\
\hline Design/Risk of Bias & Study Arms & & Outcome and Time Period & Results \\
\hline $\begin{array}{l}\text { Pindolia et al., } 2009^{30} \\
\text { Cohort/High }\end{array}$ & $\begin{array}{l}\text { G1: Telephone based MTM } \\
\text { program (acceptors) } \\
\text { G2: Usual medical care (opt- } \\
\text { out) }\end{array}$ & $\begin{array}{l}\text { G1: } 292 \\
\text { G2: } 1,081\end{array}$ & $\begin{array}{l}\text { Increase in the overall use of } \\
\text { generic drugs }\end{array}$ & $\begin{array}{l}\text { G1: } 6 \% \\
\text { G2: } 3 \% \\
\text { p not calculated because baseline } \\
\text { percentages not provided }\end{array}$ \\
\hline $\begin{array}{l}\text { Winston and Lin, } 2009^{43} \\
\text { Cohort/High }\end{array}$ & $\begin{array}{l}\text { G1: Community pharmacy } \\
\text { MTM } \\
\text { G2: Pharmacist-staffed call } \\
\text { center-based MTM } \\
\text { G3: Educational mailings }\end{array}$ & $\begin{array}{l}\text { G1: } 21,336 \\
\text { G2: } 3,436 \\
\text { G3: } 49,021\end{array}$ & $\begin{array}{l}\text { Weighted generic substitution } \\
\text { ratio: } 30 \text {-day equivalent claims } \\
\text { divided by total number of } \\
\text { claims }\end{array}$ & $\begin{array}{l}\text { Pre-MTM (Jan } 1 \text { 2007-April 30, 2007) } \\
\text { G1: } 60.1(29.8) \\
\text { G2: } 58.6(25.7) \\
\text { G3: } 58.7(27.6) \\
\text { p: NR }\end{array}$ \\
\hline & & & & $\begin{array}{l}\text { Post-MTM (Jan } 1 \text { 2008-April 30, 2008) } \\
\text { G1: } 65.7(32.5) \\
\text { G2: } 64.6(30.5) \\
\text { G3: } 63.5(32.2) \\
\text { p: NR }\end{array}$ \\
\hline & & & & $\begin{array}{l}\text { Calculated SMD for G1 vs. G3: }-0.04 \\
(95 \% \mathrm{Cl},-0.06 \text { to }-0.02 ; p<0.001)\end{array}$ \\
\hline & & & & $\begin{array}{l}\text { Calculated SMD for G2 vs. G3: }-0.03 \\
(95 \% \mathrm{Cl},-0.06 \text { to } 0.01 ; p=0.134)\end{array}$ \\
\hline
\end{tabular}

Abbreviations: $\mathrm{CI}=$ confidence interval; $\mathrm{G}=$ group; MTM = Medication Therapy Management; NR = not reported; SMD: standardized mean difference; vs. = versus. 
Table E30. Patient co-payments: Summary of results

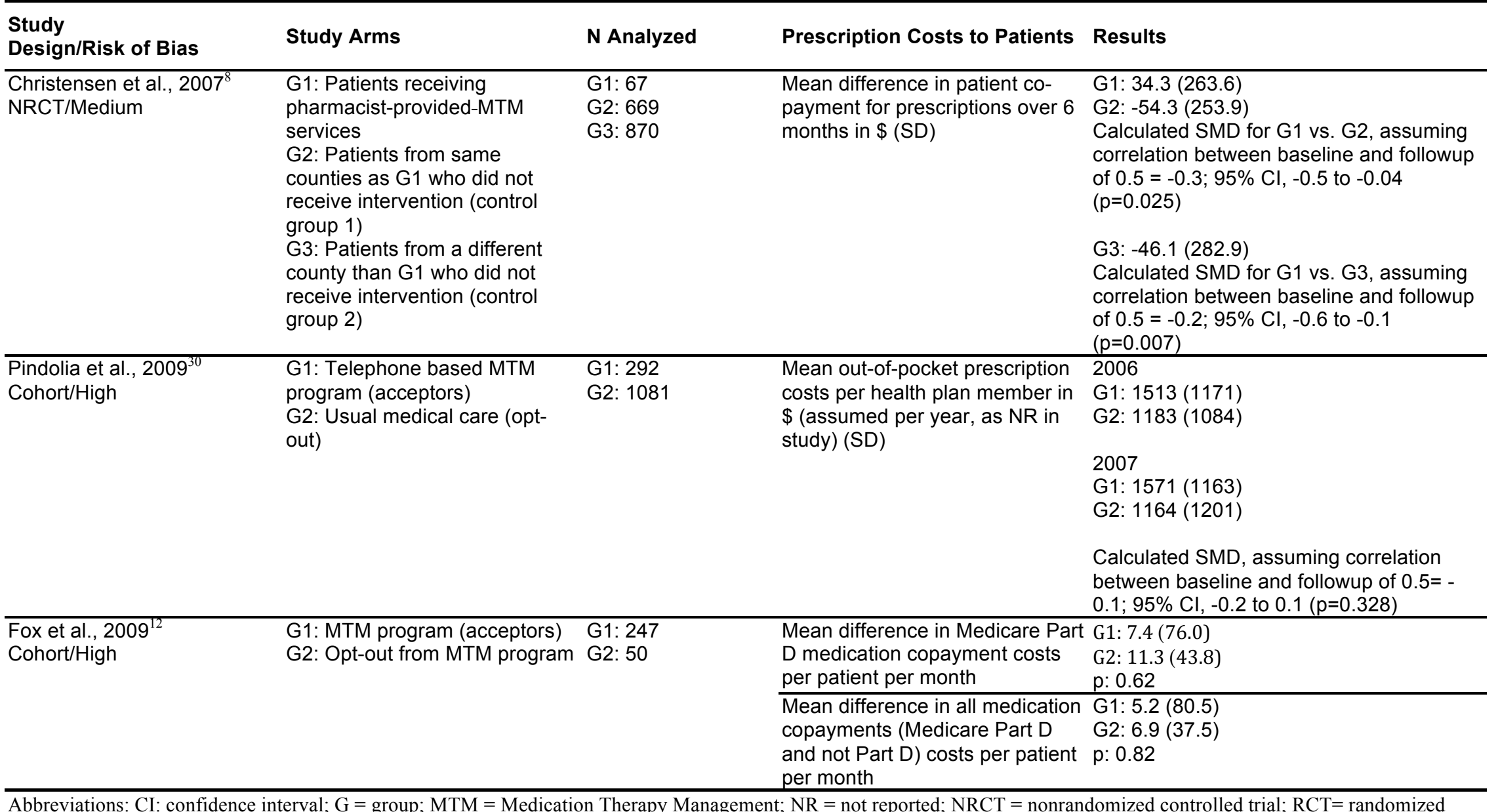

Abbreviations: CI: confidence interval; $\mathrm{G}=$ group; $\mathrm{MTM}=$ Medication Therapy Management; $\mathrm{NR}=$ not reported; NRCT = nonrandomized controlled trial; RCT= randomized

controlled trial; $\mathrm{SD}=$ standard deviation; SMD: standardized mean difference; vs. = versus 
Table E31. Total expenditures on medications by health plans: Summary of results

\begin{tabular}{|c|c|c|c|c|}
\hline $\begin{array}{l}\text { Study } \\
\text { Design/Risk of Bias }\end{array}$ & Study Arms & N Analyzed & $\begin{array}{l}\text { Prescription Costs to Health } \\
\text { Plans }\end{array}$ & Results \\
\hline $\begin{array}{l}\text { Jameson, VanNoord, and } \\
\text { Vanderwoud, } 1995^{17} \\
\text { RCT/Medium }\end{array}$ & $\begin{array}{l}\text { G1: Pharmacotherapy } \\
\text { consultation } \\
\text { G2: Usual care }\end{array}$ & $\begin{array}{l}\text { G1: } 27 \\
\text { G2: } 29\end{array}$ & $\begin{array}{l}\text { Change in cost (USD) of } \\
\text { prescription drugs over } 6 \text { months, } \\
\text { based on maximum allowable } \\
\text { cost for Medicaid reimbursement }\end{array}$ & $\begin{array}{l}\text { G1: }-130 \\
\text { G2: } 163 \\
\text { Calculated mean difference: }-293,95 \% \mathrm{Cl} \text {, } \\
-501.5 \text { to }-84.5 \\
p<0.01\end{array}$ \\
\hline $\begin{array}{l}\text { Sellors et al., } 2003^{33} \\
\text { RCT/Medium }\end{array}$ & $\begin{array}{l}\text { G1: Pharmacist consultation } \\
\text { program } \\
\text { G2: Usual care }\end{array}$ & $\begin{array}{l}\text { G1: } 379 \\
\text { G2: } 409\end{array}$ & $\begin{array}{l}\text { Mean daily medication costs per } \\
\text { patient to the Ontario Drug } \\
\text { Benefit Program (assumed CAD) } \\
\text { at } 5 \text { months }\end{array}$ & $\begin{array}{l}\text { G1: } 3.6 \\
\text { G2: } 3.8 \\
\text { Calculated mean difference: } 0.19,95 \% \mathrm{Cl} \text {, } \\
-1.5 \text { to } 1.1 \\
\text { p: } 0.78\end{array}$ \\
\hline $\begin{array}{l}\text { Chrischilles et al., } 2004^{7} \\
\text { Cohort/High }\end{array}$ & $\begin{array}{l}\text { G1: PCM-eligible patients who } \\
\text { received PCM services } \\
\text { G2: PCM-eligible patients who } \\
\text { did not receive PCM services }\end{array}$ & $\begin{array}{l}\text { G1: } 524 \\
\text { G2: } 1,687\end{array}$ & $\begin{array}{l}\text { Mean amount billed per patient } \\
\text { for active drugs in USD (based } \\
\text { on Medicaid claims) (SD) at } \\
\text { baseline and at } 9 \text { months }\end{array}$ & $\begin{array}{l}\text { Baseline } \\
\text { G1: } 488.4(20.8) \\
\text { G2: } 441.9(14.5) \\
\text { Followup } \\
\text { G1: } 525.0(22.1) \\
\text { G2: } 477.6(15.5) \\
\text { Calculated mean difference: }-0.95 \\
95 \% \mathrm{Cl},-58.7 \text { to } 56.8, \mathrm{p}: 0.974 \\
\end{array}$ \\
\hline $\begin{array}{l}\text { Christensen et al., } 2007^{8} \\
\text { NRCT/Medium }\end{array}$ & $\begin{array}{l}\text { G1: Patients receiving } \\
\text { pharmacist-provided-MTM } \\
\text { services } \\
\text { G2: Patients from same } \\
\text { counties as G1 who did not } \\
\text { receive intervention (control } \\
\text { group 1) } \\
\text { G3: Patients from a different } \\
\text { county than G1 who did not } \\
\text { receive intervention (control } \\
\text { group 2) }\end{array}$ & $\begin{array}{l}\text { G1: } 67 \\
\text { G2: } 669 \\
\text { G3: } 870\end{array}$ & $\begin{array}{l}\text { Mean difference in amount } \\
\text { insurer paid for prescriptions over } \\
6 \text { months in USD (SD) }\end{array}$ & $\begin{array}{l}\text { G1: }-90.1(793.0) \\
\text { G2: }-35.4(939.5) \\
\text { G3: }-97.3(907.4) \\
\text { Calculated mean difference for } \mathrm{G} 1 \text { vs. } \mathrm{G} 2 \text {, } \\
\text { assuming correlation between baseline } \\
\text { and followup of } 0.5=-54.7 \\
95 \% \mathrm{Cl},-287.6 \text { to } 178.2(p=0.645) \\
\text { Calculated mean difference for } \mathrm{G} 1 \text { vs. G3, } \\
\text { assuming correlation between baseline } \\
\text { and followup of } 0.5=-7.2 ; \\
95 \% \mathrm{Cl},-230.8 \text { to } 216.4(p=0.950)\end{array}$ \\
\hline
\end{tabular}


Table E31. Total expenditures on medications by health plans: Summary of results (continued)

\begin{tabular}{|c|c|c|c|c|}
\hline $\begin{array}{l}\text { Study } \\
\text { Design/Risk of Bias }\end{array}$ & Study Arms & N Analyzed & $\begin{array}{l}\text { Prescription Costs to Health } \\
\text { Plans }\end{array}$ & Results \\
\hline $\begin{array}{l}\text { Moczygemba et al., } 2011^{25} \\
\text { Moczygemba et al., } 2008^{26} \\
\text { Cohort/Medium }\end{array}$ & $\begin{array}{l}\text { G1: MTM-eligible patients who } \\
\text { opting-in to a telephone MTM } \\
\text { program (acceptors) } \\
\text { G2: MTM- eligible patients who } \\
\text { did not opt-in to the MTM } \\
\text { program (opt-out) }\end{array}$ & $\begin{array}{l}\text { G1: } 60 \\
\text { G2: } 60\end{array}$ & $\begin{array}{l}\text { Mean Part D drug costs in USD } \\
\text { (based on prescription claim } \\
\text { records, excludes non-Part D } \\
\text { drug costs) (SD) at baseline and } \\
6 \text { months }\end{array}$ & $\begin{array}{l}\text { Baseline } \\
\text { G1: } \$ 2289(\$ 887) \\
\text { G2: } \$ 2131(\$ 1273) \\
\text { p: NR } \\
\text { Follow up } \\
\text { G1: } \$ 2311(\$ 1148) \\
\text { G2: } \$ 2429(\$ 1697) \\
\text { Adjusted p: } 0.80 \\
\text { Calculated mean difference: }-276.0,95 \% \\
\text { Cl, }-751.3 \text { to } 199.3, p: 0.26\end{array}$ \\
\hline
\end{tabular}

Abbreviations: $\mathrm{CAD}=$ Canadian dollar; $\mathrm{CI}=$ confidence interval; $\mathrm{G}=$ group; $\mathrm{MTM}=$ medication therapy management; NR $=$ not reported; NRCT $=$ nonrandomized controlled trial;

$\mathrm{PCM}=$ pharmaceutical case management; $\mathrm{RCT}=$ randomized controlled trial; $\mathrm{SD}=$ standard deviation; $\mathrm{USD}=\mathrm{US}$ dollar 
Table E32. Total expenditures on medications by patients and health plans: Summary of results

\begin{tabular}{|c|c|c|c|c|}
\hline $\begin{array}{l}\text { Study } \\
\text { Design/Risk of Bias }\end{array}$ & Study Arms & N Analyzed & Prescription Costs & Results \\
\hline $\begin{array}{l}\text { Malone et al., } 2000^{20} \text {; } \\
\text { Ellis et al., 2000 } \\
\text { (interventions); } \\
\text { Malone et al., } 2001^{22} \text {; } \\
\text { Ellis et al., } 2000^{23} \\
\text { RCT/Medium }\end{array}$ & $\begin{array}{l}\text { G1: Pharmaceutical care } \\
\text { G2: Usual care }\end{array}$ & $\begin{array}{l}\text { G1: } 523 \\
\text { G2: } 531\end{array}$ & $\begin{array}{l}\text { Mean change in annual drug } \\
\text { costs in USD (calculated from } \\
\text { Denver VAMC pharmacy } \\
\text { department, individual sites, or } \\
\text { the VA Pharmacy Benefits } \\
\text { Management group) }\end{array}$ & $\begin{array}{l}\text { G1: }+203 \\
\text { G2: }+140 \\
\text { Calculated mean difference: } 63 \text {, } \\
95 \% \mathrm{Cl},-5.1 \text { to } 131.1 ; \mathrm{p}: 0.07\end{array}$ \\
\hline $\begin{array}{l}\text { Sellors et al., } 2003^{33} \\
\text { RCT/Medium }\end{array}$ & $\begin{array}{l}\text { G1: Pharmacist consultation } \\
\text { program } \\
\text { G2: Usual care }\end{array}$ & $\begin{array}{l}\text { G1: } 379 \\
\text { G2: } 409\end{array}$ & $\begin{array}{l}\text { Mean daily medication costs per } \\
\text { patient at } 5 \text { months (assumed } \\
\text { CAD) }\end{array}$ & $\begin{array}{l}\mathrm{G} 1: 5.01 \\
\mathrm{G} 2: 4.82 \\
\text { Calculated mean difference: } 0.2 \text {, } \\
95 \% \mathrm{Cl},-0.8 \text { to } 1.2 ; \mathrm{p}=0.72\end{array}$ \\
\hline $\begin{array}{l}\text { Williams et al., } 2004^{42} \\
\text { RCT/Medium }\end{array}$ & $\begin{array}{l}\text { G1: Modification of patient's } \\
\text { medication regimen by an } \\
\text { interdisciplinary medication } \\
\text { adjustment team } \\
\text { G2: Usual medical care }\end{array}$ & $\begin{array}{l}\text { G1: } 57 \\
\text { G2: } 76\end{array}$ & $\begin{array}{l}\text { Average monthly wholesale price } \\
\text { (USD) of prescription and non- } \\
\text { prescription drugs in USD }\end{array}$ & $\begin{array}{l}\text { G1: }-26.92 \\
\text { G2: }-0.68 \\
\text { Reported mean difference: }-20.2 \text {, } \\
95 \% \mathrm{Cl}, 5.8 \text { to } 34.5 \\
\text { p: } 0.006\end{array}$ \\
\hline $\begin{array}{l}\text { Krska et al., } 2001^{19} \\
\text { RCT/High }\end{array}$ & $\begin{array}{l}\text { G1: Pharmacist-led medication } \\
\text { review } \\
\text { G2: Usual care including } \\
\text { identification of pharmaceutical } \\
\text { care issues, but no plan }\end{array}$ & $\begin{array}{l}\text { G1: } 168 \\
\text { G2: } 164\end{array}$ & $\begin{array}{l}\text { Average monthly costs of } \\
\text { prescribed medication per patient } \\
\text { in British? pounds (SD) at } 3 \\
\text { months (calculated using } \\
\text { information from patient on actual } \\
\text { use) }\end{array}$ & $\begin{array}{l}\text { Baseline: } \\
\text { G1: } 39.3(29.1) \\
\text { G2: } 42.8(33.5) \\
\\
\text { Followup } \\
\text { G1: } 38.8(29.6) \\
\text { G2: } 42.6(31.8) \\
\text { Calculated mean difference: }-0.2,95 \% \mathrm{Cl} \text {, } \\
-6.7 \text { to } 6.5) \\
p=0.956\end{array}$ \\
\hline
\end{tabular}


Table E32. Total expenditures on medications by patients and health plans: Summary of results (continued)

\begin{tabular}{|c|c|c|c|c|}
\hline $\begin{array}{l}\text { Study } \\
\text { Design/Risk of Bias }\end{array}$ & Study Arms & N Analyzed & Prescription Costs & Results \\
\hline $\begin{array}{l}\text { Pai et al., } 2009^{27} \text {; Pai et al., } \\
2009^{28} \\
\text { RCT/High }\end{array}$ & $\begin{array}{l}\text { G1: Pharmaceutical care } \\
\text { G2: Usual care }\end{array}$ & $\begin{array}{l}\text { G1: NR } \\
\text { G2: NR }\end{array}$ & $\begin{array}{l}\text { Mean drug costs in USD } \\
\text { (calculated from average } \\
\text { wholesale price) over } 2 \text { years }\end{array}$ & $\begin{array}{l}\text { Baseline: } \\
\text { G1: } 430(197) \\
\text { G2: } 451(267) \\
\text { Followup: } \\
\text { Pharmaceutical care reduced mean drug } \\
\text { costs by } \$ 6.21 \text { compared with the } \\
\text { standard of care group, } \mathrm{p}=\mathrm{NS} \text {, no absolute } \\
\text { costs or other details reported }\end{array}$ \\
\hline $\begin{array}{l}\text { Fox et al., } 2009^{12} \\
\text { Cohort/High }\end{array}$ & $\begin{array}{l}\text { G1: MTM program (acceptors) } \\
\text { G2: Opt-out from MTM program } \\
\text { (opt-out) }\end{array}$ & $\begin{array}{l}\text { G1: } 247 \\
\text { G2: } 50\end{array}$ & $\begin{array}{l}\text { Mean difference in annual } \\
\text { Medicare Part D drug cost in } \\
\text { USD (patient } \\
\text { copay + insurance plan } \\
\text { medication costs + dispensing } \\
\text { fee) }\end{array}$ & $\begin{array}{l}\text { G1: }-76.7(350.8) \\
\text { G2: }-49.0(92.8) \\
\text { Calculated mean difference: }-27.8 \\
95 \% \mathrm{Cl},-125.8 \text { to } 26.6 \\
\text { p: } 0.57\end{array}$ \\
\hline $\begin{array}{l}\text { Pindolia et al., } 2009^{30} \\
\text { Cohort/High }\end{array}$ & $\begin{array}{l}\text { G1: Telephone based MTM } \\
\text { program (acceptors) } \\
\text { G2: Usual medical care (opt- } \\
\text { out) }\end{array}$ & $\begin{array}{l}\text { G1: } 292 \\
\text { G2: } 1081\end{array}$ & $\begin{array}{l}\text { Total annual prescription drug } \\
\text { cost per health plan member in } \\
\text { USD }\end{array}$ & $\begin{array}{l}\text { Pre-enrollment (\$) (January-June 2006) } \\
\text { G1: } 576.3(394.3) \\
\text { G2: } 468.1(335.9) \\
\text { Post-enrollment }(\$) \text { (July-December 2006) } \\
\text { G1: } 480.7(404.3) \\
\text { G2: } 434.7(421.4) \\
\text { Calculated mean difference: }-62.2 \\
95 \% \mathrm{Cl},-112.5 \text { to }-12.0 ; p=0.015 \\
\end{array}$ \\
\hline $\begin{array}{l}\text { Staresinic et al., } 2007^{35} \\
\text { Cohort/High }\end{array}$ & $\begin{array}{l}\text { G1: MTP program (acceptors) } \\
\text { G2: Usual care (opt-out) }\end{array}$ & $\begin{array}{l}\text { G1: } 282 \\
\text { G2: } 1544\end{array}$ & $\begin{array}{l}\text { Total prescription cost per MTMP } \\
\text { beneficiary per month in USD } \\
\text { (gross drug cost=ingredient cost } \\
\text { paid + dispensing fee }+ \text { sales } \\
\text { tax/member months in part D } \\
\text { contract) }\end{array}$ & $\begin{array}{l}\text { Participants spent less on prescription } \\
\text { medications on average (described as per } \\
\text { member per month drug spending) than } \\
\text { non-participants. Figure provided suggests } \\
\text { a decrease spend of between } 100 \text { and } 150 \\
\text { in the intervention group, but exact } \\
\text { numbers not reported. }\end{array}$ \\
\hline
\end{tabular}


Table E32. Total expenditures on medications by patients and health plans: Summary of results (continued)

\begin{tabular}{|c|c|c|c|c|}
\hline $\begin{array}{l}\text { Study } \\
\text { Design/Risk of Bias }\end{array}$ & Study Arms & N Analyzed & Prescription Costs & Results \\
\hline $\begin{array}{l}\text { Welch et al., } 2009^{41} \\
\text { Cohort/High }\end{array}$ & $\begin{array}{l}\text { G1: MTM program provided to } \\
\text { home-based beneficiaries } \\
\text { G2: No-MTM control group } \\
\text { (voluntary opt-out) }\end{array}$ & $\begin{array}{l}\text { G1: } 459 \\
\text { G2: } 336\end{array}$ & $\begin{array}{l}\text { Mean change in medication costs } \\
\text { per day in USD at } 6 \text { months. No } \\
\text { SD reported. (from data on study } \\
\text { beneficiaries' purchases } \\
\text { of ambulatory prescription } \\
\text { medications) } \\
\text { Mean percent increase in } \\
\text { medication costs per day in USD } \\
\text { at } 6 \text { months (no SD reported) }\end{array}$ & $\begin{array}{l}\text { G1: } 0.3 \\
\text { G2: }-3.3 \\
\text { Adjusted p: } 0.203 \\
\text { NOTE: Age, sex, chronic disease score, } \\
\text { and preperiod drug cost included in } \\
\text { multivariate regression modeling for } \\
\text { adjusted P } \\
\text { G1: } 49.7 \\
\text { G2: } 39.9 \\
\text { p: } 0.006 \\
\text { Adjusted OR (95\% CI): } 1.4(1.1 \text { to } 1.9) \\
\text { NOTE: Model adjusted for age, sex, } \\
\text { chronic disease score, and baseline } \\
\text { medication cost }\end{array}$ \\
\hline $\begin{array}{l}\text { Winston and Lin, } 2009^{43} \\
\text { Cohort/High }\end{array}$ & $\begin{array}{l}\text { G1: Community pharmacy MTM } \\
\text { G2: Pharmacist-staffed call } \\
\text { center-based MTM } \\
\text { G3: Educational mailings }\end{array}$ & $\begin{array}{l}\text { G1: } 21,336 \\
\text { G2: } 3,436 \\
\text { G3: } 49,021\end{array}$ & $\begin{array}{l}\text { Mean (SD) drug cost per patient } \\
\text { per month in USD after } 8 \text { months } \\
\text { of services (based drug claims } \\
\text { processing data, total allowed } \\
\text { charges, including } \\
\text { ingredient cost paid, dispensing } \\
\text { fee, and sales tax, prior to } \\
\text { subtracting } \\
\text { any patient cost-sharing } \\
\text { amounts) }\end{array}$ & $\begin{array}{l}\text { Pre-MTM period (Jan } 1 \text { 2007-April 30, } \\
\text { 2007) } \\
\text { G1: } 669(461) \\
\text { G2: } 676(463) \\
\text { G3: } 698(513) \\
\text { Post-MTM period (Jan } 1 \text { 2008-April 30, } \\
\text { 2008) } \\
\text { G1: } 634(512) \\
\text { G2: } 661(494) \\
\text { G3: } 698(556)\end{array}$ \\
\hline
\end{tabular}

Calculated mean difference for G1 vs. G3: $-35.0,95 \% \mathrm{Cl},-43.4$ to $-26.6 ; \mathrm{p}<0.001$

Calculated mean difference for $\mathrm{G} 2$ vs. G3: $-15.0,95 \% \mathrm{Cl},-33.4$ to $3.4 ; \mathrm{p}=0.11$

Abbreviations: $\mathrm{CAD}=$ Canadian dollar; $\mathrm{CI}=$ confidence interval; $\mathrm{G}=$ group; MTM = Medication Therapy Management; MTMP= Medication Therapy Management Program; NR $=$ not reported; $\mathrm{NS}=$ not sufficient; $\mathrm{OR}=$ odds ratio; $\mathrm{RCT}=$ randomized controlled trial; $\mathrm{SD}=$ standard deviation; $\mathrm{USD}=\mathrm{US}$ dollar; $\mathrm{VA}=\mathrm{Veterans} \mathrm{Administration}$; $\mathrm{VAMC}=$

Veterans Affairs Medical Center; vs. = versus. 
Table E33. Medication and other costs: Summary of results

\begin{tabular}{|c|c|c|c|c|}
\hline $\begin{array}{l}\text { Study } \\
\text { Design/Risk of Bias }\end{array}$ & Study Arms & N Analyzed & Prescription Costs to Patients & Results \\
\hline \multirow[t]{2}{*}{$\begin{array}{l}\text { Sellors et al., } 2003^{33} \\
\text { RCT/Medium }\end{array}$} & $\begin{array}{l}\text { G1: Pharmacist consultation } \\
\text { program } \\
\text { G2: Usual care }\end{array}$ & $\begin{array}{l}\text { G1: } 379 \\
\text { G2: } 409\end{array}$ & $\begin{array}{l}\text { Mean cost (SE) of health care } \\
\text { resources per patient, including } \\
\text { all hospital stays at } 5 \text { months } \\
\text { (CAD assumed) }\end{array}$ & $\begin{array}{l}\text { G1: } 1894.1(200.7) \\
\text { G2: } 1644.7(220.8) \\
p=0.83 \\
\text { Calculated mean difference: } 249.4 \text {, } \\
95 \% \mathrm{Cl},-338.4 \text { to } 837.2\end{array}$ \\
\hline & & & $\begin{array}{l}\text { Mean cost (SE) of health care } \\
\text { resources per patient, including } \\
\text { only drug-related hospital stays } \\
\text { at } 5 \text { months (CAD assumed) }\end{array}$ & $\begin{array}{l}\text { G1: } 1281.3(101.4) \\
\text { G2: } 1299.4(154.7) \\
p=0.45 \\
\text { Calculated mean difference: }-18.1 \text {, } \\
95 \% \mathrm{Cl},-386.7 \text { to } 350.5\end{array}$ \\
\hline $\begin{array}{l}\text { Triller and Hamilton, } 2007^{38} \\
\text { RCT/Medium }\end{array}$ & $\begin{array}{l}\text { G1: Visiting nurse association } \\
\text { home visit services plus } \\
\text { comprehensive pharmaceutical } \\
\text { care services } \\
\text { G2: Visiting nurse association } \\
\text { home visit services }\end{array}$ & $\begin{array}{l}\text { G1: NR } \\
\text { G2: NR }\end{array}$ & $\begin{array}{l}\text { Aggregate health system costs } \\
\text { Home care agency costs }\end{array}$ & $\begin{array}{l}\text { Values not reported, but results state that } \\
\text { costs did not significantly differ between } \\
\text { the two groups. }\end{array}$ \\
\hline $\begin{array}{l}\text { Bernsten et al., } 2001^{1,2} \\
\text { RCT/High }\end{array}$ & $\begin{array}{l}\text { G1: Structured community } \\
\text { pharmacy-based } \\
\text { pharmaceutical care program } \\
\text { G2: Usual community pharmacy } \\
\text { services }\end{array}$ & $\begin{array}{l}\text { Baseline } \\
\text { G1: } 867 \\
\text { G2: } 748 \\
\text { 6 months } \\
\text { G1: NR } \\
\text { G2: NR } \\
\text { 12 months } \\
\text { G1: NR } \\
\text { G2: NR } \\
\text { 18 months } \\
\text { G1: NR } \\
\text { G2: NR }\end{array}$ & $\begin{array}{l}\text { Mean total cost per patient } \\
\text { including (1) cost associated } \\
\text { with additional time spent by } \\
\text { pharmacists; (2) cost } \\
\text { associated with contacts with } \\
\text { GPs, specialists and nurses; and } \\
\text { (3) cost of hospitalizations and } \\
\text { drugs }\end{array}$ & $\begin{array}{l}\text { Cost data not pooled and analyzed for costs } \\
\text { because of differing health care systems } \\
\text { between countries. However, no significant } \\
\text { between-group differences in any country } \\
\text { (p=NS) }\end{array}$ \\
\hline $\begin{array}{l}\text { Fischer et al., } 2002^{11} \\
\text { NRCT/Medium }\end{array}$ & $\begin{array}{l}\text { G1: Pharmaceutical care } \\
\text { G2: Usual care }\end{array}$ & $\begin{array}{l}\text { G1: } 231 \\
\text { G2: } 444\end{array}$ & $\begin{array}{l}\text { Change in total charges (in USD) } \\
\text { for inpatient care, outpatient } \\
\text { care, and pharmacy }\end{array}$ & $\begin{array}{l}\text { G1: }-900 \\
\text { G2: }-2000 \\
\text { 95\% CI: NR } \\
\text { p: NS, no details reported } \\
\text { Calculated mean difference: } 1100 .\end{array}$ \\
\hline
\end{tabular}

Abbreviations: $\mathrm{CAD}=$ Canadian dollar; $\mathrm{CI}=$ confidence interval; $\mathrm{G}=$ group; $\mathrm{GP}=$ general practitioner; $\mathrm{NR}=$ not reported; $\mathrm{NS}=$ not significant; $\mathrm{RCT}=$ randomized controlled trial; $\mathrm{SE}=$ standard error; USD = US Dollar 
Table E34. Number of outpatient visits: Summary of results

\begin{tabular}{|c|c|c|c|c|}
\hline $\begin{array}{l}\text { Study } \\
\text { Design/Risk of Bias }\end{array}$ & Study Arms & N Analyzed & Outcome and Time Period & Results \\
\hline $\begin{array}{l}\text { Malone, 2000 } \\
\text { Ellis, 2000 } \\
\text { (interventions); } \\
\text { Malone, 2001 } 1^{22} \text { (detailed } \\
\text { QOL outcomes); } \\
\text { Ellis, 2000 }{ }^{23} \\
\text { RCT/Medium }\end{array}$ & $\begin{array}{l}\text { G1: Pharmaceutical care } \\
\text { G2: Usual care }\end{array}$ & $\begin{array}{l}\text { G1: } 523 \\
\text { G2: } 531\end{array}$ & $\begin{array}{l}\text { Mean change in number of clinic } \\
\text { visits (including visits with the } \\
\text { pharmacists in the intervention } \\
\text { arm) }\end{array}$ & $\begin{array}{l}\text { G1: }+4.8 \\
\text { G2: }+2.8 \\
\text { p: } 0.003\end{array}$ \\
\hline $\begin{array}{l}\text { Sellors et al., } 2003^{33} \\
\text { RCT/Medium }\end{array}$ & $\begin{array}{l}\text { G1: Pharmacist consultation } \\
\text { program } \\
\text { G2: Usual care }\end{array}$ & $\begin{array}{l}\text { G1: } 379 \\
\text { G2: } 409\end{array}$ & Number of clinic visits (SE) & $\begin{array}{l}\text { G1: } 0.3(0.15) \\
\text { G2: } 0.3(0.6) \\
\text { p: } 0.40\end{array}$ \\
\hline $\begin{array}{l}\text { Sidel, } 1990^{34} \\
\text { RCT/medium }\end{array}$ & $\begin{array}{l}\text { G1: received at least } 2 \\
\text { pharmacist visits involving } \\
\text { medication review, patient } \\
\text { specific education and } \\
\text { counseling; follow up patient } \\
\text { phone calls and contact of } \\
\text { physicians as needed } \\
\text { G2: only contacted for to } \\
\text { complete the survey. }\end{array}$ & $\begin{array}{l}\text { G1: } 92 \\
\text { G2: } 104\end{array}$ & $\begin{array}{l}\text { Change in number of ambulatory } \\
\text { visits over } 3 \text { months }\end{array}$ & $\begin{array}{l}\text { G1: }-1.2 \\
\text { G2: } 0.3 \\
\text { p: } 0.08\end{array}$ \\
\hline $\begin{array}{l}\text { Touchette et al., } 2012^{37} \\
\text { RCT/Medium }\end{array}$ & $\begin{array}{l}\text { G1: MTM basic (comprehensive } \\
\text { medication review and DRP } \\
\text { assessment) } \\
\text { G2: MTM enhanced (MTM plus } 2 \\
\text { page clinical summary } \\
\text { abstracted from patient's medical } \\
\text { chart). } \\
\text { G3: Usual care }\end{array}$ & $\begin{array}{l}\text { G1: } 183 \\
\text { G2: } 190 \\
\text { G3: } 183\end{array}$ & $\begin{array}{l}0-3 \text { months } \\
\text { G1: } 180 \\
\text { G2: } 190 \\
\text { G3: } 193 \\
\text { 3-6 months } \\
\text { G1: } 183 \\
\text { G2: } 190 \\
\text { G3: } 183\end{array}$ & $\begin{array}{l}0-3 \text { months } \\
\text { G1: } 2.6(2.2) \\
\text { G2: } 2.7(2.3) \\
\text { G3: } 2.6(2.2) \\
\text { G1 vs. G3: } \\
(p=0.646) \\
\text { G2 vs. G3: } \\
(p=0.816)\end{array}$ \\
\hline & & & & $\begin{array}{l}\text { 3-6 months } \\
\text { G1: } 2.2(2.1) \\
\text { G2: } 2.1(2.1) \\
\text { G3: } 2.2(2.2) \\
\text { G1 vs. G3: } \\
\text { (p=0.760) } \\
\text { G2 vs. G3: } \\
\text { (p=0.458) } \\
\end{array}$ \\
\hline
\end{tabular}


Table E34. Number of outpatient visits: Summary of results (continued)

\begin{tabular}{|c|c|c|c|c|}
\hline $\begin{array}{l}\text { Study } \\
\text { Design/Risk of Bias }\end{array}$ & Study Arms & N Analyzed & Outcome and Time Period & Results \\
\hline $\begin{array}{l}\text { Bernsten et al., } 2001^{1,2} \\
\text { RCT/High }\end{array}$ & $\begin{array}{l}\text { G1: Structured community } \\
\text { pharmacy-based pharmaceutical } \\
\text { care program } \\
\text { G2: Usual community pharmacy } \\
\text { services }\end{array}$ & $\begin{array}{l}\text { Baseline } \\
\text { G1: } 1290 \\
\text { G2: } 1164 \\
6 \text { months } \\
\text { G1: } 1024 \\
\text { G2: } 953 \\
12 \text { months } \\
\text { G1: } 863 \\
\text { G2: } 764 \\
18 \text { months } \\
\text { G1: } 704 \\
\text { G2: } 636\end{array}$ & $\begin{array}{l}\text { Mean number of contacts with } \\
\text { primary care providers, including } \\
\text { home visits and office } \\
\text { appointments (SD) }\end{array}$ & $\begin{array}{l}\text { Baseline } \\
\text { G1: } 4.8(8.4) \\
\text { G2: } 4.3(6.2) \\
\text { p: NS } \\
6 \text { months } \\
\text { G1: } 4.0(5.7) \\
\text { G2: } 3.6(4.6) \\
\text { p: NS } \\
12 \text { months } \\
\text { G1: } 4.0(7.0) \\
\text { G2: } 3.5(5.5) \\
\text { p: NS } \\
18 \text { months } \\
\text { G1: } 4.3(8.0) \\
\text { G2: } 3.2(4.0) \\
\text { p: NS }\end{array}$ \\
\hline $\begin{array}{l}\text { Krska et al., } 2001^{19} \\
\text { RCT/High }\end{array}$ & $\begin{array}{l}\text { G1: Pharmacist-led medication } \\
\text { review } \\
\text { G2: Usual care including } \\
\text { identification of pharmaceutical } \\
\text { care issues, but no plan }\end{array}$ & $\begin{array}{l}\text { G1: NR } \\
\text { G2: NR }\end{array}$ & $\begin{array}{l}\text { Hospital clinic attendance, use of } \\
\text { social services or contacts with } \\
\text { district nurses and health visitors } \\
\text { before and after the pharmacist } \\
\text { review }\end{array}$ & No differences, details NR \\
\hline $\begin{array}{l}\text { Fischer et al., } 2002^{11} \\
\text { NRCT/High }\end{array}$ & $\begin{array}{l}\text { G1: Pharmaceutical care } \\
\text { G2: Usual care }\end{array}$ & $\begin{array}{l}\text { G1: } 231 \\
\text { G2: } 444\end{array}$ & $\begin{array}{l}\text { Changes in number of clinic visits } \\
\text { over } 1 \text { year }\end{array}$ & $\begin{array}{l}\text { Intention-to-treat analysis } \\
\text { Adjusted between-group difference not } \\
\text { significant, details NR }\end{array}$ \\
\hline $\begin{array}{l}\text { Carter et al., } 1997^{4,5} \\
\text { Cohort/High }\end{array}$ & $\begin{array}{l}\text { G1: Pharmaceutical care } \\
\text { G2: Usual care }\end{array}$ & $\begin{array}{l}\text { G1: } 25 \\
\text { G2: } 26\end{array}$ & $\begin{array}{l}\text { Number of distinct dates of } \\
\text { service over } 6 \text { months }\end{array}$ & $\begin{array}{l}\mathrm{G} 1: 2.2(2.4) \\
\mathrm{G} 2: 1.0(1.0) \\
\mathrm{p}=0.07\end{array}$ \\
\hline $\begin{array}{l}\text { Chrischilles et al., } 2004^{7} \\
\text { Cohort/High }\end{array}$ & $\begin{array}{l}\text { G1: PCM-eligible patients who } \\
\text { received PCM services } \\
\text { G2: PCM-eligible patients who } \\
\text { did not receive PCM services }\end{array}$ & $\begin{array}{l}\text { G1: } 524 \\
\text { G2: } 1,687\end{array}$ & $\begin{array}{l}\text { No. of outpatient facility claims at } \\
12 \text { months }\end{array}$ & Results NR, $p=0.121$ \\
\hline
\end{tabular}

sufficient; $\mathrm{QOL}=$ quality of life; $\mathrm{RCT}=$ randomized controlled trial; $\mathrm{SD}=$ standard deviation; $\mathrm{SE}=$ standard error; vs. = versus 
Table E35. Costs of outpatient visits: Summary of results

\begin{tabular}{|c|c|c|c|c|}
\hline $\begin{array}{l}\text { Study } \\
\text { Design/Risk of Bias }\end{array}$ & Study Arms & N Analyzed & Outcome and Time Period & Results \\
\hline $\begin{array}{l}\text { Malone et al., } 2000^{20} ; \\
\text { Ellis et al., 2000 } \\
\text { (interventions); } \\
\text { Malone et al., } 2001^{22} \\
\text { (detailed QOL outcomes); } \\
\text { Ellis et al., 2000 } \\
\text { RCT/Medium }\end{array}$ & $\begin{array}{l}\text { G1: Pharmaceutical care } \\
\text { G2: Usual care }\end{array}$ & $\begin{array}{l}\text { G1: } 523 \\
\text { G2: } 531\end{array}$ & $\begin{array}{l}\text { Mean change in annual cost of } \\
\text { clinic visits in US } \$\end{array}$ & $\begin{array}{l}\text { G1: }+231 \\
\text { G2: }+333 \\
95 \% \mathrm{Cl}: \mathrm{NR} \\
\text { p: } 0.02\end{array}$ \\
\hline \multirow[t]{3}{*}{$\begin{array}{l}\text { Sellors et al., } 2003^{33} \\
\text { RCT/Medium }\end{array}$} & $\begin{array}{l}\text { G1: Pharmacist consultation } \\
\text { program } \\
\text { G2: Usual care }\end{array}$ & $\begin{array}{l}\text { G1: } 379 \\
\text { G2: } 409\end{array}$ & $\begin{array}{l}\text { Mean cost of physician visits in in } \\
\$ \text { (assumed CAD) (SE) at } 5 \\
\text { months }\end{array}$ & $\begin{array}{l}\text { G1: } 204.0(11.1) \\
\text { G2: } 198.3(10.4) \\
95 \% \mathrm{Cl} \text { (calculated for standardized difference } \\
\text { in means): }-0.11 \text { to } 0.12 \\
\mathrm{p} \text { (calculated for standardized difference in } \\
\text { means): } 0.71\end{array}$ \\
\hline & & & $\begin{array}{l}\text { Mean cost of clinic visits in in } \$ \\
\text { (assumed CAD) (SE) at } 5 \text { months }\end{array}$ & $\begin{array}{l}\mathrm{G} 1: 18.8(8.1) \\
\mathrm{G} 2: 20.9 \text { (5.0) } \\
95 \% \mathrm{Cl} \text { (calculated for standardized difference } \\
\text { in means): }-0.16 \text { to } 0.12 \\
\mathrm{p} \text { (calculated for standardized difference in } \\
\text { means): } 0.82\end{array}$ \\
\hline & & & $\begin{array}{l}\text { Mean cost of other health care } \\
\text { services/visits to health care } \\
\text { professionals in in } \$ \text { (assumed } \\
\text { CAD) (SE) at } 5 \text { months }\end{array}$ & $\begin{array}{l}\mathrm{G} 1: 288.30(40.02) \\
\mathrm{G} 2: 293.00(55.25) \\
95 \% \mathrm{Cl}(\mathrm{calculated} \text { for standardized difference } \\
\text { in means): }-0.145 \text { to } 0.135 \\
\mathrm{p} \text { (calculated for standardized difference in } \\
\text { means): } 0.97\end{array}$ \\
\hline $\begin{array}{l}\text { Chrischilles et al., } 2004^{7} \\
\text { Cohort/High }\end{array}$ & $\begin{array}{l}\text { G1: PCM-eligible patients who } \\
\text { received PCM services } \\
\text { G2: PCM-eligible patients who } \\
\text { did not receive PCM services }\end{array}$ & $\begin{array}{l}\text { G1: } 524 \\
\text { G2: } 1,687\end{array}$ & $\begin{array}{l}\text { Outpatient facility claims at } 12 \\
\text { months }\end{array}$ & $\begin{array}{l}\text { Results NR } \\
\text { p: } 0.107\end{array}$ \\
\hline \multirow[t]{2}{*}{$\begin{array}{l}\text { Carter et al., } 1997^{4,5} \\
\text { Cohort/High }\end{array}$} & $\begin{array}{l}\text { G1: Pharmaceutical care } \\
\text { G2: Usual care }\end{array}$ & $\begin{array}{l}\text { G1: } 25 \\
\text { G2: } 26\end{array}$ & $\begin{array}{l}\text { Hypertension-related charges in \$ } \\
(\mathrm{SD}) \text { at } 6 \text { months }\end{array}$ & $\begin{array}{l}\mathrm{G} 1: 122(124) \\
\mathrm{G} 2: 52(65) \\
p=0.03\end{array}$ \\
\hline & & & $\begin{array}{l}\text { Mean visit charges in } \$(S D) \text { at } 6 \\
\text { months }\end{array}$ & $\begin{array}{l}\mathrm{G} 1: 823(1,123) \\
\mathrm{G} 2: 336(246) \\
\mathrm{p}=0.02\end{array}$ \\
\hline
\end{tabular}

Abbreviations: $\mathrm{CAD}=$ Canadian dollars; $\mathrm{CI}=$ confidence interval $\mathrm{G}=$ group $\mathrm{NR}=$ not reported $\mathrm{PCM}=$ pharmaceutical case management; $\mathrm{QOL}=$ quality of life; $\mathrm{RCT}=$ randomized controlled trial; $\mathrm{SD}=$ standard deviation; $\mathrm{SE}=$ standard error; US = United States 
Table E36. Number of laboratory tests: Summary of results

\begin{tabular}{|c|c|c|c|c|}
\hline $\begin{array}{l}\text { Study } \\
\text { Design/Risk of Bias }\end{array}$ & Study Arms & N Analyzed & Outcome and Time Period & Results \\
\hline $\begin{array}{l}\text { Sellors et al., } 2003^{33} \\
\text { RCT/Medium }\end{array}$ & $\begin{array}{l}\text { G1: Pharmacist consultation } \\
\text { program } \\
\text { G2: Usual care }\end{array}$ & $\begin{array}{l}\text { G1: } 379 \\
\text { G2: } 409\end{array}$ & $\begin{array}{l}\text { Mean number of laboratory tests } \\
\text { and imaging procedures at } 5 \\
\text { months }\end{array}$ & $\begin{array}{l}\text { G1: } 8.7(0.6) \\
\mathrm{G} 2: 8.6(0.1) \\
95 \% \mathrm{Cl} \text { (calculated for standardized } \\
\text { difference in means): }-0.12 \text { to } 0.16 \\
\text { p (calculated for standardized difference in } \\
\text { means): } 0.791\end{array}$ \\
\hline $\begin{array}{l}\text { Malone et al., } 2000^{20} ; \\
\text { Ellis et al., } 2000^{21} \\
\text { (interventions); } \\
\text { Malone et al., } 2001^{22} \\
\text { (detailed QOL outcomes); } \\
\text { Ellis et al., } 2000^{23} \\
\text { RCT/Medium }\end{array}$ & $\begin{array}{l}\text { G1: Pharmaceutical care } \\
\text { G2: Usual care }\end{array}$ & $\begin{array}{l}\mathrm{G} 1: 523 \\
\mathrm{G} 2: 531\end{array}$ & $\begin{array}{l}\text { Mean change in annual number o } \\
\text { laboratory tests }\end{array}$ & $\begin{array}{l}\mathrm{G} 1:+3.1 \\
\mathrm{G} 2:+4.7 \\
95 \% \mathrm{Cl}: \mathrm{NR} \\
\text { p: } 0.001\end{array}$ \\
\hline
\end{tabular}


Table E37. Costs of laboratory tests: Summary of results

\begin{tabular}{|c|c|c|c|c|}
\hline $\begin{array}{l}\text { Study } \\
\text { Design/Risk of Bias }\end{array}$ & Study Arms & N Analyzed & Outcome and Time Period & Results \\
\hline $\begin{array}{l}\text { Sellors et al., } 2003^{33} \\
\text { RCT/Medium }\end{array}$ & $\begin{array}{l}\text { G1: Pharmacist consultation } \\
\text { program } \\
\text { G2: Usual care }\end{array}$ & $\begin{array}{l}\text { G1: } 379 \\
\text { G2: } 409\end{array}$ & $\begin{array}{l}\text { Mean cost of all lab and imaging } \\
\text { procedures at } 5 \text { months } \$ \\
\text { (assumed CAD) (SE) }\end{array}$ & $\begin{array}{l}\mathrm{G} 1: 249.3(20.8) \\
\mathrm{G} 2: 243.1(17.2) \\
95 \% \mathrm{Cl} \text { (calculated for standardized } \\
\text { difference in means): }-0.12 \text { to } 0.16 \\
\text { p (calculated for standardized difference in } \\
\text { means): } 0.816\end{array}$ \\
\hline $\begin{array}{l}\text { Malone et al., } 2000^{20} ; \\
\text { Ellis et al., } 2000^{21} \\
\text { (interventions); } \\
\text { Malone et al., } 2001^{22} \\
\text { (detailed QOL outcomes); } \\
\text { Ellis et al., 2000 } \\
\text { RCT/Medium }\end{array}$ & $\begin{array}{l}\text { G1: Pharmaceutical care } \\
\text { G2: Usual care }\end{array}$ & $\begin{array}{l}\text { G1: } 523 \\
\text { G2: } 531\end{array}$ & $\begin{array}{l}\text { Mean change in annual costs for } \\
\text { laboratory tests in US } \$\end{array}$ & $\begin{array}{l}\mathrm{G} 1:+\$ 43 \\
\mathrm{G} 2:+\$ 76 \\
95 \% \mathrm{Cl}, \mathrm{NR} \\
\mathrm{p}: 0.05\end{array}$ \\
\hline
\end{tabular}

Abbreviations: $\mathrm{CAD}=$ Canadian dollar; $\mathrm{CI}=$ confidence interval; $\mathrm{G}=$ group $\mathrm{NR}=$ not reported; $\mathrm{QOL}=$ quality of life; $\mathrm{RCT}=$ randomized controlled trial; $\mathrm{SE}=$ standard deviation; US = United States 


\section{Table E38. ED visits: Summary of results}

\begin{tabular}{|c|c|c|c|c|}
\hline $\begin{array}{l}\text { Study } \\
\text { Design/Risk of Bias }\end{array}$ & Study Arms & N Analyzed & Outcome and Time Period & Results \\
\hline $\begin{array}{l}\text { Touchette et al., } 2012^{37} \\
\text { RCT/Medium }\end{array}$ & $\begin{array}{l}\text { G1: MTM basic (comprehensive } \\
\text { medication review and DRP } \\
\text { assessment) } \\
\text { G2: MTM enhanced (MTM plus } 2 \\
\text { page clinical summary } \\
\text { abstracted from patient's medical } \\
\text { chart). } \\
\text { G3: Usual care }\end{array}$ & $\begin{array}{l}\text { G1: } 211 \\
\text { G2: } 218 \\
\text { G3: } 208\end{array}$ & $\begin{array}{l}\text { Mean number of ED visits per } \\
\text { participant }\end{array}$ & $\begin{array}{l}0 \text { to } 3 \text { months } \\
\text { G1: } 0.3(0.6) \\
\text { G2: } 0.2(0.6) \\
\text { G3: } 0.2(0.5) \\
\text { G1 vs. G3: } \\
\text { (p=0.735) } \\
\text { G2 vs. G3: } \\
\text { ( } p=0.963) \\
3 \text { to } 6 \text { months } \\
\text { G1: } 0.2(0.5) \\
\text { G2: } 0.2(0.6) \\
\text { G3: } 0.4(0.8) \\
G 1 \text { vs. G3: } \\
\text { (p=0.077) } \\
\text { G2 vs. G3: } \\
(p=0.057)\end{array}$ \\
\hline $\begin{array}{l}\text { Taylor, Byrd, and Krueger, } \\
2003^{36} \\
\text { RCT/High }\end{array}$ & $\begin{array}{l}\text { G1: Pharmaceutical care group } \\
\text { G2: Standard care }\end{array}$ & $\begin{array}{l}\text { G1: } 33 \\
\text { G2: } 36\end{array}$ & $\begin{array}{l}\text { Change in no, of ED visits from } 12 \\
\text { months before baseline through } \\
12 \text { months after }\end{array}$ & $\begin{array}{l}\text { G1: }-12 \\
\text { G2: } 0 \\
p=0.044\end{array}$ \\
\hline $\begin{array}{l}\text { Welch et al., } 2009^{41} \\
\text { Retrospective cohort } \\
\text { study/Medium }\end{array}$ & $\begin{array}{l}\text { G1: MTM program provided to } \\
\text { home-based beneficiaries } \\
\text { G2: No-MTM control group (opt- } \\
\text { out) }\end{array}$ & $\begin{array}{l}\text { G1: } 459 \\
\text { G2: } 336\end{array}$ & $\begin{array}{l}\text { Adjusted OR of ED visit from } 6 \\
\text { month before MTM through } 6 \\
\text { months after (adjusted for age, } \\
\text { sex, chronic disease score, } \\
\text { specific baseline utilization) (95\% } \\
\text { Cl) }\end{array}$ & Adjusted OR: 0.9 (0.6 to 1.3) \\
\hline $\begin{array}{l}\text { Sellors et al., } 2003^{33} \\
\text { RCT/Medium }\end{array}$ & $\begin{array}{l}\text { G1: Pharmacist consultation } \\
\text { program } \\
\text { G2: Usual care }\end{array}$ & $\begin{array}{l}\text { G1: } 379 \\
\text { G2: } 409\end{array}$ & $\begin{array}{l}\text { Mean number of ED/urgent care } \\
\text { visits and ambulance use (SE) at } \\
5 \text { months }\end{array}$ & $\begin{array}{l}\text { G1: } 0.2(0.03) \\
\text { G2: } 0.2(0.03)\end{array}$ \\
\hline
\end{tabular}

Abbreviations: $\mathrm{CI}=$ confidence interval; DRP = drug related problems; $\mathrm{ED}=$ emergency department; $\mathrm{G}=$ group; $\mathrm{MTM}=\mathrm{Medication}$ Therapy Management; OR = odds ratio; RCT $=$ randomized controlled trial; $\mathrm{SE}=$ standard error; vs. = versus. 
Table E39. Costs of emergency department visits: Summary of results

\begin{tabular}{|c|c|c|c|c|}
\hline $\begin{array}{l}\text { Study } \\
\text { Design/Risk of Bias }\end{array}$ & Study Arms & N Analyzed & Outcome and Time Period & Results \\
\hline $\begin{array}{l}\text { Sellors et al., } 2003^{33} \\
\text { RCT/Medium }\end{array}$ & $\begin{array}{l}\text { G1: Pharmacist consultation } \\
\text { program } \\
\text { G2: Usual care }\end{array}$ & $\begin{array}{l}\text { G1: } 379 \\
\text { G2: } 409\end{array}$ & $\begin{array}{l}\text { Mean cost of ED/urgent care } \\
\text { visits and ambulance use at } 5 \\
\text { months in } \$ \text { (assumed CAD) (SE) }\end{array}$ & $\begin{array}{l}\text { G1: } 0.2(0.03) \\
\mathrm{G} 2: 0.2(0.03) \\
95 \% \mathrm{Cl} \text { (calculated for standardized } \\
\text { difference in means): }-0.19 \text { to } 0.10 \\
\text { p (calculated for standardized difference in } \\
\text { means): } 0.53\end{array}$ \\
\hline $\begin{array}{l}\text { Chrischilles et al., } 2004^{7} \\
\text { Cohort/High }\end{array}$ & $\begin{array}{l}\text { G1: PCM-eligible patients who } \\
\text { received PCM services } \\
\text { G2: PCM-eligible patients who } \\
\text { did not receive PCM services }\end{array}$ & $\begin{array}{l}\text { G1: } 524 \\
\text { G2: } 1,687\end{array}$ & $\begin{array}{l}\text { Charges of ED claims at } 12 \\
\text { months }\end{array}$ & $\begin{array}{l}\text { Results NR } \\
\text { p: } 0.513\end{array}$ \\
\hline
\end{tabular}

Abbreviations: CAD: Canadian dollar; $\mathrm{CI}=$ confidence interval; $\mathrm{ED}=$ Emergency department; $\mathrm{G}=$ group; $\mathrm{NR}=$ not reported; $\mathrm{PCM}=$ pharmaceutical case management; $\mathrm{RCT}=$

randomized controlled trials; $\mathrm{SE}=$ standard error 
Table E40. Number of hospitalizations: Summary of results

\begin{tabular}{|c|c|c|c|c|}
\hline $\begin{array}{l}\text { Study } \\
\text { Design/Risk of Bias }\end{array}$ & Study Arms & N Analyzed & Outcome and Time Period & Results \\
\hline $\begin{array}{l}\text { Malone et al., } 2000^{20} ; \\
\text { Ellis et al., 2000 } \\
\text { (interventions); } \\
\text { Malone et al., } 2001^{22} \\
\text { (detailed QOL outcomes); } \\
\text { Ellis et al., 2000 } \\
\text { RCT/Medium }\end{array}$ & $\begin{array}{l}\text { G1: Pharmaceutical care } \\
\text { G2: Usual care }\end{array}$ & $\begin{array}{l}\text { G1: } 523 \\
\text { G2: } 531\end{array}$ & $\begin{array}{l}\text { Mean change in number of } \\
\text { hospitalizations }\end{array}$ & $\begin{array}{l}\text { G1: }+0.1 \\
\text { G2: }+0.2 \\
\text { p: } 0.29\end{array}$ \\
\hline \multirow[t]{2}{*}{$\begin{array}{l}\text { Sellors et al., } 2003^{33} \\
\text { RCT/Medium }\end{array}$} & $\begin{array}{l}\text { G1: Pharmacist consultation } \\
\text { program } \\
\text { G2: Usual care }\end{array}$ & $\begin{array}{l}\text { G1: } 379 \\
\text { G2: } 409\end{array}$ & $\begin{array}{l}\text { Mean all-cause hospitalizations } \\
\text { (SE) }\end{array}$ & $\begin{array}{l}\text { G1: } 0.1(0.02) \\
\text { G2: } 0.1(0.02) \\
\text { p: } 0.77\end{array}$ \\
\hline & & & $\begin{array}{l}\text { Mean drug-related } \\
\text { hospitalizations (SE) }\end{array}$ & $\begin{array}{l}\text { G1: } 0.04(0.01) \\
\text { G2: } 0.04(0.01) \\
\text { p: } 0.08\end{array}$ \\
\hline $\begin{array}{l}\text { Touchette et al., } 2012^{37} \\
\text { RCT/Medium }\end{array}$ & $\begin{array}{l}\text { G1: MTM basic (comprehensive } \\
\text { medication review and DRP } \\
\text { assessment) } \\
\text { G2: MTM enhanced (MTM plus } \\
2 \text { page clinical summary } \\
\text { abstracted from patient's medical } \\
\text { chart). } \\
\text { G3: Usual care }\end{array}$ & $\begin{array}{l}\text { Time One } \\
\text { G1: } 180 \\
\text { G2: } 190 \\
\text { G3: } 193\end{array}$ & $\begin{array}{l}\text { Percent of participants with at } \\
\text { least one hospital visit }\end{array}$ & $\begin{array}{l}\text { Time One } \\
\text { G1: } 13.9 \\
\text { G2: } 7.9 \\
\text { G3: } 10.4 \\
\text { G1 vs. G3: } 1.6(p=0.350) \\
\text { G2 vs. } G 3: 0.6(p=0.370) \\
\text { G2 vs. } G 1: 0.4(p=0.080)\end{array}$ \\
\hline & & $\begin{array}{l}\text { Time Two } \\
\text { G1: } 183 \\
\text { G2: } 190 \\
\text { G3: } 183\end{array}$ & & $\begin{array}{l}\text { Time Two } \\
\text { G1: } 17.6 \\
\text { G2: } 12.1 \\
\text { G3: } 9.3\end{array}$ \\
\hline & & & & $\begin{array}{l}\text { G1 vs. G3: } 2.6(p=0.049) \\
\text { G2 vs. } G 3: 1.4(p=0.484) \\
\text { G2 vs. } G 1: 0.3(p=0.214)\end{array}$ \\
\hline
\end{tabular}


Table E40. Number of hospitalizations: Summary of results (continued)

\begin{tabular}{|c|c|c|c|c|}
\hline $\begin{array}{l}\text { Study } \\
\text { Design/Risk of Bias }\end{array}$ & Study Arms & N Analyzed & Outcome and Time Period & Results \\
\hline $\begin{array}{l}\text { Touchette et al., } 2012^{37} \\
\text { RCT/Medium } \\
\text { (continued) }\end{array}$ & & & $\begin{array}{l}\text { Mean number of hospital visits } \\
\text { per participant }\end{array}$ & $\begin{array}{l}\text { Time One } \\
\text { G1: } 0.2(0.5) \\
\text { G2: } 0.1(0.4) \\
\text { G3: } 0.1(0.4) \\
\text { G1 vs. } G 3:(p=0.265) \\
\text { G2 vs. } G 3:(p=0.619) \\
\text { G2 vs. } G 1:(p=0.109) \\
\text { Time Two } \\
\text { G1: } 0.2(0.5) \\
\text { G2: } 0.1(0.4) \\
\text { G3: } 0.1(0.4) \\
\text { G1 vs. } G 3:(p=0.056) \\
\text { G2 vs. } G 3:(p=0.547) \\
\text { G2 vs. } G 1:(p=0.174)\end{array}$ \\
\hline $\begin{array}{l}\text { Triller and Hamilton, } 2007^{38} \\
\text { RCT/Medium }\end{array}$ & $\begin{array}{l}\text { G1: Visiting nurse association } \\
\text { home visit services plus } \\
\text { comprehensive pharmaceutical } \\
\text { care services } \\
\text { G2: Visiting nurse association } \\
\text { home visit services }\end{array}$ & $\begin{array}{l}\text { G1: NR } \\
\text { G2: NR }\end{array}$ & $\begin{array}{l}\text { Percent with any hospitalization, } \\
\text { all causes } \\
\text { Percent with any hospitalization, } \\
\text { heart-failure related }\end{array}$ & $\begin{array}{l}\text { G1: } 55 \\
\text { G2: } 58 \\
\text { RR: } 0.9 \\
95 \% \text { Cl: NR } \\
\text { p: } 0.63 \\
\text { G1: } 42 \\
\text { G2: } 55 \\
\text { RR: } 0.8 \\
\text { 95\% Cl: NR } \\
\text { p: } 0.26\end{array}$ \\
\hline
\end{tabular}


Table E40. Number of hospitalizations: Summary of results (continued)

\begin{tabular}{|c|c|c|c|c|}
\hline $\begin{array}{l}\text { Study } \\
\text { Design/Risk of Bias }\end{array}$ & Study Arms & N Analyzed & Outcome and Time Period & Results \\
\hline $\begin{array}{l}\text { Bernsten et al., } 2001^{1,2} \\
\text { RCT/High }\end{array}$ & $\begin{array}{l}\text { G1: Structured community } \\
\text { pharmacy-based pharmaceutical } \\
\text { care program } \\
\text { G2: Usual community pharmacy } \\
\text { services }\end{array}$ & $\begin{array}{l}\text { Baseline } \\
\text { G1: } 867 \\
\text { G2: } 748 \\
\text { 6 months } \\
\text { G1: NR } \\
\text { G2: NR } \\
\text { 12 months } \\
\text { G1: NR } \\
\text { G2: NR } \\
\text { 18 months } \\
\text { G1: NR } \\
\text { G2: NR }\end{array}$ & $\begin{array}{l}\text { Percent with } \geq 1 \text { hospitalization in } \\
\text { the prior } 18 \text { months }\end{array}$ & $\begin{array}{l}\text { Pooled sample } \\
\text { Baseline (during } 18 \text { months prior to study) } \\
\text { Overall: NR } \\
\text { G1: } 41.7 \\
\text { G2: } 41.3 \\
\text { p=NS } \\
18 \text { months } \\
\text { Overall: NR } \\
\text { G1: } 35.6 \\
\text { G2: } 40.4 \\
p=N S\end{array}$ \\
\hline $\begin{array}{l}\text { Pai et al., } 2009^{27} \text {; Pai et al., } \\
2009^{28} \\
\text { RCT/High }\end{array}$ & $\begin{array}{l}\text { G1: Pharmaceutical care } \\
\text { G2: Usual care }\end{array}$ & $\begin{array}{l}\text { G1: NR } \\
\text { G2: NR }\end{array}$ & All-cause hospitalizations & $\begin{array}{l}\text { G1: } 1.8(2.4) \\
\text { G2: } 3.1(3.0) \\
p: 0.02\end{array}$ \\
\hline & & & $\begin{array}{l}\text { Cumulative hospitalized time } \\
\text { (days) }\end{array}$ & $\begin{array}{l}\text { G1: } 9.7(14.7) \\
\text { G2: } 15.5(16.3) \\
\text { p: } 0.06\end{array}$ \\
\hline $\begin{array}{l}\text { Roughead et al., } 2009^{32} \\
\text { Cohort/Medium }\end{array}$ & $\begin{array}{l}\text { G1: Collaborative home-based } \\
\text { medication review } \\
\text { G2: No medication review } \\
\text { received }\end{array}$ & $\begin{array}{l}\text { G1: } 273 \\
\text { G2: } 5444\end{array}$ & $\begin{array}{l}\text { Rate of hospitalization for } \mathrm{HF} \text { at } \\
\text { any time during study }\end{array}$ & $\begin{array}{l}\text { Adjusted HR }(95 \% \mathrm{Cl}): 0.6 \text { (0.4 to } 0.8) \\
\text { p: NR } \\
\text { NOTE: Model adjusted for age, sex, } \\
\text { comorbidity, SES, season, region of } \\
\text { residence, and Ns of prescriptions, } \\
\text { prescribers, pharmacies, changes in } \\
\text { medications, hospitalizations, occupational } \\
\text { therapy visits, and speech therapy visits }\end{array}$ \\
\hline $\begin{array}{l}\text { Welch et al., } 2009^{41} \\
\text { Cohort study/Medium }\end{array}$ & $\begin{array}{l}\text { G1: MTM program provided to } \\
\text { home-based beneficiaries } \\
\text { G2: No-MTM control group } \\
\text { (voluntary opt-out) }\end{array}$ & $\begin{array}{l}\text { G1: } 459 \\
\text { G2: } 336\end{array}$ & $\begin{array}{l}\text { Adjusted OR of hospitalization } \\
\text { from } 6 \text { month before MTM } \\
\text { through } 6 \text { months after (adjusted } \\
\text { for age, sex, chronic disease } \\
\text { score, specific baseline utilization) } \\
(95 \% \mathrm{Cl})\end{array}$ & $\begin{array}{l}\text { Adjusted OR }(95 \% \mathrm{Cl}): 1.4 \text { (1.1 to } 2.0) \\
\text { NOTE: Model adjusted for age, sex, } \\
\text { Chronic Disease Score, specific baseline } \\
\text { utilization }\end{array}$ \\
\hline
\end{tabular}

Abbreviations: $\mathrm{CI}=$ confidence interval; $\mathrm{DRP}=$ drug related problem; $\mathrm{G}=$ group; $\mathrm{HR}=$ heart failure; $\mathrm{MTM}=$ Medication Therapy Management; $\mathrm{NR}=$ not reported; NS = not significant; $\mathrm{OR}=$ odds ratio; $\mathrm{QOL}=$ quality of life; $\mathrm{RCT}=$ randomized controlled trial; $\mathrm{SE}=$ standard error; vs. $=$ versus 
Table E41. Costs of Hospitalization: summary of results

\begin{tabular}{|c|c|c|c|c|}
\hline $\begin{array}{l}\text { Study } \\
\text { Design/Risk of Bias }\end{array}$ & Study Arms & N Analyzed & Outcome and Time Period & Results \\
\hline $\begin{array}{l}\text { Sellors et al., } 2003^{33} \\
\text { RCT/Medium }\end{array}$ & $\begin{array}{l}\text { G1: Pharmacist consultation } \\
\text { program } \\
\text { G2: Usual care }\end{array}$ & $\begin{array}{l}\text { G1: } 379 \\
\text { G2: } 409\end{array}$ & $\begin{array}{l}\text { Mean cost of all admissions to } \\
\text { hospital \$ (assumed CAD) (SE) }\end{array}$ & $\begin{array}{l}\text { G1: } 753.7(183.1) \\
\mathrm{G} 2: 594.9 \text { (135.2) } \\
95 \% \mathrm{Cl} \text { (calculated for standardized } \\
\text { difference in means), }-0.09 \text { to } 0.20 \\
\mathrm{p} \text { (calculated for standardized difference in } \\
\text { means): } 0.479\end{array}$ \\
\hline $\begin{array}{l}\text { Malone, } 2000^{20} ; \\
\text { Ellis, } 2000^{21} \\
\text { (interventions); } \\
\text { Malone, } 2001^{22} \text { (detailed } \\
\text { QOL outcomes); } \\
\text { Ellis, } 2000^{23} \\
\text { RCT/Medium } \\
\end{array}$ & $\begin{array}{l}\text { G1: Pharmaceutical care } \\
\text { G2: Usual care }\end{array}$ & $\begin{array}{l}\text { G1: } 523 \\
\text { G2: } 531\end{array}$ & $\begin{array}{l}\text { Mean change in annual } \\
\text { hospitalization costs in US \$ }\end{array}$ & $\begin{array}{l}\mathrm{G} 1:+542 \\
\mathrm{G} 2:+763 \\
\text { Variance not reported } \\
95 \% \mathrm{Cl}, \mathrm{NR} \\
\text { p: } 0.21\end{array}$ \\
\hline $\begin{array}{l}\text { Chrischilles et al., } 2004^{7} \\
\text { Cohort/High }\end{array}$ & $\begin{array}{l}\text { G1: PCM-eligible patients who } \\
\text { received PCM services } \\
\text { G2: PCM-eligible patients who } \\
\text { did not receive PCM services }\end{array}$ & $\begin{array}{l}\text { G1: } 524 \\
\text { G2: } 1,687\end{array}$ & Charges of inpatient claims & $\begin{array}{l}\text { Results NR } \\
\text { p: } 0.937\end{array}$ \\
\hline
\end{tabular}

Abbreviations: $\mathrm{CAD}=$ Canadian dollar; $\mathrm{CI}=$ confidence interval; $\mathrm{G}=$ group; $\mathrm{NR}=$ not reported; $\mathrm{PCM}=$ pharmaceutical case management; $\mathrm{QOL}=$ quality of life; $\mathrm{RCT}=$

L randomized controlled trial; $\mathrm{SE}=$ standard error; US = United States; RCT = randomized controlled trial. 
Table E42. Applicability

\begin{tabular}{|c|c|c|c|c|c|}
\hline $\begin{array}{l}\text { Author, } \\
\text { Year } \\
\text { Trial name }\end{array}$ & $\begin{array}{l}\text { Interventions and Comparator } \\
\text { Descriptions }\end{array}$ & $\begin{array}{l}\text { Study Population broadly } \\
\text { applicable? (e.g., not selected } \\
\text { using narrow eligibility criteria, } \\
\text { similarity in demographics } \\
\text { between study population and } \\
\text { community patients) }\end{array}$ & $\begin{array}{l}\text { Intervention broadly } \\
\text { applicable? (e.g., } \\
\text { design of } \\
\text { interventions } \\
\text { reflected in current } \\
\text { practice) }\end{array}$ & $\begin{array}{l}\text { Comparator(s) } \\
\text { broadly applicable? } \\
\text { (e.g., alternative } \\
\text { therapy or usual care } \\
\text { reflective of current } \\
\text { practice) }\end{array}$ & $\begin{array}{l}\text { Outcomes broadly } \\
\text { applicable? (e.g., not } \\
\text { limited to short-term, } \\
\text { surrogate, or } \\
\text { composite outcomes) }\end{array}$ \\
\hline $\begin{array}{l}\text { Bernsten et } \\
\text { al., } 2001^{1} \text {; } \\
\text { Sturgess et } \\
\text { al., } 2003^{2}\end{array}$ & $\begin{array}{l}\text { G1: Structured community } \\
\text { pharmacy-based pharmaceutical } \\
\text { care program } \\
\text { G2: Normal pharmaceutical Usual } \\
\text { community pharmacy services }\end{array}$ & Yes & Yes & Yes & Yes \\
\hline $\begin{array}{l}\text { Blennerhass } \\
\text { ett et al., } \\
2007^{3}\end{array}$ & $\begin{array}{l}\text { Implementation of a Home } \\
\text { Medication Review (HMR) into a } \\
\text { chronic heart failure collaborative } \\
\text { care model. HMRs were conducted } \\
\text { by accredited pharmacists. } \\
\text { G2: No HMR }\end{array}$ & Yes & $\begin{array}{l}\text { No } \\
\text { Appears to be very } \\
\text { specific to the Heartlink } \\
\text { program in NSW; } \\
\text { involves developing and } \\
\text { maintaining } \\
\text { communication } \\
\text { pathways between } \\
\text { hospital and community } \\
\text { pharmacy which would } \\
\text { require more time and } \\
\text { investment. }\end{array}$ & Yes & $\begin{array}{l}\text { Unclear or NR } \\
\text { Appears to be very } \\
\text { specific to the population } \\
\text { in NSW and the } \\
\text { Heartlink program } \\
\text { developed there. Unclear } \\
\text { about how widely } \\
\text { applicable this would be. }\end{array}$ \\
\hline $\begin{array}{l}\text { Carter et al., } \\
1997^{4}, \\
\text { Barnette et } \\
\text { al., } 1996^{5}\end{array}$ & $\begin{array}{l}\text { G1: Pharmaceutical care provided } \\
\text { by pharmacists within an } \\
\text { interdisciplinary practice model. } \\
\text { Patient education (lifestyle, risk } \\
\text { factor modifications, and drug } \\
\text { therapy) was standardized. } \\
\text { G2: Usual care }\end{array}$ & $\begin{array}{l}\text { No } \\
\text { Rural population }\end{array}$ & Yes & Yes & Yes \\
\hline
\end{tabular}


Table E42. Applicability (continued)

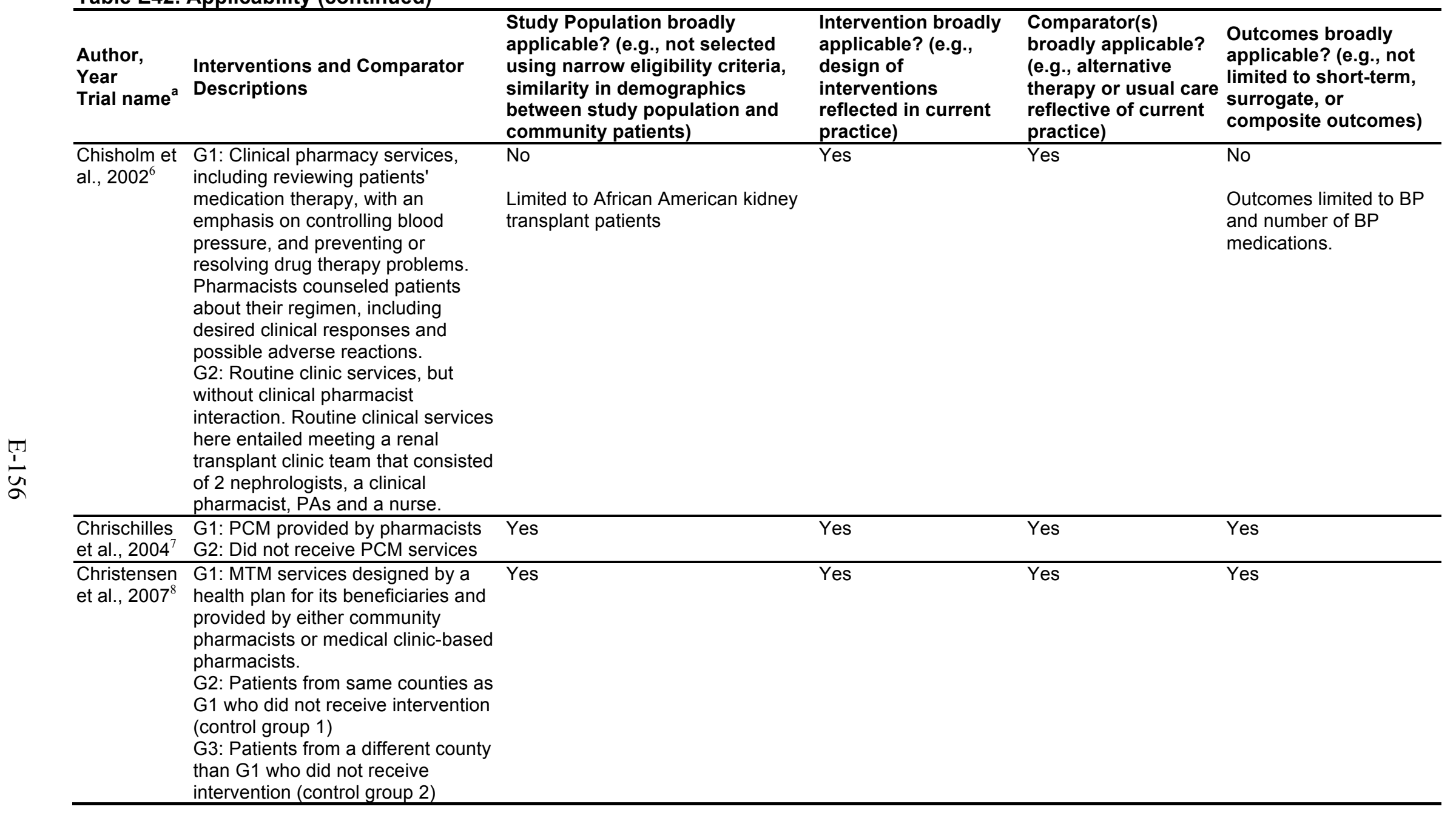


Table E42. Applicability (continued)

\begin{tabular}{|c|c|c|c|c|c|}
\hline $\begin{array}{l}\text { Author, } \\
\text { Year } \\
\text { Trial name }\end{array}$ & $\begin{array}{l}\text { Interventions and Comparator } \\
\text { Descriptions }\end{array}$ & $\begin{array}{l}\text { Study Population broadly } \\
\text { applicable? (e.g., not selected } \\
\text { using narrow eligibility criteria, } \\
\text { similarity in demographics } \\
\text { between study population and } \\
\text { community patients) }\end{array}$ & $\begin{array}{l}\text { Intervention broadly } \\
\text { applicable? (e.g., } \\
\text { design of } \\
\text { interventions } \\
\text { reflected in current } \\
\text { practice) }\end{array}$ & $\begin{array}{l}\text { Comparator(s) } \\
\text { broadly applicable? } \\
\text { (e.g., alternative } \\
\text { therapy or usual care } \\
\text { reflective of current } \\
\text { practice) }\end{array}$ & $\begin{array}{l}\text { Outcomes broadly } \\
\text { applicable? (e.g., not } \\
\text { limited to short-term, } \\
\text { surrogate, or } \\
\text { composite outcomes) }\end{array}$ \\
\hline $\begin{array}{l}\text { Clifford et } \\
\text { al., } 2002^{9}\end{array}$ & $\begin{array}{l}\text { G1: Pharmaceutical care provided } \\
\text { by a clinical pharmacist, which } \\
\text { included a comprehensive review } \\
\text { relating to pharmacotherapy and } \\
\text { diabetes, use of proprietary and } \\
\text { non-proprietary medications, such } \\
\text { as complementary medicines, and } \\
\text { identification of drug therapy } \\
\text { problems. } \\
\text { G2: Standard outpatient care for } \\
\text { diabetes }\end{array}$ & Yes & Yes & Yes & Yes \\
\hline $\begin{array}{l}\text { Fischer et } \\
\text { al., } 2000^{10}\end{array}$ & $\begin{array}{l}\text { G1: Pharmaceutical care based on } \\
\text { the Encara Practice System } \\
\text { provided by onsite health } \\
\text { maintenance organization staff } \\
\text { pharmacists. } \\
\text { G2: Standard Community Pharmacy } \\
\text { Practice } \\
\text { G3: A set of refusers surveyed and } \\
\text { included in some analyses among } \\
\text { those who were at eligible clinics but } \\
\text { initially declined to participate. }\end{array}$ & Yes & Yes & Yes & $\begin{array}{l}\text { No } \\
\text { The outcomes are very } \\
\text { intermediate (receipt of } \\
\text { information, use of } \\
\text { reminders to take } \\
\text { medication, and } \\
\text { awareness of side } \\
\text { effects) }\end{array}$ \\
\hline $\begin{array}{l}\text { Fischer et } \\
\text { al., } 2002^{11}\end{array}$ & $\begin{array}{l}\text { Pharmaceutical care based on the } \\
\text { Encara Practice System provided by } \\
\text { pharmacists. Communication of } \\
\text { pharmacist with the patient's } \\
\text { physician about drug therapy } \\
\text { problems identified by the } \\
\text { pharmacist. } \\
\text { G2: Usual care with no additional } \\
\text { interventions. }\end{array}$ & Yes & $\begin{array}{l}\text { Unclear or NR } \\
\text { Pharmacies } \\
\text { volunteered to } \\
\text { participate in the } \\
\text { intervention group. Not } \\
\text { clear how } \\
\text { representative they are } \\
\text { of community } \\
\text { pharmacies in general. }\end{array}$ & Yes & Yes \\
\hline
\end{tabular}


Table E42. Applicability (continued)

\begin{tabular}{|c|c|c|c|c|c|}
\hline $\begin{array}{l}\text { Author, } \\
\text { Year } \\
\text { Trial name }\end{array}$ & $\begin{array}{l}\text { Interventions and Comparator } \\
\text { Descriptions }\end{array}$ & $\begin{array}{l}\text { Study Population broadly } \\
\text { applicable? (e.g., not selected } \\
\text { using narrow eligibility criteria, } \\
\text { similarity in demographics } \\
\text { between study population and } \\
\text { community patients) }\end{array}$ & $\begin{array}{l}\text { Intervention broadly } \\
\text { applicable? (e.g., } \\
\text { design of } \\
\text { interventions } \\
\text { reflected in current } \\
\text { practice) }\end{array}$ & $\begin{array}{l}\text { Comparator(s) } \\
\text { broadly applicable? } \\
\text { (e.g., alternative } \\
\text { therapy or usual care } \\
\text { reflective of current } \\
\text { practice) }\end{array}$ & $\begin{array}{l}\text { Outcomes broadly } \\
\text { applicable? (e.g., not } \\
\text { limited to short-term, } \\
\text { surrogate, or } \\
\text { composite outcomes) }\end{array}$ \\
\hline $\begin{array}{l}\text { Fox et al., } \\
2009^{12}\end{array}$ & $\begin{array}{l}\text { G1: Florida Health Care Plans MTM } \\
\text { program, consisting of a medication } \\
\text { therapy review and evaluation by a } \\
\text { clinical pharmacist that was } \\
\text { documented and sent to the } \\
\text { patient's physician through health } \\
\text { plan review } \\
\text { G2: Opt-out from MTM program }\end{array}$ & Yes & Yes & Yes & Yes \\
\hline $\begin{array}{l}\text { Gattis et al., } \\
1999^{13}\end{array}$ & $\begin{array}{l}\text { G1: Clinical pharmacy services, } \\
\text { including an assessment of } \\
\text { prescribed regimen, compliance, } \\
\text { and adverse effects, and symptoms } \\
\text { and response to therapy. Providing } \\
\text { patient education about the purpose } \\
\text { of each drug and reinforcing } \\
\text { adherence. Detailed written } \\
\text { information was also provided to } \\
\text { patients. } \\
\text { G2: Usual medical care }\end{array}$ & $\begin{array}{l}\text { No } \\
\text { Study population limited to patients } \\
\text { with moderate to severe heart } \\
\text { failure. }\end{array}$ & Yes & Yes & Yes \\
\hline $\begin{array}{l}\text { Hanlon et } \\
\text { al., } 1996^{14}\end{array}$ & $\begin{array}{l}\text { G1: Pharmaceutical care provided } \\
\text { by a clinical pharmacist } \\
\text { G2: Usual care in the General } \\
\text { Medicine Clinic }\end{array}$ & $\begin{array}{l}\text { No } \\
\text { All male VA patients. }\end{array}$ & Yes & Yes & Yes \\
\hline $\begin{array}{l}\text { Harrison et } \\
\text { al., } 2012^{15}\end{array}$ & $\begin{array}{l}\text { G1: Pharmaceutical care provided } \\
\text { by a clinical pharmacist for the } \\
\text { purpose of identifying and resolving } \\
\text { actual and potential drug therapy } \\
\text { problems, medication teaching, } \\
\text { adherence optimization, medication } \\
\text { reconciliation, and provision of drug } \\
\text { information. } \\
\text { G2: Retrospective historical control } \\
\text { of matched patients who received } \\
\text { standard care at a routine medical } \\
\text { visit within } 8 \text { months prior to study } \\
\text { period }\end{array}$ & $\begin{array}{l}\text { No } \\
\text { Study population limited to recent } \\
\text { lung transplant recipients. }\end{array}$ & $\begin{array}{l}\text { Unclear or NR } \\
\text { What was done could } \\
\text { be applicable, although } \\
\text { given the highly } \\
\text { specialized nature of } \\
\text { the clinical condition, } \\
\text { the pharmacists were } \\
\text { likely specialized, and } \\
\text { as a result, the } \\
\text { intervention may not } \\
\text { have been applicable. }\end{array}$ & $\begin{array}{l}\text { No } \\
\text { Same issue as } \\
\text { population applicability } \\
\text { because the transplant } \\
\text { population is highly } \\
\text { selected and requires } \\
\text { specialized care. }\end{array}$ & Yes \\
\hline
\end{tabular}




\section{Table E42. Applicability (continued)}

\begin{tabular}{|c|c|c|c|c|c|}
\hline $\begin{array}{l}\text { Author, Year } \\
\text { Trial name }^{a}\end{array}$ & $\begin{array}{l}\text { Interventions and Comparator } \\
\text { Descriptions }\end{array}$ & $\begin{array}{l}\text { Study Population broadly } \\
\text { applicable? (e.g., not selected } \\
\text { using narrow eligibility criteria, } \\
\text { similarity in demographics } \\
\text { between study population and } \\
\text { community patients) }\end{array}$ & $\begin{array}{l}\text { Intervention broadly } \\
\text { applicable? (e.g., } \\
\text { design of } \\
\text { interventions } \\
\text { reflected in current } \\
\text { practice) }\end{array}$ & $\begin{array}{l}\text { Comparator(s) } \\
\text { broadly applicable? } \\
\text { (e.g., alternative } \\
\text { therapy or usual care } \\
\text { reflective of current } \\
\text { practice) }\end{array}$ & $\begin{array}{l}\text { Outcomes broadly } \\
\text { applicable? (e.g., not } \\
\text { limited to short-term, } \\
\text { surrogate, or } \\
\text { composite outcomes) }\end{array}$ \\
\hline $\begin{array}{l}\text { Isetts et al., } \\
2008^{16}\end{array}$ & $\begin{array}{l}\text { G1: MTM services provided by } \\
\text { staff pharmacists, including the } \\
\text { establishment of goals of therapy, } \\
\text { in collaboration with primary care } \\
\text { providers. } \\
\text { G2: Usual medical care without } \\
\text { MTM }\end{array}$ & Yes & Yes & Yes & Yes \\
\hline $\begin{array}{l}\text { Jameson, } \\
\text { VanNoord, } \\
\text { and } \\
\text { Vanderwoud, } \\
1995^{17}\end{array}$ & $\begin{array}{l}\text { Pharmacotherapy consultation and } \\
\text { followup provided by clinical } \\
\text { ambulatory care pharmacist. } \\
\text { G2: Standard office-based primary } \\
\text { care. }\end{array}$ & Yes & Yes & Yes & Yes \\
\hline $\begin{array}{l}\text { Jeong et al., } \\
2007^{18}\end{array}$ & $\begin{array}{l}\text { G1: Pharmacist-managed MTMP } \\
\text { provided by ambulatory care } \\
\text { pharmacists and healthcare } \\
\text { support staff } \\
\text { G2: Eligible for Part D MTMP but } \\
\text { declined enrollment } \\
\text { G3: Patients without Part D as their } \\
\text { primary drug benefit }\end{array}$ & Yes & $\begin{array}{l}\text { No } \\
\text { MTM intervention itself } \\
\text { may be applicable, but } \\
\text { it was delivered within } \\
\text { Kaiser Permanente's } \\
\text { integrated healthcare } \\
\text { system, which does not } \\
\text { reflect organization of } \\
\text { most healthcare } \\
\text { systems in the U.S. }\end{array}$ & Yes & Yes \\
\hline $\begin{array}{l}\text { Krska et al., } \\
2001^{19}\end{array}$ & $\begin{array}{l}\text { G1: Medication reviews led by } \\
\text { clinically-trained pharmacists. } \\
\text { G2: Usual care involving interviews } \\
\text { and identification of pharmaceutical } \\
\text { care issues but with no } \\
\text { pharmaceutical care plan } \\
\text { implemented. }\end{array}$ & No & Yes & Yes & Yes \\
\hline
\end{tabular}


Table E42. Applicability (continued)

\begin{tabular}{|c|c|c|c|c|c|}
\hline $\begin{array}{l}\text { Author, Year } \\
\text { Trial name }\end{array}$ & $\begin{array}{l}\text { Interventions and Comparator } \\
\text { Descriptions }\end{array}$ & $\begin{array}{l}\text { Study Population broadly } \\
\text { applicable? (e.g., not selected } \\
\text { using narrow eligibility criteria, } \\
\text { similarity in demographics } \\
\text { between study population and } \\
\text { community patients) }\end{array}$ & $\begin{array}{l}\text { Intervention broadly } \\
\text { applicable? (e.g., } \\
\text { design of } \\
\text { interventions } \\
\text { reflected in current } \\
\text { practice) }\end{array}$ & $\begin{array}{l}\text { Comparator(s) } \\
\text { broadly applicable? } \\
\text { (e.g., alternative } \\
\text { therapy or usual care } \\
\text { reflective of current } \\
\text { practice) }\end{array}$ & $\begin{array}{l}\text { Outcomes broadly } \\
\text { applicable? (e.g., not } \\
\text { limited to short-term, } \\
\text { surrogate, or } \\
\text { composite outcomes) }\end{array}$ \\
\hline $\begin{array}{l}\text { Malone et al., } \\
2000^{20}\end{array}$ & $\begin{array}{l}\text { G1: Pharmaceutical care provided } \\
\text { by clinical pharmacists practicing }\end{array}$ & Yes & No & Yes & No \\
\hline $\begin{array}{l}\text { Ellis et al., } \\
2000^{21} \\
\text { Malone et al., } \\
2001^{22} \text {; } \\
\text { Ellis et al., } \\
2000^{23}\end{array}$ & $\begin{array}{l}\text { according to scope of practice } \\
\text { within their respective health care } \\
\text { facilities. } \\
\text { G2: Usual care without } \\
\text { pharmaceutical care }\end{array}$ & & $\begin{array}{l}\text { VA is a large integrated } \\
\text { health system with on- } \\
\text { site pharmacy and } \\
\text { highly trained clinical } \\
\text { pharmacists who are } \\
\text { embedded within } \\
\text { ambulatory care clinics. }\end{array}$ & & $\begin{array}{l}\text { Unclear how applicable } \\
\text { VA costing methods and } \\
\text { systems are to the rest } \\
\text { of the healthcare system. }\end{array}$ \\
\hline IMPROVE & & & $\begin{array}{l}\text { This is not typical of } \\
\text { most primary care } \\
\text { practices. }\end{array}$ & & \\
\hline $\begin{array}{l}\text { McDonough } \\
\text { et al., } 2005^{24}\end{array}$ & $\begin{array}{l}\text { G1: Pharmaceutical care provided } \\
\text { by community pharmacists. Drug } \\
\text { therapy monitoring focused on } 5 \\
\text { drug therapy problems: } \\
\text { appropriateness of does, proper } \\
\text { regimen, potential interactions, } \\
\text { nonadherence, and adverse } \\
\text { effects. Patient education also } \\
\text { provided. } \\
\text { G2: Usual care }\end{array}$ & Yes & Yes & Yes & Yes \\
\hline $\begin{array}{l}\text { Moczygemba } \\
\text { et al., } 2011^{25} \\
\text { Moczygemba } \\
\text { et al., } 2008^{26}\end{array}$ & $\begin{array}{l}\text { G1: Opt-in telephone-based MTM } \\
\text { program, in which MTM services } \\
\text { provided by clinical pharmacists or } \\
\text { a managed care pharmacy } \\
\text { resident based on the American } \\
\text { Pharmacists Association and } \\
\text { National Association of Chain Drug } \\
\text { Stores Foundation MTM } \\
\text { framework. } \\
\text { G2: No-MTM control group }\end{array}$ & Yes & Yes & Yes & Yes \\
\hline
\end{tabular}


Table E42. Applicability (continued)

\begin{tabular}{|c|c|c|c|c|c|}
\hline $\begin{array}{l}\text { Author, } \\
\text { Year } \\
\text { Trial name }^{a}\end{array}$ & $\begin{array}{l}\text { Interventions and Comparator } \\
\text { Descriptions }\end{array}$ & $\begin{array}{l}\text { Study Population broadly } \\
\text { applicable? (e.g., not selected } \\
\text { using narrow eligibility criteria, } \\
\text { similarity in demographics } \\
\text { between study population and } \\
\text { community patients) }\end{array}$ & $\begin{array}{l}\text { Intervention broadly } \\
\text { applicable? (e.g., } \\
\text { design of } \\
\text { interventions } \\
\text { reflected in current } \\
\text { practice) }\end{array}$ & $\begin{array}{l}\text { Comparator(s) } \\
\text { broadly applicable? } \\
\text { (e.g., alternative } \\
\text { therapy or usual care } \\
\text { reflective of current } \\
\text { practice) }\end{array}$ & $\begin{array}{l}\text { Outcomes broadly } \\
\text { applicable? (e.g., not } \\
\text { limited to short-term, } \\
\text { surrogate, or } \\
\text { composite outcomes) }\end{array}$ \\
\hline $\begin{array}{l}\text { Pai et al., } \\
2009^{27} \text {; Pai } \\
\text { et al., } 2009^{28}\end{array}$ & $\begin{array}{l}\text { G1: Pharmaceutical care including } \\
\text { drug therapy reviews conducted by } \\
\text { a nephrology-trained clinical } \\
\text { pharmacist with the patient. Also } \\
\text { included patient and health care } \\
\text { provider education. } \\
\text { G2: Standard of care, consisting of } \\
\text { brief therapy reviews conducted by } \\
\text { a nurse }\end{array}$ & $\begin{array}{l}\text { No } \\
\text { Narrow eligibility - Adults with ESRD } \\
\text { who were undergoing a stable } \\
\text { hemodialysis. }\end{array}$ & Yes & Yes & Yes \\
\hline $\begin{array}{l}\text { Park et al., } \\
1996^{29}\end{array}$ & $\begin{array}{l}\text { G1: Comprehensive } \\
\text { pharmaceutical services including } \\
\text { drug therapy monitoring and patient } \\
\text { education provided by a community } \\
\text { pharmacy resident. } \\
\text { G2: Usual care }\end{array}$ & Yes & Yes & Yes & Yes \\
\hline $\begin{array}{l}\text { Pindolia et } \\
\text { al., } 2009^{30}\end{array}$ & $\begin{array}{l}\text { G1: Telephone-based MTM services } \\
\text { provided as part of a Medicare Part } \\
\text { D MTM program by pharmacy care } \\
\text { management clinical pharmacists. } \\
\text { G2: Usual medical care }\end{array}$ & Yes & Yes & Yes & Yes \\
\hline $\begin{array}{l}\text { Planas et al., } \\
2009^{31}\end{array}$ & $\begin{array}{l}\text { G1: MTM services provided by } \\
\text { community pharmacists. Also } \\
\text { included patient education on diet } \\
\text { and lifestyle modifications to lower } \\
\text { blood pressure. } \\
\text { G2: No MTM received, but only } \\
\text { informed of blood pressure goals for } \\
\text { patients with diabetes }\end{array}$ & Yes & Yes & Yes & Yes \\
\hline $\begin{array}{l}\text { Roughead et } \\
\text { al., } 2009^{32}\end{array}$ & $\begin{array}{l}\text { G1: Home Medication Reviews } \\
\text { (HMR), a collaborative model of } \\
\text { pharmaceutical care, conducted by } \\
\text { accredited pharmacists. } \\
\text { G2: No medication review received }\end{array}$ & Yes & $\begin{array}{l}\text { Unclear or NR } \\
\text { Australia's health care } \\
\text { system is different than } \\
\text { the U.S. health care } \\
\text { system, so it is unclear } \\
\text { how generalizable } \\
\text { these results are to the } \\
\text { U.S. }\end{array}$ & Yes & Yes \\
\hline
\end{tabular}


Table E42. Applicability (continued)

\begin{tabular}{|c|c|c|c|c|c|}
\hline $\begin{array}{l}\text { Author, } \\
\text { Year } \\
\text { Trial name }\end{array}$ & $\begin{array}{l}\text { Interventions and Comparator } \\
\text { Descriptions }\end{array}$ & $\begin{array}{l}\text { Study Population broadly } \\
\text { applicable? (e.g., not selected } \\
\text { using narrow eligibility criteria, } \\
\text { similarity in demographics } \\
\text { between study population and } \\
\text { community patients) }\end{array}$ & $\begin{array}{l}\text { Intervention broadly } \\
\text { applicable? (e.g., } \\
\text { design of } \\
\text { interventions } \\
\text { reflected in current } \\
\text { practice) }\end{array}$ & $\begin{array}{l}\text { Comparator(s) } \\
\text { broadly applicable? } \\
\text { (e.g., alternative } \\
\text { therapy or usual care } \\
\text { reflective of current } \\
\text { practice) }\end{array}$ & $\begin{array}{l}\text { Outcomes broadly } \\
\text { applicable? (e.g., not } \\
\text { limited to short-term, } \\
\text { surrogate, or } \\
\text { composite outcomes) }\end{array}$ \\
\hline $\begin{array}{l}\text { Sellors et al., } \\
2003^{33}\end{array}$ & $\begin{array}{l}\text { G1: Clinical pharmacist } \\
\text { consultations provided to family } \\
\text { physicians and their patients by } \\
\text { community pharmacists. } \\
\text { G2: Usual care for family physicians } \\
\text { and their patients from matched } \\
\text { postal codes. }\end{array}$ & $\begin{array}{l}\text { Yes and No } \\
\text { Yes for Canada, but may not be for } \\
\text { U.S. }\end{array}$ & Yes & Yes & Yes \\
\hline $\begin{array}{l}\text { Sidel et al., } \\
1990^{34}\end{array}$ & $\begin{array}{l}\text { G1: Home visits by pharmacists } \\
\text { and, when needed, consultations } \\
\text { with physicians to identify and } \\
\text { correct problems associated with } \\
\text { medication use. } \\
\text { G2: Standard care without any visits } \\
\text { or information provided to G1. }\end{array}$ & $\begin{array}{l}\text { Unclear or NR } \\
\text { Narrow eligibility based on excluding } \\
\text { low and medium risk patients and } \\
\text { those considered to be "difficult". }\end{array}$ & Yes & Yes & $\begin{array}{l}\text { No } \\
\text { Short-term, most } \\
\text { subjective and not } \\
\text { broadly applicable, most } \\
\text { surrogate outcomes. }\end{array}$ \\
\hline $\begin{array}{l}\text { Staresinic et } \\
\text { al., } 2007^{35}\end{array}$ & $\begin{array}{l}\text { G1: MTM services provided as part } \\
\text { of a Medicare Part D MTM program } \\
\text { by an MTM Coordinator (non-clinical } \\
\text { staff) and a pharmacist } \\
\text { G2: Usual care provided to MTM- } \\
\text { eligible enrollees who chose not to } \\
\text { participate }\end{array}$ & Yes & Yes & Yes & Unclear or NR \\
\hline $\begin{array}{l}\text { Taylor, Byrd, } \\
\text { and Krueger, } \\
2003^{36}\end{array}$ & $\begin{array}{l}\text { G1: Pharmaceutical care provided } \\
\text { by pharmacists } \\
\text { G2: Standard care without advice or } \\
\text { recommendations given to patients } \\
\text { or physicians }\end{array}$ & Yes & Yes & Yes & Yes \\
\hline $\begin{array}{l}\text { Touchette et } \\
\text { al., } 2012^{37}\end{array}$ & $\begin{array}{l}\text { G1: MTM basic (comprehensive } \\
\text { medication review and DRP } \\
\text { assessment) } \\
\text { G2: MTM enhanced (MTM plus 2- } \\
\text { page clinical summary abstracted } \\
\text { from patient's medical chart) } \\
\text { G3: Usual care, consisting of } \\
\text { medication counseling per clinic's } \\
\text { normal routine but no formal MTM } \\
\text { from a study pharmacist }\end{array}$ & Yes & Yes & Yes & Yes \\
\hline
\end{tabular}


Table E42. Applicability (continued)

\begin{tabular}{|c|c|c|c|c|c|}
\hline $\begin{array}{l}\text { Author, } \\
\text { Year } \\
\text { Trial name }^{\mathrm{a}}\end{array}$ & $\begin{array}{l}\text { Interventions and Comparator } \\
\text { Descriptions }\end{array}$ & $\begin{array}{l}\text { Study Population broadly } \\
\text { applicable? (e.g., not selected } \\
\text { using narrow eligibility criteria, } \\
\text { similarity in demographics } \\
\text { between study population and } \\
\text { community patients) }\end{array}$ & $\begin{array}{l}\text { Intervention broadly } \\
\text { applicable? (e.g., } \\
\text { design of } \\
\text { interventions } \\
\text { reflected in current } \\
\text { practice) }\end{array}$ & $\begin{array}{l}\text { Comparator(s) } \\
\text { broadly applicable? } \\
\text { (e.g., alternative } \\
\text { therapy or usual care } \\
\text { reflective of current } \\
\text { practice) }\end{array}$ & $\begin{array}{l}\text { Outcomes broadly } \\
\text { applicable? (e.g., not } \\
\text { limited to short-term, } \\
\text { surrogate, or } \\
\text { composite outcomes) }\end{array}$ \\
\hline $\begin{array}{l}\text { Triller and } \\
\text { Hamilton, } \\
2007^{38}\end{array}$ & $\begin{array}{l}\text { G1: Visiting nurse association } \\
\text { home visit services plus } \\
\text { comprehensive pharmaceutical care } \\
\text { services } \\
\text { G2: Visiting nurse association home } \\
\text { visit services only }\end{array}$ & Yes & Yes & Yes & Yes \\
\hline $\begin{array}{l}\text { Volume et } \\
\text { al., } 2001^{39} \text {; } \\
\text { Kassam et } \\
\text { al., } 2001^{40} \\
\text { PREP }\end{array}$ & $\begin{array}{l}\text { G1: Comprehensive pharmaceutical } \\
\text { care services using a nine-step } \\
\text { process as defined by Hepler and } \\
\text { Strand provided by community } \\
\text { pharmacists. } \\
\text { G2: Traditional pharmacy care }\end{array}$ & Yes & Yes & Yes & Yes \\
\hline $\begin{array}{l}\text { Welch et al., } \\
2009^{41}\end{array}$ & $\begin{array}{l}\text { G1: MTM program provided to } \\
\text { home-based beneficiaries as part of } \\
\text { a Medicare Part D MTM program } \\
\text { G2: No-MTM control group } \\
\text { (voluntary opt-out) }\end{array}$ & Yes & $\begin{array}{l}\text { Yes } \\
\text { KPCO's level of } \\
\text { integration not } \\
\text { widespread, but } \\
\text { intervention itself is } \\
\text { applicable }\end{array}$ & Yes & Yes \\
\hline $\begin{array}{l}\text { Williams et } \\
\text { al., } 2004^{42}\end{array}$ & $\begin{array}{l}\text { G1: Medication review and } \\
\text { optimization of patient's medication } \\
\text { regimen conducted by an } \\
\text { interdisciplinary medication } \\
\text { adjustment team in addition to usual } \\
\text { medical care and "Bound for Health" } \\
\text { booklet. } \\
\text { G2: Usual medical care plus } \\
\text { provision of "Bound for Health" } \\
\text { booklet }\end{array}$ & Yes & Yes & Yes & Yes \\
\hline
\end{tabular}


Table E42. Applicability (continued)

\begin{tabular}{|c|c|c|c|c|c|}
\hline $\begin{array}{l}\text { Author, } \\
\text { Year } \\
\text { Trial name }\end{array}$ & $\begin{array}{l}\text { Interventions and Comparator } \\
\text { Descriptions }\end{array}$ & $\begin{array}{l}\text { Study Population broadly } \\
\text { applicable? (e.g., not selected } \\
\text { using narrow eligibility criteria, } \\
\text { similarity in demographics } \\
\text { between study population and } \\
\text { community patients) }\end{array}$ & $\begin{array}{l}\text { Intervention broadly } \\
\text { applicable? (e.g., } \\
\text { design of } \\
\text { interventions } \\
\text { reflected in current } \\
\text { practice) }\end{array}$ & $\begin{array}{l}\text { Comparator(s) } \\
\text { broadly applicable? } \\
\text { (e.g., alternative } \\
\text { therapy or usual care } \\
\text { reflective of current } \\
\text { practice) }\end{array}$ & $\begin{array}{l}\text { Outcomes broadly } \\
\text { applicable? (e.g., not } \\
\text { limited to short-term, } \\
\text { surrogate, or } \\
\text { composite outcomes) }\end{array}$ \\
\hline $\begin{array}{l}\text { Winston and } \\
\text { Lin, } 2009^{43}\end{array}$ & $\begin{array}{l}\text { G1: MTM provided in a community } \\
\text { pharmacy (i.e., care in face-to-face } \\
\text { meetings or by telephone) as part of } \\
\text { a Medicare Part D MTM program } \\
\text { G2: MTM provided by pharmacist- } \\
\text { staffed call centers as part of a } \\
\text { Medicare Part D MTM program } \\
\text { G3: Educational mailings (i.e., } \\
\text { mailed letter containing patient- } \\
\text { specific medication related } \\
\text { information, personal medication } \\
\text { record, and tips to save money on } \\
\text { prescriptions) }\end{array}$ & Yes & Yes & Yes & Yes \\
\hline $\begin{array}{l}\text { Witry, } \\
\text { Doucette, } \\
\text { and Gainer, } \\
2011^{44}\end{array}$ & $\begin{array}{l}\text { G1: PCM provided by community } \\
\text { pharmacists to lowa Medicaid } \\
\text { enrollees } \\
\text { G2: PCM provided by community } \\
\text { pharmacists to patients with private } \\
\text { individual-group insurance }\end{array}$ & Yes & Yes & Yes & Yes \\
\hline $\begin{array}{l}\text { Abbreviations: } \\
\text { Effectiveness I } \\
\text { management; } 1 \\
\text { OR = odds rati } \\
\text { RCT = random }\end{array}$ & $\begin{array}{l}\text { : BMI = body mass index; } \mathrm{CMR}=\text { compre } \\
\text { Data and Information Set; HMR = home } \mathrm{n} \\
\text { MTMP = medication therapy management } \\
\text { io; PA = physician assistant; PCM = pharn } \\
\text { nized controlled trial; VA = Veterans Affa }\end{array}$ & $\begin{array}{l}\text { ehensive medication review; DRP }=\mathrm{dr} \\
\text { medication review; ITT }=\text { intention-to- } \\
\text { it program; } \mathrm{N}=\text { sample or group size; } \\
\text { maceutical case management; PDP }=\mathrm{p} \\
\text { airs }\end{array}$ & $\begin{array}{l}\text { regimen problem; DTP } \\
\text { t; } \text { KPCO = Kaiser Perma } \\
=\text { not applicable; } \mathrm{NR}=\mathrm{n} \\
\text { cription drug plan; PREP }\end{array}$ & $\begin{array}{l}\text { rug therapy problem; } \mathrm{G}=\mathrm{g} \\
\text { nte Colorado; MTM = mec } \\
\text { reported; NRCT = non-ran } \\
\text { Pharmaceutical Care Rese }\end{array}$ & $\begin{array}{l}\text { group; HEDIS = Healthcare } \\
\text { edication therapy } \\
\text { ndomized controlled trial; } \\
\text { earch and Education Project; }\end{array}$ \\
\hline
\end{tabular}




\section{References}

1. Bernsten C, Bjorkman I, Caramona M, et al. Improving the well-being of elderly patients via community pharmacy-based provision of pharmaceutical care: a multicentre study in seven European countries. Drugs Aging. 2001;18(1):63-77. PMID: 11232739.

2. Sturgess IK, McElnay JC, Hughes CM, et al. Community pharmacy based provision of pharmaceutical care to older patients. Pharm World Sci. 2003 Oct;25(5):218-26. PMID: 14584229.

3. Blennerhassett JD, Cusack BM, Smith CD, et al. A novel medicines management pathway. Journal of Pharmacy Practice and Research (Australia). 2006;36(Mar):175-9. PMID: 44-00783.

4. Carter BL, Barnette DJ, Chrischilles E, et al. Evaluation of hypertensive patients after care provided by community pharmacists in a rural setting. Pharmacotherapy. 1997 NovDec;17(6):1274-85. PMID: 9399611.

5. Barnette DJ, Murphy CM, Carter BL. Clinical skill development for community pharmacists. J Am Pharm Assoc (Wash). 1996 Sep;NS36(9):573-80. PMID: 8824077.

6. Chisholm MA, Mulloy LL, Jagadeesan M, et al. Effect of clinical pharmacy services on the blood pressure of African-American renal transplant patients. Ethn Dis. 2002 Summer;12(3):392-7. PMID: 12148711.

7. Chrischilles EA, Carter BL, Lund BC, et al. Evaluation of the Iowa Medicaid pharmaceutical case management program. $\mathrm{J}$ Am Pharm Assoc (2003). 2004 MayJun;44(3):337-49. PMID: 15191244.

8. Christensen DB, Roth M, Trygstad T, et al. Evaluation of a pilot medication therapy management project within the North Carolina State Health Plan. J Am Pharm Assoc (2003). 2007 Jul-Aug;47(4):471-83. PMID: 17616493.

9. Clifford RM, Batty KT, Davis TME, et al. A randomised controlled trial of a pharmaceutical care programme in high-risk diabetic patients in an outpatient clinic. Int $\mathrm{J}$ Pharm Pract. 2002;10(2):85-9.

10. Fischer LR, Scott LM, Boonstra DM, et al. Pharmaceutical care for patients with chronic conditions. J Am Pharm Assoc
(Wash). 2000 Mar-Apr;40(2):174-80. PMID: 10730021.

11. Fischer LR, Defor TA, Cooper S, et al. Pharmaceutical care and health care utilization in an HMO. Eff Clin Pract. 2002 Mar-Apr;5(2):49-57. PMID: 11990212.

12. Fox D, Ried LD, Klein GE, et al. A medication therapy management program's impact on low-density lipoprotein cholesterol goal attainment in Medicare Part D patients with diabetes. J Am Pharm Assoc (2003). 2009 Mar-Apr;49(2):192-9. PMID: 19289345.

13. Gattis WA, Hasselblad V, Whellan DJ, et al. Reduction in heart failure events by the addition of a clinical pharmacist to the heart failure management team: results of the Pharmacist in Heart Failure Assessment Recommendation and Monitoring (PHARM) Study. Arch Intern Med. 1999 Sep 13;159(16):1939-45. PMID: 10493325.

14. Hanlon JT, Weinberger M, Samsa GP, et al. A randomized, controlled trial of a clinical pharmacist intervention to improve inappropriate prescribing in elderly outpatients with polypharmacy. Am J Med. 1996 Apr;100(4):428-37. PMID: 8610730.

15. Harrison JJ, Wang J, Cervenko J, et al. Pilot study of a pharmaceutical care intervention in an outpatient lung transplant clinic. Clin Transplant. 2012 Mar-Apr;26(2):E149-57. PMID: 22507355.

16. Isetts BJ, Schondelmeyer SW, Artz MB, et al. Clinical and economic outcomes of medication therapy management services: the Minnesota experience. J Am Pharm Assoc. 2008 Mar-Apr;48(2):203-11; 3 p following 11. PMID: 18359733.

17. Jameson J, VanNoord G, Vanderwoud K. The impact of a pharmacotherapy consultation on the cost and outcome of medical therapy. J Fam Pract. 1995 Nov;41(5):469-72. PMID: 7595265.

18. Jeong EW, Gallagher MW, Fredriks D, et al. Impact of a pharmacist-managed medication therapy management (MTM) program. ASHP Midyear Clinical Meeting. 2007;42(DEC)PMID: 45-05344. 
19. Krska J, Cromarty JA, Arris F, et al. Pharmacist-led medication review in patients over 65: a randomized, controlled trial in primary care. Age Ageing. 2001 May;30(3):205-11. PMID: 11443021.

20. Malone DC, Carter BL, Billups SJ, et al. An economic analysis of a randomized, controlled, multicenter study of clinical pharmacist interventions for high-risk veterans: the IMPROVE study. Impact of Managed Pharmaceutical Care Resource Utilization and Outcomes in Veterans Affairs Medical Centers. Pharmacotherapy. 2000 Oct;20(10):1149-58. PMID: 11034037.

21. Ellis SL, Billups SJ, Malone DC, et al. Types of interventions made by clinical pharmacists in the IMPROVE study. Impact of Managed Pharmaceutical Care on Resource Utilization and Outcomes in Veterans Affairs Medical Centers. Pharmacotherapy. 2000 Apr;20(4):429-35. PMID: 10772374.

22. Malone DC, Carter BL, Billups SJ, et al. Can clinical pharmacists affect SF-36 scores in veterans at high risk for medicationrelated problems? Med Care. 2001 Feb;39(2):113-22. PMID: 11176549.

23. Ellis SL, Carter BL, Malone DC, et al. Clinical and economic impact of ambulatory care clinical pharmacists in management of dyslipidemia in older adults: the IMPROVE study. Impact of Managed Pharmaceutical Care on Resource Utilization and Outcomes in Veterans Affairs Medical Centers. Pharmacotherapy. 2000 Dec;20(12):150816. PMID: 11130223.

24. McDonough RP, Doucette WR, Kumbera P, et al. An evaluation of managing and educating patients on the risk of glucocorticoid-induced osteoporosis. Value Health. 2005 Jan-Feb;8(1):24-31. PMID: 15841891 .

25. Moczygemba LR, Barner JC, Lawson KA, et al. Impact of telephone medication therapy management on medication and health-related problems, medication adherence, and Medicare Part D drug costs: a 6-month follow up. Am J Geriatr Pharmacother. 2011 Oct;9(5):328-38. PMID: 21865093.
26. Moczygemba LR, Barner JC, Gabrillo ER, et al. Development and implementation of a telephone medication therapy management program for Medicare beneficiaries. Am J Health Syst Pharm. 2008 Sep 1;65(17):1655-60. PMID: 18714113.

27. Pai AB, Boyd A, Depczynski J, et al. Reduced drug use and hospitalization rates in patients undergoing hemodialysis who received pharmaceutical care: a 2-year, randomized, controlled study. Pharmacotherapy. 2009 Dec;29(12):143340. PMID: 19947803.

28. Pai AB, Boyd A, Chavez A, et al. Healthrelated quality of life is maintained in hemodialysis patients receiving pharmaceutical care: a 2-year randomized, controlled study. Hemodial Int. 2009 Jan;13(1):72-9. PMID: 19210281.

29. Park JJ, Kelly P, Carter BL, et al. Comprehensive pharmaceutical care in the chain setting. J Am Pharm Assoc (Wash). 1996 Jul;NS36(7):443-51. PMID: 8840744.

30. Pindolia VK, Stebelsky L, Romain TM, et al. Mitigation of medication mishaps via medication therapy management. Ann Pharmacother. 2009 Apr;43(4):611-20. PMID: 19336646.

31. Planas LG, Crosby KM, Mitchell KD, et al. Evaluation of a hypertension medication therapy management program in patients with diabetes. J Am Pharm Assoc (2003). 2009 Mar-Apr;49(2):164-70. PMID: 19289342.

32. Roughead EE, Barratt JD, Ramsay E, et al. The effectiveness of collaborative medicine reviews in delaying time to next hospitalization for patients with heart failure in the practice setting: results of a cohort study. Circ Heart Fail. 2009 Sep;2(5):424-8. PMID: 19808372.

33. Sellors J, Kaczorowski J, Sellors C, et al. A randomized controlled trial of a pharmacist consultation program for family physicians and their elderly patients. CMAJ. 2003 Jul 8;169(1):17-22. PMID: 12847034.

34. Sidel VW, Beizer JL, Lisi-Fazio D, et al. Controlled study of the impact of educational home visits by pharmacists to high-risk older patients. J Community Health. 1990 Jun;15(3):163-74. PMID: 2195066. 
35. Staresinic AG. Early experience with medication management - Description and preliminary outcomes of a Medicare Part D medication therapy management program. Journal of the Pharmacy Society of Wisconsin (USA). 2007(SEP-OCT):8-0. PMID: 45-01183.

36. Taylor CT, Byrd DC, Krueger K. Improving primary care in rural Alabama with a pharmacy initiative. Am J Health Syst Pharm. 2003 Jun 1;60(11):1123-9. PMID: 12816022 .

37. Touchette DR, Masica AL, Dolor RJ, et al. Safety-focused medication therapy management: a randomized controlled trial. J Am Pharm Assoc (2003). 2012 SepOct;52(5):603-12. PMID: 23023840.

38. Triller DM, Hamilton RA. Effect of pharmaceutical care services on outcomes for home care patients with heart failure. Am J Health Syst Pharm. 2007 Nov 1;64(21):2244-9. PMID: 17959576.

39. Volume CI, Farris KB, Kassam R, et al. Pharmaceutical care research and education project: patient outcomes. J Am Pharm Assoc (Wash). 2001 May-Jun;41(3):411-20. PMID: 11372906.
40. Kassam R, Farris KB, Burback L, et al. Pharmaceutical care research and education project: pharmacists' interventions. J Am Pharm Assoc (Wash). 2001 MayJun;41(3):401-10. PMID: 11372905.

41. Welch EK, Delate T, Chester EA, et al. Assessment of the impact of medication therapy management delivered to homebased Medicare beneficiaries. Ann Pharmacother. 2009 Apr;43(4):603-10. PMID: 19318600.

42. Williams ME, Pulliam CC, Hunter R, et al. The short-term effect of interdisciplinary medication review on function and cost in ambulatory elderly people. J Am Geriatr Soc. 2004 Jan;52(1):93-8. PMID: 14687321

43. Winston S, Lin YS. Impact on drug cost and use of Medicare part D of medication therapy management services delivered in 2007. J Am Pharm Assoc (2003). 2009 NovDec;49(6):813-20. PMID: 19858047.

44. Witry MJ, Doucette WR, Gainer KL. Evaluation of the pharmaceutical case management program implemented in a private sector health plan. J Am Pharm Assoc (2003). 2011 Sep-Oct;51(5):631-5. PMID: 21896463. 


\section{Appendix F. Risk of Bias Evaluations and Rationale}

Table F1. Risk of bias domains and ratings

\begin{tabular}{|c|c|c|c|c|c|c|c|c|c|c|c|}
\hline $\begin{array}{l}\text { Author, Year } \\
\text { Trial Name }\end{array}$ & $\begin{array}{l}\text { Intervention and } \\
\text { Comparator } \\
\text { Descriptions }\end{array}$ & $\begin{array}{l}\text { Study } \\
\text { Design }\end{array}$ & $\begin{array}{l}\text { Randomi- } \\
\text { zation } \\
\text { method } \\
\text { adequate? } \\
\text { (RCTs } \\
\text { only) }\end{array}$ & $\begin{array}{l}\text { Allocation } \\
\text { conceal- } \\
\text { ment ad- } \\
\text { equate? } \\
\text { (RCTs } \\
\text { only) }\end{array}$ & $\begin{array}{l}\text { Recruitment } \\
\text { strategy for } \\
\text { study } \\
\text { different } \\
\text { across } \\
\text { groups? }\end{array}$ & $\begin{array}{l}\text { Groups } \\
\text { similar at } \\
\text { baseline, or } \\
\text { differences } \\
\text { adjusted } \\
\text { for in } \\
\text { analysis? }\end{array}$ & $\begin{array}{l}\text { Outcome } \\
\text { asse- } \\
\text { ssors } \\
\text { blinded? } \\
\text { (RCTs or } \\
\text { NRCTs } \\
\text { only) }\end{array}$ & $\begin{array}{l}\text { Impact from } \\
\text { concurrent } \\
\text { intervention or } \\
\text { unintended } \\
\text { exposure ruled } \\
\text { out by } \\
\text { researchers? }\end{array}$ & $\begin{array}{l}\text { Did variation } \\
\text { from study } \\
\text { protocol } \\
\text { compromise } \\
\text { conclusions? } \\
\text { (RCTs or } \\
\text { NRCTs only) }\end{array}$ & $\begin{array}{l}\text { High } \\
\text { overall (i.e., } \\
\geq 20 \% \text { ) or } \\
\text { differential } \\
\text { (i.e., } \geq 15 \% \text { ) } \\
\text { attrition }\end{array}$ & $\begin{array}{l}\text { Did attrition } \\
\text { result in } \\
\text { differences in } \\
\text { group } \\
\text { charac- } \\
\text { teristics? }\end{array}$ \\
\hline $\begin{array}{l}\text { Bernsten et al., } \\
\text { 20011; } \\
\text { Sturgess, } \\
20032\end{array}$ & $\begin{array}{l}\text { G1: Structured } \\
\text { community } \\
\text { pharmacy- } \\
\text { based } \\
\text { pharmaceutical } \\
\text { care program } \\
\text { G2: Usual } \\
\text { community } \\
\text { pharmacy } \\
\text { services }\end{array}$ & $\begin{array}{l}\text { RCT: } \\
\text { cluster- } \\
\text { rando- } \\
\text { mized }\end{array}$ & $\begin{array}{l}\text { Unclear or } \\
\text { NR }\end{array}$ & $\begin{array}{l}\text { Unclear or } \\
\text { NR }\end{array}$ & No & Yes & Yes & Unclear or NR & Yes & Yes & Yes \\
\hline $\begin{array}{l}\text { Blennerhassett } \\
\text { et al., } 20073\end{array}$ & $\begin{array}{l}\text { G1: } \\
\text { Implementation } \\
\text { of HMR, a } \\
\text { collaborative } \\
\text { model of } \\
\text { pharmaceutical } \\
\text { care, into a } \\
\text { chronic heart } \\
\text { failure } \\
\text { collaborative } \\
\text { care model, } \\
\text { conducted by } \\
\text { accredited } \\
\text { pharmacists } \\
\text { G2: No HMR }\end{array}$ & Cohort & NA & NA & Yes & No & NA & No & NA & Yes & $\begin{array}{l}\text { Unclear or } \\
\text { NR }\end{array}$ \\
\hline
\end{tabular}


Table F1. Risk of bias domains and ratings (continued)

\begin{tabular}{|c|c|c|c|c|c|c|c|c|c|c|c|}
\hline $\begin{array}{l}\text { Author, Year } \\
\text { Trial Name }\end{array}$ & $\begin{array}{l}\text { Intervention } \\
\text { and } \\
\text { Comparator } \\
\text { Descriptions }\end{array}$ & $\begin{array}{l}\text { Study } \\
\text { Design }\end{array}$ & $\begin{array}{l}\text { Randomi- } \\
\text { zation } \\
\text { method } \\
\text { adequate? } \\
\text { (RCTs } \\
\text { only) }\end{array}$ & $\begin{array}{l}\text { Allocation } \\
\text { conceal- } \\
\text { ment ad- } \\
\text { ? equate? } \\
\text { (RCTs } \\
\text { only) }\end{array}$ & $\begin{array}{l}\text { Recruitment } \\
\text { strategy for } \\
\text { study } \\
\text { different } \\
\text { across } \\
\text { groups? }\end{array}$ & $\begin{array}{l}\text { Groups } \\
\text { similar at } \\
\text { baseline, } \\
\text { or } \\
\text { differences } \\
\text { adjusted } \\
\text { for in } \\
\text { analysis? }\end{array}$ & $\begin{array}{l}\text { Outcome } \\
\text { asse- } \\
\text { ssors } \\
\text { blinded? } \\
\text { (RCTs or } \\
\text { NRCTs } \\
\text { only) }\end{array}$ & $\begin{array}{l}\text { Impact from } \\
\text { concurrent } \\
\text { intervention } \\
\text { or unintended } \\
\text { exposure } \\
\text { ruled out by } \\
\text { researchers? }\end{array}$ & $\begin{array}{l}\text { Did variation } \\
\text { from study } \\
\text { protocol } \\
\text { compromise } \\
\text { conclusions? } \\
\text { (RCTs or } \\
\text { NRCTs only) }\end{array}$ & $\begin{array}{l}\text { High } \\
\text { overall } \\
\text { (i.e., } \geq 20 \% \text { ) } \\
\text { or } \\
\text { ? differential } \\
\text { (i.e., } \geq 15 \% \text { ) } \\
\text { attrition }\end{array}$ & $\begin{array}{l}\text { Did attrition } \\
\text { result in } \\
\text { differences } \\
\text { in group } \\
\text { charac- } \\
\text { teristics? }\end{array}$ \\
\hline $\begin{array}{l}\text { Carter et al., } \\
1997^{4} \text {; } \\
\text { Barnette et al., } \\
1996^{5}\end{array}$ & $\begin{array}{l}\text { G1: } \\
\text { Pharmaceutical } \\
\text { care provided by } \\
\text { pharmacists } \\
\text { within } \\
\text { interdisciplinary } \\
\text { practice model. } \\
\text { Standardized } \\
\text { patient } \\
\text { education } \\
\text { (lifestyle, risk } \\
\text { factor } \\
\text { modifications, } \\
\text { and drug } \\
\text { therapy). } \\
\text { G2: Usual care } \\
\end{array}$ & Cohort & NA & NA & Yes & No & Yes & Unclear or NR & NA & No & NA \\
\hline $\begin{array}{l}\text { Chisholm et al., } \\
2002^{6}\end{array}$ & $\begin{array}{l}\text {, G1: Clinical } \\
\text { pharmacy } \\
\text { services, } \\
\text { including } \\
\text { reviewing } \\
\text { patients' } \\
\text { medication } \\
\text { therapy, with } \\
\text { emphasis on } \\
\text { controlling BP, } \\
\text { and preventing } \\
\text { or resolving } \\
\text { DTPs. } \\
\text { Pharmacists } \\
\text { counseled } \\
\text { patients about } \\
\text { their regimen, }\end{array}$ & $\begin{array}{l}\text { RCT: } \\
\text { parallel, } \\
\text { not } \\
\text { clustered }\end{array}$ & $\begin{array}{l}\text { Unclear or } \\
\text { NR }\end{array}$ & $\begin{array}{l}\text { Unclear or } \\
\text { NR }\end{array}$ & No & Yes & $\begin{array}{l}\text { Unclear or } \\
\text { NR }\end{array}$ & Unclear or NR & Unclear or NR & $\mathrm{No}$ & Unclear or NR \\
\hline
\end{tabular}


Table F1. Risk of bias domains and ratings (continued)

\begin{tabular}{|c|c|c|c|c|c|c|c|c|c|c|}
\hline $\begin{array}{l}\text { Author, Year } \\
\text { Trial Name }\end{array}$ & $\begin{array}{l}\text { Intervention } \\
\text { and } \\
\text { Comparator } \\
\text { Descriptions }\end{array}$ & $\begin{array}{l}\text { Randomi- } \\
\text { zation } \\
\text { method } \\
\text { adequate? } \\
\text { (RCTs } \\
\text { only) }\end{array}$ & $\begin{array}{l}\text { Allocation } \\
\text { conceal- } \\
\text { ment ad- } \\
\text { ? equate? } \\
\text { (RCTs } \\
\text { only) }\end{array}$ & $\begin{array}{l}\text { Recruitment } \\
\text { strategy for } \\
\text { study } \\
\text { different } \\
\text { across } \\
\text { groups? }\end{array}$ & $\begin{array}{l}\text { Groups } \\
\text { similar at } \\
\text { baseline, } \\
\text { or } \\
\text { differences } \\
\text { adjusted } \\
\text { for in } \\
\text { analysis? }\end{array}$ & $\begin{array}{l}\text { Outcome } \\
\text { asse- } \\
\text { ssors } \\
\text { blinded? } \\
\text { (RCTs or } \\
\text { NRCTs } \\
\text { only) }\end{array}$ & $\begin{array}{l}\text { Impact from } \\
\text { concurrent } \\
\text { intervention } \\
\text { or unintended } \\
\text { exposure } \\
\text { ruled out by } \\
\text { researchers? }\end{array}$ & $\begin{array}{l}\text { Did variation } \\
\text { from study } \\
\text { protocol } \\
\text { compromise } \\
\text { conclusions? } \\
\text { (RCTs or } \\
\text { NRCTs only) }\end{array}$ & $\begin{array}{l}\text { High } \\
\text { overall } \\
\text { (i.e., } \geq 20 \% \text { ) } \\
\text { or } \\
\text { ? differential } \\
\text { (i.e., } \geq 15 \% \text { ) } \\
\text { attrition }\end{array}$ & $\begin{array}{l}\text { Did attrition } \\
\text { result in } \\
\text { differences } \\
\text { in group } \\
\text { charac- } \\
\text { teristics? }\end{array}$ \\
\hline $\begin{array}{l}\text { Chisholm et al. } \\
2002^{6} \\
\text { (continued) }\end{array}$ & $\begin{array}{l}\text {, including desired } \\
\text { clinical } \\
\text { responses and } \\
\text { possible adverse } \\
\text { reactions. } \\
\text { G2: Routine } \\
\text { clinic services, } \\
\text { but without } \\
\text { clinical } \\
\text { pharmacist } \\
\text { interaction. } \\
\text { Routine clinical } \\
\text { services here } \\
\text { entailed meeting } \\
\text { renal transplant } \\
\text { clinic team } \\
\text { consisting of } 2 \\
\text { nephrologists, } \\
\text { clinical } \\
\text { pharmacist, PAs } \\
\text { and nurse. }\end{array}$ & & & & & & & & & \\
\hline $\begin{array}{l}\text { Chrischilles et } \\
\text { al., } 2004^{7}\end{array}$ & $\begin{array}{l}\text { G1: PCM-eligibleCohort } \\
\text { patients who } \\
\text { received PCM } \\
\text { services } \\
\text { G2: PCM-eligible } \\
\text { patients who did } \\
\text { not receive PCM } \\
\text { services }\end{array}$ & NA & NA & Yes & No & $\begin{array}{l}\text { Unclear or } \\
\text { NR }\end{array}$ & Unclear or NR & NA & No & No \\
\hline
\end{tabular}


Table F1. Risk of bias domains and ratings (continued)

\begin{tabular}{|c|c|c|c|c|c|c|c|c|c|c|c|}
\hline $\begin{array}{l}\text { Author, Year } \\
\text { Trial Name }\end{array}$ & $\begin{array}{l}\text { Intervention } \\
\text { and } \\
\text { Comparator } \\
\text { Descriptions }\end{array}$ & $\begin{array}{l}\text { Study } \\
\text { Design }\end{array}$ & $\begin{array}{l}\text { Randomi- } \\
\text { zation } \\
\text { method } \\
\text { adequate? } \\
\text { (RCTs } \\
\text { only) }\end{array}$ & $\begin{array}{l}\text { Allocation } \\
\text { conceal- } \\
\text { ment ad- } \\
\text { equate? } \\
\text { (RCTs } \\
\text { only) }\end{array}$ & $\begin{array}{l}\text { Recruitment } \\
\text { strategy for } \\
\text { study } \\
\text { different } \\
\text { across } \\
\text { groups? }\end{array}$ & $\begin{array}{l}\text { Groups } \\
\text { similar at } \\
\text { baseline, } \\
\text { or } \\
\text { differences } \\
\text { adjusted } \\
\text { for in } \\
\text { analysis? }\end{array}$ & $\begin{array}{l}\text { Outcome } \\
\text { asse- } \\
\text { ssors } \\
\text { blinded? } \\
\text { (RCTs or } \\
\text { NRCTs } \\
\text { only) }\end{array}$ & $\begin{array}{l}\text { Impact from } \\
\text { concurrent } \\
\text { intervention } \\
\text { or unintended } \\
\text { exposure } \\
\text { ruled out by } \\
\text { researchers? }\end{array}$ & $\begin{array}{l}\text { Did variation } \\
\text { from study } \\
\text { protocol } \\
\text { compromise } \\
\text { conclusions? } \\
\text { (RCTs or } \\
\text { NRCTs only) }\end{array}$ & $\begin{array}{l}\text { High } \\
\text { overall } \\
\text { (i.e., } \geq 20 \% \text { ) } \\
\text { or } \\
\text { ? differential } \\
\text { (i.e., } \geq 15 \% \text { ) } \\
\text { attrition }\end{array}$ & $\begin{array}{l}\text { Did attrition } \\
\text { result in } \\
\text { differences } \\
\text { in group } \\
\text { charac- } \\
\text { teristics? }\end{array}$ \\
\hline $\begin{array}{l}\text { Christensen et } \\
\text { al., } 2007^{8}\end{array}$ & $\begin{array}{l}\text { G1: MTM } \\
\text { services } \\
\text { designed by a } \\
\text { health plan for } \\
\text { beneficiaries and } \\
\text { provided by } \\
\text { either } \\
\text { community } \\
\text { pharmacists or } \\
\text { medical clinic- } \\
\text { based } \\
\text { pharmacists. } \\
\text { G2: Patients } \\
\text { from same } \\
\text { counties as G1 } \\
\text { who did not } \\
\text { receive } \\
\text { intervention. } \\
\text { G3: Patients } \\
\text { from a different } \\
\text { county than G1 } \\
\text { who did not } \\
\text { receive } \\
\text { intervention. }\end{array}$ & NRCT & NA & NA & Yes & $\begin{array}{l}\text { Unclear or } \\
\text { NR }\end{array}$ & $\begin{array}{l}\text { Unclear or } \\
\text { NR }\end{array}$ & No & Yes & Yes & Unclear or NR \\
\hline
\end{tabular}


Table F1. Risk of bias domains and ratings (continued)

\begin{tabular}{|c|c|c|c|c|c|c|c|c|c|c|c|}
\hline $\begin{array}{l}\text { Author, Year } \\
\text { Trial Name }\end{array}$ & $\begin{array}{l}\text { Intervention } \\
\text { and } \\
\text { Comparator } \\
\text { Descriptions }\end{array}$ & $\begin{array}{l}\text { Study } \\
\text { Design }\end{array}$ & $\begin{array}{l}\text { Randomi- } \\
\text { zation } \\
\text { method } \\
\text { adequate? } \\
\text { (RCTs } \\
\text { only) }\end{array}$ & $\begin{array}{l}\text { Allocation } \\
\text { conceal- } \\
\text { ment ad- } \\
\text { ? equate? } \\
\text { (RCTs } \\
\text { only) }\end{array}$ & $\begin{array}{l}\text { Recruitment } \\
\text { strategy for } \\
\text { study } \\
\text { different } \\
\text { across } \\
\text { groups? }\end{array}$ & $\begin{array}{l}\text { Groups } \\
\text { similar at } \\
\text { baseline, } \\
\text { or } \\
\text { differences } \\
\text { adjusted } \\
\text { for in } \\
\text { analysis? }\end{array}$ & $\begin{array}{l}\text { Outcome } \\
\text { asse- } \\
\text { ssors } \\
\text { blinded? } \\
\text { (RCTs or } \\
\text { NRCTs } \\
\text { only) }\end{array}$ & $\begin{array}{l}\text { Impact from } \\
\text { concurrent } \\
\text { intervention } \\
\text { or unintended } \\
\text { exposure } \\
\text { ruled out by } \\
\text { researchers? }\end{array}$ & $\begin{array}{l}\text { Did variation } \\
\text { from study } \\
\text { protocol } \\
\text { compromise } \\
\text { conclusions? } \\
\text { (RCTs or } \\
\text { NRCTs only) }\end{array}$ & $\begin{array}{l}\text { High } \\
\text { overall } \\
\text { (i.e., } \geq 20 \% \text { ) } \\
\text { or } \\
\text { ?differential } \\
\text { (i.e., } \geq 15 \% \text { ) } \\
\text { attrition }\end{array}$ & $\begin{array}{l}\text { Did attrition } \\
\text { result in } \\
\text { differences } \\
\text { in group } \\
\text { charac- } \\
\text { teristics? }\end{array}$ \\
\hline $\begin{array}{l}\text { Clifford et al., } \\
2002^{9}\end{array}$ & $\begin{array}{l}\text { G1: } \\
\text { Pharmaceutical } \\
\text { care provided by } \\
\text { a clinical } \\
\text { pharmacist, } \\
\text { including a } \\
\text { comprehensive } \\
\text { review relating to } \\
\text { pharma- } \\
\text { cotherapy and } \\
\text { diabetes, use of } \\
\text { proprietary and } \\
\text { non-proprietary } \\
\text { medications, } \\
\text { such as } \\
\text { complementary } \\
\text { medicines, and } \\
\text { identification of } \\
\text { DTPs. } \\
\text { G2: Standard } \\
\text { outpatient care } \\
\text { for diabetes }\end{array}$ & $\begin{array}{l}\text { RCT: } \\
\text { parallel, } \\
\text { not } \\
\text { clustered } \\
\end{array}$ & Yes & $\begin{array}{l}\text { Unclear or } \\
\text { NR }\end{array}$ & No & Yes & $\begin{array}{l}\text { Unclear or } \\
\text { NR }\end{array}$ & No & No & $\begin{array}{l}\text { Unclear or } \\
\text { NR }\end{array}$ & Unclear or NR \\
\hline
\end{tabular}


Table F1. Risk of bias domains and ratings (continued)

\begin{tabular}{|c|c|c|c|c|c|c|c|c|c|c|c|}
\hline $\begin{array}{l}\text { Author, Year } \\
\text { Trial Name }\end{array}$ & $\begin{array}{l}\text { Intervention } \\
\text { and } \\
\text { Comparator } \\
\text { Descriptions }\end{array}$ & $\begin{array}{l}\text { Study } \\
\text { Design }\end{array}$ & $\begin{array}{l}\text { Randomi- } \\
\text { zation } \\
\text { method } \\
\text { adequate? } \\
\text { (RCTs } \\
\text { only) }\end{array}$ & $\begin{array}{l}\text { Allocation } \\
\text { conceal- } \\
\text { ment ad- } \\
\text { ? equate? } \\
\text { (RCTs } \\
\text { only) }\end{array}$ & $\begin{array}{l}\text { Recruitment } \\
\text { strategy for } \\
\text { study } \\
\text { different } \\
\text { across } \\
\text { groups? }\end{array}$ & $\begin{array}{l}\text { Groups } \\
\text { similar at } \\
\text { baseline, } \\
\text { or } \\
\text { differences } \\
\text { adjusted } \\
\text { for in } \\
\text { analysis? }\end{array}$ & $\begin{array}{l}\text { Outcome } \\
\text { asse- } \\
\text { ssors } \\
\text { blinded? } \\
\text { (RCTs or } \\
\text { NRCTs } \\
\text { only) }\end{array}$ & $\begin{array}{l}\text { Impact from } \\
\text { concurrent } \\
\text { intervention } \\
\text { or unintended } \\
\text { exposure } \\
\text { ruled out by } \\
\text { researchers? }\end{array}$ & $\begin{array}{l}\text { Did variation } \\
\text { from study } \\
\text { protocol } \\
\text { compromise } \\
\text { conclusions? } \\
\text { (RCTs or } \\
\text { NRCTs only) }\end{array}$ & $\begin{array}{l}\text { High } \\
\text { overall } \\
\text { (i.e., } \geq 20 \% \text { ) } \\
\text { or } \\
\text { ? differential } \\
\text { (i.e., } \geq 15 \% \text { ) } \\
\text { attrition }\end{array}$ & $\begin{array}{l}\text { Did attrition } \\
\text { result in } \\
\text { differences } \\
\text { in group } \\
\text { charac- } \\
\text { teristics? }\end{array}$ \\
\hline $\begin{array}{l}\text { Fischer et al., } \\
2000^{10}\end{array}$ & $\begin{array}{l}\text { G1: } \\
\text { Pharmaceutical } \\
\text { care based on } \\
\text { Encara Practice } \\
\text { System provided } \\
\text { by onsite health } \\
\text { maintenance } \\
\text { organization } \\
\text { staff pharmacists } \\
\text { (acceptors). } \\
\text { G2: Standard } \\
\text { Community } \\
\text { Pharmacy } \\
\text { Practice. } \\
\text { G3: A set of } \\
\text { those at eligible } \\
\text { clinics who } \\
\text { initially declined } \\
\text { to participate } \\
\text { (opt-out). }\end{array}$ & $\begin{array}{l}\text { NRCT } \\
\text { s } \\
\text { s } \\
\end{array}$ & NA & NA & No & Yes & No & Unclear or NR & Unclear or NR & $\begin{array}{l}\text { Unclear or } \\
\text { NR }\end{array}$ & Yes \\
\hline $\begin{array}{l}\text { Fischer et al., } \\
2002^{11}\end{array}$ & $\begin{array}{l}\text { G1: } \\
\text { Pharmaceutical } \\
\text { care based on } \\
\text { Encara Practice } \\
\text { System provided } \\
\text { by pharmacists. } \\
\text { Communication } \\
\text { of pharmacist } \\
\text { with the patient's } \\
\text { physician about } \\
\text { drug therapy } \\
\text { problems } \\
\text { identified by the } \\
\text { pharmacist. } \\
\text { G2: Usual care. }\end{array}$ & NRCT & NA & NA & Yes & Yes & $\begin{array}{l}\text { Unclear or } \\
\text { NR }\end{array}$ & & Unclear or NR & $\begin{array}{l}\text { Unclear or } \\
\text { NR }\end{array}$ & Unclear or NR \\
\hline
\end{tabular}


Table F1. Risk of bias domains and ratings (continued)

\begin{tabular}{|c|c|c|c|c|c|c|c|c|c|c|c|}
\hline $\begin{array}{l}\text { Author, Year } \\
\text { Trial Name }\end{array}$ & $\begin{array}{l}\text { Intervention } \\
\text { and } \\
\text { Comparator } \\
\text { Descriptions }\end{array}$ & $\begin{array}{l}\text { Study } \\
\text { Design }\end{array}$ & $\begin{array}{l}\text { Randomi- } \\
\text { zation } \\
\text { method } \\
\text { adequate? } \\
\text { (RCTs } \\
\text { only) }\end{array}$ & $\begin{array}{l}\text { Allocation } \\
\text { conceal- } \\
\text { ment ad- } \\
\text { equate? } \\
\text { (RCTs } \\
\text { only) }\end{array}$ & $\begin{array}{l}\text { Recruitment } \\
\text { strategy for } \\
\text { study } \\
\text { different } \\
\text { across } \\
\text { groups? }\end{array}$ & $\begin{array}{l}\text { Groups } \\
\text { similar at } \\
\text { baseline, } \\
\text { or } \\
\text { differences } \\
\text { adjusted } \\
\text { for in } \\
\text { analysis? }\end{array}$ & $\begin{array}{l}\text { Outcome } \\
\text { asse- } \\
\text { ssors } \\
\text { blinded? } \\
\text { (RCTs or } \\
\text { NRCTs } \\
\text { only) }\end{array}$ & $\begin{array}{l}\text { Impact from } \\
\text { concurrent } \\
\text { intervention } \\
\text { or unintended } \\
\text { exposure } \\
\text { ruled out by } \\
\text { researchers? }\end{array}$ & $\begin{array}{l}\text { Did variation } \\
\text { from study } \\
\text { protocol } \\
\text { compromise } \\
\text { conclusions? } \\
\text { (RCTs or } \\
\text { NRCTs only) }\end{array}$ & $\begin{array}{l}\text { High } \\
\text { overall } \\
\text { (i.e., } \geq 20 \% \text { ) } \\
\text { or } \\
\text { ? differential } \\
\text { (i.e., } \geq 15 \% \text { ) } \\
\text { attrition }\end{array}$ & $\begin{array}{l}\text { Did attrition } \\
\text { result in } \\
\text { differences } \\
\text { in group } \\
\text { charac- } \\
\text { teristics? }\end{array}$ \\
\hline $\begin{array}{l}\text { Fox et al., } \\
2009^{12}\end{array}$ & $\begin{array}{l}\text { G1: Florida } \\
\text { Health Care } \\
\text { Plans MTM } \\
\text { program, } \\
\text { consisting of } \\
\text { medication } \\
\text { therapy review } \\
\text { and evaluation } \\
\text { by a clinical } \\
\text { pharmacist that } \\
\text { was documented } \\
\text { and sent to } \\
\text { patient's } \\
\text { physician } \\
\text { through health } \\
\text { plan review } \\
\text { (acceptors) } \\
\text { G2: Opt-out from } \\
\text { MTM program }\end{array}$ & Cohort & NA & NA & Yes & Yes & NA & Unclear or NR & NA & No & No \\
\hline
\end{tabular}


Table F1. Risk of bias domains and ratings (continued)

\begin{tabular}{|c|c|c|c|c|c|c|c|c|c|c|c|}
\hline $\begin{array}{l}\text { Author, Year } \\
\text { Trial Name }\end{array}$ & $\begin{array}{l}\text { Intervention } \\
\text { and } \\
\text { Comparator } \\
\text { Descriptions }\end{array}$ & $\begin{array}{l}\text { Study } \\
\text { Design }\end{array}$ & $\begin{array}{l}\text { Randomi- } \\
\text { zation } \\
\text { method } \\
\text { adequate? } \\
\text { (RCTs } \\
\text { only) }\end{array}$ & $\begin{array}{l}\text { Allocation } \\
\text { conceal- } \\
\text { ment ad- } \\
\text { equate? } \\
\text { (RCTs } \\
\text { only) }\end{array}$ & $\begin{array}{l}\text { Recruitment } \\
\text { strategy for } \\
\text { study } \\
\text { different } \\
\text { across } \\
\text { groups? }\end{array}$ & $\begin{array}{l}\text { Groups } \\
\text { similar at } \\
\text { baseline, } \\
\text { or } \\
\text { differences } \\
\text { adjusted } \\
\text { for in } \\
\text { analysis? }\end{array}$ & $\begin{array}{l}\text { Outcome } \\
\text { asse- } \\
\text { ssors } \\
\text { blinded? } \\
\text { (RCTs or } \\
\text { NRCTs } \\
\text { only) }\end{array}$ & $\begin{array}{l}\text { Impact from } \\
\text { concurrent } \\
\text { intervention } \\
\text { or unintended } \\
\text { exposure } \\
\text { ruled out by } \\
\text { researchers? }\end{array}$ & $\begin{array}{l}\text { Did variation } \\
\text { from study } \\
\text { protocol } \\
\text { compromise } \\
\text { conclusions? } \\
\text { (RCTs or } \\
\text { NRCTs only) }\end{array}$ & $\begin{array}{l}\text { High } \\
\text { overall } \\
\text { (i.e., } \geq 20 \% \text { ) } \\
\text { or } \\
\text { ? differential } \\
\text { (i.e., } \geq 15 \% \text { ) } \\
\text { attrition }\end{array}$ & $\begin{array}{l}\text { Did attrition } \\
\text { result in } \\
\text { differences } \\
\text { in group } \\
\text { charac- } \\
\text { teristics? }\end{array}$ \\
\hline $\begin{array}{l}\text { Gattis et al., } \\
1999^{13}\end{array}$ & $\begin{array}{l}\text { G1: Clinical } \\
\text { pharmacy } \\
\text { services, } \\
\text { including } \\
\text { assessment of } \\
\text { prescribed } \\
\text { regimen, } \\
\text { compliance, and } \\
\text { adverse effects, } \\
\text { and symptoms } \\
\text { and response to } \\
\text { therapy. } \\
\text { Providing patient } \\
\text { education about } \\
\text { purpose of each } \\
\text { drug and } \\
\text { reinforcing } \\
\text { adherence. } \\
\text { Detailed written } \\
\text { information also } \\
\text { provided to } \\
\text { patients. } \\
\text { G2: Usual } \\
\text { medical care }\end{array}$ & $\begin{array}{l}\text { RCT: } \\
\text { parallel, } \\
\text { not } \\
\text { clustered } \\
\\
\end{array}$ & Yes & Yes & No & Yes & No & No & No & No & No \\
\hline $\begin{array}{l}\text { Hanlon et al., } \\
1996^{14}\end{array}$ & $\begin{array}{l}\text { G1: } \\
\text { Pharmaceutical } \\
\text { care provided by } \\
\text { clinical } \\
\text { pharmacist. } \\
\text { G2: Usual care } \\
\text { in the General } \\
\text { Medicine Clinic }\end{array}$ & $\begin{array}{l}\text { RCT: } \\
\text { parallel, } \\
\text { not } \\
\text { clustered } \\
\end{array}$ & Yes & $\begin{array}{l}\text { Unclear or } \\
\text { NR }\end{array}$ & No & Yes & Yes & Unclear or NR & Unclear or NR & No & Unclear or NR \\
\hline
\end{tabular}


Table F1. Risk of bias domains and ratings (continued)

\begin{tabular}{|c|c|c|c|c|c|c|c|c|c|c|c|}
\hline $\begin{array}{l}\text { Author, Year } \\
\text { Trial Name }\end{array}$ & $\begin{array}{l}\text { Intervention } \\
\text { and } \\
\text { Comparator } \\
\text { Descriptions }\end{array}$ & $\begin{array}{l}\text { Study } \\
\text { Design }\end{array}$ & $\begin{array}{l}\text { Randomi- } \\
\text { zation } \\
\text { method } \\
\text { adequate? } \\
\text { (RCTs } \\
\text { only) }\end{array}$ & $\begin{array}{l}\text { Allocation } \\
\text { conceal- } \\
\text { ment ad- } \\
\text { equate? } \\
\text { (RCTs } \\
\text { only) }\end{array}$ & $\begin{array}{l}\text { Recruitment } \\
\text { strategy for } \\
\text { study } \\
\text { different } \\
\text { across } \\
\text { groups? }\end{array}$ & $\begin{array}{l}\text { Groups } \\
\text { similar at } \\
\text { baseline, } \\
\text { or } \\
\text { differences } \\
\text { adjusted } \\
\text { for in } \\
\text { analysis? }\end{array}$ & $\begin{array}{l}\text { Outcome } \\
\text { asse- } \\
\text { ssors } \\
\text { blinded? } \\
\text { (RCTs or } \\
\text { NRCTs } \\
\text { only) }\end{array}$ & $\begin{array}{l}\text { Impact from } \\
\text { concurrent } \\
\text { intervention } \\
\text { or unintended } \\
\text { exposure } \\
\text { ruled out by } \\
\text { researchers? }\end{array}$ & $\begin{array}{l}\text { Did variation } \\
\text { from study } \\
\text { protocol } \\
\text { compromise } \\
\text { conclusions? } \\
\text { (RCTs or } \\
\text { NRCTs only) }\end{array}$ & $\begin{array}{l}\text { High } \\
\text { overall } \\
\text { (i.e., } \geq 20 \% \text { ) } \\
\text { or } \\
\text { ? differential } \\
\text { (i.e., } \geq 15 \% \text { ) } \\
\text { attrition }\end{array}$ & $\begin{array}{l}\text { Did attrition } \\
\text { result in } \\
\text { differences } \\
\text { in group } \\
\text { charac- } \\
\text { teristics? }\end{array}$ \\
\hline $\begin{array}{l}\text { Harrison et al., } \\
2012^{15}\end{array}$ & $\begin{array}{l}\text { G1: } \\
\text { Pharmaceutical } \\
\text { care provided by } \\
\text { a clinical } \\
\text { pharmacist for } \\
\text { the purpose of } \\
\text { identifying and } \\
\text { resolving actual } \\
\text { and potential } \\
\text { DTPs, } \\
\text { medication } \\
\text { teaching, } \\
\text { adherence } \\
\text { optimization, } \\
\text { medication } \\
\text { reconciliation, } \\
\text { and provision of } \\
\text { drug information. } \\
\text { G2: } \\
\text { Retrospective } \\
\text { historical control } \\
\text { of matched } \\
\text { patients who } \\
\text { received } \\
\text { standard care at } \\
\text { a routine } \\
\text { medical visit } \\
\text { within } 8 \text { months } \\
\text { prior to study } \\
\text { period }\end{array}$ & Cohort & NA & NA & Yes & Yes & $\begin{array}{l}\text { Unclear or } \\
\text { NR }\end{array}$ & No & Unclear or NR & No & No \\
\hline
\end{tabular}


Table F1. Risk of bias domains and ratings (continued)

\begin{tabular}{|c|c|c|c|c|c|c|c|c|c|c|c|}
\hline $\begin{array}{l}\text { Author, Year } \\
\text { Trial Name }\end{array}$ & $\begin{array}{l}\text { Intervention } \\
\text { and } \\
\text { Comparator } \\
\text { Descriptions }\end{array}$ & $\begin{array}{l}\text { Study } \\
\text { Design }\end{array}$ & $\begin{array}{l}\text { Randomi- } \\
\text { zation } \\
\text { method } \\
\text { adequate? } \\
\text { (RCTs } \\
\text { only) }\end{array}$ & $\begin{array}{l}\text { Allocation } \\
\text { conceal- } \\
\text { ment ad- } \\
\text { equate? } \\
\text { (RCTs } \\
\text { only) }\end{array}$ & $\begin{array}{l}\text { Recruitment } \\
\text { strategy for } \\
\text { study } \\
\text { different } \\
\text { across } \\
\text { groups? }\end{array}$ & $\begin{array}{l}\text { Groups } \\
\text { similar at } \\
\text { baseline, } \\
\text { or } \\
\text { differences } \\
\text { adjusted } \\
\text { for in } \\
\text { analysis? }\end{array}$ & $\begin{array}{l}\text { Outcome } \\
\text { asse- } \\
\text { ssors } \\
\text { blinded? } \\
\text { (RCTs or } \\
\text { NRCTs } \\
\text { only) }\end{array}$ & $\begin{array}{l}\text { Impact from } \\
\text { concurrent } \\
\text { intervention } \\
\text { or unintended } \\
\text { exposure } \\
\text { ruled out by } \\
\text { researchers? }\end{array}$ & $\begin{array}{l}\text { Did variation } \\
\text { from study } \\
\text { protocol } \\
\text { compromise } \\
\text { conclusions? } \\
\text { (RCTs or } \\
\text { NRCTs only) }\end{array}$ & $\begin{array}{l}\text { High } \\
\text { overall } \\
\text { (i.e., } \geq 20 \% \text { ) } \\
\text { or } \\
\text { ? differential } \\
\text { (i.e., } \geq 15 \% \text { ) } \\
\text { attrition }\end{array}$ & $\begin{array}{l}\text { Did attrition } \\
\text { result in } \\
\text { differences } \\
\text { in group } \\
\text { charac- } \\
\text { teristics? }\end{array}$ \\
\hline $\begin{array}{l}\text { Isetts et al., } \\
2008^{16}\end{array}$ & $\begin{array}{l}\text { G1: MTM } \\
\text { services } \\
\text { provided by staff } \\
\text { pharmacists, } \\
\text { including the } \\
\text { establishment of } \\
\text { goals of therapy, } \\
\text { in collaboration } \\
\text { with primary } \\
\text { care providers. } \\
\text { G2: Usual } \\
\text { medical care } \\
\text { without MTM. } \\
\end{array}$ & Cohort & NA & NA & Yes & $\begin{array}{l}\text { Unclear or } \\
\text { NR }\end{array}$ & No & No & Unclear or NR & $\begin{array}{l}\text { Unclear or } \\
\text { NR }\end{array}$ & Unclear or NR \\
\hline $\begin{array}{l}\text { Jameson et al., } \\
1995^{17}\end{array}$ & $\begin{array}{l}\text { G1: Pharmaco- } \\
\text { therapy } \\
\text { consultation and } \\
\text { follow-up } \\
\text { provided by } \\
\text { clinical } \\
\text { ambulatory care } \\
\text { pharmacist. } \\
\text { G2: Standard } \\
\text { office-based } \\
\text { primary care. }\end{array}$ & $\begin{array}{l}\text { RCT: } \\
\text { parallel, } \\
\text { not } \\
\text { clustered } \\
\end{array}$ & Yes & $\begin{array}{l}\text { Unclear or } \\
\text { NR }\end{array}$ & No & Yes & No & Unclear or NR & No & No & Unclear or NR \\
\hline
\end{tabular}


Table F1. Risk of bias domains and ratings (continued)

\begin{tabular}{|c|c|c|c|c|c|c|c|c|c|c|c|}
\hline $\begin{array}{l}\text { Author, Year } \\
\text { Trial Name }\end{array}$ & $\begin{array}{l}\text { Intervention } \\
\text { and } \\
\text { Comparator } \\
\text { Descriptions }\end{array}$ & $\begin{array}{l}\text { Study } \\
\text { Design }\end{array}$ & $\begin{array}{l}\text { Randomi- } \\
\text { zation } \\
\text { method } \\
\text { adequate? } \\
\text { (RCTs } \\
\text { only) }\end{array}$ & $\begin{array}{l}\text { Allocation } \\
\text { conceal- } \\
\text { ment ad- } \\
\text { equate? } \\
\text { (RCTs } \\
\text { only) }\end{array}$ & $\begin{array}{l}\text { Recruitment } \\
\text { strategy for } \\
\text { study } \\
\text { different } \\
\text { across } \\
\text { groups? }\end{array}$ & $\begin{array}{l}\text { Groups } \\
\text { similar at } \\
\text { baseline, } \\
\text { or } \\
\text { differences } \\
\text { adjusted } \\
\text { for in } \\
\text { analysis? }\end{array}$ & $\begin{array}{l}\text { Outcome } \\
\text { asse- } \\
\text { ssors } \\
\text { blinded? } \\
\text { (RCTs or } \\
\text { NRCTs } \\
\text { only) }\end{array}$ & $\begin{array}{l}\text { Impact from } \\
\text { concurrent } \\
\text { intervention } \\
\text { or unintended } \\
\text { exposure } \\
\text { ruled out by } \\
\text { researchers? }\end{array}$ & $\begin{array}{l}\text { Did variation } \\
\text { from study } \\
\text { protocol } \\
\text { compromise } \\
\text { conclusions? } \\
\text { (RCTs or } \\
\text { NRCTs only) }\end{array}$ & $\begin{array}{l}\text { High } \\
\text { overall } \\
\text { (i.e., } \geq 20 \% \text { ) } \\
\text { or } \\
\text { ? differential } \\
\text { (i.e., } \geq 15 \% \text { ) } \\
\text { attrition }\end{array}$ & $\begin{array}{l}\text { Did attrition } \\
\text { result in } \\
\text { differences } \\
\text { in group } \\
\text { charac- } \\
\text { teristics? }\end{array}$ \\
\hline $\begin{array}{l}\text { Jeong et al., } \\
2007^{18}\end{array}$ & $\begin{array}{l}\text { G1: Pharmacist- } \\
\text { managed MTMP } \\
\text { provided by } \\
\text { ambulatory care } \\
\text { pharmacists and } \\
\text { healthcare } \\
\text { support staff } \\
\text { (acceptors) } \\
\text { G2: Eligible for } \\
\text { Part D MTMP } \\
\text { but declined } \\
\text { enrollment } \\
\text { (refusers) } \\
\text { G3: Patients } \\
\text { without Part D } \\
\text { as their primary } \\
\text { drug benefit }\end{array}$ & Cohort & NA & NA & Yes & $\begin{array}{l}\text { Unclear or } \\
\text { NR }\end{array}$ & $\begin{array}{l}\text { Unclear or } \\
\text { NR }\end{array}$ & No & NA & Yes & Unclear or NR \\
\hline $\begin{array}{l}\text { Krska et al., } \\
2001^{19}\end{array}$ & $\begin{array}{l}\text { G1: Medication } \\
\text { reviews led by } \\
\text { clinically-trained } \\
\text { pharmacists. } \\
\text { G2: Usual care } \\
\text { involving } \\
\text { interviews and } \\
\text { identification of } \\
\text { pharmaceutical } \\
\text { care issues but } \\
\text { with no } \\
\text { pharmaceutical } \\
\text { care plan } \\
\text { implemented. }\end{array}$ & $\begin{array}{l}\text { RCT: } \\
\text { parallel, } \\
\text { not } \\
\text { clustered } \\
\end{array}$ & $\begin{array}{l}\text { Unclear or } \\
\text { NR }\end{array}$ & $\begin{array}{l}\text { Unclear or } \\
\text { NR }\end{array}$ & No & No & $\begin{array}{l}\text { Unclear or } \\
\text { NR }\end{array}$ & Unclear or NR & Unclear or NR & No & Unclear or NR \\
\hline
\end{tabular}


Table F1. Risk of bias domains and ratings (continued)

\begin{tabular}{|c|c|c|c|c|c|c|c|c|c|c|c|}
\hline $\begin{array}{l}\text { Author, Year } \\
\text { Trial Name }\end{array}$ & $\begin{array}{l}\text { Intervention } \\
\text { and } \\
\text { Comparator } \\
\text { Descriptions }\end{array}$ & $\begin{array}{l}\text { Study } \\
\text { Design }\end{array}$ & $\begin{array}{l}\text { Randomi- } \\
\text { zation } \\
\text { method } \\
\text { adequate? } \\
\text { (RCTs } \\
\text { only) }\end{array}$ & $\begin{array}{l}\text { Allocation } \\
\text { conceal- } \\
\text { ment ad- } \\
\text { ? equate? } \\
\text { (RCTs } \\
\text { only) }\end{array}$ & $\begin{array}{l}\text { Recruitment } \\
\text { strategy for } \\
\text { study } \\
\text { different } \\
\text { across } \\
\text { groups? }\end{array}$ & $\begin{array}{l}\text { Groups } \\
\text { similar at } \\
\text { baseline, } \\
\text { or } \\
\text { differences } \\
\text { adjusted } \\
\text { for in } \\
\text { analysis? }\end{array}$ & $\begin{array}{l}\text { Outcome } \\
\text { asse- } \\
\text { ssors } \\
\text { blinded? } \\
\text { (RCTs or } \\
\text { NRCTs } \\
\text { only) }\end{array}$ & $\begin{array}{l}\text { Impact from } \\
\text { concurrent } \\
\text { intervention } \\
\text { or unintended } \\
\text { exposure } \\
\text { ruled out by } \\
\text { researchers? }\end{array}$ & $\begin{array}{l}\text { Did variation } \\
\text { from study } \\
\text { protocol } \\
\text { compromise } \\
\text { conclusions? } \\
\text { (RCTs or } \\
\text { NRCTs only) }\end{array}$ & $\begin{array}{l}\text { High } \\
\text { overall } \\
\text { (i.e., } \geq 20 \% \text { ) } \\
\text { or } \\
\text { ? differential } \\
\text { (i.e., } \geq 15 \% \text { ) } \\
\text { attrition }\end{array}$ & $\begin{array}{l}\text { Did attrition } \\
\text { result in } \\
\text { differences } \\
\text { in group } \\
\text { charac- } \\
\text { teristics? }\end{array}$ \\
\hline $\begin{array}{l}\text { Malone et al., } \\
2000^{20} ; \\
\text { Ellis, } 2000^{21} ; \\
\text { Malone, } 2001^{22} \text {; } \\
\text { Ellis, } 2000^{23} \\
\text { IMPROVE }\end{array}$ & $\begin{array}{l}\text { G1: } \\
\text { Pharmaceutical } \\
\text { care provided by } \\
\text {; clinical } \\
\text { pharmacists } \\
\text { practicing } \\
\text { according to } \\
\text { scope of practice } \\
\text { within their } \\
\text { respective health } \\
\text { care facilities } \\
\text { G2: Usual care } \\
\text { without } \\
\text { pharmaceutical } \\
\text { care }\end{array}$ & $\begin{array}{l}\text { RCT: } \\
\text { parallel, } \\
\text { not } \\
\text { clustered } \\
\end{array}$ & Yes & $\begin{array}{l}\text { Unclear or } \\
\text { NR }\end{array}$ & No & Yes & \multicolumn{2}{|c|}{$\begin{array}{l}\text { Unclear or No } \\
\text { NR }\end{array}$} & No & No & Unclear or NR \\
\hline $\begin{array}{l}\text { McDonough et } \\
\text { al., 2005 } \\
\end{array}$ & $\begin{array}{l}\text { G1: } \\
\text { Pharmaceutical } \\
\text { care provided by } \\
\text { community } \\
\text { pharmacists. } \\
\text { Drug therapy } \\
\text { monitoring } \\
\text { focused on } 5 \\
\text { DTPs: } \\
\text { appropriateness } \\
\text { of dose, proper } \\
\text { regimen, } \\
\text { potential } \\
\text { interactions, } \\
\text { nonadherence, } \\
\text { and adverse } \\
\text { effects. Patient } \\
\text { education also } \\
\text { provided. } \\
\text { G2: Usual care }\end{array}$ & $\begin{array}{l}\text { RCT: } \\
\text { cluster- } \\
\text { rando- } \\
\text { mized }\end{array}$ & $\begin{array}{l}\text { Unclear or } \\
\text { NR }\end{array}$ & $\begin{array}{l}\text { Unclear or } \\
\text { NR }\end{array}$ & No & No & \multicolumn{2}{|c|}{$\begin{array}{l}\text { Unclear or No } \\
\text { NR }\end{array}$} & \multicolumn{2}{|c|}{ Unclear or NR No } & Unclear or NR \\
\hline
\end{tabular}


Table F1. Risk of bias domains and ratings (continued)

\begin{tabular}{|c|c|c|c|c|c|c|c|c|c|c|c|}
\hline $\begin{array}{l}\text { Author, Year } \\
\text { Trial Name }\end{array}$ & $\begin{array}{l}\text { Intervention } \\
\text { and } \\
\text { Comparator } \\
\text { Descriptions }\end{array}$ & $\begin{array}{l}\text { Study } \\
\text { Design }\end{array}$ & $\begin{array}{l}\text { Randomi- } \\
\text { zation } \\
\text { method } \\
\text { adequate? } \\
\text { (RCTs } \\
\text { only) }\end{array}$ & $\begin{array}{l}\text { Allocation } \\
\text { conceal- } \\
\text { ment ad- } \\
\text { ? equate? } \\
\text { (RCTs } \\
\text { only) }\end{array}$ & $\begin{array}{l}\text { Recruitment } \\
\text { strategy for } \\
\text { study } \\
\text { different } \\
\text { across } \\
\text { groups? }\end{array}$ & $\begin{array}{l}\text { Groups } \\
\text { similar at } \\
\text { baseline, } \\
\text { or } \\
\text { differences } \\
\text { adjusted } \\
\text { for in } \\
\text { analysis? }\end{array}$ & $\begin{array}{l}\text { Outcome } \\
\text { asse- } \\
\text { ssors } \\
\text { blinded? } \\
\text { (RCTs or } \\
\text { NRCTs } \\
\text { only) }\end{array}$ & $\begin{array}{l}\text { Impact from } \\
\text { concurrent } \\
\text { intervention } \\
\text { or unintended } \\
\text { exposure } \\
\text { ruled out by } \\
\text { researchers? }\end{array}$ & $\begin{array}{l}\text { Did variation } \\
\text { from study } \\
\text { protocol } \\
\text { compromise } \\
\text { conclusions? } \\
\text { (RCTs or } \\
\text { NRCTs only) }\end{array}$ & $\begin{array}{l}\text { High } \\
\text { overall } \\
\text { (i.e., } \geq 20 \% \text { ) } \\
\text { or } \\
\text { ? differential } \\
\text { (i.e., } \geq 15 \% \text { ) } \\
\text { attrition }\end{array}$ & $\begin{array}{l}\text { Did attrition } \\
\text { result in } \\
\text { differences } \\
\text { in group } \\
\text { charac- } \\
\text { teristics? }\end{array}$ \\
\hline $\begin{array}{l}\text { Moczygemba } \epsilon \\
\text { al., 2011 } \\
\text { Moczygemba } \\
\text { al., } 2008^{26}\end{array}$ & $\begin{array}{l}\text { tG1: Opt-in } \\
\text { telephone-based } \\
\text { tMTM program, } \\
\text { in which MTM } \\
\text { services } \\
\text { provided by } \\
\text { clinical } \\
\text { pharmacists or } \\
\text { managed care } \\
\text { pharmacy } \\
\text { resident based } \\
\text { on American } \\
\text { Pharmacists } \\
\text { Association and } \\
\text { National } \\
\text { Association of } \\
\text { Chain Drug } \\
\text { Stores } \\
\text { Foundation MTM } \\
\text { framework } \\
\text { (acceptors) } \\
\text { G2: No-MTM } \\
\text { control group } \\
\text { (opt-out) }\end{array}$ & Cohort & NA & NA & Yes & Yes & $\begin{array}{l}\text { Unclear or } \\
\text { NR }\end{array}$ & Unclear or NR & Unclear or NR & Yes & Unclear or NR \\
\hline
\end{tabular}


Table F1. Risk of bias domains and ratings (continued)

\begin{tabular}{|c|c|c|c|c|c|c|c|c|c|c|c|}
\hline $\begin{array}{l}\text { Author, Year } \\
\text { Trial Name }\end{array}$ & $\begin{array}{l}\text { Intervention } \\
\text { and } \\
\text { Comparator } \\
\text { Descriptions }\end{array}$ & $\begin{array}{l}\text { Study } \\
\text { Design }\end{array}$ & $\begin{array}{l}\text { Randomi- } \\
\text { zation } \\
\text { method } \\
\text { adequate? } \\
\text { (RCTs } \\
\text { only) }\end{array}$ & $\begin{array}{l}\text { Allocation } \\
\text { conceal- } \\
\text { ment ad- } \\
\text { ? equate? } \\
\text { (RCTs } \\
\text { only) }\end{array}$ & $\begin{array}{l}\text { Recruitment } \\
\text { strategy for } \\
\text { study } \\
\text { different } \\
\text { across } \\
\text { groups? }\end{array}$ & $\begin{array}{l}\text { Groups } \\
\text { similar at } \\
\text { baseline, } \\
\text { or } \\
\text { differences } \\
\text { adjusted } \\
\text { for in } \\
\text { analysis? }\end{array}$ & $\begin{array}{l}\text { Outcome } \\
\text { asse- } \\
\text { ssors } \\
\text { blinded? } \\
\text { (RCTs or } \\
\text { NRCTs } \\
\text { only) }\end{array}$ & $\begin{array}{l}\text { Impact from } \\
\text { concurrent } \\
\text { intervention } \\
\text { or unintended } \\
\text { exposure } \\
\text { ruled out by } \\
\text { researchers? }\end{array}$ & $\begin{array}{l}\text { Did variation } \\
\text { from study } \\
\text { protocol } \\
\text { compromise } \\
\text { conclusions? } \\
\text { (RCTs or } \\
\text { NRCTs only) }\end{array}$ & $\begin{array}{l}\text { High } \\
\text { overall } \\
\text { (i.e., } \geq 20 \% \text { ) } \\
\text { or } \\
\text { ? differential } \\
\text { (i.e., } \geq 15 \% \text { ) } \\
\text { attrition }\end{array}$ & $\begin{array}{l}\text { Did attrition } \\
\text { result in } \\
\text { differences } \\
\text { in group } \\
\text { charac- } \\
\text { teristics? }\end{array}$ \\
\hline $\begin{array}{l}\text { Pai et al., } \\
2009^{27} \text {; } \\
\text { Pai et al., } \\
2009^{28}\end{array}$ & $\begin{array}{l}\text { G1: } \\
\text { Pharmaceutical } \\
\text { care including } \\
\text { drug therapy } \\
\text { reviews } \\
\text { conducted by } \\
\text { nephrology- } \\
\text { trained clinical } \\
\text { pharmacist with } \\
\text { patient. Also } \\
\text { included patient } \\
\text { and health care } \\
\text { provider } \\
\text { education. } \\
\text { G2: Standard of } \\
\text { care, consisting } \\
\text { of brief therapy } \\
\text { reviews } \\
\text { conducted by } \\
\text { nurse }\end{array}$ & $\begin{array}{l}\text { RCT: } \\
\text { cluster- } \\
\text { rando- } \\
\text { mized }\end{array}$ & No & Yes & No & Yes & $\begin{array}{l}\text { Unclear or } \\
\text { NR }\end{array}$ & Unclear or NR & Unclear or NR & Yes & No \\
\hline $\begin{array}{l}\text { Park et al., } \\
1996^{29}\end{array}$ & $\begin{array}{l}\text { G1: } \\
\text { Comprehensive } \\
\text { pharmaceutical } \\
\text { services, } \\
\text { including drug } \\
\text { therapy } \\
\text { monitoring and } \\
\text { patient } \\
\text { education } \\
\text { provided by } \\
\text { community } \\
\text { pharmacy } \\
\text { resident. } \\
\text { G2: Usual care }\end{array}$ & $\begin{array}{l}\text { RCT: } \\
\text { parallel, } \\
\text { not } \\
\text { clustered }\end{array}$ & $\begin{array}{l}\text { Unclear or } \\
\text { NR }\end{array}$ & $\begin{array}{l}\text { Unclear or } \\
\text { NR }\end{array}$ & No & No & No & Unclear or NR & No & No & Unclear or NR \\
\hline
\end{tabular}


Table F1. Risk of bias domains and ratings (continued)

\begin{tabular}{|c|c|c|c|c|c|c|c|c|c|c|c|}
\hline $\begin{array}{l}\text { Author, Year } \\
\text { Trial Name }\end{array}$ & $\begin{array}{l}\text { Intervention } \\
\text { and } \\
\text { Comparator } \\
\text { Descriptions }\end{array}$ & $\begin{array}{l}\text { Study } \\
\text { Design }\end{array}$ & $\begin{array}{l}\text { Randomi- } \\
\text { zation } \\
\text { method } \\
\text { adequate? } \\
\text { (RCTs } \\
\text { only) }\end{array}$ & $\begin{array}{l}\text { Allocation } \\
\text { conceal- } \\
\text { ment ad- } \\
\text { equate? } \\
\text { (RCTs } \\
\text { only) }\end{array}$ & $\begin{array}{l}\text { Recruitment } \\
\text { strategy for } \\
\text { study } \\
\text { different } \\
\text { across } \\
\text { groups? }\end{array}$ & $\begin{array}{l}\text { Groups } \\
\text { similar at } \\
\text { baseline, } \\
\text { or } \\
\text { differences } \\
\text { adjusted } \\
\text { for in } \\
\text { analysis? }\end{array}$ & $\begin{array}{l}\text { Outcome } \\
\text { asse- } \\
\text { ssors } \\
\text { blinded? } \\
\text { (RCTs or } \\
\text { NRCTs } \\
\text { only) }\end{array}$ & $\begin{array}{l}\text { Impact from } \\
\text { concurrent } \\
\text { intervention } \\
\text { or unintended } \\
\text { exposure } \\
\text { ruled out by } \\
\text { researchers? }\end{array}$ & $\begin{array}{l}\text { Did variation } \\
\text { from study } \\
\text { protocol } \\
\text { compromise } \\
\text { conclusions? } \\
\text { (RCTs or } \\
\text { NRCTs only) }\end{array}$ & $\begin{array}{l}\text { High } \\
\text { overall } \\
\text { (i.e., } \geq 20 \% \text { ) } \\
\text { or } \\
\text { ? differential } \\
\text { (i.e., } \geq 15 \% \text { ) } \\
\text { attrition }\end{array}$ & $\begin{array}{l}\text { Did attrition } \\
\text { result in } \\
\text { differences } \\
\text { in group } \\
\text { charac- } \\
\text { teristics? }\end{array}$ \\
\hline $\begin{array}{l}\text { Pindolia et al., } \\
2009^{30}\end{array}$ & $\begin{array}{l}\text { G1: Telephone- } \\
\text { based MTM } \\
\text { services } \\
\text { provided as part } \\
\text { of Medicare Part } \\
\text { D MTM program } \\
\text { by pharmacy } \\
\text { care } \\
\text { management } \\
\text { clinical } \\
\text { pharmacists } \\
\text { (acceptors) } \\
\text { G2: Usual } \\
\text { medical care } \\
\text { (opt-out) }\end{array}$ & Cohort & NA & NA & Yes & No & NA & Unclear or NR & NA & $\begin{array}{l}\text { Unclear or } \\
\text { NR }\end{array}$ & Unclear or NR \\
\hline $\begin{array}{l}\text { Planas et al., } \\
2009^{31}\end{array}$ & $\begin{array}{l}\text { G1: MTM } \\
\text { services } \\
\text { provided by } \\
\text { community } \\
\text { pharmacists. } \\
\text { Also included } \\
\text { patient } \\
\text { education on } \\
\text { diet and lifestyle } \\
\text { modifications to } \\
\text { lower blood } \\
\text { pressure. } \\
\text { G2: No MTM } \\
\text { received, but } \\
\text { only informed of } \\
\text { blood pressure } \\
\text { goals for } \\
\text { patients with } \\
\text { diabetes }\end{array}$ & $\begin{array}{l}\text { RCT: } \\
\text { parallel, } \\
\text { not } \\
\text { clustered } \\
\end{array}$ & Yes & Yes & No & No & $\begin{array}{l}\text { Unclear or } \\
\text { NR }\end{array}$ & Unclear or NR & Unclear or NR & Yes & Unclear or NR \\
\hline
\end{tabular}


Table F1. Risk of bias domains and ratings (continued)

\begin{tabular}{|c|c|c|c|c|c|c|c|c|c|c|c|}
\hline $\begin{array}{l}\text { Author, Year } \\
\text { Trial Name }\end{array}$ & $\begin{array}{l}\text { Intervention } \\
\text { and } \\
\text { Comparator } \\
\text { Descriptions }\end{array}$ & $\begin{array}{l}\text { Study } \\
\text { Design }\end{array}$ & $\begin{array}{l}\text { Randomi- } \\
\text { zation } \\
\text { method } \\
\text { adequate? } \\
\text { (RCTs } \\
\text { only) }\end{array}$ & $\begin{array}{l}\text { Allocation } \\
\text { conceal- } \\
\text { ment ad- } \\
\text { equate? } \\
\text { (RCTs } \\
\text { only) }\end{array}$ & $\begin{array}{l}\text { Recruitment } \\
\text { strategy for } \\
\text { study } \\
\text { different } \\
\text { across } \\
\text { groups? }\end{array}$ & $\begin{array}{l}\text { Groups } \\
\text { similar at } \\
\text { baseline, } \\
\text { or } \\
\text { differences } \\
\text { adjusted } \\
\text { for in } \\
\text { analysis? }\end{array}$ & $\begin{array}{l}\text { Outcome } \\
\text { asse- } \\
\text { ssors } \\
\text { blinded? } \\
\text { (RCTs or } \\
\text { NRCTs } \\
\text { only) }\end{array}$ & $\begin{array}{l}\text { Impact from } \\
\text { concurrent } \\
\text { intervention } \\
\text { or unintended } \\
\text { exposure } \\
\text { ruled out by } \\
\text { researchers? }\end{array}$ & $\begin{array}{l}\text { Did variation } \\
\text { from study } \\
\text { protocol } \\
\text { compromise } \\
\text { conclusions? } \\
\text { (RCTs or } \\
\text { NRCTs only) }\end{array}$ & $\begin{array}{l}\text { High } \\
\text { overall } \\
\text { (i.e., } \geq 20 \% \text { ) } \\
\text { or } \\
\text { ? differential } \\
\text { (i.e., } \geq 15 \% \text { ) } \\
\text { attrition }\end{array}$ & $\begin{array}{l}\text { Did attrition } \\
\text { result in } \\
\text { differences } \\
\text { in group } \\
\text { charac- } \\
\text { teristics? }\end{array}$ \\
\hline $\begin{array}{l}\text { Roughead et } \\
\text { al., } 2009^{32}\end{array}$ & $\begin{array}{l}\text { G1: HMRs } \\
\text { conducted by } \\
\text { accredited } \\
\text { pharmacists } \\
\text { G2: No } \\
\text { medication } \\
\text { review received }\end{array}$ & Cohort & NA & NA & No & Yes & $\begin{array}{l}\text { Unclear or } \\
\text { NR }\end{array}$ & Unclear or NR & NA & $\begin{array}{l}\text { Unclear or } \\
\text { NR }\end{array}$ & Unclear or NR \\
\hline $\begin{array}{l}\text { Sellors et al., } \\
2003^{33}\end{array}$ & $\begin{array}{l}\text { G1: Clinical } \\
\text { pharmacist } \\
\text { consultations } \\
\text { provided to } \\
\text { family physicians } \\
\text { and their } \\
\text { patients by } \\
\text { community } \\
\text { pharmacists. } \\
\text { G2: Usual care } \\
\text { for family } \\
\text { physicians and } \\
\text { their patients } \\
\text { from matched } \\
\text { postal codes. }\end{array}$ & $\begin{array}{l}\text { RCT: } \\
\text { cluster- } \\
\text { rando- } \\
\text { mized } \\
5\end{array}$ & Yes & Yes & No & Yes & Yes & No & Unclear or NR & No & Unclear or NR \\
\hline
\end{tabular}


Table F1. Risk of bias domains and ratings (continued)

\begin{tabular}{|c|c|c|c|c|c|c|c|c|c|c|c|}
\hline $\begin{array}{l}\text { Author, Year } \\
\text { Trial Name }\end{array}$ & $\begin{array}{l}\text { Intervention } \\
\text { and } \\
\text { Comparator } \\
\text { Descriptions }\end{array}$ & $\begin{array}{l}\text { Study } \\
\text { Design }\end{array}$ & $\begin{array}{l}\text { Randomi- } \\
\text { zation } \\
\text { method } \\
\text { adequate? } \\
\text { (RCTs } \\
\text { only) }\end{array}$ & $\begin{array}{l}\text { Allocation } \\
\text { conceal- } \\
\text { ment ad- } \\
\text { ? equate? } \\
\text { (RCTs } \\
\text { only) }\end{array}$ & $\begin{array}{l}\text { Recruitment } \\
\text { strategy for } \\
\text { study } \\
\text { different } \\
\text { across } \\
\text { groups? }\end{array}$ & $\begin{array}{l}\text { Groups } \\
\text { similar at } \\
\text { baseline, } \\
\text { or } \\
\text { differences } \\
\text { adjusted } \\
\text { for in } \\
\text { analysis? }\end{array}$ & $\begin{array}{l}\text { Outcome } \\
\text { asse- } \\
\text { ssors } \\
\text { blinded? } \\
\text { (RCTs or } \\
\text { NRCTs } \\
\text { only) }\end{array}$ & $\begin{array}{l}\text { Impact from } \\
\text { concurrent } \\
\text { intervention } \\
\text { or unintended } \\
\text { exposure } \\
\text { ruled out by } \\
\text { researchers? }\end{array}$ & $\begin{array}{l}\text { Did variation } \\
\text { from study } \\
\text { protocol } \\
\text { compromise } \\
\text { conclusions? } \\
\text { (RCTs or } \\
\text { NRCTs only) }\end{array}$ & $\begin{array}{l}\text { High } \\
\text { overall } \\
\text { (i.e., } \geq 20 \% \text { ) } \\
\text { or } \\
\text { ? differential } \\
\text { (i.e., } \geq 15 \% \text { ) } \\
\text { attrition }\end{array}$ & $\begin{array}{l}\text { Did attrition } \\
\text { result in } \\
\text { differences } \\
\text { in group } \\
\text { charac- } \\
\text { teristics? }\end{array}$ \\
\hline $\begin{array}{l}\text { Sidel et al., } \\
1990^{34}\end{array}$ & $\begin{array}{l}\text { G1: Home visits } \\
\text { by pharmacists } \\
\text { and, when } \\
\text { needed, } \\
\text { consultations } \\
\text { with physicians } \\
\text { to identify and } \\
\text { correct problems } \\
\text { associated with } \\
\text { medication use. } \\
\text { G2: Standard } \\
\text { care without any } \\
\text { visits or } \\
\text { information } \\
\text { provided to G1. }\end{array}$ & $\begin{array}{l}\text { RCT: } \\
\text { parallel, } \\
\text { not } \\
\text { clustered } \\
\end{array}$ & $\begin{array}{l}\text { Unclear or } \\
\text { NR }\end{array}$ & $\begin{array}{l}\text { Unclear or } \\
\text { NR }\end{array}$ & No & Yes & Yes & No & NA & Yes & Unclear or NR \\
\hline $\begin{array}{l}\text { Staresinic et } \\
\text { al., } 2007^{35}\end{array}$ & $\begin{array}{l}\text { G1: MTM } \\
\text { services } \\
\text { provided as part } \\
\text { of a Medicare } \\
\text { Part D MTM } \\
\text { program by } \\
\text { MTM } \\
\text { Coordinator } \\
\text { (non-clinical } \\
\text { staff) and } \\
\text { pharmacist } \\
\text { (acceptors) } \\
\text { G2: Usual care } \\
\text { provided to } \\
\text { MTM-eligible } \\
\text { enrollees who } \\
\text { chose not to } \\
\text { participate (opt- } \\
\text { out) }\end{array}$ & Cohort & NA & NA & No & No & NA & Unclear or NR & NA & Yes & Unclear or NR \\
\hline
\end{tabular}


Table F1. Risk of bias domains and ratings (continued)

\begin{tabular}{|c|c|c|c|c|c|c|c|c|c|c|c|}
\hline $\begin{array}{l}\text { Author, Year } \\
\text { Trial Name }\end{array}$ & $\begin{array}{l}\text { Intervention } \\
\text { and } \\
\text { Comparator } \\
\text { Descriptions }\end{array}$ & $\begin{array}{l}\text { Study } \\
\text { Design }\end{array}$ & $\begin{array}{l}\text { Randomi- } \\
\text { zation } \\
\text { method } \\
\text { adequate? } \\
\text { (RCTs } \\
\text { only) }\end{array}$ & $\begin{array}{l}\text { Allocation } \\
\text { conceal- } \\
\text { ment ad- } \\
\text { ? equate? } \\
\text { (RCTs } \\
\text { only) }\end{array}$ & $\begin{array}{l}\text { Recruitment } \\
\text { strategy for } \\
\text { study } \\
\text { different } \\
\text { across } \\
\text { groups? }\end{array}$ & $\begin{array}{l}\text { Groups } \\
\text { similar at } \\
\text { baseline, } \\
\text { or } \\
\text { differences } \\
\text { adjusted } \\
\text { for in } \\
\text { analysis? }\end{array}$ & $\begin{array}{l}\text { Outcome } \\
\text { asse- } \\
\text { ssors } \\
\text { blinded? } \\
\text { (RCTs or } \\
\text { NRCTs } \\
\text { only) }\end{array}$ & $\begin{array}{l}\text { Impact from } \\
\text { concurrent } \\
\text { intervention } \\
\text { or unintended } \\
\text { exposure } \\
\text { ruled out by } \\
\text { researchers? }\end{array}$ & $\begin{array}{l}\text { Did variation } \\
\text { from study } \\
\text { protocol } \\
\text { compromise } \\
\text { conclusions? } \\
\text { (RCTs or } \\
\text { NRCTs only) }\end{array}$ & $\begin{array}{l}\text { High } \\
\text { overall } \\
\text { (i.e., } \geq 20 \% \text { ) } \\
\text { or } \\
\text { ?ifferential } \\
\text { (i.e., } \geq 15 \% \text { ) } \\
\text { attrition }\end{array}$ & $\begin{array}{l}\text { Did attrition } \\
\text { result in } \\
\text { differences } \\
\text { in group } \\
\text { charac- } \\
\text { teristics? }\end{array}$ \\
\hline $\begin{array}{l}\text { Taylor, Byrd, } \\
\text { and Krueger, } \\
2003^{36}\end{array}$ & $\begin{array}{l}\text { G1: } \\
\text { Pharmaceutical } \\
\text { care provided by } \\
\text { pharmacists } \\
\text { G2: Standard } \\
\text { care without } \\
\text { advice or recom- } \\
\text { mendations } \\
\text { given to patients } \\
\text { or physicians } \\
\end{array}$ & $\begin{array}{l}\text { RCT: } \\
\text { parallel, } \\
\text { not } \\
\text { clustered } \\
\\
\end{array}$ & $\begin{array}{l}\text { Unclear or } \\
\text { NR }\end{array}$ & $\begin{array}{l}\text { Unclear or } \\
\text { NR }\end{array}$ & No & No & $\begin{array}{l}\text { Unclear or } \\
\text { NR }\end{array}$ & Unclear or NR & Unclear or NR & No & Unclear or NR \\
\hline $\begin{array}{l}\text { Touchette et } \\
\text { al., } 2012^{37}\end{array}$ & $\begin{array}{l}\text { G1: MTM basic } \\
\text { (comprehensive } \\
\text { medication } \\
\text { review and DRP } \\
\text { assessment) } \\
\text { G2: MTM } \\
\text { enhanced (MTM } \\
\text { plus } 2 \text { page } \\
\text { clinical summary } \\
\text { abstracted from } \\
\text { patient's medical } \\
\text { chart) } \\
\text { G3: Usual care, } \\
\text { consisting of } \\
\text { medication } \\
\text { counseling per } \\
\text { clinic's normal } \\
\text { routine but no } \\
\text { formal MTM } \\
\text { from a study } \\
\text { pharmacist }\end{array}$ & $\begin{array}{l}\text { RCT: } \\
\text { parallel, } \\
\text { not } \\
\text { clustered } \\
\\
\end{array}$ & Yes & Yes & No & Yes & Yes & Unclear or NR & No & No & Unclear or NR \\
\hline
\end{tabular}


Table F1. Risk of bias domains and ratings (continued)

\begin{tabular}{|c|c|c|c|c|c|c|c|c|c|c|c|}
\hline $\begin{array}{l}\text { Author, Year } \\
\text { Trial Name }\end{array}$ & $\begin{array}{l}\text { Intervention } \\
\text { and } \\
\text { Comparator } \\
\text { Descriptions }\end{array}$ & $\begin{array}{l}\text { Study } \\
\text { Design }\end{array}$ & $\begin{array}{l}\text { Randomi- } \\
\text { zation } \\
\text { method } \\
\text { adequate? } \\
\text { (RCTs } \\
\text { only) }\end{array}$ & $\begin{array}{l}\text { Allocation } \\
\text { conceal- } \\
\text { ment ad- } \\
\text { equate? } \\
\text { (RCTs } \\
\text { only) }\end{array}$ & $\begin{array}{l}\text { Recruitment } \\
\text { strategy for } \\
\text { study } \\
\text { different } \\
\text { across } \\
\text { groups? }\end{array}$ & $\begin{array}{l}\text { Groups } \\
\text { similar at } \\
\text { baseline, } \\
\text { or } \\
\text { differences } \\
\text { adjusted } \\
\text { for in } \\
\text { analysis? }\end{array}$ & $\begin{array}{l}\text { Outcome } \\
\text { asse- } \\
\text { ssors } \\
\text { blinded? } \\
\text { (RCTs or } \\
\text { NRCTs } \\
\text { only) }\end{array}$ & $\begin{array}{l}\text { Impact from } \\
\text { concurrent } \\
\text { intervention } \\
\text { or unintended } \\
\text { exposure } \\
\text { ruled out by } \\
\text { researchers? }\end{array}$ & $\begin{array}{l}\text { Did variation } \\
\text { from study } \\
\text { protocol } \\
\text { compromise } \\
\text { conclusions? } \\
\text { (RCTs or } \\
\text { NRCTs only) }\end{array}$ & $\begin{array}{l}\text { High } \\
\text { overall } \\
\text { (i.e., } \geq 20 \% \text { ) } \\
\text { or } \\
\text { ? differential } \\
\text { (i.e., } \geq 15 \% \text { ) } \\
\text { attrition }\end{array}$ & $\begin{array}{l}\text { Did attrition } \\
\text { result in } \\
\text { differences } \\
\text { in group } \\
\text { charac- } \\
\text { teristics? }\end{array}$ \\
\hline $\begin{array}{l}\text { Triller et al., } \\
2007^{38}\end{array}$ & $\begin{array}{l}\text { G1: VNA home } \\
\text { visit services } \\
\text { plus } \\
\text { comprehensive } \\
\text { pharmaceutical } \\
\text { care services } \\
\text { G2: VNA home } \\
\text { visit services } \\
\text { only }\end{array}$ & $\begin{array}{l}\text { RCT: } \\
\text { parallel, } \\
\text { not } \\
\text { clustered }\end{array}$ & Yes & No & No & No & $\begin{array}{l}\text { Unclear or } \\
\text { NR }\end{array}$ & NA & Yes & No & Unclear or NR \\
\hline $\begin{array}{l}\text { Volume et al., } \\
\text { 2001, PREP } \\
\text { Kassam et al., } \\
2001^{40}\end{array}$ & $\begin{array}{l}\text { G1: } \\
\text { Comprehensive } \\
\text { pharmaceutical } \\
\text { care services } \\
\text { using a nine- } \\
\text { step process as } \\
\text { defined by } \\
\text { Hepler and } \\
\text { Strand provided } \\
\text { by community } \\
\text { pharmacists } \\
\text { G2: Traditional } \\
\text { pharmacy care }\end{array}$ & RCT & $\begin{array}{l}\text { Unclear or } \\
\text { NR }\end{array}$ & Yes & Unclear or NR & Yes & No & Unclear or NR & No & Yes & Unclear or NR \\
\hline $\begin{array}{l}\text { Welch et al., } \\
2009^{41}\end{array}$ & $\begin{array}{l}\text { G1: MTM } \\
\text { program } \\
\text { provided to } \\
\text { home-based } \\
\text { beneficiaries as } \\
\text { part of Medicare } \\
\text { Part D MTM } \\
\text { program } \\
\text { (acceptors) } \\
\text { G2: No-MTM } \\
\text { control group } \\
\text { (voluntary opt- } \\
\text { out) }\end{array}$ & Cohort & NA & NA & Yes & No & NA & Unclear or NR & NA & No & No \\
\hline
\end{tabular}


Table F1. Risk of bias domains and ratings (continued)

\begin{tabular}{|c|c|c|c|c|c|c|c|c|c|c|c|}
\hline $\begin{array}{l}\text { Author, Year } \\
\text { Trial Name }\end{array}$ & $\begin{array}{l}\text { Intervention } \\
\text { and } \\
\text { Comparator } \\
\text { Descriptions }\end{array}$ & $\begin{array}{l}\text { Study } \\
\text { Design }\end{array}$ & $\begin{array}{l}\text { Randomi- } \\
\text { zation } \\
\text { method } \\
\text { adequate? } \\
\text { (RCTs } \\
\text { only) }\end{array}$ & $\begin{array}{l}\text { Allocation } \\
\text { conceal- } \\
\text { ment ad- } \\
\text { ? equate? } \\
\text { (RCTs } \\
\text { only) }\end{array}$ & $\begin{array}{l}\text { Recruitment } \\
\text { strategy for } \\
\text { study } \\
\text { different } \\
\text { across } \\
\text { groups? }\end{array}$ & $\begin{array}{l}\text { Groups } \\
\text { similar at } \\
\text { baseline, } \\
\text { or } \\
\text { differences } \\
\text { adjusted } \\
\text { for in } \\
\text { analysis? }\end{array}$ & $\begin{array}{l}\text { Outcome } \\
\text { asse- } \\
\text { ssors } \\
\text { blinded? } \\
\text { (RCTs or } \\
\text { NRCTs } \\
\text { only) }\end{array}$ & $\begin{array}{l}\text { Impact from } \\
\text { concurrent } \\
\text { intervention } \\
\text { or unintended } \\
\text { exposure } \\
\text { ruled out by } \\
\text { researchers? }\end{array}$ & $\begin{array}{l}\text { Did variation } \\
\text { from study } \\
\text { protocol } \\
\text { compromise } \\
\text { conclusions? } \\
\text { (RCTs or } \\
\text { NRCTs only) }\end{array}$ & $\begin{array}{l}\text { High } \\
\text { overall } \\
\text { (i.e., } \geq 20 \% \text { ) } \\
\text { or } \\
\text { ? differential } \\
\text { (i.e., } \geq 15 \% \text { ) } \\
\text { attrition }\end{array}$ & $\begin{array}{l}\text { Did attrition } \\
\text { result in } \\
\text { differences } \\
\text { in group } \\
\text { charac- } \\
\text { teristics? }\end{array}$ \\
\hline $\begin{array}{l}\text { Williams et al., } \\
2004^{42}\end{array}$ & $\begin{array}{l}\text { G1: Modification } \\
\text { of patient's } \\
\text { medication } \\
\text { regimen } \\
\text { conducted by } \\
\text { interdisciplinary } \\
\text { medication } \\
\text { adjustment team } \\
\text { in addition to } \\
\text { usual medical } \\
\text { care and "Bound } \\
\text { for Health" } \\
\text { booklet. } \\
\text { G2: Usual } \\
\text { medical care } \\
\text { plus provision of } \\
\text { "Bound for } \\
\text { Health" booklet }\end{array}$ & $\begin{array}{l}\text { RCT: } \\
\text { parallel, } \\
\text { not } \\
\text { clustered } \\
\\
\end{array}$ & $\begin{array}{l}\text { Unclear or } \\
\text { NR }\end{array}$ & $\begin{array}{l}\text { Unclear or } \\
\text { NR }\end{array}$ & No & Yes & $\begin{array}{l}\text { Unclear or } \\
\text { NR }\end{array}$ & No & No & No & Unclear or NR \\
\hline
\end{tabular}


Table F1. Risk of bias domains and ratings (continued)

\begin{tabular}{|c|c|c|c|c|c|c|c|c|c|c|c|}
\hline $\begin{array}{l}\text { Author, Year } \\
\text { Trial Name }\end{array}$ & $\begin{array}{l}\text { Intervention } \\
\text { and } \\
\text { Comparator } \\
\text { Descriptions }\end{array}$ & $\begin{array}{l}\text { Study } \\
\text { Design }\end{array}$ & $\begin{array}{l}\text { Randomi- } \\
\text { zation } \\
\text { method } \\
\text { adequate? } \\
\text { (RCTs } \\
\text { only) }\end{array}$ & $\begin{array}{l}\text { Allocation } \\
\text { conceal- } \\
\text { ment ad- } \\
\text { ? equate? } \\
\text { (RCTs } \\
\text { only) }\end{array}$ & $\begin{array}{l}\text { Recruitment } \\
\text { strategy for } \\
\text { study } \\
\text { different } \\
\text { across } \\
\text { groups? }\end{array}$ & $\begin{array}{l}\text { Groups } \\
\text { similar at } \\
\text { baseline, } \\
\text { or } \\
\text { differences } \\
\text { adjusted } \\
\text { for in } \\
\text { analysis? }\end{array}$ & $\begin{array}{l}\text { Outcome } \\
\text { asse- } \\
\text { ssors } \\
\text { blinded? } \\
\text { (RCTs or } \\
\text { NRCTs } \\
\text { only) }\end{array}$ & $\begin{array}{l}\text { Impact from } \\
\text { concurrent } \\
\text { intervention } \\
\text { or unintended } \\
\text { exposure } \\
\text { ruled out by } \\
\text { researchers? }\end{array}$ & $\begin{array}{l}\text { Did variation } \\
\text { from study } \\
\text { protocol } \\
\text { compromise } \\
\text { conclusions? } \\
\text { (RCTs or } \\
\text { NRCTs only) }\end{array}$ & $\begin{array}{l}\text { High } \\
\text { overall } \\
\text { (i.e., } \geq 20 \% \text { ) } \\
\text { or } \\
\text { ? differential } \\
\text { (i.e., } \geq 15 \% \text { ) } \\
\text { attrition }\end{array}$ & $\begin{array}{l}\text { Did attrition } \\
\text { result in } \\
\text { differences } \\
\text { in group } \\
\text { charac- } \\
\text { teristics? }\end{array}$ \\
\hline $\begin{array}{l}\text { Winston and } \\
\text { Lin, } 2009^{43}\end{array}$ & $\begin{array}{l}\text { G1: MTM } \\
\text { provided in } \\
\text { community } \\
\text { pharmacy (i.e., } \\
\text { care in face-to- } \\
\text { face meetings or } \\
\text { by telephone) as } \\
\text { part of Medicare } \\
\text { Part D MTM } \\
\text { program } \\
\text { G2: MTM } \\
\text { provided by } \\
\text { pharmacist- } \\
\text { staffed call } \\
\text { centers as part } \\
\text { of Medicare Part } \\
\text { D MTM program } \\
\text { G3: Educational } \\
\text { mailings (i.e., } \\
\text { mailed letter } \\
\text { containing } \\
\text { patient-specific } \\
\text { medication } \\
\text { related } \\
\text { information, } \\
\text { personal } \\
\text { medication } \\
\text { record, and tips } \\
\text { to save money } \\
\text { on prescriptions) }\end{array}$ & Cohort & NA & NA & Yes & Yes & NA & Unclear or NR & Unclear or NR & R NA & NA \\
\hline
\end{tabular}


Table F1. Risk of bias domains and ratings (continued)

\begin{tabular}{|c|c|c|c|c|c|c|c|c|c|c|}
\hline $\begin{array}{l}\text { Author, Year } \\
\text { Trial Name }\end{array}$ & $\begin{array}{l}\text { Intervention } \\
\text { and } \\
\text { Comparator } \\
\text { Descriptions }\end{array}$ & $\begin{array}{l}\text { Study } \\
\text { Design }\end{array}$ & $\begin{array}{l}\text { Randomi- } \\
\text { zation } \\
\text { method } \\
\text { adequate? } \\
\text { (RCTs } \\
\text { only) }\end{array}$ & $\begin{array}{l}\text { Allocation } \\
\text { conceal- } \\
\text { ment ad- } \\
\text { equate? } \\
\text { (RCTs } \\
\text { only) }\end{array}$ & $\begin{array}{l}\text { Recruitment } \\
\text { strategy for } \\
\text { study } \\
\text { different } \\
\text { across } \\
\text { groups? }\end{array}$ & $\begin{array}{l}\text { Groups } \\
\text { similar at } \\
\text { baseline, } \\
\text { or } \\
\text { differences } \\
\text { adjusted } \\
\text { for in } \\
\text { analysis? }\end{array}$ & $\begin{array}{l}\text { Outcome } \\
\text { asse- } \\
\text { ssors } \\
\text { blinded? } \\
\text { (RCTs or } \\
\text { NRCTs } \\
\text { only) }\end{array}$ & $\begin{array}{l}\text { Impact from } \\
\text { concurrent } \\
\text { intervention } \\
\text { or unintended } \\
\text { exposure } \\
\text { ruled out by } \\
\text { researchers? }\end{array}$ & $\begin{array}{ll}\text { Did variation } & \text { High } \\
\text { from study } & \text { overall } \\
\text { protocol } & \text { (i.e., } \geq 20 \%) \\
\text { compromise } & \text { or } \\
\text { conclusions? } & \text { differential } \\
\text { (RCTs or } & \text { (i.e., } \geq 15 \%) \\
\text { NRCTs only) } & \text { attrition }\end{array}$ & $\begin{array}{l}\text { Did attrition } \\
\text { result in } \\
\text { differences } \\
\text { in group } \\
\text { charac- } \\
\text { teristics? }\end{array}$ \\
\hline $\begin{array}{l}\text { Witry, } \\
\text { Doucette, and } \\
\text { Gainer, } 2011^{44}\end{array}$ & $\begin{array}{l}\text { G1: PCM } \\
\text { provided by } \\
\text { community } \\
\text { pharmacists to } \\
\text { lowa Medicaid } \\
\text { enrollees } \\
\text { G2: PCM } \\
\text { provided to } \\
\text { patients with } \\
\text { private } \\
\text { individual-group } \\
\text { insurance }\end{array}$ & Cohort & NA & NA & No & No & $\begin{array}{l}\text { Unclear or } \\
\text { NR }\end{array}$ & Unclear or NR & $\begin{array}{c}\text { Unclear or NR Unclear or } \\
\text { NR }\end{array}$ & Unclear or NR \\
\hline
\end{tabular}


Table F2. Risk of bias domains and ratings

\begin{tabular}{|c|c|c|c|c|c|c|c|c|c|c|}
\hline $\begin{array}{l}\text { Author, Year } \\
\text { Trial Name }^{a}\end{array}$ & $\begin{array}{l}\text { Interventions/ } \\
\text { Comparator } \\
\text { Descriptions }\end{array}$ & $\begin{array}{l}\text { Study } \\
\text { Design }\end{array}$ & ITT? & $\begin{array}{l}\text { Eligibility } \\
\text { criteria } \\
\text { measured } \\
\text { consistently } \\
\text { using valid } \\
\text { and reliable } \\
\text { measures? }\end{array}$ & $\begin{array}{l}\text { Intermediate } \\
\text { Outcomes: } \\
\text { assessed } \\
\text { consistently } \\
\text { using valid and } \\
\text { reliable } \\
\text { measures? }\end{array}$ & $\begin{array}{l}\text { Patient-Centered } \\
\text { Outcomes: } \\
\text { assessed } \\
\text { consistently } \\
\text { using valid and } \\
\text { reliable } \\
\text { measures? }\end{array}$ & $\begin{array}{l}\text { Utilization } \\
\text { Outcomes: } \\
\text { assessed } \\
\text { consistently } \\
\text { using valid } \\
\text { and reliable } \\
\text { measures? }\end{array}$ & $\begin{array}{l}\text { Potential } \\
\text { outcome } \\
\text { pre- } \\
\text { specified } \\
\text { and } \\
\text { reported? }\end{array}$ & $\begin{array}{l}\text { Were important } \\
\text { confounding and } \\
\text { modifying } \\
\text { variables taken } \\
\text { into account in } \\
\text { design and/or } \\
\text { analysis? }\end{array}$ & Risk of Bias \\
\hline $\begin{array}{l}\text { Bernsten et al., } \\
2001^{1} \text {; } \\
\text { Sturgess, } \\
2003^{2}\end{array}$ & $\begin{array}{l}\text { G1: Structured } \\
\text { community } \\
\text { pharmacy-based } \\
\text { pharmaceutical } \\
\text { care program } \\
\text { G2: Usual } \\
\text { community } \\
\text { pharmacy } \\
\text { services }\end{array}$ & $\begin{array}{l}\text { RCT: } \\
\text { cluster- } \\
\text { rando- } \\
\text { mized } \\
\end{array}$ & No & Yes & Yes & Yes & Yes & Yes & $\begin{array}{l}\text { Partial (some } \\
\text { variables were } \\
\text { taken in to } \\
\text { account) }\end{array}$ & High \\
\hline $\begin{array}{l}\text { Blennerhassett } \\
\text { et al., } 2007^{3}\end{array}$ & $\begin{array}{l}\text { G1: } \\
\text { Implementation } \\
\text { of HMR, a } \\
\text { collaborative } \\
\text { model of } \\
\text { pharmaceutical } \\
\text { care, into a } \\
\text { chronic heart } \\
\text { failure } \\
\text { collaborative } \\
\text { care model, } \\
\text { conducted by } \\
\text { accredited } \\
\text { pharmacists } \\
\text { G2: No HMR }\end{array}$ & Cohort & NA & $\begin{array}{l}\text { Unclear or } \\
\text { NR }\end{array}$ & NA & NA & NA & Yes & $\begin{array}{l}\text { No (Not } \\
\text { accounted for or } \\
\text { not identified) }\end{array}$ & High \\
\hline
\end{tabular}


Table F2. Risk of bias domains and ratings (continued)

\begin{tabular}{|c|c|c|c|c|c|c|c|c|c|c|}
\hline $\begin{array}{l}\text { Author, Year } \\
\text { Trial Name }^{a}\end{array}$ & $\begin{array}{l}\text { Interventions/ } \\
\text { Comparator } \\
\text { Descriptions }\end{array}$ & $\begin{array}{l}\text { Study } \\
\text { Design }\end{array}$ & ITT? & $\begin{array}{l}\text { Eligibility } \\
\text { criteria } \\
\text { measured } \\
\text { consistently } \\
\text { using valid } \\
\text { and reliable } \\
\text { measures? } \\
\end{array}$ & $\begin{array}{l}\text { Intermediate } \\
\text { Outcomes: } \\
\text { assessed } \\
\text { consistently } \\
\text { using valid and } \\
\text { reliable } \\
\text { measures? }\end{array}$ & $\begin{array}{l}\text { Patient-Centered } \\
\text { Outcomes: } \\
\text { assessed } \\
\text { consistently } \\
\text { using valid and } \\
\text { reliable } \\
\text { measures? }\end{array}$ & $\begin{array}{l}\text { Utilization } \\
\text { Outcomes: } \\
\text { assessed } \\
\text { consistently } \\
\text { using valid } \\
\text { and reliable } \\
\text { measures? }\end{array}$ & $\begin{array}{l}\text { Potential } \\
\text { outcome } \\
\text { pre- } \\
\text { specified } \\
\text { and } \\
\text { reported? }\end{array}$ & $\begin{array}{l}\text { Were important } \\
\text { confounding and } \\
\text { modifying } \\
\text { variables taken } \\
\text { into account in } \\
\text { design and/or } \\
\text { analysis? }\end{array}$ & Risk of Bias \\
\hline $\begin{array}{l}\text { Carter et al., } \\
1997^{4} \text {; } \\
\text { Barnette et } \\
\text { al., } 1996^{5}\end{array}$ & $\begin{array}{l}\text { G1: } \\
\text { Pharmaceutical } \\
\text { care provided by } \\
\text { pharmacists within } \\
\text { interdisciplinary } \\
\text { practice model. } \\
\text { Standardized } \\
\text { patient education } \\
\text { (lifestyle, risk } \\
\text { factor } \\
\text { modifications, and } \\
\text { drug therapy). } \\
\text { G2: Usual care }\end{array}$ & Cohort & No & Yes & No & Yes & Yes & $\begin{array}{l}\text { Unclear or } \\
\text { NR }\end{array}$ & $\begin{array}{l}\text { No (Not } \\
\text { accounted for or } \\
\text { not identified) }\end{array}$ & High \\
\hline $\begin{array}{l}\text { Chisholm et } \\
\text { al., } 2002^{6}\end{array}$ & $\begin{array}{l}\text { G1: Clinical } \\
\text { pharmacy } \\
\text { services, including } \\
\text { reviewing patients' } \\
\text { medication } \\
\text { therapy, with } \\
\text { emphasis on } \\
\text { controlling BP, } \\
\text { and preventing or } \\
\text { resolving DTPs. } \\
\text { Pharmacists } \\
\text { counseled } \\
\text { patients about } \\
\text { their regimen, } \\
\text { including desired } \\
\text { clinical }\end{array}$ & $\begin{array}{l}\text { RCT: } \\
\text { parallel, } \\
\text { not } \\
\text { clus- } \\
\text { tered } \\
\end{array}$ & No & Yes & Yes & NA & $\begin{array}{l}\text { Unclear or } \\
\text { NR }\end{array}$ & Yes & Yes & Medium \\
\hline
\end{tabular}


Table F2. Risk of bias domains and ratings (continued)

\begin{tabular}{|c|c|c|c|c|c|c|c|c|c|c|}
\hline $\begin{array}{l}\text { Author, Year } \\
\text { Trial Name }^{a}\end{array}$ & $\begin{array}{l}\text { Interventions/ } \\
\text { Comparator } \\
\text { Descriptions }\end{array}$ & $\begin{array}{l}\text { Study } \\
\text { Design }\end{array}$ & ITT? & $\begin{array}{l}\text { Eligibility } \\
\text { criteria } \\
\text { measured } \\
\text { consistently } \\
\text { using valid } \\
\text { and reliable } \\
\text { measures? }\end{array}$ & $\begin{array}{l}\text { Intermediate } \\
\text { Outcomes: } \\
\text { assessed } \\
\text { consistently } \\
\text { using valid and } \\
\text { reliable } \\
\text { measures? }\end{array}$ & $\begin{array}{l}\text { Patient-Centered } \\
\text { Outcomes: } \\
\text { assessed } \\
\text { consistently } \\
\text { using valid and } \\
\text { reliable } \\
\text { measures? }\end{array}$ & $\begin{array}{l}\text { Utilization } \\
\text { Outcomes: } \\
\text { assessed } \\
\text { consistently } \\
\text { using valid } \\
\text { and reliable } \\
\text { measures? }\end{array}$ & $\begin{array}{l}\text { Potential } \\
\text { outcome } \\
\text { pre- } \\
\text { specified } \\
\text { and } \\
\text { reported? }\end{array}$ & $\begin{array}{l}\text { Were important } \\
\text { confounding and } \\
\text { modifying } \\
\text { variables taken } \\
\text { into account in } \\
\text { design and/or } \\
\text { analysis? }\end{array}$ & Risk of Bias \\
\hline $\begin{array}{l}\text { Chisholm et } \\
\text { al., } 2002^{6} \\
\text { (continued) }\end{array}$ & $\begin{array}{l}\text { responses and } \\
\text { possible adverse } \\
\text { reactions. } \\
\text { G2: Routine } \\
\text { clinic services, } \\
\text { but without } \\
\text { clinical } \\
\text { pharmacist } \\
\text { interaction. } \\
\text { Routine clinical } \\
\text { services here } \\
\text { entailed meeting } \\
\text { renal transplant } \\
\text { clinic team } \\
\text { consisting of } 2 \\
\text { nephrologists, } \\
\text { clinical } \\
\text { pharmacist, PAs } \\
\text { and nurse. }\end{array}$ & & & & & & & & & \\
\hline $\begin{array}{l}\text { Chrischilles et } \\
\text { al., } 2004^{7}\end{array}$ & $\begin{array}{l}\text { G1: PCM-eligible } \\
\text { patients who } \\
\text { received PCM } \\
\text { services } \\
\text { G2: PCM-eligible } \\
\text { patients who did } \\
\text { not receive PCM } \\
\text { services }\end{array}$ & Cohort & No & Yes & Yes & NA & Yes & Yes & $\begin{array}{l}\text { No (Not } \\
\text { accounted for or } \\
\text { not identified) }\end{array}$ & High \\
\hline
\end{tabular}


Table F2. Risk of bias domains and ratings (continued)

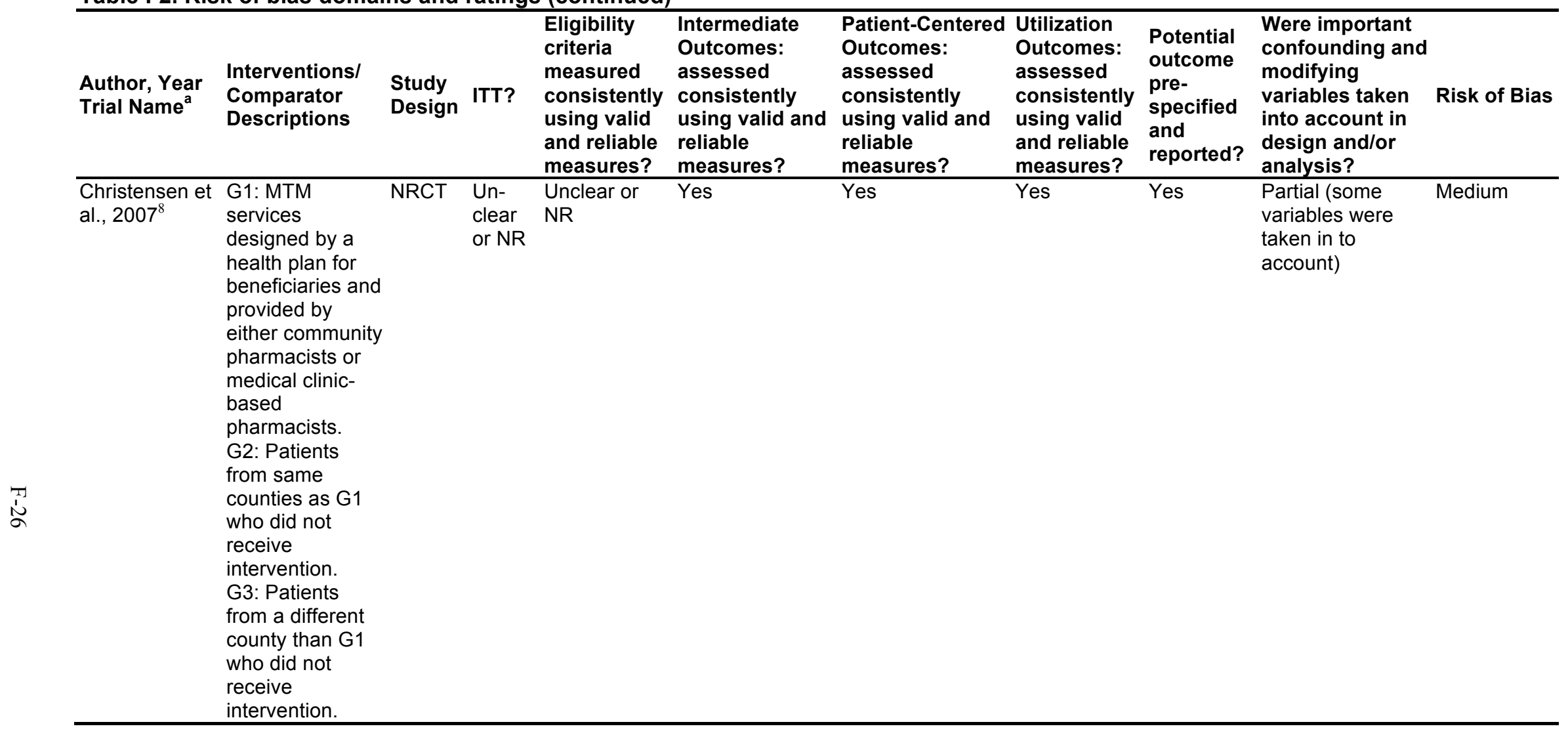


Table F2. Risk of bias domains and ratings (continued)

\begin{tabular}{|c|c|c|c|c|c|c|c|c|c|c|}
\hline $\begin{array}{l}\text { Author, Year } \\
\text { Trial Name }^{a}\end{array}$ & $\begin{array}{l}\text { Interventions/ } \\
\text { Comparator } \\
\text { Descriptions }\end{array}$ & $\begin{array}{l}\text { Study } \\
\text { Design }\end{array}$ & ITT? & $\begin{array}{l}\text { Eligibility } \\
\text { criteria } \\
\text { measured } \\
\text { consistently } \\
\text { using valid } \\
\text { and reliable } \\
\text { measures? }\end{array}$ & $\begin{array}{l}\text { Intermediate } \\
\text { Outcomes: } \\
\text { assessed } \\
\text { consistently } \\
\text { using valid and } \\
\text { reliable } \\
\text { measures? }\end{array}$ & $\begin{array}{l}\text { Patient-Centered } \\
\text { Outcomes: } \\
\text { assessed } \\
\text { consistently } \\
\text { using valid and } \\
\text { reliable } \\
\text { measures? }\end{array}$ & $\begin{array}{l}\text { Utilization } \\
\text { Outcomes: } \\
\text { assessed } \\
\text { consistently } \\
\text { using valid } \\
\text { and reliable } \\
\text { measures? }\end{array}$ & $\begin{array}{l}\text { Potential } \\
\text { outcome } \\
\text { pre- } \\
\text { specified } \\
\text { and } \\
\text { reported? }\end{array}$ & $\begin{array}{l}\text { Were important } \\
\text { confounding and } \\
\text { modifying } \\
\text { variables taken } \\
\text { into account in } \\
\text { design and/or } \\
\text { analysis? }\end{array}$ & Risk of Bias \\
\hline $\begin{array}{l}\text { Clifford et al., } \\
2002^{9}\end{array}$ & $\begin{array}{l}\text { G1: } \\
\text { Pharmaceutical } \\
\text { care provided by } \\
\text { a clinical } \\
\text { pharmacist, } \\
\text { including a } \\
\text { comprehensive } \\
\text { review relating to } \\
\text { pharmaco- } \\
\text { therapy and } \\
\text { diabetes, use of } \\
\text { proprietary and } \\
\text { non-proprietary } \\
\text { medications, } \\
\text { such as } \\
\text { complementary } \\
\text { medicines, and } \\
\text { identification of } \\
\text { DTPs. } \\
\text { G2: Standard } \\
\text { outpatient care } \\
\text { for diabetes }\end{array}$ & $\begin{array}{l}\text { RCT: } \\
\text { parallel, } \\
\text { not } \\
\text { clus- } \\
\text { tered }\end{array}$ & Yes & Yes & Yes & Yes & Yes & Yes & $\begin{array}{l}\text { Partial (some } \\
\text { variables were } \\
\text { taken in to } \\
\text { account) }\end{array}$ & Medium \\
\hline
\end{tabular}


Table F2. Risk of bias domains and ratings (continued)

\begin{tabular}{|c|c|c|c|c|c|c|c|c|c|c|}
\hline $\begin{array}{l}\text { Author, Year } \\
\text { Trial Name }^{a}\end{array}$ & $\begin{array}{l}\text { Interventions/ } \\
\text { Comparator } \\
\text { Descriptions }\end{array}$ & $\begin{array}{l}\text { Study } \\
\text { Design }\end{array}$ & ITT? & $\begin{array}{l}\text { Eligibility } \\
\text { criteria } \\
\text { measured } \\
\text { consistently } \\
\text { using valid } \\
\text { and reliable } \\
\text { measures? }\end{array}$ & $\begin{array}{l}\text { Intermediate } \\
\text { Outcomes: } \\
\text { assessed } \\
\text { consistently } \\
\text { using valid and } \\
\text { reliable } \\
\text { measures? }\end{array}$ & $\begin{array}{l}\text { Patient-Centered } \\
\text { Outcomes: } \\
\text { assessed } \\
\text { consistently } \\
\text { using valid and } \\
\text { reliable } \\
\text { measures? }\end{array}$ & $\begin{array}{l}\text { Utilization } \\
\text { Outcomes: } \\
\text { assessed } \\
\text { consistently } \\
\text { using valid } \\
\text { and reliable } \\
\text { measures? }\end{array}$ & $\begin{array}{l}\text { Potential } \\
\text { outcome } \\
\text { pre- } \\
\text { specified } \\
\text { and } \\
\text { reported? }\end{array}$ & $\begin{array}{l}\text { Were important } \\
\text { confounding and } \\
\text { modifying } \\
\text { variables taken } \\
\text { into account in } \\
\text { design and/or } \\
\text { analysis? }\end{array}$ & Risk of Bias \\
\hline $\begin{array}{l}\text { Fischer et al., } \\
2000^{10}\end{array}$ & $\begin{array}{l}\text { G1: } \\
\text { Pharmaceutical } \\
\text { care based on } \\
\text { Encara Practice } \\
\text { System provided } \\
\text { by onsite health } \\
\text { maintenance } \\
\text { organization staff } \\
\text { pharmacists } \\
\text { (acceptors). } \\
\text { G2: Standard } \\
\text { Community } \\
\text { Pharmacy } \\
\text { Practice. } \\
\text { G3: A set of } \\
\text { those at eligible } \\
\text { clinics who } \\
\text { initially declined } \\
\text { to participate } \\
\text { (opt-out). }\end{array}$ & NRCT & $\begin{array}{l}\text { Un- } \\
\text { clear } \\
\text { or NR }\end{array}$ & Yes & No & No & NA & $\begin{array}{l}\text { Unclear or } \\
\text { NR }\end{array}$ & Yes & Medium \\
\hline
\end{tabular}


Table F2. Risk of bias domains and ratings (continued)

\begin{tabular}{|c|c|c|c|c|c|c|c|c|c|c|}
\hline $\begin{array}{l}\text { Author, Year } \\
\text { Trial Name }^{a}\end{array}$ & $\begin{array}{l}\text { Interventions/ } \\
\text { Comparator } \\
\text { Descriptions }\end{array}$ & $\begin{array}{l}\text { Study } \\
\text { Design }\end{array}$ & ITT? & $\begin{array}{l}\text { Eligibility } \\
\text { criteria } \\
\text { measured } \\
\text { consistently } \\
\text { using valid } \\
\text { and reliable } \\
\text { measures? }\end{array}$ & $\begin{array}{l}\text { Intermediate } \\
\text { Outcomes: } \\
\text { assessed } \\
\text { consistently } \\
\text { using valid and } \\
\text { reliable } \\
\text { measures? }\end{array}$ & $\begin{array}{l}\text { Patient-Centered } \\
\text { Outcomes: } \\
\text { assessed } \\
\text { consistently } \\
\text { using valid and } \\
\text { reliable } \\
\text { measures? }\end{array}$ & $\begin{array}{l}\text { Utilization } \\
\text { Outcomes: } \\
\text { assessed } \\
\text { consistently } \\
\text { using valid } \\
\text { and reliable } \\
\text { measures? }\end{array}$ & $\begin{array}{l}\text { Potential } \\
\text { outcome } \\
\text { pre- } \\
\text { specified } \\
\text { and } \\
\text { reported? }\end{array}$ & $\begin{array}{l}\text { Were important } \\
\text { confounding and } \\
\text { modifying } \\
\text { variables taken } \\
\text { into account in } \\
\text { design and/or } \\
\text { analysis? }\end{array}$ & Risk of Bias \\
\hline $\begin{array}{l}\text { Fischer et al., } \\
2002^{11}\end{array}$ & $\begin{array}{l}\text { G1: } \\
\text { Pharmaceutical } \\
\text { care based on } \\
\text { Encara Practice } \\
\text { System provided } \\
\text { by pharmacists. } \\
\text { Communication } \\
\text { of pharmacist } \\
\text { with the patient's } \\
\text { physician about } \\
\text { drug therapy } \\
\text { problems } \\
\text { identified by the } \\
\text { pharmacist. } \\
\text { G2: Usual care. }\end{array}$ & NRCT & No & Yes & NA & NA & Yes & Yes & Yes & Medium \\
\hline
\end{tabular}


Table F2. Risk of bias domains and ratings (continued)

\begin{tabular}{|c|c|c|c|c|c|c|c|c|c|c|}
\hline $\begin{array}{l}\text { Author, Year } \\
\text { Trial Name }^{a}\end{array}$ & $\begin{array}{l}\text { Interventions/ } \\
\text { Comparator } \\
\text { Descriptions }\end{array}$ & $\begin{array}{l}\text { Study } \\
\text { Design }\end{array}$ & ITT? & $\begin{array}{l}\text { Eligibility } \\
\text { criteria } \\
\text { measured } \\
\text { consistently } \\
\text { using valid } \\
\text { and reliable } \\
\text { measures? }\end{array}$ & $\begin{array}{l}\text { Intermediate } \\
\text { Outcomes: } \\
\text { assessed } \\
\text { consistently } \\
\text { using valid and } \\
\text { reliable } \\
\text { measures? }\end{array}$ & $\begin{array}{l}\text { Patient-Centered } \\
\text { Outcomes: } \\
\text { assessed } \\
\text { consistently } \\
\text { using valid and } \\
\text { reliable } \\
\text { measures? }\end{array}$ & $\begin{array}{l}\text { Utilization } \\
\text { Outcomes: } \\
\text { assessed } \\
\text { consistently } \\
\text { using valid } \\
\text { and reliable } \\
\text { measures? }\end{array}$ & $\begin{array}{l}\text { Potential } \\
\text { outcome } \\
\text { pre- } \\
\text { specified } \\
\text { and } \\
\text { reported? }\end{array}$ & $\begin{array}{l}\text { Were important } \\
\text { confounding and } \\
\text { modifying } \\
\text { variables taken } \\
\text { into account in } \\
\text { design and/or } \\
\text { analysis? }\end{array}$ & Risk of Bias \\
\hline $\begin{array}{l}\text { Fox et al., } \\
2009^{12}\end{array}$ & $\begin{array}{l}\text { G1: Florida } \\
\text { Health Care } \\
\text { Plans MTM } \\
\text { program, } \\
\text { consisting of } \\
\text { medication } \\
\text { therapy review } \\
\text { and evaluation } \\
\text { by a clinical } \\
\text { pharmacist that } \\
\text { was documented } \\
\text { and sent to } \\
\text { patient's } \\
\text { physician } \\
\text { through health } \\
\text { plan review } \\
\text { (acceptors) } \\
\text { G2: Opt-out from } \\
\text { MTM program }\end{array}$ & Cohort & NA & Yes & Unclear or NR & NA & Yes & Yes & $\begin{array}{l}\text { No (Not } \\
\text { accounted for or } \\
\text { not identified) }\end{array}$ & High \\
\hline
\end{tabular}


Table F2. Risk of bias domains and ratings (continued)

\begin{tabular}{|c|c|c|c|c|c|c|c|c|c|c|}
\hline $\begin{array}{l}\text { Author, Year } \\
\text { Trial Name }^{a}\end{array}$ & $\begin{array}{l}\text { Interventions/ } \\
\text { Comparator } \\
\text { Descriptions }\end{array}$ & $\begin{array}{l}\text { Study } \\
\text { Design }\end{array}$ & ITT? & $\begin{array}{l}\text { Eligibility } \\
\text { criteria } \\
\text { measured } \\
\text { consistently } \\
\text { using valid } \\
\text { and reliable } \\
\text { measures? }\end{array}$ & $\begin{array}{l}\text { Intermediate } \\
\text { Outcomes: } \\
\text { assessed } \\
\text { consistently } \\
\text { using valid and } \\
\text { reliable } \\
\text { measures? }\end{array}$ & $\begin{array}{l}\text { Patient-Centered } \\
\text { Outcomes: } \\
\text { assessed } \\
\text { consistently } \\
\text { using valid and } \\
\text { reliable } \\
\text { measures? }\end{array}$ & $\begin{array}{l}\text { Utilization } \\
\text { Outcomes: } \\
\text { assessed } \\
\text { consistently } \\
\text { using valid } \\
\text { and reliable } \\
\text { measures? }\end{array}$ & $\begin{array}{l}\text { Potential } \\
\text { outcome } \\
\text { pre- } \\
\text { specified } \\
\text { and } \\
\text { reported? }\end{array}$ & $\begin{array}{l}\text { Were important } \\
\text { confounding and } \\
\text { modifying } \\
\text { variables taken } \\
\text { into account in } \\
\text { design and/or } \\
\text { analysis? }\end{array}$ & Risk of Bias \\
\hline $\begin{array}{l}\text { Gattis et al., } \\
1999^{13}\end{array}$ & $\begin{array}{l}\text { G1: Clinical } \\
\text { pharmacy } \\
\text { services, } \\
\text { including } \\
\text { assessment of } \\
\text { prescribed } \\
\text { regimen, } \\
\text { compliance, and } \\
\text { adverse effects, } \\
\text { and symptoms } \\
\text { and response to } \\
\text { therapy. } \\
\text { Providing patient } \\
\text { education about } \\
\text { purpose of each } \\
\text { drug and } \\
\text { reinforcing } \\
\text { adherence. } \\
\text { Detailed written } \\
\text { information also } \\
\text { provided to } \\
\text { patients. } \\
\text { G2: Usual } \\
\text { medical care }\end{array}$ & $\begin{array}{l}\text { RCT: } \\
\text { parallel, } \\
\text { not } \\
\text { clus- } \\
\text { tered }\end{array}$ & Yes & Yes & Yes & No & NA & Yes & Yes & Medium \\
\hline $\begin{array}{l}\text { Hanlon et al., } \\
1996^{14}\end{array}$ & $\begin{array}{l}\text { G1: } \\
\text { Pharmaceutical } \\
\text { care provided by } \\
\text { clinical } \\
\text { pharmacist. } \\
\text { G2: Usual care } \\
\text { in the General } \\
\text { Medicine Clinic }\end{array}$ & $\begin{array}{l}\text { RCT: } \\
\text { parallel, } \\
\text { not } \\
\text { clus- } \\
\text { tered }\end{array}$ & Yes & Yes & Yes & Yes & Yes & Yes & Yes/No/Partial & Low \\
\hline
\end{tabular}


Table F2. Risk of bias domains and ratings (continued)

\begin{tabular}{|c|c|c|c|c|c|c|c|c|c|c|}
\hline $\begin{array}{l}\text { Author, Year } \\
\text { Trial Name }^{a}\end{array}$ & $\begin{array}{l}\text { Interventions/ } \\
\text { Comparator } \\
\text { Descriptions }\end{array}$ & $\begin{array}{l}\text { Study } \\
\text { Design }\end{array}$ & ITT? & $\begin{array}{l}\text { Eligibility } \\
\text { criteria } \\
\text { measured } \\
\text { consistently } \\
\text { using valid } \\
\text { and reliable } \\
\text { measures? }\end{array}$ & $\begin{array}{l}\text { Intermediate } \\
\text { Outcomes: } \\
\text { assessed } \\
\text { consistently } \\
\text { using valid and } \\
\text { reliable } \\
\text { measures? }\end{array}$ & $\begin{array}{l}\text { Patient-Centered } \\
\text { Outcomes: } \\
\text { assessed } \\
\text { consistently } \\
\text { using valid and } \\
\text { reliable } \\
\text { measures? }\end{array}$ & $\begin{array}{l}\text { Utilization } \\
\text { Outcomes: } \\
\text { assessed } \\
\text { consistently } \\
\text { using valid } \\
\text { and reliable } \\
\text { measures? }\end{array}$ & $\begin{array}{l}\text { Potential } \\
\text { outcome } \\
\text { pre- } \\
\text { specified } \\
\text { and } \\
\text { reported? }\end{array}$ & $\begin{array}{l}\text { Were important } \\
\text { confounding and } \\
\text { modifying } \\
\text { variables taken } \\
\text { into account in } \\
\text { design and/or } \\
\text { analysis? }\end{array}$ & Risk of Bias \\
\hline $\begin{array}{l}\text { Harrison et al., } \\
2012^{15}\end{array}$ & $\begin{array}{l}\text { G1: } \\
\text { Pharmaceutical } \\
\text { care provided by } \\
\text { a clinical } \\
\text { pharmacist for } \\
\text { the purpose of } \\
\text { identifying and } \\
\text { resolving actual } \\
\text { and potential } \\
\text { DTPs, } \\
\text { medication } \\
\text { teaching, } \\
\text { adherence } \\
\text { optimization, } \\
\text { medication } \\
\text { reconciliation, } \\
\text { and provision of } \\
\text { drug information. } \\
\text { G2: } \\
\text { Retrospective } \\
\text { historical control } \\
\text { of matched } \\
\text { patients who } \\
\text { received } \\
\text { standard care at } \\
\text { a routine medical } \\
\text { visit within } 8 \\
\text { months prior to } \\
\text { study period }\end{array}$ & Cohort & $\begin{array}{l}\text { Un- } \\
\text { clear } \\
\text { or NR }\end{array}$ & $\begin{array}{l}\text { Unclear or } \\
\text { NR }\end{array}$ & Unclear or NR & NA & NA & Yes & $\begin{array}{l}\text { Partial (some } \\
\text { variables were } \\
\text { taken in to } \\
\text { account) }\end{array}$ & Medium \\
\hline
\end{tabular}


Table F2. Risk of bias domains and ratings (continued)

\begin{tabular}{|c|c|c|c|c|c|c|c|c|c|c|}
\hline $\begin{array}{l}\text { Author, Year } \\
\text { Trial Name }^{a}\end{array}$ & $\begin{array}{l}\text { Interventions/ } \\
\text { Comparator } \\
\text { Descriptions }\end{array}$ & $\begin{array}{l}\text { Study } \\
\text { Design }\end{array}$ & ITT? & $\begin{array}{l}\text { Eligibility } \\
\text { criteria } \\
\text { measured } \\
\text { consistently } \\
\text { using valid } \\
\text { and reliable } \\
\text { measures? }\end{array}$ & $\begin{array}{l}\text { Intermediate } \\
\text { Outcomes: } \\
\text { assessed } \\
\text { consistently } \\
\text { using valid and } \\
\text { reliable } \\
\text { measures? }\end{array}$ & $\begin{array}{l}\text { Patient-Centered } \\
\text { Outcomes: } \\
\text { assessed } \\
\text { consistently } \\
\text { using valid and } \\
\text { reliable } \\
\text { measures? }\end{array}$ & $\begin{array}{l}\text { Utilization } \\
\text { Outcomes: } \\
\text { assessed } \\
\text { consistently } \\
\text { using valid } \\
\text { and reliable } \\
\text { measures? }\end{array}$ & $\begin{array}{l}\text { Potential } \\
\text { outcome } \\
\text { pre- } \\
\text { specified } \\
\text { and } \\
\text { reported? }\end{array}$ & $\begin{array}{l}\text { Were important } \\
\text { confounding and } \\
\text { modifying } \\
\text { variables taken } \\
\text { into account in } \\
\text { design and/or } \\
\text { analysis? }\end{array}$ & Risk of Bias \\
\hline $\begin{array}{l}\text { Isetts et al., } \\
2008^{16}\end{array}$ & $\begin{array}{l}\text { G1: MTM } \\
\text { services } \\
\text { provided by staff } \\
\text { pharmacists, } \\
\text { including the } \\
\text { establishment of } \\
\text { goals of therapy, } \\
\text { in collaboration } \\
\text { with primary care } \\
\text { providers. } \\
\text { G2: Usual } \\
\text { medical care } \\
\text { without MTM. }\end{array}$ & Cohort & $\begin{array}{l}\text { Un- } \\
\text { clear } \\
\text { or NR }\end{array}$ & $\begin{array}{l}\text { Unclear or } \\
\text { NR }\end{array}$ & Yes & NA & NA & $\begin{array}{l}\text { Unclear or } \\
\text { NR }\end{array}$ & $\begin{array}{l}\text { No (Not } \\
\text { accounted for or } \\
\text { not identified) }\end{array}$ & High \\
\hline $\begin{array}{l}\text { Jameson et al., } \\
1995^{17}\end{array}$ & $\begin{array}{l}\text { G1: Pharmaco- } \\
\text { therapy } \\
\text { consultation and } \\
\text { follow-up } \\
\text { provided by } \\
\text { clinical } \\
\text { ambulatory care } \\
\text { pharmacist. } \\
\text { G2: Standard } \\
\text { office-based } \\
\text { primary care. }\end{array}$ & $\begin{array}{l}\text { RCT: } \\
\text { parallel, } \\
\text { not } \\
\text { clus- } \\
\text { tered }\end{array}$ & No & Yes & Yes & NA & Yes & Yes & $\begin{array}{l}\text { No (Not } \\
\text { accounted for or } \\
\text { not identified) }\end{array}$ & Medium \\
\hline
\end{tabular}


Table F2. Risk of bias domains and ratings (continued)

\begin{tabular}{|c|c|c|c|c|c|c|c|c|c|c|}
\hline $\begin{array}{l}\text { Author, Year } \\
\text { Trial Name }^{a}\end{array}$ & $\begin{array}{l}\text { Interventions/ } \\
\text { Comparator } \\
\text { Descriptions }\end{array}$ & $\begin{array}{l}\text { Study } \\
\text { Design }\end{array}$ & ITT? & $\begin{array}{l}\text { Eligibility } \\
\text { criteria } \\
\text { measured } \\
\text { consistently } \\
\text { using valid } \\
\text { and reliable } \\
\text { measures? }\end{array}$ & $\begin{array}{l}\text { Intermediate } \\
\text { Outcomes: } \\
\text { assessed } \\
\text { consistently } \\
\text { using valid and } \\
\text { reliable } \\
\text { measures? }\end{array}$ & $\begin{array}{l}\text { Patient-Centered } \\
\text { Outcomes: } \\
\text { assessed } \\
\text { consistently } \\
\text { using valid and } \\
\text { reliable } \\
\text { measures? }\end{array}$ & $\begin{array}{l}\text { Utilization } \\
\text { Outcomes: } \\
\text { assessed } \\
\text { consistently } \\
\text { using valid } \\
\text { and reliable } \\
\text { measures? }\end{array}$ & $\begin{array}{l}\text { Potential } \\
\text { outcome } \\
\text { pre- } \\
\text { specified } \\
\text { and } \\
\text { reported? }\end{array}$ & $\begin{array}{l}\text { Were important } \\
\text { confounding and } \\
\text { modifying } \\
\text { variables taken } \\
\text { into account in } \\
\text { design and/or } \\
\text { analysis? }\end{array}$ & Risk of Bias \\
\hline $\begin{array}{l}\text { Jeong et al., } \\
2007^{18}\end{array}$ & $\begin{array}{l}\text { G1: Pharmacist- } \\
\text { managed MTMP } \\
\text { provided by } \\
\text { ambulatory care } \\
\text { pharmacists and } \\
\text { healthcare } \\
\text { support staff } \\
\text { (acceptors) } \\
\text { G2: Eligible for } \\
\text { Part D MTMP } \\
\text { but declined } \\
\text { enrollment } \\
\text { (refusers) } \\
\text { G3: Patients } \\
\text { without Part D as } \\
\text { their primary } \\
\text { drug benefit }\end{array}$ & Cohort & NA & Yes & Yes & NA & NA & $\begin{array}{l}\text { Unclear or } \\
\text { NR }\end{array}$ & $\begin{array}{l}\text { No (Not } \\
\text { accounted for or } \\
\text { not identified) }\end{array}$ & High \\
\hline $\begin{array}{l}\text { Krska et al., } \\
2001^{19}\end{array}$ & $\begin{array}{l}\text { G1: Medication } \\
\text { reviews led by } \\
\text { clinically-trained } \\
\text { pharmacists. } \\
\text { G2: Usual care } \\
\text { involving } \\
\text { interviews and } \\
\text { identification of } \\
\text { pharmaceutical } \\
\text { care issues but } \\
\text { with no } \\
\text { pharmaceutical } \\
\text { care plan } \\
\text { implemented. }\end{array}$ & $\begin{array}{l}\text { RCT: } \\
\text { parallel, } \\
\text { not } \\
\text { clus- } \\
\text { tered }\end{array}$ & No & Yes & Yes & Yes & $\begin{array}{l}\text { Unclear or } \\
\text { NR }\end{array}$ & Yes & $\begin{array}{l}\text { No (Not } \\
\text { accounted for or } \\
\text { not identified) }\end{array}$ & Medium \\
\hline
\end{tabular}


Table F2. Risk of bias domains and ratings (continued)

\begin{tabular}{|c|c|c|c|c|c|c|c|c|c|c|}
\hline $\begin{array}{l}\text { Author, Year } \\
\text { Trial Name }^{a}\end{array}$ & $\begin{array}{l}\text { Interventions/ } \\
\text { Comparator } \\
\text { Descriptions }\end{array}$ & $\begin{array}{l}\text { Study } \\
\text { Design }\end{array}$ & ITT? & $\begin{array}{l}\text { Eligibility } \\
\text { criteria } \\
\text { measured } \\
\text { consistently } \\
\text { using valid } \\
\text { and reliable } \\
\text { measures? }\end{array}$ & $\begin{array}{l}\text { Intermediate } \\
\text { Outcomes: } \\
\text { assessed } \\
\text { consistently } \\
\text { using valid and } \\
\text { reliable } \\
\text { measures? }\end{array}$ & $\begin{array}{l}\text { Patient-Centered } \\
\text { Outcomes: } \\
\text { assessed } \\
\text { consistently } \\
\text { using valid and } \\
\text { reliable } \\
\text { measures? }\end{array}$ & $\begin{array}{l}\text { Utilization } \\
\text { Outcomes: } \\
\text { assessed } \\
\text { consistently } \\
\text { using valid } \\
\text { and reliable } \\
\text { measures? }\end{array}$ & $\begin{array}{l}\text { Potential } \\
\text { outcome } \\
\text { pre- } \\
\text { specified } \\
\text { and } \\
\text { reported? }\end{array}$ & $\begin{array}{l}\text { Were important } \\
\text { confounding and } \\
\text { modifying } \\
\text { variables taken } \\
\text { into account in } \\
\text { design and/or } \\
\text { analysis? }\end{array}$ & Risk of Bias \\
\hline $\begin{array}{l}\text { Malone et al., } \\
2000^{20} ; \\
\text { Ellis, } 2000^{21} ; \\
\text { Malone, } \\
2001^{22} ; \\
\text { Ellis, } 2000^{23} \\
\text { IMPROVE }\end{array}$ & $\begin{array}{l}\text { G1: } \\
\text { Pharmaceutical } \\
\text { care provided by } \\
\text { clinical } \\
\text { pharmacists } \\
\text { practicing } \\
\text { according to } \\
\text { scope of practice } \\
\text { within their } \\
\text { respective health } \\
\text { care facilities } \\
\text { G2: Usual care } \\
\text { without } \\
\text { pharmaceutical } \\
\text { care }\end{array}$ & $\begin{array}{l}\text { RCT: } \\
\text { parallel, } \\
\text { not } \\
\text { clus- } \\
\text { tered } \\
\end{array}$ & Yes & Yes & Yes & Yes & Yes & $\begin{array}{l}\text { Unclear or } \\
\text { NR }\end{array}$ & Yes & Medium \\
\hline $\begin{array}{l}\text { McDonough et } \\
\text { al., } 2005^{24}\end{array}$ & $\begin{array}{l}\text { G1: } \\
\text { Pharmaceutical } \\
\text { care provided by } \\
\text { community } \\
\text { pharmacists. } \\
\text { Drug therapy } \\
\text { monitoring } \\
\text { focused on } 5 \\
\text { DTPs: } \\
\text { appropriateness } \\
\text { of dose, proper } \\
\text { regimen, } \\
\text { potential } \\
\text { interactions, } \\
\text { nonadherence, } \\
\text { and adverse } \\
\text { effects. Patient } \\
\text { education also } \\
\text { provided. } \\
\text { G2: Usual care }\end{array}$ & $\begin{array}{l}\text { RCT: } \\
\text { cluster- } \\
\text { rando- } \\
\text { mized }\end{array}$ & Yes & Yes & Yes & NA & NA & $\begin{array}{l}\text { Unclear or } \\
\text { NR }\end{array}$ & $\begin{array}{l}\text { No (Not } \\
\text { accounted for or } \\
\text { not identified) }\end{array}$ & Medium \\
\hline
\end{tabular}


Table F2. Risk of bias domains and ratings (continued)

\begin{tabular}{|c|c|c|c|c|c|c|c|c|c|c|}
\hline $\begin{array}{l}\text { Author, Year } \\
\text { Trial Name }^{a}\end{array}$ & $\begin{array}{l}\text { Interventions/ } \\
\text { Comparator } \\
\text { Descriptions }\end{array}$ & $\begin{array}{l}\text { Study } \\
\text { Design }\end{array}$ & ITT? & $\begin{array}{l}\text { Eligibility } \\
\text { criteria } \\
\text { measured } \\
\text { consistently } \\
\text { using valid } \\
\text { and reliable } \\
\text { measures? }\end{array}$ & $\begin{array}{l}\text { Intermediate } \\
\text { Outcomes: } \\
\text { assessed } \\
\text { consistently } \\
\text { using valid and } \\
\text { reliable } \\
\text { measures? }\end{array}$ & $\begin{array}{l}\text { Patient-Centered } \\
\text { Outcomes: } \\
\text { assessed } \\
\text { consistently } \\
\text { using valid and } \\
\text { reliable } \\
\text { measures? }\end{array}$ & $\begin{array}{l}\text { Utilization } \\
\text { Outcomes: } \\
\text { assessed } \\
\text { consistently } \\
\text { using valid } \\
\text { and reliable } \\
\text { measures? }\end{array}$ & $\begin{array}{l}\text { Potential } \\
\text { outcome } \\
\text { pre- } \\
\text { specified } \\
\text { and } \\
\text { reported? }\end{array}$ & $\begin{array}{l}\text { Were important } \\
\text { confounding and } \\
\text { modifying } \\
\text { variables taken } \\
\text { into account in } \\
\text { design and/or } \\
\text { analysis? }\end{array}$ & Risk of Bias \\
\hline $\begin{array}{l}\text { Moczygemba } \\
\text { et al., } 2011^{25} \text {; } \\
\text { Moczygemba } \\
\text { et al., } 2008^{26}\end{array}$ & $\begin{array}{l}\text { G1: Opt-in } \\
\text { telephone-based } \\
\text { MTM program, in } \\
\text { which MTM } \\
\text { services } \\
\text { provided by } \\
\text { clinical } \\
\text { pharmacists or } \\
\text { managed care } \\
\text { pharmacy } \\
\text { resident based } \\
\text { on American } \\
\text { Pharmacists } \\
\text { Association and } \\
\text { National } \\
\text { Association of } \\
\text { Chain Drug } \\
\text { Stores } \\
\text { Foundation MTM } \\
\text { framework } \\
\text { (acceptors) } \\
\text { G2: No-MTM } \\
\text { control group } \\
\text { (opt-out) }\end{array}$ & Cohort & $\begin{array}{l}\text { Un- } \\
\text { clear } \\
\text { or NR }\end{array}$ & NA & Yes & NA & Yes & Yes & $\begin{array}{l}\text { Partial (some } \\
\text { variables were } \\
\text { taken in to } \\
\text { account) }\end{array}$ & Medium \\
\hline
\end{tabular}


Table F2. Risk of bias domains and ratings (continued)

\begin{tabular}{|c|c|c|c|c|c|c|c|c|c|c|}
\hline $\begin{array}{l}\text { Author, Year } \\
\text { Trial Name }^{a}\end{array}$ & $\begin{array}{l}\text { Interventions/ } \\
\text { Comparator } \\
\text { Descriptions }\end{array}$ & $\begin{array}{l}\text { Study } \\
\text { Design }\end{array}$ & ITT? & $\begin{array}{l}\text { Eligibility } \\
\text { criteria } \\
\text { measured } \\
\text { consistently } \\
\text { using valid } \\
\text { and reliable } \\
\text { measures? }\end{array}$ & $\begin{array}{l}\text { Intermediate } \\
\text { Outcomes: } \\
\text { assessed } \\
\text { consistently } \\
\text { using valid and } \\
\text { reliable } \\
\text { measures? }\end{array}$ & $\begin{array}{l}\text { Patient-Centered } \\
\text { Outcomes: } \\
\text { assessed } \\
\text { consistently } \\
\text { using valid and } \\
\text { reliable } \\
\text { measures? }\end{array}$ & $\begin{array}{l}\text { Utilization } \\
\text { Outcomes: } \\
\text { assessed } \\
\text { consistently } \\
\text { using valid } \\
\text { and reliable } \\
\text { measures? }\end{array}$ & $\begin{array}{l}\text { Potential } \\
\text { outcome } \\
\text { pre- } \\
\text { specified } \\
\text { and } \\
\text { reported? }\end{array}$ & $\begin{array}{l}\text { Were important } \\
\text { confounding and } \\
\text { modifying } \\
\text { variables taken } \\
\text { into account in } \\
\text { design and/or } \\
\text { analysis? }\end{array}$ & Risk of Bias \\
\hline $\begin{array}{l}\text { Pai et al., } \\
2009^{27} \text {; } \\
\text { Pai et al., } \\
2009^{28}\end{array}$ & $\begin{array}{l}\text { G1: } \\
\text { Pharmaceutical } \\
\text { care including } \\
\text { drug therapy } \\
\text { reviews } \\
\text { conducted by } \\
\text { nephrology- } \\
\text { trained clinical } \\
\text { pharmacist with } \\
\text { patient. Also } \\
\text { included patient } \\
\text { and health care } \\
\text { provider } \\
\text { education. } \\
\text { G2: Standard of } \\
\text { care, consisting } \\
\text { of brief therapy } \\
\text { reviews } \\
\text { conducted by } \\
\text { nurse }\end{array}$ & $\begin{array}{l}\text { RCT: } \\
\text { cluster- } \\
\text { rando- } \\
\text { mized }\end{array}$ & No & Yes & Yes & $\mathrm{NA}$ & Yes & Yes & Yes & High \\
\hline $\begin{array}{l}\text { Park et al., } \\
1996^{29}\end{array}$ & $\begin{array}{l}\text { G1: } \\
\text { Comprehensive } \\
\text { pharmaceutical } \\
\text { services, } \\
\text { including drug } \\
\text { therapy } \\
\text { monitoring and } \\
\text { patient education } \\
\text { provided by } \\
\text { community } \\
\text { pharmacy } \\
\text { resident. } \\
\text { G2: Usual care }\end{array}$ & $\begin{array}{l}\text { RCT: } \\
\text { parallel, } \\
\text { not } \\
\text { clus- } \\
\text { tered } \\
\end{array}$ & Yes & Yes & Yes & Yes & NA & $\begin{array}{l}\text { Unclear or } \\
\text { NR }\end{array}$ & $\begin{array}{l}\text { No (Not } \\
\text { accounted for or } \\
\text { not identified) }\end{array}$ & High \\
\hline
\end{tabular}


Table F2. Risk of bias domains and ratings (continued)

\begin{tabular}{|c|c|c|c|c|c|c|c|c|c|c|}
\hline $\begin{array}{l}\text { Author, Year } \\
\text { Trial Name }^{a}\end{array}$ & $\begin{array}{l}\text { Interventions/ } \\
\text { Comparator } \\
\text { Descriptions }\end{array}$ & $\begin{array}{l}\text { Study } \\
\text { Design }\end{array}$ & ITT? & $\begin{array}{l}\text { Eligibility } \\
\text { criteria } \\
\text { measured } \\
\text { consistently } \\
\text { using valid } \\
\text { and reliable } \\
\text { measures? }\end{array}$ & $\begin{array}{l}\text { Intermediate } \\
\text { Outcomes: } \\
\text { assessed } \\
\text { consistently } \\
\text { using valid and } \\
\text { reliable } \\
\text { measures? }\end{array}$ & $\begin{array}{l}\text { Patient-Centered } \\
\text { Outcomes: } \\
\text { assessed } \\
\text { consistently } \\
\text { using valid and } \\
\text { reliable } \\
\text { measures? }\end{array}$ & $\begin{array}{l}\text { Utilization } \\
\text { Outcomes: } \\
\text { assessed } \\
\text { consistently } \\
\text { using valid } \\
\text { and reliable } \\
\text { measures? }\end{array}$ & $\begin{array}{l}\text { Potential } \\
\text { outcome } \\
\text { pre- } \\
\text { specified } \\
\text { and } \\
\text { reported? }\end{array}$ & $\begin{array}{l}\text { Were important } \\
\text { confounding and } \\
\text { modifying } \\
\text { variables taken } \\
\text { into account in } \\
\text { design and/or } \\
\text { analysis? }\end{array}$ & Risk of Bias \\
\hline $\begin{array}{l}\text { Pindolia et al., } \\
2009^{30}\end{array}$ & $\begin{array}{l}\text { G1: Telephone- } \\
\text { based MTM } \\
\text { services } \\
\text { provided as part } \\
\text { of Medicare Part } \\
\text { D MTM program } \\
\text { by pharmacy } \\
\text { care } \\
\text { management } \\
\text { clinical } \\
\text { pharmacists } \\
\text { (acceptors) } \\
\text { G2: Usual } \\
\text { medical care } \\
\text { (opt-out) }\end{array}$ & Cohort & Yes & Yes & Unclear or NR & Unclear or NR & Yes & Yes & $\begin{array}{l}\text { No (Not } \\
\text { accounted for or } \\
\text { not identified) }\end{array}$ & High \\
\hline $\begin{array}{l}\text { Planas et al., } \\
2009^{31}\end{array}$ & $\begin{array}{l}\text { G1: MTM } \\
\text { services } \\
\text { provided by } \\
\text { community } \\
\text { pharmacists. } \\
\text { Also included } \\
\text { patient education } \\
\text { on diet and } \\
\text { lifestyle } \\
\text { modifications to } \\
\text { lower blood } \\
\text { pressure. } \\
\text { G2: No MTM } \\
\text { received, but } \\
\text { only informed of } \\
\text { blood pressure } \\
\text { goals for patients } \\
\text { with diabetes }\end{array}$ & $\begin{array}{l}\text { RCT: } \\
\text { parallel, } \\
\text { not } \\
\text { clus- } \\
\text { tered }\end{array}$ & Yes & Yes & Unclear or NR & NA & NA & Yes & $\begin{array}{l}\text { No (Not } \\
\text { accounted for or } \\
\text { not identified) }\end{array}$ & High \\
\hline
\end{tabular}


Table F2. Risk of bias domains and ratings (continued)

\begin{tabular}{|c|c|c|c|c|c|c|c|c|c|c|}
\hline $\begin{array}{l}\text { Author, Year } \\
\text { Trial Name }^{\mathrm{a}}\end{array}$ & $\begin{array}{l}\text { Interventions/ } \\
\text { Comparator } \\
\text { Descriptions }\end{array}$ & $\begin{array}{l}\text { Study } \\
\text { Design }\end{array}$ & ITT? & $\begin{array}{l}\text { Eligibility } \\
\text { criteria } \\
\text { measured } \\
\text { consistently } \\
\text { using valid } \\
\text { and reliable } \\
\text { measures? }\end{array}$ & $\begin{array}{l}\text { Intermediate } \\
\text { Outcomes: } \\
\text { assessed } \\
\text { consistently } \\
\text { using valid and } \\
\text { reliable } \\
\text { measures? }\end{array}$ & $\begin{array}{l}\text { Patient-Centered } \\
\text { Outcomes: } \\
\text { assessed } \\
\text { consistently } \\
\text { using valid and } \\
\text { reliable } \\
\text { measures? }\end{array}$ & $\begin{array}{l}\text { Utilization } \\
\text { Outcomes: } \\
\text { assessed } \\
\text { consistently } \\
\text { using valid } \\
\text { and reliable } \\
\text { measures? }\end{array}$ & $\begin{array}{l}\text { Potential } \\
\text { outcome } \\
\text { pre- } \\
\text { specified } \\
\text { and } \\
\text { reported? }\end{array}$ & $\begin{array}{l}\text { Were important } \\
\text { confounding and } \\
\text { modifying } \\
\text { variables taken } \\
\text { into account in } \\
\text { design and/or } \\
\text { analysis? }\end{array}$ & Risk of Bias \\
\hline $\begin{array}{l}\text { Roughead et } \\
\text { al., } 2009^{32}\end{array}$ & $\begin{array}{l}\text { G1: HMRs } \\
\text { conducted by } \\
\text { accredited } \\
\text { pharmacists } \\
\text { G2: No } \\
\text { medication } \\
\text { review received }\end{array}$ & Cohort & $\begin{array}{l}\text { Un- } \\
\text { clear } \\
\text { or NR }\end{array}$ & Yes & NA & NA & Yes & Yes & Yes & Medium \\
\hline $\begin{array}{l}\text { Sellors et al., } \\
2003^{33}\end{array}$ & $\begin{array}{l}\text { G1: Clinical } \\
\text { pharmacist } \\
\text { consultations } \\
\text { provided to } \\
\text { family physicians } \\
\text { and their patients } \\
\text { by community } \\
\text { pharmacists. } \\
\text { G2: Usual care } \\
\text { for family } \\
\text { physicians and } \\
\text { their patients } \\
\text { from matched } \\
\text { postal codes. }\end{array}$ & $\begin{array}{l}\text { RCT: } \\
\text { cluster- } \\
\text { randomi } \\
\text { zed }\end{array}$ & $\begin{array}{l}\text { Un- } \\
\text { clear } \\
\text { or NR }\end{array}$ & Yes & NA & Yes & Yes & Yes & Yes & Medium \\
\hline $\begin{array}{l}\text { Sidel et al., } \\
1990^{34}\end{array}$ & $\begin{array}{l}\text { G1: Home visits } \\
\text { by pharmacists } \\
\text { and, when } \\
\text { needed, } \\
\text { consultations } \\
\text { with physicians } \\
\text { to identify and } \\
\text { correct problems } \\
\text { associated with } \\
\text { medication use. } \\
\text { G2: Standard } \\
\text { care without any } \\
\text { visits or } \\
\text { information } \\
\text { provided to G1. }\end{array}$ & $\begin{array}{l}\text { RCT: } \\
\text { parallel, } \\
\text { not } \\
\text { clus- } \\
\text { tered }\end{array}$ & No & No & Yes & Yes & Yes & Yes & $\begin{array}{l}\text { No (Not } \\
\text { accounted for or } \\
\text { not identified) }\end{array}$ & Medium \\
\hline
\end{tabular}


Table F2. Risk of bias domains and ratings (continued)

\begin{tabular}{|c|c|c|c|c|c|c|c|c|c|c|}
\hline $\begin{array}{l}\text { Author, Year } \\
\text { Trial Name }^{\mathrm{a}}\end{array}$ & $\begin{array}{l}\text { Interventions/ } \\
\text { Comparator } \\
\text { Descriptions }\end{array}$ & $\begin{array}{l}\text { Study } \\
\text { Design }\end{array}$ & ITT? & $\begin{array}{l}\text { Eligibility } \\
\text { criteria } \\
\text { measured } \\
\text { consistently } \\
\text { using valid } \\
\text { and reliable } \\
\text { measures? }\end{array}$ & $\begin{array}{l}\text { Intermediate } \\
\text { Outcomes: } \\
\text { assessed } \\
\text { consistently } \\
\text { using valid and } \\
\text { reliable } \\
\text { measures? }\end{array}$ & $\begin{array}{l}\text { Patient-Centered } \\
\text { Outcomes: } \\
\text { assessed } \\
\text { consistently } \\
\text { using valid and } \\
\text { reliable } \\
\text { measures? }\end{array}$ & $\begin{array}{l}\text { Utilization } \\
\text { Outcomes: } \\
\text { assessed } \\
\text { consistently } \\
\text { using valid } \\
\text { and reliable } \\
\text { measures? }\end{array}$ & $\begin{array}{l}\text { Potential } \\
\text { outcome } \\
\text { pre- } \\
\text { specified } \\
\text { and } \\
\text { reported? }\end{array}$ & $\begin{array}{l}\text { Were important } \\
\text { confounding and } \\
\text { modifying } \\
\text { variables taken } \\
\text { into account in } \\
\text { design and/or } \\
\text { analysis? }\end{array}$ & Risk of Bias \\
\hline $\begin{array}{l}\text { Staresinic et } \\
\text { al., } 2007^{35}\end{array}$ & $\begin{array}{l}\text { G1: MTM } \\
\text { services } \\
\text { provided as part } \\
\text { of a Medicare } \\
\text { Part D MTM } \\
\text { program by MTM } \\
\text { Coordinator } \\
\text { (non-clinical } \\
\text { staff) and } \\
\text { pharmacist } \\
\text { (acceptors) } \\
\text { G2: Usual care } \\
\text { provided to } \\
\text { MTM-eligible } \\
\text { enrollees who } \\
\text { chose not to } \\
\text { participate (opt- } \\
\text { out) }\end{array}$ & Cohort & Yes & Yes & Yes & NA & Yes & $\begin{array}{l}\text { Unclear or } \\
\text { NR }\end{array}$ & $\begin{array}{l}\text { No (Not } \\
\text { accounted for or } \\
\text { not identified) }\end{array}$ & High \\
\hline $\begin{array}{l}\text { Taylor, Byrd, } \\
\text { and Krueger, } \\
2003^{36}\end{array}$ & $\begin{array}{l}\text { G1: } \\
\text { Pharmaceutical } \\
\text { care provided by } \\
\text { pharmacists } \\
\text { G2: Standard } \\
\text { care without } \\
\text { advice or recom- } \\
\text { mendations } \\
\text { given to patients } \\
\text { or physicians }\end{array}$ & $\begin{array}{l}\text { RCT: } \\
\text { parallel, } \\
\text { not } \\
\text { clus- } \\
\text { tered } \\
\end{array}$ & $\begin{array}{l}\text { Un- } \\
\text { clear } \\
\text { or NR }\end{array}$ & Yes & Yes & Yes & Yes & Yes & $\mathrm{N} / \mathrm{A}$ & Medium \\
\hline
\end{tabular}


Table F2. Risk of bias domains and ratings (continued)

\begin{tabular}{|c|c|c|c|c|c|c|c|c|c|c|}
\hline $\begin{array}{l}\text { Author, Year } \\
\text { Trial Name }^{a}\end{array}$ & $\begin{array}{l}\text { Interventions/ } \\
\text { Comparator } \\
\text { Descriptions }\end{array}$ & $\begin{array}{l}\text { Study } \\
\text { Design }\end{array}$ & ITT? & $\begin{array}{l}\text { Eligibility } \\
\text { criteria } \\
\text { measured } \\
\text { consistently } \\
\text { using valid } \\
\text { and reliable } \\
\text { measures? }\end{array}$ & $\begin{array}{l}\text { Intermediate } \\
\text { Outcomes: } \\
\text { assessed } \\
\text { consistently } \\
\text { using valid and } \\
\text { reliable } \\
\text { measures? }\end{array}$ & $\begin{array}{l}\text { Patient-Centered } \\
\text { Outcomes: } \\
\text { assessed } \\
\text { consistently } \\
\text { using valid and } \\
\text { reliable } \\
\text { measures? }\end{array}$ & $\begin{array}{l}\text { Utilization } \\
\text { Outcomes: } \\
\text { assessed } \\
\text { consistently } \\
\text { using valid } \\
\text { and reliable } \\
\text { measures? }\end{array}$ & $\begin{array}{l}\text { Potential } \\
\text { outcome } \\
\text { pre- } \\
\text { specified } \\
\text { and } \\
\text { reported? }\end{array}$ & $\begin{array}{l}\text { Were important } \\
\text { confounding and } \\
\text { modifying } \\
\text { variables taken } \\
\text { into account in } \\
\text { design and/or } \\
\text { analysis? }\end{array}$ & Risk of Bias \\
\hline $\begin{array}{l}\text { Touchette et } \\
\text { al., } 2012^{37}\end{array}$ & $\begin{array}{l}\text { G1: MTM basic } \\
\text { (comprehensive } \\
\text { medication } \\
\text { review and DRP } \\
\text { assessment) } \\
\text { G2: MTM } \\
\text { enhanced (MTM } \\
\text { plus } 2 \text { page } \\
\text { clinical summary } \\
\text { abstracted from } \\
\text { patient's medical } \\
\text { chart) } \\
\text { G3: Usual care, } \\
\text { consisting of } \\
\text { medication } \\
\text { counseling per } \\
\text { clinic's normal } \\
\text { routine but no } \\
\text { formal MTM from } \\
\text { a study } \\
\text { pharmacist }\end{array}$ & $\begin{array}{l}\text { RCT: } \\
\text { parallel, } \\
\text { not } \\
\text { clus- } \\
\text { tered } \\
\end{array}$ & Yes & Yes & No & Yes & Yes & Yes & Yes & Low \\
\hline $\begin{array}{l}\text { Triller et al., } \\
2007^{38}\end{array}$ & $\begin{array}{l}\text { G1: VNA home } \\
\text { visit services } \\
\text { plus } \\
\text { comprehensive } \\
\text { pharmaceutical } \\
\text { care services } \\
\text { G2: VNA home } \\
\text { visit services } \\
\text { only }\end{array}$ & $\begin{array}{l}\text { RCT: } \\
\text { parallel, } \\
\text { not } \\
\text { clus- } \\
\text { tered }\end{array}$ & Yes & Yes & NA & Yes & Yes & Yes & $\begin{array}{l}\text { No (Not } \\
\text { accounted for or } \\
\text { not identified) }\end{array}$ & Medium \\
\hline
\end{tabular}


Table F2. Risk of bias domains and ratings (continued)

\begin{tabular}{|c|c|c|c|c|c|c|c|c|c|c|}
\hline $\begin{array}{l}\text { Author, Year } \\
\text { Trial Name }^{a}\end{array}$ & $\begin{array}{l}\text { Interventions/ } \\
\text { Comparator } \\
\text { Descriptions }\end{array}$ & $\begin{array}{l}\text { Study } \\
\text { Design }\end{array}$ & ITT? & $\begin{array}{l}\text { Eligibility } \\
\text { criteria } \\
\text { measured } \\
\text { consistently } \\
\text { using valid } \\
\text { and reliable } \\
\text { measures? }\end{array}$ & $\begin{array}{l}\text { Intermediate } \\
\text { Outcomes: } \\
\text { assessed } \\
\text { consistently } \\
\text { using valid and } \\
\text { reliable } \\
\text { measures? }\end{array}$ & $\begin{array}{l}\text { Patient-Centered } \\
\text { Outcomes: } \\
\text { assessed } \\
\text { consistently } \\
\text { using valid and } \\
\text { reliable } \\
\text { measures? }\end{array}$ & $\begin{array}{l}\text { Utilization } \\
\text { Outcomes: } \\
\text { assessed } \\
\text { consistently } \\
\text { using valid } \\
\text { and reliable } \\
\text { measures? }\end{array}$ & $\begin{array}{l}\text { Potential } \\
\text { outcome } \\
\text { pre- } \\
\text { specified } \\
\text { and } \\
\text { reported? }\end{array}$ & $\begin{array}{l}\text { Were important } \\
\text { confounding and } \\
\text { modifying } \\
\text { variables taken } \\
\text { into account in } \\
\text { design and/or } \\
\text { analysis? }\end{array}$ & Risk of Bias \\
\hline $\begin{array}{l}\text { Volume et al., } \\
2001, \text { PREP } \\
\text { Kassam et al., } \\
2001^{40}\end{array}$ & $\begin{array}{l}\text { G1: } \\
\text { Comprehensive } \\
\text { pharmaceutical } \\
\text { care services } \\
\text { using a nine-step } \\
\text { process as } \\
\text { defined by } \\
\text { Hepler and } \\
\text { Strand provided } \\
\text { by community } \\
\text { pharmacists } \\
\text { G2: Traditional } \\
\text { pharmacy care }\end{array}$ & $\begin{array}{l}\text { RCT: } \\
\text { cluster- } \\
\text { rando- } \\
\text { mized } \\
\end{array}$ & $\begin{array}{l}\text { Un- } \\
\text { clear } \\
\text { or NR }\end{array}$ & Yes & Yes & Yes & NA & Yes & $\begin{array}{l}\text { No (Not } \\
\text { accounted for or } \\
\text { not identified) }\end{array}$ & Medium \\
\hline $\begin{array}{l}\text { Welch et al., } \\
2009^{41}\end{array}$ & $\begin{array}{l}\text { G1: MTM } \\
\text { program } \\
\text { provided to } \\
\text { home-based } \\
\text { beneficiaries as } \\
\text { part of Medicare } \\
\text { Part D MTM } \\
\text { program } \\
\text { (acceptors) } \\
\text { G2: No-MTM } \\
\text { control group } \\
\text { (voluntary opt- } \\
\text { out) }\end{array}$ & Cohort & $\begin{array}{l}\text { Un- } \\
\text { clear } \\
\text { or NR }\end{array}$ & Yes & Unclear or NR & Yes & Yes & Yes & $\begin{array}{l}\text { Partial (some } \\
\text { variables were } \\
\text { taken in to } \\
\text { account) }\end{array}$ & Medium \\
\hline
\end{tabular}


Table F2. Risk of bias domains and ratings (continued)

\begin{tabular}{|c|c|c|c|c|c|c|c|c|c|c|}
\hline $\begin{array}{l}\text { Author, Year } \\
\text { Trial Name }^{a}\end{array}$ & $\begin{array}{l}\text { Interventions/ } \\
\text { Comparator } \\
\text { Descriptions }\end{array}$ & $\begin{array}{l}\text { Study } \\
\text { Design }\end{array}$ & ITT? & $\begin{array}{l}\text { Eligibility } \\
\text { criteria } \\
\text { measured } \\
\text { consistently } \\
\text { using valid } \\
\text { and reliable } \\
\text { measures? }\end{array}$ & $\begin{array}{l}\text { Intermediate } \\
\text { Outcomes: } \\
\text { assessed } \\
\text { consistently } \\
\text { using valid and } \\
\text { reliable } \\
\text { measures? }\end{array}$ & $\begin{array}{l}\text { Patient-Centered } \\
\text { Outcomes: } \\
\text { assessed } \\
\text { consistently } \\
\text { using valid and } \\
\text { reliable } \\
\text { measures? }\end{array}$ & $\begin{array}{l}\text { Utilization } \\
\text { Outcomes: } \\
\text { assessed } \\
\text { consistently } \\
\text { using valid } \\
\text { and reliable } \\
\text { measures? }\end{array}$ & $\begin{array}{l}\text { Potential } \\
\text { outcome } \\
\text { pre- } \\
\text { specified } \\
\text { and } \\
\text { reported? }\end{array}$ & $\begin{array}{l}\text { Were important } \\
\text { confounding and } \\
\text { modifying } \\
\text { variables taken } \\
\text { into account in } \\
\text { design and/or } \\
\text { analysis? }\end{array}$ & Risk of Bias \\
\hline $\begin{array}{l}\text { Williams et al., } \\
2004^{42}\end{array}$ & $\begin{array}{l}\text { G1: Modification } \\
\text { of patient's } \\
\text { medication } \\
\text { regimen } \\
\text { conducted by } \\
\text { interdisciplinary } \\
\text { medication } \\
\text { adjustment team } \\
\text { in addition to } \\
\text { usual medical } \\
\text { care and "Bound } \\
\text { for Health" } \\
\text { booklet. } \\
\text { G2: Usual } \\
\text { medical care } \\
\text { plus provision of } \\
\text { "Bound for } \\
\text { Health" booklet }\end{array}$ & $\begin{array}{l}\text { RCT: } \\
\text { parallel, } \\
\text { not } \\
\text { clus- } \\
\text { tered }\end{array}$ & $\begin{array}{l}\text { Un- } \\
\text { clear } \\
\text { or NR }\end{array}$ & Yes & Yes & Yes & No & Yes & $\begin{array}{l}\text { No (Not } \\
\text { accounted for or } \\
\text { not identified) }\end{array}$ & Medium \\
\hline
\end{tabular}


Table F2. Risk of bias domains and ratings (continued)

\begin{tabular}{|c|c|c|c|c|c|c|c|c|c|c|}
\hline $\begin{array}{l}\text { Author, Year } \\
\text { Trial Name }^{\mathrm{a}}\end{array}$ & $\begin{array}{l}\text { Interventions/ } \\
\text { Comparator } \\
\text { Descriptions }\end{array}$ & $\begin{array}{l}\text { Study } \\
\text { Design }\end{array}$ & ITT? & $\begin{array}{l}\text { Eligibility } \\
\text { criteria } \\
\text { measured } \\
\text { consistently } \\
\text { using valid } \\
\text { and reliable } \\
\text { measures? }\end{array}$ & $\begin{array}{l}\text { Intermediate } \\
\text { Outcomes: } \\
\text { assessed } \\
\text { consistently } \\
\text { using valid and } \\
\text { reliable } \\
\text { measures? }\end{array}$ & $\begin{array}{l}\text { Patient-Centered } \\
\text { Outcomes: } \\
\text { assessed } \\
\text { consistently } \\
\text { using valid and } \\
\text { reliable } \\
\text { measures? }\end{array}$ & $\begin{array}{l}\text { Utilization } \\
\text { Outcomes: } \\
\text { assessed } \\
\text { consistently } \\
\text { using valid } \\
\text { and reliable } \\
\text { measures? }\end{array}$ & $\begin{array}{l}\text { Potential } \\
\text { outcome } \\
\text { pre- } \\
\text { specified } \\
\text { and } \\
\text { reported? }\end{array}$ & $\begin{array}{l}\text { Were important } \\
\text { confounding and } \\
\text { modifying } \\
\text { variables taken } \\
\text { into account in } \\
\text { design and/or } \\
\text { analysis? }\end{array}$ & Risk of Bias \\
\hline $\begin{array}{l}\text { Winston and } \\
\text { Lin, } 2009^{43}\end{array}$ & $\begin{array}{l}\text { G1: MTM } \\
\text { provided in } \\
\text { community } \\
\text { pharmacy (i.e., } \\
\text { care in face-to- } \\
\text { face meetings or } \\
\text { by telephone) as } \\
\text { part of Medicare } \\
\text { Part D MTM } \\
\text { program } \\
\text { G2: MTM } \\
\text { provided by } \\
\text { pharmacist- } \\
\text { staffed call } \\
\text { centers as part } \\
\text { of Medicare Part } \\
\text { D MTM program } \\
\text { G3: Educational } \\
\text { mailings (i.e., } \\
\text { mailed letter } \\
\text { containing } \\
\text { patient-specific } \\
\text { medication } \\
\text { related } \\
\text { information, } \\
\text { personal } \\
\text { medication } \\
\text { record, and tips } \\
\text { to save money } \\
\text { on prescriptions) }\end{array}$ & Cohort & No & Yes & NA & NA & Yes & $\begin{array}{l}\text { Unclear or } \\
\text { NR }\end{array}$ & $\begin{array}{l}\text { No (Not } \\
\text { accounted for or } \\
\text { not identified) }\end{array}$ & High \\
\hline
\end{tabular}


Table F2. Risk of bias domains and ratings (continued)

\begin{tabular}{|c|c|c|c|c|c|c|c|c|c|c|}
\hline $\begin{array}{l}\text { Author, Year } \\
\text { Trial Name }^{a}\end{array}$ & $\begin{array}{l}\text { Interventions/ } \\
\text { Comparator } \\
\text { Descriptions }\end{array}$ & $\begin{array}{l}\text { Study } \\
\text { Design }\end{array}$ & ITT? & $\begin{array}{l}\text { Eligibility } \\
\text { criteria } \\
\text { measured } \\
\text { consistently } \\
\text { using valid } \\
\text { and reliable } \\
\text { measures? }\end{array}$ & $\begin{array}{l}\text { Intermediate } \\
\text { Outcomes: } \\
\text { assessed } \\
\text { consistently } \\
\text { using valid and } \\
\text { reliable } \\
\text { measures? }\end{array}$ & $\begin{array}{l}\text { Patient-Centered } \\
\text { Outcomes: } \\
\text { assessed } \\
\text { consistently } \\
\text { using valid and } \\
\text { reliable } \\
\text { measures? }\end{array}$ & $\begin{array}{l}\text { Utilization } \\
\text { Outcomes: } \\
\text { assessed } \\
\text { consistently } \\
\text { using valid } \\
\text { and reliable } \\
\text { measures? }\end{array}$ & $\begin{array}{l}\text { Potential } \\
\text { outcome } \\
\text { pre- } \\
\text { specified } \\
\text { and } \\
\text { reported? }\end{array}$ & $\begin{array}{l}\text { Were important } \\
\text { confounding and } \\
\text { modifying } \\
\text { variables taken } \\
\text { into account in } \\
\text { design and/or } \\
\text { analysis? }\end{array}$ & Risk of Bias \\
\hline $\begin{array}{l}\text { Witry, } \\
\text { Doucette, and } \\
\text { Gainer, } 2011^{44}\end{array}$ & $\begin{array}{l}\text { G1: PCM } \\
\text { provided by } \\
\text { community } \\
\text { pharmacists to } \\
\text { lowa Medicaid } \\
\text { enrollees } \\
\text { G2: PCM } \\
\text { provided to } \\
\text { patients with } \\
\text { private } \\
\text { individual-group } \\
\text { insurance }\end{array}$ & Cohort & $\begin{array}{l}\text { Un- } \\
\text { clear } \\
\text { or NR }\end{array}$ & Yes & Unclear or NR & NA & NA & Yes & $\begin{array}{l}\text { No (Not } \\
\text { accounted for or } \\
\text { not identified) }\end{array}$ & High \\
\hline
\end{tabular}

Abbreviations: $\mathrm{BP}=$ blood pressure; DTP = drug therapy problem; $\mathrm{G}=$ group; HMR = home medication review; IMPROVE = specific name of the MTM trial that was done in the Veterans Affairs health system; ITT = intention-to-treat; MTM = medication therapy management; MTMP = medication therapy management program; NA = not applicable; NR = not reported; NRCT $=$ non-randomized controlled trial; PA = physician assistant; PCM = pharmaceutical case management; PREP = Pharmaceutical Care Research and Education Project; $\mathrm{RCT}=$ randomized controlled trial; VNA = visiting nurse association. 
Table F3. Rationale for high and medium risk of bias ratings

\begin{tabular}{ll}
\hline $\begin{array}{l}\text { Author, Year } \\
\text { Trial Name }\end{array}$ & Risk of Bias \\
\hline $\begin{array}{l}\text { Bernsten et al., 2001; } \\
\text { Sturgess, } 2003^{2}\end{array}$ & Rationale for Rating \\
& High \\
& $\begin{array}{c}\text { Potential for performance and selective outcome reporting bias: } \\
\text { - Issues concerning site and country-specific variation in pooled analyses } \\
\text { - Some selective reporting of country-specific outcomes when statistically significant }\end{array}$
\end{tabular}

- Some selective reporting of country-specific outcomes when statistically significant

Potential for attrition bias:

- High overall attrition and no strategies used to take into account baseline differences between patients LTFU and study completers

Potential for selection bias:

- Some important potential confounders not measured at baseline, like baseline disease severity and co-morbidity

Blennerhassett et al., $2007^{3} \quad$ High

Potential for selection bias:

- $\quad$ Different methods of recruiting into each arm (self-selection)

- Differences between HMR and non-HMR groups which are not accounted for clearly, and exclusion criteria not specified Potential for attrition bias:

- Differential and high attrition rates

Potential for measurement bias:

- Unvalidated outcome measure for eligible outcome (Table 3: "information received confused them")

Missing information:

- Intervention with patients not clearly defined or explained

Carter et al., $1997^{4}$; Barnette et High

al., $1996^{5}$

Potential for selection bias:

- No accounting for differences in recruitment strategies or for baseline differences

Chisholm et al., $2002^{6} \quad$ Medium

Potential for attrition bias:

- Did not use ITT analysis.

Potential for measurement bias:

- Unclear that all outcome assessments blinded

Missing information:

- $\quad$ Lack of information about how utilization outcomes were measured

Chrischilles et al., 2004 High

Potential for selection bias:

- High risk of confounding from the pharmacist potentially selecting patients for the intervention who were on high risk medications

- Differences in the prevalence of high risk medications at baseline not controlled for the analysis. 


\section{Table F3. Rationale for high and medium risk of bias ratings (continued)}

\begin{tabular}{|c|c|}
\hline $\begin{array}{l}\text { Author, Year } \\
\text { Trial Name }^{\mathrm{a}}\end{array}$ & $\begin{array}{l}\text { Risk of Bias } \\
\text { Rationale for Rating }\end{array}$ \\
\hline Christensen et al., $2007^{8}$ & $\begin{array}{l}\text { Medium } \\
\text { Potential for selection bias: } \\
\text { - Group assignment not randomized } \\
\text { Intervention group patients had more prescriptions at baseline, suggesting difference in severity of disease between } \\
\text { intervention group and both control groups } \\
\text { Potential for measurement bias: } \\
\text { - No outcomes presented in the study actually provided comparison among study groups. DTPs identified and resolved } \\
\text { and patient satisfaction only provided as pre/post for the study group, and economic outcomes presented as pre/post for } \\
\text { each of the three groups, but they were not compared to each other. }\end{array}$ \\
\hline Clifford et al., $2002^{9}$ & $\begin{array}{l}\text { Medium } \\
\text { Potential for selection bias: } \\
\text { - Not clear how groups compare in terms of comorbidity or number of medications at baseline. } \\
\text { Still, measures taken to reduce bias in other domains, like having the same pharmacist provide the intervention to all patients. }\end{array}$ \\
\hline Fischer et al., $2000^{10}$ & $\begin{array}{l}\text { Medium } \\
\text { Potential for selection bias: } \\
\text { - Unclear reporting of N's in outcome analyses makes fully determining selection bias difficult } \\
\text { Potential for measurement bias: } \\
\text { - Outcome measures, although piloted and assessed for face validity prior to study, were unvalidated and relied on self- } \\
\text { - } \quad \text { While authors claim research questions a priori included assessment of "awareness of side effects", they apparently } \\
\text { found it paradoxical that intervention arm reported more side effects and so post hoc decided to interpret this as } \\
\text { "increased awareness" making it very difficult to draw a valid conclusion. }\end{array}$ \\
\hline Fischer et al., $2002^{11}$ & $\begin{array}{l}\text { Medium } \\
\text { Potential for selection bias: } \\
\quad \quad \text { Lack of randomization }\end{array}$ \\
\hline Fox et al., $2009^{12}$ & $\begin{array}{l}\text { High } \\
\begin{array}{l}\text { Potential for selection bias: } \\
\text { - No baseline clinical data provided about patients, in particular number of diagnosed conditions, number of medications } \\
\quad \text { prescribed, and healthcare utilization }\end{array}\end{array}$ \\
\hline
\end{tabular}




\section{Table F3. Rationale for high and medium risk of bias ratings (continued)}

\begin{tabular}{|c|c|}
\hline $\begin{array}{l}\text { Author, Year } \\
\text { Trial Name }^{a}\end{array}$ & $\begin{array}{l}\text { Risk of Bias } \\
\text { Rationale for Rating }\end{array}$ \\
\hline Gattis et al., $1999^{13}$ & $\begin{array}{l}\text { Medium } \\
\text { Potential for measurement bias: } \\
\text { - Lack of blinded outcome assessment } \\
\text { - Additional potential source of bias because intervention pharmacist was responsible for assessing control group's } \\
\text { outcomes } \\
\text { Missing information } \\
\text { - Unclear to what extent included patients had care outside of Duke }\end{array}$ \\
\hline Harrison et al., $2012^{15}$ & $\begin{array}{l}\text { Medium } \\
\text { Potential for selection bias: } \\
\quad \quad \text { Differences in recruitment } \\
\text { Potential for measurement bias: } \\
\text { - Lack of information regarding how outcomes measured and degree of blinding of assessors }\end{array}$ \\
\hline Isetts et al., $2008^{16}$ & $\begin{array}{l}\text { High } \\
\text { Potential for selection bias: } \\
\text { - Differences in recruitment methods, but no evidence that any methods used to adjust for these differences } \\
\text { Unclear how clinics with MTM differed from clinics without MTM in terms of patient populations served and other } \\
\text { - } \quad \text { Unclear how HEDIS comparison group was identified } \\
\text { Potential for measurement bias: } \\
\text { - Did not take into account different confounding and modifying variables into a multivariate analysis } \\
\text { Missing information } \\
\text { - Data on baseline covariates between intervention and HEDIS control group not presented }\end{array}$ \\
\hline Jameson et al., $1995^{17}$ & $\begin{array}{l}\text { Medium } \\
\text { Potential for detection and attrition bias: } \\
\qquad \quad \text { Outcome assessment not blinded and no ITT analysis conducted } \\
\end{array}$ \\
\hline Jeong et al., $2007^{18}$ & $\begin{array}{l}\text { High } \\
\text { Potential for selection bias: } \\
\text { - Cohort study in which patients self-selected group assignment and appropriate statistical controls for selection bias not } \\
\text { in place } \\
\text { Baseline characteristics did not capture important variables that could potentially bias results, such as burden of co- } \\
\text { morbidity, number of prescriptions, and multiple demographic variables }\end{array}$ \\
\hline
\end{tabular}




\section{Table F3. Rationale for high and medium risk of bias ratings (continued)}

\begin{tabular}{|c|c|}
\hline $\begin{array}{l}\text { Author, Year } \\
\text { Trial Name }^{a}\end{array}$ & $\begin{array}{l}\text { Risk of Bias } \\
\text { Rationale for Rating }\end{array}$ \\
\hline Krska et al., $2001^{19}$ & $\begin{array}{l}\text { Medium } \\
\text { Potential for selection and measurement bias: } \\
\text { - No details on randomization or allocation concealment } \\
\text { - No details about blinding. }\end{array}$ \\
\hline $\begin{array}{l}\text { Malone et al., } 2000^{20} \\
\text { Ellis, } 2000^{21} ; \\
\text { Malone, } 2001^{22} \\
\text { Ellis, } 2000^{23} \\
\text { IMPROVE }\end{array}$ & $\begin{array}{l}\text { Medium } \\
\text { Potential for selection bias: } \\
\text { - Lack of information about allocation concealment } \\
\text { Potential for detection bias: } \\
\text { - Blinding of outcome assessors unclear. } \\
\text { Potential for performance bias: } \\
\text { - Numerous concurrent changes within the VA clinical setting may have impacted either the intervention patients, control } \\
\quad \text { patients, or both }\end{array}$ \\
\hline McDonough et al., $2005^{24}$ & $\begin{array}{l}\text { Medium } \\
\text { Potential for selection and detection bias: } \\
\text { - Differences in outcome at baseline not adjusted for in analysis } \\
\text { - Uncertain whether outcome assessors blinded } \\
\text { - Outcome measurement based on self-report only }\end{array}$ \\
\hline $\begin{array}{l}\text { Moczygemba et al., } 2011^{25} \text {; } \\
\text { Moczygemba et al., } 2008^{26}\end{array}$ & $\begin{array}{l}\text { Medium } \\
\text { Potential for selection bias: } \\
\qquad \quad 16.7 \% \text { of patients allocated to the intervention group withdrew, and attrition not fully accounted for in design }\end{array}$ \\
\hline $\begin{array}{l}\text { Pai et al., } 2009^{27} \text {; Pai et al., } \\
2009^{28}\end{array}$ & $\begin{array}{l}\text { High } \\
\text { Potential for selection bias: } \\
\qquad \quad \text { Inadequate sequence generation } \\
\quad>50 \% \text { attrition }\end{array}$ \\
\hline
\end{tabular}




\section{Table F3. Rationale for high and medium risk of bias ratings (continued)}

\begin{tabular}{|c|c|}
\hline $\begin{array}{l}\text { Author, Year } \\
\text { Trial Name }^{a}\end{array}$ & $\begin{array}{l}\text { Risk of Bias } \\
\text { Rationale for Rating }\end{array}$ \\
\hline Park et al., $1996^{29}$ & High \\
\hline & $\begin{array}{l}\text { Potential for selection bias and contamination: } \\
\text { - Lack of cluster randomization increasing likelihood of contamination of the usual care arm at each site } \\
\text { - Method of randomization and whether allocation concealment used NR } \\
\text { - Differences in important factors at baseline despite randomization, with no statistical adjustment } \\
\text { Potential for performance bias: }\end{array}$ \\
\hline
\end{tabular}

Potential for performance bias:

- $\quad$ Potential for secular effects or uncontrolled confounding from other interventions or exposures because the intervention was conducted at separate time points at the two separate sites, using two different interventionists

Potential for detection bias:

- $\quad$ Lack of outcome assessor blinding

Pindolia et al., $2009^{30} \quad$ High

Potential for selection bias:

- Neither baseline differences in health utilization characteristics nor important confounders (e.g., polypharmacy, number of conditions) accounted for in statistical analysis

Potential for detection bias:

- Not clear that outcome assessors were blinded

\begin{tabular}{ccc}
\hline Planas et al., $2009^{31}$ & High \\
& $\begin{array}{c}\text { Potential for selection bias: } \\
\text { • No steps taken to }\end{array}$
\end{tabular}

- No steps taken to control for baseline differences in demographic characteristics and BMI that were measured, and other important potential confounders not measured at all. Important risk of confounding despite use of ITT analysis and RCT design.

Potential for detection bias:

- Not clear that outcome assessors were blinded

\begin{tabular}{ll}
\hline Roughead et al., $2009^{32}$ & Medium \\
& Missing information:
\end{tabular}

- Lack of clarity on various risk of bias criteria

Sellors et al., $2003^{33} \quad$ Medium

Potential for selection bias and contamination:

- Unclear if ITT analysis used or if investigators controlled for potential co-interventions 


\section{Table F3. Rationale for high and medium risk of bias ratings (continued)}

\begin{tabular}{|c|c|}
\hline $\begin{array}{l}\text { Author, Year } \\
\text { Trial Name }^{a}\end{array}$ & $\begin{array}{l}\text { Risk of Bias } \\
\text { Rationale for Rating }\end{array}$ \\
\hline Sidel et al., $1990^{34}$ & $\begin{array}{l}\text { Medium } \\
\text { Potential for selection and detection bias: } \\
\text { - High attrition } \\
\text { - Outcomes all self-reported and not validated } \\
\text { - Unclear if or how researchers blinded when obtaining questionnaires } \\
\text { - No confounders taken into account in analysis }\end{array}$ \\
\hline Staresinic et al., $2007^{35}$ & $\begin{array}{l}\text { High } \\
\text { Potential for selection bias: } \\
\qquad \quad \text { Group assignment based on self-selection, since intervention group formed from those who returned a survey }\end{array}$ \\
\hline $\begin{array}{l}\text { Taylor, Byrd, and Krueger, } \\
2003^{36}\end{array}$ & 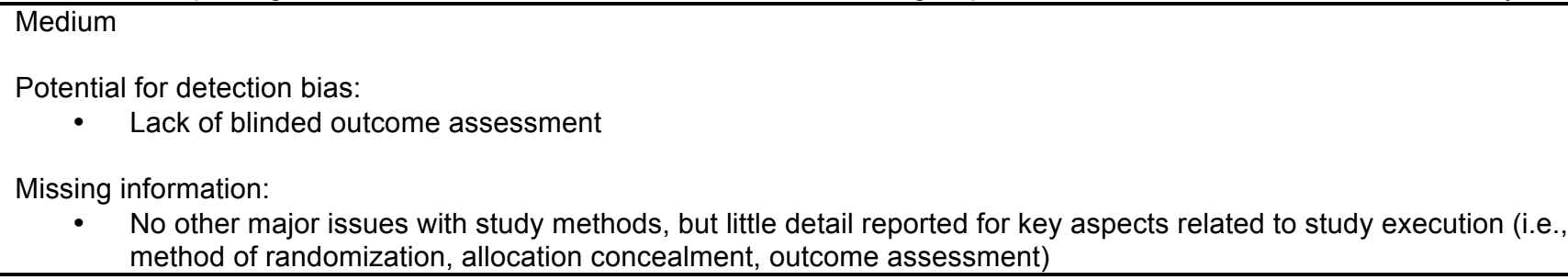 \\
\hline Triller et al., $2007^{38}$ & $\begin{array}{l}\text { Medium } \\
\text { Potential for selection and detection bias: } \\
\text { - Baseline differences in medication use imply differing disease severity among groups } \\
\text { - No adjustment of results for baseline differences in age or disease severity or medication usage } \\
\text { Note (not a source of bias): study powered based on assumption of a } 40 \% \text { relative reduction in composite outcome }\end{array}$ \\
\hline $\begin{array}{l}\text { Volume et al., } 2001^{39} \\
\text { Kassam et al., } 2001^{40}\end{array}$ & $\begin{array}{l}\text { Medium } \\
\text { Potential for performance bias: }\end{array}$ \\
\hline $\begin{array}{l}\text { PREP (Pharmaceutical Care } \\
\text { Research and Education } \\
\text { Project) }\end{array}$ & $\begin{array}{l}\text { - Intervention provided at different pharmacy sites by different interventionists with no mention of measures used to } \\
\text { ensure fidelity of intervention } \\
\text { Potential for selection bias: } \\
\text { - Lack of adjustment for differences at baseline } \\
\text { - Borderline high attrition and possibility of selection bias due to pharmacist control over patient recruitment }\end{array}$ \\
\hline
\end{tabular}




\section{Table F3. Rationale for high and medium risk of bias ratings (continued)}

\begin{tabular}{|c|c|}
\hline $\begin{array}{l}\text { Author, Year } \\
\text { Trial Name }^{a}\end{array}$ & $\begin{array}{l}\text { Risk of Bias } \\
\text { Rationale for Rating }\end{array}$ \\
\hline Welch et al., $2009^{41}$ & $\begin{array}{l}\text { Medium } \\
\text { Potential for detection bias: } \\
\text { - Adjusted ORs most reliable outcomes to use because other non-OR outcomes not adjusted for baseline differences with } \\
\text { - } \quad \text { Validity and reliability of sources for outcome data unclear. }\end{array}$ \\
\hline Williams et al., $2004^{42}$ & $\begin{array}{l}\text { Medium } \\
\text { Potential for selection bias: } \\
\text { - Although randomized design used, method of randomization and allocation concealment not reported } \\
\text { - Unclear whether outcome assessors blinded } \\
\text { Potential for measurement bias: } \\
\text { - Questionable methods used for calculating costs of drugs, particularly if intervention only } 6 \text { weeks long }\end{array}$ \\
\hline Winston and Lin, $2009^{43}$ & $\begin{array}{l}\text { High } \\
\text { Potential for selection bias: } \\
\text { - Study does not control underlying confounders leading to patients' selection of pharmacies } \\
\text { - Pharmacies' inability to provide MTM leading to other modalities and outcomes }\end{array}$ \\
\hline $\begin{array}{l}\text { Witry, Doucette, and Gainer, } \\
2011^{44}\end{array}$ & $\begin{array}{l}\text { High } \\
\text { Potential for selection bias: } \\
\text { - Use of historical control group with much larger } \mathrm{N}, \text { not addressed in design } \\
\text { - No attempts to adjust for potential and actual differences in confounders and baseline characteristics, including baseline } \\
\text { comorbidities, age, and sex } \\
\text { - No reporting of attrition at all } \\
\text { Missing information: } \\
\text { - Lack of reporting about major aspects of study design }\end{array}$ \\
\hline
\end{tabular}

Abbreviations: HEDIS = Healthcare Effectiveness Data and Information Set; HMR = home medication review; IMPROVE = specific name of the MTM trial that was done in the Veterans Affairs health system; ITT = intention-to-treat; MTM = medication therapy management; $\mathrm{N}=$ sample or group size; $\mathrm{NR}=$ not reported; OR $=$ odds ratio; RCT $=$ randomized controlled trial; VA = Veterans Affairs. 


\section{References}

1. Bernsten C, Bjorkman I, Caramona M, et al. Improving the well-being of elderly patients via community pharmacy-based provision of pharmaceutical care: a multicentre study in seven European countries. Drugs Aging. 2001;18(1):63-77. PMID: 11232739.

2. Sturgess IK, McElnay JC, Hughes CM, et al. Community pharmacy based provision of pharmaceutical care to older patients. Pharm World Sci. 2003 Oct;25(5):218-26. PMID: 14584229.

3. Blennerhassett JD, Cusack BM, Smith CD, et al. A novel medicines management pathway. Journal of Pharmacy Practice and Research (Australia). 2006;36(Mar):175-9. PMID: 44-00783.

4. Carter BL, Barnette DJ, Chrischilles E, et al. Evaluation of hypertensive patients after care provided by community pharmacists in a rural setting. Pharmacotherapy. 1997 NovDec;17(6):1274-85. PMID: 9399611.

5. Barnette DJ, Murphy CM, Carter BL. Clinical skill development for community pharmacists. J Am Pharm Assoc (Wash). 1996 Sep;NS36(9):573-80. PMID: 8824077.

6. Chisholm MA, Mulloy LL, Jagadeesan M, et al. Effect of clinical pharmacy services on the blood pressure of African-American renal transplant patients. Ethn Dis. 2002 Summer;12(3):392-7. PMID: 12148711.

7. Chrischilles EA, Carter BL, Lund BC, et al. Evaluation of the Iowa Medicaid pharmaceutical case management program. $\mathrm{J}$ Am Pharm Assoc (2003). 2004 MayJun;44(3):337-49. PMID: 15191244.

8. Christensen DB, Roth M, Trygstad T, et al. Evaluation of a pilot medication therapy management project within the North Carolina State Health Plan. J Am Pharm Assoc (2003). 2007 Jul-Aug;47(4):471-83. PMID: 17616493.

9. Clifford RM, Batty KT, Davis TME, et al. A randomised controlled trial of a pharmaceutical care programme in high-risk diabetic patients in an outpatient clinic. Int $\mathrm{J}$ Pharm Pract. 2002;10(2):85-9.

10. Fischer LR, Scott LM, Boonstra DM, et al. Pharmaceutical care for patients with chronic conditions. J Am Pharm Assoc
(Wash). 2000 Mar-Apr;40(2):174-80. PMID: 10730021.

11. Fischer LR, Defor TA, Cooper S, et al. Pharmaceutical care and health care utilization in an HMO. Eff Clin Pract. 2002 Mar-Apr;5(2):49-57. PMID: 11990212.

12. Fox D, Ried LD, Klein GE, et al. A medication therapy management program's impact on low-density lipoprotein cholesterol goal attainment in Medicare Part D patients with diabetes. J Am Pharm Assoc (2003). 2009 Mar-Apr;49(2):192-9. PMID: 19289345.

13. Gattis WA, Hasselblad V, Whellan DJ, et al. Reduction in heart failure events by the addition of a clinical pharmacist to the heart failure management team: results of the Pharmacist in Heart Failure Assessment Recommendation and Monitoring (PHARM) Study. Arch Intern Med. 1999 Sep 13;159(16):1939-45. PMID: 10493325.

14. Hanlon JT, Weinberger M, Samsa GP, et al. A randomized, controlled trial of a clinical pharmacist intervention to improve inappropriate prescribing in elderly outpatients with polypharmacy. Am J Med. 1996 Apr;100(4):428-37. PMID: 8610730.

15. Harrison JJ, Wang J, Cervenko J, et al. Pilot study of a pharmaceutical care intervention in an outpatient lung transplant clinic. Clin Transplant. 2012 Mar-Apr;26(2):E149-57. PMID: 22507355.

16. Isetts BJ, Schondelmeyer SW, Artz MB, et al. Clinical and economic outcomes of medication therapy management services: the Minnesota experience. J Am Pharm Assoc. 2008 Mar-Apr;48(2):203-11; 3 p following 11. PMID: 18359733.

17. Jameson J, VanNoord G, Vanderwoud K. The impact of a pharmacotherapy consultation on the cost and outcome of medical therapy. J Fam Pract. 1995 Nov;41(5):469-72. PMID: 7595265.

18. Jeong EW, Gallagher MW, Fredriks D, et al. Impact of a pharmacist-managed medication therapy management (MTM) program. ASHP Midyear Clinical Meeting. 2007;42(DEC)PMID: 45-05344. 
19. Krska J, Cromarty JA, Arris F, et al. Pharmacist-led medication review in patients over 65: a randomized, controlled trial in primary care. Age Ageing. 2001 May;30(3):205-11. PMID: 11443021.

20. Malone DC, Carter BL, Billups SJ, et al. An economic analysis of a randomized, controlled, multicenter study of clinical pharmacist interventions for high-risk veterans: the IMPROVE study. Impact of Managed Pharmaceutical Care Resource Utilization and Outcomes in Veterans Affairs Medical Centers. Pharmacotherapy. 2000 Oct;20(10):1149-58. PMID: 11034037.

21. Ellis SL, Billups SJ, Malone DC, et al. Types of interventions made by clinical pharmacists in the IMPROVE study. Impact of Managed Pharmaceutical Care on Resource Utilization and Outcomes in Veterans Affairs Medical Centers. Pharmacotherapy. 2000 Apr;20(4):429-35. PMID: 10772374.

22. Malone DC, Carter BL, Billups SJ, et al. Can clinical pharmacists affect SF-36 scores in veterans at high risk for medicationrelated problems? Med Care. 2001 Feb;39(2):113-22. PMID: 11176549.

23. Ellis SL, Carter BL, Malone DC, et al. Clinical and economic impact of ambulatory care clinical pharmacists in management of dyslipidemia in older adults: the IMPROVE study. Impact of Managed Pharmaceutical Care on Resource Utilization and Outcomes in Veterans Affairs Medical Centers. Pharmacotherapy. 2000 Dec;20(12):150816. PMID: 11130223.

24. McDonough RP, Doucette WR, Kumbera P, et al. An evaluation of managing and educating patients on the risk of glucocorticoid-induced osteoporosis. Value Health. 2005 Jan-Feb;8(1):24-31. PMID: 15841891 .

25. Moczygemba LR, Barner JC, Lawson KA, et al. Impact of telephone medication therapy management on medication and health-related problems, medication adherence, and Medicare Part D drug costs: a 6-month follow up. Am J Geriatr Pharmacother. 2011 Oct;9(5):328-38. PMID: 21865093.
26. Moczygemba LR, Barner JC, Gabrillo ER, et al. Development and implementation of a telephone medication therapy management program for Medicare beneficiaries. Am J Health Syst Pharm. 2008 Sep 1;65(17):1655-60. PMID: 18714113.

27. Pai AB, Boyd A, Depczynski J, et al. Reduced drug use and hospitalization rates in patients undergoing hemodialysis who received pharmaceutical care: a 2-year, randomized, controlled study.

Pharmacotherapy. 2009 Dec;29(12):143340. PMID: 19947803.

28. Pai AB, Boyd A, Chavez A, et al. Healthrelated quality of life is maintained in hemodialysis patients receiving pharmaceutical care: a 2-year randomized, controlled study. Hemodial Int. 2009 Jan;13(1):72-9. PMID: 19210281.

29. Park JJ, Kelly P, Carter BL, et al. Comprehensive pharmaceutical care in the chain setting. J Am Pharm Assoc (Wash). 1996 Jul;NS36(7):443-51. PMID: 8840744.

30. Pindolia VK, Stebelsky L, Romain TM, et al. Mitigation of medication mishaps via medication therapy management. Ann Pharmacother. 2009 Apr;43(4):611-20. PMID: 19336646.

31. Planas LG, Crosby KM, Mitchell KD, et al. Evaluation of a hypertension medication therapy management program in patients with diabetes. J Am Pharm Assoc (2003). 2009 Mar-Apr;49(2):164-70. PMID: 19289342.

32. Roughead EE, Barratt JD, Ramsay E, et al. The effectiveness of collaborative medicine reviews in delaying time to next hospitalization for patients with heart failure in the practice setting: results of a cohort study. Circ Heart Fail. 2009 Sep;2(5):424-8. PMID: 19808372.

33. Sellors J, Kaczorowski J, Sellors C, et al. A randomized controlled trial of a pharmacist consultation program for family physicians and their elderly patients. CMAJ. 2003 Jul 8;169(1):17-22. PMID: 12847034.

34. Sidel VW, Beizer JL, Lisi-Fazio D, et al. Controlled study of the impact of educational home visits by pharmacists to high-risk older patients. J Community Health. 1990 Jun;15(3):163-74. PMID: 2195066. 
35. Staresinic AG. Early experience with medication management - Description and preliminary outcomes of a Medicare Part D medication therapy management program. Journal of the Pharmacy Society of Wisconsin (USA). 2007(SEP-OCT):8-0. PMID: 45-01183.

36. Taylor CT, Byrd DC, Krueger K. Improving primary care in rural Alabama with a pharmacy initiative. Am J Health Syst Pharm. 2003 Jun 1;60(11):1123-9. PMID: 12816022 .

37. Touchette DR, Masica AL, Dolor RJ, et al. Safety-focused medication therapy management: a randomized controlled trial. J Am Pharm Assoc (2003). 2012 SepOct;52(5):603-12. PMID: 23023840.

38. Triller DM, Hamilton RA. Effect of pharmaceutical care services on outcomes for home care patients with heart failure. Am J Health Syst Pharm. 2007 Nov 1;64(21):2244-9. PMID: 17959576.

39. Volume CI, Farris KB, Kassam R, et al. Pharmaceutical care research and education project: patient outcomes. J Am Pharm Assoc (Wash). 2001 May-Jun;41(3):411-20. PMID: 11372906.
40. Kassam R, Farris KB, Burback L, et al. Pharmaceutical care research and education project: pharmacists' interventions. J Am Pharm Assoc (Wash). 2001 MayJun;41(3):401-10. PMID: 11372905.

41. Welch EK, Delate T, Chester EA, et al. Assessment of the impact of medication therapy management delivered to homebased Medicare beneficiaries. Ann Pharmacother. 2009 Apr;43(4):603-10. PMID: 19318600.

42. Williams ME, Pulliam CC, Hunter R, et al. The short-term effect of interdisciplinary medication review on function and cost in ambulatory elderly people. J Am Geriatr Soc. 2004 Jan;52(1):93-8. PMID: 14687321.

43. Winston S, Lin YS. Impact on drug cost and use of Medicare part D of medication therapy management services delivered in 2007. J Am Pharm Assoc (2003). 2009 NovDec;49(6):813-20. PMID: 19858047.

44. Witry MJ, Doucette WR, Gainer KL. Evaluation of the pharmaceutical case management program implemented in a private sector health plan. J Am Pharm Assoc (2003). 2011 Sep-Oct;51(5):631-5. PMID: 21896463. 


\section{Appendix G. Meta-analyses}

Figure G1: Effect of MTM on LDL Cholesterol

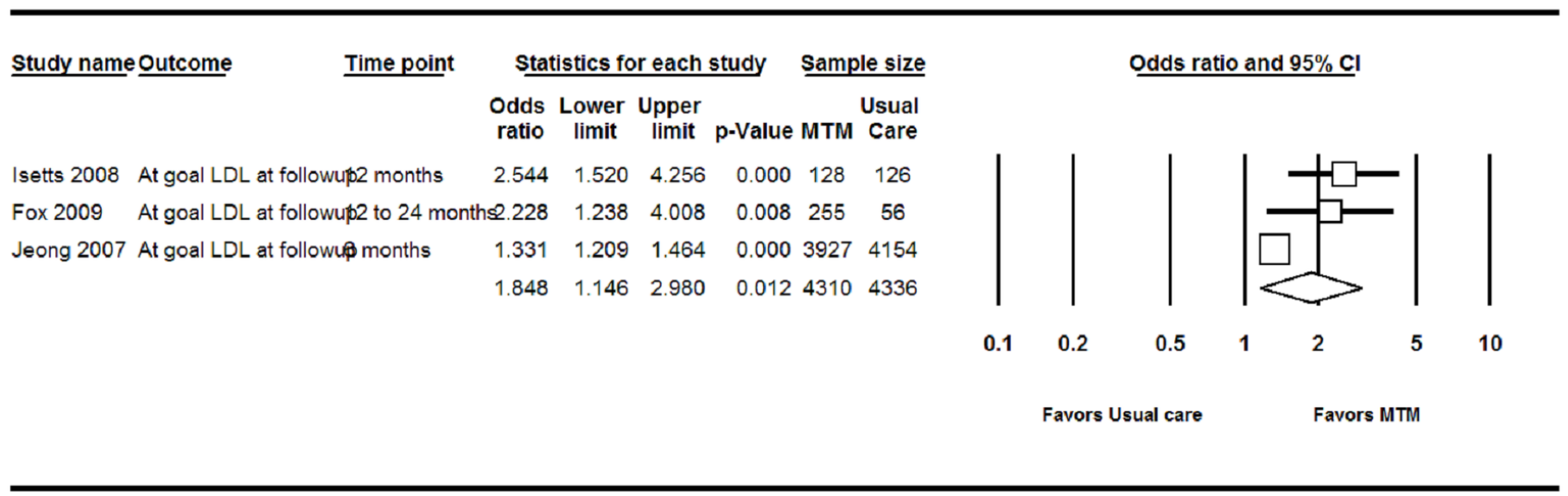

\section{Cohort studies}

Figure G2: Effect of MTM on achieving hypertension goals

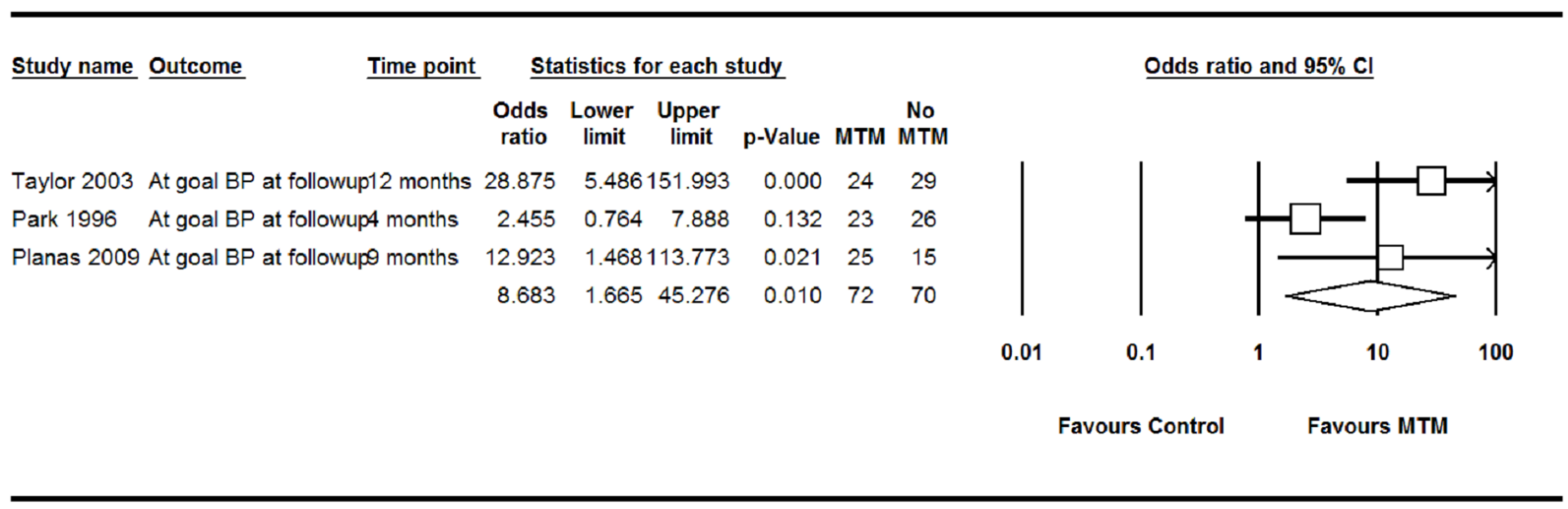

Medium and high risk of bias RCTs 
Figure G3: Effect of MTM on systolic blood pressure

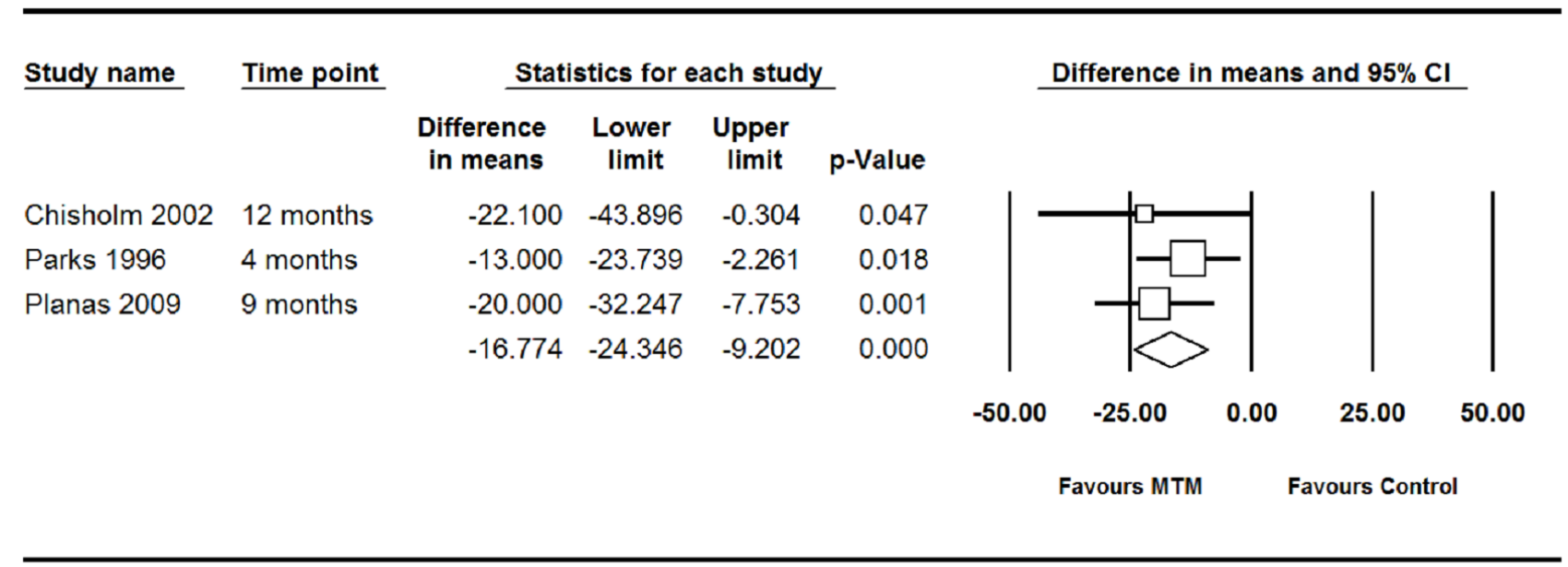

Medium and high risk-of-bias RCTs

Figure G4: Effect of MTM on SF-36 Physical Functioning Domain

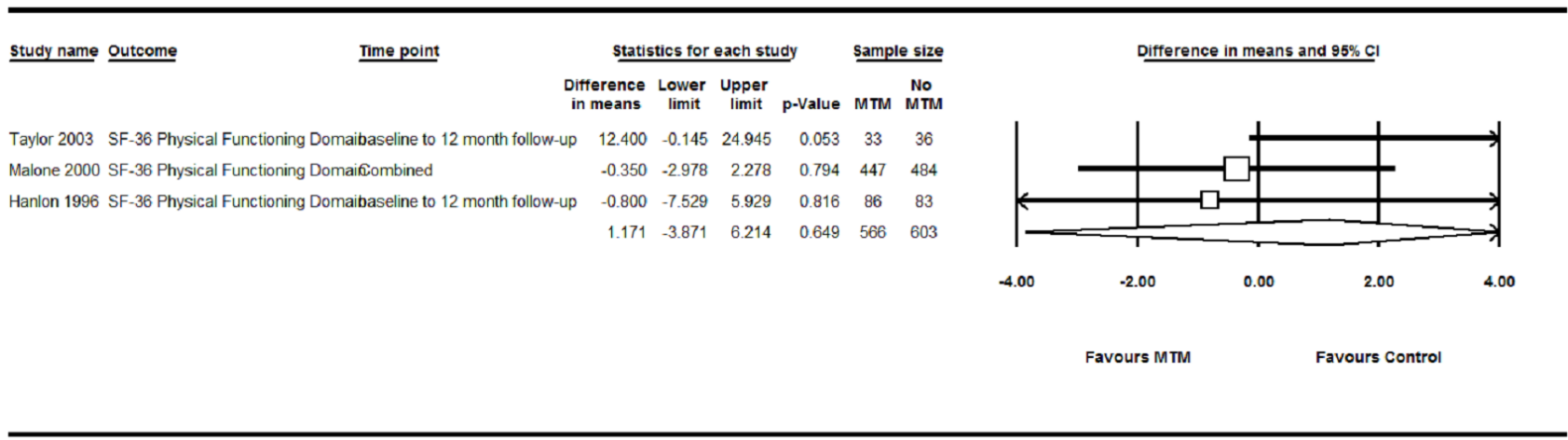

Low or medium risk of bias

Figure G5: Effect of MTM on SF-36 Role Physical Domain

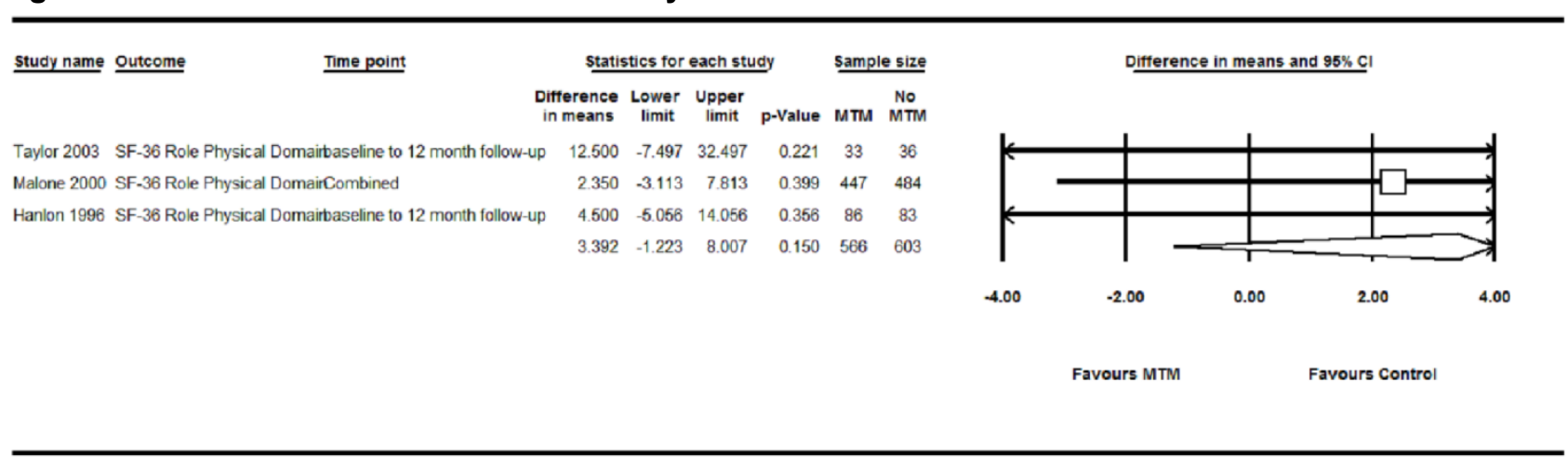

Low or medium risk of bias 
Figure G6: Effect of MTM on SF-36 Bodily Pain Domain

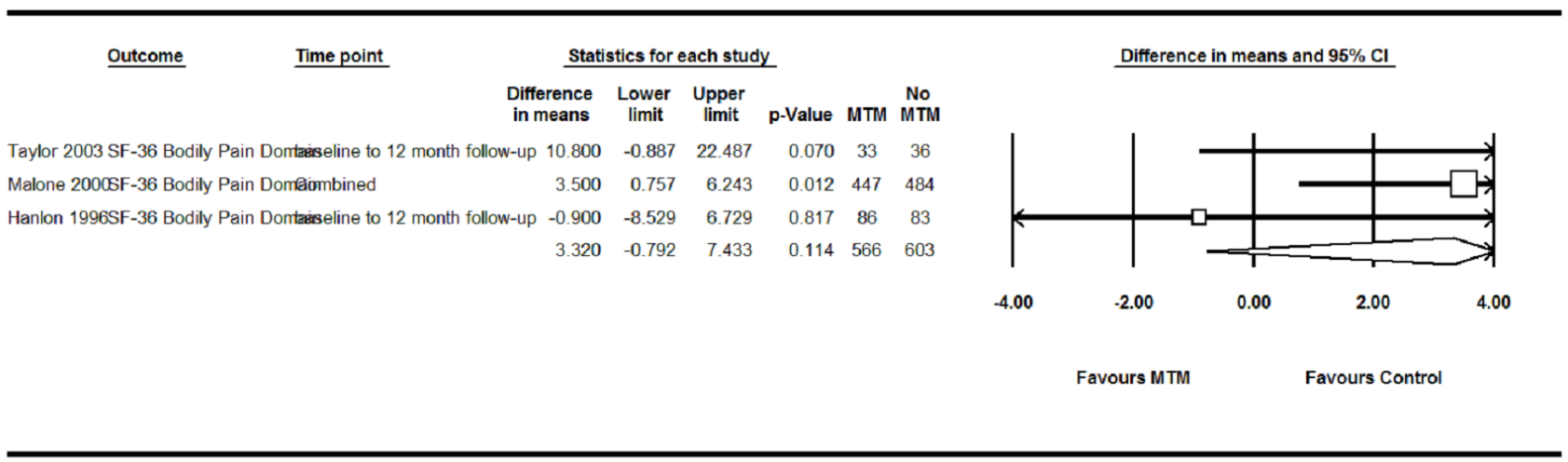

Low or medium risk of bias

Figure G7: Effect of MTM on SF-36 General Health Perception Domain

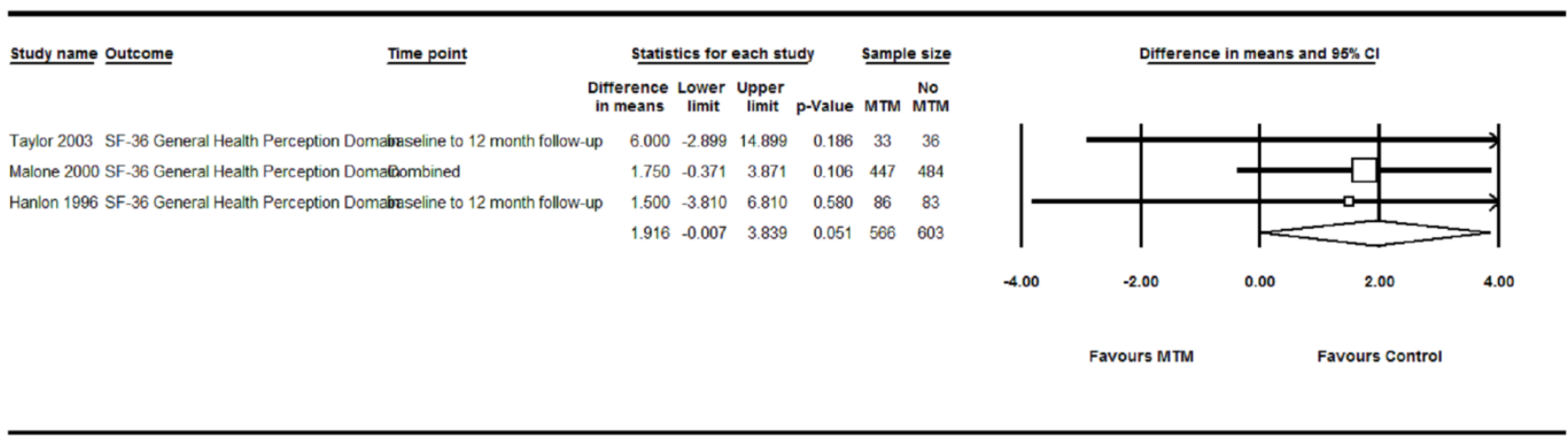

Low or medium risk of bias

Figure G8: Effect of MTM on SF-36 Vitality Domain

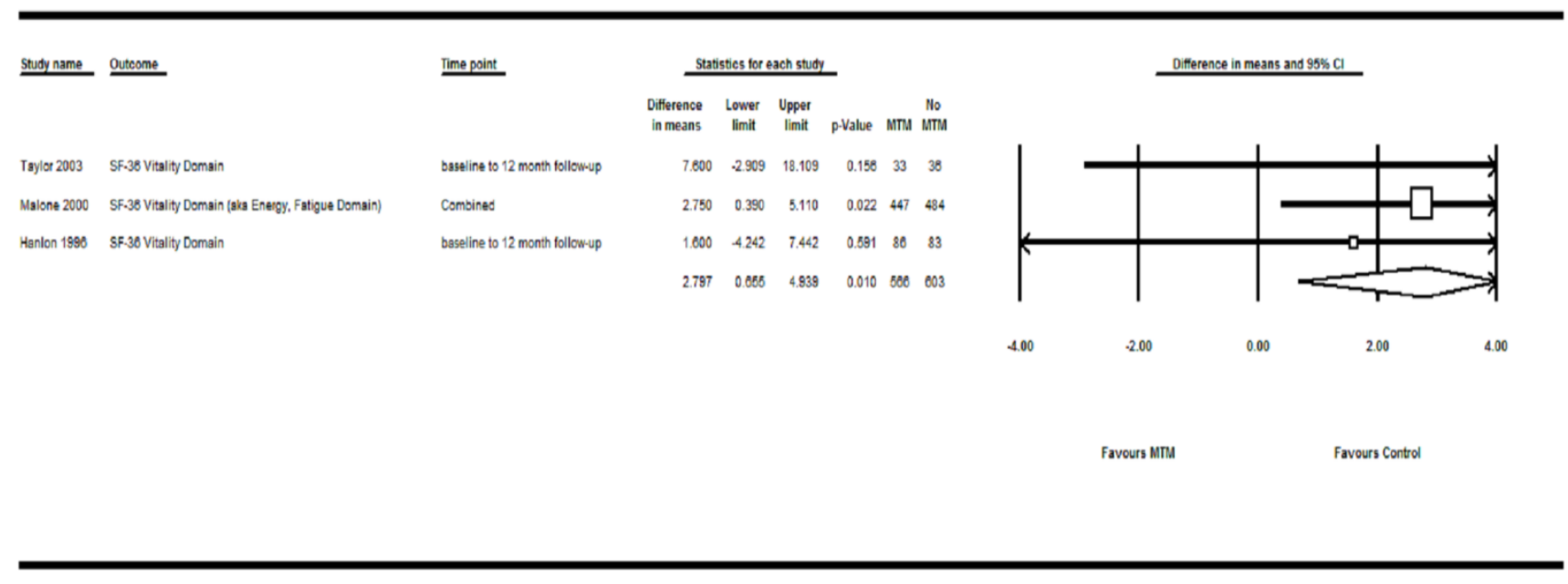


Figure G9: Effect of MTM on SF-36 Social Functioning Domain

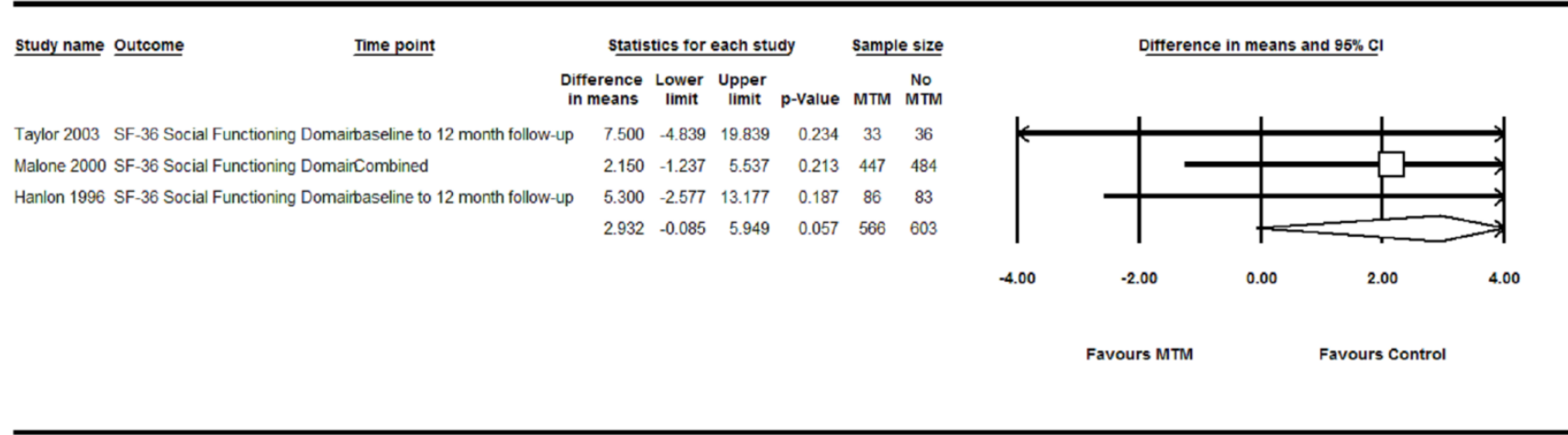

Low or medium risk of bias

Figure G10: Effect of MTM on SF-36 Role Emotional Domain

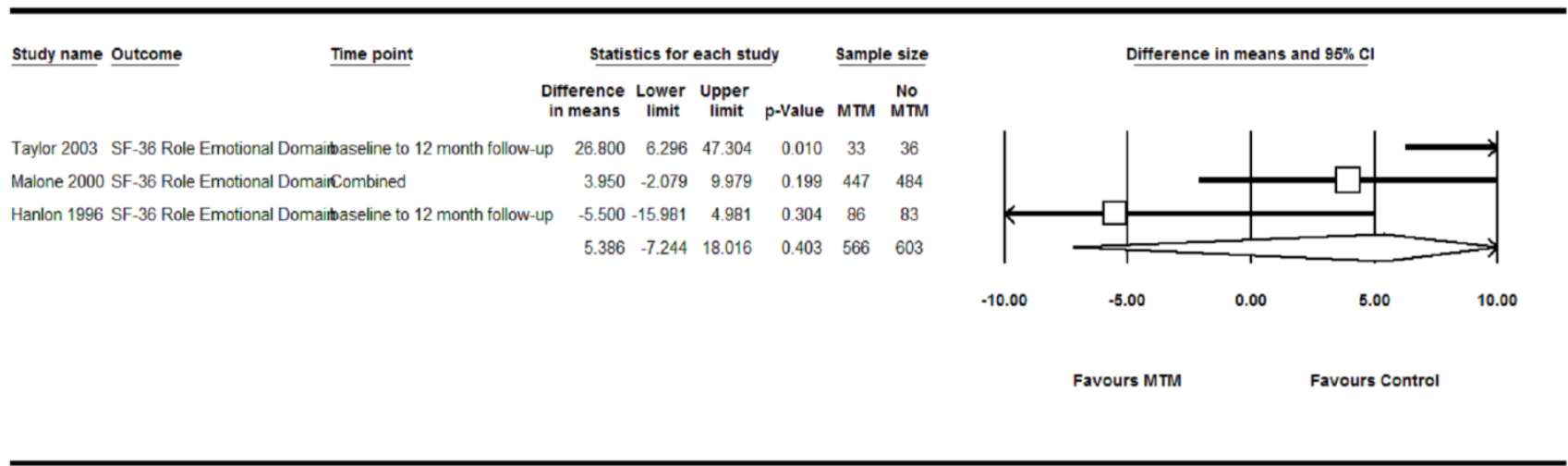

Low or medium risk of bias

Figure G11: Effect of MTM on SF-36 Mental Health Domain

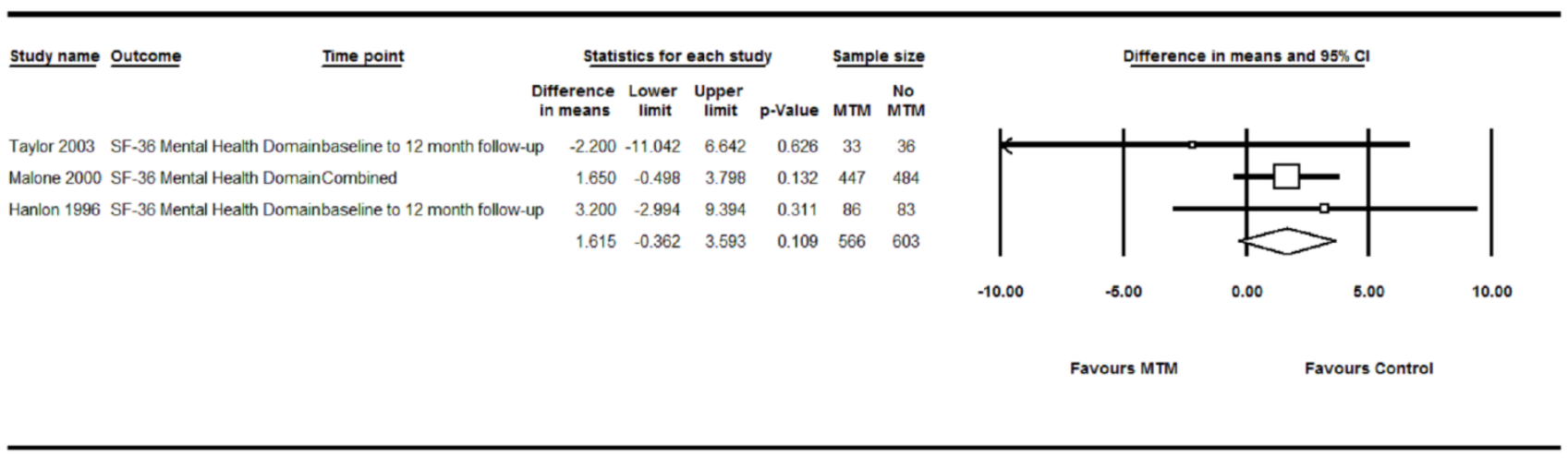

Low or medium risk of bias 
Figure G12: Effect of MTM on health plan expenditures

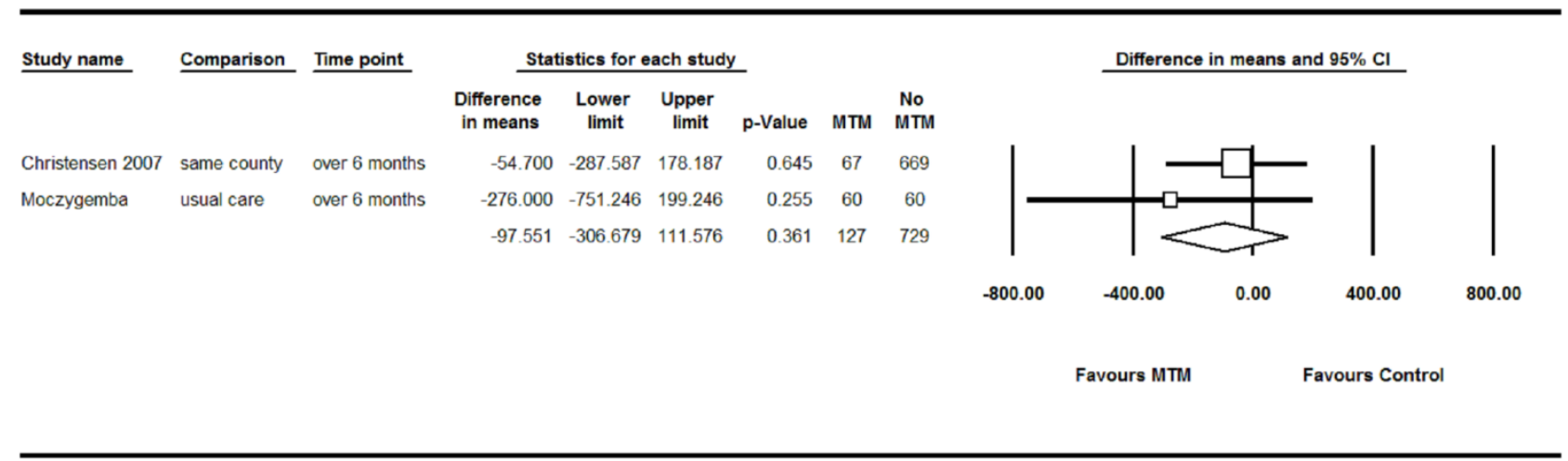

Medium risk-of-bias non-randomized studies

Figure G13: Effect of MTM on outpatient visits

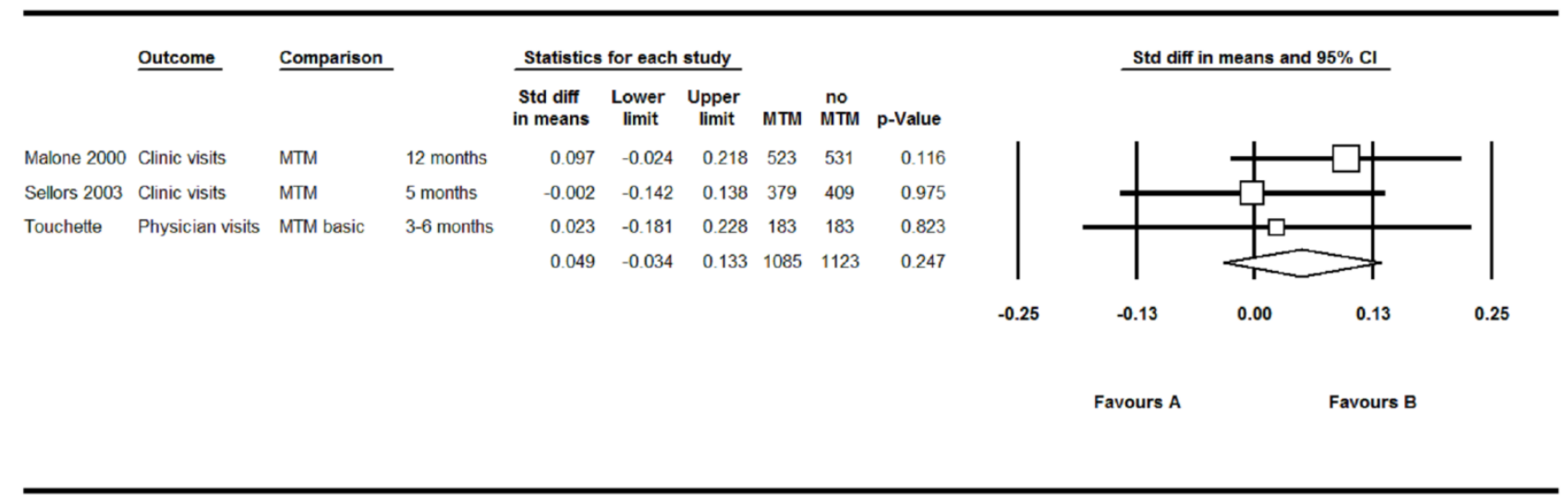

Medium risk-of-bias RCTs

Figure G14: Effect of MTM on mean number of hospitalizations

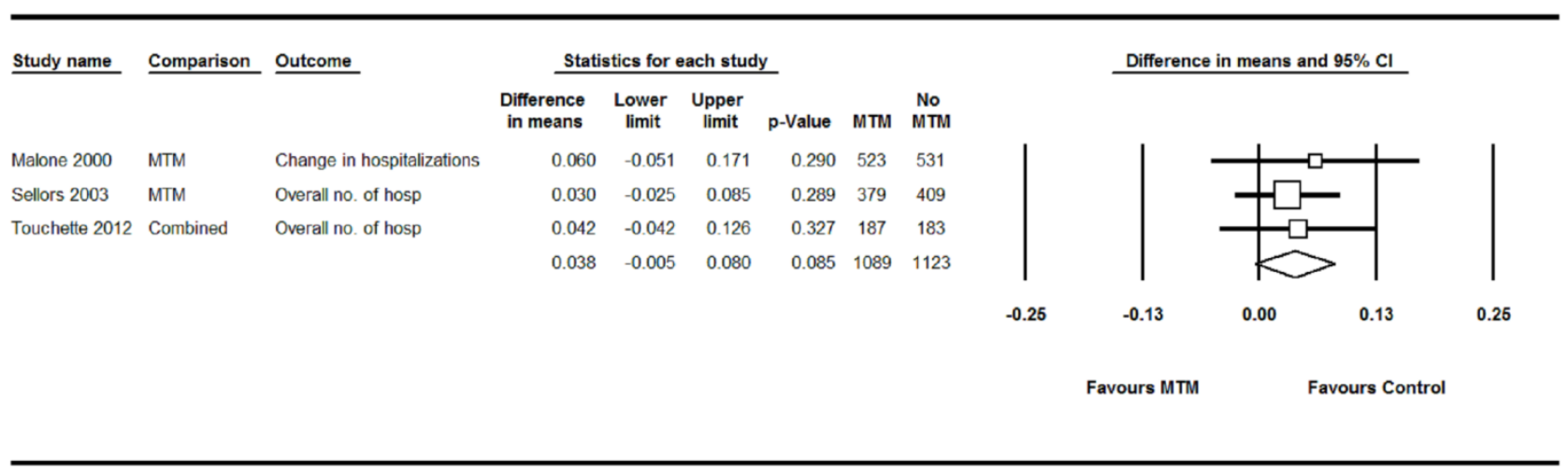

Medium risk-of-bias RCTs 